Portland State University

PDXScholar

\title{
An Empirical Assessment of the Center-Periphery Hypothesis in International Economic Relations
}

Fazlollah Bonakdar Shirazi

Portland State University

Follow this and additional works at: https://pdxscholar.library.pdx.edu/open_access_etds Let us know how access to this document benefits you.

\section{Recommended Citation}

Shirazi, Fazlollah Bonakdar, "An Empirical Assessment of the Center-Periphery Hypothesis in International Economic Relations" (1988). Dissertations and Theses. Paper 1206.

https://doi.org/10.15760/etd.1205

This Dissertation is brought to you for free and open access. It has been accepted for inclusion in Dissertations and Theses by an authorized administrator of PDXScholar. Please contact us if we can make this document more accessible: pdxscholar@pdx.edu. 
AN EMPIRICAL ASSESSMENT OF THE CENTER-PERIPHERY HYPOTHESIS IN INTERNATIONAL ECONOMIC RELATIONS

by

FAZLOLLAH BONAKDAR SHIRAZI

A dissertation submitted in partial fulfillment of the requirements for the degree of

DOCTOR OF PHILOSOPHY

in

SYSTEMS SCIENCE

Portland State University

(c) 1988 


\section{TO THE OFFICE OF GRADUATE STUDIES:}

The members of the Committee approve the dissertation of Fazlollah Bonakdar Shirazi presented May 27, 1988.

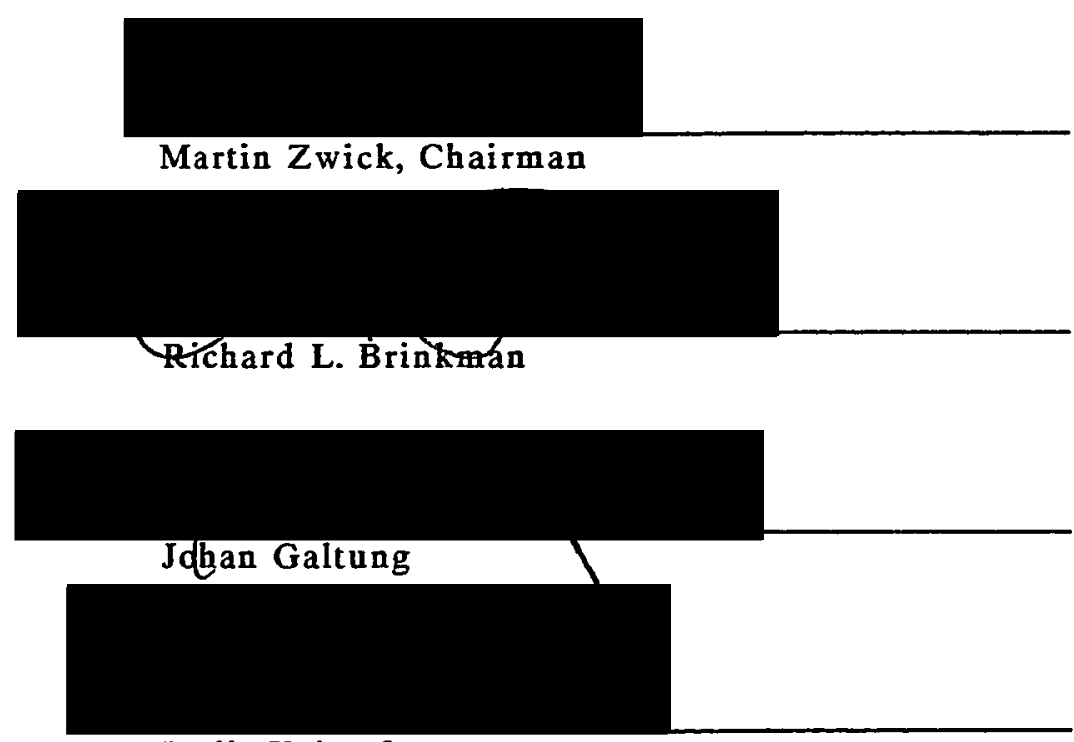

Ladis Kristof

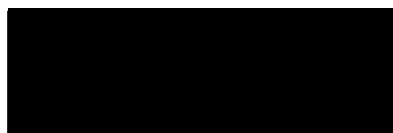

AbduY Qayum

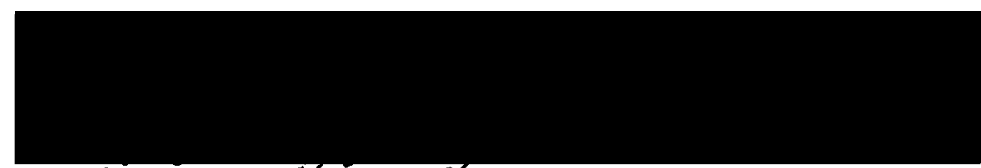

Helen Youngelłon-Neą

\section{APPROVED:}

Martin Zwick, Coordinator, Systems Science

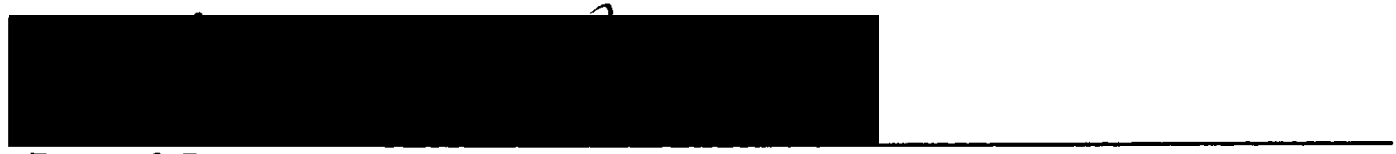

Bernard Ross, Vice Provost for Graduate Studies 
AN ABSTRACT OF THE DISSERTATION OF Fazlollah Bonakdar Shirazi for the Doctor of Philosophy in Systems Science presented May 27, 1988.

Title: An Empirical Assessment of the Center-Periphery

Hypothesis in International Economic Relations.

APPROVED BY MEMBERS OF THE DISSERTATION COMMITTEE:

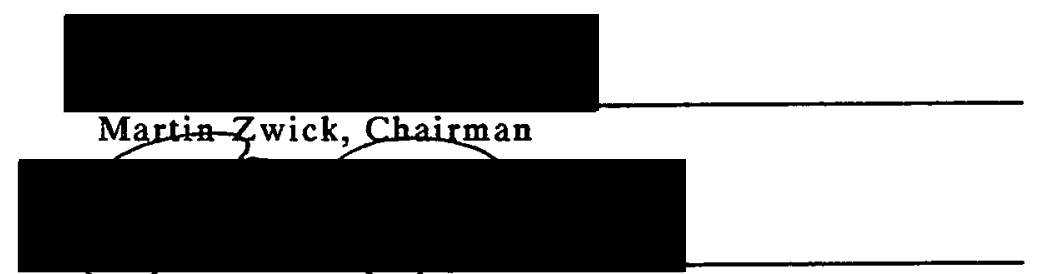

Richard L. Brinkman

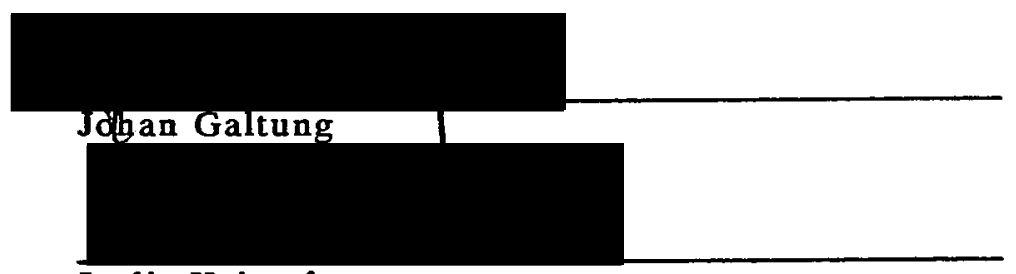

Ladis Kristof

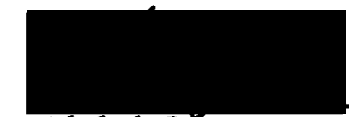

Abdul Qayum

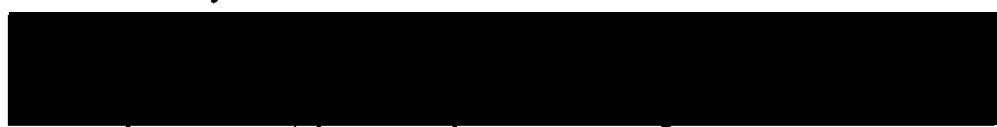

Helen Youngelson-Near

There are two leading perspectives on trade and economic development: the classical view based on the ideas of free trade and comparative advantage, which regards the international division of labor through free trade as supporting eccromic development; and the dependency theory view regards the international 
division of labor as an obstacle to the economic development of the nowunderdeveloped countries. The purpose of this study is to investigate hypotheses advanced by dependency theory, and, more particularly, by Galtung's Structural Theory of Imperialism.

According to Galtung's theory, the world is divided into center and periphery countries, themselves divided into center and periphery sectors. The distinction between center and periphery is based on differences among nations in trade partner concentration, export commodity concentration, vertical trade, and quality of life. A periphery country is said to have most of its trade with one center country, while a center country is free to trade with many partners. A periphery country tends to export a small number of primary products, while a center country has a greater diversity of exports, which are principally manufactured goods. These factors reflect a dependence of the periphery on the center and produce a gap in the quality of life between the two.

The synchronic properties of the center-periphery relationship are tested for 127 countries for the years 1962, 1970, and 1980 with bivariate correlation calculations among ten variables: 1- Trade Partner Concentration (EPC), 2- Total Trade Linkages (TTL), 3- Import Partner Concentration (IPC), 4- Export Commodity Concentration (ECC), 5- Import Commodity Concentration (ICC), 6Vertical Trade (VT), 7- Physical Quality of Life Index (PQLI), 8- Percent share of GDP in Agriculture (AGR/GDP), 9- GNP per capita (GNP), and 10- Export Dependency (ED).

The diachronic properties of the world system at the regional and global levels are investigated by: 1- developing export trade hierarchies to identify center and associated periphery countries; 2- comparing regional and global averages for the national variables; 3- conducting decomposition analysis 
of export/import activity to assess diversities within and among regions; and 4calculating system-wide variables, Global Polarization (GP) and Global Concentration (GC), based also on import/export data.

At the national level, all hypothesized relationships among the ten variables are confirmed (are statistically significant at the .05 level), except for all relationships involving ICC and some relationships involving ED. The ICC results support the contention of Michaely that import and export commodity concentrations are positively correlated, in contradiction to assertions made by Leontief. All correlations between TPC, ECC, VT, and PQLI agree with the propositions of dependency theory.

At the regional level, the study reveals the continued existence of differences between the industrialized. region and the other regions of the world, despite improvements for some regions in some variables (e.g., EPC, VT, and PQLI). Regions are more homogeneous with respect to member countries than the world is with respect to regions. At the global level five major hierarchies (United States, United Kingdom, France, West Germany, and Soviet Union) are identified. From 1962 to 1980, the United States' hierarchy grew, mostly at the expense of that of the United Kingdom. Japan's hierarchy, nonexistent in 1962, emerged strongly by 1980. The systemic variables, GP and GC showed moderate to high, but constant, levels.

No clear trend is apparent over this study period for the world system as a whole. While global averages and averages for the non-industrialized regions show changes in many variables in the direction of reduced world system differentiation, the systemic variables and the results of the decomposition analysis show constancy over time. However, an increased differentiation is suggested by GNP/capita data. 
In summary, although systemic changes over time are complex and individual countries may show ascent or decline, the general pattern of differentiation between center and periphery, as proposed by Galtung and others, holds true for the post World War II period. 


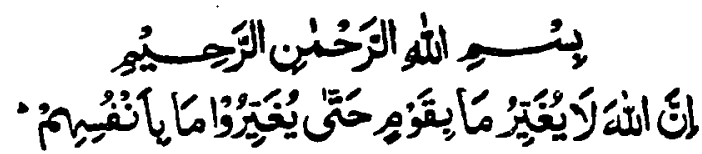

In the name of God, Most Gracious, Most Merciful.

Verily never will God change the condition of a people until they change it themselves. Holy Qur'an, Ra'd 11.

\section{ACKNOWLEDGMENTS}

I am greatly indebted to many people in this endeavor. I was privileged to have a thesis committee that included Abdul Qayum, Richard Brinkman, Johan Galtung, Helen Youngelson-Neal, and Ladis Kristof. I am very appreciative of their support and criticisms and I thank them all. I am especially indebted to my chairman Martin Zwick, whose guidance and drive for excellence helped create this thesis out of my often chaotic manuscripts. (But I take full responsibility for its content.) My deepest appreciation and thanks for his support which extended throughout all my academic years at Portland State.

I also thank the Mostazafan Foundation of New York and Systems Science Ph.D. Program for their moral and generous financial support without which completing this task would have been impossible.

In the not-so-modern world system in which I live in, it is no hypothesis that I (the "center") intellectually benefited from graduate experience, while my wife Mahroo, and my children, Sara and Amir, and my mother Zivar suffered the most (always the fate of the "periphery"). I thank them very much for their great endurance and support and hope to be able to share any benefits that I may have gained with them.

In the real world system, where there is tremendous resistance to change, I believe (and am saddened by this belief) that it is already too late to expect a 
voluntary restructuring based on principles of equity and justice. Any change in the existing center-periphery structure will require an increased awareness in the periphery of the mechanisms and properties of the world system. I hope that this work contributes to this end, however small the contribution may be. Therefore, I wish to dedicate this work to those at the periphery of the periphery who have suffered the most and who are the ones who will have to change this structure (hopefully) in a not too distant future. 


\title{
TABLE OF CONTENTS
}

\begin{abstract}
PAGE
ACKNOWLEDGMENTS $\ldots \ldots \ldots \ldots \ldots \ldots \ldots \ldots \ldots \ldots \ldots \ldots \ldots \ldots \ldots \ldots$

LIST OF TABLES $\ldots \ldots \ldots \ldots \ldots \ldots \ldots \ldots \ldots \ldots \ldots \ldots \ldots \ldots \ldots \ldots \ldots$

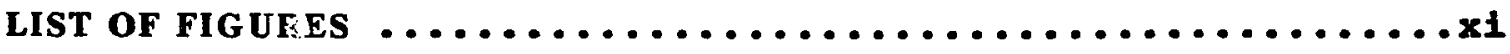

CHAPTER
\end{abstract}

I- INTRODUCTION $\ldots \ldots \ldots \ldots \ldots \ldots \ldots \ldots \ldots \ldots \ldots \ldots \ldots \ldots$

- The Classical View .....................4

- The Dependency Theory View ................

- Domain of the Investigation $\ldots \ldots \ldots \ldots \ldots \ldots \ldots \ldots$

- Organization of the Study $\ldots \ldots \ldots \ldots \ldots \ldots \ldots$

II- LITERATURE REVIEW ...................... 11

- Mercantile Capitalism and The First

European Expansion(1500-1870) .............11

- The New Imperialism and The Second

European Expansion(1870-1914) ............17

- The Age of Neocolonialism(1947-Present) $\ldots \ldots \ldots \ldots . . .25$

- Classical Theories of Development and

Colonialism ...................27

- Adam Smith ....................27

- John Stuart Mill ..................28

- Classical Thecries of Imperialism $\ldots \ldots \ldots \ldots \ldots \ldots . . .29$

- J. A. Hobson $\ldots \ldots \ldots \ldots \ldots \ldots \ldots \ldots \ldots \ldots \ldots \ldots . \ldots \ldots$

- V. I. Lenin ...................... 32

- Structural Theory of Imperialism $\ldots \ldots \ldots \ldots \ldots \ldots \ldots . \ldots$

- J. Galtung ............................ 34 
- Structural Theory of Cumulative Causation ........40

- G. Myrdal $\ldots \ldots \ldots \ldots \ldots \ldots \ldots \ldots \ldots \ldots \ldots \ldots$

- The Economic Commission for Latin America and the Dependency Theory $\ldots \ldots \ldots \ldots \ldots \ldots \ldots$

- R. Prebisch $\ldots \ldots \ldots \ldots \ldots \ldots \ldots \ldots \ldots \ldots \ldots \ldots$. . . . . 1

- T. Dos Santos ....................42

- A. G. Frank ....................... 44

- World Systems Analysis ..................46

- I. Wallerstein $\ldots \ldots \ldots \ldots \ldots \ldots \ldots \ldots \ldots \ldots \ldots$

- Previous Tests of Dependency Theory $\ldots \ldots \ldots \ldots \ldots . .47$

III- METHODOLOGY $\ldots \ldots \ldots \ldots \ldots \ldots \ldots \ldots \ldots \ldots \ldots$

- Operationalizing the National Measures ...........54

- Export Partner Concentration $\ldots \ldots \ldots \ldots \ldots \ldots \ldots . \ldots 56$

- Total Trade Linkages ..................58

- Import Partner Concentration .............58

- Export Commodity Concentration ...........59

- Import Commodity Concentration ........... 59

- Vertical Trade .....................660

- Economic Dependence on Agriculture ..........61

- Physical Quality of Life Index ...........61

- GNP/Capita .......................62

- Export Dependency .................66

- Operationalizing the System-wide Studies $\ldots \ldots \ldots \ldots . .63$

- Trade Hierarchies ..................64

- Regional Averages $\ldots \ldots \ldots \ldots \ldots \ldots \ldots \ldots \ldots$

- Regional Decomposition ..............66

- Global Polarization ...................

- Global Concentration .................69

- Data Sources .........................

- Present Study ...................... 71

- National Study ....................... 71

- System-wide Study $\ldots \ldots \ldots \ldots \ldots \ldots \ldots \ldots \ldots$

IV- RESULTS OF NATIONAL STUDIES $\ldots \ldots \ldots \ldots \ldots \ldots$

- Discussion of Correlation Results $\ldots \ldots \ldots \ldots \ldots \ldots \ldots 77$ 
- Diachronic Investigations .................80

- Trade Partner Concentration .............80

- Total Trade Linkages ..................82

- Concentration of Exports and Imports ...........91

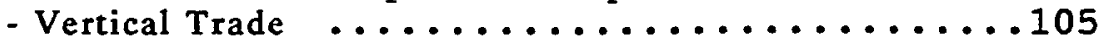

- Physical Quality of Life Index ............... 109

- Economic Dependence on Agriculture ..........114

- GNP Per Capita . . . . . . . . . . . . . . . 119

- Export Dependency ...................121

- Ascent and Decline in the World System ...........121

- Deviations from Center-Periphery

Hypothesis . . . . . . . . . . . . . . . 127

\section{V- RESULTS OF THE SYSTEM-WIDE AND}

REGIONAL STUDIES . . . . . . . . . . . . . . . . 129

- International Trade Hierarchies ...............129

- Results of Regional Comparisons .............148

- Regional Averages ................... 148

- Regional Diversity Calculations ............. 160

- Results of System-wide Studies .............. 168

- Global Polarization ...................168

- Global Concentration .................168

- Global Averages .....................169

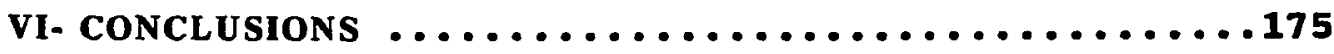

- Theoretical Context....................175

- Discussion of National Hypotheses .............179

- Discussion of Global Investigations............. 182

- Directions for Future Research.............. 187

BIBLIOGRAPHY $\ldots \ldots \ldots \ldots \ldots \ldots \ldots \ldots \ldots$

APPENDIX A- COUNTRIES USED IN THE STUDY ................. 194

APPENDIX B- COMPARISON OF CALCULATIONS BETWEEN ENTROPY AND

HIRSCHMANN INDECES OF CONCENTRATION ......... 199

APPENDIX C- CORRELATION RESULTS (OIL EXPORTING COUNTRIES

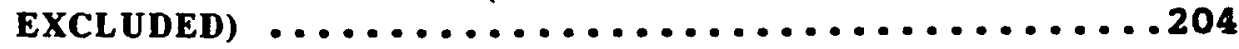

APPENDIX D- SUMMARY OF NATIONAL STATISTICS .............224 


\section{LIST OF TABLES}

TABLE

PAGE

I- Some Quantitative Indicators of

Global Differentiation .....................

II- Gaps in Terms of Real GNP per Capita .................

III- Multilateral Trade in the 18 th Century $\ldots \ldots \ldots \ldots \ldots \ldots \ldots$

IV-Summary of Variables .......................55

V-Statistical Sources $\ldots \ldots \ldots \ldots \ldots \ldots \ldots \ldots \ldots \ldots \ldots \ldots$

VI- Variables and Their Data Sources ..................73

VII- Countries Ranked by Export Partner Concentration

A- Year $1938 \ldots \ldots \ldots \ldots \ldots \ldots \ldots \ldots \ldots \ldots$

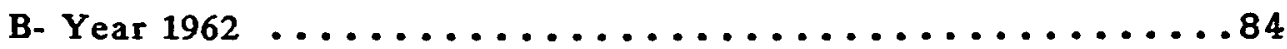

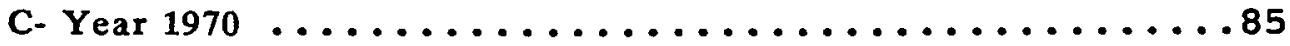

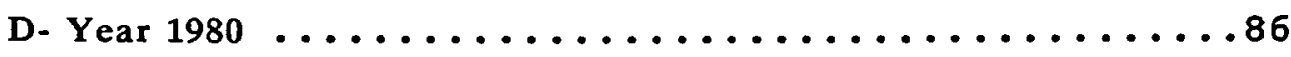

VIII- Countries Ranked by Import Partner Concentration

A- Year $1938 \ldots \ldots \ldots \ldots \ldots$. . . . . . . . . . . . . . 87

B- Year $1962 \ldots \ldots \ldots \ldots \ldots \ldots$. . . . . . . . . . . . 888

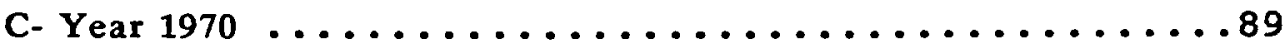

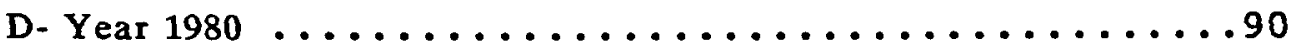

IX- Countries Ranked by Total Trade Linkages
A- Year 1938
.92
B- Year $1962 \ldots \ldots \ldots \ldots \ldots \ldots$

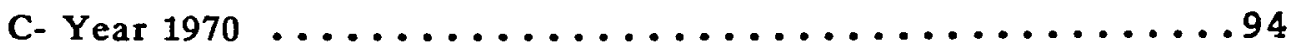

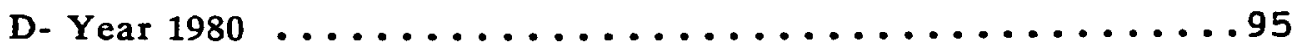


X- Countries Ranked by Export Commodity Concentration
A- Year 1962
.97
B- Year 1970 .98
C- Year 1980 .99

XI- Countries Ranked by Import Commodity Concentration
A- Year 1962
.............................. 100
B- Year 1970 .101
C- Year 1980

XII- Countries Ranked by Vertical Trade
A- Year 1962 .106

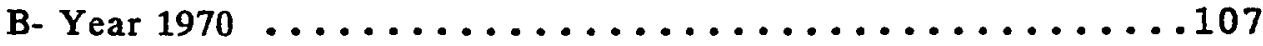
C- Year $1980 \ldots \ldots \ldots \ldots \ldots \ldots \ldots$........................ 108

XIII- COUNTRIES RANKED BY P.Q.L.I.
A- Year 1962
B- Year 1970
C- Year 1980 .113

XIV-A Average PQLI in Top Ten EPC Rankings in 1962 . . . . . . . . 115

XIV-B Average PQLI in Bottom Ten EPC Rankings in 1962 . . . . . . 115

XV-Countries Ranked by AGR in GDP
A- Year 1962
B- Year 1970
C- Year 1980 
XVI- Countries Ranked by GNP/Capita

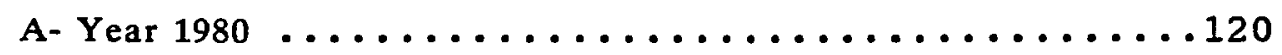

XVII- Countires Ranked by Percent GDP to Largest Market

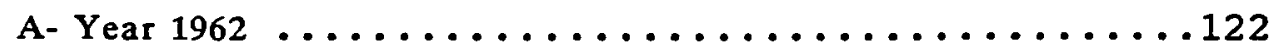

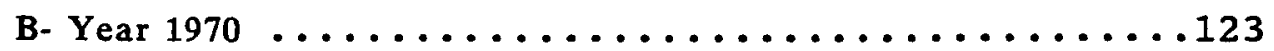

C. Year $1980 \ldots \ldots \ldots \ldots \ldots \ldots \ldots \ldots$. . . . . . . . . . . . 124

Summary of Regional Statistics

XVIII- Export Partner Concentration . . . . . . . . . . . . . . . . 149

XIX-Import Partner Concentration $\ldots \ldots \ldots \ldots \ldots \ldots 1$

XX-IMP/EXP Ratios ........................ 152

XXI- Total Trade Linkages ....................... . 153

XXII- Export Commodity Concentration ................. 155

XXIII-Import Commodity Concentration $\ldots \ldots \ldots \ldots \ldots \ldots \ldots \ldots$

XXIV-Vertical Trade Statistics $\ldots \ldots \ldots \ldots \ldots \ldots \ldots \ldots$.............. 158

XXV-Quality of Life $\ldots \ldots \ldots \ldots \ldots \ldots . \ldots \ldots \ldots$

XXVI- Economic Dependance on Agriculture $\ldots \ldots \ldots \ldots \ldots 16 \ldots \ldots$

XXVII- Summary of Regional Diversity Calculations

A- Export Diversities .......................... 162

B-Import Diversities $\ldots \ldots \ldots \ldots \ldots \ldots \ldots 2$

XXVIII- Summary of Regional Diversity Calculations

A- Export, Year 1962 ... . . . . . . . . . . . . . 163

B- Import, Year $1962 \ldots \ldots \ldots \ldots \ldots \ldots$. . . . . . . . . 163

XXIX-Summary of Regional Diversity Calculations

A- Export, Year 1970 ... . . . . . . . . . . . . . . 164

B-Import, Year $1970 \quad \ldots \ldots \ldots \ldots \ldots \ldots$. . . . . . . . 164 
XXX-Summary of Regioanl Diversity Calculations

A- Export, Year $1980 \ldots \ldots \ldots \ldots \ldots \ldots$

B- Import, Year 1980 . . . . . . . . . . . . . . . 165

XXXI- Summary of Global Statistics ................ 172 


\section{LIST GF FIGURES}

$\begin{array}{ll}\text { FIGURE } & \text { PAGE }\end{array}$

1- Studies of Center-Periphery Hypothesis ...................49

2- Previous Tests of the Dependency Theory $\ldots \ldots \ldots \ldots \ldots \ldots \ldots \ldots \ldots \ldots$

3- Static Input-Output Table of International Trade Flows $\ldots \ldots \ldots \ldots \ldots \ldots$

4- Correlation Analysis $\ldots \ldots \ldots \ldots \ldots \ldots \ldots \ldots \ldots \ldots \ldots \ldots \ldots \ldots$

5- Summary of Pearson Correlation Results Among Variables ..........78

6- Correlation Coefficients Between TPC and TTL $\ldots \ldots \ldots \ldots \ldots$

7- National Variables for South Korea ..................... 126

8- The World Trade Hierarchies in Year $1938 \ldots \ldots \ldots \ldots \ldots \ldots$

9- United States Trade Hierarchies

1- 1962 Trade Hierarchy ........................133

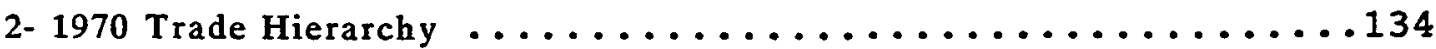

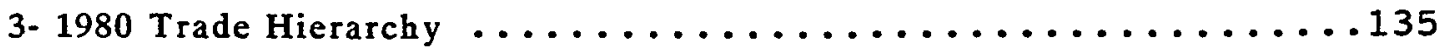

10- United Kingdom Trade Hierarchies

1- 1962 Trade Hierarchy . . . . . . . . . . . . . . . . . 138

2- 1970 Trade Hierarchy ... . . . . . . . . . . . . . . . 139

3- 1980 Trade Hierarchy ..........................139

11- German Trade Hierarchies

1- 1962 Trade Hierarchy .........................141

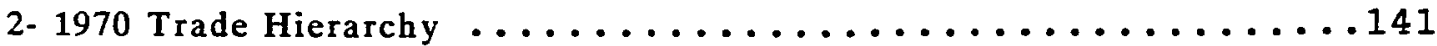

3- 1980 Trade Hierarchy $\ldots \ldots \ldots \ldots \ldots \ldots \ldots \ldots \ldots \ldots$. . . . . . . . 141 
12- French Trade Hierarchies $\quad$ xiii

1- 1962 Trade Hierarchy ..........................142

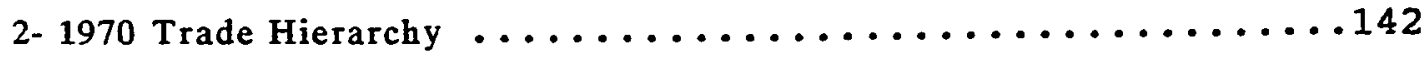

3- 1980 Trade Hierarchy ......................... 142

13- Soviet Union Trade Hierarchies

1- 1962 Trade Hierarchy ..........................144

2- 1970 Trade Hierarchy ... . . . . . . . . . . . . . . . . . . 144

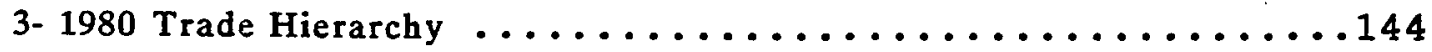

14- Center/Periphery-Capitalist/Socialist Axes $\ldots \ldots \ldots \ldots \ldots \ldots \ldots \ldots$

15- Global Measures of Polarization and Concentration $\ldots \ldots \ldots \ldots \ldots \ldots \ldots$

16- Global Averages for National Variables .................. 171 


\section{CHAPTER I}

\section{INTROD UCTION}

Since the colonial era, which came to an end around the Second World War, newly independent countries have become the focus of world attention. Due to their colonial history and newly acquired political independence, many poor countries have expressed discontent with their "dependence" on producing primary products for export markets. Such dependence has often been seen as resulting from "foreign domination" or "exploitation"1.

It has also become customary to treat global disparities of income in terms of differences between "rich" industrialized countries and "poor" primaryproducing countries. The gap in welfare and income between the few developed industrialized countries and the many underdeveloped countries has been the focus of many recent studies. These studies $2,3,4$ suggest that extensive global differentiation is a phenomenon of the last several centuries in the development of a world economy which began with European overseas expansion and the

1- G.M. Meier, Leading Issues in Economic Development (N.Y. :Ox ford University Press, 1984), F. 5.

2- P. Donaldson, Worlds Apart: The Economic Gulf Between Nations (New York: Viking Press, 1973)

3- P. Bairoch, Disparities in Economic Development Since the Industrial Revolution (St. Martin Press, 1978).

4- S. Patel, "The Economic Distance Between Nations: Its Origin, Measurement, and Outlook," Economic Journal, (March 1974), p. 119-131. 
articulation of an international economy based on the flow of goods and people to and from Europe.

Moreover, it has been said that the existing gap between developed and underdeveloped countries in terms of income have been widening, suggesting an ever increasing differentiation of the world system. For example, recent study by Bairoch (Tables $I$ and II) indicates that the GNP per capita ratio between poor and rich countries was 1:2 prior to the industrial revolution ${ }^{5}$. Prior to industrialization, rich countries like the United States and Jamaica had per capita GNPs in the range of $\$ 200-\$ 300$ whereas poor countries were in the $\$ 90-\$ 200$ range. When an economic region such as Europe was compared to India and China, the gap was reduced to a ratio of $1: 1.2-1.3^{6}$. However, by the late $1970 \mathrm{~s}$, the gap widened to $1: 30$ as suggested in Table II.

Countries that are today as economically diverse as France, Sweden, Egypt, Iran, and Mexico all had similar standards of living at one time ${ }^{7}$. The data in Table I, therefore, suggest that since 1800 there has existed an ever-increasing differentiation within the capitalist world system that has produced well-being at the center (the developed countries), and relative impoverishment at the periphery (the underdeveloped countries) ${ }^{8}$. The data show systemic differentiation: the developed countries have high incomes per capita, relatively low inequality, and small proportions of their labor forces in agriculture; the underdeveloped

5- P. Bairoch, and Levy-Leboyer, M. Disparities in Economic Development Since the Industrial Revolution (St. Martin Press, 1978), p. 3-17.

6- Ibid. p. 8.

7- Ibid. p. 8.

8- H. Addo, "Foreign Policy Strategies for Achieving the NIEO: A Third World Perspective," in C.W. Kegley, Jr. and P. McGowan(eds.) The Political Economy of Foreign Policy Behavior (Beverly Hills: Sage, 1980) 
TABLE I

SOME QUANTITATIVE INDICATORS OF GLOBAL DIFFERENTIATION

\begin{tabular}{|c|c|c|c|c|c|}
\hline Country & \multicolumn{2}{|c|}{$\begin{array}{l}\text { GNP per Capita } \\
\text { before the } \\
\text { ndustrial Revolution }\end{array}$} & $\begin{array}{l}\text { GNP per } \\
\text { Capita } \\
1977^{b}\end{array}$ & $\begin{array}{l}\text { Percentage } \\
\text { of Population } \\
\text { with one-half of } \\
\text { National Income } \\
\text { early } 1960 \mathrm{~s}^{c}\end{array}$ & $\begin{array}{c}\text { percentage } \\
\text { of Population } \\
\text { in Agriculture } \\
1970^{d} \text {. }\end{array}$ \\
\hline \multicolumn{6}{|l|}{ I. "Rich": } \\
\hline U.K. & 1700 & $\$ 160-200$ & $\$ 3976$ & $47.3 \%$ & $3.0 \%$ \\
\hline U.S.A. & 1710 & $200-260$ & 8188 & 42.0 & 4.0 \\
\hline France & $1780-1790$ & $170-200$ & 6728 & 33.6 & 15.0 \\
\hline Sweden & 1860 & $190-230$ & 8690 & 46.5 & 9.0 \\
\hline Japan & 1885 & $160-200$ & 5155 & 40.0 & 17.0 \\
\hline $\begin{array}{l}\text { U.S.S.R. } \\
\text { 11. "Poor" }\end{array}$ & 1860 & $160-200$ & 3835 & - & 32.0 \\
\hline Egypt & 1887 & $170-200$ & 285 & 27.4 & 57.0 \\
\hline Ghana & 1891 & $90-150$ & 352 & - & 58.0 \\
\hline India & 1800 & $160-210$ & 140 & 35.0 & 73.0 \\
\hline Iran & 1900 & $140-200$ & 1846 & - & 42.0 \\
\hline Jamaica & 1832 & $240-280$ & 1033 & 13.2 & 36.0 \\
\hline Mexico & 1803 & $160-260$ & 1055 & 15.9 & 46.0 \\
\hline Philippine & es 1902 & $170-210$ & 419 & 24.8 & 57.0 \\
\hline
\end{tabular}

SOURCE:

a- P. Bairoch (1979: 147) values in 1960 U.S. dollars

b- U.S. Arms Control and Disarmament Agency (1979)

c- Taylor and Hudson (1972: 263-265)

d- U.S. Department of State

TABLE II

GAPS IN TERMS OF REAL GNP PER CAPITA (1962 US DOLlars)

\begin{tabular}{|c|c|c|c|c|c|c|c|}
\hline & veloped & \multirow{3}{*}{$\begin{array}{l}\text { countries } \\
\text { Most } \\
\text { developed } \\
\text { B }\end{array}$} & \multicolumn{2}{|c|}{ Third World } & \multicolumn{3}{|c|}{$\begin{array}{l}\text { Gaps between developed } \\
\text { countries and 3rd world }\end{array}$} \\
\hline & \multirow{2}{*}{$\begin{array}{c}\text { Total } \\
\text { A }\end{array}$} & & \multirow{2}{*}{$\begin{array}{c}\text { Total } \\
\text { C }\end{array}$} & \multirow{2}{*}{$\begin{array}{c}\text { Less } \\
\text { developed } \\
D\end{array}$} & \multirow[b]{2}{*}{$A / C$} & \multirow[b]{2}{*}{$B / C$} & \multirow[b]{2}{*}{$B / D$} \\
\hline & & & & & & & \\
\hline 1750 & 182 & 230 & 188 & 130 & 1.0 & 1.2 & 1.8 \\
\hline 1800 & 198 & 240 & 188 & 130 & 1.1 & 1.3 & 1.8 \\
\hline 1830 & 237 & 360 & 183 & 130 & 1.3 & 2.0 & 2.8 \\
\hline 1860 & 324 & 580 & 174 & 130 & 1.9 & 3.3 & 4.5 \\
\hline 1913 & 662 & 1350 & 192 & 130 & 3.4 & 7.0 & 10.4 \\
\hline 1950 & 1054 & 2420 & 203 & 135 & 5.2 & 11.9 & 17.9 \\
\hline 1960 & 1453 & 2800 & 250 & 140 & 5.8 & 11.2 & 20.0 \\
\hline 1970 & 2229 & 3600 & 308 & 140 & 7.2 & 11.7 & 25.7 \\
\hline 1977 & 2737 & 4220 & 355 & 145 & 3.7 & 11.9 & 29.1 \\
\hline
\end{tabular}


countries have low incomes that are unequally distributed and a largely agricultural population 9 .

In order to formulate a development theory that could explain tòe sharp differences between developed and underdeveloped countries, it is of fundamental importance to give attention to those aspects of their economic and social histories that gave rise to such systemic inequality in the world system.

There are two leading perspectives on trade and development. The ClassicalNeoclassical view argues that free trade based upon comparative advantage promotes the economic development of both developed and underdeveloped countries. The dependency theory view argues that the economic well-being of the now developed countries has been attained at the expense of underdeveloped countries through the mechanisms of a world capitalist system over the past several centuries. These contrasting views are presented below:

\section{THE CLASSICAL VIEW OF ECONOMIC DEVELOPMENT}

Classical economic theory received its inspirations from the writings of Adam Smith, David Ricardo, J.S. Mill, and Alfred Marshall.

The orthodox interpretation was that foreign trade could be a leading force in the process of development. On this notion, Marshall went so far as to state that "the causes which determine the economic progress of nations belong to the study of international trade ${ }^{10}$.

The two major ideas that emerged from the writings of Smith and Mill regarding the international trade were: the "vent for surplus" theory, which stated

9- P.J. McGowan, and B. Kordan, "Imperialism in World system Perspective: Britain 1870-1914," in World System Structure: Continuity and Change Edited by: W.L. Hollist, and J.N., Rosenau (Beverly Hills:Sage, 1981), p.57.

10- A. Marshall, Principles of Economics (London: 1920), 8th ed.,p. 270. 
that international trade overcame the narrowness of the domestic market and provided an outlet for the surplus above the domestic requirements; and the "productivity" theory, which stated that by widening the extent of the market, international trade would improve the division of labor and raise the general level of productivity within a country.

According to these views, if trade increased the capacity for development, then the larger the volume of trade, the greater the potential for development should be. Therefore, the leading factors that promote economic well-being and increase national income are the country's ability to participate in international trade and the international division of labor because these factors permit countries to specialize and to trade commodities that they can produce cheaply in exchange for commodities that their trading partners can produce cheaply.

This theory further states that free trade would raise the participating countries' level of welfare, as well as to lead to a factor price equalization between countries. The wage differences between the developed and the less developed countries would, for example, be reduced, which in turn, would lead to a more equal international distribution of income.

In summary, the positive view of trade and development thus emphasizes the direct gain that came from international specialization plus additional support to a country's development through a number of spread effects within the domestic economy. The fundamental contention of classical-neoclassical trade theory is that real income of each country would be higher with trade than without ${ }^{11}$.

11- G.M. Meier, Leading Issues in Economic Development ( N.Y.: Oxford University Press, 1984), p. 492. 
THE DEPENDENCY THEORY VIEW OF ECONOMIC DEVELOPMENT

The formation of dependency theory was inspired in large by the early writings of H.W. Singer, Raul Prebisch, and Gunnar Myrdal. Dependency theory later grew to include the works of A.G. Frank, Dos Santos, Cardoso, Sunkel, and Furtado. The ideas of world system analysis of Immanuel Wallerstein and the structural theory of imperialism by Johan Galtung were also complementary to this school.

Originally Singer ${ }^{12}$ questioned the effects of international trade on development. According to Singer, in the international division of labor, underdeveloped countries were producing and exporting raw materials while the developed countries were producing manufactured goods. This division of labor was seen by Singer to be an obstacle to the economic development of the underdeveloped countries. Since the underdeveloped countries producing raw materials are subjected to deteriorating terms of trade with the industrialized countries, the gains from trade are concentrated in the developed countries. Further, contrary to what classical economists advocated as free trade and comparative advantage, Singer suggested that underdeveloped countries should not specialize in commodities with which they had comparative advantage, but should instead attempt to change the whole structure of comparative advantage and should invest in industrialization.

Myrdal $^{13}$ asserted that not only did free trade not equalize incomes in different countries but it also actually aggravated their differences. Myrdal stated

12- H.W. Singer, "The Distribution of Gains Between Investing and Borrowing Countries", American Economic Review, Vol II, no 2.

13- G. Myrdal, Economic Theory and Underdeveloped Regions ( London: Gerald Duckworth, 1957). 
that contrary to the perceived view, international trade involved a cumulative process moving away from equilibrium in factor productions and increased the disparities in the distribution of facilities for production between developed and underdeveloped countries ${ }^{14}$.

One of the most important causes of these disparities according to Galtung $^{15}$ is the vertical division of labor. He assu:ts that the vertical division of labor leads to a widening material gap between the developed and underdeveloped countries. In the case of the division of labor, different forms of production affect societal organizations, technological development, and education and research differently. An advanced form of production has positive secondary spin off effects on a number of other sectors in the community, while a more primitive form of production does not. According to this argument, the theory of comparative advantage fails to reveal this kind of relationship, which, from a development theory point of view, is fundamental.

In the dependency theory formulation of international trade relations, countries are divided into distinct categories of center and periphery with a third category of semi-periphery occupying the middle ground. The following distinctions between the center and periphery countries are then made: center countries produce manufactured products, whereas periphery countries produce primary and agricultural products; center countries enjoy a much higher standard of living than the periphery countries; at any time, center countries exploit other center countries the least and peripheral countries the most, with semi-periphery countries falling somewhere in between the two.

14- G. Myrdal, An International Economy: Problems and Prospects (New York: Harper and Row, 1969), p. 225.

15- J. Galtung, "A Structural Theory of Imperialism", Journal of Peace Research, Vol VIII, No 2. 
The formation of a center-periphery relationship among countries is said to have started with the expansion of the European capitalist system which over the past centuries integrated the other regions of the world into its sphere of influence by political domination and colonization. These peripheral countries, within this new economic system, were denied new technologies, were prevented from setting up their own industries, and were prohibited from selling their products to other countries. According to Frank ${ }^{16}$, underdevelopment was not an original stage, but rather a created condition. Frank supported this assertion by pointing out the British deindustrialization of India, the destructive effects of the slave trade on African societies, and the obliteration of the Indian civilization in Central and South America.

The income data presented earlier in Table $I$, in light of dependency theory, suggests that since the integration of a world economy, there has existed an everincreasing differentiation within the capitalist world system that has produced well-being at the center and relative impoverishment at the periphery.

According to Galtung, this differentiation manifests in a number of ways. First, there is concentration of trade partners, with the periphery having most of its trade with one center country, while that nation is free to extend its trade relations in any direction. Second, there is commodity concentration, with periphery nations having only few primary commodities to export. And third, center-periphery coupling has produced a gap in the quality of living conditions between these nations. The present research is designed to test the hypotheses set forth by dependency theory in general and by Johan Galtung in particular.

16- A.G. Frank, "The Development of Underdevelopment" in Imperialism and Underdevelopment: A Reader, ed. R.I. Rhodes, p.4. 


\section{DOMAIN OF THE INVESTIGATION}

To test the hypothesis that the world is hierarchically structured with center countries occupying the top and the periphery countries the bottom of the hierarchy, one would ideally develop a model of the world system structure since 1500. However, unavailability of data limits the range of this study to the post World War II period. The study period is very critical to any study of dependency theory since most periphery countries achieved their political independence around the Second World War. Thus diachronic investigation will examine if political independence for the peripheries has been followed by economic independence and economic development.

This study considers the synchronic and diachronic patterns of interaction among a large number of countries (127) by using international trade statistics and other data for the years $1938,1962,1970$, and 1980 . The overall goal is to test the usefulness of the center-periphery concept, to see if it can be operationalized and, if so, if it has expected relationships between the certain economic variables which can be confirmed empirically. These variables at the national level are: export partner concentration, total trade linkages, import partner concentration, export commodity concentration, import commodity concentration, vertical trade, agriculture/GDP, quality of life, G.N.P./Capita, and export dependency ${ }^{17}$. At the

17- At the national level, ten other variables will also be calculated but due to space limitations they will only appear as additional information in their appropriate appendices. These variables are: ratio of export partner concentration to import partner concentration, ratio of export commodity concentration to import commodity concentration, percent exports of manufacture, percent exports of nonmanufacture, percent imports of manufacture, percent imports of nonmanufacture, total exports, total imports, percent exports to largest trading partner, and percent imports from largest trading partner. 
global level, they are: world system centrality, global centralization, and regional decomposition.

At the systemwide level of analysis, trade hierarchies among nations will be constructed and comparisons of regional and global averages of variables used in the national study will also be made.

Although tests of dependency theory have previously been conducted, this study undertakes synchronic tests of the center-periphery hypothesis which are more extensive than what has been done so far. New variables and techniques are introduced to overcome the limitations of the previous models. In addition, this research investigates empirically the diachronic characteristics of the world system as a whole, a level of analysis which has been only minimally explored -in a quantitative way- in the past.

\section{ORGANIZATION OF THE STUDY}

Chapter II presents an overview of the literature on international trade and development by: first, providing a short history of the international economic relations during each of the significant periods between 1500 to present; second, reviewing classical and dependency theories; and third, presenting the previous tests of dependency theory.

Chapter III operationalizes the variables used in the national and systemwide studies and then formulates the proposed hypotheses and investigations that are carried out in this research.

Chapter IV presents the results of investigations at the national level.

Chapter $\mathrm{V}$ presents the results of the systemwide studies.

Chapter VI presents conclusions, discusses the limitations of this study, and suggests some directions for future research. 


\section{CHAPTER II}

\section{LITERATURE REVIEW}

\section{MERCANTILE CAPITALISM AND THE FIRST EUROPEAN EXPANSION (1500-1870)}

The discovery of America, and that of the passage to the East Indies by the Cape of Good Hope, are the two greatest events recorded in the history of mankind. Their consequences have already been very great... By uniting, in some measure, the most distant parts of the world, by enabling them to relieve one another's wants, to increase one another's enjoyments, and to encourage one another's industry, their general tendency would seem to be beneficial. To the natives, however, both of the East and the West Indies, all the commercial benefits which can have resulted from those events have been sunk and lost in the dreadful misfortunes which they have occasioned.

- Adam Smith, An Inquiry into the Nature and Causes of the Wealth of Nations(1776).

The first great European expansion overseas began with the sea explorations of the Portuguese and Spanish in the second half of the fifteenth century. Soon, the European merchants and governments realized the possibilities for increasing their power and wealth. Commercial companies were chartered and financed, with military and naval expeditions frequently sent out after them to ensure political control of overseas territories. Gradually, this led to the establishment of many European colonies in the Americas.

Later on, this practice of increasing state power and security by government regulation of commerce life was called mercantilism. Colonies became very desirable since they of fered an opportunity to shut out competition by allowing the colonial countries to monopolize as many of these overseas mercantile opportunities as possible. 
Mercantile regulations were designed to give the European countries the monopoly of colonial trade and wealth. According to Fieldhouse, there were common European traditions. There was a remarkable similarity among the practices of different states. All excluded foreign ships from colonial ports; insisted that colonial trade pass through metropolitan ports in both directions; and forbade colonial products or manufactures to compete with those of the metropolis. These practices were later rationalized into a logical system by theorists; the eighteenth century called this mercantilism, and assumed that colonial possessions were to serve the European powers by bringing them wealth ${ }^{1}$.

The restrictions imposed on colonies under mercantilism were of two categories: (1) they prohibited or limited the production of manufactures, such as textiles or hardware, to preserve a market for European exports; (2) they prevented the processing of primary colonial products before export to Europe. This second category was far more important as the natural line of development for primary producers was to undertake the more advanced processing of their commodities before export.

These regulations had an adverse ef fect on colonial wealth and slowed down the development of the colonies towards a more complex economic structure. Fieldhouse ${ }^{2}$ stated that the Spanish and Portuguese colonies suffered most from imperial monopoly since neither country had the industrial strength to supply its colonies with manufactures, and neither consumed a large proportion of its colonial exports. 1967), p. 85 .

1- D.K. Fieldhouse, The colonial Empires ( New york:Delacorte Press, 2- Ibid, p. 91. 
Adam Smith, writing on the effects of mercantilism on the colonies, observed that the natural growth of these countries were severely restricted by the monopolization of colonial trade by exclusive companies that obliged the colonies to limit their production based on the needs of the mother countries ${ }^{3}$.

In addition to this, there was the economic effect of the slave triangle. With ships sailing from Europe to West Africa, taking slaves to the Americas and then returning to Europe with bullions and sugar, a triangular trade system took shape. Sixteenth century Europe thus witnessed the growth of the center-periphery structure from its earliest embryonic form. With the slave trade and the colonization of the Americas more of the world was brought under the influence of Europe's trade. This was the beginning of the international division of labor, with slave labor in Americas providing primary commodities for European markets. The gold and silver from Central America which flowed through the trading arteries of European merchant cities allowed the accumulation of money and commercial capital. This eventually was to lay the basis for investment in the early manufacturing industries of Europe. The pattern of this international division of labor is illustrated in Table III.

The expansion of trade during the eighteenth century commercial revolution was credited to this so-called triangular trade. The triangular trade was a tricontinental trade relationship in which Europe supplied the exports and ships, Africa supplied the slaves, and American colonial plantations supplied the raw materials. The trade originated in Europe, with ships sailing to African coasts

3- A. Smith, An Inquiry into the Nature and Causes of The Wealth of Nations ,Edwin Canan(ed.), (New York: The Modern Library, 1937), p. 542. 
TABLE III

MULTILATERAL TRADE IN 18TH CENTURY

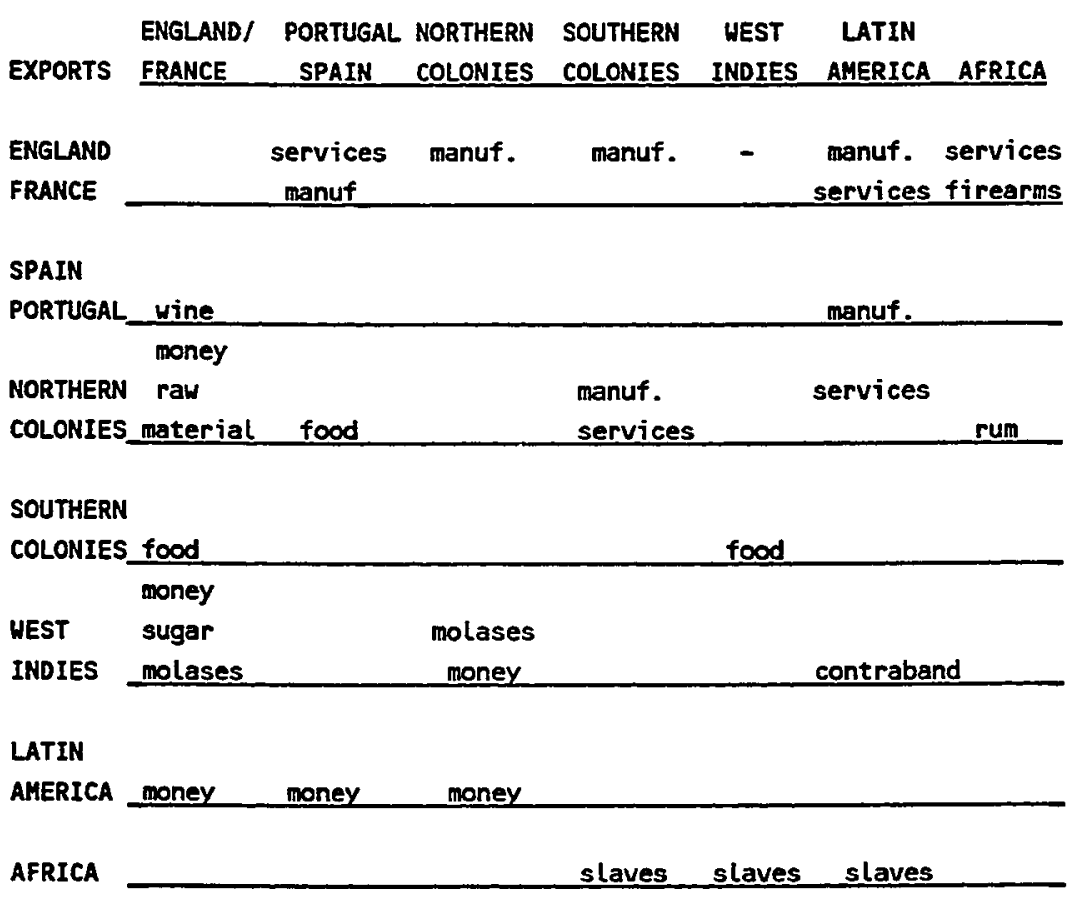

Manufacture: especially textiles

Food: including tobacco, fish Services: especially shipping

Money: coin, bullion, drafts

Raw material: timber, iron, etc.

SOURCE: Adapted from F. Mauro, 1961 
containing European goods that were exchanged on the coasts of Africa for slaves. Those same ships then sailed to colonial plantations and traded the slaves for cargoes of colonial products which were taken to European markets. During each trade cycle, European merchants received triple profits, all of which were accumulated in European countries. As the intensity of trade grew so did the accumulation of capital ${ }^{4}$.

The kingpin in the triangle of European manufactures, was African labor, and American colonial produce paid for the supply, transport, sale, exploitation, and replacement of the black slaves whose work ultimately supported the entire system. The most widely cited estimates of the number of African slaves imported into the Americas are 900,000 in the sixteenth century, 2.75 million in the seventeenth century, 7 million in the eighteenth century and 4 million in the nineteenth century ${ }^{5}$. Philip Curtin's estimate for the eighteenth century is 5.5 million ${ }^{6}$.

Meanwhile, as triangular trade boosted the British economy, Friedri $-\mathrm{il}_{\mathrm{L}}$ List $^{7}$ commented favorably on the success of British commercial policy. As he stated, the British were adept at buying raw materials and selling manufactured products. Karl Marx also noted in Capital that "the colonies secured a market for the budding manufactures and, through the monopoly of the market, an increased accumulation. The treasures captured outside Europe by undisguised looting, pp. 51-52.

4- E. Williams, Capitalism and Slavery (New York: Perigee Books, 1944),

5- J.D. Fage, An Introduction to the History of West Africa (Cambridge: Cambridge University Press, 1962), p. 83.

6- P. Bohannon, and P. Curtin, Africa and Africans (New York: Natural History Press, 1971), p. 269.

7- F. List, National System of Political Economy (Philadelphia: J.B. Lippincot, 1856), pp. 114-120. 
enslavement, and murder, floated back to the mother-country and were there turned into capital ${ }^{8}$.

But perhaps the most forceful argument was that of Eric Williams, in his

\section{Capitalism and Slavery:}

The triangular trade thereby gave a triple stimulus to British industry. The Negroes were purchased with British manufactures; transported to the plantations, they produced sugar, cotton indigo, molasses and other tropical products, the processing of which created new industries in England; while the maintenance of the Negroes and their owners on the plantations provided another market for British industry: New England agriculture and Newfoundland fisheries. By 1750 there was hardly a trading or manufacturing town in England which was not in some way connected with the triangular or direct colonial trade. The profits obtained provided one of the main streams of that accumulation of capital in England which financed the Industrial Revolution ${ }^{9}$.

Thus, the triangular trade made an enormous contribution to Britain's industrial development. The profits from this trade fertilized the entire productive system of the country.

According to Williams, many of the eighteenth century banks that were established in Liverpool and Manchester, the slaving metropolis and cotton capital, respectively, were directly associated with triangular trade. Businesses like Barclays Bank and British Leyland were among those whose investments in African trade dated back to the last quarter of the eighteenth century ${ }^{10}$.

Heavy industries played an important role in the progress of the industrial revolution and the development of triangular trade. The capital which financed

8- K. Marx, Capital (Moscow: Foreign Languages Publishing House, 1962), pp. 753-754.

9- E. Williams, Capitalism and Slavery (New York: Perigee Books, 1944), pp. $52,57,60-61,64,68,70-73,78,81-84$.

10- Ibid, p. 101. 
the growth of the metropolitan industries came directly from the wealth that was accumulated from triangular trade. Williams stated that it was capital investments from the West Indian plantations that made James Watt's steam engine possible ${ }^{11}$.

However, it must not be inferred that triangular trade was solely and entirely responsible for British economic development. The growth of the internal market in England, the ploughing-in of the profits from industry to generate still further capital and achieve still greater expansion, also played a large part.

\section{THE NEW IMPERIALISM AND THE SECOND EUROPEAN EXPANSION} (1870-1914)

All theories of economic imperialism basically attempt to explain the reasons behind the expansion of the new imperialism which took place between 1870-1914. While the proportion of the world's land under European control including the old or existing colonies was at 35 per cent in 1800 , it reached to 84.4 per cent in $1914^{12}$. The new imperialism characteristically was different from the old because the older colonies were for the most part created as a result of European settlements and the establishment of European style societies or plantation communities in the Americas. However, the new ones were colonies created as a result of military occupation and political control by European countries in Africa, Asia and the Pacific. Therefore, there was a swing towards dominion of European empires over non-European people.

The intervention of the European countries gave the impression that the stimulus to colonization was unprecedentedly strong and general among the European powers. The colonization of new territories was always followed by vast

11- Ibid, p. 102.

12- D.K. Fieldhouse, Economic and Empire: 1830-1914 (Ithaca: Cornell University Press, 1973), pp. 10-11. 
amount of investments by Europeans which also suggested the existence of extraordinary surplus capital in European countries.

Fieldhouse on the subject of imperialism and trade wrote that the growth of imperialism can be explained by the accelerating need for new overseas markets and sources of new materials during a period of expanding industrialization and commercial competition. The resultant competition for limited markets, especially for textiles, iron, steel and metallurgical products, had consequences which according to Fieldhouse were relevant to the growth of economic imperialism ${ }^{13}$. These consequences were: (1) the existence of prolonged cyclical depressions in European countries around the 1870-1890s; (2) the necessity of protecting domestic markets by raising tariffs was felt by all major governments in Europe, along with the United States; (3) the increase importance of markets of the less developed world to European trade which caused intense rivalry among European countries, and finally, (4) the need for colonies as extensions for capital investment, which resulted in a scramble for overseas expansion and possession of new colonies ${ }^{14}$, was simultaneously felt in all European countries.

\section{Cyclical Depressions}

To examine the hypothesis that postulated serious deterioration in overseas market opportunities in the last quarter of the century, Fieldhouse examined the official trade statistics of Britain, France and Germany from 1870 to 1900 to investigate the existence of secular or cyclical slumps in the value of exports from the industrial states. The data he obtained fully supported the general assumption

13- Ibid, p. 10-11.

14- Ibid, p. 11. 
that the last thirty years of the nineteenth century were a difficult period for the export-oriented economies of Western Europe ${ }^{15}$.

For Britain, exports suffered a prolonged decline lasting from 1873 to 1890 followed by yet a further decline from 1890 to 1898. For the world's largest industrialized state to run an adverse trading balance of over 100 million pounds a year for most of the period 1876-1900 implied a serious inability to compete in the overseas market. This may have well stimulated British interests in new overseas markets and in new colonies, for these were necessary to secure a vent for British products $^{16}$. France also felt the effects of three cyclical depressions in the period of 1877-80, 1883-88, and 1892-96 which caused significant adverse balance on visible trade throughout these years. Moreover, since French colonizing activity was particularly evident during the second of these cyclical slumps, it was quite possible that there may have been a correlation between declining exports and the search for new overseas markets.

According to Fieldhouse, Germany's cyclical depression in comparison to the others was slight. German exports did not significantly seem to rise above the level of 1883 until 1895; also after 1888, Germany had a significant and growing adverse balance of trade 17 .

According to Fieldhouse, key British and French interests were deeply concerned about the length and severity of these trade depressions and their effects on the domestic economy they became convinced that monopoly of the trade of existing possessions was a partial remed ${ }^{18}$.

15- Ibid, p. 14.

16- Ibid, p. 14.

17- Ibid, p. 19.

18- Ibid, p. 19. 
Growth of Tariff Protection

For both Germany and France severe protectionist tariffs followed periods of relatively free trade. Germany which was moving towards free trade principles in the 1860s, under pressure from industrialists, introduced a new tariff in 1879 imposing relatively low duties on imported manufactures and heavier duties on agricultural produce. The climax of high tariffs for Germany came in the year 1902 when duties on goods from Britain were raised to 25 per cent and on goods imported from Russia to 131 per cent ${ }^{19}$.

The protectionist policies of France were similar to those of Germany. While the 1860 s and 1870 s were a period of economic liberalism, in 1881 a new general tariff gave effective protection to a range of manufactures while leaving agricultural products and raw materials unprotected. By 1892, France had introduced a general protective tariff system where certain raw materials were still allowed free entry but others and certain semi-manufactures which were produced in France were made dutiable for the first time since the 1860s. This made France one of the most severely protectionist states, though still less than the United States whose average duties rose from 47 percent in 1869 , to 49.5 per cent in 1890 and to 57 per cent in $1897^{20}$.

It follows that it is perfectly possible that protectionism and imperialism may have had a direct relationship given the fact that the age of the new imperialism coincided with the resurgence of protectionism in France and Germany and the intensification of protectionism in the United States, Russia and other countries such as Portugal, Spain, and Italy 21 .

19- Ibid, p. 20.

20- Ibid, p. 20.

21- Ibid, p. 21. 
Expansion of the Market

By the mid-1880s French metropolitan opinion was that, France, whose exports were held back by ever increasing competition, must find guaranteed markets for her primary and industrial products. Further, these new dependencies would only be desirable if they provided a monopolistic outlet for French products $^{22}$.

By the latter 1880 s, the dominant mercantile opinion was that colonies were only useful if protected from foreign trade competition, and that further imperial expansion could be justified provided that new colonies could be made into monopolistic markets.

Perhaps the most logical explanation of the economic case for tropical colonization by a protectionist European state was that of Jules Ferry made in 1890. He viewed colonization as essential to the survival of industrialized countries that were facing increasing competition from other industrialized countries ${ }^{23}$.

Prior to Germany's interest in imperialism, the question of colonies and their significance to the metropolitan country was basically limited to the academic spheres. As far back as 1821, Hegel had adopted concepts from Smith and other classical economists, arguing that "colonization was desirable for bourgeois economy because it opened up new markets and relieved domestic pressures resulting from underconsumption ${ }^{24 " . ~ B u t ~ t h e ~ r e a l ~ c r e a t o r ~ o f ~ t h e ~ G e r m a n ~ c o l o n i a l ~}$ tradition was Friedrich List. His primary objective was that:

free trade was a system designed and propagated in her own interest by Britain as the first power to develop modern

22- Ibid, p. 23.

23- P. Roubiquet, Discours et Opinions de Jules Ferry, trans. D.K. Fieldhouse, pp. 556-57.

24- G.W.F. Hegel, Philosophy of Right (Oxford: 1942), pp. 242-249. 
industries. For other countries that wished to follow the same path, free trade was an obstacle because without protection their own nascent industries could not compete with the cheaper imports of the more advanced manufacturer ${ }^{25}$.

List strongly believed that if Germany wanted to become a great nation, it should have its own colonies.

Meanwhile, British imperialism in the 1880 s and 1890 s was a reaction to the protectionism of others, particularly when foreign tariffs threatened the exclusion of British traders from regions of Africa and the East. The commercial value of colonies to Britain was acknowledged well before the $1880 \mathrm{~s}^{26}$. E.G. Wakefield, leader of the colonial reformers during the 1830 s and $1840 \mathrm{~s}$, expressed the idea that the colonies were natural exporting communities. The colonies produced what the old country wanted, and the old country produced what the colonies wanted. The old country and the colony, therefore, were naturally each other's best customers $^{27}$.

Such arguments, which were instrumental in the establishment of white settlement colonies in North America, South Africa, and Australasia, lost their luster especially after the adoption of the protective tariffs in Canada in 1859 which were later copied by several Australasian colonies. As a result, it had to be assumed that self-governing colonies would eventually cease to offer special commercial advantages to the parent state 28 .

25- F. List, National System of Political Economy (Philadelphia: J.B. Lippincot, 1856)

26- D.K. Fieldhouse, Economic and Empire: 1830-1914 (Ithaca: Cornell University Press, 1973), pp. 32.

83.

27- E.G. Wakefield, A View of the Art of Colonization (London: 1849), p.

28- D.K. Fieldhouse, Economic and Empire: 1830-1914 (Ithaca: Cornell University Press, 1973), p. 33. 
Conditions for British foreign trade had changed dramatically by early 1880s. British exports were dropping in the face of foreign competition in established markets and the rise of protectionism in Europe. On the other hand, while France and other rival powers were expanding their colonial annexation and imposing differential tariffs to exclude British trade, new colonies had to be deliberately acquired in order to protect openings for new British trade in Africa and the East.

In 1896, Joseph Chamberlain, notoriously the most expansionist British statesman, stated categorically that if Britain did not occupy most parts of Africa, it would be done by British rivals, who would proceed to close this great commercial market to the British empire ${ }^{29}$.

Such statements suggest that British expansion after 1880 was affected, if not directly caused, by concern for overseas markets 30 .

\section{Imperialism of Capital}

The process of capital accumulation was primarily achieved by reinvesting the profits in productive activities with the expectation that adequate profit from the new investment could be received. It appeared however, that by the end of the nineteenth century, the incentive to invest this capital within national boundaries had diminished due to the decline of profit rates. Therefore, in order to avoid the eventual economic stagnation, European capitalists looked for new rewarding fields of investment abroad.

J.S. Mill, on the subject of surplus capital, wrote that the supply of cheap food and cheap raw materials could only be done by the export of capital which

29- W.K. Hancock, "Survey of British Commonwealth Affairs," II, ii, Problems of Economic Policy, 1918-39, London: 1942, p. 82.

30- D.K. Fieldhouse, Economic and Empire: 1830-1914 (Ithaca: Cornell University Press, 1973), p. 29. 
led to increasing capital investment in local manufactured goods with which to pay for the supply of raw materials. Thus, the exportation of capital, to Mill, was an agent of great efficiency in extending the field of employment. The more capital was sent away, the more capital accumulation could be retained at home ${ }^{31}$.

This statement should be taken in conjunction with his view that trade with colonies be regarded as akin to trade within a country: As he stated "the colonies were simply regions where Britain found it convenient to produce certain raw materials, and to which British capital flowed freely ${ }^{32 n}$.

Jules Ferry, stressing the commercial and financial attractiveness of overseas' territories at that time wrote, that for countries like France, which by the very character of its industry was tied to large markets, the purpose of the foundation of a colony was to create a market ${ }^{33}$.

The ideal environment appeared to be in areas where economic conditions differed substantially with those of Europe, particularly in places where there were ample raw materials and non-European labor to be exploited. Investment of surplus capital in such regions not only earned a higher rate of profit than was possible at home, but also reduced the pressures of capital seeking utilization at home.

A number of European countries, according to Fieldhouse, simultaneously felt the need for colonies as fields for investment after 1870. A competitive rush

31- J.S. Mill, Principles of Political Economics (London, 1848), p. 739.

32- Ibid, p. 685-686. Fieldhouse.

33- P. Roubiquet, Discours et Opinions de Jules Ferry trans. D.K. 
for overseas possessions occurred which resulted in the partition of the other regions of the world a nong the European countries ${ }^{34}$.

The central arguments of capitalist imperialism have been theoretically credited to the two most influential proponents, J.A. Hobson and V.I. Lenin. Their contributions to the theoretical bases of capitalist imperialism are discussed elsewhere in this study.

\section{THE AGE OF NEOCOLONIALISM}

(1947- PRESENT)

The decolonization which followed the Second World War can be attributed to at least three factors: the decline in economic and military power of the traditional imperial countries of Europe vis-a-vis the United States; the growth of liberation movements and the threat of revolution in colonial countries demanding independence; and the emergence of the United States' hegemony over the European capitalist countries and with United States' insistence on opening up the colonial and protected imperial markets to obtain vital raw materials, markets and investment outlets.

This new international order provided an opportunity to destroy the existing patterns of colonial dependency. However, by this time, the colonial countries had become fixed as economic peripheries of the center countries and their independence not only did not signify the end of colonialism but also heralded a new state of neocolonialism. Fieldhouse asserts that decolonization took place only because the capitalists felt that the socio-economic structures of their

34- D.K. Fieldhouse, Economic and Empire: 1830-1914 (Ithaca: Cornell University Press), 1973, p. 39. 
colonies were so dependent that their own interests could be preserved even without their continued formal political control ${ }^{35}$.

The grounds for this confidence were that while the newly independent governments were in theory free to restructure their economic and social systems, few of them in fact were able or willing to do this. Fieldhouse proposed two factors that inhibited such change:

1. The end of a formal empire did not necessarily alter the fact that most large scale commercial enterprises were owned and run by foreigners, usually foreign based 36 .

2. The new indigenous rulers might consider that their own private interests would be best served by continuing to act as allies- compradors is the term often used- of foreign capital, particularly the great multinational companies which would pay them well(bribes, salaries, directorships, etc.) for their collaboration 37 .

Thus, the newly independent countries' economic structures were seen as an extension of the previous cósonial periods. This assertion led to the emergence of a new school economic thought based on dependency theory. The theories proposed by this school, which form the core of the present study, are discussed elsewhere in this chapter.

35- Ibid, p. 10.

36- Ibid, p. 10.

37- Ibid, p. 10. 


\section{CLASSICAL THEORIES AND COLONIALISM}

\section{ADAM SMITH AND THE WEALTH OF NATIONS}

The orthodox interpretation of foreign trade by classical and neoclassical economists is that it could act as a major propelling force toward development. Adam Smith's model of foreign trade consisted of two main ideas. First, was the idea of surplus productive capacity: if a country could open idle land and a labor force to the world market, then the excess resources could be used to produce a surplus of goods for export and trade, thereby "venting" the surplus productive capacity that otherwise would have been unused. Second, by providing an extension to the world market, international trade could also improve the division of labor and raise the general level of productivity within a country.

Smith clearly distinguished the good effects of trade and the bad effects of monopoly and regulations controlling the trade of the colonies with each other and the mother country. Smith's coinage of the term mercantile system was intended to characterize policies which he believed were designed to further the interests of the merchant and manufacturing classes at the expense of other members of the community.

The good effects of trade followed from the extension of the market; thus the discovery of Americas was the most important element of European trade expansion.

All members of society in European countries, according to Smith, stood to gain since the stimulus to growth in manufacturing provided by the colonial trade increased demand for domestic agricultural products 38 .

38- Ibid, p. 575. 
Smith also asserted that the natural benefits of trade with the colonies had been reduced and in some cases completely nullified by the attempts of European countries to confine such benefits to themselves. In the case of the American colonies, Smith asserted that the exclusive trade regulations of England had kept the industrial development of her American colonies below what it could have been and that the trade barrier had not allowed the colony to benefit from trade with other countries 39 .

\section{J. S. MILL AND PRINCIPLES OF POLITICAL ECONOMY}

Classical economists considered Ricardo's concept of comparative advantage to be important in determining the pattern of trade. This suggested that it was not only the use of surplus resources, but the more efficient allocation of resources which allowed trade to benefit a country by promoting a more efficient international allocation of resources. Trading countries were able to enjoy a higher real income by specializing in production according to their comparative advantage. Exports had instrumental significance as the intermediate goods used for the indirect production of imports, and allowed the country to buy imports on more favorable terms than if produced directly at home.

Thus, specialization according to comparative advantage yielded the direct benefits of international exchange. Classical and neoclassical economists saw no conflict between a country's conformity with its comparative advantage and the acceleration of its development. Indeed, John Stuart Mill stated that trade, according to comparative advantage, resulted in a "more efficient employment of the production forces of the world", and that this might be considered the "direct economic advantage of foreign trade." But there were, in addition, indirect effects,

39- Ibid, p. 558-559. 
which must be counted as benefits of a high order. Mill further suggested that by widening the extent of the market through foreign trade, a small country could overcome its diseconomies while providing indirect benefits of inducing innovations and increasing productivity 40 .

Another important consideration, according to Mill, mainly applicable in early stages of industrialization was that through foreign trade, the diversity of products that previously was not attainable would induce people to work harder and be more productive so that they would be able to attain their new tastes. Mill believed that trade in some cases could induce people to save and accumulate capital, and this could be regarded as another kind of industrial revolution ${ }^{41}$.

For Mill colonization was an economic necessity, and as such required government support and control. It is therefore easy to understand how John Stuart Mill, who was so deeply committed to the support of colonization, could claim that it involved, the future and permanent interests of civilization itself $\mathrm{f2}^{\mathbf{2}}$.

\section{CLASSICAL THEORIES OF IMPERIALISM}

\section{J.A. HOBSON AND THE TAP-ROOT OF IMPERIALISM}

The first general and systematic explanation linking late nineteenth century colonial expansion with the dynamics of capitalist development was given in Imperialism by J.A. Hobson ${ }^{43}$. Hobson clearly distinguished colonization from imperialism. Colonization was defined as the migration of white colonists to

40- J.S. Mill, Principles of Political Economics (London, 1848).

41- Ibid, Vol. II, book III, sec. 5, chapter 17.

42- Ibid, p. 970.

Press, 1965)

43- J.A. Hobson, Imperialism (Ann Arbor: The University of Michigan 
sparsely peopled foreign lands which would eventually obtain some form of selfgovernment. Imperialism, on the other hand, was inspired by a nationalistic trend and involved the absorption of tropical or subtropical lands where white men would not settle with their families.

Hobson argued that since 1870 Britain obtained colonies, which held very few commercial attractions, provided little room for emigration and gave minimal trade opportunities. Why then did Britain pursue this policy of imperialism? Hobson's reply was three fold. First, certain sectional interests had guided foreign policy to their own ends. There were clearly identifiable interest groups which benefited from aggressive imperial policy. Hobson believed that, "it is not too much to say that the modern foreign policy of Great Britain has been primarily a struggle for profitable markets of investment ${ }^{44 n}$. Second, there were the economic aspects of foreign investment. For example, the income derived from interest on foreign investments enormously exceeded those derived from profits from the ordinary export and import trade. In addition, while foreign and colonial trade, and presumably the income from it, was growing slowly, the share of import values representing income from foreign investment was growing very rapidly 45 . In Hobson's view, aggressive imperialism only benefited those investors who could not find profitable markets at home and it forced the government to secure profitable investment opportunities abroad ${ }^{46}$. Moreover, the colonial economy was structured such that it would minimize the risks of investments and secure favorable returns ${ }^{47}$. Third, Imperialism could benefit a country by naturally striving to

44- Ibid, p. 53.

45- Ibid, p. 53.

46- Ibid, p. 62.

47- Ibid, p. 56. 
fasten to the mother country the markets of each new territorial acquisition, convinced that only by such separate increments can the aggregate of trade grow.

According to Hobson, the assumption that the home demand is a fixed amount, and that any commodity made in excess of this amount had to find a foreign market, or remain unsold, was quite unwarranted ${ }^{48}$. To Hobson, there was not a limit to the quantity of capital and labor that could be employed in supplying the home markets, provided the effective demand for the goods that were produced was so distributed that every increase in production stimulated a corresponding increase in consumption ${ }^{49}$. Hobson believed that it was not necessary to expand foreign markets because he believed that whatever was produced in England could be consumed in England-- if income there were properly distributed ${ }^{50}$. This argument, according to Hobson, did not mean that Great Britain could disregard her external markets however. Some considerable foreign markets were viewed by Hobson as an economic necessity. Britain, through her exports made purchases of food and material which she could not produce, or could only produce at a great disadvantage. This fact, according to Hobson, made a considerable external market a matter of vital importance to Britain 51 .

Therefore, "the economic taproot of imperialism", as Hobson described it, was an increase in the volume of production and capital accumulation on the one hand and an unequal distribution of income on the other. As Hobson stated:

It is this economic condition of affairs that forms the taproot of imperialism. If the consuming public in this country raised its standards of consumption to keep pace with every rise of

48- Ibid, p. 29.

49- Ibid, p. 29.

50- Ibid, p. 88.

51- Ibid, p. 29. 
productive powers, there could be no excess of goods or capital clamorous to use imperialism in order to find markets: foreign trade would indeed exist, but there would be no difficulty in exchanging a small surplus of our manufactures for the food and raw material we annually absorbed, and all the savings that we made could find employment, if we chose, in home industries ${ }^{52}$.

Therefore, to Hobson, it was not industrial progress that demanded the new foreign markets, but the mal-distribution of consumer power which prevented the consumption of capital and commodities within the British economy ${ }^{53}$.

\section{V.I. LENIN AND IMPERIALISM, THE HIGHEST STAGE OF CAPITALISM}

Lenin's theory of imperialism drew heavily from the works of Hobson and Hilferding. Hobson's contribution was the notion of surplus capital and the fact that groups of investors influenced government policies to secure profitable investment opportunities. Hilferding's contribution was the notion of finance capitalism which was defined as the increasing concentration of economic activity and banking into larger and larger combines so producing a new form of capitalism in which the banks controlled the flow of investments into large-scale industrial concerns.

Hilferding's explanation of why capital had to be exported by the finance capitalist was modified by Lenin as follows:

It goes without saying that if capitalism could develop agriculture, which today lags far behind industry everywhere, if it could raise the standard of living of the masses, who are everywhere still poverty-stricken and underfed... there could be no talk of a superabundance of capital... but if capitalism did these things it would not be capitalism; for unequal development and wretched conditions of the masses are fundamental and inevitable conditions and premises of this mode of production. As long as capitalism remains what it is, surplus capital will never be utilized for the purposes of raising the standard of living of the masses in a given

52- Ibid, p. 81.

53- Ibid, p. 85. 
country, for this would mean a decline in profits for the capitalists; it will be used for the purpose of increasing those profits by exporting capital abroad to the backward countries 54 .

On this basis Lenin produced his formal definition of imperialism. To Lenin, imperialism was the monopoly stage of capitalism. Imperialism according to Lenin had five basic features: the concentration of production and capital developing to such a high stage that it creates monopolies which play a decisive role in economic life; the merging of bank capital with industrial capital, and the creation, on the basis of this "finance capital," of financial oligarchies; the export of capital as distinguished from the export of commodities acquiring exceptional importance; the formation of international monopolist capitalist combines which share the world among themselves; the completion of territorial division of the whole world among the largest capitalist powers 55 .

Once at this stage of imperialism, all that could follow was the intensification of competition between rival imperialist states, the partition of the world among the most powerful of them, and finally their destruction in war and revolution.

Therefore, to Lenin, imperialism was not a simple repairable distortion in mature capitalist economies as Hobson had suggested, but was an essential, inherent flaw, which could only be removed by eliminating capitalism. 1916), p. 70.

54- V.I. Lenin, Imperialism, the Highest Stage of Capitalism (Moscow:

55- Ibid, p. 105-106. 


\section{STRUCTURAL THEORY OF IMPERIALISM JOHAN GALT UNG}

More recently, Galtung, in an article entitled "A Structural Theory of Imperialism," has pointed out two important facts about the world: (1) the tremendous inequality, within and between countries, in all dimensions of human living conditions, and (2) the resistance of this inequality to change ${ }^{56}$. Galtung divides the world's countries into Center nations and Periphery nations, each with its own center and periphery. Thus, in Center countries there is a center of the Center and a periphery of the Center. And in Peripheral countries, there is a center of the Periphery and a periphery of the Periphery 57.

Galtung believes that the mechanism underlying this discrepancy is imperialism 58 . Imperialism, according to Galtung has three criteria, two mechanisms, five types, and three phases:

\section{Defining Imperialism}

The three criteria that define imperialism as a relationship between a Center and a Periphery country are ${ }^{59}$ :

1. There is harmony between the interests of the center in the Center nation and the center in the Periphery nation;

2. There is more disharmony of interests within the Periphery nation than within the Center nation;

56- J. Galtung, "A Structural Theory of Imperialism," Journal of Peace Research, Vol. 8, No. 2, 1971, p. 81.

57- Ibid, p. 81.

58- Ibid, p. 81.

59- Ibid, p. 83. 
3. There is disharmony of interests between the periphery in the Center nation and the periphery in the Periphery nation.

An essential element for center-periphery structure Galtung states, is that the Center nation establishes a bridgehead in the center of the Periphery. If there is no such relation, there is no imperialism by this definition ${ }^{60}$.

\section{The Two Mechanisms of Imperialism}

There are two basic mechanism of imperialism that are concerned with center-periphery interaction: the principle of vertical interaction, asd the feudal interaction structure ${ }^{61}$.

Vertical interaction, according to Galtung is the major cause of inequality in this world, whether it take the form of looting or highly unequal exchange, or whether it produces highly differential spin-off effects due to the processing gap $^{62}$. Galtung sees a major distinction between the productive activities of the Center and the Periphery because of the existence of international division of labor. Agriculture or extractive industries like mining that are relatively primitive, intensive in labor, low in required labor skill, and relatively low in capital equipment, are performed by dependent Periphery countries while activities that are very high in skill and capital equipment take place in Center countries.

Feudal interaction structure is the factor that maintains and reinforces this inequality by protecting it. Galtung distinguishes four rules that define this structure ${ }^{63}$ :

60- Ibid, p. 85 .

61- Ibid, p. 85.

62-Ibid, p. 89.

63- Ibid, p. 89. 
1. Interaction between Center and Periphery is vertical,

2. Interaction between Periphery and Periphery is missing,

3. Multilateral interaction involving all Center and

Periphery countries is missing,

4. Interaction with the outside world is monopolized

by the Center, with two implications:

a) Periphery interaction with other Center nations is missing,

b) Center as well as Feriphery interaction with

Periphery nations belonging to other Center nations is missing.

There are two important tendencies in this feudal structure ${ }^{64}$ :

1. There is trade partner concentration, which means that the center countries have high levels of transactions with a wide range of different partners so that no one trade partner has a monopoly. Center countries trade a great deal with each other while countries in the Periphery are victims of this monopoly. These latter countries concentrate largely on one partner, on whom they are more or less dependent. Empirically, it is observed that there are high levels of import partner concentration as well as export partner concentration in the Periphery, as opposed to the Center, which is more free to extend its trade relations in almost any direction, except in the pure case, with the Peripheries of other Center nations ${ }^{65}$.

64- Ibid, p. 90.

65- Ibid, p. 90. 
2. There is commodity concentration, which implies that the center countries are highly diversified in commodities they export whereas the Peripheral countries are under great pressure to concentrate on a very small range of commodities.

According to Galtung, a Periphery's concentration is not based on its comparative advantage:

There is a historical rather than a geographical explanation to this. A territory may have been exploited for the raw materials most easily available and/or most needed in the Center, and this, in turn, led to a certain social structure, to communication lines to the deposits, to trade structures, to the emergence of certain groups (often based on ownership of that particular material), and so on. To start exploiting a new kind of raw material in the same territory might upset carefully designed local balances; hence, it might be easier to have a fresh start for that raw material in virgin territory with no bridgehead already prepared for the imperialist. In order to substantiate this hypothesis we would have to demonstrate that there are particularly underutilized and systematically underexplored deposits precisely in countries where one type of raw material has already been exploited ${ }^{66}$.

The combined effects of these two tendencies is a dependency of the Periphery on the Center ${ }^{67}$.

\section{The Five Types of Imperialism}

The international structure, which Galtung calls imperialist, consists of more than relations of economic dependence. Galtung emphasizes not only economic factọrs, but also political, military, communications and cultural dimensions as well. These dimensions are all part of a generalized imperialism, and in Galtung's opinion imperialism can result from any of these factors and lead to other forms of dependency, i.e. they are convertible ${ }^{68}$. Political imperialism may, for instance, change int economic imperialism via dictated terms of trade; the

66- Ibid, p. 90.

67- Ibid, p. 90.

68-Ibid, p. 91. 
imperialism of communication may change into cultural imperialism via control of the flow of information, and cultural imperialism may change into economic imperialism via the export of development models.

In addition, economic imperialism may lead to military imperialism. For example, Galtung makes a distinction between spin-off effects and spill-over effects in vertical interaction. Describing the former, he writes that 'when a nation exchanges tractors for oil it develops a tractor-producing capacity. One possible spin-off effect is a tank-producing capacity, and this becomes a spill-over effect the moment that capacity is converted into military imperialism ${ }^{69}$.

On convertibility of one form of imperialism to another, Galtung says that:

It leads us to reject the assumption of one type of imperialism as more basic than the others. It is the mutual reinforcement, the positive feedback between these types rather than any simple reductionist causal chain, that seems to be the dominant characteristic. If economic, political, and military imperialism seem so dominant today, this may be an artifact due to our training that emphasizes these factors rather than communication and cultural factors ${ }^{70}$.

Galtung has since increased the complexity of the model by adding the concepts of social imperialism and subimperialism ${ }^{71}$. Social imperialism is a centerperiphery relationship in which the Center forces a certain social structure upon the Periphery so that it can act as a bridgehead for the Center. The concept itself originated in the Chinese political debate and is best illustrated by the relationship between the Soviet Union and China during the first ten years after the Chinese Revolution, when China relied heavily on the Soviet Union.

69- Ibid, P. 98.

70- Ibid, p. 99.

71- J. Galtung, "Conflict on a Global Scale: Social Imperialism and the Sub-imperialism-Continuities in the Structural Theory of Imperialism," World Development, Vol. 4, No. 3. 
Galtung defines subimperialism as imperialism by proxy, which can be illustrated by the relations between the United States and Brazil, and a Latin America dominated by Brazil.

The Three Phases of Imperialism

The basic idea, according to Galtung, is that the Center country establishes a bridgehead in the Periphery nation, and more particularly, in the center of the Periphery country ${ }^{72}$. Imperialism has evolved through three phases: colonialism, which took the form of occupation with the center of the Periphery actually consisting of people from the center of the Center who engaged in occupation of the periphery; neo-colonialism, in which the center of the Center interacted with the center of the Periphery through international organizations; neo-neocolonialism, in which the center of the Center interacts with the center of the Periphery via international communication ${ }^{73}$.

Galtung points out that:

"imperialism is so splendidly self-supporting that it no longer has any need for direct political control. An empire that controls a colony is an inefficient empire. There is not the slightest need to control a colony with foreign troops if the bridgehead bourgeoise of the center of the Periphery will do the job free of charge. Why send in the marines if you have, say, the Chilean army? It is only when an imperial system is in danger of breaking down that direct intervention is at all necessary ${ }^{74}$.

Therefore, according to Galtung, the imperialist system is self-perpetuating and dependence is permanent.

72- J. Galtung, "A Structural Theory of Imperialism," Journal of Peace Research, Vol. 8, No. 2, 1971, p. 94.

73- Ibid, p. 94.

74- K.W. Deutsch, "Theories of Imperialism and Neocolonialism," in Testing Theories of Economic Imperialism, edited by: Rosen, S.J. and Kurth J.R. (Toronto: Lexington Books, 1974), p. 27. 


\section{STRUCTURAL THEORY OF CUMULATIVE CAUSATION GUNNAR MYRDAL}

The hypothesis of cumulative causation as an explanation of the lagging growth of developing nations is associated with Gunnar Myrdal. Myrdal contended that in the context of development both economic and social forces have tendencies towards disequilibrium, and that the assumption in economic theory that economic systems invariably tend towards equilibrium is false ${ }^{75}$. If this were not so, how could the widening gap in living standards between developed and underdeveloped nations be explained?

International trade, according to Myrdal, does not by itself necessarily work for equality; on the contrary, it may have strong backwash effects on the underdeveloped countries.

Most underdeveloped countries have either been under the political domination of a metropolitan power, or are economically dominated from abroad so that their economies closely resemble those of the colonial era.

A metropolitan country not only has an interest in using the dependent country as a market for its own manufacturing products but it also has an interest in monopolizing the dependent country as far as possible for its own interests, both in the export and import market. Therefore, enforced bilateralism is another phenomenon characteristic of all colonial empires ${ }^{76}$. The advantages of this bilateral tendency are very substantial to the metropolitan countries while to dependent countries it means a considerable economic disadvantage since it tends

75- G. Myrdal, Asian Drama (New York: Pantheon Books,1968).

76- G. Myrdal, "Poverty- Imperialism- Poverty", in The Economic Causes of Imperialism edited by M. Wolfe, (New York; John Wiley and Sons, 1972), p. 135. 
to worsen their terms of trade by the restrictions imposed on the market where they buy and sell.

According to Myrdal, the most important effect of colonialism is related to the negative fact that the colony is deprived of effective nationhood, and has no government of its own which could take constructive measures to promote the balanced growth of a national economy:

The country and the people were laid bare and defenseless to the play of the market forces as redirected only by the interests of the foreign metropolitan power. This by itself thwarted individual initiatives, at the same time as it prevented the formation of a public policy motivated by the common interests of the people.... For all these reasons, colonialism meant primarily only a strengthening of all the forces in the markets which anyhow were working towards internal and international inequalities. It built itself into, and gave an extra impetus and a peçuliar character to, the circular causation of the cumulative process 77 .

\section{THE ECONOMIC COMMISSION FOR LATIN AMERICA AND DEPENDENCY THEORISTS}

\section{RA UL PREBISCH}

The distinction between center and periphery in the world economy was originally made by Raul Prebisch ${ }^{78}$, who later became head of United Nations' Economic Commission for Latin America(ECLA).

To Prebisch, the causes of Latin American underdevelopment were to be found in the system of international free trade. He supported theoretically the existing international division of labor, in which periphery had to specialize in the production of primary products and the center in the production of industrial goods. He also felt that this division of labor would result in the greatest possible 
advantage to all countries. However, his own empirical studies revealed that after 1880, while the terms of trade for Britain, which was an importer of primary products, improved, the terms of trade for the primary products exporting peripheries, deteriorated. Therefore, he concluded, the system was only beneficial to industrialized countries.

In addition, ECLA tried to present a complete theory of development which particularly emphasized the structural imbalances between center and periphery countries. To ECLA, there was a distinction between underdeveloped and undeveloped countries. Underdevelopment was thought to be the result of specific processes in the international trade system that led to underdevelopment in one part of the world and development in another.

Historically, the ECLA school of economic thought was at the center of the great debate that eventually led to dependency theory .

\section{DOS SANTOS AND THE STRUCTURE OF DEPENDENCE}

Unequal development, according to Dos Santos, must be seen as an integral part of the world capitalist system. Such a systemic inequality is an inevitable fact because center countries develop at the expense of the periphery by the transfer of the economic surplus through monopoly power in trade and financial relations 79 .

According to Dos Santos, dependence is:

A situation in which the economy of certain countries is conditioned by the development and expansion of another economy to which the former is subjected. The relation is such that some countries can expand and can be self-sustaining, while others can do this only as a reflection of expansion ${ }^{80}$.

79- T. Dos Santos, "The Structure of Dependence", in Readings in U.S. Imperialism edited by K.T. Fann, and D.C. Hodges, (Boston: Porter Sargent Publisher,1971), p. 225.

80- Ibid, p. 226. 
Dos Santos distinguishes three different forms of dependence ${ }^{81}$ :

1. There is colonial dependence based on the domination of the periphery's economy by a trade monopoly of European states.

2. There is financial-industrial dependence, which consolidated itself at the end of nineteenth century, and which has geared the economic structure of dependent nations to the needs of the center by the expansion of foreign capital investments in the production of raw materials and agricultural products for the consumption of center countries.

3. There is a new type of dependence which has emerged in the post-war era since 1945 based on multinational corporations which have begun to invest in industries geared to the internal market of the developing countries. This is technological-industrial dependence.

Dos Santos also maintains that dependency is not simply an external phenomenon; it also has to do with the supportive power groups within the poor countries themselves who find the status quo profitable:

The income inequality and conspicuous consumption of the wealthy classes, a dependency mentality and the ingrained habit of seeking outside help, and the unholy alliance between the domestic elite and foreign interests, all conspire to impede internal development.... If dependency defines the internal situation and is structurally linked to it, a country cannot break out of it simply by isolating itself from external influence; such action would simply provoke chaos in a society which is of essence dependent. The only solution therefore would be to change its internal structure, a course which necessarily leagds to confrontation with the existing international structure ${ }^{82}$.

81- Ibid, p. 227.

82- T. Dos Santos, "The Crisis of Development Theory and the Problems of Dependence in Latin America," in H. Bernstein (ed.), Underdevelopment and Development, (Harmondsworth: Penguin, 1973) 


\section{ANDRE GUNDER FRANK AND THE DEVELOPMENT OF UNDER DEVELOPMENT}

Andre Gunder Frank, a leading advocate for the school of dependency theory, argues that the relationship between the developed world and the underdeveloped world is inherently exploitative. That is to say, it leads to the continued en richment of some nations and the perpetual stagnation and poverty of other nations 83 .

Frank also states that "the present underdevelopment of Latin America and other underdeveloped regions is the result of their century-long participation in the process of world capitalist development ${ }^{84 n}$. Further, "underdevelopment of these regions was and still is generated by the very same historical processes which also generated economic development of the center nations ${ }^{85}$. To Frank, any industrial development in underdeveloped economies under center-periphery conditions will not break the cycle of the periphery's underdevelopment.

Using Brazil and Chile as case studies, Frank developed three hypotheses addressing the diachronic characteristics of the center-periphery structure:

1. Within a world-embracing center-periphery structure, the centers tend to develop and the peripheries to underdevelop ${ }^{86}$. Frank states that:

underdevelopment is not original or traditional and that neither the past nor the present of the underdeveloped countries resembles in any important respect the past of the now-developed

83- A.G. Frank, "The development of Underdevelopment" in Imperialism and Underdevelopment: A reader," ed. R.I. Rhodes, p. 8.

84- Ibid, p. 8.

85- Ibid, p. 9.

86- Ibid, p. 9. 
countries. The now-developed countries were never underdeveloped, though they may have been undeveloped 87 .

Consequently, he argues that underdevelopment in the peripheries is due to their integration into the world capitalist system.

2. Peripheries experience their greatest economic development and especially their most classically capitalist industrial development if and when their ties to their centers are weakest ${ }^{88}$.

According to Frank, this hypothesis suggests that:

the generally accepted thesis that development in the underdeveloped countries follows from the greatest degree of contact with metropolitan developed countries is not correct ${ }^{89}$.

Internationally, the classic example of industrialization through nonparticipation as a periphery in the capitalist world system is Japan after Meiji restoration. One may ask, why was resource-poor but unsatellized Japan able to industrialize so quickly at the end of the century, while resource-rich Latin American countries were not able to do so ${ }^{90}$ ? Frank suggests that the fundamental reason is that Japan was not satellized either during the Tokugawa or the Meiji period, and therefore did not have its development structurally limited as did the countries which were so satellized 91 .

3. Regions which are the most underdeveloped and feudal-seeming today are the ones which had the closest ties to the centers in the past. They are the regions which were the greatest exporters of primary products and the largest sources of

87- Ibid, p. 9.

88- Ibid, p. 10.

89- Ibid, p. 10.

90- Ibid, p. 11.

91- Ibid, P. 11. 
capital for the world center, and which were abandoned by the center when for one reason or another business declined. This hypothesis also contradicts the generally held thesis that the source of a regions' underdevelopment is its isolation and pre-capitalist institutions ${ }^{92}$.

\section{THE WORLD SYSTEM APPROACH IMMAN UEL WALLERSTEIN}

The World System Approach is usually associated with Immanuel Wallerstein. According to Wallerstein's perspective, there is an integrated world capitalist system existing in the twentieth century, encompassing even the socialist countries. Market forces integrate all countries, primarily through trade but also through the flow of capital and labor, generating and reproducing four interdependent parts: the core-states (corresponding to the dependency school's center, or metropolis), the semiperiphery, the periphery and the external arena. The semiperiphery consists of an intermediate but functionally important category of countries which are either turning into core states, or losing their status as such, and disappearing out into the periphery. The external arena are those countries that in the past were not incorporated into the European capitalist system, but in the long historical process of world integration were all incorporated or are being incorporated in the world system(peripheralization).

Wallerstein sees the world capitalist system, which originated around 1500 , going through four stages ${ }^{93}$ : first, the emergence of the European world economy that encompassed the Western European dominated parts of the world(1450-1640); second, the period of retrenchment caused by a systemwide depression

92- Ibid, p. 13.

1974)

93- I. Wallerstein, The Modern World System, (New York: Academic Press, 
characterized by the dominance of mercantilism and the emergence of a single dominant state within the system(1640-1730); third, the industrial revolution in which the European World system eliminated other world systems to incorporate the entire globe(1730-1917); fourth, the period of consolidation of the world capitalist system and the upsurge of revolutions(1917 to the present). Although it is only in the last two stages that the core has specialized in industrial goods in its trade with the periphery, in all phases the core has appropriated wealth from the periphery. Wallerstein is in agreement with Frank in believing that the European world has been capitalist since around 1500 and that there has been essential continuity in the direction of resource flow during the entire period.

In world-system studies the center-periphery relationship is central to the operation and development of the capitalist world economy ${ }^{94}$. Hopkins asserts that what is "ground" in dependency studies becomes "figure" in world system studies 95.

In historical terms, for world-system studies the center-periphery division and integration occur despite continual shifts in the areas and processes forming the system's center, semiperiphery, and periphery 96 .

\section{PREVIOUS TESTS OF DEPENDENCY THEORY}

The applicability of the center and periphery hypothesis to the world system has been partially examined in other empirical studies(Galtung 97 ,

94- T.K. Hopkins, World-System Analysis: Theory and Methodology (Beverly Hills: Sage publications, 1982), p. 20.

95- Ibid. p. 20.

96- Ibid. p. 21.

97- J. Galtung, "A Structural Theory of Imperialism," Journal of Peace Research, Vol. 8, No. 2, 1971. 
Gidengil $^{98}$, Snyder and Dick ${ }^{99}$ ). These studies can be divided into two categories: major studies on the center-periphery hypothesis, and studies that tested the hypotheses put forward by dependency theorists. Figures 1 and 2 give summarized description of these studies including the types of variables used, the methods employed, and a short description of the results.

Major Tests of Dependency Theory, Findings and Results

Galtung. In an article called "A Structural Theory of Imperialism," Galtung (1971) empirically tested the hypotheses that he and others had developed in terms of center and periphery dichotomy. His study was conducted for 61 countries, for the year 1967 using correlation analysis. His test incorporated four types of variables: (1) development variables of GNP/cap and percentage employed in nonprimary sectors; (2) inequality variables of Gini index of income distribution and Gini index of land distribution; (3) vertical trade variable of trade composition index; and (4) feudal trade variables of partner concentration index and commodity concentration index.

All correlations were in the expected direction, and most of them were rather substantial (for example the correlation coefficients obtained between EPC and VT, GNP/cap, and ECC was $-0.97,-0.89$, and 0.35 respectively). Galtung regarded that the hypotheses were very well confirmed. He suggested that the theory was rich and that it could provide ample opportunity basis for empirical research employing synchronic statistical methods as well as diachronic case

98- E. Gidengil, "Centers and Peripheries: An Empirical Test of Galtung's Theory of Imperialism," Journal of Peace Research, Vol. 15, No. 1, 1978.

99- D. Snyder, E.L. Kick, "Structural Position in the World System and Economic Growth 1955-70: A Multiple Network Analysis of Transnational Interaction." American Journal of Sociology 84(1979):1096-126. 


\begin{tabular}{|c|c|c|c|c|c|}
\hline Author & Variables used & Year & Countries & Method & Results \\
\hline Galtung: & $\begin{array}{l}\text {-Trade partner concentration } \\
\text { (defined as the proportion of } \\
\text { of trade with most important } \\
\text { partner) } \\
\text {-Trade commodity concentration } \\
\text { (defined as the proportion of the } \\
\text { three most important commodities } \\
\text { to the total export) } \\
\text {-G.N.P./capita, } \\
\text {-Vertical trade index, } \\
\text {-Gini of income distribution, } \\
\text {-Gini of land distribution. }\end{array}$ & 1967 & 61 & Correlation & Confirmed hyp. \\
\hline Gidengil: & $\begin{array}{l}\text {-Trade partner concentration } \\
\text { (Michaely index) } \\
\text {-Trade commodity concentration } \\
\text { (Michaely index) } \\
\text {-G.D.P./capita, } \\
\text {-Inequality (defined as percent di } \\
\text { of total product and total Labor }\end{array}$ & 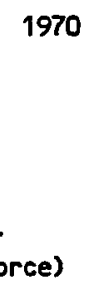 & 68 & $\begin{array}{l}\text { Cluster } \\
\text { Analysis }\end{array}$ & Confirmed hyp. \\
\hline Snyder: & $\begin{array}{l}\text {-Trade data(defined by exports), } \\
\text {-Military interventions, } \\
\text {-Number of diplomats, } \\
\text {-International treaties. }\end{array}$ & 1965 & 118 & $\begin{array}{l}\text { Block } \\
\text { Modeling }\end{array}$ & Confirmed hyp. \\
\hline
\end{tabular}

Figure 1. Studies of center-periphery hypothesis. 
studies. Galtung's study has been treated as the most comprehensive theoretical work conducted on the concept of the center and periphery dichotomy.

Gidengil. This study was conducted using 1970 data for 68 countries. using cluster analysis. The variables used were: (1) trade partner concentration; (2) trade commodity concentration; (3) GDP per capita; and (4) inequality (defined as percent distribution of the labor force in various economic sectors, i.e., agriculture, mining, manufacturing, etc.). Various cluster analysis techniques were used in operationalization of the variables used.

The study confirmed that certain countries can indeed be identified with Galtung's ideal types of center and periphery areas. The study identified one center group comprising twenty countries which were high on development measures, centrally located in the interaction structure, on top of the vertical interaction relation, and having relatively low disharmony of interest domestically. Then there were thirteen, predominantly Latin American, periphery countries which exhibited precisely the opposite pattern. The author suggested that repeating this type of analysis at regular intervals would make it possible to identify countries that were changing their position in the center-periphery network and could provide a fruitful source for theories about the dynamics of imperialism.

Snyder. Unlike the previous studies, this study put emphasis on the importance of non-economic transactions. Following Galtung's definition that the world system is essentially a joint function of economic, military, political, and cultural relations, the study attempted to derive the structural location of nations in a multidimensional setting. This study was conducted using block model analysis on 118 countries for the year 1965. The variables used were: (1) export data for considering the economic dimension; (2) military intervention data for considering the military dimension; (3) number of diplomats and international treaties data 
for considering the political dimension. Particular blocks were identified that could be interprated as center, semiperiphery, and periphery groupings, and thus confirming the existence of the center-semiperiphery-periphery structure in the world system.

These studies while supporting proposed ideas about center and periphery, suffered from certain weaknesses: First, the models used a small sample of nations. Second, although they all declared the usefulness of diachronic investigations, the studies were actually conducted for only one year, while one would ideally wish to see if such a pattern existed over a period of several decades. Also, the variables used in these models were not at best ideal: the variables that Galtung used to measure the trade partner concentration and export commodity concentration were based on the amount of trade with one partner or the amount of trade in only a few commodities. Therefore significant information about the countries' trade structures was lost. Although the Gini index of land and income distributions are good measures of inequality, these measures depended heavily on data provided by individual countries. Thus, lack of accurate data for many countries limit their usefulness, especially for diachronic studies. In addition, Gidengil's use of intersectoral income inequalities instead of the Gini index also caused the exclusion of a few countries because of the lack of comparable data. Snyder's study failed to show any overall hierarchy in the world system except for the military dominance of the center countries. The lack of identification of an economic and trade hierarchy was a serious weakness of this study. Furthermore, a deficiency in the use of block modeling was the identification of the U.S.S.R, and the Eastern European countries alongside Cuba, Kenya, Iran, Lebanon, Jordan and Israel as semiperiphery countries, when according to the center periphery 
hypothesis, Cuba is expected to have been identified as a periphery to the U.S.S.R. and Eastern Europe.

\section{OTHER TESTS OF DEPENDENCY THEORY}

There have also been numerous studies that have attempted to test the external dependence of the periphery countries on particular center countries. Figure 2 summarizes the more important quantitative studies relating to the dependency theory. 


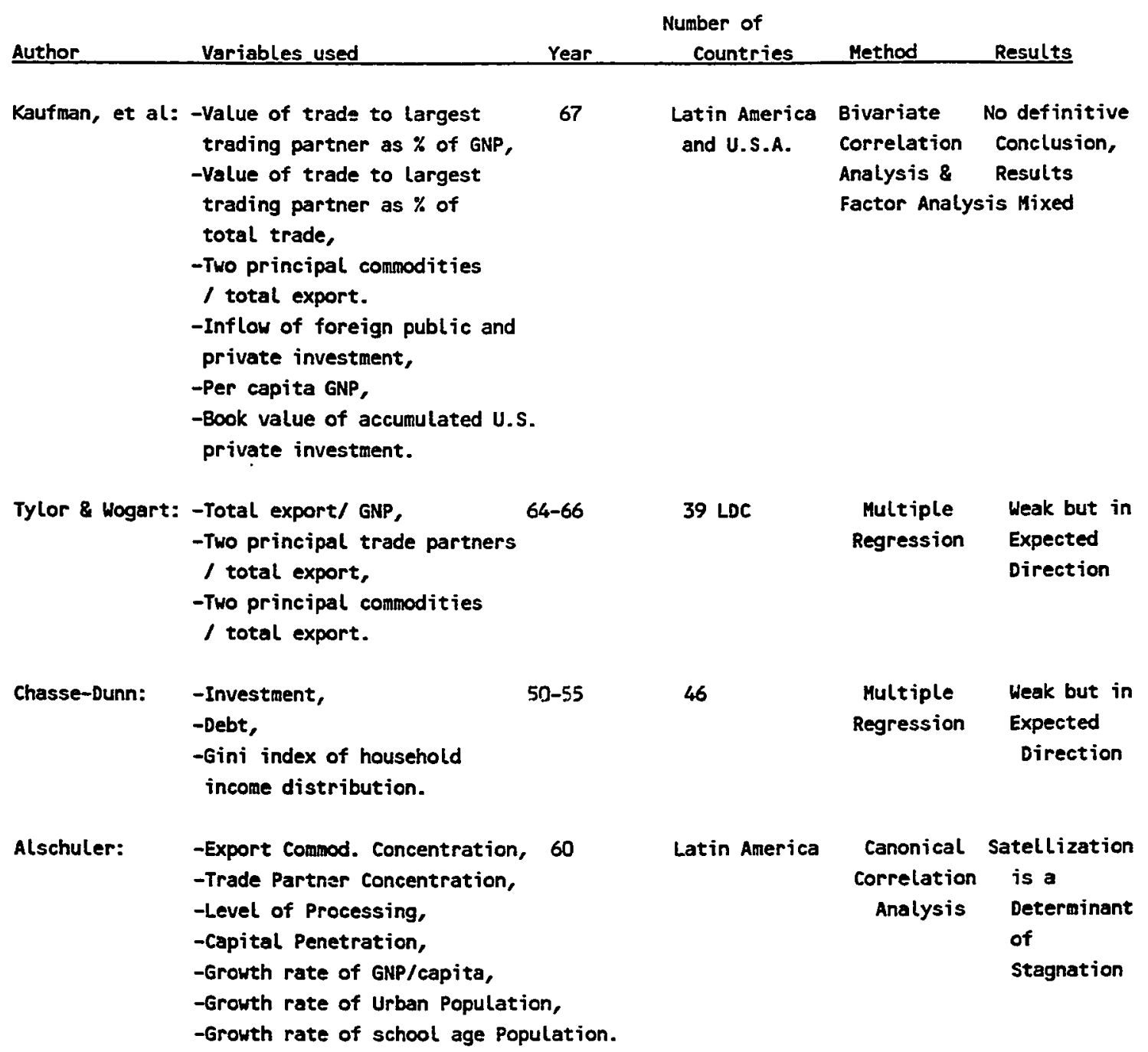

Figure 2. Previous tests of the dependency theory. 


\section{CHAPTER III}

\section{METHODOLOGY}

The following sections discuss the actual variables that are used and how they are operationalized (calculated from data sources listed below). The variables are divided into national and systemwide measures. The majority of these variables are based on trade statistics. Other variables use measures of national economy (G.N.P.), trade commodity structure, and development statistics. Many variables operationalized in this study are new to dependency theory and world systems analysis; for old variables new relationships are also tested. Table IV summarizes the variables that are operationalized in this study. Column one indicates the type of data used to derive these measures. Column two indicates the theory which the method of calculation is based on. Column three indicates if the variable or the measure was used in any previous study and column five provides information on the variables hypothesized direction of association with the measure of centralness or peripheralness.

\section{-OPERATIONALIZING THE NATIONAL STUDIES}

At the national level of investigation ten variables are operationalized. The overall goal is to see if the relationships among these variables are in the direction expected by the center-periphery hypotheses. The variables used for 1962 , 1970, and 1980 studies are: Export Partner Concentration (EPC), Total Trade Linkages (TTL), Import Partner Concentration (IPC), Export Commodity Concentration (ECC), Import Commodity Concentration (ICC), Vertical Trade (VT), 
TABLE IV

SUMMARY OF VARIABLES

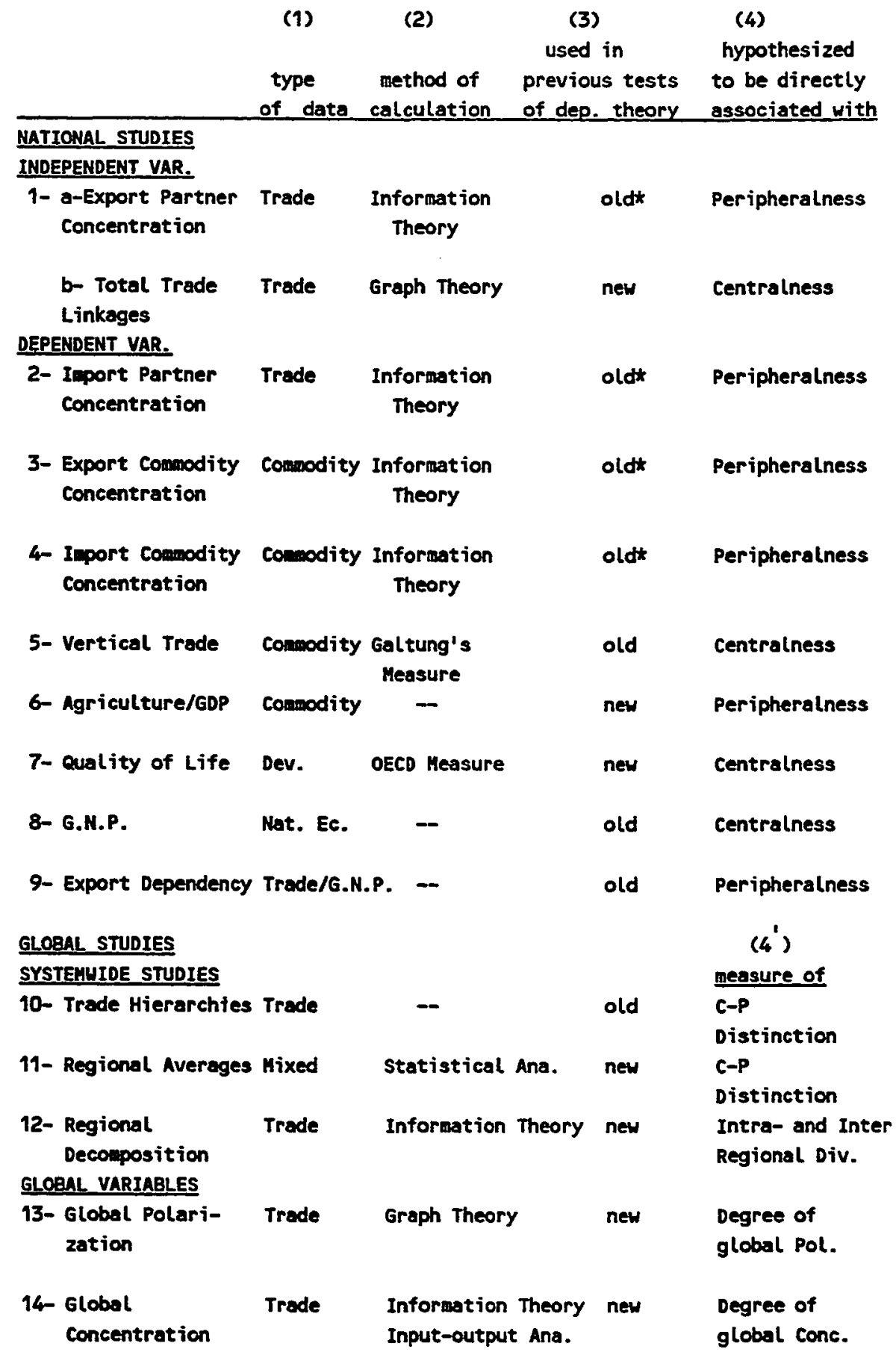

* - Although the measures have been used before, the information theoretic methods of calculations are new for this particular application. 
Agriculture/GDP, Physical Quality of Life (PQLI), G.N.P., and Export Dependency (ED). Variables used for the 1938 study are Export Partner Concentration(EPC), Import Partner Concentration(IPC), the Ratio of EPC to IPC (REI), total imports (TI), total exports (TE), the export share of largest partner (LPS), and Total Trade Linkages (TTL).

At the national level this study is focused on the relationships that exist among all of these variable. But since the center-periphery hypothesis views trade partner concentration as a leading factor in maintaining external dependence, this variable is used as the independent variable. It should be emphasized that the relationships studied here are not intended to suggest any particular causal relationships between any two variables. The independent/dependent variable distinction is employed only as a convenient tool for the analyses of the relationships among these variables.

\section{THE INDEPENDENT VARIABLE}

\section{Export Partner Concentration}

Center countries are defined as having greater multilateral export interactions. Periphery countries are said to have much fewer multilateral trade interactions than the center countries since center countries have historically limited the periphery countries' trade opportunities to their own markets. This aspect of feudal interaction is measured by doing entropy calculations to measure the concentration of trading partners of the countries under study. The greater the concentration, the more peripheral a country is said to be. Therefore, Export Partner Concentration is used as a measure of a country's peripheralness.

A common measure of concentration is the Gini-Hirschman index. The Gini-Hirschman index denoted by $C_{i x}$, is defined as the sum of the squared 
proportions of one country's exports and those of another country to the first country's total exports:

$$
c_{i x}=100 \sum_{j}\left(x_{i j} / x_{i}\right)^{2} \text {, }
$$

where $x_{i j}$ is the value of country i's exports to country $j$ 's, and $X_{i}$ represents the total value of exports of country $i$ to the world. Concentration by this method is a direct function of the relative inequality of dispersion and an inverse function of the number of countries.

An alternative concentration measure is the entropy coefficient (actually a diversity, rather than a concentration measure) which has been derived from information theory.

DIVERSITY $=-\sum \mathbf{P}_{\mathbf{i}} \log _{2} \mathbf{P}_{\mathbf{i}}$, where $\mathbf{P}_{\mathbf{i}}$ is the fraction of exports to country i.

CONCENTRATION= (DIVERSITY ${ }^{\text {max }}$-DIVERSITY $) /$ DIVERSITY $\max$

$$
=1-\left(\mathrm{H} / \mathrm{H}^{\mathrm{max}}\right)
$$

When $\mathrm{H}=\mathrm{H}^{\max }$ (maximum diversity) $\mathrm{C}=\mathrm{O}$

$$
\mathrm{H}=0 \quad \text { (minimum diversity) } \mathrm{C}=1
$$

The maximum value of the entropy measure for diversity is given by $\log _{2}(n)$, which occurs when exports are spread evenly over $n$ other countries. Its minimum value of zero occurs when all exports are concentrated in one country.

While the figures in appendix $C$ provide a correlation coefficient of .97 between entropy based concentration and Hirschman's Index of Concentration, the results are not identical( ordering of countries is not exactly same by the two measure.) However, because of the later use of information content of the global 
input-output analysis( Global Concentration) and the use of entropy in the decomposition analysis, entropy calculations are used as measures of concentration in this study.

\section{Total Trade Linkages}

An important structural effect of colonialism on the peripheries has been the monopolization of their trade by mother countries. Total Trade Linkages is calculated by the Point Centrality Index derived from graph theory ${ }^{1,2}$. In this manner, an index of TTL which measures the degree of centralness or peripheralness can be computed. The TTL Index calculates the number of export linkages that a country has. The lower this number, the more peripheral a country is said to be. Total Trade Linkages treats all trade ties equally(assuming trade to be greater than some threshold) while the Trade Partner Concentration index relies strongly on the number of countries and the volume of trade in calculations. If all trade volumes were to be equal, then $E P C=\log _{2}$ TTL. To consider a linkage between two countries a threshold value of 10,000 dollars is used.

\section{DEPENDENT VARIABLES}

\section{Import Partner Concentration}

It has also been stated that import partner concentration is another characteristic of periphery countries due to the fact that their markets have

1- Measures of Point Centrality are based entirely on the tendency of a single point to be more central than all other points in the network. Using Nieminen's measure the TTL index can be calculated as the count of the degree or number of adjacencies for a point, $P_{k}$ :

$\mathrm{C}_{\mathrm{d}}\left(\mathbf{P}_{\mathrm{k}}\right)=\sum_{\mathrm{i}=1}^{\sum_{1}} \mathrm{a}\left(\mathbf{P}_{\mathrm{i}}, \mathbf{P}_{\mathrm{k}}\right)$,

where $a\left(P_{i}, P_{k}\right)=1$ IFF $P_{i}$ and $P_{k}$ are connected else 0 .

2- J. Nieminen, "On Centrality in a Graph." Scandinavian Journal of Psychology, 15:322-336. 
historically been monopolized by the center countries. The method of calculation for this variable is same as for Export Partner Concentration.

\section{Export Commodity Concentration}

Periphery countries are said to be dependent on exports of only a few commodities whereas the center countries export many different commodities. This variable is a measure of how concentrated a country's exports are.

The degree of dependence of a country on a few commodities for export is measured by entropy calculations in a manner similar to that indicated for trade partner concentration. The larger the measure, the more a country's export trade is concentrated in fewer commodities. Five commodity sectors ( 3 raw material and 2 manufacture) were considered in these calculations: Food and beverages, Nonfood agriculture, Fuels, minerals, and metals, Machinery and equipment, and other manufactures. While it is desirable to calculate diversity by including more sectors( such as United Nations data which is given in 12 sectors), reluctantly, the World Bank's data is used instead since data is available for more countries.

\section{Import Commodity Concentration}

It is expected that the more concentrated the exports, the more diversified will be the imports, since a high concentration of exports indicates a high degree of specialization in production which means, a large variety of goods will need to be imported ${ }^{3}, 4$. Therefore it can be expected that center countries import a smaller

3- M. Michaely, Concentration in International Trade (Amsterdam: North-Holland Publishing Company, 1967), p.17.

4- J. Galtung, "A Structural Theory of Imperialism," Journal of Peace Research, Vol. 8, No. 2, 1971, p. 81. 
range of commodities than periphery countries ${ }^{5}$. This measure was similarly derived by entropy calculations. Six commodity sectors (4 raw material and 2 manufacture) are considered in these calculations: Food and beverages, Nonfood agriculture, Fuel and lubricants, Nonfuel minerals and metals, Machinery and equipment, and other manufactures.

The high degree of aggregation in commodity data for both imports and exports dictated that caution be used in interpretation of results since great diversity within any of these categories was completely ignored.

\section{Vertical Trade}

According to the center-periphery model there is an international division of labor. At the top of this structure are industrialized center countries which transform their raw material imports into manufactured products for exports. At the bottom of this structure are material producing peripheral countries which export their unprocessed mineral and agricultural commodities in order to pay for manufactured goods which they must import.

Galtung's index of vertical trade is used to locate a nation's position in the international division of labor:

\section{VERTICAL TRADE INDEX $=[(A+D)-(B+C)] /[(A+D)+(B+C)]$}

Where:

$$
\begin{aligned}
& \mathbf{A}=\text { value of raw materials imported, } \\
& \mathbf{B}=\text { value of raw materials exported, } \\
& \mathbf{C}=\text { value of processed goods imported, }
\end{aligned}
$$

and $D=$ value of processed goods exported.

5- W. Leontief, "The Structure of Development," Scientific America. September:1963, p.164. 
This indicator varies from -1 to +1 . The higher the value of the index, the higher the position of a country in the international division of labor.

\section{Economic Dependence on Agriculture}

The extent to which a country's economy is oriented to agriculture was also expected to be associated with the degree of peripheralness. The greater the share of agriculture in a country's GDP, the more peripheral that country is likely to be. The association of economic dependence on agriculture is an alternative indicator of the level of processing in any given country and is closely related to Galtung's vertical trade index.

\section{Physical Quality of Life Index}

While per capita GNP can measure the general economic performance of countries, it is unable to show how output is distributed among people in poor countries. If societies are to improve their ability to increase per capita income ( in the broadest sense), they must undergo substantial structural transformations that cannot be limited to economic matters; major economic, social, and political changes need to complement one another. $6,7,8$

A single quantitative measure that can capture the complexity and subtlety of the basic needs concept for a single country must be non-

6- McGranahan, et al. Contents and Measurement of Socioeconomic Development (N.Y.: Praeger Publishers, 1972)

7- I. Adleman, C.T. Morris, Society, Politics and Economic Development (Baltimore: John Hopkins Press, 1971)

8- H. Chenery, M. Syrquin, Patterns of Development 1950-70 (London: Oxford Univ. Press, 1975) 
ethnocentric $^{9}$. This excludes the use of measures based on value systems, population structure, land ownership, employment, and housing which would introduce ethnocentric biases in the research 10 .

Excluding such variables leaves at least three variables which do not exhibit any ethnocentricity ${ }^{11}$ : infant mortality, life expectancy, and basic literacy.

The PQLI is based on a simple indexing system. For each indicator, the performance of individual countries is placed on a scale of 0 to 100 , where 0 represents an explicitly defined 'worst' performance and 100 represents a 'best' performance ever observed for any country. Once performance for each indicator is scaled to this common measure, a composite index can be calculated by averaging the three indicators, giving equal weight to each of them. The resulting PQLI is thus scaled automatically on an index of 0 to $100^{12}$. In rare instances, however, it is possible to obtain a PQLI index which is outside this range, since these "worst" and "best" performances are based intuitively by the authors upon their observed samples and any individual country could conceivably achieve greater literacy, life expectancy or infant mortality than is expected.

The quality of life index produces a hierarchical structure of the world based on the level of well being of the people of each country.

\section{GNP Per Capita}

The Gross National Product is defined as a dollar flow of total product for a nation: it is the sum of consumption plus investment (foreign and domestic) plus

9- D.M. Morris, Measuring the Condition of the World's Poor (N.Y.: Pergamon Press, 1979), p. 26.

10- Ibid, P. 26.

11- Ibid, p. 31.

12- Ibid, P. 109. 
government expenditures on goods and services. Dividing this figure by the population of each country, the per capita GNP can be obtained, which is a better measure for this study than the GNP. This figure is calculated by all governments and therefore no calculations are necessary.

\section{Export Dependency}

This measure of economic dependence is the percentage of a nation's Gross Domestic Product(GDP) represented by exports to the largest trading partner. There exists a high level, medium level, and a low level of external dependency if respectively, more than 10 percent, between 5-10 percent, and below 5 percent of a country's GDP is accounted for by the exports to its largest trading partner. These cutof $f$ points have been suggested by Richardson ${ }^{13}$ and Singer ${ }^{14}$. These thresholds have no particular theoretical origin but it does satisfy the need for an empirical criterion detecting the presence or absence of a dominant trade partner.

\section{OPERATIONALIZING THE SYSTEM-WIDE STUDIES}

In this section the regional and global variables are discussed: (1) trade hierarchies are constructed based on the percent share of exports to the largest export partner for each country; (2) regional averages are calculated for all variables studied; (3) regional diversities based on decomposition analysis that uses import/export activity of countries are calculated; (4) two measures of Global Polarization and Systemic Concentrations that calculate the degrees of world

13- N.R. Richardson, "Political Compliance and U.S. Trade Development." American Political Science Review, vol. 70, No. 4, 1976, pp. 10981109.

14- M. Singer, Weak States in a World of Powers: The Dynamics of International Relationshios ( New York: Free Press, 1972) 
system centralization and concentration are discussed; and, (5) global averages for all variables are calculated.

\section{Trade Hierarchies}

An important property of the center-periphery relationship is the existence of international trade hierarchies. Trade hierarchies are based on the fact that trade relationships among nations are not equal. An important characteristic of trade hierarchies lies in their ability to identify countries that are "dominant" or are "dominated". A dominated country is one whose exports are primarily concentrated to one country or to a few. A dominant country, on the other hand, is one that serves as major partner for several other countries but, itself has low export partner concentration, and thus is not dependent on other countries as much as the dominated ones. Therefore, trade hierarchies can be regarded as another indicator of center and periphery distinction. In such hierarchies, center countries are identified as countries whose dependence on any country as an export market is not high while periphery country's exports are primarily directed at one or a few center countries.

In order to obtain international trade hierarchies, the per cent export share of all countries to their largest export partner is calculated and then trade hierarchies for each dominant country are drawn graphically.

\section{Regional Averages}

Calculations of regional averages are based on the division of the world into seven distinct regions: African countries, Asian countries, Eastern European countries, Developing European countries, Latin American countries, Middle Eastern countries, and Industrialized European countries with Japan and the United States included. 
To calculate the characteristics of each region, national variables are averaged for all the countries in the region. Comparisons are done (a) for each region between the averages at different times $(1962,1970,1980)$ and (b) between each region and the industrialized region for the averages at each time. In addition, (c) global averages at different times are also compared.

To conduct these difference of means tests, two different methods are utilized:

1- For comparing regions with the industrialized region, the Student-t distribution (for small samples) is used. in this case the null hypothesis is stated as follows:

Null Hypothesis: The difference of means is zero.

Alternate Hypothesis: The difference of means is not zero.

The statistic for conducting a significance test is given by:

$t=\left(\bar{x}_{1}-\bar{x}_{2}\right) \sqrt{\frac{\left(n_{1}-1\right) s_{1}^{2}+\left(n_{2}-1\right) s_{2}^{2} 1}{\left(n_{1}-1\right)+\left(n_{2}-1\right)} \frac{1}{n_{1}} n_{2}}$

where, $x_{1}$ and $x_{2}$ are the two sample means, $n_{1}$ and $n_{2}$

are sample sizes, and $S_{1}$ and $S_{2}$ are the sample standard

deviations.

This is compared with the Student-t distribution for $\left[n_{1}+n_{2}-2\right]$ degrees of freedom, for a two tailed test, at a 0.05 significance level.

2- For comparing regional or global averages at two different times, the paired ttest is utilized. The mean and the standard deviation of the differences between each pair of related values are calculated and then a t-test is applied. In this case the null hypothesis is constructed as follows:

Null Hypothesis: The average difference is zero.

Alternate Hypothesis: The average difference is not zero.

The $t$ statistic is: 


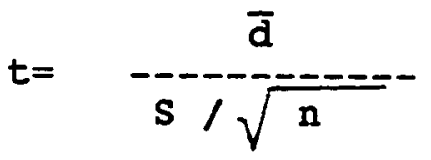

where, $n=$ number of pairs; $d=$ mean of the difference;

$S=$ standard deviation of the differences.

This is compared with the Student t-distribution for (n-1) degrees of freedom, for a two tailed test, at a 0.05 significance level.

The paired t-test assumes that the two samples are selected from a population whose variance is the same at the two sampling times. In order to see if the population variance is indeed constant, the F-test is conducted. The null hypothesis is that the population variance at the two times are equal. The alternate hypothesis is that the two variances are not equal. This test is applied to the variances for the global averages (for 127 countries) of the national variables. If the null hypothesis for the F-test is rejected, the paired t-test is not conducted. (In such cases, a non-parametric test could be conducted, but such tests are not done in this study.)

\section{Regional Diversity Decomposition Analysis}

While previous measures operationalize the national variables and the system-wide structural indicators, this measure considers the structure of world trade at the regional level.

This regional study is designed to reveal for import and export activities: the level of diversity within the regions ( referred to as the "within-set" diversity), the level of diversity between these regions( "between-set" diversity), and the level of economic diversity for the entire world. 
While the within-set diversity measures the diversity of each region independently, the between-set measure identifies the extent to which exporting activity is equally distributed among the seven regions. The relative weight of each of the regions determine the contribution of its diversification to the degree of export diversification in the whole world.

Diversity measures are normalized by dividing them by their maximum possible values; this neutralizes the effect of the number of categories(regions, nations) on the diversity calculations. Normalized diversities thus represent essentially measures of homogeneity, e.g. a region with high normalized export diversity has export activity nearly uniformly distributed among its member nations.

The data used in the calculation of regional diversities is import and export data from each country in the study. Decomposition of entropy into between-set and within-set components have been utilized previously in other studies 15 .

The entropy indicies of diversity are as follows:

Let $E_{i}$ be the amount of export of country $i$ in region $r$, where $i=1, \ldots, n_{r}$, where $n_{r}$ is the number of countries in regions, and $E_{r}$ is the total for region $E$. Then within-set entropies were calculated using the following formula:

$$
\begin{aligned}
& \text { UITHIN-SET } r \\
& \text { Let each region's relative share of the total global exports be } E_{r} / E_{G} \text {, }
\end{aligned}
$$
where $E_{G}$ is the total global exports.

The average within-set entropies were then calculated by:

15- H. Theil, Economics and In formation Theory (Chicago: North Holland Publishing Company, 1967) 


$$
D(W)=A \text { VERAGE WITHIN-SET }=\sum_{r=1} E_{r} / E_{G} * \text { WITHIN-SET } r
$$

The normalized within-set entropies were calculated by:

$$
\begin{aligned}
\text { NORHALIZED WITHIN-SET } & =\text { UITHIN-SET } r / \text { UITHIN-SET } r \max \\
& =\text { UITHIN-SET }_{r} / \log _{2}(n r) \text { (3) }
\end{aligned}
$$

where, nr was the number of countries in region $\mathrm{r}$.

The entropy measure of diversification for the between-sets was then expressed as:

$D(B)=B E T H E E N-S E T=-\sum_{r=1} E_{r} / E_{G} \log _{2} E_{r} / E_{G}$

The normalized between is expressed as:

MORHALIZED BETUEEN-SET= BETUEEN-SET/ $\log _{2}(n)$

The entropy measure of economic diversity for the entire world was then obtained by:

$$
=-\sum_{r=1}^{D=D(B)+D(H)} E_{r} / E_{G} \log _{2} E_{r} / E_{G}+\sum_{r=1} E_{r} / E_{G}\left[-\prod_{i=1} E_{i} / E_{r} \log _{2} E_{i} / E_{r}\right]
$$

Replacing the export data with import data, equations 1 through 6 were used to calculate regional diversities of imports.

\section{Global Polarization}

The degree of world system centralness depends on the tendency of a single country to be more central than all other countries in the trade network. Measures of this type are based on differences between centrality of the most central country and that of all others. 
A graph theoretic formula for determining the centrality of a network is ${ }^{16}$ :

$C N=\frac{\sum_{i=1}\left[C\left(P^{\star}\right)-C\left(P_{i}\right)\right]}{n^{2}-3 n+2}$

where $C\left(P_{i}\right)$ is the centrality of point, $P_{i}$, where $P^{*}$ is the most central point, and where $n$ is the number of points in the network.

Measures of point centrality, $C$, are based entirely or in part on the tendency of a single point to be more central than all other points in the network. Nieminen's 17 measure is the count of the degree or number of adjacencies for a point, Pk. Thus: $C(P k)=\sum a(P i, P k)$, where $a(P i, P k)=1$ if, and only if, $P i$ and Pk are connected; else 0 .

A given point, $\mathrm{Pk}$, can at most be adjacent to $\mathrm{n-1}$ other points in a graph. The maximum of $\mathrm{C}\left(\mathrm{Pk}^{*}\right)$, therefore, is $\mathrm{n-1}$ for a point that is adjacent to all other points.

If the graph is a star, each of the other points will have $C(P i)=1$ and the differences will be (n-1)-1=n-2 for each of the $n-1$ comparisons. Thus, the difference sum will be $(n-2)(n-1)$ for the star. Thus, from the perspective of degree, the star is the most centralized graph. It yields the largest difference sum, $n^{2}-3 n+2$. A link is sàid to exist between two countries if, and only if, there is more than $10,000.00$ dollars of trade between them.

\section{Global Concentration}

An approach to measure the degree of global centralization is to treat it as a systemic concentration, i.e., as a property of the distribution of trade behavior over

16- L.C. Freeman, "Centrality in Social Networks: Conceptual Clarifications." Social Networks, 1 (1978-79): p. 215-39.

17- J. Nieminen, "On Centrality in a Graph." Scandinavian Journal of Psychology, 15:322-336. 
the entire globe. According to this approach, centralization increases as trade become increasingly concentrated among fewer and fewer countries. This measure is different from the previous measure. While Global Polarization concentrates on the tendency of a nation to be more central than the others, the Global concentration is interested more on the distribution of trade activities in the entire trade network.

The information content of the input-output table of international trade is used to measure the Global Concentration.

Let $\mathrm{Xij}$ be the amount of import (or export) from nation $\mathrm{i}$ to nation $\mathrm{j}$, and let $\mathrm{T}=\sum \sum \mathrm{Xij}$ be the total trade flows in the matrix. We can normalize the original input-output matrix of trade flow to form a bivariate probability distribution(Pij) matrix (Figure 3 ) where $P i j$ is calculated as: $P i j=X i j / T$, for $i, j=1, \ldots, n$, as internation flows.

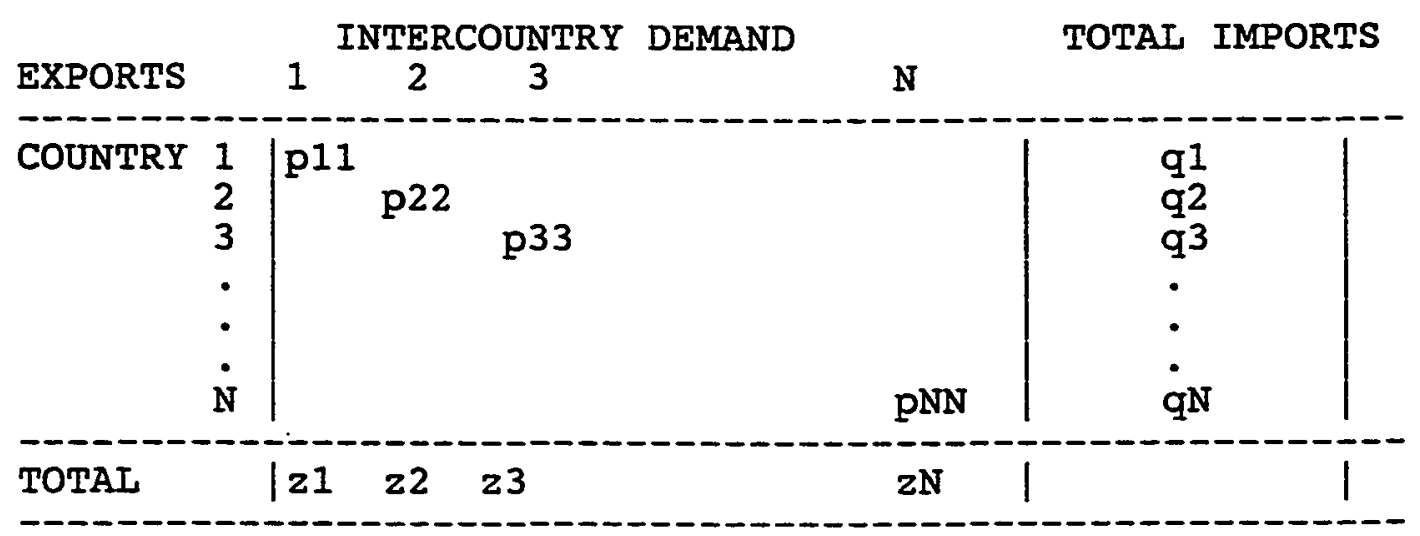

Figure 3. Static input-output table of international trade flows.

Now the expected mutual information of the bivariate array( $\mathrm{Pij}$ ) can be written as:

$$
\mathrm{u}=-\sum \sum \text { pij } \log _{2} \text { pij, }
$$


This is called the information content of the input-output table ${ }^{18}$. This measure (6) is Shannon's measure of uncertainty. The maximum $U$ is obtained when all nations have equal amounts of trade and export to all other nations. A measure of systemic concentration (C) can be defined: $C=1-\left(U / U_{\max }\right)$. $C$ increases as the observations become increasingly concentrated into fewer nations. This variable, however, does not reveal whether a center-periphery structure exists among the trading nations; it merely measures the number and relative strength of trade interactions.

\section{DATA SOURCES FOR CALCULATIONS}

This section offers information about the major sources for the data used in this research study. Table $V$ shows the sources of statistics on international trade and other data needed for this study. Table VI shows the particular data source (as listed in table V) for each of the variables used in this study.

While the number of countries used in calculating each measure were 64 for 1938, 127 for 1962, 142 for 1970, and 147 for 1980, correlation analyses for 1962, 1970, and 1980, used only 127 countries that were common to all those years. In doing so, the correlation results and their longitudinal comparisons could be made more accurately than with results obtained from various sample sizes.

\section{PRESENT ST UDY}

\section{NATIONAL STUDIES / TEST OF THE CENTER-PERIPHERY IDEA}

Dependency theory asserts particular relationships among variables, and these relationships are tested in this study by bivariate correlation calculations.

18- H. Theil, Economics and Information Theory (Chicago: North Holland Publishing Company, 1967) 
There are ten major national variables of which eight are dependent. The independent variables are: (1) Export Partner Concentration, and (2) Total Trade Linkages which is closely related to the first variable. Figure 4. is a summary matrix of the expected correlations among these variables. In listing the national hypotheses, only hypotheses relating the independent variable, Export Partner Concentration (E.P.C.), to other variables are listed. However, other hypotheses shown in the correlation matrix are also tested in this study.

The following hypotheses are formulated in terms of relation to centralness, but since some variables measure properties expected to be associated with peripheralness, those related hypotheses are rephrased where necessary.

TABLE V

STATISTICAL SOURCES

a)- DIRECTION OF TRADE source: International Monetary Fund

b)- THE WORLD TABLES source: The World Bank

c)- NETWORK OF WORLD TRADE source: The League of Nations

d)- THE UNITED STATES AND WORLD TRADE source: Green, T.R. and Lutz, J.M. 
TABLE VI

\section{VARIABLES AMD THEIR DATA SOURCES}

\begin{tabular}{l|c} 
VARIABLES & DATA SOURCES \\
\hline 1a- EXPORT PARTNER CONC. & $\mathrm{a}, \mathrm{c}$ \\
1b- TOTAI TRADE IINKAGES & $\mathrm{a}, \mathrm{c}$ \\
2- IMPORT PARTNER CONC. & $\mathrm{a}, \mathrm{c}$ \\
3- EXPORT COMMODITY CONC. & $\mathrm{b}$ \\
4- IMPORT COMMODITY CONC. & $\mathrm{b}$ \\
5- VERTICAI TRADE & $\mathrm{b}$ \\
6- AGRICULTURE/GDP & $\mathrm{b}$ \\
7- QUAITT OF LIFE & $\mathrm{b}$ \\
8- G.N.P./CAPITA & $\mathrm{b}$ \\
9- EXPORT DEPENDENCY & $\mathrm{d}$ \\
10- TRADE HIERARCHIES & $\mathrm{a}$ \\
11- REGIONAL AVERAGES & $\mathrm{a}, \mathrm{b}, \mathrm{c}$ \\
12- REGIONAL DECOMPOSITION & $\mathrm{a}$ \\
13- GLOBAI POLARIZATION & $\mathrm{a}$ \\
14- GLOBAL CONCENTRATION & $\mathrm{a}$
\end{tabular}

The correlation calculations examine the following hypotheses:

H1- Centralness is associated with diversification of import partners whereas peripheralness is associated with concentration of import partners. More specifically, the Export Partner Concentration(EPC) index is positively correlated with the index of Import Partner Concentration(IPC).

H2- Center countries are associated with a high number of export trade linkages with other countries whereas periphery countries are associated with fewer trade linkages. More specifically, EPC is negatively correlated with the index of Total Trade Linkages(TTL).

H3- Centralness is associated with the export of diversified commodities whereas peripheralness is associated with the export of only a few commodities. More specifically, the EPC index is positively correlated with Export Commodity Concentration(ECC).

H4- Centralness is associated with the concentrated importing of commodities (mainly raw materials) whereas peripheralness is associated with a diversity of commodity imports (mainly processed goods). More specifically, EPC is negatively correlated with Import Commodity Concentration(ICC).

H5- Center countries are expected to have a high position and periphery countries a low position in the international division of labor. More specifically, EPC will be negatively correlated with Vertical Trade(VT). 
H6- Center countries have better living conditions than periphery countries. More specifically, the EPC index is negatively correlated with the Physical Quality of Life Index(PQLI).

H7- Centralness is associated with a lower share of agriculture in a nation's GDP whereas the peripheralness is associated with a higher share of agriculture in GDP. More specifically, EPC index is positively correlated with the ratio of Agriculture/GDP(A/G).

H8- Center countries have high GNP/capita whereas periphery countries have a low GNP/capita. More specifically, the EPC index is negatively correlated with the GNP/capita.

H9- Center countries have lower export trade dependency whereas periphery countries have high export dependency. More specifically, the EPC index is positively correlated with Export Dependency(ED).

The relationships among national variables and their direction of correlations are summarized in Figure 4 below. The two independent variables are placed first and last in this figure to facilitate inspection in relation to other variables.

PATTERN OF CORRELATIONS ACCORDING TO HYPOTHESES

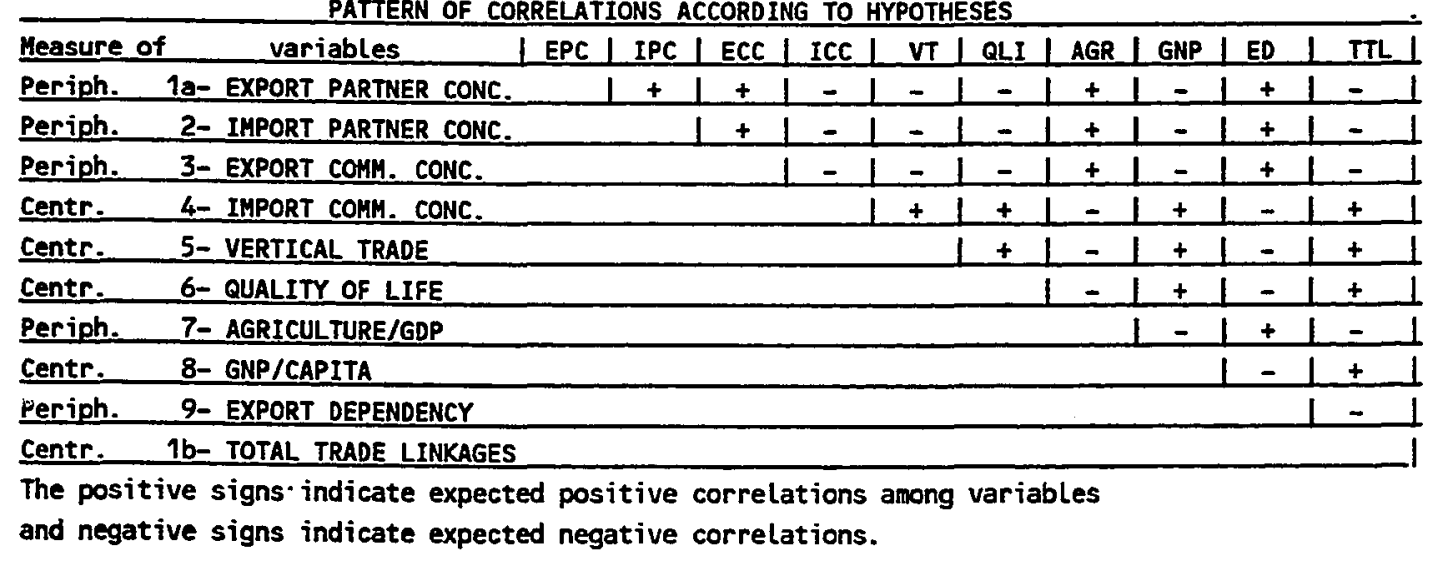

Figure 4. Correlation analysis.

These hypotheses are tested using the Pearson Correlation (PEARSON CORR) routine of the Statistical Package for the Social Sciences(SPSS). This routine computes a one tailed test of Pearson Product-Moment correlations for 
pairs of variables. Significance tests are reported for each coefficient and are derived from the use of Student's t-test with N-2 degrees of freedom.

\section{NATIONAL STUdIES / DIACHRONIC INVESTIGATIONS}

In addition to testing specific hypotheses associated with the distinction between center and periphery nations, longitudinal studies are done to examine other aspects of dependency theory and world system analysis.

The previous testing of hypotheses are done for three different years, but essentially those tests are a synchronic study, i.e. those tests are not concerned with trends in the correlations, except a little. Data is made possible to carry out longitudinal studies for individual nations. As an example, a longitudinal look at South Korea is provided.

\section{SYSTEM-WIDE STUDIES / GLOBAL HIERARCHIES}

Dependency theory and world system analysis assert the existence of a world system with a particular structure. Yet, previous studies have only focused on the national level and have not addressed the question of the existence (and properties) of such a world system. In this part of study, the idea of an overall global system is examined to see if the assertions of dependency theory and the world system school could be supported.

It has been claimed that one of the most important properties of the centerperiphery relationship is the hierarchical nature of the international trade system in which all peripheral countries are linked to only a few center countries which are at the top of the trade hierarchies. Often the bulk of a periphery nation's trade is tied to a particular center country which produces, by definition, a condition of dependency of the periphery nation on its center country. The relationship among 
center countries is classified as one of interdependence in which their level of dependence on each other is mutual. To test the preceding assertions that were made about the international trade system, the total share of trade interaction within and between each group, was calculated and compared for each year of the study. Therefore, the trade hierarchies based on the percent share of trade among countries were constructed.

\section{SYSTEMWIDE STUDIES/ REGIONAL ANALYSES}

Regional studies are carried out by two different approaches: (1) national data are used to calculate regional averages. In doing so, each region's characteristic is compared to other regions for each national variable; and, (2) entropy diversities among and within each region of the world are calculated for the years of 1962,1970 , and 1980 , so that the diachronic changes could be examined.

\section{SYSTEMWIDE STUDIES/ GLOBAL ANALYSES}

Global analyses are also divided into two distinct studies: (1) Global averages are calculated for each national variable and their results are analyzed; and (2) Global Polarization and Global Concentration are calculated and results are analyzed. These analyses provide information about the diachronic characteristics of the world system and allow to determine whether polarization in the world system has increased from 1938 to the present (i.e. to see if the systemic concentration has been increasing). 


\section{CHAPTER IV}

\section{RESULTS OF THE NATIONAL STUDIES}

\section{DISCUSSION OF CORRELATION RESULTS}

The hypotheses listed in the theoretical section of this research suggest particular relationships among the dependency measures. In brief, we expect that some countries, which would commonly be regarded as "developed", would be on the top of what Galtung calls the vertical trade but that they would be low on feudal interaction, namely, trade partner concentration and trade commodity concentration. Other countries, commonly regarded as "undeveloped", would be low on the vertical trade but high on the feudal interaction index.

There are ten major national variables of which eight are treated as dependent. The two independent variables chosen for this study are the Export Partner Concentration (EPC), and the Total Trade Linkages (TTL). TTL measures the number of export partners of individual countries without considering the trade volume. While TPC is a measure of peripheralness, TTL measures the degree of centralness. .

To calculate the bivariate Pearson correlations between these ten variables, 127 countries are selected. Data for these countries are available for all the years selected for this study.

Figure 5 presents the Pearson correlation coefficients ( $r$ ) measuring the association between the previously discussed variables ( see Figure 3 for expected signs of correlations and abbreviation of variable labels). A Second set of correlation calculations that excludes the oil producing countries, is also conducted 


\begin{tabular}{|c|c|c|c|c|c|c|}
\hline \multirow[b]{2}{*}{ Variables } & \multicolumn{6}{|c|}{ YEAR $=1938$} \\
\hline & 1 EPC $\mid I P C$ & $\perp$ REI & $1 T I$ & $\perp \mathrm{TE}$ & $\perp$ LPS & $\perp \mathrm{TTL}$ \\
\hline EPC & 1.85 & $1-.32$ & $1-.49$ & $1-.53$ & 1.95 & $1-.86$ \\
\hline IPC & & $1-.18$ & $1-.52$ & $1-.54$ & 1.78 & $1-.78$ \\
\hline REI & & & 1.06 & 1.27 & $1-.17$ & 1.42 \\
\hline II & & & & 1.89 & $1-.41$ & 1.69 \\
\hline$\underline{T E}$ & & & & & $1-.43$ & 1.73 \\
\hline LPS & & & & & & $1-.72$ \\
\hline$\underline{T L}$ & & & & & & \\
\hline
\end{tabular}

\begin{tabular}{|c|c|}
\hline \multirow[b]{2}{*}{ Variables } & YEAR=1962 \\
\hline & 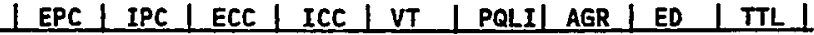 \\
\hline EPC & $1.85|.34| .42 *|-.57|-.48|.42| .57|-.76|$ \\
\hline IPC & $\perp .37|.53 *|-.46|-.53| .48|.50|-.65 \mid$ \\
\hline ECC & $\perp .48 *|-.44|-.32|-.25| .28|-.43|$ \\
\hline$\underline{\mathrm{ICC}}$ & $|-.63 *|-.63 *|. .66 *| .30 *|-.56 *|$ \\
\hline vT & $1.59|-.51|-.15|.81|$ \\
\hline PQLI & $|-.78|-.02|.58|$ \\
\hline AGR/GDP & $|-.15 *|-.53 \mid$ \\
\hline ED & $|-.38|$ \\
\hline \multirow[t]{2}{*}{$\underline{T T L}$} & \\
\hline & $\underline{\text { YEAR }=1970}$ \\
\hline Variables & $\perp E P C \perp I P C \perp E C C|I C C \perp V T| P Q L I \mid$ AGR $\mid$ ED $|T T L|$ \\
\hline EPC & 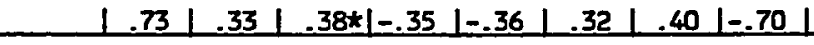 \\
\hline IPC & 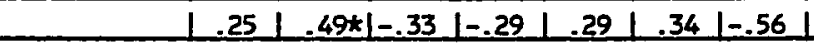 \\
\hline$\underline{\text { ECC }}$ & $|.47 *|-.44|-.34| .21|.40|-.38 \mid$ \\
\hline$\underline{I C C}$ & $|-.64 *|-.47 *|.31 *| .27 * \mid-.54 * 1$ \\
\hline vT & $\perp .55|-.49|-.00 \mid .66 \perp$ \\
\hline PQLI & $|-.74|-.03|.57|$ \\
\hline AGR/GDP & $|-.14 *|-.55 \mid$ \\
\hline ED & $|-.21|$ \\
\hline TIL & \\
\hline
\end{tabular}

\section{YEAR=1980}

\begin{tabular}{l|l|l|l|l|l|l|l|l|l|l|l|l|l|l|l|l|} 
Variables & EPC & IPC & $E C C$ & ICCE & VT & PQLI & AGR & ED & GNP & TTL \\
\hline
\end{tabular} \begin{tabular}{l|l|l|l|l|l|l|l|l|l|l|l|l|l|l|l|l|l|l|l|l|}
\hline EPC & .68 & .63 & $.43 *$ & -.50 & -.45 & .25 & .55 & -.33 & -.72 \\
\hline
\end{tabular}

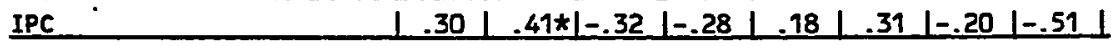
\begin{tabular}{l|l|l|l|l|l|l|l|l|}
\hline ECC & $.51 *|-.47|-.32|.02|-.45 \mid$ & $-.03|-.45|$
\end{tabular} ICC $\quad|-.68 *|-.43 *|-.01 *|-36 *|-.06 *|-.53 * 1$

\begin{tabular}{|c|c|}
\hline VT & \begin{tabular}{|l|l|l|}
$.54|-34|-.38|.28| .63 \mid$ & $|.28|$
\end{tabular} \\
\hline PQLI & $|-.66|-.08|.58| .66 \mid$ \\
\hline AGR/GDP & $|-.12 *|-.66|-.52|$ \\
\hline ED & $|.05 *|-.31 \mid$ \\
\hline GNP & 1.581 \\
\hline$\Pi \mathrm{IT}$ & \\
\hline
\end{tabular}

Figure 5. Summary of Pearson correlation results among variables. 
and its results are compiled in Appendix $C$. While the correlation coefficients of this set are in general higher than the coefficients found in Figure 5, thier results, except for GNP, are not discussed.

The results support the hypotheses advocated by the dependency theory school. For all the study years, all correlation coefficients between the independent variable and the dependent variables are in the expected directions, and are statistically significant at the 0.05 level. One variable that consistently gives results in a direction opposite to what is predicted is the Import Commodity Concentration. Results opposite to those expected are also found with the Export Dependency variable. An * is placed next to any correlation coefficient found to be in the opposite direction of what is predicted. These results are discussed below.

As a test of hypotheses, the findings provided positive confirmation of the center-periphery hypothesis. These results can not, of course, be taken to confirm all of the propositions made by these theories, more specifically, the propositions not explicitly tested in this study. It is not within the province of this study to ascertain whether and, if so, degree to which these findings could be assimilated into a neoclassical perspective. The point is that the tested relations are explicitly proposed by the dependency theories, these relations are confirmed by the present study, i.e., the results are found to be in the direction proposed by dependency theory and theory of structural imperialism as presented by Galtung and others.

The following sections of this chapter will review each hypothesis in turn and will also include detailed discussions on the diachronic characteristics of the variables over the study period. 


\title{
DIACHRONIC INVESTIGATIONS
}

\section{TRADE PARTNER CONCENTRATION}

\begin{abstract}
HYPOTHESIS- Centralness is associated with diversification of import partners whereas peripheralness is associated with concentration of import partners. More specifically, the Export Partner Concentration(EPC) index is positively correlated with the Import Partner Concentration(IPC).
\end{abstract}

As Figure 6 indicates, the correlation coef ficients between EPC and IPC are substantial and in a positive direction thus confirming the hypothesis. The correlation coefficient are 0.85 for $1938,0.84$ for $1962,0.73$ for 1970 , and 0.68 for 1980, suggesting that although the correlation coefficients for trade partner concentration appear to be decreasing, concentration has remained very strong for all four years sampled in the study.

Historically, the term "center countries" has referred to the so-called "mother countries" of Europe whereas the term "periphery countries" has referred to countries that at one time or another were European colonial possessions. In various eras in the history of the modern world under the old mercantile system or in the new age of imperialism of the late 1800 s, foreign trade of the periphery countries were limited to trade mainly with only one dominant center country (following Galtung's ideal type, in which a periphery country's interaction with other countries is missing). 


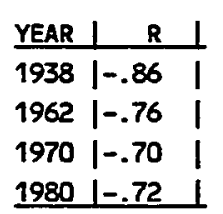

B- SUMMARY OF CORRELATION COEFFICIENTS

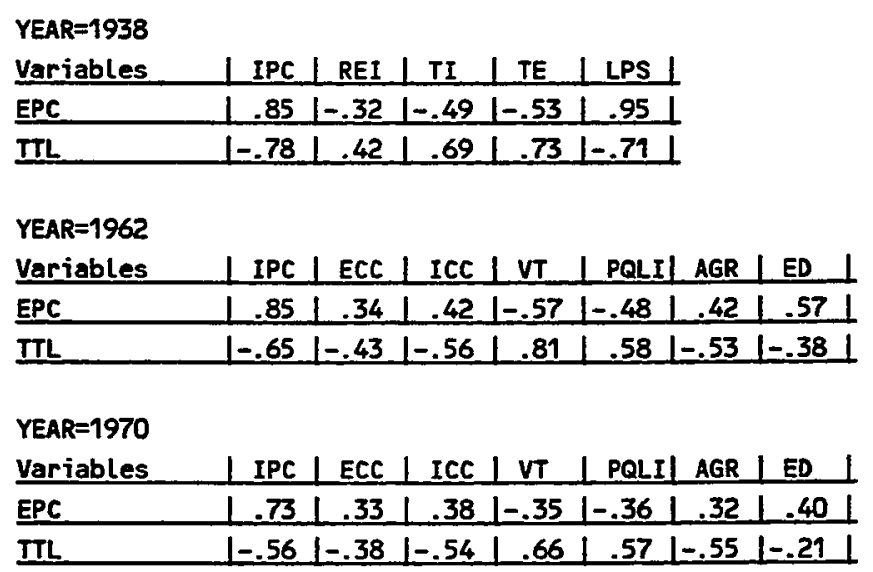

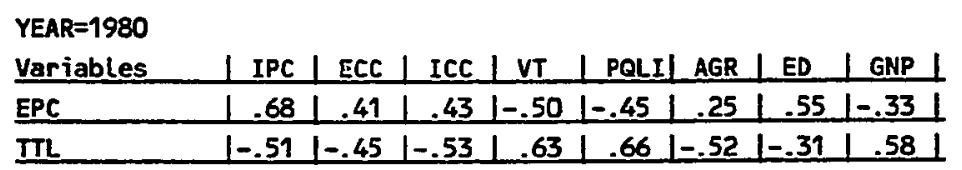

Figure 6. Corrolation coefficients between EPC, TTL, and other variables. 
For perfect concentration, export and import partner concentration for the periphery countries would have been close to 1.0. In evaluating how independent a periphery has become, it is necessary to show whether these variables deviated substantially or not from such perfect monopolization.

From the results of Table VII the following observations can be made: At the national level, while the value for average EPC for the top ten countries at the periphery end was .99 in 1962, for the center end this figure was as low as .30 . These figures for 1970 were 0.87 for the periphery end and 0.32 for the center end. In 1980 the figures were 0.79 for the periphery end and 0.30 for the center end. From these figures it seems that while at the periphery end the direction for export partner concentration has been towards more diversification, the magnitude of the changes has not been considerable, suggesting that concentration of export partners may be a permanent structural property for the periphery countries.

For import partner concentration the same figures were 0.96 and 0.34 for $1962,0.79$ and 0.35 for 1970 , and 0.77 and 0.34 for 1980. Although the averages for IPC were lower than those of EPC, the same general statement can be made for import partner concentration.

\section{TOTAL TRADE LINKAGES}

The most important structural effect of colonialism on periphery's economy was that of monopolization of the periphery's trade by the mother country. While center countries were free to trade with other countries, the periphery countries were limited to trading with only one center country. Therefore, the number of countries a center could trade with was much greater than the number a periphery had to trade with. Thus, a good indicator to determine if a country was a center or 
TABLE VII-A

COUNTRIES RANKED BY EXPORT PARTNER CONCENTRATION IN 1938

\begin{tabular}{|c|c|c|c|c|c|}
\hline & COUNTRY & EPC & & COUNTRY & EPC \\
\hline 1 & PANAYAA & 1.00 & 33 & MOROCCO & 0.58 \\
\hline 2 & FALKLAND & 1.00 & 34 & ECUADOR & 0.56 \\
\hline 3 & IRELAND & 0.92 & 35 & BULGARIA & 0.55 \\
\hline 4 & HONDORAS & 0.89 & 36 & FINLAND & 0.55 \\
\hline 5 & NEU ZEALAND & 0.84 & 37 & MALAYSIA & 0.51 \\
\hline 6 & ALGERIA & 0.83 & 38 & SPAIN & 0.51 \\
\hline 7 & BOLIVIA & 0.83 & 39 & TURKEY & 0.51 \\
\hline 8 & GUATEMALA & 0.80 & 40 & IRAN & 0.51 \\
\hline 9 & PHILIPPINES & 0.80 & 41 & BRAZIL & 0.50 \\
\hline 10 & cuan & 0.79 & 42 & URUGUAY & 0.50 \\
\hline 1 & EL SALVADOR & 0.76 & 43 & CHILE & 0.49 \\
\hline 12 & MICARAGUA & 0.75 & 44 & PORTUGAL & 0.48 \\
\hline 13 & COSTA RICA & 0.74 & 45 & GREECE & 0.48 \\
\hline 14 & JAMAICA & 0.74 & 46 & EGYPT & 0.48 \\
\hline 15 & THAILAMD & 0.73 & 47 & PERU & 0.48 \\
\hline 16 & TRIMIDAD & 0.73 & 48 & U.S.S.R. & 0.48 \\
\hline 17 & IRAO & 0.73 & 49 & YUGOSLAVIA & 0.47 \\
\hline 18 & COLOHBIA & 0.71 & 50 & ARGENTINE & 0.45 \\
\hline 19 & PARAGUAY & 0.71 & 51 & NORHAY & 0.42 \\
\hline 20 & HAITI & 0.70 & 52 & HUNGARY & 0.42 \\
\hline 21 & VENEZUELA & 0.69 & 53 & SHEDEN & 0.39 \\
\hline 22 & DOHINICAN R & 0.69 & 54 & ROHAINIA & 0.38 \\
\hline 23 & TUNISIA & 0.66 & 55 & NETHERLANDS & 0.38 \\
\hline 24 & CEYLON & 0.65 & 56 & POLAND & 0.37 \\
\hline 25 & S. AFRICA & 0.64 & 57 & AUSTRIA & 0.33 \\
\hline 26 & DENMARK & 0.64 & 58 & FRANCE & 0.32 \\
\hline 27 & NIGERIA & 0.64 & 59 & ITALY & 0.29 \\
\hline 28 & HOHG KONG & 0.63 & 60 & U.S.A. & 0.29 \\
\hline 29 & CHIMA & 0.63 & 61 & SWITZERLAND & 0.29 \\
\hline 30 & MEXICO & 0.62 & 62 & CZECHOSLOV & 0.28 \\
\hline 31 & AUSTRALI & 0.61 & 63 & U.K. & 22 \\
\hline 32 & CANADA & 0.61 & 64 & GERMANY & 0. \\
\hline
\end{tabular}


TABLE VII-B

COUNTRIES RANKED BY EXPORT PARTNER CONCENTRATION IN 1962

\begin{tabular}{|c|c|c|c|c|c|c|c|c|}
\hline & COUNTRY & EPC & & COUNTRY & EPC & & COUNTRY & EPC \\
\hline 1 & MARTINIQUE & 1.00 & 44 & HAITI & 0.68 & 87 & YEMEN, PEO & 0.50 \\
\hline 2 & oman & 1.00 & 45 & PHILIPPINE & 0.67 & 88 & ICELAND & 0.49 \\
\hline 5 & CAPE VERDI & 1.00 & 46 & EAST GERMA & 0.67 & ونة & BRAZIL & 0.48 \\
\hline 4 & MAURITANIA & 1.00 & 47 & FIJI & 0.65 & 90 & MOROCCO & 0.48 \\
\hline 5 & NEW CALEDO & 1.00 & 48 & COLOMBIA & 0.65 & 91 & IRAQ & 0.46 \\
\hline 6 & BENIN & 1.00 & 49 & NEW ZEALAN & 0.65 & 92 & BURMA & 0.45 \\
\hline 7 & GUADALOUPE & 0.99 & 50 & TUNISIA & 0.64 & 93 & MALAYSIA & 0.45 \\
\hline 8 & CENT. AFR. & 0.98 & 51 & NIGERIA & 0.64 & 94 & POLAND & 0.45 \\
\hline$?$ & BAHAKAS & 0.95 & 52 & CUBA & 0.64 & 95 & THAILAND & 0.45 \\
\hline & REUNION & 0.95 & 53 & AFGHANISTA & 0.64 & 96 & TURKEY & 0.44 \\
\hline & SENEGAL & 0.88 & 54 & IVORY COAS & 0.64 & 97 & DENMARK & 0.44 \\
\hline & MAURITIUS & 0.87 & 55 & EL SALVAdo & 0.63 & 98 & URUGUAY & 0.44 \\
\hline & NEW GUINEA & 0.87 & 56 & LIBYA & 0.63 & 99 & FIMLAND & 0.43 \\
\hline & SOMALI & 0.87 & 57 & CANADA & 0.63 & 100 & SUDNN & 0.43 \\
\hline & GAMBIA & 0.86 & 58 & NICARAGUA & 0.62 & 101 & SIMGAPORE & 0.42 \\
\hline & MIGER & 0.86 & 59 & QATAR & 0.62 & 102 & BELGIUM & 0.42 \\
\hline & MALI & 0.83 & 60 & TRINIDAD & 0.62 & 103 & ARGENTIMA & 0.42 \\
\hline & ALGERIA & 0.83 & 61 & CONGO & 0.62 & 104 & HOHG KONG & 0.42 \\
\hline & BARBADOS & 0.83 & 62 & CYPRUS & 0.61 & 105 & SAUDI ARAB & 0.42 \\
\hline & SIERRA LEO & 0.82 & 63 & VIET NAM & 0.60 & 106 & U.S.S.R. & 0.42 \\
\hline & GREENLAND & 0.81 & 64 & RUHANIA & 0.59 & 107 & AUSTRRLIA & 0.42 \\
\hline & IRELAND & 0.81 & 65 & BERMUDA & 0.59 & 108 & MORLAY & 0.41 \\
\hline & Lios & 0.80 & 66 & HUNGARY & 0.59 & 109 & IRAS & 0.41 \\
\hline & MACAO & 0.80 & 67 & MOZAMBIQUE & 0.58 & 110 & AUSTRIA & 0.41 \\
\hline & DOMINICAN & 0.78 & 68 & CZECHOSLOV & 0.58 & 111 & ISRAEL & 0.41 \\
\hline & BRUNEI & 0.78 & 69 & ETHIOPIA & 0.58 & 112 & SYRIA & 0.40 \\
\hline & CANEROON & 0.78 & 70 & JORDAN & 0.57 & 113 & NETHERLAND & 0.40 \\
\hline 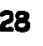 & CHAD & 0.77 & 71 & KUWAIT & 0.57 & 114 & GREECE & 0.39 \\
\hline & GABOH & 0.76 & 72 & ANGOLA & 0.56 & 115 & PORTUGAL & 0.39 \\
\hline 30 & MADAGASCAR & 0.75 & 73 & LEBANON & 0.56 & 116 & NETHERLAND & 0.39 \\
\hline & BOLIVIA & 0.75 & 74 & CHIMA & 0.56 & 117 & SPAIN & 0.38 \\
\hline & SURIMin & 0.74 & 75 & CHILE & 0.56 & 118 & PAKISTAN & 0.37 \\
\hline 33 & MALTA & 0.73 & 76 & GUINEA & 0.55 & 119 & EGYPT & 0.37 \\
\hline & ECUADOR & 0.72 & 77 & VENEZUELA & 0.55 & 120 & SUEDEN & 0.37 \\
\hline & COSTA RICA & 0.71 & 78 & TANZANIA & 0.54 & 121 & INDIA & 0.36 \\
\hline 60 & MEXICO & 0.71 & 79 & GHANA & 0.54 & 122 & YuGOSLAVIA & 0.36 \\
\hline & YEMEN, ARA & 0.70 & 80 & PERU & 0.53 & 123 & JAPAN & 0.32 \\
\hline 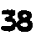 & HONDORAS & 0.69 & 81 & UGANDA & 0.52 & 124 & ITALY & 0.32 \\
\hline 39 & KOREA & 0.69 & 82 & PANAMA & 0.52 & 125 & SUITZERLAN & 0.32 \\
\hline & PARAGUAY & 0.69 & 83 & BAHREIN & 0.51 & 126 & FRANCE & 0.31 \\
\hline & BULGARIA & 0.69 & 84 & KENYA & 0.51 & 127 & GERMANY & 0.30 \\
\hline & JAHAICA & 0.68 & 85 & SOUTH AFRI & 0.51 & 128 & U.S.A. & 0.29 \\
\hline & guATEMALA & 0.68 & 86 & INDONESIA & 0.50 & 129 & U.K. & 0.22 \\
\hline
\end{tabular}


TABLE VII-C

COUNTRIES RANKED BY EXPORT PARTNER CONCENTRATION IN 1970

\begin{tabular}{|c|c|c|c|c|c|c|c|c|}
\hline & COUNTRY & EPC & & COUNTRY & EPC & & COUNTRY & EPC \\
\hline 1 & BERMUDA & 0.93 & 50 & BULGARIA & 0.61 & 92 & YEMEN, PEO & 0.47 \\
\hline 2 & MARTINIQUE & 0.93 & 51 & SURINAM & 0.61 & 93 & IRAN & 0.47 \\
\hline 3 & BRUNEI & 0.89 & 52 & NETHERLAND & 0.60 & 94 & CZECHOSLOV & 0.46 \\
\hline 4 & REUNION & 0.88 & 53 & PARAGUAY & 0.60 & 95 & MOROCCO & 0.46 \\
\hline 5 & GREENLAND & 0.87 & 54 & BENIN & 0.60 & 96 & KUWAIT & 0.46 \\
\hline 6 & SEYCHELLES & 0.87 & 55 & QATAR & 0.59 & 97 & POLAND & 0.46 \\
\hline 7 & BURUNDI & 0.86 & 56 & GABON & 0.59 & 98 & THAILAND & 0.45 \\
\hline 8 & DOHINICAN & 0.85 & 57 & IVORY COAS & 0.59 & 99 & NETHERLAND & 0.45 \\
\hline 9 & GUADALOUPE & 0.84 & 58 & MOZAMBIQUE & 0.58 & 100 & SRI LANKA & 0.45 \\
\hline 10 & GUINEA FR. & 0.83 & 59 & EL SALVADO & 0.58 & 101 & IRAO & 0.45 \\
\hline 11 & MAURITIUS & 0.80 & 60 & NEN 2EALAN & 0.58 & 102 & EGYPT & 0.44 \\
\hline 2 & SIERRA LEO & 0.79 & 61 & ETHIOPIA & 0.58 & 103 & MALAYSIA & 0.43 \\
\hline 13 & neu caledo & 0.79 & 62 & GUINEA REP & 0.58 & 104 & HONG KONG & 0.43 \\
\hline 14 & CHAD & 0.79 & 63 & OMAN & 0.56 & 105 & MORUAY & 0.43 \\
\hline 15 & SOHALIA & 0.77 & 64 & ECUADOR & 0.56 & 106 & SYRIA & 0.43 \\
\hline 16 & YEMEN, ARA & 0.77 & 65 & LIBYA & 0.56 & 107 & PORTUGAL & 0.43 \\
\hline 17 & BNHAMAS & 0.75 & 66 & CAMEROON & 0.56 & 108 & SAUOI ARAB & 0.43 \\
\hline 18 & SENEGAL & 0.75 & 67 & EAST GER & 0.55 & 109 & SUDAN & 0.43 \\
\hline 19 & PAwara & 0.75 & 68 & SOUTH AFRI & 0.55 & 110 & ROHANIA & 0.42 \\
\hline 0 & BARBADOS & 0.73 & 69 & VENEZUELA & 0.55 & 111 & UGANDA & 0.42 \\
\hline 21 & NEPAL & 0.73 & 70 & ANGOLA & 0.55 & 112 & BURAR & 0.41 \\
\hline 22 & GNiBIA & 0.71 & 71 & MALTA & 0.55 & 113 & U.S.S.R. & 0.41 \\
\hline 3 & maLAUI & 0.71 & 72 & COSTA RICA & 0.54 & 114 & FINLAND & 0.41 \\
\hline 24 & LAOS & 0.70 & 73 & JORDAN & 0.54 & 115 & KERYA & 0.41 \\
\hline 25 & MEXICO & 0.70 & 74 & NICARAGUA & 0.54 & 116 & AUSTRALIA & 0.41 \\
\hline 26 & IRELAHD & 0.69 & 75 & TAIUAN & 0.53 & 117 & DENMARK & 0.40 \\
\hline 27 & PHILIPPINE & 0.69 & 76 & CUBA & 0.53 & 118 & TANZANIA & 0.39 \\
\hline 28 & HAITI & 0.69 & $\pi$ & NIGERIA & 0.53 & 119 & LEBANON & 0.39 \\
\hline 29 & JAMAICA & 0.69 & 78 & ZAMBIA & 0.52 & 120 & TURKEY & 0.39 \\
\hline 30 & NIGER & 0.69 & 79 & MACAO & 0.52 & 121 & GREECE & 0.39 \\
\hline 31 & CENT. AF. & 0.68 & 80 & PERU & 0.52 & 122 & BRAZIL & 0.39 \\
\hline 32 & NORTH VIET & 0.68 & 81 & GUATEMALA & 0.51 & 123 & URUGUAY & 0.39 \\
\hline 33 & BOLIVIA & 0.68 & 82 & CYPRUS & 0.51 & 124 & ARGENTIMA & 0.38 \\
\hline 34 & KOREA & 0.67 & 83 & HUNGARY & 0.50 & 125 & rugoslavia & 0.38 \\
\hline 35 & TRIHIDAD & 0.66 & 84 & COLOHBIA & 0.50 & 126 & ISRAEL & 0.38 \\
\hline 36 & PAPAU N. G & 0.66 & 85 & GHANA & 0.50 & 127 & SINGAPORE & 0.38 \\
\hline 37 & CANADA & 0.65 & 86 & TUNISIA & 0.50 & 128 & AUSTRIA & 0.38 \\
\hline 38 & FIJI & 0.65 & 87 & ICELAND & 0.50 & 129 & CHIM & 0.37 \\
\hline 39 & VIET MAH & 0.65 & 88 & CONGO & 0.49 & 130 & SHEDEN & 0.36 \\
\hline 40 & INDONESIA & 0.64 & 89 & CHILE & 0.49 & 131 & SPAIK & 0.36 \\
\hline 41 & ALGERIA & 0.64 & 90 & BAHREIN & 0.48 & 132 & INDIA & 0.35 \\
\hline 42 & MALI & 0.63 & 91 & BELGIUH & 0.48 & 133 & ITALY & 0.35 \\
\hline 43 & HONDORAS & 0.63 & 92 & YEMEN, PEO & 0.47 & 134 & JAPAN & 0.35 \\
\hline 44 & ZAIRE & 0.63 & 93 & IRAN & 0.47 & 135 & FRAMCE & 0.34 \\
\hline 45 & U.A.E. & 0.63 & 94 & CZECHOSLOV & 0.46 & 136 & GERHANY & 0.33 \\
\hline 46 & NORTH KORE & 0.69 & 95 & MOROCCO & 0.46 & 137 & U.S.A. & 0.31 \\
\hline 47 & AFGHANISTA & 0.61 & 96 & KUWAIT & 0.46 & 138 & SUITZERLAN & 0.31 \\
\hline 48 & MAURITANIA & 0.61 & 97 & POLAND & 0.46 & 139 & PAKISTAN & 0.28 \\
\hline 49 & GUYANA & 0.61 & 98 & THAILAND & 0.45 & 140 & U.K. & 0.23 \\
\hline
\end{tabular}


TABLE VII-D

COUNTRIES RANKED BY EXPORT PARTNER CONCENTRATION IN 1980

\begin{tabular}{|c|c|c|c|c|c|c|c|c|}
\hline & COUNTRY & EPC & & COUNTRY & EPC & & COUNTRY & EPC \\
\hline 1 & REUNION & 0.89 & 50 & ECUADOR & 0.58 & 99 & IRAN & 0.45 \\
\hline 2 & ZAIRE & 0.83 & 51 & NIGERIA & 0.58 & 100 & HUNGARY & 0.44 \\
\hline 3 & GUADALOUPE & 0.82 & 52 & JAMAICA & 0.58 & 101 & KUWAIT & 0.44 \\
\hline 4 & NEW CALEdo & 0.80 & 53 & CHAD & 0.57 & 102 & POLAND & 0.44 \\
\hline 5 & MARTINIQUE & 0.79 & 54 & LIBYA & 0.57 & 103 & PERU & 0.44 \\
\hline 6 & BRUNEI & 0.78 & 55 & CAMEROON & 0.56 & 104 & mozarbique & 0.44 \\
\hline 7 & SOHALIA & 0.77 & 56 & GABON & 0.56 & 105 & EGYPT & 0.43 \\
\hline 8 & SIERRA LEO & 0.76 & 57 & VIETNAM & 0.56 & 106 & IRAQ & 0.42 \\
\hline 9 & MAURITIUS & 0.74 & 58 & BOLIVIA & 0.56 & 107 & SUDAN & 0.42 \\
\hline 10 & EQU. GUINE & 0.74 & 59 & GUINEA & 0.56 & 108 & KOREA & 0.42 \\
\hline 11 & BELIZE & 0.74 & 60 & NICARAGUA & 0.56 & 109 & SAUDI ARAB & 0.41 \\
\hline 12 & CAPE VERDI & 0.72 & 61 & BENIN & 0.55 & 110 & CHILE & 0.41 \\
\hline 13 & OALN & 0.70 & 62 & MALTA & 0.54 & 111 & URUGUAY & 0.41 \\
\hline 14 & AFGHANISTA & 0.70 & 63 & MALANI & 0.54 & 112 & BURMA & 0.41 \\
\hline 5 & HAITI & 0.69 & 64 & NORTH KORE & 0.54 & 113 & FINLAND & 0.41 \\
\hline 16 & MEXICO & 0.68 & 65 & NEPAL & 0.53 & 114 & CYPRUS & 0.41 \\
\hline 17 & SEYCHELLES & 0.68 & 66 & UGANDA & 0.53 & 115 & ISRAEL & 0.40 \\
\hline 18 & TRINIDAD & 0.68 & 67 & MALI & 0.53 & 116 & AUSTRIA & 0.40 \\
\hline 19 & DOMINICAN & 0.67 & 68 & NORWAY & 0.53 & 117 & YUGOSLAVIA & 0.40 \\
\hline 20 & BURUNDI & 0.67 & 69 & MADAGASCAR & 0.53 & 118 & MOROCCO & 0.39 \\
\hline 21 & BAHAMAS & 0.67 & 70 & & 0.53 & 119 & NEW ZEALAN & 0.39 \\
\hline 22 & BARBADOS & 0.66 & 71 & SURINAME & 0.53 & 120 & AUSTRALIA & 0.39 \\
\hline 23 & CUBA & 0.65 & 72 & GHANA & 0.52 & 21 & U.S.S.R. & 0.39 \\
\hline 24 & CEN. AFR. & 0.65 & 73 & MACAO & 0.51 & 122 & DENHARK & 0.39 \\
\hline 5 & GREENLAND & 0.65 & 74 & IRELAND & 0.51 & 123 & THAILAND & 0.39 \\
\hline 26 & INDONESIA & 0.65 & 75 & TUNISIA & 0.51 & 124 & HONG KONG & 0.38 \\
\hline 27 & NIGER & 0.65 & 76 & PARAGUAY & 0.51 & 125 & TANZANIA & 0.38 \\
\hline 28 & PAUPA N GU & 0.64 & $\pi$ & A RICA & 0.51 & 126 & ARGENTINA & 0.37 \\
\hline 29 & PANARA & 0.64 & 78 & PHILIPPINE & 0.50 & 127 & EAST GERHA & 0.37 \\
\hline 30 & SYRIA & 0.64 & 79 & VENEZUELA & & 28 & PORTUGAL & 0.37 \\
\hline 31 & EL SALVADO & 0.63 & 80 & LEBANON & 0.50 & 129 & TURKEY & 0.37 \\
\hline 32 & DJIBOUTI & 0.63 & 81 & QATAR & 0.49 & 130 & KENYA & 0.35 \\
\hline 33 & MALDIVES & 0.63 & 82 & H AFRI & 0.49 & 131 & ROHANIA & 0.34 \\
\hline 34 & GREMADA & 0.63 & 83 & ZAMBIA & 0.49 & 132 & GREECE & 0.34 \\
\hline 35 & ALGERIA & 0.63 & 84 & GUATEMALA & 0.49 & 133 & SINGAPORE & 0.34 \\
\hline 36 & HONDORAS & 0.62 & 85 & U.A.E. & 0.48 & 134 & SWEDEN & 0.33 \\
\hline 37 & CAMDA & 0.62 & 86 & JORDAN & 0.48 & 135 & ITALY & 0.33 \\
\hline 38 & CONGO & 0.61 & 87 & CZECHOSLOV & 0.48 & 136 & SHITZERLAN & 0.33 \\
\hline 39 & GUIANA, FR & 0.61 & 88 & SENEGAL & 0.48 & 137 & GERHANY & 0.33 \\
\hline 40 & L10 & 0.61 & 89 & IVORY COAS & 0.47 & 138 & INDIA & 0.32 \\
\hline 41 & ANGOLA & 0.61 & 90 & MALAYSIA & 0.47 & 139 & FRANCE & 0.31 \\
\hline 42 & BURKINA FA & 0.61 & 91 & BAMRAIN & 0.47 & 140 & SPAIN & 0.31 \\
\hline 43 & BULGARIA & 0.60 & 92 & ETHIOPIA & 0.46 & 141 & JAPAN & 0.31 \\
\hline 44 & YEHEN, A.R & 0.60 & 93 & ICELAND & 0.46 & 142 & SRI LANKA & 0.31 \\
\hline 45 & GUINEA-BIS & 0.60 & 94 & BERMUDA & 0.46 & 143 & BRAZIL & 0.30 \\
\hline 46 & YEMEN, A.D & 0.60 & 95 & NETHERLAND & 0.45 & 144 & U.S.A. & 0.29 \\
\hline 47 & FIJI & 0.59 & 96 & COLOMBIA & 0.45 & 145 & PAKISTAN & 0.29 \\
\hline 48 & METHR. ANT & 0.59 & 97 & CHINA & 0.45 & 146 & BANGLADESH & 0.29 \\
\hline & MAURITANIA & 0.59 & 98 & BELGIUH & 0.45 & 147 & UK & 0.28 \\
\hline
\end{tabular}


TABLE VIII-A

COUNTRIES RANKED BY IMPORT PARTNER CONCENTRATION IN 1938

\begin{tabular}{|c|c|c|c|c|c|}
\hline & COUNTRY & IPC & & COUNTRY & IPC \\
\hline 1 & TUNISIA & 1.00 & 33 & DENMARK & 0.46 \\
\hline 2 & FALKLAND & 1.00 & 34 & SPAIN & 0.45 \\
\hline 3 & ALGERIA & 0.78 & 35 & GREECE & 0.44 \\
\hline 4 & PHILIPPINES & 0.75 & 36 & ARGENTINE & 0.44 \\
\hline 5 & CUBA & 0.74 & 37 & F INLAND & 0.44 \\
\hline 6 & CANADA & 0.72 & 38 & SWITZERLAND & 0.43 \\
\hline 7 & IRELAND & 0.71 & 39 & AUSTRIA & 0.40 \\
\hline 8 & ECUADOR & 0.70 & 40 & NORWAY & 0.40 \\
\hline 9 & NEH ZEALAND & 0.67 & 41 & SWEDEN & 0.39 \\
\hline 10 & COLOMBIA & 0.67 & 42 & POLAND & 0.37 \\
\hline 11 & PARAgUAY & 0.67 & 43 & EGYPT & 0.36 \\
\hline 12 & VENEZUELA & 0.67 & 44 & NETHERLANDS & 0.36 \\
\hline 13 & MEXICO & 0.65 & 45 & CZECHOSLOV & 0.31 \\
\hline 14 & AUSTRALIA & 0.60 & 46 & ITALY & 0.29 \\
\hline 15 & BOLIVIA & 0.59 & 47 & FRANCE & 0.27 \\
\hline 16 & HONG KONG & 0.58 & 48 & U.K. & 0.23 \\
\hline 17 & U.S.S.R. & 0.58 & 49 & U.S.A. & 0.22 \\
\hline 18 & S. AFRICA & 0.57 & 50 & GERMANY & 0.17 \\
\hline 19 & PERU & 0.56 & & & \\
\hline 20 & THAILAKD & 0.56 & & & \\
\hline 21 & HOROCCO & 0.54 & & & \\
\hline 22 & BULGARIA & 0.53 & & & \\
\hline 23 & MALAYSIA & 0.52 & & & \\
\hline 24 & TURKEY & 0.52 & & & \\
\hline 25 & CHILE & 0.50 & & & \\
\hline 26 & ROHAINIA & 0.50 & & & \\
\hline 27 & YUGOSLAVIA & 0.50 & & & \\
\hline 28 & CHINA & 0.49 & & & \\
\hline 29 & BRAZIL & 0.49 & & & \\
\hline 30 & HUNGARY & 0.48 & & & \\
\hline 31 & PORTUGAL & 0.48 & & & \\
\hline 32 & URUGUAY & 0.46 & & & \\
\hline
\end{tabular}


TABLE VIII-B

COUNTRIES RANKED BY IMPORT PARTNER CONCENTRATION IN 1962

\begin{tabular}{|c|c|c|c|c|c|c|c|c|}
\hline & 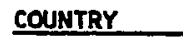 & IPC & & COUNTRY & IPC & & COUNTRY & IPC \\
\hline 1 & GUADALOUPE & 1.00 & 44 & LAOS & 0.66 & 87 & ETHIOPIA & 0.50 \\
\hline 2 & CENT. AFR. & 1.00 & 45 & SURINAM & 0.66 & 88 & THAILAND & .50 \\
\hline 3 & CHAD & 1.00 & 46 & JAMAICA & 0.66 & 89 & MOROCCO & 0.49 \\
\hline 4 & NIGER & 1.00 & 47 & MALTA & 0.64 & 90 & ICELAND & 0.49 \\
\hline 5 & BENIN & 1.00 & 48 & TANZANIA & 0.64 & 91 & CHILE & 0.49 \\
\hline 6 & MARTINIQUE & 0.99 & 49 & MAURITIUS & 0.64 & 92 & IRAN & 0.49 \\
\hline 7 & macao & 0.99 & 50 & TRINIDAD & 0.63 & 93 & mozambIQue & 0.48 \\
\hline 8 & MAURITANIA & 0.91 & 51 & NICARAGUA & 0.63 & 94 & BURHA & 0.48 \\
\hline 9 & REUMION & 0.90 & 52 & DOMINICAN & 0.63 & 95 & SOUTH AFRI & 0.47 \\
\hline 10 & GABON & 0.90 & 53 & VIET NAM & 0.62 & 96 & SAUDI ARAB & 0.47 \\
\hline 11 & NEW CALEDO & 0.89 & 54 & IRELAND & 0.62 & 97 & FINLAND & 0.47 \\
\hline 12 & YEMEN, ARA & 0.88 & 55 & BAHREIN & 0.60 & 98 & AUSTRALIA & 0.46 \\
\hline 13 & GAMBIA & 0.88 & 56 & VENEZUELA & 0.59 & 99 & ARGENTINA & 0.46 \\
\hline 14 & NEW GUINEA & 0.86 & 57 & GUATEMALA & 0.59 & 100 & JORDAN & 0.46 \\
\hline 15 & BRUNEI & 0.85 & 58 & RUMANIA & 0.58 & 101 & TURKEY & 0.46 \\
\hline 16 & IVORY COAS & 0.84 & 59 & HUNGARY & 0.58 & 102 & INDONESIA & 0.46 \\
\hline 17 & METHERLAND & 0.83 & 60 & NEW ZEALAN & 0.58 & 103 & INDIA & 0.45 \\
\hline 18 & GREENLAND & 0.83 & 61 & COSTA RICA & 0.58 & 104 & NORWAY & 0.45 \\
\hline 19 & MALI & 0.81 & 62 & PHILIPPINE & 0.58 & 105 & HONG KONG & 0.45 \\
\hline 20 & ALGERIA & 0.79 & 63 & KENYA & 0.58 & 106 & POLAND & 0.4 \\
\hline 21 & CAPE VERDI & 0.78 & 64 & GUINEA & & 107 & SUDAN & 1.44 \\
\hline 22 & MADAGASCAR & 0.78 & 65 & ECUADOR & 0.57 & 108 & YEHEN, PEO & 0.44 \\
\hline 23 & BERHUDA & 0.76 & 66 & BOLIVIA & 0.57 & 109 & DENHARK & 0.4 \\
\hline 24 & OMAN & 0.76 & 67 & LIBYA & 0.57 & 110 & EGYPT & 0.4 \\
\hline 25 & BARBADOS & 0.75 & 68 & COLOMBIA & 0.57 & 111 & URUGUAY & 0.4 \\
\hline 26 & UGANDA & 0.74 & 69 & ANGOLA & & 112 & BRAZIL & 0.42 \\
\hline 27 & CUBA & 0.73 & 70 & PANAMA & 0.56 & 113 & BELGIUM & 0.4 \\
\hline 28 & EAST GERMA & 0.73 & 71 & EL SALY & 0.55 & 114 & SWEDEN & 0.4 \\
\hline 29 & FIJI & 0.73 & 72 & PERU & 0.54 & 115 & PORTUGAL & 0.41 \\
\hline 30 & KOREA & 0.72 & 73 & PAKISTAN & 0.53 & 116 & LEBANON & 0.4 \\
\hline 31 & HAITI & 0.72 & 74 & AUSTRIA & 0.53 & 117 & IRAQ & 0.4 \\
\hline 32 & SENEGAL & 0.71 & 75 & CYPRUS & 0.53 & 118 & U.S.S.R. & 0.4 \\
\hline 33 & HEXICO & 0.71 & 76 & NIGERIA & 0.53 & 119 & SYRIA & 0.3 \\
\hline 34 & BULGARIA & 0.71 & 77 & KUWAIT & 0.53 & 120 & GREECE & 0.3 \\
\hline 35 & AFGHLNISTA & 0.70 & 78 & PARAguay & 0.53 & 121 & YUGOSLAVIA & 0.3 \\
\hline 36 & Barharis & 0.70 & 79 & CONGO & 0.53 & 122 & JAPAK & 0.3 \\
\hline 37 & HONDORAS & 0.70 & 80 & CZECHOSLOV & 0.51 & 123 & NETHERLAND & 0.3 \\
\hline 38 & SIERRA LEO & 0.69 & 81 & MALAYSIA & 0.51 & 124 & SPAIH & 0.3 \\
\hline 39 & SOHALI & 0.69 & 82 & ISRAEL & 0.51 & 125 & ITALY & 0.3 \\
\hline 40 & CAMEROON & 0.69 & 83 & GHANA & 0.51 & 126 & FRANCE & 0.3 \\
\hline 41 & CANADA & 0.68 & 84 & CHINA & 0.51 & 127 & U.S.A. & 0.3 \\
\hline 42 & QATAR & 0.68 & 85 & SINGAPORE & 0.50 & 128 & GERHANY & 0.3 \\
\hline 43 & TUNISIA & 0.67 & 86 & SWITZERLAN & 0.50 & 129 & U.K. & 0.2 \\
\hline
\end{tabular}


TABLE VIII-C

COUNTRIES RANKED BY IMPORT PARTNER CONCENTRATION IN 1970

\begin{tabular}{|c|c|c|c|c|c|c|c|c|}
\hline & COUNTRY & IPC & & COUNTRY & IPC & & COUNTRY & IPC \\
\hline 1 & GREENLAND & 0.88 & 50 & VENEZUELA & 0.57 & 92 & PAKISTAN & 0.46 \\
\hline 2 & GUADALOUPE & 0.83 & 51 & SENEGAL & 0.57 & 93 & TURKEY & 0.46 \\
\hline 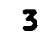 & MaCAO & 0.82 & 52 & PHILIPPINE & 0.56 & 94 & JORDAN & 0.46 \\
\hline 4 & MEPAL & 0.80 & 53 & GUINEA REP & 0.56 & 95 & GHANA & 0.46 \\
\hline 5 & REUMION & 0.79 & 54 & ALGERIA & 0.56 & 96 & PERU & 0.46 \\
\hline 6 & PAPAU N. G & 0.78 & 55 & ANGOLA & 0.55 & 97 & YEMEN, ARA & 0.45 \\
\hline 7 & MARTINIQUE & 0.77 & 56 & PARAgUAY & 0.55 & 98 & ICELAKD & 0.45 \\
\hline 8 & NORTH KORE & 0.74 & 57 & COLOMBIA & 0.55 & 99 & ETHIOPIA & 0.45 \\
\hline 9 & GUINEA FR. & 0.73 & 58 & MALAUI & 0.54 & 100 & AUSTRALIA & 0.45 \\
\hline 10 & SEYCHELLES & 0.73 & 59 & MALTA & 0.54 & 101 & KENYA & 0.45 \\
\hline 11 & BERHUDA & 0.73 & 60 & NICARAGUA & 0.54 & 102 & MALAYSIA & 0.44 \\
\hline 2 & NORTH VIET & 0.72 & 61 & ISRAEL & 0.54 & 103 & BRAZIL & 0.44 \\
\hline 13 & NEU CALEDO & 0.72 & 62 & ECUADOR & 0.54 & 104 & ZAMBIA & 0.44 \\
\hline 14 & NETHERLAND & 0.71 & 63 & AUSTRIA & 0.54 & 105 & CYPRUS & 0.44 \\
\hline 15 & MALI & 0.71 & 64 & EL SALVADO & 0.53 & 106 & CHINA & 0.44 \\
\hline 16 & CANADA & 0.70 & 65 & TUNISIA & 0.53 & 107 & HONG KONG & 0.44 \\
\hline 17 & BAHAMAS & 0.69 & 66 & BOLIVIA & 0.53 & 108 & ARGENTINA & 0.44 \\
\hline 18 & OMAN & 0.68 & 67 & INDONESIA & 0.53 & 109 & LIBYA & 0.44 \\
\hline 19 & CENT. AF. & 0.68 & 68 & TRINIDAD & 0.53 & 110 & MAURITIUS & 0.44 \\
\hline 20 & MAURITANIA & 0.67 & 69 & GUATEMALA & 0.53 & 111 & FINLAND & 0.43 \\
\hline 21 & NIGER & 0.67 & 70 & UGANDA & 0.53 & 112 & TANZANIA & 0.43 \\
\hline 22 & GABON & 0.67 & 71 & NEW ZEALAN & 0.52 & 113 & NORWAY & 0.43 \\
\hline 23 & MEXICO & 0.66 & 72 & PANAMA & 0.51 & 114 & DENMARK & 0.43 \\
\hline 24 & CONGO & 0.66 & 73 & GAMBIA & 0.51 & 115 & URUGUAY & 0.43 \\
\hline 25 & BRUNEI & 0.65 & 74 & THAILAND & 0.50 & 116 & SINGAPORE & 0.42 \\
\hline 26 & KOREA & 0.65 & 75 & COSTA RICA & 0.50 & 117 & PORTUGAL & 0.42 \\
\hline 27 & LAOS & 0.63 & 76 & IRAN & 0.49 & 118 & ROMANIA & 0.42 \\
\hline 28 & QUTAR & 0.63 & $\pi$ & SOUTH AFRI & 0.49 & 119 & YEMEN, PEO & 0.41 \\
\hline 39 & CHAD & 0.63 & 78 & CHILE & 0.49 & 120 & INDIA & 0.41 \\
\hline 30 & IRELAND & 0.63 & 79 & CZECHOSLOV & 0.49 & 121 & SAUDI ARAB & 0.41 \\
\hline 31 & TAIHAN & 0.62 & 80 & SWITZERLAN & 0.49 & 122 & NETHERLAND & 0.40 \\
\hline 32 & EAHREIN & 0.62 & 81 & HUNGARY & 0.48 & 123 & GREECE & 0.40 \\
\hline 3 & BENIN & 0.62 & 82 & ZAIRE & 0.48 & 124 & KUWAIT & 0.40 \\
\hline 34 & U.A.E. & 0.61 & 83 & POLAND & 0.48 & 125 & SWEDEN & 0.40 \\
\hline 35 & cuen & 0.61 & 84 & BURUNDI & 0.47 & 126 & U.S.S.R. & 0.40 \\
\hline 36 & JAMuICA & 0.61 & 85 & BURMA & 0.47 & 127 & SUDAN & 0.40 \\
\hline 37 & DOMINICAN & 0.61 & 86 & NIGERIA & 0.47 & 128 & FRANCE & 0.39 \\
\hline 38 & CAMEROOAN & 0.60 & 87 & SIERRA LEO & 0.47 & 129 & YUGOSLAVIA & 0.39 \\
\hline 39 & BARBADOS & 0.60 & 88 & SOMALIA & 0.47 & 130 & U.S.A. & 0.39 \\
\hline 40 & VIET MAH & 0.59 & 89 & BELGIUH & 0.46 & 131 & SRI LANKA & 0.39 \\
\hline 41 & FIJI & 0.59 & 90 & MOROCCO & 0.46 & 132 & JAPAN & 0.37 \\
\hline 42 & HAITI & 0.59 & 91 & MOZAMBIQUE & 0.46 & 133 & GERMANY & 0.36 \\
\hline 43 & IVORY COAS & 0.59 & 92 & PAKISTAN & 0.46 & 134 & SPAIN & 0.36 \\
\hline 44 & SURINAY & 0.59 & 93 & TURKEY & 0.46 & 135 & LEBANON & 0.35 \\
\hline 45 & HONDORAS & 0.58 & 94 & JORDAN & 0.46 & 136 & EGYPT & 0.35 \\
\hline 46 & GUYANA & 0.58 & 95 & GHANA & 0.46 & 137 & ITALY & 0.35 \\
\hline 47 & EAST GER & 0.58 & 96 & PERU & 0.46 & 138 & SYRIA & 0.35 \\
\hline 48 & BULGARIA & 0.57 & 97 & YEMEN, ARA & 0.45 & 139 & IRAQ & 0.33 \\
\hline 49 & AFGHANISTA & 0.57 & 98 & ICELAND & 0.45 & 140 & U.K. & 0.26 \\
\hline
\end{tabular}


TABLE VIII-D

COUNTRIES RANKED BY IMPORT PARTNER CONCENTRATION IN 1980

\begin{tabular}{|c|c|c|c|c|c|c|c|c|}
\hline & COUNTRY & IPC & & COUNTRY & IPC & & COUNTRY & IPC \\
\hline 1 & EQU. GUINE & 0.90 & 50 & CZECHOSLOV & 0.54 & 99 & NIGERIA & 0.44 \\
\hline 2 & MACAO & 0.87 & 51 & SOMALIA & 0.54 & 100 & NORUAY & 0.44 \\
\hline 3 & GREEKLAND & 0.83 & 52 & VENEZUELA & 0.54 & 101 & VIETNAM & 0.44 \\
\hline 4 & MARTINIQUE & 0.80 & 53 & BARBADOS & 0.54 & 102 & QATAR & 0.44 \\
\hline 5 & GUADALOUPE & 0.79 & 54 & BERMUDA & 0.54 & 103 & FINLAND & 0.44 \\
\hline 6 & REUNION & 0.72 & 55 & MAURITANIA & 0.53 & 104 & SWITZERLAM & 0.44 \\
\hline 7 & GUIANA, FR & 0.70 & 56 & BURUNDI & 0.53 & 105 & ANGOLA & 0.44 \\
\hline 8 & MEXICO & 0.69 & 57 & GUINEA & 0.53 & 106 & URUGUAY & 0.43 \\
\hline 9 & NETHR. ANT & 0.68 & 58 & AUSTRIA & 0.53 & 107 & MAURITIUS & 0.43 \\
\hline 10 & CAMADA & 0.68 & 59 & SOUTH AFRI & 0.52 & 108 & DENHARK & 0.43 \\
\hline . & GRENADA & 0.68 & 60 & BURMA & 0.52 & 109 & CHIM & 0.43 \\
\hline 12 & PAUPA N GU & 0.67 & 61 & GUINEA-BIS & 0.52 & 110 & ARGENTINA & 0.42 \\
\hline 3 & BAHRAIN & 0.67 & 62 & NICARAGUA & 0.52 & 111 & SAUDI ARAB & 0.42 \\
\hline 14 & BRUNEI & 0.67 & 63 & BOLIVIA & 0.52 & 112 & AUSTRALIA & 0.42 \\
\hline 5 & GABON & 0.66 & 64 & IVORY COAS & 0.51 & 113 & SINGAPORE & 0.42 \\
\hline 16 & AFGHANISTA & 0.66 & 65 & DJIBOUTI & 0.51 & 114 & ETHIOPIA & 0.42 \\
\hline 7 & CEN. AFR. & 0.65 & 66 & SENEGAL & 0.51 & 115 & CYPRUS & 0.41 \\
\hline 18 & LAO & 0.65 & 67 & GAMBIA & 0.51 & 116 & U.A.E. & 0.41 \\
\hline 19 & BURKIMA FA & 0.65 & 68 & ZAIRE & 0.51 & 117 & KUWAIT & 0.41 \\
\hline 20 & BULGARIA & 0.64 & 69 & ECUADOR & 0.51 & 118 & SUDAN & 0.40 \\
\hline 1 & CHAD & 0.63 & 70 & MALTA & 0.51 & 119 & JORDAN & 0.40 \\
\hline 2 & HAITI & 0.63 & 71 & COSTA RICA & 0.50 & 120 & EGYPT & 0.40 \\
\hline 3 & NEU CALEDo & 0.63 & 72 & SIERRA LEO & 0.50 & 121 & BANGLADESH & 0.39 \\
\hline 34 & cuan & 0.63 & 73 & GUATEMALA & 0.50 & 122 & TURKEY & 0.39 \\
\hline .5 & MALI & 0.62 & 74 & maLAHI & 0.50 & 123 & SHEDEN & 0.39 \\
\hline 86 & NEPAL & 0.62 & 75 & COLOMBIA & 0.50 & 124 & YUGOSLAVIA & 0.39 \\
\hline 27 & CAPE VERDI & 0.62 & 76 & ISRAEL & 0.50 & 125 & YEMEN, A.R & 0.39 \\
\hline 88 & BELIZE & 0.62 & 77 & HONG KONG & 0.49 & 126 & GREECE & 0.39 \\
\hline 29 & DOMIHICAN & 0.61 & 78 & INDONESIA & 0.49 & 127 & TANZANIA & 0.39 \\
\hline 30 & MALDIVES & 0.61 & 79 & TUNISIA & 0.48 & 128 & IRAQ & 0.39 \\
\hline 31 & IRELANO & 0.61 & 80 & POLAND & 0.48 & 129 & BRAZIL & 0.39 \\
\hline 52 & SURINAME & 0.61 & 81 & PERU & 0.48 & 130 & mozAmbiaUe & 0.38 \\
\hline 33 & NIGER & 0.61 & 82 & GHANA & 0.48 & 131 & SRI LANKA & 0.38 \\
\hline 34 & FIJI & 0.60 & 83 & BENIN & 0.48 & 132 & PAKISTAN & 0.37 \\
\hline 35 & ZAMBIA & 0.59 & 84 & MALAYSIA & 0.48 & 133 & FRANCE & 0.37 \\
\hline 36 & TRINIDAD & 0.58 & 85 & YEMEN, A.D & 0.47 & 134 & LEBANON & 0.37 \\
\hline 37 & conso & 0.58 & 86 & ALGERIA & 0.46 & 135 & SYRIA & 0.37 \\
\hline 38 & PARAgUAY & 0.58 & 87 & BELGIUH & 0.46 & 136 & NETHERLAND & 0.37 \\
\hline 39 & JANAICA & 0.58 & 88 & NEW ZEALAN & 0.46 & 137 & PORTUGAL & 0.37 \\
\hline 40 & UGANDA & 0.57 & 89 & PANAMA & 0.46 & 138 & JAPAN & 0.36 \\
\hline 41 & BAHAMAS & 0.57 & 90 & PHILIPPINE & 0.45 & 139 & U.S.S.R. & 0.36 \\
\hline 42 & EAST GERMA & 0.56 & 91 & LIBYA & 0.45 & 140 & INDIA & 0.35 \\
\hline 43 & EL SALVADO & 0.56 & 92 & OKAN & 0.45 & 141 & ROHANIA & 0.35 \\
\hline 44 & MORTH KORE & 0.56 & 93 & MOROCCO & 0.45 & 142 & ITALY & 0.34 \\
\hline 45 & SEYCHELLES & 0.56 & 94 & KENYA & 0.45 & 143 & IRAN & 0.34 \\
\hline 46 & CAHEROON & 0.55 & 95 & ICELAND & 0.45 & 144 & U.S.A. & 0.33 \\
\hline 47 & KOREA & 0.55 & 96 & HUNGARY & 0.44 & 145 & GERHANY & 0.33 \\
\hline 48 & MADAGASCAR & 0.54 & 97 & CHILE & 0.44 & 146 & SPAIN & 0.33 \\
\hline 49 & HONDORAS & 0.54 & 98 & THAILAND & 0.44 & 147 & UK & 0.33 \\
\hline
\end{tabular}


a periphery was to observe the number of trade ties it had with other countries.

The hypothesis was formulated as:

HYPOTHESIS- Center countries is associated with a high number of export trade linkages with other countries whereas periphery countries is associated with fewer trade linkages. More specifically, EPC is negatively correlated with TTL.

Correlation coefficients between EPC and other variables
\begin{tabular}{|l|c|c|c|c|c|c|c|}
\hline EPC VS. & IPC & ECC & ICC & VT & POLI & AGR & ED \\
\hline 1962 & .85 & .34 & .42 & -.57 & -.48 & .42 & .57 \\
\hline 1970 & .73 & .33 & .38 & -.35 & -.36 & .32 & .40 \\
\hline 1980 & .68 & .41 & .43 & -.50 & -.45 & .25 & .55 \\
\hline
\end{tabular}

Empirically, not only is this hypothesis confirmed but it is also supported by one of the highest correlation coefficients with EPC. Infact, a look at the summary figures above (derived from Figure 5) indicates that the TTL correlation coefficients with other variables are also the strongest in the entire list. This finding is however expected because TTL and EPC use different methods of investigating the diversity of trade ties.

Tables IX-A through IX-D clearly demonstrate that the industrialized countries occupy the center end of these tables in all study years.

In identifying center as opposed to periphery countries, TTL clearly reveals the existence of inequality in world trade structure. Therefore, this variable can be considered as an independent variable in the study of centerperiphery relationships.

\section{CONCENTRATION OF EXPORTS AND IMPORTS}

As stated earlier, dependency theory asserts that countries with high export partner concentration, namely the periphery countries, exhibit higher export commodity concentration than do center countries. From this expectation the following hypothesis is presented: 
TABLE IX

COUNTRIES RANKED BY TOTAL TRADE LINKAGES IN 1938

\begin{tabular}{|c|c|c|c|}
\hline COUNTRY & TTL & COUNTRY & $\mathrm{TTL}$ \\
\hline 1- U.K. & 48 & 32- PHILIPPINES & 14 \\
\hline 2- U.S.A. & 47 & 33- MEXICO & 13 \\
\hline 3- GERMANY & 47 & 34- URUGUAY & 13 \\
\hline 4- FRANCE & 42 & 35- CEYLON & 1 \\
\hline 5- METHERLANDS & 42 & 36- CHINA & 18 \\
\hline 6- ITALY & 40 & 37- HONG KONG & 18 \\
\hline 7- SUITZERLAND & 40 & 38- PORTUGAL & 18 \\
\hline 8- CZECHOSLOV & 36 & 39- ALGERIA & 11 \\
\hline 9- SWEDEN & 36 & 40- MOROCCO & 1 \\
\hline 10- CAMADA & 33 & 41- CUBA & 11 \\
\hline 11- ARGENTIME & 29 & 42- S. AFRICA & 1 \\
\hline 12- AUSTRIA & 28 & 43- NEW ZEALAND & 10 \\
\hline 13- POLAKD & 28 & 44- THAILAND & \\
\hline 14- AUSTRALIA & 27 & 45- TUNISIA & 8 \\
\hline 15- BRAZIL & 25 & 46- NIGERIA & \\
\hline 16- MORWAY & 25 & 47- ECUADOR & \\
\hline 17- HUHGARY & 23 & 48- VENEZUELA & 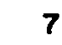 \\
\hline 18- EGYPT & 22 & 49- COLOHBIA & \\
\hline 19- malaysia & 22 & 50- TRINIDAD & \\
\hline 20- DENMARK & 21 & 51- BOLIVIA & \\
\hline 21- ROMAINIA & 21 & 52- DOMINICAN R & \\
\hline 22- TURKEY & 20 & 53- JAMAICA & \\
\hline 23- FINLAND & 19 & 54- IRAQ & \\
\hline 24- GREECE & 19 & 55- IRELAND & \\
\hline 25- YugosLavia & 19 & 56- HAITI & \\
\hline 26- CHILE & 18 & 57- GUATEMALA & \\
\hline 27- U.S.S.R. & 17 & 58- EL SALVADOR & \\
\hline 28- IRAN & 15 & 59- PARAGUAY & \\
\hline 29- BULgARIA & 15 & 60- COSTA RICA & \\
\hline 30- SPAIN & 15 & 61- NICARAGUA & \\
\hline \multirow[t]{2}{*}{ 31- PERU } & 14 & 62- HONDORAS & \\
\hline & & 63- FALKLAND & \\
\hline
\end{tabular}


TABLE IX-B

COUNTRIES RANKED BY TOTAL TRADE LINKAGES IN 1962

\begin{tabular}{|c|c|c|c|c|c|c|c|c|}
\hline & COUNTRY & $\mathrm{TTL}$ & & COUNTRY & TTL & & COUNTRY & TIL \\
\hline 1 & U.K. & 112 & 44 & SAUDI ARAB & 28 & 87 & CYPRUS & 8 \\
\hline 2 & JAPAN & 102 & 45 & THAILAND & 28 & 88 & QATAR & 8 \\
\hline 3 & GERMANY & 101 & 46 & INDONESIA & 27 & 89 & GUINEA & 8 \\
\hline 4 & U.S.A. & 96 & 47 & BULGARIA & 25 & 90 & VIET NAM & 8 \\
\hline 5 & FRANCE & 95 & 48 & PERU & 25 & 91 & FIJI & 8 \\
\hline 6 & NETHERLAND & 95 & 49 & SYRIA & 24 & 92 & HAITI & 7 \\
\hline 7 & ITALY & 91 & 50 & CZECHOSLOV & 23 & 93 & HONDORAS & 7 \\
\hline 8 & BELGIUH & 90 & 51 & GHANA & 22 & 94 & JORDAN & 7 \\
\hline 9 & SUITZERLAN & 74 & 52 & CUBA & 21 & 95 & GABON & 7 \\
\hline 10 & SWEDEN & 73 & 53 & SUDAN & 21 & 96 & LIBYA & 7 \\
\hline 11 & CANADA & 71 & 54 & COLOMBIA & 20 & 97 & BOLIVIA & 6 \\
\hline 12 & INDIA & 65 & 55 & URUGUAY & 20 & 98 & CAMEROOH & 6 \\
\hline 13 & DENMARK & 63 & 56 & CHILE & 19 & 99 & MADAGASCAR & 6 \\
\hline 14 & NETHERLAND & 58 & 57 & BURMA & 19 & 100 & PARAGUAY & 5 \\
\hline 15 & AUSTRALIA & 58 & 58 & PHILIPPINE & 19 & 101 & BERHUDA & 5 \\
\hline 16 & AUSTRIA & 53 & 59 & KUWAIT & 17 & 102 & SURINAM & 5 \\
\hline 17 & SPAIN & 52 & 60 & NEW ZEALAN & 17 & 103 & SEREGAL & 5 \\
\hline 18 & NORWAY & 51 & 61 & KENYA & 17 & 104 & KOREA & 5 \\
\hline 19 & U.S.S.R. & 49 & 62 & ICELAND & 16 & 105 & YEHEN, ARA & 4 \\
\hline 20 & HOHG KONG & 47 & 63 & PANAMA & 16 & 106 & MAURITIUS & 4 \\
\hline 21 & SIHGAPORE & 45 & 64 & BAHREIN & 16 & 107 & BRUNEI & 4 \\
\hline 22 & VEREZUELA & 44 & 65 & LEBANON & 16 & 108 & BARBADOS & 3 \\
\hline 23 & FINLAND & 43 & 66 & YEMEN, PEO & 15 & 109 & CHAD & 3 \\
\hline 24 & rucoslaVia & 42 & 67 & ALGERIA & 15 & 110 & SIERRA LEO & 3 \\
\hline 25 & POLAND & 42 & 68 & TUNISIA & 15 & 111 & MACAO & 3 \\
\hline 26 & ARGENTIMA & 40 & 69 & UGANDA & 15 & 112 & NEW GUINEA & 3 \\
\hline 27 & BRAZIL & 40 & 70 & TANZANIA & 15 & 113 & MALTA & 2 \\
\hline 28 & SOUTH AFRI & 40 & 71 & DOMINICAN & 14 & 114 & GREENLAND & 2 \\
\hline 29 & PAKISTAN & 39 & 72 & NIGERIA & 13 & 195 & GAMBIA & 2 \\
\hline 30 & IRAN & 38 & 73 & CONGO & 13 & 116 & MALI & 2 \\
\hline 31 & maLAYSIA & 35 & 74 & ANGOLA & 12 & 117 & NIGER & 2 \\
\hline 32 & PORTUGAL & 34 & 75 & ETHIOPIA & 12 & 118 & REUNION & 2 \\
\hline 33 & EAST GERHA & 34 & 76 & IVORY COAS & 12 & 119 & SOHALI & 2 \\
\hline 34 & MOROCCO & 34 & 77 & MOZAMBIQUE & 12 & 120 & BAHAMAS & 1 \\
\hline 35 & EGYPT & 33 & 78 & IRELAND & 11 & 121 & GUADALOUPE & 1 \\
\hline 36 & CHINA & 33 & 79 & ECUADOR & 11 & 122 & MARTINIQUE & 1 \\
\hline 37 & TURKEY & 32 & 80 & NICARAGUA & 11 & 123 & CENT. AFR. & 1 \\
\hline 38 & IRAO & 31 & 81 & EL SALVADO & 10 & 124 & MAURITANIA & 1 \\
\hline 39 & GREECE & 29 & 82 & TRINIDAD & 10 & 125 & NEH CALEDO & 1 \\
\hline 40 & HUNGARY & 29 & 83 & gUATEMALA & 9 & 126 & BENIN & 1 \\
\hline 41 & MEXICO & 29 & 84 & JAMAICA & 9 & 127 & OHAN & 0 \\
\hline 42 & RUMANIA & 28 & 85 & AFGHANISTA & 9 & 128 & CAPE VERDI & 0 \\
\hline 43 & ISRAEL & 28 & 86 & COSTA RICA & 8 & 129 & LAOS & 0 \\
\hline
\end{tabular}


TABLE IX-C

COUNTRIES RANKEO BY TOTAL TRADE LINKAGES IN 1970

\begin{tabular}{|c|c|c|c|c|c|c|c|c|}
\hline & COUNTRY & $\mathrm{TTL}$ & & COUNTRY & $\pi$ & & COUNTRY & ITL \\
\hline 1 & U.K. & 126 & 50 & THAILAND & 32 & 92 & HONDORAS & 13 \\
\hline 2 & FRANCE & 125 & 51 & KENYA & 32 & 93 & ZAIRE & 13 \\
\hline 3 & GERMANY & 125 & 52 & NIGERIA & 30 & 94 & SURINAM & 12 \\
\hline 4 & JAPAN & 124 & 53 & IRELAND & 29 & 95 & ORAN & 12 \\
\hline 5 & NETHERLAND & 117 & 54 & SOUTH AFRI & 29 & 96 & GUYANA & 11 \\
\hline 6 & ITALY & 116 & 55 & TANZANIA & 29 & 97 & NORTH KORE & 11 \\
\hline 7 & U.S.A. & 115 & 56 & UGANDA & 28 & 98 & CATAR & 10 \\
\hline 8 & BELGIUH & 111 & 57 & ZAMBIA & 28 & 99 & U.A.E. & 10 \\
\hline 9 & SHITZERLAN & 98 & 58 & URUGUAY & 27 & 100 & SENEGAL & 10 \\
\hline 10 & SUEDEN & 92 & 59 & SRI LANKA & 26 & 101 & BOLIVIA & 9 \\
\hline 11 & DEAHARK & 90 & 60 & NEW ZEALAN & 25 & 102 & PARAGUAY & 9 \\
\hline 12 & CAHADA & 90 & 61 & SYRIA & 25 & 103 & macao & 9 \\
\hline 13 & AUSTRIA & 81 & 62 & SUDAN & 25 & 104 & GUINEA REP & 9 \\
\hline 14 & AUSTRALIA & 80 & 63 & CUBA & 25 & 105 & DOMINICAN & 8 \\
\hline 15 & HONG KONG & 75 & 64 & GHANA & 24 & 106 & AFGHANISTA & 8 \\
\hline 16 & CZECHOSLOV & 72 & 65 & CHILE & 23 & 107 & BENIN & 8 \\
\hline 17 & INDIA & 71 & 66 & PHILIPPINE & 23 & 108 & MALAWI & 8 \\
\hline 18 & SINGAPORE & 71 & 67 & LEBANON & 22 & 109 & MALTA & 7 \\
\hline 19 & U.S.S.R. & 69 & 68 & ICELAND & 21 & 110 & PANAMA & 7 \\
\hline 20 & NORUAY & 66 & 69 & KOREA & 21 & 111 & JORDAN & 7 \\
\hline 21 & SPAIN & 62 & 70 & ALGERIA & 21 & 112 & MAURITANIA & 7 \\
\hline 22 & POLAKD & 61 & 71 & TUNISIA & 21 & 113 & SIERRA LEO & 7 \\
\hline 23 & BRAZIL & 60 & 72 & YEHEN, PEO & 20 & 114 & FIJI & 7 \\
\hline 24 & CHIMA & 58 & 73 & BURMA & 20 & 115 & PAPAU N. G & 7 \\
\hline 25 & PAKISTAN & 56 & 74 & ANGOLA & 20 & 116 & HAITI & 6 \\
\hline 26 & FINLAND & 55 & 75 & GUATEMALA & 19 & 117 & CENT. AF. & 6 \\
\hline 27 & YUGOSLAVIA & 54 & 76 & LIBYA & 19 & 118 & NEW CALEdo & 6 \\
\hline 28 & ARGENTINA & 54 & 77 & COSTA RICA & 18 & 119 & BAHAMAS & 5 \\
\hline 29 & MALAYSIA & 49 & 78 & ECUADOR & 18 & 120 & NIGER & 5 \\
\hline 30 & IRAN & 48 & 79 & TRINIDAD & 18 & 121 & BARBADOS & 4 \\
\hline 31 & HUNGARY & 48 & 80 & IVORY COAS & 18 & 122 & NEPAL & 4 \\
\hline 32 & BULGARIA & 47 & 81 & CAMEROON & 17 & 123 & GAYBIA & 4 \\
\hline 33 & ISRAEL & 46 & 82 & NICARAGUA & 16 & 124 & MALI & 4 \\
\hline 34 & EAST GER & 46 & 83 & BAHREIN & 16 & 125 & MAURITIUS & 4 \\
\hline 35 & EGYPT & 45 & 84 & CYPRUS & 15 & 126 & NORTH VIET & 4 \\
\hline 36 & VENEZUELLA & 44 & 85 & GABON & 15 & 127 & BRUNEI & 3 \\
\hline 37 & ROAnHIA & 43 & 86 & JAYAICA & 14 & 128 & LAOS & 3 \\
\hline 38 & NETHERLAND & 42 & 87 & INDONESIA & 14 & 129 & CHAD & 3 \\
\hline 39 & SAUDI ARAB & 42 & 88 & CONGO & 14 & 130 & SOHALIA & 3 \\
\hline 40 & TURKEY & 39 & 89 & ETHIOPIA & 14 & 131 & BERHUDA & 2 \\
\hline 41 & GREECE & 38 & 90 & mozambique & 14 & 132 & GREENLAND & 2 \\
\hline 42 & PORTUGAL & 38 & 91 & EL SALVADO & 13 & 133 & GUADALOUPE & 2 \\
\hline 43 & PERU & 38 & 92 & HONDORAS & 13 & 134 & MARTINIQUE & 2 \\
\hline 44 & COLOMBIA & 37 & 93 & ZAIRE & 13 & 135 & BURUNDI & 2 \\
\hline 45 & IRAQ & 37 & 94 & SURINAM & 12 & 136 & REUMION & 2 \\
\hline 46 & MOROCCO & 36 & 95 & OMAN & 12 & 137 & GUINEA FR. & 1 \\
\hline 47 & MEXICO & 35 & 96 & GUYANA & 11 & 138 & YEHEN, ARA & 1 \\
\hline 48 & TAIWAN & 35 & 97 & NORTH KORE & 11 & 139 & VIET NAM & 1 \\
\hline 49 & KUHAIT & 33 & 98 & CATAR & 10 & 140 & SEYCHELLES & 0 \\
\hline
\end{tabular}


TABLE IX-D

COUNTRIES RANKED BY TOTAL TRADE LINKAGES IN 1980

\begin{tabular}{|c|c|c|c|c|c|c|c|c|}
\hline & COUNTRY & $\pi L$ & & COUNTRY & TTL & & COUNTRY & TTL \\
\hline 1 & GERHANY & 141 & 50 & SRI LANKA & 52 & 99 & SURIMAME & 20 \\
\hline 2 & UK & 141 & 51 & IRAN & 52 & 100 & UGANDA & 19 \\
\hline 3 & FRANCE & 140 & 52 & HUNGARY & 50 & 101 & YEMEN, A.D & 19 \\
\hline 4 & NETHERLAND & 139 & 53 & TURKEY & 50 & 102 & PARAGUAY & 19 \\
\hline 5 & JAPAN & 138 & 54 & NETHR. ANT & 50 & 103 & CONGO & 18 \\
\hline 6 & BELGIUH & 135 & 55 & U.A.E. & 49 & 104 & BOLIVIA & 18 \\
\hline 7 & ITALY & 134 & 56 & EGYPT & 48 & 105 & NICARAGUA & 18 \\
\hline 8 & U.S.A. & 131 & 57 & INDONESIA & 47 & 106 & PANAMA & 18 \\
\hline 9 & SPAIN & 118 & 58 & TUNISIA & 42 & 107 & MADAGASCAR & 17 \\
\hline 10 & SUITZERLAN & 118 & 59 & TRINIDAD & 41 & 108 & EL SALVADO & 16 \\
\hline 11 & SUEDEN & 115 & 60 & ALGERIA & 40 & 109 & GUINEA & 15 \\
\hline 12 & HONG KONG & 115 & 61 & KUWAIT & 40 & 110 & MALI & 15 \\
\hline 13 & CANADA & 112 & 62 & CHILE & 40 & 111 & PAUPA N GU & 15 \\
\hline 14 & DENMARK & 110 & 63 & URUGUAY & 40 & 112 & GREENLAND & 13 \\
\hline 15 & BRAZIL & 105 & 64 & CUBA & 40 & 113 & CEN. AFR. & 12 \\
\hline 16 & AUSTRIA & 101 & 65 & CYPRUS & 39 & 114 & MAURITIUS & 12 \\
\hline 17 & NORWAY & 98 & 66 & SOUTH AFRI & 38 & 115 & ZAIRE & 12 \\
\hline 18 & KOREA & 98 & 67 & IVORY COAS & 37 & 116 & VIETNAY & 12 \\
\hline 19 & INDIA & 95 & 68 & TANZANIA & 37 & 117 & OHAN & 12 \\
\hline 20 & SINGAPORE & 93 & 69 & LEBANON & 37 & 118 & MAURITANIA & 11 \\
\hline 21 & IRELAND & 90 & 70 & NIGERIA & 36 & 119 & MEPAL & 11 \\
\hline 22 & AUSTRALIA & 89 & 71 & BAHRAIN & 36 & 120 & BENIN & 10 \\
\hline 23 & ARGENTIMA & 88 & 72 & COSTA RICA & 35 & 121 & BURKIMA FA & 10 \\
\hline 24 & YUEOSLAVIA & 84 & 73 & SENEGAL & 34 & 122 & FIJI & 10 \\
\hline 25 & FINLAND & 83 & 74 & SUDAN & 34 & 123 & NIGER & 9 \\
\hline 26 & U.S.S.R. & 83 & 75 & CATAR & 33 & 124 & BRUNEI & 9 \\
\hline 27 & PORTUGAL & 80 & 76 & ECUADOR & 33 & 125 & HAITI & 9 \\
\hline 28 & CZECHOSLOV & 80 & $\pi$ & BURMA & 32 & 126 & CHAD & 8 \\
\hline 29 & POLAND & 79 & 78 & GUATEMALA & 31 & 127 & GAMBIA & 8 \\
\hline 30 & MEN ZEALAN & 78 & 79 & MOZAMBIQUE & 30 & 128 & SOHALIA & 8 \\
\hline 31 & MALAYSIA & $\pi$ & 80 & LIBYA & 30 & 129 & BELIZE & 8 \\
\hline 32 & PAKISTAN & 77 & 81 & SYRIA & 30 & 130 & BURUNDI & 7 \\
\hline 33 & ThaILANo & 73 & 82 & ZAMBIA & 29 & 131 & BARBADOS & 7 \\
\hline 34 & ROMANIA & 70 & 83 & ICELAND & 28 & 132 & DJIBOUTI & 6 \\
\hline 35 & GREECE & 69 & 84 & NORTH KORE & 27 & 133 & YEHEN, A.R & 6 \\
\hline 36 & PHILIPPINE & 64 & 85 & CAMEROON & 25 & 134 & GUIANA, FR & 6 \\
\hline 37 & BULGARIA & 64 & 86 & BAHAMAS & 25 & 135 & GUIHEA-BIS & 5 \\
\hline 38 & SAUDI ARAB & 63 & 87 & DOMINICAN & 25 & 136 & SIERRA LEO & 5 \\
\hline 39 & EAST GERMA & 63 & 88 & JAMAICA & 25 & 137 & LAO & 5 \\
\hline 40 & ISRAEL & 62 & 89 & ETHIOPIA & 24 & 138 & BERHUDA & 5 \\
\hline 41 & KENYA & 60 & 90 & HONDORAS & 24 & 139 & GRENADA & 5 \\
\hline 42 & HOROCCO & 56 & 91 & MALTA & 23 & 140 & MARTINIQUE & 5 \\
\hline 43 & BAKGLADESH & 56 & 92 & JORDAN & 23 & 141 & EQU. GUINE & 4 \\
\hline 44 & CHIMA & 56 & 93 & ANGOLA & 22 & 142 & REUNION & 4 \\
\hline 45 & COLOHBIA & 56 & 94 & HACAO & 22 & 143 & NEH CALEDO & 4 \\
\hline 46 & MEXICO & 55 & 95 & GHANA & 21 & 144 & GUADALOUPE & 4 \\
\hline 47 & VENEZUELA & 54 & 96 & MALAWI & 21 & 145 & MALDIVES & 3 \\
\hline 48 & IRAQ & 53 & 97 & AFGHANISTA & 21 & 146 & SEYCHELLES & 2 \\
\hline 49 & PERU & 53 & 98 & GABON & 20 & 147 & CAPE VERDI & 1 \\
\hline
\end{tabular}


HYPOTHESIS (H2)- Centralness is associated with the diversified export of commodities whereas peripheralness is associated with the export of only a few commodities. More specifically, EPC is positively correlated with ECC.

Correlation coefficients between EPC and other variables
\begin{tabular}{|l|c|c|c|c|c|c|c|}
\hline EPC VS. & IPC & ECC & ICC & VT & POLI & AGR & ED \\
\hline 1962 & .85 & .34 & .42 & -.57 & -.48 & .42 & .57 \\
\hline 1970 & .73 & .33 & .38 & -.35 & -.36 & .32 & .40 \\
\hline 1980 & .68 & .41 & .43 & -.50 & -.45 & .25 & .55 \\
\hline
\end{tabular}

The calculated correlation coefficients between EPC and ECC support the hypothesis. The correlation coefficient which was .34 in 1962 increased to .41 for 1980 which suggests that the relationship between these two variables has been getting stronger.

The second hypothesis tested the assertion that countries with high export partner concentration would have lower import commodity concentration. This hypothesis was stated as:

HYPOTHESIS (H3)- Centralness is associated with the concentrated importing of commodities(mainly raw materials) whereas peripheralness is associated with diverse imports of commodities( mainly processed goods). More specifically, EPC is negatively correlated with ICC.

The calculated correlation coefficients(Figure 5) between EPC and ICC (.42 in $1962, .38$ in 1970 , and .43 in 1980) are moderately sized but in the opposite direction of what was expected. This rejection of the hypothesis is consistent in all three sample years of the study. More discussion on this finding will be made in the next section. 
TABLE X-A

COUNTRIES RANKED BY EXPORT COMMODITY CONCENTRATION IN 1962

\begin{tabular}{|c|c|c|c|c|c|c|c|c|}
\hline & COUNTRY & ECC & & COUNTRY & ECC & & COUNTRY & ECC \\
\hline 1 & GAMBIA & 1.00 & 44 & ISRAEL & 0.43 & 87 & MEXICO & 0.14 \\
\hline 2 & SAUDI ARAB & 0.98 & 45 & JAMAICA & 0.42 & 88 & KOREA & 0.14 \\
\hline 3 & NETHERLAND & 0.96 & 46 & PARAGUAY & 0.41 & 89 & SHEDEN & 0.13 \\
\hline 4 & BARBADOS & 0.90 & 47 & KENYA & 0.41 & 90 & KUWAIT & 0.12 \\
\hline 5 & FIJI & 0.89 & 48 & NICARAGUA & 0.40 & 91 & U.S.A. & 0.12 \\
\hline 6 & LIBYA & 0.88 & 49 & SWITZERLAN & 0.40 & 92 & SOUTH AFRI & 0.09 \\
\hline 7 & ECUADOR & 0.88 & 50 & AFGHANISTA & 0.39 & 93 & NETHERLAND & 0.07 \\
\hline 8 & ICELAND & 0.83 & 51 & PAKISTAN & 0.39 & 94 & YUCOSLAVIA & 0.07 \\
\hline 9 & VENEZUELA & 0.80 & 52 & IRELAND & 0.39 & 95 & MORWAY & 0.05 \\
\hline 10 & IRAQ & 0.80 & 53 & JAPAN & 0.38 & 96 & SINGAPORE & 0.05 \\
\hline 11 & BENIN & 0.80 & 54 & TUNISIA & 0.38 & 97 & CAMADA & 0.04 \\
\hline 12 & DOHINICAN & 0.78 & 55 & THAILAND & 0.37 & & MALTA & $N / A$ \\
\hline 13 & NIGER & 0.76 & 56 & JORDAN & 0.37 & & U.S.S.R. & N/A \\
\hline 14 & Costa RICA & 0.76 & 57 & NIGERIA & 0.36 & & BULGARIA & N/A \\
\hline 15 & BOLIVIA & 0.75 & 58 & PHILIPPINE & 0.36 & & CZECHOSLOV & N/A \\
\hline 16 & SEMEGAL & 0.72 & 59 & ANGOLA & 0.35 & & EAST GERMA & $N / A$ \\
\hline 17 & IRAN & 0.69 & 60 & INDONESIA & 0.35 & & HUNGARY & $N / A$ \\
\hline 18 & TRINIOAD & 0.69 & 61 & GERHANY & 0.33 & & POLAND & $N / A$ \\
\hline 19 & CHILE & 0.67 & 62 & CAHERCON & 0.32 & & RURANIA & $N / A$ \\
\hline 20 & HONG KONG & 0.67 & 63 & PERU & 0.31 & & CUBA & $N / A$ \\
\hline 21 & SOHALI & 0.66 & 64 & MOZAMBIQUE & 0.31 & & HAITI & $N / A$ \\
\hline 22 & ETHIOPIA & 0.66 & 65 & GABON & 0.31 & & BERHUDA & N/A \\
\hline 23 & mALI & 0.64 & 66 & LAOS & 0.30 & & GREENLAND & $N / A$ \\
\hline 24 & SURINAM & 0.62 & 67 & SIERRA LEO & 0.30 & & GUADALOUPE & N/A \\
\hline 25 & IVORY COAS & 0.58 & 68 & GREECE & 0.29 & & MART INIQUE & $N / A$ \\
\hline 26 & BURMA & 0.57 & 69 & MOROCCO & 0.29 & & YENEN, PEO & N/A \\
\hline 27 & MADAGASCAR & 0.57 & 70 & SYRIA & 0.28 & & BAHREIN & $N / A$ \\
\hline 28 & SUDAN & 0.57 & 71 & CENT. AFR. & 0.28 & & OHAN & N/A \\
\hline 29 & GUATEMALA & 0.56 & 72 & PORTUGAL & 0.28 & & QATAR & N/A \\
\hline 30 & HONDORAS & 0.56 & 73 & EGYPT & 0.27 & & YENEN, ARA & N/A \\
\hline 31 & COLOHBIA & 0.54 & 74 & INDIA & 0.27 & & ALGERIA & $N / A$ \\
\hline 32 & ARGENTIMA & 0.52 & 75 & BELGIUM & 0.26 & & CAPE VERDI & N/A \\
\hline 33 & CHAD & 0.51 & 76 & U.K. & 0.25 & & GUINEA & N/A \\
\hline 34 & GHANA & 0.50 & 77 & DENMARK & 0.25 & & MAURITANIA & N/A \\
\hline 35 & NEW ZEALAN & 0.49 & 78 & FINLAND & 0.25 & & MAURITIUS & N/A \\
\hline 36 & PANAMA & 0.48 & 79 & SPAIN & 0.23 & & REUNIOH & N/A \\
\hline 37 & URUGUAY & 0.48 & 80 & CONGO & 0.21 & & BRUNEI & $N / A$ \\
\hline 38 & EL SALVAdO & 0.48 & 81 & AUSTRIA & 0.21 & & CHINA & N/A \\
\hline 39 & TURKEY & 0.46 & 82 & AUSTRALIA & 0.21 & & MACAO & $N / A$ \\
\hline 40 & CYPRUS & 0.45 & 83 & ITALY & 0.20 & & MALAYSIA & $N / A$ \\
\hline 41 & UGANDA & 0.45 & 84 & FRANCE & 0.16 & & VIET NAM & $\mathbf{N} / \mathbf{A}$ \\
\hline 42 & BRAzIL & 0.45 & 85 & BAHAMAS & 0.16 & & NEW CALEDO & $N / A$ \\
\hline 43 & NEU GUINEA & 0.43 & 86 & LEBANON & 0.16 & & TANZANIA & $\mathbf{N} / \mathbf{A}$ \\
\hline
\end{tabular}


TABLE $X-B$

COUNTRIES RANKED BY EXPORT COMMODITY CONCENTRATION IN 1970

\begin{tabular}{|c|c|c|c|c|c|c|c|c|}
\hline & COUNTRY & ECC & & COUNTRY & ECC & & COUNTRY & ECC \\
\hline 1 & GAMBIA & 0.99 & 50 & JAPAN & 0.41 & 92 & AFGHANISTA & 0.20 \\
\hline 2 & SAUDI ARAB & 0.99 & 51 & SIERRA LEO & 0.39 & 93 & LAOS & 0.20 \\
\hline 3 & oman & 0.98 & 52 & ISRAEL & 0.38 & 94 & INDIA & 0.20 \\
\hline 4 & ZAMBIA & 0.96 & 53 & GABON & 0.38 & 95 & SYRIA & 0.19 \\
\hline 5 & U.A.E. & 0.96 & 54 & NEW ZEALAN & 0.38 & 96 & GREECE & 0.19 \\
\hline 6 & VENEZUELA & 0.89 & 55 & SWITZERLAN & 0.37 & 97 & SUEDEN & 0.19 \\
\hline 7 & FIJI & 0.86 & 56 & PAKISTAN & 0.36 & 98 & FRANCE & 0.19 \\
\hline 8 & IRAQ & 0.84 & 57 & GUATEMALA & 0.36 & 99 & LEBANON & 0.17 \\
\hline 9 & METHERLAND & 0.84 & 58 & KOREA & 0.36 & 100 & SPAIN & 0.16 \\
\hline 10 & ECUADOR & 0.83 & 59 & EL SALVADO & 0.34 & 101 & вAHAMAS & 0.16 \\
\hline 1 & KUHAIT & 0.82 & 60 & NIGERIA & 0.34 & 102 & HUNGARY & 0.1 \\
\hline 2 & BOLIVIA & 0.79 & 61 & GERMANY & 0.34 & 103 & DENMARK & 0.1 \\
\hline 13 & SURINAY & 0.77 & 62 & KENYA & 0.33 & 104 & U.S.A. & 0.14 \\
\hline 14 & MIGER & 0.76 & 63 & JAMAICA & 0.33 & 105 & NETHERLAND & 0.1 \\
\hline 15 & BERHUDA & 0.76 & 64 & SENEGAL & 0.33 & 106 & MEXICO & 0.09 \\
\hline 16 & IRAN & 0.72 & 65 & NICARAGUA & 0.33 & 107 & YUGOSLAVIA & 0.09 \\
\hline 17 & DOHINICAN & 0.71 & 66 & CAMERCON & 0.32 & 108 & AUSTRALIA & 0.0 \\
\hline 18 & MALAWI & 0.71 & 67 & URUGUAY & 0.31 & 109 & SOUTH AFRI & 0.0 \\
\hline 19 & CHILE & 0.70 & 68 & CONGO & 0.31 & 110 & NORWAY & 0.0 \\
\hline 20 & ETHIOPIA & 0.68 & 69 & MOROCCO & 0.31 & 111 & CANADA & 0.0 \\
\hline 21 & SOHALIA & 0.65 & 70 & INDONESIA & 0.31 & 112 & SINGAPORE & 0.0 \\
\hline 22 & BURUNDI & 0.65 & 71 & CENT. AF. & 0.31 & & GREENLAND & N/A \\
\hline 23 & HOHG KONG & 0.65 & 72 & JORDAN & 0.30 & & GUADALOUPE & $N / A$ \\
\hline 24 & PAPAU N. G & 0.64 & 73 & BRAZIL & 0.29 & & GUINEA FR. & $N / A$ \\
\hline 25 & YEHEN, ARA & 0.63 & 74 & TURKEY & 0.28 & & MARTIMIQUE & $N / A$ \\
\hline 26 & SUDAK & 0.62 & 75 & CYPRUS & 0.28 & & QATAR & $N / A$ \\
\hline 27 & COSTA RICA & 0.60 & 76 & HAITI & 0.28 & & YEMEN, PEO & $N / A$ \\
\hline 28 & ICELANO & 0.59 & 77 & TANZANIA & 0.28 & & BRUNEI & $N / A$ \\
\hline 29 & GHAKA & 0.56 & 78 & THAILAND & 0.27 & & TAIHAN & $N / A$ \\
\hline 30 & TRINIDAD & 0.55 & 79 & MOZAMBIQUE & 0.27 & & MACAO & N/A \\
\hline 31 & Pakama & 0.55 & 80 & ITALY & 0.27 & & NEPAL & $N / A$ \\
\hline 32 & BAHREIN & 0.54 & 81 & IRELAND & 0.27 & & VIET NAH & $N / A$ \\
\hline 33 & ZAIRE & 0.53 & 82 & U.K. & 0.26 & & GUINEA REP & N/A \\
\hline 34 & CHAD & 0.52 & 83 & TUNISIA & 0.26 & & LIBYA & $N / A$ \\
\hline 35 & SRI LANKA & 0.50 & 84 & AUSTRIA & 0.26 & & MAURITANIA & $N / A$ \\
\hline 36 & ALGERIA & 0.50 & 85 & MALAYSIA & 0.25 & & MAURITIUS & $N / A$ \\
\hline 37 & EArendos & 0.49 & 86 & BELGIUM & 0.24 & & REUNION & $\mathrm{N} / \mathrm{A}$ \\
\hline 38 & UGANDA & 0.48 & 87 & EGYPT & 0.23 & & SEYCHELLES & $N / A$ \\
\hline 39 & COLOMBIA & 0.47 & 88 & PHILIPPINE & 0.22 & & NEH CALEdo & $N / A$ \\
\hline 40 & ARGENTINA & 0.46 & 89 & PORTUGAL & 0.22 & & BULGARIA & N/A \\
\hline 41 & PARAGUAY & 0.46 & 90 & FINLAND & 0.22 & & CHIMA & N/A \\
\hline 42 & BENIN & 0.46 & 91 & ANGOLA & 0.21 & & CUBA & $\mathbf{N} / \mathbf{A}$ \\
\hline 43 & GUYANA & 0.46 & 92 & AFGHANISTA & 0.20 & & CZECHOSLOV & N/A \\
\hline 44 & BURMA & 0.46 & 93 & LAOS & 0.20 & & EAST GER & N/A \\
\hline 45 & IVORY COAS & 0.46 & 94 & INDIA & 0.20 & & NORTH KORE & $\mathbf{N} / \mathbf{A}$ \\
\hline 46 & MALI & 0.44 & 95 & SYRIA & 0.19 & & NORTH VIET & N/A \\
\hline 47 & HONDORAS & 0.44 & 96 & GREECE & 0.19 & & POLAND & N/A \\
\hline 48 & PERU & 0.41 & 97 & SWEDEN & 0.19 & & ROMANIA & N/A \\
\hline 促 & MALTA & 0.41 & 98 & FRANCE & 0.19 & & U.S.S.R. & $\mathbf{N} /$ \\
\hline
\end{tabular}


TABLE $\mathrm{X}-\mathrm{C}$

COUNTRIES RANKED BY EXPORT COMMODITY CONCENTRATION IN 1980

\begin{tabular}{|c|c|c|c|c|c|c|c|}
\hline & COUNTRY & ECC & & COUNTRY & ECC & COUNTRY & ECC \\
\hline 1 & SAUDI ARAB & 0.97 & 50 & ITALY & 0.30 & DJIBOUTI & N/A \\
\hline 2 & ALGERIA & 0.96 & 51 & CHILE & 0.30 & EQU. GUINE & $N / A$ \\
\hline 3 & VENEZUELA & 0.92 & 52 & CAMEROON & 0.28 & GAMBIA & N/A \\
\hline 4 & BAHRAIN & 0.88 & 53 & TANZANIA & 0.28 & GHANA & N/A \\
\hline 5 & FIJI & 0.87 & 54 & AUSTRIA & 0.28 & GUINEA & N/A \\
\hline 6 & MIGERIA & 0.86 & 55 & NORWAY & 0.28 & GUINEA-BIS & N/A \\
\hline 7 & QATAR & 0.86 & 56 & CZECHOSLOV & 0.27 & MALI & N/A \\
\hline 8 & BURUNDI & 0.86 & 57 & MOROCCO & 0.27 & MAURITANIA & $N / A$ \\
\hline 9 & TRINIDAD & 0.80 & 58 & JORDAN & 0.27 & MAURITIUS & $N / A$ \\
\hline 10 & MALAUI & 0.77 & 59 & CYPRUS & 0.27 & mozambique & $N / A$ \\
\hline 11 & GABON & 0.77 & 60 & INDIA & 0.26 & NIGER & N/A \\
\hline 12 & SOHALIA & 0.73 & 61 & YEMEN, A.R & 0.26 & REUMION & $N / A$ \\
\hline 13 & MULAT & 0.73 & 62 & GUATEMALA & 0.25 & SEYCHELLES & N/A \\
\hline 14 & BOLIVIA & 0.65 & 63 & UK & 0.25 & SIERRA LEO & N/A \\
\hline 15 & CONGO & 0.65 & 64 & KENYA & 0.25 & UGANDA & N/A \\
\hline 16 & MALTA & 0.61 & 65 & SENEGAL & 0.24 & ZAIRE & $N / A$ \\
\hline 17 & HONG KONG & 0.60 & 66 & URUGUAY & 0.23 & ZAMBIA & N/A \\
\hline 18 & DOMINICAN & 0.55 & 67 & EL SALVADO & 0.23 & AFGHANISTA & $N / \boldsymbol{A}$ \\
\hline 19 & ETHIOPIA & 0.54 & 68 & BELGIUM & 0.23 & BRUNEI & N/A \\
\hline 20 & ICELAND & 0.53 & 69 & IRELAND & 0.23 & BURMA & N/A \\
\hline 21 & INDONESIA & 0.51 & 70 & GREECE & 0.23 & CHIMA & N/A \\
\hline 22 & MADAGASCAR & 0.51 & 71 & PORTUGAL & 0.23 & LAO & N/A \\
\hline 23 & SUDAN & 0.50 & 72 & SWEDEN & 0.21 & MACAO & $N / A$ \\
\hline 24 & HONDORAS & 0.49 & 73 & TURKEY & 0.21 & MALDIVES & N/A \\
\hline 25 & MICARAGUA & 0.48 & 74 & PAKISTAN & 0.21 & MEN CALEDO & $N / A$ \\
\hline 26 & PANAMA & 0.48 & 75 & FINLAND & 0.21 & VIETNAM & N/A \\
\hline 27 & BERHUDA & 0.46 & 76 & NEH ZEALAN & 0.20 & ROMANIA & $N / A$ \\
\hline 28 & ECUADOR & 0.46 & 77 & SRI LANKA & 0.20 & IRAN & $N / A$ \\
\hline 29 & SYRIA & 0.46 & 78 & SPAIN & 0.19 & IRAQ & $N / A$ \\
\hline 30 & JAPAN & 0.46 & 79 & SOUTH AFRI & 0.19 & LEBANON & N/A \\
\hline 31 & BAHGLADESH & 0.46 & 80 & FRANCE & 0.18 & LIBYA & $N / A$ \\
\hline 32 & COSTA RICA & 0.46 & 81 & PHILIPPINE & 0.18 & U.A.E. & N/A \\
\hline 33 & KOREA & 0.45 & 82 & YUGOSLAVIA & 0.16 & YEMEN, A.D & $\mathbf{M} / \mathbf{A}$ \\
\hline 34 & COLOMBIA & 0.45 & 83 & BRAZIL & 0.15 & BAHARAS & $M / A$ \\
\hline 35 & OALAN & 0.43 & 84 & THAILAND & 0.15 & BELIZE & N/A \\
\hline 36 & PAUPA N GU & 0.41 & 85 & DENHARK & 0.14 & GREENLAND & N/A \\
\hline 37 & SAMAICA & 0.41 & 86 & HUNGARY & 0.14 & GRENADA & M/A \\
\hline 38 & IVORY COAS & 0.39 & 87 & U.S.A. & 0.13 & GUADALOUPE & N/A \\
\hline 39 & ISRAEL & 0.39 & 88 & MEXICO & 0.12 & GUIANA, FR & M/A \\
\hline 40 & PARAGUAY & 0.39 & 89 & AUSTRALIA & 0.10 & HAITI & N/A \\
\hline 41 & EGYPT & 0.38 & 90 & NETHERLAND & 0.10 & MARTIMIQUE & N/A \\
\hline 42 & SHITZERLAN & 0.38 & 91 & MALAYSIA & 0.09 & NETHR. ANT & N/A \\
\hline 43 & TUNISIA & 0.37 & 92 & SINGAPORE & 0.07 & SURINAME & N/A \\
\hline 44 & BARBADOS & 0.35 & & CANADA & 0.04 & BULGARIA & $N / A$ \\
\hline 45 & NEPAL & 0.35 & & ANGOLA & $N / A$ & CUBA & $N / A$ \\
\hline 46 & PERU & 0.34 & & BENIN & N/A & EAST GERMA & $\mathbf{N} / \mathbf{A}$ \\
\hline 47 & ARGENTIMA & 0.32 & & BURKINA FA & N/A & NORTH KORE & N/A \\
\hline 48 & CEN. AFR. & 0.32 & & CAPE VERDI & N/A & POLAND & N/A \\
\hline 49 & GERHAKY & 0.30 & & CHAD & $N / A$ & U.S.S.R. & $\mathbf{N} / \mathbf{A}$ \\
\hline
\end{tabular}


TABLE XI-A

COUNTRIES RANKED BY IMPORT COMMODITY CONCENTRATION IN 1962

\begin{tabular}{|c|c|c|c|c|c|c|c|c|}
\hline & COUNTRY & ICC & & COUNTRY & $I C C$ & & COUNTRY & ICC \\
\hline 1 & NETHERLAND & 0.66 & 44 & JORDAN & 0.25 & 87 & BELGIUK & 0.05 \\
\hline 2 & AFGHANISTA & 0.56 & 45 & CYPRUS & 0.24 & 88 & SPAIN & 0.04 \\
\hline 3 & UGANDA & 0.56 & 46 & MOZAMBIQUE & 0.24 & 89 & FRANCE & 0.03 \\
\hline 4 & MADAGASCAR & 0.36 & 47 & SURINAM & 0.23 & 90 & ITALY & 0.02 \\
\hline 5 & COSTA RICA & 0.35 & 48 & NIGER & 0.23 & 91 & JAPAN & 0.02 \\
\hline 6 & BURMA & 0.34 & 49 & HONG KONG & 0.23 & & MALTA & $N / A$ \\
\hline 7 & HONDORAS & 0.34 & 50 & BARBADOS & 0.23 & & U.S.S.R. & N/A \\
\hline 8 & ANGOLA & 0.34 & 51 & NEW ZEALAN & 0.23 & & BULGARIA & N/A \\
\hline 9 & LIBYA & 0.33 & 52 & FIJI & 0.22 & & CZECHOSLOV & N/A \\
\hline 10 & NICARAEUA & 0.33 & 53 & TUNISIA & 0.22 & & EAST GERHA & N/A \\
\hline 11 & SAUDI ARAB & 0.32 & 54 & MOROCCO & 0.22 & & HUNGARY & N/A \\
\hline 12 & GAMBIA & 0.32 & 55 & CHAD & 0.21 & & POLAND & N/A \\
\hline 13 & CENT. AFR. & 0.32 & 56 & JAMAICA & 0.21 & & RUMANIA & N/A \\
\hline 14 & KUUAIT & 0.32 & 57 & PARAGUAY & 0.21 & & CUBA & $N / A$ \\
\hline 15 & MEU GUINEA & 0.31 & 58 & PAKISTAN & 0.20 & & HAITI & N/A \\
\hline 16 & VENEZUELA & 0.31 & 59 & AUSTRALIA & 0.19 & & BaHAnAs & $N / A$ \\
\hline 17 & MEXICO & 0.31 & 60 & LEBANON & 0.19 & & BERMUDA & N/A \\
\hline 18 & IRAQ & 0.31 & 61 & CHILE & 0.19 & & GREENLAND & $N / A$ \\
\hline 19 & ECUADOR & 0.31 & 62 & CANADA & 0.18 & & GUADALOUPE & $N / A$ \\
\hline 20 & ETHIOPIA & 0.30 & 63 & ICELAND & 0.18 & & MARTINIQUE & $N / \mathfrak{R}$ \\
\hline 21 & SENEGAL & 0.30 & 64 & TURKEY & 0.18 & & YENEN, PEO & $N / A$ \\
\hline 22 & ARGENTINA & 0.30 & 65 & GREECE & 0.17 & & BAHREIN & N/A \\
\hline 23 & IVORY COAS & 0.30 & 66 & FINLAND & 0.17 & & IRAN & N/A \\
\hline 24 & NIGERIA & 0.30 & 67 & SWITZERLAN & 0.16 & & OHAN & N/A \\
\hline 25 & CONEO & 0.30 & 68 & IRELAND & 0.15 & & QATAR & N/A \\
\hline 26 & SUDAN & 0.29 & 69 & NORWAY & 0.15 & & YEMEN, ARA & N/A \\
\hline 27 & GABON & 0.29 & 70 & ISRAEL & 0.15 & & SOUTH AFRI & N/A \\
\hline 28 & GHANA & 0.29 & 71 & KOREA & 0.14 & & ALGERIA & N/A \\
\hline 29 & THAILAND & 0.29 & 72 & DENMARK & 0.14 & & CAPE VERDI & N/A \\
\hline 30 & SOHALI & 0.29 & 73 & BRAZIL & 0.12 & & GUINEA & $N / A$ \\
\hline 31 & CAMEROON & 0.28 & 74 & SWEDEN & 0.12 & & KENYA & N/A \\
\hline 32 & EL SALVADO & 0.28 & 75 & EGYPT & 0.12 & & MAURITANIA & N/A \\
\hline 33 & PERU & 0.28 & 76 & YUGOSLAVIA & 0.12 & & MAURITIUS & $N / A$ \\
\hline 34 & COLOHBIA & 0.28 & $\pi$ & URUGUAY & 0.12 & & REUNION & $N / A$ \\
\hline 35 & GUATERALA & 0.27 & 78 & AUSTRIA & 0.12 & & SIERRA LEO & N/A \\
\hline 36 & SYRIA & 0.27 & 79 & NETHERLAND & 0.11 & & BRUNEI & $N / A$ \\
\hline 37 & BOLIVIA & 0.26 & 80 & SINGAPORE & 0.11 & & CHINA & H/A \\
\hline 38 & LAOS & 0.26 & 81 & INDIA & 0.10 & & INDONESIA & N/A \\
\hline 39 & BENIN & 0.26 & 82 & PHILIPPINE & 0.08 & & MACAO & N/A \\
\hline 40 & MALI & 0.26 & 83 & PORTUGAL & 0.08 & & MALAYSIA & N/A \\
\hline 41 & TRINIDAD & 0.26 & 84 & U.K. & 0.08 & & VIET HAN & N/A \\
\hline 42 & PANAMA & 0.26 & 85 & GERHANY & 0.08 & & NEH CALEDO & N/A \\
\hline 43 & DOHINICAN & 0.25 & 86 & U.S.A. & 0.06 & & TANZANIA & $N / A$ \\
\hline
\end{tabular}


TABLE XI-B

COUNTRIES RANKED BY IMPORT COMHODITY CONCENTRATION IN 1970

\begin{tabular}{|c|c|c|c|c|c|c|c|c|}
\hline & COUNTRY & ICC & & COUNTRY & ICC & & COUNTRY & ICC \\
\hline 1 & OHAN & 0.56 & 50 & ZAIRE & 0.27 & 92 & HONDORAS & $\overline{0.15}$ \\
\hline 2 & NETHERLAND & 0.48 & 51 & MOZAMBIQUE & 0.27 & 93 & SURINAM & 0.15 \\
\hline 3 & UGANDA & 0.41 & 52 & HONG KONG & 0.26 & 94 & NETHERLAND & 0.14 \\
\hline 4 & YEMEN, ARA & 0.39 & 53 & CYPRUS & 0.26 & 95 & MALAYSIA & 0.13 \\
\hline 5 & AFGHANISTA & 0.38 & 54 & THAILAND & 0.25 & 96 & U.S.A. & 0.13 \\
\hline 6 & GABON & 0.37 & 55 & BARBADOS & 0.25 & 97 & BURUNDI & 0.13 \\
\hline 7 & GUATEMALA & 0.36 & 56 & JORDAN & 0.25 & 98 & PORTUGAL & 0.13 \\
\hline 8 & NIGERIA & 0.35 & 57 & PANAMA & 0.25 & 99 & PERU & 0.12 \\
\hline 9 & CENT. AF. & 0.35 & 58 & GHANA & 0.25 & 100 & SINGAPORE & 0.12 \\
\hline 10 & IRAN & 0.34 & 59 & MALTA & 0.25 & 101 & EGYPT & 0.11 \\
\hline 11 & BURMA & 0.34 & 60 & FIJI & 0.25 & 102 & URUGUAY & 0.11 \\
\hline 12 & KUWAIT & 0.34 & 61 & JAMAICA & 0.24 & 103 & HUNGARY & 0.10 \\
\hline 13 & ECUADOR & 0.34 & 62 & SENEGAL & 0.24 & 104 & BELGIUN & 0.09 \\
\hline 14 & conso & 0.34 & 63 & SUDAN & 0.23 & 105 & GERMANY & 0.09 \\
\hline 15 & BAHREIN & 0.34 & 64 & TURKEY & 0.23 & 106 & FRANCE & 0.09 \\
\hline 16 & CAMEROON & 0.34 & 65 & MEN ZEALAN & 0.23 & 107 & KOREA & 0.08 \\
\hline 17 & COSTA RICA & 0.33 & 66 & PARAGUAY & 0.22 & 108 & INDIA & 0.07 \\
\hline 18 & EL SALVADO & 0.33 & 67 & DOMINICAN & 0.22 & 109 & SPAIN & 0.06 \\
\hline 19 & BENIN & 0.33 & 68 & CHILE & 0.22 & 110 & U.K. & 0.05 \\
\hline 20 & BoLIVIA & 0.33 & 69 & SOMALIA & 0.22 & 111 & ITALY & 0.03 \\
\hline 21 & MICARAGUA & 0.33 & 70 & SWITZERLAN & 0.22 & 112 & JAPAN & 0.01 \\
\hline 22 & IRAQ & 0.32 & 71 & GREECE & 0.21 & & BERHUDA & $N / \boldsymbol{A}$ \\
\hline 23 & VENEZUELA & 0.32 & 72 & ISRAEL & 0.21 & & GREENLAND & $H / A$ \\
\hline 24 & U.A.E. & 0.32 & 73 & LAOS & 0.21 & & GUADALOUPE & N/A \\
\hline 25 & GAMEIA & 0.32 & 74 & BAHAMAS & 0.20 & & GUINEA FR. & N/A \\
\hline 26 & PAPAU N. G. & 0.32 & 75 & CHAD & 0.20 & & MARTINIQUE & $N / A$ \\
\hline 27 & COLOMBIA & 0.32 & 76 & ARGENTINA & 0.19 & & YEMEN, PEO & N/A \\
\hline 28 & TANZANZA & 0.32 & 77 & IRELAND & 0.19 & & BRUNEI & $N / A$ \\
\hline 29 & ANGOLA & 0.32 & 78 & SYRIA & 0.19 & & TAIHAN & N/A \\
\hline 30 & QATAR & 0.31 & 79 & PAKISTAN & 0.19 & & INDONESIA & $N / A$ \\
\hline 31 & TRINIOAD & 0.30 & 80 & MALI & 0.18 & & MACAO & $N / A$ \\
\hline 32 & SIERRA LEO & 0.30 & 81 & ICELAND & 0.18 & & NEPAL & N/A \\
\hline 33 & SAUDI ARAB & 0.30 & 82 & DENMARK & 0.18 & & VIET NAR & N/A \\
\hline 34 & SOUTH AFRI & 0.30 & 83 & BRAZIL & 0.18 & & GUINEA REP & $N / A$ \\
\hline 35 & SRI LANKA & 0.30 & 84 & FINLAND & 0.17 & & MAURITANIA & $N / A$ \\
\hline 36 & HAITI & 0.30 & 85 & LEBANON & 0.17 & & MAURITIUS & N/A \\
\hline 37 & NIGER & 0.30 & 86 & NORWAY & 0.17 & & REUNIOH & $N / A$ \\
\hline 38 & IVORY COAS & 0.29 & 87 & TUNISIA & 0.17 & & SEYCHELLES & $N / A$ \\
\hline 39 & AUSTRALIA & 0.29 & 88 & SHEDEN & 0.17 & & NEW CALEDO & $N / A$ \\
\hline 40 & AUSTRIA & 0.29 & 89 & PHILIPPINE & 0.16 & & BULGARIA & $N / A$ \\
\hline 41 & ALGERIA & 0.29 & 90 & MOROCCO & 0.16 & & CHINA & $N / A$ \\
\hline 42 & ETHIOPIA & 0.29 & 91 & YUGOSLAVIA & 0.16 & & cuBn & $\mathbf{N} / \mathbf{A}$ \\
\hline 43 & KENYA & 0.29 & 92 & HONDORAS & 0.15 & & CZECHOSLOV & $N / A$ \\
\hline 44 & LIBYA & 0.29 & 93 & SURINAM & 0.15 & & EAST GER & $N / A$ \\
\hline 45 & GUYANA & 0.28 & 94 & METHERLAND & 0.14 & & NORTH KORE & $N / \boldsymbol{A}$ \\
\hline 46 & MEXICO & 0.28 & 95 & MALAYSIA & 0.13 & & NORTH VIET & $N / A$ \\
\hline 47 & MALAHI & 0.28 & 96 & U.S.A. & 0.13 & & POLAND & $N / A$ \\
\hline 48 & CAMADA & 0.28 & 97 & BURUNDI & 0.13 & & Ronanta & $N / A$ \\
\hline 49 & ZAMBIA & 0.27 & 98 & PORTUGAL & 0.13 & & U.S.S.R. & $N / A$ \\
\hline
\end{tabular}


TABLE XI-C

COUNTRIES RANKED BY IMPORT COMMODITY CONCENTRATION IN 1980

\begin{tabular}{|c|c|c|c|c|c|c|c|}
\hline & COUNTRY & ICC & & COUNTRY & ICC & COUNTRY & ICC \\
\hline 1 & BOLIVIA & 0.40 & 50 & ICELAND & 0.20 & CHAD & $N / A$ \\
\hline 2 & SOUTH AFRI & 0.38 & 51 & NEW 2EALAN & 0.20 & DJIBOUTI & $N / A$ \\
\hline 3 & ECUADOR & 0.37 & 52 & TANZANIA & 0.20 & EQU. GUINE & N/A \\
\hline 4 & SAUDI ARAB & 0.36 & 53 & IRELAND & 0.20 & GAMBIA & $N / A$ \\
\hline 5 & KWWAIT & 0.36 & 54 & BARBADOS & 0.19 & GHAKA & $\mathbf{N} / \mathbf{A}$ \\
\hline 6 & BAHRAIN & 0.34 & 55 & BRAZIL & 0.19 & GUIMEA & N/A \\
\hline 7 & LIBYA & 0.34 & 56 & SENEGAL & 0.19 & GUINEA-BIS & $\mathbf{N} / \mathbf{A}$ \\
\hline 8 & GABON & 0.33 & 57 & COLOMBIA & 0.19 & MALI & N/A \\
\hline 9 & MEXICO & 0.32 & 58 & PHILIPPINE & 0.19 & MAURITANIA & $\mathbf{N} / \mathbf{A}$ \\
\hline 10 & NIGERIA & 0.31 & 59 & JORDAN & 0.19 & MAURITIUS & $N / A$ \\
\hline 11 & CEN. AFR. & 0.30 & 60 & THAILAND & 0.18 & MOZAHBIQUE & N/A \\
\hline 12 & MEPAL & 0.30 & 61 & AUSTRIA & 0.18 & NIGER & N/A \\
\hline 13 & QATAR & 0.30 & 62 & MALAYSIA & 0.18 & REUNION & $N / A$ \\
\hline 14 & VENEZUELA & 0.30 & 63 & NORWAY & 0.18 & SEYCHELLES & N/A \\
\hline 15 & CAMEROON & 0.29 & 64 & SWEDEN & 0.18 & SIERRA LEO & N/A \\
\hline 16 & BURUNDI & 0.28 & 65 & JAPAN & 0.17 & UGAYDA & $N / \boldsymbol{A}$ \\
\hline 17 & U.A.E. & 0.28 & 66 & CHILE & 0.17 & ZAIRE & N/A \\
\hline 18 & HONG KONG & 0.27 & 67 & SRI LANKA & 0.17 & ZAMBIA & N/A \\
\hline 19 & TURKEY & 0.27 & 68 & SYRIA & 0.17 & AFGHANISTA & $N / A$ \\
\hline 20 & NICARAGUA & 0.27 & 69 & INDONESIA & 0.17 & BRUNEI & N/A \\
\hline 21 & YENEN, A.R & 0.27 & 70 & ISRAEL & 0.16 & BURHA & $\mathbf{N} / \mathbf{A}$ \\
\hline 22 & CONGO & 0.26 & 71 & DOMINICAN & 0.16 & CHINA & N/A \\
\hline 23 & MALAWI & 0.26 & 72 & FINLAND & 0.16 & LAO & N/A \\
\hline 24 & PERU & 0.26 & 73 & URUGUAY & 0.16 & MACAO & $N / A$ \\
\hline 25 & HONDORAS & 0.26 & 74 & U.S.A. & 0.16 & MALDIVES & N/A \\
\hline 26 & ALGERIA & 0.26 & 75 & GREECE & 0.16 & NEW CALEDO & $N / A$ \\
\hline 27 & COSTA RICA & 0.25 & 76 & SINGAPORE & 0.16 & PAUPA N GU & N/A \\
\hline 28 & AUSTRALIA & 0.25 & 77 & DENHARK & 0.15 & VIETNAH & N/A \\
\hline 29 & Orar & 0.25 & 78 & PAKISTAN & 0.14 & ROHNNIA & $N / A$ \\
\hline 30 & BERHUDA & 0.25 & 79 & INDIA & 0.14 & IRAN & $N / A$ \\
\hline 31 & ARGENTINA & 0.24 & 80 & NETHERLAND & 0.14 & IRAQ & N/A \\
\hline 32 & PANarh & 0.24 & 81 & BANGLADESH & 0.13 & LEBANON & N/A \\
\hline 33 & EGYPT & 0.24 & 82 & FRANCE & 0.13 & YEMEN, A.D & N/A \\
\hline 34 & IVORY COAS & 0.24 & 83 & BELGIUM & 0.13 & BAHAMAS & N/A \\
\hline 35 & MADAGASCAR & 0.23 & 84 & TUNISIA & 0.13 & BELIZE & N/A \\
\hline 36 & MLTA & 0.23 & 85 & SPAIN & 0.12 & GREENLAND & N/A \\
\hline 37 & GUATEHALA & 0.23 & 86 & GERMANY & 0.12 & GRENADA & N/A \\
\hline 38 & PARAGUAY & 0.23 & 87 & UK & 0.12 & GUADALOUPE & $N / A$ \\
\hline 39 & KENYA & 0.23 & 88 & HUNGARY & 0.12 & GUIANA, FR & N/A \\
\hline 40 & EL SALVADO & 0.23 & 89 & YUGOSLAVIA & 0.11 & HAITI & N/A \\
\hline 41 & CAMADA & 0.22 & 90 & PORTUGAL & 0.09 & MARTINIQUE & N/A \\
\hline 42 & FIJI & 0.22 & 91 & CZECHOSLOV & 0.09 & NETHR. ANT & $\mathbf{N} / \mathbf{A}$ \\
\hline 43 & ETHIOPIA & 0.22 & 92 & MOROCCO & 0.09 & SURINANE & $N / A$ \\
\hline 44 & TRINIDAD & 0.21 & 93 & ITALY & 0.08 & BULGARIA & $N / A$ \\
\hline 45 & JAMAICA & 0.21 & 94 & KOREA & 0.07 & CUBA & $\mathbf{N} / \mathbf{A}$ \\
\hline 46 & SUDAH & 0.21 & & ANGOLA & $M / A$ & EAST GERMA & $N / A$ \\
\hline 47 & SOHALIA & 0.21 & & BENIN & N/A & NORTH KORE & N/A \\
\hline 18 & CYPRUS & 0.20 & & BURKINA FA & N/A & POLAND & $N / A$ \\
\hline 99 & SUITZERLAN & 0.20 & & CAPE VERDI & N/A & U.S.S.R. & N/A \\
\hline
\end{tabular}




\section{OTHER TESTS OF HYPOTHESES ON COMMODITY CONCENTRATION}

1- One of the widely held views on the structure of trade commodity composition has been based on the ideas of Leontief ${ }^{1}$. Leontief states that,

"each of the underdeveloped countries specializes in the massive export of a few agricultural and mineral commodities and depends on imports for the supply of a broad spectrum of manufactured goods... The U.S. economy, on the other hand, exports a great diversity of manufactured goods and imports a few agricultural and mineral commodities... An underdeveloped economy is consequently the mirror-image of an advanced economy. ${ }^{2}$

Leontief's hypothesis are tested and the following results are found: The correlation coefficients between ECC and ICC are +0.48 for $1962,+0.47$ for 1970 , and +0.51 for 1980 . Therefore, Leontief's hypothesis that countries with higher ECCs should be associated with lower ICCs and lower ECCs be associated with higher ICCs is rejected. This result is interesting since the hypothesized pattern of concentration in trade has conventionally dominated in economic thought. As has previously been stated the aggregation of data did not seem to play a major factor in the results obtained here. Michaely's study which was conducted at a much lower degree of aggregation still rejected this hypothesis.

2- Michaely's analysis of the 1954 data on 44 countries made two general observations: Exports were more concentrated than imports, and there was a much larger variation among countries with regard to the degree of commodity concentration of exports than with regard to the commodity concentration of imports.

1- W. Leontief, Input-Output Economics (N.Y:: Oxford University Press, 1966), p.64.

2- W. Leontief, "The Structure of Development," Scientific American. 1963:September:164. 
In comparing the present results (tables $X$ through XII) with those of Michaely's, the following is observed: Exports are more concentrated than imports in 78 of the 91 countries studied in 1962,88 of the 110 countries studied in 1970 , and 77 of 92 countries studied in 1980 . The results here are found to be similar to those found in the earlier study conducted by Michaely. However, according to Michaely, finding so many countries that had import commodity concentrations greater than export commodity concentrations is contrary to conventional thinking in economics.

The second observation of Michaely that the variation among countries was larger in export commodity concentration than in import commodity concentration is also supported by the results of this study. In 1962, ECC ranged from 1.0 to 0.04 , while ICC varied from 0.66 to .02 . These variations for 1970 were 0.99 to 0.07 for ECC and 0.56 to 0.01 for ICC, and in 1980, they were 0.97 to 0.04 for ECC and 0.4 to 0.07 for ICC . Michaely suggested that the major cause of variation with regard to commodity concentrations was that, "the more developed the economy in terms of higher per capita income, the more diversified are the exports. ${ }^{3}$ However, the correlation coefficient of -.03 between ECC and GNP was very small and insignificant; therefore, such an assumption becomes questionable.

3- Simon Kuznets ${ }^{4}$ suggested that economic activities would be more diversified in countries with a higher GNP per capita even if two underdeveloped countries were compared. His assertion was formulated as the following hypothesis:

3- Ibid, p. 13.

4- S. Kuznets, Economic Growth of Small Nations. The Challenge of Development (Jerusalem: The Eliezer Kaplan School of Economics and Social Sciences, The Hebrew University, 1958). 
HYPOTHESIS- The more developed an economy, the more diversified is its exports. More specifically, the GNP per capita is negatively correlated with Export Commodity Concentration.

The correlation coefficient between GNP per capita and ECC for the year 1980 is -0.03 . The correlation coefficient in this case is too small to support the hypothesis. A possible reason for such a low correlation could be related to the fact that oil producing countries in recent years have obtained very high GNPs per capita without any fundamental structural changes in their economy. Therefore, it seems that as long as oil producing countries are present in the correlation studies, GNP per capita would not be an adequate variable.

\section{VERTICAL TRADE}

This section will test Galtung's hypothesis on the international division of labor. According to Galtung, at the top of this structure are the industrialized center countries which transform their raw material imports into manufactured products for export. At the bottom of this structure are the material producing peripheral countries who export their raw materials in order to pay for manufactured goods which they import. This hypothesis was stated as:

HYPOTHESIS (H4)- Center countries are expected to have a high position and periphery countries a low position in the international division of labor. More specifically, EPC is negatively correlated with VT.

Correlation coefficlents between EPC and other variables

\begin{tabular}{|l|c|c|c|c|c|c|c|}
\hline EPC V8. & IPC & ECC & ICC & VT & POLI & AGR & ED \\
\hline 1962 & .85 & .34 & .42 & -.57 & -.48 & .42 & .57 \\
\hline 1970 & .73 & .33 & .38 & -.35 & -.36 & .32 & .40 \\
\hline 1980 & .68 & .41 & .43 & -.50 & -.45 & .25 & .55 \\
\hline
\end{tabular}

Empirically, the correlation coefficients between EPC and VT are strong and in the expected direction. they are -0.57 for $1962,-0.34$ for 1970 , and -0.50 for 1980 , and thus confirm Galtung's hypothesis. In tables XII-A, XII-B, and XII-C, at 
TABLE XII-A

COUNTRIES RANKED BY VERTICAL TRADE IN 1962

\begin{tabular}{|c|c|c|c|c|c|c|c|c|}
\hline & COUNTRY & vT & & COUNTRY & VT & & COUNTRY & VT \\
\hline 1 & JAPAN & 0.64 & 44 & GABON & -0.57 & 87 & COLOHBIA & -0.77 \\
\hline 2 & U.K. & 0.56 & 45 & CENT. AFR. & -0.58 & 88 & ECUADOR & -0.78 \\
\hline 3 & GERMANY & 0.44 & 46 & SOMALI & -0.58 & 89 & ETHIOPIA & -0.80 \\
\hline 4 & HONG KONG & 0.36 & 47 & GREECE & -0.59 & 90 & LIBYA & -0.83 \\
\hline 5 & ITALY & 0.31 & 48 & BENIN & -0.60 & 91 & UGANDA & -0.96 \\
\hline 6 & FRANCE & 0.31 & 49 & FIJI & -0.60 & & MALTA & N/A \\
\hline 7 & SHITZERLAN & 0.25 & 50 & AUSTRALIA & -0.61 & & U.S.S.R. & H/A \\
\hline 8 & U.S.A. & 0.23 & 51 & NIGER & -0.61 & & BULGARIA & N/A \\
\hline 9 & BELGIUM & 0.22 & 52 & SURINAM & -0.62 & & CZECHOSLOV & N/A \\
\hline & AUSTRIA & 0.11 & 53 & SAUDI ARAB & -0.62 & & EAST GERMA & N/A \\
\hline 1 & SHEDEN & 0.04 & 54 & CHILE & -0.63 & & HUNGARY & N/A \\
\hline & PORTUGAL & 0.02 & 55 & CHAD & -0.63 & & POLAND & $N / A$ \\
\hline 13 & ISRAEL & 0.01 & 56 & ANGOLA & -0.63 & & RUHANIA & N/A \\
\hline 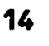 & NETHERLAND & -0.07 & 57 & BOLIVIA & -0.63 & & CuBA & $N / A$ \\
\hline & rugosLaVIA & -0.07 & 58 & EL SALVAdo & -0.63 & & HAITI & $N / A$ \\
\hline 16 & SIMGAPORE & -0.07 & 59 & PANAMA & -0.64 & & Baranias & N/A \\
\hline $\mid$ & NETHERLAND & -0.11 & 60 & SYRIA & -0.65 & & BERHUDA & N/A \\
\hline 18 & FINLAND & -0.14 & 61 & ICELAND & -0.66 & & GREENLAND & N/A \\
\hline 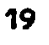 & INDIA & -0.15 & 62 & TURKEY & -0.67 & & GUADALOUPE & N/A \\
\hline 2 & SPAIN & -0.18 & 63 & CYPRUS & -0.67 & & MARTINIQUE & N/A \\
\hline 2 & MORUAY & -0.22 & 64 & GAMBIA & -0.67 & & YEHEN, PEO & N/A \\
\hline & DENHARK & -0.24 & 65 & CAMEROON & -0.68 & & BAHREIN & N/A \\
\hline 2 & KUWAIT & -0.24 & 66 & MEXICO & -0.68 & & IRAN & $\mathbf{N} / \mathbf{A}$ \\
\hline 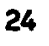 & CONGO & -0.33 & 67 & GUATEMALA & -0.68 & & OHAN & N/A \\
\hline 2 & IRELAND & -0.34 & 68 & mozambique & -0.68 & & & N/A \\
\hline 26 & KOREA & -0.34 & 69 & NIGERIA & -0.69 & & YEMEN, ARA & N/A \\
\hline 2 & TRINIDAD & -0.34 & 70 & IRAQ & -0.69 & & SOUTH AFRI & N/A \\
\hline 28 & EGYPT & -0.35 & 71 & GHANA & -0.70 & & ALGERIA & N/A \\
\hline 2 & LAOS & -0.37 & 72 & DOHINICAN & -0.70 & & CAPE VERDI & N/A \\
\hline 30 & CAMADA & -0.38 & 73 & AFGHANISTA & -0.71 & & GUINEA & N/A \\
\hline 31 & LEEANON & -0.46 & 74 & MALI & -0.71 & & KENYA & $N / A$ \\
\hline 32 & PAKISTAK & -0.46 & 75 & COSTA RICA & -0.72 & & MAURETANIA & $H / A$ \\
\hline 33 & PARAgUAY & -0.50 & 76 & VENEZUELA & -0.72 & & MAURITIUS & $N / A$ \\
\hline 34 & MOROCCO & -0.51 & $\pi$ & NEW ZEALAN & -0.72 & & REUNION & N/A \\
\hline 35 & BARBADOS & -0.51 & 78 & IVORY COAS & -0.73 & & SIERRA LEO & N/A \\
\hline 36 & PHILIPPINE & -0.53 & 79 & MADAGASCAR & -0.75 & & BRUNEI & $N / A$ \\
\hline 37 & NEU GUINEA & -0.54 & 80 & THAILAND & -0.75 & & CHINR & $N / A$ \\
\hline 38 & BRAZIL & -0.54 & 81 & PERU & -0.75 & & INDONESIA & $N / A$ \\
\hline 39 & TUNISIA & -0.54 & 82 & HONDORAS & -0.77 & & MACAO & N/A \\
\hline 40 & JORDAN & -0.54 & 83 & SUDAN & -0.77 & & MALAYSIA & $N / A$ \\
\hline 41 & JAMAICA & -0.56 & 84 & NICARAGUA & -0.77 & & VIET NAH & N/A \\
\hline 2 & SENEGAL & -0.57 & 85 & ARGENTINA & -0.77 & & NEW CALEDO & N/A \\
\hline 3 & URUGUAY & -0.57 & 86 & BURHA & -0.77 & & TANZANIA & N/A \\
\hline
\end{tabular}


TABLE XII-B

COUNTRIES RANKED BY VERTICAL TRADE IN 1970

\begin{tabular}{|c|c|c|c|c|c|c|c|c|}
\hline & COUNTRY & VT & & COUNTRY & VT & & COUNTRY & vT \\
\hline 1 & JAPAN & 0.68 & 50 & CONGO & -0.48 & 92 & GUYANA & -0.71 \\
\hline 2 & U.K. & 0.38 & 51 & MEXICO & -0.48 & 93 & LIBYA & -0.72 \\
\hline 3 & ITALY & 0.37 & 52 & PERU & -0.50 & 94 & QATAR & -0.72 \\
\hline 4 & GERMANY & 0.34 & 53 & EL SALVADO & -0.51 & 95 & CAYIEROON & -0.73 \\
\hline 5 & HOAG KONG & 0.28 & 54 & MALAYSIA & -0.51 & 96 & ALGERIA & -0.73 \\
\hline 6 & KOREA & 0.22 & 55 & MOROCCO & -0.54 & 97 & KUHAIT & -0.73 \\
\hline 7 & BELGIUH & 0.17 & 56 & HONDORAS & -0.54 & 98 & BOLIVIA & -0.74 \\
\hline 8 & SHITZERLAN & 0.17 & 57 & BRAZIL & -0.55 & 99 & NIGER & -0.74 \\
\hline 9 & FRANCE & 0.16 & 58 & BURUNDI & -0.55 & 100 & GABON & -0.74 \\
\hline 0 & MALTA & 0.14 & 59 & GUATERALA & -0.55 & 101 & COLOHBIA & -0.75 \\
\hline & SHEDEN & 0.06 & 60 & PARAGUAY & -0.56 & 102 & IRAQ & -0.76 \\
\hline & U.S.A. & 0.06 & 61 & CHAD & -0.56 & 103 & ZAMBIA & -0.77 \\
\hline 13 & HUNGARY & 0.06 & 62 & CYPRUS & -0.57 & 104 & U.A.E. & -0.77 \\
\hline 4 & LEBAHON & 0.01 & 63 & ANGOLA & -0.57 & 105 & ETHIOPIA & -0.78 \\
\hline 5 & PORTUGAL & 0.00 & 64 & ARGENTINA & -0.59 & 106 & IRAN & -0.81 \\
\hline 6 & INDIA & 0.00 & 65 & BAHREIN & -0.60 & 107 & VENEZUELA & -0.81 \\
\hline 7 & SPAIH & -0.01 & 66 & AFGHANISTA & -0.61 & 108 & ECUADOR & -0.82 \\
\hline 8 & AUSTRIA & -0.02 & 67 & PHILIPPINE & -0.61 & 109 & BURMA & -0.82 \\
\hline 9 & ISRAEL & -0.02 & 68 & COSTA RICA & -0.62 & 110 & NIGERIA & -0.87 \\
\hline 20 & FIMLAND & -0.03 & 69 & GAMBIA & -0.62 & 111 & UGANDA & -0.89 \\
\hline 1 & NETHERLAND & -0.07 & 70 & FIJI & -0.63 & 112 & OMAN & -0.92 \\
\hline 2 & SIERRA LEO & -0.07 & 71 & SURINAM & -0.63 & & BERIMUDA & $N / A$ \\
\hline 3 & PAKISTAN & -0.09 & 72 & SAUDI ARAB & -0.63 & & GREENLAND & $N / A$ \\
\hline 34 & Yugoslavia & -0.10 & 73 & AUSTRALIA & -0.63 & & GUADALOUPE & $N / A$ \\
\hline 25 & BAHAHAS & -0.16 & 74 & NICARAGUA & -0.65 & & GUINEA FR. & $N / A$ \\
\hline 26 & NORWAY & -0.16 & 75 & BENIN & -0.65 & & MARTINIQUE & $N / A$ \\
\hline 27 & DENMARK & -0.16 & 76 & NEW ZEALAN & -0.65 & & YEMEN, PEO & $N / A$ \\
\hline 88 & METHERLAND & -0.18 & 77 & ICELAND & -0.66 & & BRUNEI & $N / A$ \\
\hline 99 & LAOS & -0.21 & 78 & PANAMA & -0.66 & & TAIWAN & N/A \\
\hline 0 & TRINIDAD & -0.21 & 79 & MOZAMBIQUE & -0.66 & & INDONESIA & N/A \\
\hline 1 & JAMAICA & -0.26 & 80 & SUDAN & -0.67 & & MACAO & $N / A$ \\
\hline 22 & SINGAPORE & -0.28 & 81 & KENYA & -0.67 & & NEPAL & N/A \\
\hline 3 & CAMADA & -0.28 & 82 & CHILE & -0.68 & & VIET MAY & N/A \\
\hline 14 & EGYPT & -0.29 & 83 & TURKEY & -0.68 & & GUINEA REP & N/A \\
\hline 35 & YEHEN, ARA & -0.29 & 84 & ZAIRE & -0.68 & & MAURITANIA & N/A \\
\hline 36 & IRELAND & -0.29 & 85 & DOMINI & -0.69 & & MAURITIUS & $N / A$ \\
\hline 8 & CENT. AF. & -0.36 & 86 & GHANA & -0.69 & & REUNION & N/A \\
\hline 38 & SRI LANKA & -0.37 & 87 & TANZANIA & -0.70 & & SEYCHELLES & N/A \\
\hline 39 & GREECE & -0.38 & 88 & MALAWI & -0.70 & & NEW CALEDO & $N / A$ \\
\hline 40 & TUNISIA & -0.39 & 89 & THAILAND & -0.71 & & BULGARIA & $M / A$ \\
\hline 41 & URUGUAY & -0.40 & 90 & PAPAU N. G & -0.71 & & CHINA & $N / A$ \\
\hline 42 & BARBADOS & -0.41 & 91 & IVORY COAS & -0.71 & & CUBA & N/A \\
\hline 43 & SOUTH AFRI & -0.42 & 92 & GUYANA & -0.71 & & CZECHOSLOV & N/A \\
\hline 44 & JORDAN & -0.43 & 93 & LIBYA & -0.72 & & EAST GER & N/A \\
\hline 45 & SEAEGAL & -0.44 & 94 & QATAR & -0.72 & & NORTH KORE & N/A \\
\hline 46 & SYRIA & -0.45 & 95 & CAMEROON & -0.73 & & MORTH VIET & N/A \\
\hline 47 & HAITI & -0.46 & 96 & ALGERIA & -0.73 & & POLAND & N/A \\
\hline 48 & MALI & -0.46 & 97 & KUHAIT & -0.73 & & ROIANIA & $\mathbf{N} / \mathbf{A}$ \\
\hline 49 & SOHAL. & -0.48 & 98 & BOLIVIA & -0.74 & & U.S.S.R. & $N / A$ \\
\hline
\end{tabular}


TABLE XII-C

COUNTRIES RANKED BY VERTICAL TRADE IN 1980

\begin{tabular}{|c|c|c|c|c|c|c|c|}
\hline & COUNTRY & vT & & COUNTRY & vT & COUNTRY & vT \\
\hline 1 & JAPAN & 0.76 & 50 & NORWAY & -0.35 & CHAD & N/A \\
\hline 2 & KOREA & 0.47 & 51 & COSTA RICA & -0.38 & DJIBOUTI & $N / A$ \\
\hline 3 & ITALY & 0.39 & 52 & GUATEMALA & -0.40 & EQU. GUINE & N/A \\
\hline 4 & SPAIK & 0.34 & 53 & CHILE & -0.41 & GAHBIA & N/A \\
\hline 5 & GERIANY & 0.31 & 54 & MEXICO & -0.44 & GHakn & $N / A$ \\
\hline 6 & CZECHOSLOV & 0.29 & 55 & TRINIDAD & -0.44 & GUINEA & N/A \\
\hline 7 & MALTA & 0.28 & 56 & KENYA & -0.44 & GUINEA-BIS & $N / A$ \\
\hline 8 & ISRAEL & 0.25 & 57 & NEH ZEALAN & -0.46 & MALI & $\mathbf{N} / \mathbf{A}$ \\
\hline 9 & JARAICA & 0.23 & 58 & MEPAL & -0.46 & MAURITANIA & $N / A$ \\
\hline 10 & HONG KONG & 0.21 & 59 & MALAYSIA & -0.48 & MAURITIUS & $N / A$ \\
\hline 11 & PORTUGAL & 0.20 & 60 & EGYPT & -0.48 & mOZAMBIQUE & $\mathbf{N} / \mathbf{A}$ \\
\hline 12 & FRANCE & 0.19 & 61 & CER. AFR. & -0.48 & MIGER & $N / A$ \\
\hline 13 & SUITZERLAN & 0.19 & 62 & TANZANIA & -0.49 & REUNION & $N / A$ \\
\hline 14 & SHEDEN & 0.17 & 63 & PANATIA & -0.49 & SEYCHELLES & $\boldsymbol{N} / \boldsymbol{A}$ \\
\hline 15 & rugosLAVIA & 0.16 & 64 & NICARAGUA & -0.49 & SIERRA LEO & $M / A$ \\
\hline 16 & U.S.A. & 0.16 & 65 & SYRIA & -0.50 & VGAKDA & $N / A$ \\
\hline 17 & UK & 0.14 & 66 & COLOHBIA & -0.50 & ZAIRE & $N / A$ \\
\hline 18 & AUSTRIA & 0.14 & 67 & AUSTRALIA & -0.50 & ZAMBIA & $N / A$ \\
\hline 19 & BELGIUY & 0.14 & 68 & PARAGUAY & -0.50 & AFGHARISTA & $N / A$ \\
\hline 20 & FIMLAND & 0.14 & 69 & ARGENTINA & -0.54 & BRUNEI & $M / A$ \\
\hline 21 & INDIA & 0.11 & 70 & PERU & -0.56 & BURMA & N/A \\
\hline 22 & BANGLADESH & 0.11 & 71 & CONGO & -0.58 & CHIM & $N / A$ \\
\hline 23 & OHan & 0.05 & 72 & FIJI & -0.58 & LAO & N/A \\
\hline 24 & HUMGARY & 0.04 & 73 & SUDAN & -0.59 & MACAO & $N / A$ \\
\hline 25 & SINGAPORE & -0.01 & 74 & HONDORAS & -0.60 & MALOIVES & N/A \\
\hline 26 & DENMARK & -0.02 & 75 & ICELAND & -0.60 & NEH CALEDO & N/A \\
\hline 27 & BRAZIL & -0.02 & 76 & IVORY COAS & -0.63 & PAUPA N GU & N/A \\
\hline 28 & NETHERLAND & -0.04 & 77 & INDONESIA & -0.63 & VIETNAY & N/A \\
\hline 29 & PAKISTAN & -0.05 & 78 & ETHIOPIA & -0.64 & ROHANIA & N/A \\
\hline 30 & BERAIUDA & -0.06 & 79 & MADAGASCAR & -0.65 & IRAN & N/A \\
\hline 31 & CYPRUS & -0.08 & 80 & SOMALIA & -0.67 & IRAQ & N/A \\
\hline 32 & IRELAND & -0.10 & 81 & MALAHI & -0.68 & LEEANON & N/A \\
\hline 33 & BAREADOS & -0.11 & 82 & ALGERIA & -0.71 & YEHEN, A.D & $\mathbf{N} / \mathbf{A}$ \\
\hline 34 & GREECE & -0.13 & 83 & KUHAIT & -0.72 & ванаNAS & N/A \\
\hline 35 & YEMEN, A.R & -0.13 & 84 & QATAR & -0.73 & BELIZE & N/A \\
\hline 36 & TURKEY & -0.16 & 85 & BURUNDI & -0.74 & GREENLAND & $N / A$ \\
\hline 37 & URUGUAY & -0.18 & 86 & CAMEROON & -0.74 & GRENAOA & $N / A$ \\
\hline 38 & PHILIPPINE & -0.22 & 87 & GABON & -0.75 & GUADRLOUPE & N/A \\
\hline 39 & TUNISIA & -0.22 & 88 & MIGERIA & -0.77 & GUIANA, FR & N/A \\
\hline 40 & MOROCCO & -0.23 & 89 & U.A.E. & -0.77 & HAITI & N/A \\
\hline 41 & CANADA & -0.25 & 90 & VENEZUELA & -0.77 & MARTINIQUE & N/A \\
\hline 42 & EL SALVADO & -0.25 & 91 & LIBYA & -0.78 & NETHR. ANT & N/A \\
\hline 43 & JORDAN & -0.28 & 92 & SAUDI ARAB & -0.82 & SURINAME & N/A \\
\hline 44 & THAILAND & -0.29 & 93 & ECUADOR & -0.84 & BULGARIA & N/A \\
\hline 45 & BAHRAIN & -0.30 & 94 & BOLIVIA & -0.85 & CUBA & N/A \\
\hline 46 & DOHIHICAN & -0.30 & & ANGOLA & $N / A$ & EAST GERMA & N/A \\
\hline 47 & SEREGAL & -0.34 & & BENIN & $N / A$ & NORTH KORE & N/A \\
\hline 48 & SRI LANKA & -0.34 & & BURKINA FA & $N / A$ & POLAND & N/A \\
\hline 49 & SOUTH AFRI & -0.35 & & CAPE VERDI & $N / A$ & U.S.S.R. & N/A \\
\hline
\end{tabular}


the center end of the hierarchy are the expected industrialized countries of Western Europe, Japan and the United States. At the periphery end are the regions of Latin America and Africa. It should however be stated that like all other variables this distinction is not complete and there are countries that show up in the center end(and at the periphery end) which are expected to be in the other end. For example Jamaica with vertical trade indices of $-.56,-.26$, and .23 for the years 1962, 1970, and 1980 and Oman's dramatic jump from -.92 to .23 are highly questionable.

In all three years of the study, Japan stayed at the top of the vertical trade index while the United Kingdom, which ranked second in 1962 and 1970 dropped dramatically to number 17 in 1980 . This serves as yet another indication of the loss of the economic dominance which the United Kingdom once had. The United States has also witnessed such decline and ranks 16th in 1980.

Another significant development in the vertical trade structure is the ascendance of South Korea. While South Korea ranked 26th in 1962, by 1970 it ranked 6th, and in 1980 it surpassed all industrialized countries except Japan. The ascendance of South Korea cannot however be generalized to other members of the so called "Pacific Rim" countries of Hong Kong, Taiwan, Singapore, and Malaysia. They all witnessed declines in their position during the time period of this study.

\section{THE PHYSICAL QUALITY OF LIFE INDEX}

The Physical Quality of Life Index produces a hierarchy of the world based on the level of well-being of the people of each country. The PQLI is a composite index based on the levels of infant mortality, life expectancy, and basic literacy in the countries under study. The PQLI hypothesis is: 
HYPOTHESIS (5)- Center countries have better living conditions than periphery countries. More specifically, the EPC is negatively correlated with PQLI.

Correlation coefficients between EPC and other variables

\begin{tabular}{|l|c|c|c|c|c|c|c|}
\hline EPC VS. & IPC & ECC & ICC & VT & PQLI & AGR & ED \\
\hline 1962 & .85 & .34 & .42 & -.57 & -.48 & .42 & .57 \\
\hline 1970 & .73 & .33 & .38 & -.35 & -.36 & .32 & .40 \\
\hline 1980 & .68 & .41 & .43 & -.50 & -.45 & .25 & .55 \\
\hline
\end{tabular}

The empirical correlation results confirm the hypothesis that the center countries would enjoy better levels of living conditions. The correlation coefficients between EPC and PQLI are -.48 in $1962,-.36$ in 1970 and -.45 in 1980 . The bivariate correlations between PQLI and VT, TTL, and GNP also confirm the expectation that countries with high PQLIs are the center countries.

The PQLI shows a consistent improvement over time for the developing countries. The average PQLI for the world as a whole rose from 51.46 in 1962 to 63.48 in 1970 and to 66.29 in 1980 (Table XXIII). For all the countries which are studied in 1962 through 1980, only the Yemen Democratic Republic declined in PQLI from 18.2 to 16.5 (Tables XIII-A, XIII-B, and XIII-C). This drop, on closer examination, is attributable to the rise in infant mortality of Yemen from 177.4 in 1970 to 190 in 1980.

The experience of the developing countries with respect to PQLIs and level of well-being compared favorably with earlier levels in industrialized countries. By the late 1960s, life expectancy in periphery countries was 49 years, a level that was reached by the industrialized countries in $1900^{1}$. With respect to PQLI, the gap between industrialized countries and some developing countries has narrowed since 1962; however, this is in large due to the nature of this index which has natural

1- D. Morawetz, Twenty-five Years of Economic Development: 1950 to 1975 (Washington: The World Bank, 1977), p. 48. 
TABLE XIII-A

COUNTRIES RANKED EY P.Q.L.1. IN 1962

\begin{tabular}{|c|c|c|c|c|c|c|c|c|}
\hline & COUNTRY & P.Q.L.I & & COUNTRY & P.Q.L.I & & COUNTRY & Q.L.I \\
\hline 1 & ICELAND & 95.48 & 44 & VENEZUELA & 59.47 & 87 & CAMEROON & 15.69 \\
\hline 2 & SWEDEN & 95.18 & 45 & COSTA RICA & 56.29 & 88 & ETHIOPIA & 15.66 \\
\hline 3 & NORWAY & 95.09 & 46 & PHILIPPINE & 55.28 & 89 & SUDAN & 14.98 \\
\hline 4 & NETHERLAND & 95.07 & 47 & KUWAIT & 55.25 & 90 & NIGERIA & 12.64 \\
\hline 5 & DENHARK & 93.67 & 48 & MALAYSIA & 54.15 & 91 & HAITI & 12.64 \\
\hline 6 & NEW ZEALAH & 93.08 & 49 & THAILAND & 53.88 & 92 & SAUDI ARAB & 11.85 \\
\hline 7 & SHITZERLAN & 92.88 & 50 & COLOMBIA & 53.00 & 93 & IVORY COAS & 9.21 \\
\hline 8 & AUSTRALIA & 92.76 & 51 & BRAZIL & 51.39 & 94 & SENEGAL & 8.35 \\
\hline 9 & FRANCE & 91.15 & 52 & PANAMA & 51.20 & 95 & MAURITANIA & 7.71 \\
\hline 10 & U.S.A. & 91.02 & 53 & PARAGUAY & 50.82 & 96 & SOHALI & 6.12 \\
\hline 11 & U.K. & 90.82 & 54 & MEXICO & 49.02 & 97 & NIGER & 5.34 \\
\hline 12 & BELGIUH & 90.58 & 55 & DOMINICAN & 48.60 & 98 & MALI & 5.25 \\
\hline 13 & FINLAND & 90.50 & 56 & ECUADOR & 46.84 & 99 & CENT. AFR. & 4.76 \\
\hline 14 & CZECHOSLOV & 90.07 & 57 & SOUTH AFRI & 45.84 & 100 & CHAD & 4.50 \\
\hline 15 & IRELAND & 89.60 & 58 & EL SALVADO & 41.78 & 101 & BENIN & 4.40 \\
\hline 16 & GERYANY & 89.50 & 59 & GUATEMALA & 40.08 & 102 & SIERRA LEO & 0.82 \\
\hline 17 & U.S.S.R. & 88.49 & 60 & BAHREIN & 39.84 & 103 & AFGHANISTA & -1.93 \\
\hline 18 & JAPAN & 88.33 & 61 & CAPE VERDI & 37.28 & & CAMADA & N/A \\
\hline 19 & EAST GERMA & 88.31 & 62 & NICARAGUA & 36.85 & & CUBA & N/A \\
\hline 20 & AUSTRIA & 88.17 & 63 & PERU & 36.76 & & BAHAMAS & N/A \\
\hline 21 & HUNGARY & 85.27 & 64 & BURMA & 35.61 & & BERHUDA & N/A \\
\hline 22 & ITALY & 84.95 & 65 & HONDORAS & 35.04 & & GREENLAND & N/A \\
\hline 23 & ISRAEL & 84.22 & 66 & SYRIA & 34.53 & & GUADALOUPE & N/A \\
\hline 24 & BULGARIA & 83.93 & 67 & JORDAN & 33.00 & & MARTINIQUE & N/A \\
\hline 25 & POLAND & 82.84 & 68 & EGYPT & 30.83 & & NETHERLAND & N/A \\
\hline 26 & SPAIK & 82.15 & 69 & TURKEY & 29.34 & & YENEN, PEO & $N / \boldsymbol{A}$ \\
\hline 27 & URUGUAY & 81.85 & 70 & IRAQ & 29.31 & & LEBANON & N/A \\
\hline 28 & GREECE & 81.75 & 71 & GHAMA & 27.86 & & Onan & N/A \\
\hline 29 & CYPRUS & 81.71 & 72 & INDONESIA & 27.72 & & QATAR & N/A \\
\hline 30 & TRIMIDAD & 79.94 & 73 & BOLIVIA & 26.39 & & YEHEN, ARA & $M / A$ \\
\hline 31 & ARGENTIMA & 79.40 & 74 & CHINA & 26.17 & & ALGERIA & $N / A$ \\
\hline 32 & BARBADOS & 78.70 & 75 & LIBYA & 25.42 & & ANGOLA & N/A \\
\hline 33 & HONG KONG & 7.13 & 76 & IRAN & 25.25 & & GUIHEA & $N / A$ \\
\hline 34 & MALTA & 77.07 & 77 & UGANDA & 24.75 & & MADAGASCAR & N/A \\
\hline 35 & JAMAICA & 76.59 & 78 & TUNISIA & 24.42 & & MOZAMBIQUE & N/A \\
\hline 36 & RUMANIA & 76.10 & 79 & INDIA & 23.41 & & REUNION & $N / A$ \\
\hline 37 & SURINAR & 70.86 & 80 & KENYA & 23.18 & & BRUNEI & $N / A$ \\
\hline 38 & SINGAPORE & 70.27 & 81 & LAOS & 22.70 & & MACAO & $N / A$ \\
\hline 39 & YUGOSLAVIA & 67.81 & 82 & MOROCCO & 22.41 & & VIET NAM & N/A \\
\hline 40 & PORTUGAL & 64.86 & 83 & CONGO & 22.23 & & NEH CALEDO & $N / A$ \\
\hline 41 & MAURITIUS & 62.32 & 84 & PAKISTAN & 19.89 & & NEU GUINEA & $H / A$ \\
\hline 42 & CHILE & 61.46 & 85 & TANZANIA & 18.07 & & FIJI & N/A \\
\hline 43 & KOREA & 60.38 & 86 & GABON & 15.81 & & GAMBIA & N/A \\
\hline
\end{tabular}


TABLE XIII-B

COUNTRIES RANKED BY P.Q.L.I. IN 1970

\begin{tabular}{|c|c|c|c|c|c|c|c|c|}
\hline & & & & & & $=$ & & \\
\hline & COUNTRY & P.Q.L.1. & & COUNTRY & P.Q.L.I. & & COUNTRY & P.Q.L.I. \\
\hline 1 & SUEDEN & 97.06 & 50 & CHILE & 72.75 & 92 & ETHIOPIA & 19.83 \\
\hline 2 & MORHAY & 96.71 & 51 & PARAGUAY & 72.25 & 93 & YEHEN, PEO & 18.20 \\
\hline 3 & ICELAND & 96.38 & 52 & LEBANON & 70.49 & 94 & MALAUI & 16.27 \\
\hline 4 & NETHERLAND & 96.29 & 53 & KUWAIT & 69.34 & 95 & MAURITANIA & 15.47 \\
\hline 5 & DENMARK & 95.72 & 54 & PHILIPPINE & 68.82 & 96 & NEPAL & 15.46 \\
\hline 6 & JAPAN & 95.11 & 55 & MEXICO & 68.19 & 97 & SENEGAL & 15.09 \\
\hline 7 & SHITZERLAN & 94.73 & 56 & COLOMBIA & 67.23 & 98 & BENIN & 14.35 \\
\hline 8 & AUSTRALIA & 94.13 & 57 & THAILAND & 66.60 & 99 & MALI & 12.89 \\
\hline 9 & FRANCE & 94.08 & 58 & MALAYSIA & 65.24 & 100 & CHAD & 11.93 \\
\hline 10 & NEN ZEALAN & 94.06 & 59 & BRAZIL & 59.69 & 101 & SOHALIA & 11.66 \\
\hline 11 & CANADA & 93.75 & 60 & DOMINICAN & 59.36 & 102 & SIERRA LEO & 8.38 \\
\hline 12 & BELGIUH & 93.47 & 61 & BAHREIN & 57.23 & 103 & AFGHANISTA & 4.54 \\
\hline 13 & U.K. & 93.13 & 62 & ECUADOR & 56.41 & & SOUTH AFRI & N/A \\
\hline 14 & FIHLAND & 93.05 & 63 & EL SALVADO & 55.23 & & BAHAHAS & $N / A$ \\
\hline 15 & EAST GER & 93.01 & 64 & PERU & 53.89 & & BERTUDA & $\mathrm{N} / \mathrm{A}$ \\
\hline 16 & U.S.A. & 92.79 & 65 & SYRIA & 49.98 & & GREENLAND & N/A \\
\hline 17 & IRELAND & 92.62 & 66 & GUATEMALA & 49.24 & & GUADALOUPE & $N / A$ \\
\hline 18 & CZECHOSLOV & 91.96 & 67 & HONDORAS & 48.30 & & GUIHER FR. & $N / A$ \\
\hline 19 & GERHANY & 91.90 & 68 & NICARAGUA & 47.97 & & MARTINIQUE & $N / A$ \\
\hline 20 & AUSTRIA & 91.55 & 69 & TURKEY & 45.35 & & METHERLAND & $N / A$ \\
\hline 21 & U.S.S.R. & 91.33 & 70 & U.A.E. & 44.60 & & JORDAN & $N / A$ \\
\hline 22 & ITALY & 90.14 & 71 & INDONESIA & 43.19 & & EGYPT & $N / A$ \\
\hline 23 & BULGARIA & 90.11 & 72 & LIBYA & 43.00 & & BRUKEI & $N / \boldsymbol{A}$ \\
\hline 24 & POLAND & 89.62 & 73 & QATAR & 42.07 & & BURMA & $\mathbf{N} / \mathbf{A}$ \\
\hline 25 & SPAIH & 89.38 & 74 & CONGO & 40.86 & & TAIHAN & $N / A$ \\
\hline 26 & ISRAEL & 88.89 & 75 & IRAN & 40.46 & & LAOS & $N / A$ \\
\hline 27 & MaLTa & 88.83 & 76 & IRAQ & 39.13 & & MACAO & N/A \\
\hline 28 & HUREARY & 88.76 & 77 & ZANBIA & 37.13 & & VIET NAH & $N / A$ \\
\hline 29 & JAMAICA & 88.01 & 78 & KENYA & 36.87 & & ANGOLA & $N / A$ \\
\hline 30 & BARBADOS & 87.59 & 79 & GHANA & 36.51 & & BURUNDI & N/A \\
\hline 31 & GREECE & 86.81 & 80 & ALGERIA & 34.24 & & CENT. AF. & N/A \\
\hline 32 & CYPRUS & 86.70 & 81 & INDIA & 33.64 & & GABON & $N / A$ \\
\hline 33 & Hons Kong & 85.40 & 82 & MOROCCO & 32.87 & & GAMBIA & N/A \\
\hline 34 & TRINIDAD & 85.11 & 83 & BOLIVIA & 31.39 & & GUINEA REP & N/A \\
\hline 35 & URUGUAY & 84.21 & 84 & HAITI & 29.51 & & MOZAMBIQUE & $N / A$ \\
\hline 36 & ROHNHIA & 83.68 & 85 & PAKISTAN & 26.94 & & NIGER & $N / A$ \\
\hline 37 & ARGENTINA & 82.95 & 86 & SAUDI ARAB & 26.63 & & NIGERIA & $N / A$ \\
\hline 38 & GUYAMA & 81.03 & 87 & ZAIRE & 24.07 & & REUNION & $N / A$ \\
\hline 39 & SINGAPORE & 80.68 & 88 & OMAN & 22.17 & & SEYCHELLES & $N / A$ \\
\hline 40 & YugosLavia & 78.65 & 89 & CAMEROON & 22.03 & & TANZANIA & $N / A$ \\
\hline 41 & COSTA RICA & 78.23 & 90 & IVORY COAS & 21.84 & & TUNISIA & $N / A$ \\
\hline 42 & FIJI & 78.04 & 91 & SUDAN & 20.49 & & UGANDA & $N / A$ \\
\hline 43 & Panara & 77.40 & 92 & ETHIOPIA & 19.83 & & NEW CALEDO & N/A \\
\hline 44 & SURIMAN & 76.83 & 93 & YEMEN, PEO & 18.20 & & PAPAU N. G & $N / A$ \\
\hline 45 & KOREA & 75.37 & 94 & MALAWI & 16.27 & & CHIMA & N/A \\
\hline 46 & PORTUGAL & 74.45 & 95 & MAURITANIA & 15.47 & & CUBA & N/A \\
\hline 47 & SRI LAKKA & 73.58 & 96 & NEPAL & 15.46 & & NORTH KORE & N/A \\
\hline 48 & MURITIUS & 72.88 & 97 & SENEGAL & 15.09 & & NORTH VIET & N/A \\
\hline 49 & VENEZUELA & 72.84 & 98 & BENIN & 14.35 & & YEHEN, ARA & N/A \\
\hline
\end{tabular}


TABLE XIII-C

COUNTRIES RANKED BY P.Q.L.I. IN 1980

\begin{tabular}{|c|c|c|c|c|c|c|c|c|}
\hline & & P.Q.L.I & & COUNTRY & P.Q.L.I & & COUNTRY & Han \\
\hline 1 & ICELAND & 99.86 & 50 & MEXICO & 77.03 & 99 & IVORY COAS & 34.25 \\
\hline 2 & SUEDEN & 99.59 & 51 & KUWAIT & 77.00 & 100 & PAKISTAN & 33.77 \\
\hline 3 & JAPAN & 99.40 & 52 & SURIMAME & 76.65 & 101 & LAO & 3.65 \\
\hline 4 & NORHAY & 98.86 & 53 & THAILAND & 76.09 & 102 & SUDAN & 33.51 \\
\hline 5 & METHERLAND & 98.62 & 54 & LEBANON & 75.80 & 103 & SOIAALIA & 33.23 \\
\hline 6 & SUITZERLAN & 98.45 & 55 & COLOMBIA & 74.43 & 104 & OHAN & 32.58 \\
\hline 7 & FRANCE & 97.91 & 56 & PHILIPPINE & 72.61 & 105 & YEHEN, A.D & 31.99 \\
\hline 8 & DENHARK & 97.90 & 57 & MALAYSIA & 72.46 & 106 & BAHGLADESH & 30.78 \\
\hline 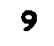 & FINLAND & 97.82 & 58 & CHINA & 70.06 & 107 & BENIK & 30.33 \\
\hline 0 & CANADA & 97.33 & 59 & BRAZIL & 69.76 & 108 & GABON & 29.97 \\
\hline & AUSTRALIK & 97.07 & 60 & ECUADOR & 69.16 & 109 & BURUNDI & 29.56 \\
\hline & U.S.A. & 97.00 & 61 & SYRIA & 67.71 & 110 & CEN. AFR. & 26.77 \\
\hline & NEU ZEALAN & 96.30 & 62 & JORDAN & 67.44 & 111 & ETHIOPIA & 24.30 \\
\hline & UK & 96.03 & 63 & U.A.E. & 66.98 & 112 & MAURITANIA & 23.84 \\
\hline 5 & BELGIUH & 95.91 & 64 & NICARAGUA & 66.71 & 113 & NEPAL & 23.06 \\
\hline 16 & GERHAWY & 95.70 & 65 & DOMINICAN & 66.68 & 114 & MALAHI & 22.54 \\
\hline 17 & EAST GERMA & 95.61 & 66 & BAHRAIN & 66.15 & 115 & NIGER & 21.44 \\
\hline 8 & IRELAND & 95.58 & 67 & EL SALYADO & 65.59 & 116 & CHAD & 21.44 \\
\hline 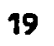 & AUSTRIA & 95.51 & 68 & PERU & 64.74 & 117 & SENEGAL & 19.05 \\
\hline 20 & ITALY & 95.45 & 69 & TUNISIA & 60.70 & 118 & GUINEA & 18.80 \\
\hline 1 & SPAIN & 94.44 & 70 & SOUTH AFRI & 59.63 & 119 & MALI & 18.29 \\
\hline 22 & HOHS KONG & 94.03 & 71 & HONDORAS & 58.55 & 120 & YEMEN, A.R & 16.50 \\
\hline 23 & CZECHOSLOV & 93.81 & 72 & GUATEMALA & 58.23 & 121 & SIERRA LEO & 13.28 \\
\hline & Poland & 93.80 & 73 & TANZANIA & 56.64 & 122 & AFGHANISTA & 9.74 \\
\hline 25 & BULGARIA & 92.84 & 74 & TURKEY & 56.46 & 123 & GAMBIA & 9.21 \\
\hline 6 & U.S.S.R. & 92.72 & 75 & CAPE YERDI & 55.52 & & ANGOLA & $N / A$ \\
\hline 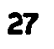 & BARBADOS & 91.75 & 76 & BURMA & 54.82 & & BURKINA FA & N/A \\
\hline 28 & ISRAEL & 91.72 & 77 & INDONESIA & 52.36 & & DJIBOUTI & H/A \\
\hline & HURGARY & 91.71 & 78 & IRAN & 52.12 & & EQU. GUINE & N/A \\
\hline 80 & ROHARIA & & 79 & KENYA & & & GUINEA-BIS & //A \\
\hline 31 & MALTA & 91.15 & 80 & LIBYA & 51.99 & & MOZAMBIQUE & N/A \\
\hline 32 & CYPRUS & 91.11 & 81 & QATAR & 51.79 & & REUNION & $/ / A$ \\
\hline 33 & GREECE & 90.57 & 82 & CONGO & 51.64 & & SEYCHELLES & N/A \\
\hline 34 & JAraich & 90.36 & 83 & EGYPT & 48.59 & & BRUNEI & $N / A$ \\
\hline 35 & TRIMIDAD & 90.10 & 84 & IRAQ & 46.43 & & MACAO & $/ / A$ \\
\hline 36 & COSTA RICA & 89.76 & 85 & BOLIYIA & 46.23 & & MALDIVES & H/A \\
\hline 37 & SIHGAPORE & 89.21 & 86 & ZAIRE & 45.55 & & NEU CALEDO & N/A \\
\hline 88 & URUGUAY & 88.13 & 87 & UGANDA & 45.35 & & VIETNAAY & N/A \\
\hline 9 & PANAMA & 87.30 & 88 & MOROCCO & 44.38 & & BAHARAS & $N / A$ \\
\hline 0 & ARGENTINA & 86.54 & 89 & ALGERIA & 44.02 & & BELIZE & N/A \\
\hline 1 & ruGosLAVIA & 85.75 & 90 & ZAMBIA & 43.73 & & BERMUUDA & N/A \\
\hline 2 & PORTUGAL & 85.39 & 91 & MADAGASCAR & 43.01 & & GREENLAND & N/A \\
\hline 33 & FIJI & 84.36 & 92 & GHANA & 42.88 & & GRENADA & N/A \\
\hline 44 & KOREA & 83.95 & 93 & CAMEROON & 42.13 & & GUADALOUPE & K/A \\
\hline 5 & CHILE & 83.02 & 94 & PAUPA N GU & 40.30 & & GUIANA, FR & N/A \\
\hline 6 & SRI LNKA & 82.23 & 95 & INDIA & 39.79 & & MARTINIOUE & N/A \\
\hline 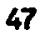 & VENEZUELA & 80.84 & 96 & SAUDI ARAB & 39.42 & & NETHR. ANT & N/A \\
\hline 0 & MAURITIUS & 79.68 & 97 & HAITI & 37.99 & & CUBA & N/A \\
\hline & PARAGUAY & 78.34 & 98 & NIGERIA & 34.61 & & HORTH KORE & $N / A$ \\
\hline
\end{tabular}


upper limits of 100 percent literacy, and a life expectancy of 77 years, which are already closely approximated by the industrialized countries.

In order to investigate the gap in PQLI among countries, average PQLI index for the year 1962 for the ten countries on the top of EPC and the ten countries on the bottom of the EPC rankings are calculated and once compared ( Tables XIV-A and XIV-B), the gap between these two groups are found to be decreasing. The gap in quality of life which in 1962 had a ratio of 1:2.5 between these two groups drops to $1: 1.81$ in 1980 .

\section{ECONOMIC DEPENDENCE ON AGRICULTURE}

The extent to which a country's economy is oriented to agriculture is another measure associated with peripheralness. The hypothesis concerning agriculture was:

HYPOTHESIS (H6)- Centralness is associated with a lower share of agriculture in a nation's GDP whereas peripheralness is associated with a higher share of agriculture in GDP. More specifically, EPC is positively correlated with AGR.

Correlation coeffictents between EPC and other vartables

\begin{tabular}{|l|c|c|c|c|c|c|c|}
\hline EPC VS. & IPC & ECC & ICC & VT & POII & AGR & ED \\
\hline 1962 & .85 & .34 & .42 & -.57 & -.48 & .42 & .57 \\
\hline 1970 & .73 & .33 & .38 & -.35 & -.36 & .32 & .40 \\
\hline 1980 & .68 & .41 & .43 & -.50 & -.45 & .25 & .55 \\
\hline
\end{tabular}

The empirical results of the correlation analysis support this hypothesis. The correlation coefficients between EPC and AGR is 0.42 for $1962,0.32$ for 1970 , and 0.26 for 1980. Also the correlation coefficient between AGR and GNP per capita in 1980 was -0.66 , a rather high coefficient, suggesting that the developed countries with high GNPs per capita are less dependent on agriculture than are countries of the periphery. 
TABLE XIV-A

AVERAge PQLI IN TOP TEN EPC RANKINGS IN 1962

\begin{tabular}{lrrr} 
& 1962 & 1970 & 1980 \\
\hline SWEDEN & 95.18 & 97.06 & 99.59 \\
INDIA & 23.41 & 33.64 & 39.79 \\
YUGOSLAVIA & 67.81 & 78.65 & 85.75 \\
JAPAN & 88.33 & 95.11 & 99.40 \\
ITALY & 84.95 & 90.14 & 95.45 \\
SHITZERLAND & 92.88 & 94.73 & 98.45 \\
FRANCE & 91.15 & 94.08 & 97.91 \\
GERMANY & 89.50 & 91.90 & 97.50 \\
U.S.A. & 91.02 & 92.79 & 97.00 \\
U.K. & 90.82 & 93.13 & 96.03 \\
\hline AVERAGE & $\mathbf{8 1 . 5 0}$ & 86.12 & 90.51
\end{tabular}

TABLE XIV-B

AVERAGE PQLI IN BOTTON TEN EPC RANKINGS IN 1962

\begin{tabular}{lrrr} 
& 1962 & 1970 & 1980 \\
\hline MAURITANIA & 7.71 & 15.47 & 23.84 \\
BENIN & 4.40 & 14.35 & 30.33 \\
SENEGAL & 8.35 & 15.09 & 19.05 \\
MAURITIUS & 62.32 & 72.88 & 79.68 \\
SOMALI & 6.12 & 11.66 & 33.23 \\
MALI & 5.25 & 12.89 & 18.29 \\
BARBADOS & 78.70 & 87.59 & 91.75 \\
IRELAND & 89.60 & 92.60 & 95.58 \\
DOMINICAN & 48.60 & 59.36 & 66.68 \\
CAMEROON & 15.69 & 22.03 & 42.13 \\
\hline AVERAGE & 32.67 & 40.40 & 50.06
\end{tabular}


TABLE XV-A

COUNTRIES RANKED BY AGR IN GDP IN 1962

\begin{tabular}{|c|c|c|c|c|c|c|c|}
\hline & COUNTRY & ARR IN G & & COUNTRY & AGR IN GDP & COUNTRY & AGR IN GDP \\
\hline 1 & SOMALI & 71.10 & 44 & MOROCCO & 23.30 & U.S.S.R. & N/A \\
\hline 2 & NIGER & 69.00 & 45 & CONGO & 23.20 & BULGARIA & N/A \\
\hline 3 & ETHIOPIA & 64.50 & 46 & PERU & 21.70 & CZECHOSLOV & $N / A$ \\
\hline 4 & NIGERIA & 64.30 & 47 & CYPRUS & 20.90 & EAST GERMA & $N / A$ \\
\hline 5 & MOZAMBIQUE & 55.70 & 48 & HUNGARY & 20.40 & POLAND & N/A \\
\hline 6 & MALI & 54.80 & 49 & PANAMA & 19.70 & RUMANIA & N/A \\
\hline 7 & BENIN & 54.80 & 50 & MEXICO & 18.90 & CUBA & N/A \\
\hline 8 & ANGOLA & 53.20 & 51 & URUGUAY & 18.00 & GUATEMALA & N/A \\
\hline 9 & UGANDA & 52.40 & 52 & MAURITIUS & 18.00 & HAITI & N/A \\
\hline 10 & CHAD & 52.00 & 53 & BRAZIL & 17.70 & BAHAMAS & N/A \\
\hline 11 & CENT. AFR. & 50.50 & 54 & ARGENTINA & 17.60 & BERYUDA & N/A \\
\hline 12 & IMDONESIA & 50.00 & 55 & IRAQ & 17.10 & GREENLAND & N/A \\
\hline 13 & INDIA & 48.90 & 56 & FINLAND & 16.70 & GUADALOUPE & N/A \\
\hline 14 & PAKISTAN & 46.20 & 57 & SURINAM & 16.10 & MARTINIQUE & N/A \\
\hline 15 & MAURITANIA & 44.40 & 58 & ALGERIA & 16.00 & NETHERLAND & N/A \\
\hline 16 & IVORY COAS & 43.40 & 59 & LIBYA & 13.70 & YEMEN, PEO & N/A \\
\hline 17 & HONDORAS & 41.90 & 60 & JAPAN & 12.60 & BAHREIK & N/A \\
\hline 18 & TURKEY & 41.40 & 61 & JAMAICA & 12.50 & JORDAN & N/A \\
\hline 19 & GHANA & 40.80 & 62 & SOUTH AFRI & 12.30 & KUWAIT & N/A \\
\hline 20 & THAILAND & 39.80 & 63 & ITALY & 12.20 & LEBANON & N/A \\
\hline 21 & GAMBIA & 39.20 & 64 & AUSTRALIA & 12.00 & OHAN & $N / A$ \\
\hline 22 & KOREA & 39.20 & 65 & DENMARK & 11.20 & QATAR & $N / A$ \\
\hline 23 & KENYA & 38.80 & 66 & ISRAEL & 11.10 & SAUDI ARAB & N/A \\
\hline 24 & PARAGUAY & 38.20 & 67 & AUSTRIA & 11.00 & SYRIA & N/A \\
\hline 25 & MADAGASCAR & 37.30 & 68 & FRANCE & 10.60 & YEMEN, ARA & $N / A$ \\
\hline 26 & EL SALVADO & 36.10 & 69 & CHILE & 9.30 & NEH ZEALAN & N/A \\
\hline 27 & HALAYSIA & 36.00 & 70 & NORWAY & 9.00 & CAHEROON & $N / A$ \\
\hline 28 & COLOMBIA & 35.60 & 71 & TRINIDAD & 8.90 & CAPE VERDI & $N / A$ \\
\hline 29 & Buran & 35.00 & 72 & BELGIUN & 6.50 & GUINEA & $N / \lambda$ \\
\hline 30 & GABON & 32.50 & 73 & MALTA & 6.40 & REUNION & $N / A$ \\
\hline 31 & EGYPT & 29.90 & 74 & VENEZUELA & 6.00 & SIERRA LEO & $N / A$ \\
\hline 32 & IRAN & 29.10 & 75 & CANADA & 5.70 & SUDAN & $N / \mathbf{A}$ \\
\hline 33 & PORTUGAL & 28.40 & 76 & GERMANY & 5.70 & TUNISIA & N/A \\
\hline 34 & ECUADOR & 27.50 & 77 & U.S.A. & 4.00 & AFGHANISTA & N/A \\
\hline 35 & GREECE & 26.80 & 78 & SINGAPORE & 3.50 & BRUNEI & N/A \\
\hline 36 & DOHINICAN & 26.70 & 79 & U.K. & 3.40 & CHIMA & N/A \\
\hline 37 & YugosLavia & 26.00 & 80 & HONG KONG & 3.40 & LAOS & N/A \\
\hline 38 & COSTA RICA & 26.00 & & NETHERLAND & $N / A$ & MACAO & N/A \\
\hline 39 & BOLIVIA & 25.70 & & ICELAND & N/A & VIET WAH & N/A \\
\hline 40 & BARBADOS & 25.70 & & IRELAND & $N / A$ & NEW CALEDO & N/A \\
\hline 41 & PHILIPPINE & 25.70 & & SPAIN & N/A & NEW GUINEA & N/A \\
\hline 42 & SEMEGAL & 24.30 & & SWEDEN & $N / A$ & FIJI & $N / A$ \\
\hline 43 & NICARAGUA & 23.70 & & SHITZERLAN & N/A & TANZANIA & N/A \\
\hline
\end{tabular}


TABLE XV-B

COUNTRIES RANKED BY AGR IN GDP IN 1970

\begin{tabular}{|c|c|c|c|c|c|c|c|c|}
\hline & COUNTRY & GR IN $\mathrm{G}$ & & COUNTRY & AGR IN GD & & COUNTRY & AGR IN GDP \\
\hline 1 & BURUNDI & 68.20 & 50 & IRAN & 23.80 & 92 & TRINIDAD & 5.50 \\
\hline 2 & NEPAL & 67.80 & 51 & ZAIRE & 23.40 & 93 & SHEDEN & 5.30 \\
\hline 3 & SOMALIA & 66.80 & 52 & GUYANA & 22.80 & 94 & BELGIUH & 5.00 \\
\hline 4 & NIGER & 65.10 & 53 & GREECE & 21.90 & 95 & CANADA & 4.80 \\
\hline 5 & ETHIOPIA & 58.50 & 54 & MOROCCO & 21.80 & 96 & GERHANY & 4.40 \\
\hline 6 & NIGERIA & 56.70 & 55 & YugosLaVIA & 21.40 & 97 & LIBYA & 3.50 \\
\hline 7 & MALAHI & 54.00 & 56 & PORTUGAL & 20.60 & 98 & U.S.A. & 3.10 \\
\hline 8 & YENEN, PEO & 52.20 & 57 & BOLIVIA & 20.50 & 99 & SINGAPORE & 2.90 \\
\hline 9 & UGANDA & 51.80 & 58 & TUNISIA & 20.50 & 100 & U.K. & 2.80 \\
\hline 10 & BENIN & 51.40 & 59 & HUNGARY & 20.00 & 101 & HONG KONG & 2.60 \\
\hline 11 & INDONESIA & 49.50 & 60 & CYPRUS & 19.60 & 102 & KUUAIT & 0.50 \\
\hline 12 & CHAD & 49.20 & 61 & CONGO & 19.50 & & SUITZERLAN & $N / A$ \\
\hline 13 & MALI & 48.60 & 62 & BAREADOS & 18.30 & & ICELAND & $N / A$ \\
\hline 14 & mozambioue & 48.50 & 63 & IRAQ & 18.00 & & NEH ZEALAN & N/A \\
\hline 15 & ANGOLA & 48.40 & 64 & MAURITIUS & 16.80 & & GUATEmaLA & $\mathbf{H} / \mathbf{A}$ \\
\hline 16 & INDIA & 48.20 & 65 & PANAMA & 16.70 & & HAITI & $\mathbf{N} / \mathbf{A}$ \\
\hline 17 & TANZANIA & 43.60 & 66 & PERU & 15.60 & & BaHamas & H/A \\
\hline 18 & GHaNa & 43.40 & 67 & IRELAND & 14.40 & & BERHUDA & $\mathbf{N} / \mathbf{A}$ \\
\hline 19 & PAPAU N. G & 43.10 & 68 & ARGENTINA & 13.60 & & GREENLAND & $\mathbf{N} / \mathbf{A}$ \\
\hline 20 & SUDAN & 41.10 & 69 & MEXICO & 13.60 & & GUADALOUPE & $\mathbf{N} / \mathbf{A}$ \\
\hline 21 & CENT. AF. & 40.70 & 70 & FINLAND & 13.50 & & GUINEA FR. & $N / A$ \\
\hline 22 & PAKISTAN & 39.90 & 71 & SPAIN & 13.10 & & MARTIMIQUE & $N / A$ \\
\hline 23 & GAMBIA & 39.30 & 72 & URUGUAY & 13.00 & & NETHERLAND & $N / A$ \\
\hline 24 & HONDORAS & 37.50 & 73 & SURINAM & 13.00 & & BAHREIN & $N / A$ \\
\hline 25 & KENYA & 36.70 & 74 & ALGERIA & 13.00 & & JORDAN & $N / A$ \\
\hline 26 & BURMA & 36.50 & 75 & BRAZIL & 11.90 & & LEBANON & N/A \\
\hline 27 & PARAGUAY & 34.90 & 76 & ZAMBIA & 11.30 & & QATAR & N/A \\
\hline 28 & TURKEY & 34.00 & 77 & SOUTH AFRI & 10.50 & & U.A.E. & N/A \\
\hline 29 & SIERRA LEO & 34.00 & 78 & ITALY & 10.30 & & YEHEN, ARA & $N / \boldsymbol{R}$ \\
\hline 30 & MAURITANIA & 33.70 & 79 & AUSTRALIA & 9.40 & & AFGHANISTA & $N / A$ \\
\hline 31 & THAILAND & 33.60 & 80 & JAMAICA & 9.20 & & BRUNEI & $N / R$ \\
\hline 32 & IVORY COAS & 33.50 & 81 & JAPAN & 8.50 & & TAIUAN & $N / A$ \\
\hline 33 & maLAYSIA & 32.10 & 82 & ISRAEL & 8.50 & & LAOS & $\mathbf{N} / \mathbf{A}$ \\
\hline 34 & KOREA & 32.00 & 83 & AUSTRIA & 8.40 & & MACAO & $N / A$ \\
\hline 35 & FIJI & 31.80 & 84 & DENHARK & 7.90 & & VIET NAM & N/A \\
\hline 36 & Onaw & 31.20 & 85 & FRANCE & 7.90 & & GUINEA REP & $N / A$ \\
\hline 37 & CAHIEROON & 30.90 & 86 & CHILE & 7.60 & & REUNION & $\mathbf{M} / \mathbf{A}$ \\
\hline 38 & COLOHBIA & 30.50 & 87 & MALTA & 7.20 & & SEYCHELLES & $\mathbf{M} / \mathbf{A}$ \\
\hline 39 & SRI LANKA & 30.00 & 88 & SAUDI ARAB & 7.00 & & NEU CALEDO & $\mathbf{N} / \mathbf{A}$ \\
\hline 40 & EL SALYAdO & 28.90 & 89 & MORWAY & 6.90 & & BULGARIA & $N / A$ \\
\hline 41 & EGYPT & 28.50 & 90 & VENEZUELA & 6.50 & & CHIMA & $N / A$ \\
\hline 42 & PHILIPPINE & 26.70 & 91 & NETHERLAND & 5.80 & & Cuen & $N / A$ \\
\hline 43 & ECUADOR & 25.20 & 92 & TRINIDAD & 5.50 & & CZECHOSLOY & $N / A$ \\
\hline 44 & GABON & 25.20 & 93 & SWEDEN & 5.30 & & EAST GER & $N / A$ \\
\hline 45 & SYRIA & 25.10 & 94 & BELGIUH & 5.00 & & NORTH KORE & $N / A$ \\
\hline 46 & DOHIMICAN & 24.80 & 95 & CAMADA & 4.80 & & HORTH VIET & $N / R$ \\
\hline 47 & SENEGAL & 24.70 & 96 & GERHANY & 4.40 & & POLAND & $\mathbf{N} / \mathbf{A}$ \\
\hline 48 & NICARAGUA & 24.30 & 97 & LIBYA & 3.50 & & ROMANIA & $N / A$ \\
\hline 49 & COSTA RICA & 23.80 & 98 & U.S.A. & 3.10 & & U.S.S.R. & $\mathbf{N} / \mathbf{A}$ \\
\hline
\end{tabular}


TABLE XV-C

COUNTRIES RANKED BY AGR IN GDP IN 1980

\begin{tabular}{|c|c|c|c|c|c|c|c|c|}
\hline & COUNTRY & AGR IN G & & COUNTRY & AGR IN GD & & COUNTRY & AGR IN GDP \\
\hline 1 & UGANDA & 73.70 & 50 & NICARAGUA & 23.70 & 99 & SHEDEN & 3.70 \\
\hline 2 & NEPAL & 63.70 & 51 & CAPE VERDI & 21.60 & 100 & U.S.A. & 3.00 \\
\hline 3 & BURUNDI & 59.60 & 52 & DOMINICAN & 20.50 & 101 & OHAN & 3.00 \\
\hline 4 & SOMALIA & 59.20 & 53 & KOREA & 20.30 & 102 & TRINIDAD & 3.00 \\
\hline 5 & GHANA & 55.20 & 54 & COSTA RICA & 20.30 & 103 & BELGIUH & 2.80 \\
\hline 6 & GUINEA-BIS & 54.60 & 55 & SYRIA & 19.30 & 104 & GERHANY & 2.80 \\
\hline 7 & BNHGLADESH & 54.30 & 56 & TUNISIA & 18.30 & 105 & UK & 2.20 \\
\hline 8 & ETHIOPIA & 51.40 & 57 & MAURITIUS & 18.20 & 106 & LIBYA & 1.90 \\
\hline 9 & CHAD & 51.20 & 58 & MOROCCO & 18.20 & 107 & SINGAPORE & 1.70 \\
\hline 10 & TANzania & 47.60 & 59 & HUNGARY & 18.10 & 108 & HOHG KOHG & 1.30 \\
\hline 11 & GUINEA & 46.40 & 60 & GREECE & 17.60 & 109 & BAHRAIK & 1.30 \\
\hline 12 & BELIZE & 45.40 & 61 & BOLIVIA & 17.60 & 110 & SAUDI ARAB & 1.30 \\
\hline 13 & MALAWI & 45.30 & 62 & YEMEN, A.D & 15.80 & 111 & U.A.E. & 0.80 \\
\hline 14 & BURMA & 44.90 & 63 & ECUADOR & 15.10 & 112 & KUWATT & 0.30 \\
\hline 15 & BENIN & 44.80 & 64 & IRELAND & 15.00 & & ICELAND & $\mathrm{H} / \mathrm{A}$ \\
\hline 16 & ANGOLA & 43.30 & 65 & ZAMBIA & 14.20 & & SUITZERLAN & $N / A$ \\
\hline 17 & HOZAMBIQUE & 43.10 & 66 & CYPRUS & 13.60 & & BURKINA FA & $N / A$ \\
\hline 18 & MALI & 41.60 & 67 & PORTUGAL & 13.60 & & DJIBOUTI & $N / A$ \\
\hline 19 & INDIA & 40.60 & 68 & CONGO & 13.40 & & EQU. GUINE & N/A \\
\hline 20 & NIGER & 39.30 & 69 & YUGOSLAVIA & 13.20 & & REUNION & $N / A$ \\
\hline 21 & SUDAN & 38.80 & 70 & BRAZIL & 13.10 & & SEYCHELLES & $N / A$ \\
\hline 22 & MADAGASCAR & 36.40 & 71 & PANAMA & 11.80 & & AFGHANISTA & N/A \\
\hline 23 & CEN. AFR. & 36.20 & 72 & NEW ZEALAN & 11.70 & & BRUNEI & N/A \\
\hline 24 & KENYA & 35.10 & 73 & SURINAME & 10.60 & & LAO & N/A \\
\hline 25 & GAMBIA & 33.90 & 74 & URUGUAY & 10.20 & & MACAO & H/A \\
\hline 26 & YEMEN, A.R & 33.80 & 75 & PERU & 9.90 & & HALDIVES & N/A \\
\hline 27 & SIERRA LEO & 33.60 & 76 & ARGENTINA & 9.70 & & NEH CALEdo & N/A \\
\hline 28 & PAUPA N GU & 33.60 & 77 & BARBADOS & 9.70 & & VIETNAM & N/A \\
\hline 29 & CHINA & 32.50 & 78 & MEXICO & 9.50 & & ROHANIA & N/A \\
\hline 30 & PAKISTAN & 32.20 & 79 & JORDAN & 9.20 & & IRAH & $N / A$ \\
\hline 31 & PARAgUAY & 31.70 & 80 & FINLAND & 8.80 & & IRAQ & $N / A$ \\
\hline 32 & GRENADA & 31.30 & 81 & SPAIN & 8.20 & & LEBANON & N/A \\
\hline 33 & CAMEROON & 30.50 & 82 & JAMAICA & 8.20 & & QATAR & N/A \\
\hline 34 & HONDORAS & 30.30 & 83 & SOUTH AFRI & 7.70 & & BaHakas & N/A \\
\hline 35 & MAURITANIA & 28.80 & 84 & CHILE & 7.60 & & BERRUDA & N/A \\
\hline 36 & ZAIRE & 28.70 & 85 & ALGERIA & 7.00 & & GREENLAND & $N / A$ \\
\hline 37 & INDCHESIA & 28.70 & 86 & GABON & 7.00 & & GUADALOUPE & $N / A$ \\
\hline 38 & SRI LARKA & 28.60 & 87 & ITALY & 6.80 & & GUATERALA & $N / A$ \\
\hline 39 & COLOHBIA & 28.60 & 88 & AUSTRALIA & 6.30 & & GUIANA, FR & H/A \\
\hline 40 & THAILAND & 27.70 & 89 & VENEZUELA & 5.90 & & HAITI & $N / A$ \\
\hline 41 & EL SALVADO & 27.20 & 90 & NORWAY & 5.30 & & MARTINIQUE & $\mathrm{N} / \boldsymbol{A}$ \\
\hline 42 & MALAYSIA & 26.40 & 91 & ISRAEL & 5.30 & & NETHR. ANT & $N / A$ \\
\hline 43 & IVORY COAS & 26.10 & 92 & DENMARK & 5.10 & & BULGARIA & N/A \\
\hline 44 & PHILIPPINE & 25.90 & 93 & MALTA & 5.10 & & CUBA & N/A \\
\hline 45 & NIGERIA & 25.40 & 94 & JAPAN & 5.00 & & CZECHOSLOV & N/A \\
\hline 46 & SENEGAL & 24.60 & 95 & AUSTRIA & 4.90 & & EAST GERHA & N/A \\
\hline 47 & EGYPT & 24.50 & 96 & FRANCE & 4.90 & & NORTH KORE & $N / A$ \\
\hline 48 & TURKEY & 23.80 & 97 & NETHERLAND & 4.30 & & POLAND & $N / A$ \\
\hline 49 & FIJI & 23.70 & 98 & CANADA & 4.00 & & U.S.S.R. & N/A \\
\hline
\end{tabular}




\section{GNP PER CAPITA}

Perhaps the most common indicator of economic growth and development is the GNP per capita. Higher per capita GNPs are often associated with economies that are industrially more advanced, and excluding the oil producing countries, this indicator has historically has been high for only the more industrialized countries. Therefore, higher GNPs per capita are associated with center countries. The hypothesis related to the test of GNP per capita was:

HYPOTHESIS (H8)- Center countries will have high GNPs per capita whereas periphery countries will be associated with low GNPs per capita. More specifically, the EPC index will be negatively correlated with the GNP per capita.

Although, the empirical results of the correlation analysis supported this hypothesis, the correlation coefficient of -0.33 with EPC was not very strong. The correlation coefficient between GNP per capita and PQLI (+0.56) was quite high and supported the hypothesis that greater levels of well-being are directly associated with greater GNPs per capita. Another correlation coefficient that was high was that of GNP per capita with the percent agriculture in GDP $(-0.66)$. This also suggested that economies that have been more industrialized and where the share of agriculture in their economy is not high, are the countries that have higher GNPs per capita and consequently have better standards of living.

The gap in per capita GNP, as exhibited by Bairoch (Table II), between the developed countries of the industrialized region and other regions has continued to widen. In 1980, the GNP gap in terms of ratios between the industrialized region and Africa was 10:1. Once the top ten richest countries were compared to the ten poorest countries, the gap increased to $49: 1$. When the gap between the richest country and the poorest country was compared, a ratio of $94: 1$ illuminated the 
TABLE XVI

COUNTRIES RANKED BY GNP/CAPITA IN 1980

\begin{tabular}{|c|c|c|c|c|c|c|c|c|}
\hline & COUNTRY & GNP/CAPITA & & COUNTRY & GNP/CAPITA & & COUNTRY GN & /CAPITA \\
\hline 1 & U.A.E. & 24660 . & 44 & POLAND & 3600 . & 87 & CONGO & 1110. \\
\hline 2 & KUWAIT & 20900. & 45 & BARBADOS & 3500. & 88 & BELIZE & 1080. \\
\hline 3 & SUITZERLAN & 17430. & 46 & BULGARIA & 3400. & 89 & CAMEROON & 880. \\
\hline 4 & SHEDEN & 14840. & 47 & GUADALOUPE & 3300. & 90 & NIGERIA & 870. \\
\hline 5 & NORHAY & 14060. & 48 & U.S.S.R. & 3200. & 91 & MOROCCO & 860. \\
\hline 6 & GERLLANY & 13450. & 49 & SURINAME & 3030. & 92 & NICARAGUA & 860. \\
\hline$\gamma$ & DERHARK & 13120. & 50 & URUGUAY & 2820 . & 93 & GRENADA & 850. \\
\hline 8 & ICELAND & 12860. & 51 & NETHR. ANT & 2800 & 94 & ANGOLA & 840. \\
\hline 9 & U.S.A. & 12820. & 52 & YUGOSLAVIA & 2790. & 95 & PAUPA N GU & 840. \\
\hline 10 & SAUDI ARAB & 12600. & 53 & SOUTH AFRI & 2770 & 96 & PHILIPPINE & 790. \\
\hline 11 & FRANCE & 12190. & 54 & ARGENTIMA & 2560. & 97 & THAILAND & 770. \\
\hline 12 & BELGIUH & 11920. & 55 & CHILE & 2560 . & 98 & EGYPT & 650. \\
\hline 13 & METHERLAND & 11790. & 56 & IRAQ & 2540. & 99 & EL SALVAdO & 650. \\
\hline 14 & CANADA & 11400. & 57 & PORTUGAL & 2520. & 100 & NORTH KORE & 640. \\
\hline 15 & AUSTRALIA & 11080. & 58 & MEXICO & 2250. & 101 & ZAMBIA & 600. \\
\hline 16 & FIMLAKD & 10680. & 59 & BRAZIL & 2220. & 102 & BOLIVIA & 600. \\
\hline 17 & AUSTRIA & 10210. & 60 & ALGERIA & 2140. & 103 & HOADORAS & 600 \\
\hline 18 & QATAR & 10100. & 61 & HUNGARY & 2100. & 104 & INDONESIA & 530. \\
\hline 19 & JAPAN & 10080. & 62 & FIJI & 2000 & 105 & YEMEN, A.D & 500. \\
\hline 20 & UK & 9110. & 63 & GUIANA, FR & 2000 & 106 & MAURITANIA & 460. \\
\hline 2 & BAHRAIN & 8960. & 64 & PANAMA & 1910. & 107 & YEMEN, A.R & 460. \\
\hline 22 & LIBYA & 8450. & 65 & ROMANIA & 1890. & 108 & SENEGAL & 430. \\
\hline 23 & NEH zEALAN & T700. & 66 & MALAYSIA & 1840. & 109 & KENYA & 420. \\
\hline 4 & BERHUDA & 7600. & 67 & IRAN & 1800. & 110 & GHANA & 400 \\
\hline 25 & ITALY & 6960. & 68 & KOREA & 1700. & 111 & DJIBOUTI & 390. \\
\hline 26 & GREENLAND & 6700. & 69 & PARAGUAY & 1630. & 112 & SUDAN & 380. \\
\hline 27 & NEW CALEDO & 6500. & 70 & JORDAN & 1620. & 113 & GAMBIA & 370. \\
\hline 28 & OHAN & 5920. & 71 & SYRIA & 1570. & 114 & mozamiBIQUE & 360. \\
\hline 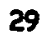 & TRIMIDAD & 5670. & 72 & TURKEY & 1540. & 115 & PAKISTAN & 350. \\
\hline 30 & SPAIK & 5640. & 73 & SEYCHELLES & 500. & 116 & CAPE VERDI & 340. \\
\hline 49 & SIKGAPORE & 5240. & 74 & MACAO & 1500. & 117 & MADAGASCAR & 330. \\
\hline 32 & IRELAND & 5230. & 75 & COSTA RICA & 1430. & 118 & NIGER & 330. \\
\hline 33 & ISRAEL & 5160. & 76 & TUNISIA & 1420. & 119 & BENIN & 320. \\
\hline 3 & HONG Kong & 5100. & 77 & COLOMBIA & 1380. & 120 & CEN. AFR. & 320. \\
\hline 35 & EAST GERHA & 5100. & 78 & MAURITIUS & 1270. & 121 & SIERRA LEO & 320. \\
\hline 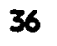 & GREECE & 4420. & 79 & DOMINICAN & 1260. & 122 & GUINEA & 300. \\
\hline 37 & CZECHOSLOY & 4400. & 80 & IVORY COAS & 1200. & 123 & CHINA & 300. \\
\hline 38 & VENEZUELA & 4220. & 81 & LEBANON & 1200. & 124 & SRI LANKA & 300. \\
\hline 59 & REUNION & 4200 & 82 & CUBA & 1200. & 125 & HAITI & 300. \\
\hline 0 & MARTINIQUE & 4000 & 83 & ECUADOR & 1180. & 126 & SOMALIA & 280. \\
\hline 1 & GABON & 3810. & 84 & JAIAICA & 1180. & 127 & TANZANIA & 280. \\
\hline 2 & CYPRUS & 3740. & 85 & PERU & 1170. & 128 & EQU. GUINE & 270. \\
\hline 3 & MALTA & 3600. & 86 & GUATEMALA & 1140. & 129 & INDIA & 260. \\
\hline
\end{tabular}


sharp differences in the GNP's per capita at both extremes of the center and periphery.

\section{EXPORT DEPENDENCY}

The percent of GDP that is accounted for by exports to the largest trading partner is the last national variable. It has been of ten asserted that the more a country's exports are dependent on a single market the more dependent that country is likely to be. The hypothesis regarding Export Dependency is stated as follows:

HYPOTHESIS (H9)- Center countries have a lower export trade dependency whereas periphery countries are highly export dependent. More specifically, the EPC index is positively correlated with Export Dependency.

Correlation coefficients between EPC and other varlables

\begin{tabular}{|l|c|c|c|c|c|c|c|}
\hline EPC VS. & IPC & ECC & ICC & VT & POII & AGR & ED \\
\hline 1962 & .85 & .34 & .42 & -.57 & -.48 & .42 & .57 \\
\hline 1970 & .73 & .33 & .38 & -.35 & -.36 & .32 & .40 \\
\hline 1980 & .68 & .41 & .43 & -.50 & -.45 & .25 & .55 \\
\hline
\end{tabular}

The empirical results for Export Dependency for all the study years confirm the hypothesis. The correlation coefficients between EPC and ED were high and significant, but ED correlations with Vertical Trade, PQLI, and AGR/GDP were found to be insignificant.

\section{ASCENT AND DECLINE IN THE WORLD SYSTEM}

Through time, the world system has witnessed the rise and decline of many nations. The decline of Spanish Empire of previous centuries and the more recent decline of the British Empire are only two examples of such downward movements in the world system. The emergence of United States as a hegemonic power after 
TABLE XVII-A

COUNTRIES RANKED BY EXPORTS AS PERCENT GDP TO LARGEST MARKET IN 1961

\begin{tabular}{|c|c|c|c|c|c|c|c|}
\hline & COUNTRY & ED AS ZGDP & & COUNTRY & AS KGDP & COUNTRY & D AS XGDP \\
\hline 1 & MARTINIQUE & 36.60 & 44 & DENMARK & 5.80 & BAHREIN & $N / A$ \\
\hline 2 & MAURITIUS & 30.70 & 45 & COLOMBIA & 5.60 & BELGIUM & $N / A$ \\
\hline 3 & SURIMAHE & 29.30 & 46 & SOUTH AFR & 5.60 & BERHUDA & $N / A$ \\
\hline 4 & GUADALOUPE & 26.70 & 47 & GUATEMALA & 5.50 & BRUNEI & N/A \\
\hline 5 & KUWAIT & 25.20 & 48 & GUINEA & 4.90 & BULGARIA & N/A \\
\hline 6 & YEMEM,A.D. & 20.60 & 49 & PHILIPPINES & 4.80 & BURMA & N/A \\
\hline 7 & GABON & 20.30 & 50 & AUSTRIA & 4.70 & CHAD & N/A \\
\hline 8 & REUHION & 20.00 & 51 & CONGO & 4.70 & CHIMA & N/A \\
\hline 9 & IRELAND & 19.40 & 52 & PANAMA & 4.40 & CUBA & N/A \\
\hline 10 & BARBADOS & 16.10 & 53 & CHILE & 4.10 & CZECHOSLOV & N/A \\
\hline 11 & SENEGAL & 15.00 & 54 & FINLAND & 4.10 & EAST GERHA & $\mathbf{N} / \mathbf{A}$ \\
\hline 12 & TRIMIDAD & 14.40 & 55 & KENYA & 4.10 & EGYPT & N/A \\
\hline 13 & IVORY COAS & 14.20 & 56 & NORWAY & 3.90 & FIJI & $N / A$ \\
\hline 14 & GAYBIA & 12.90 & 57 & MEXICO & 3.80 & GERHANY & N/A \\
\hline 15 & DOMIMICAN & 12.80 & 58 & SHITZERLAN & 3.80 & GREENLAND & N/A \\
\hline 16 & VENEzUELA & 11.70 & 59 & ETHIOPIA & 3.10 & HUHGARY & N/A \\
\hline 17 & HONG KONG & 11.50 & 60 & SWEDEN & 3.00 & IRAQ & N/A \\
\hline 18 & ALGERIA & 11.20 & 61 & AUSTRALIA & 2.90 & JORDAN & $N / A$ \\
\hline 19 & HONDORAS & 10.90 & 62 & INDONESIA & 2.80 & LAOS & $\mathbf{N} / \mathbf{A}$ \\
\hline 20 & NEW ZEALAN & 10.50 & 63 & SUDAN & 2.80 & LEBANON & $\mathbf{N} / \mathbf{A}$ \\
\hline 21 & IRAN & 9.90 & 64 & BRAZIL & 2.70 & LIBYA & $\mathbf{N} / \mathbf{A}$ \\
\hline 22 & TUNISIA & 9.80 & 65 & URUGUAY & 2.70 & MACAO & $N / A$ \\
\hline 23 & COSTA RICA & 9.40 & 66 & MALTA & 2.50 & maLAYSIA & $N / \boldsymbol{A}$ \\
\hline 24 & SIERRA LEO & 9.20 & 67 & THAILAND & 2.30 & MOZAMBIQUE & $N / A$ \\
\hline 2 & BOLIVIA & 9.10 & 68 & JAPAN & 2.10 & NETH. ANTH & $N / A$ \\
\hline 26 & CAMEROON & 9.10 & 69 & MALI & 2.10 & MEW CALEDO & $N / A$ \\
\hline 27 & JAMAICA & 8.80 & 70 & ITALY & 2.00 & OMAN & $N / A$ \\
\hline 28 & CANADA & 8.50 & 71 & PORTUGAL & 1.70 & PARAGUAY & $N / A$ \\
\hline 29 & NETHERLAND & 8.10 & 72 & FRANCE & 1.60 & PAUPA N. G & N/A \\
\hline 30 & NICARAGUA & 8.00 & 73 & ISRAEL & 1.30 & POLAND & N/A \\
\hline 31 & ICELAND & 7.50 & 74 & ARGENTINA & 1.20 & QATAR & $N / A$ \\
\hline 32 & PERU & 7.50 & 75 & GREECE & 1.10 & ROMANIA & N/A \\
\hline 33 & EL SALVADO & 7.00 & 76 & MAURITANIA & 1.10 & SINGAPORE & $N / A$ \\
\hline 34 & MOROCCO & 7.00 & $\pi$ & TURKEY & 1.10 & SONALIA & N/A \\
\hline 35 & NIGER & 6.90 & 78 & UK & 1.10 & SYRIA & $N / A$ \\
\hline 36 & BENIN & 6.70 & 79 & INDIA & 1.00 & TANZANIA & N/A \\
\hline 37 & CEN. AFR. & 6.70 & 80 & SPAIN & 1.00 & U.S.A. & N/A \\
\hline 38 & SAUDI ARAB & 6.70 & 81 & KOREA & 0.80 & U.S.S.R. & N/A \\
\hline 39 & ECUADOR & 6.50 & 82 & PAKISTAN & 0.70 & UGANDA & $N / A$ \\
\hline 40 & GHANA & 6.50 & 83 & VIETNAM & 0.60 & YEMEN,P.D. & N/A \\
\hline 41 & HAITI & 6.40 & & AFGHANISTA & $N / A$ & YUGOSLAVIA & N/A \\
\hline 42 & MIGERIA & 6.40 & & ANGOLA & N/A & & \\
\hline 43 & CYPRUS & 5.90 & & BAHAMAS & $N / A$ & & \\
\hline
\end{tabular}


TABLE XVII-B

COUNTRIES RANKED BY EXPORTS AS PERCENT GDP TO LARGEST MARKET IN 1971

\begin{tabular}{|c|c|c|c|c|c|c|c|}
\hline & COUNTRY & AS KGDP & & COUNTRY & ED AS $X G D P$ & COUNTRY & SD AS XGDP \\
\hline 1 & HOAG KONG & 30.50 & 44 & ECUADOR & 4.80 & BRUNEI & N/A \\
\hline 2 & GABON & 23.20 & 45 & EL SALVADO & 4.80 & BULGARIA & N/A \\
\hline 3 & SURINARE & 22.10 & 46 & PANAMA & 4.80 & BURMA & N/A \\
\hline 4 & IRELAND & 18.90 & 47 & AUSTRIA & 4.40 & CHINA & $N / A$ \\
\hline 5 & NETHERLAND & 17.50 & 48 & GUATEMALA & 4.40 & COAGO & $N / A$ \\
\hline 6 & MAURITIUS & 16.90 & 49 & ALGERIA & 4.10 & CUBA & $N / A$ \\
\hline 7 & HONDORAS & 16.10 & 50 & DENMARK & 4.00 & CZECHOSLOV & N/A \\
\hline 8 & GAMBIA & 14.70 & 51 & FINLAND & 4.00 & EAST GERMA & N/A \\
\hline 9 & SAUDI ARAB & 13.40 & 52 & AUSTRALIA & 3.90 & EGYPT & N/A \\
\hline I0 & SIERRA LEO & 13.40 & 53 & UGANDA & 3.90 & GERMIANY & N/A \\
\hline 19 & GUADALOUPE & 13.20 & 54 & GHANA & 3.80 & GREENLAND & N/A \\
\hline 12 & CAHADA & 12.80 & 55 & NORWAY & 3.80 & GUTNEA & H/A \\
\hline 13 & BELGIUH & 11.20 & 56 & PERU & 3.80 & HUHGARY & N/A \\
\hline 14 & KUHAIT & 11.10 & 57 & SUITZERLAN & 3.60 & IRAO & N/A \\
\hline 15 & JARAICA & 10.90 & 58 & PORTUGAL & 3.50 & JORDAN & N/A \\
\hline 16 & MUURITANIA & 10.50 & 59 & COLOMBIA & 3.40 & KENYA & N/A \\
\hline 17 & REUNION & 10.50 & 60 & ITALY & 3.40 & LAOS & N/A \\
\hline 18 & MARTINIQUE & 10.10 & 61 & JAPAN & 3.40 & LEBANON & $N / A$ \\
\hline 19 & DOHINICAN & 10.00 & 62 & MALI & 3.00 & LIBYA & N/A \\
\hline 20 & VENEZUELA & 9.80 & 63 & SOUTH AFR & 3.00 & MACAO & $N / A$ \\
\hline 21 & IVORY COAS & 9.60 & 64 & ETHIOPIA & 2.90 & MALAYSIA & $N / A$ \\
\hline 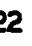 & BEMIN & 9.20 & 65 & ISRAEL & 2.90 & MOZAMBIQUE & N/A \\
\hline 23 & COSTA RICA & 9.00 & 66 & THAILAND & 2.90 & NETH. ANTH & N/A \\
\hline 24 & ICELAND & 9.00 & 67 & SWEDEN & 2.80 & NEU CALEDO & N/A \\
\hline 25 & BOLIVIA & 8.80 & 68 & FRANCE & 2.70 & OMAN & N/A \\
\hline 26 & IRAN & 8.80 & 69 & MEXICO & 2.50 & PARAGUAY & $N / A$ \\
\hline 2 & BARBADOS & 8.70 & 70 & CHAD & 1.90 & PAUPA N. G & $N / A$ \\
\hline 28 & FIJI & 8.20 & 71 & CHILE & 1.90 & POLAND & $N / \boldsymbol{A}$ \\
\hline 2 & SENEGAL & 8.20 & 72 & UK & 1.90 & QATAR & $N / A$ \\
\hline 30 & CEK. AFR. & 8.10 & 73 & BRAZIL & 1.70 & ROMANIA & $N / A$ \\
\hline 31 & MALTA & 7.80 & 74 & GREECE & 1.20 & SIMGAPORE & $N / \boldsymbol{A}$ \\
\hline 3 & CYPRUS & 7.60 & 75 & SPAIN & 1.20 & SOMALIA & $N / A$ \\
\hline 33 & MIGERIA & 7.40 & 76 & TURKEY & 1.10 & SUDAN & N/A \\
\hline 34 & NICARAGUA & 7.30 & 77 & URUGUAY & 0.80 & SYRIA & $\mathbf{N} / \mathbf{A}$ \\
\hline 35 & NIGER & 7.30 & 78 & INDIA & 0.70 & TANZANIA & N/A \\
\hline 36 & HAITI & 6.70 & 79 & PAKISTAN & 0.60 & TRINIDAD & N/A \\
\hline 37 & NEU ZEALAN & 5.90 & 80 & VIETNAM & 0.00 & TUNISIA & N/A \\
\hline 38 & PHILIPPINES & 5.90 & & AFGHANISTA & $N / A$ & U.S.A. & $N / A$ \\
\hline 39 & KOREA & 5.80 & & ANGOLA & $N / A$ & U.S.S.R. & N/A \\
\hline 40 & YEREN,A.D. & 5.60 & & ARGENTINA & $N / A$ & YEAEN,P.D. & H/A \\
\hline 4 & INDONESIA & 5.40 & & BAHAMAS & N/A & YUGOSLAVIA & N/A \\
\hline 4 & CAHEROON & 5.10 & & BAHREIN & N/A & & \\
\hline 43 & moROCCO & 4.90 & & BERHUDA & N/A & & \\
\hline
\end{tabular}


TABLE XVII-C

COUNTRIES RANKED BY EXPORTS AS PERCENT GDP TO LARGEST MARKET IN 1976

\begin{tabular}{|c|c|c|c|c|c|c|c|}
\hline & COUNTRY & D AS XGDP & & COUNTRY & DD AS XGDP & COUNTRY & AS KGDP \\
\hline 1 & MAURITIUS & 40.50 & 44 & SWITZERLAN & 4.00 & GREENLAND & $N / A$ \\
\hline 2 & IRELAND & 21.90 & 45 & MOROCCO & 3.80 & GUADALOUPE & $N / R$ \\
\hline 3 & KUWAIT & 21.00 & 46 & NEW ZEALAN & 3.60 & GUINEA & $N / A$ \\
\hline 4 & GABON & 18.90 & 47 & COLOMBIA & 3.50 & HONG KOHG & $N / A$ \\
\hline 5 & HONDORAS & 18.40 & 48 & ISRAEL & 3.50 & HUNGARY & $N / A$ \\
\hline 6 & SAUDI ARAB & 16.20 & 49 & SOUTH AFR & 3.40 & JORDAN & $\mathbf{N} / \mathbf{A}$ \\
\hline 7 & SENEGAL & 15.70 & 50 & KENYA & 3.00 & LAOS & $\mathbf{N} / \mathbf{A}$ \\
\hline 8 & SURINAYE & 15.20 & 51 & PORTUGAL & 2.90 & LEBANON & $N / A$ \\
\hline 9 & NETHERLAND & 13.90 & 52 & FRANCE & 2.80 & LIBYA & N/A \\
\hline 10 & CANADA & 13.30 & 53 & PERU & 2.80 & MACAO & N/A \\
\hline 11 & DOHINICAN & 12.70 & 54 & SWEDEN & 2.70 & MALAYSIA & N/A \\
\hline 12 & VENEZUELA & 12.50 & 55 & GREECE & 2.50 & MALI & N/A \\
\hline 13 & EL SALVADO & 12.40 & 56 & ETHIOPIA & 2.40 & MALTA & N/A \\
\hline 14 & SIERRA LEO & 11.90 & 57 & MEXICO & 2.40 & MARTINIQUE & $N / A$ \\
\hline 15 & IRAQ & 11.10 & 58 & JAPAN & 2.30 & MUURITANIA & M/A \\
\hline 16 & ghrara & 11.00 & 59 & URUGUAY & 2.20 & mOZAMBIQUE & $N / A$ \\
\hline 17 & ECUADOR & 10.90 & 60 & UK & 2.00 & METH. ANTH & $\mathbf{N} / \mathbf{A}$ \\
\hline 18 & BELGIUM & 10.60 & 61 & SPAIN & 1.20 & NEH CALEDO & $N / A$ \\
\hline 19 & COSTA RICA & 9.90 & 62 & BRAZIL & 1.10 & NIGER & N/A \\
\hline 20 & KOREA & 9.80 & 63 & TURKEY & 1.00 & MIGERIA & $N / A$ \\
\hline 21 & INDCNESIA & 9.60 & 64 & PAKISTAN & 0.70 & Onan & $\mathrm{M} / \mathrm{A}$ \\
\hline 22 & MICARAGUA & 9.30 & 65 & INDIA & 0.60 & PARAGUAY & $N / R$ \\
\hline 23 & CYPRUS & 9.20 & & AFGHANISTA & N/A & PAUPA N. G & $N / \boldsymbol{A}$ \\
\hline 24 & BARBADOS & 9.10 & & ALGERIA & N/A & POLAND & $\mathbf{N} / \mathbf{A}$ \\
\hline 25 & FIJI & 8.60 & & ANGOLA & $N / A$ & QATAR & $N / A$ \\
\hline 26 & JaraICa & 8.60 & & ARGENTINA & $N / A$ & REUMION & N/A \\
\hline 27 & CAMEROON & 8.50 & & BAHAMAS & $N / A$ & ROHANIA & $N / A$ \\
\hline 28 & IVORY COAS & 8.30 & & BAHREIN & $N / A$ & SINGAPORE & N/A \\
\hline 29 & ICELAND & 7.90 & & BENIN & $N / A$ & SOMALIA & $N / A$ \\
\hline 30 & BOLIVIA & 7.60 & & BERMUDA & N/A & SUDAN & N/A \\
\hline 31 & NORWAY & 7.40 & & BRUNEI & $N / A$ & SYRIA & N/A \\
\hline 32 & GHAKR & 6.90 & & BULGARIA & $N / A$ & TANzANIA & N/A \\
\hline 33 & HAITI & 6.50 & & BURMA & N/A & TRINIDAD & N/A \\
\hline 34 & GUATEMALA & 6.40 & & CEN. AFR. & $N / A$ & TUNISIA & N/A \\
\hline 35 & IRAN & 6.10 & & CHAD & N/A & U.S.A. & N/A \\
\hline 36 & PAKAMA & 5.50 & & CHINA & $M / A$ & U.S.S.R. & N/A \\
\hline 37 & AUSTRALIA & 5.40 & & CONGO & N/A & UGANDA & $N / A$ \\
\hline 38 & PHILIPPINES & 5.20 & & CUBA & $N / A$ & VIETNAY & $N / A$ \\
\hline 39 & AUSTRIA & 4.90 & & CZECHOSLOV & N/A & YEMEN,A.D. & $N / A$ \\
\hline 40 & CHILE & 4.80 & & EAST GERMA & $N / A$ & YEMEN,P.D. & $N / A$ \\
\hline 41 & THAILAND & 4.80 & & EGYPT & $N / A$ & YUGOSLAVIA & $N / A$ \\
\hline 42 & ITALY & 4.10 & & FINLAND & $N / A$ & & \\
\hline 43 & DENHARK & 4.00 & & GERHANY & $N / A$ & & \\
\hline
\end{tabular}


the World War II and the rise of Japan can both be regarded as ascents in the world system. Another interesting movement in the world system that has recently attracted much attention is the ascent of a few countries in the Pacific Rim region of the south east Asia. The Pacific Rim region includes the countries of Hong Kong, Taiwan, Singapore, South Korea, and Malaysia.

In the 1960 s exports of manufacture from the less developed countries, particularly from the Pacific Rim region, grew rapidly from about three billion dollars in 1960 to more than nine billion dollars in the 1970 (UNIDO, 1981). By 1980, the exports of manufactured products from the less developed countries rose to 80 billion dollars which accounted for more than nine per cent of the world total. This growth in manufactured exports, particularly from the 'open' economies of South Korea, Taiwan, Singapore and Malaysia, provided a vindication of comparative advantage and the idea that these countries that possess a relatively abundant endowments of labor should export goods that are more labor intensive. On these grounds, it was argued that, the emergence of the new industrial countries provides proof that a free market provides opportunities for exchange and specialization that can benefit every country.

Figure 7 demonstrates the economic and social dimensions of South Korea. As can be seen, South Korea was able to improve in all economic indicators that were tested in this study. It is based on these assertions that there is a continuing justification for the "open-economy" policies of the I.M.F. and the World Bank.

Some writers, such as Frank, Gantzel, Wallerstein and others, are skeptical both about the benefits from the past expansion in manufactured exports and about the possibilities from continued expansion in the future in light of world demands and protectionist regulations in the industrialized countries. Frank argues that the workers in these so called free trade zones are 'superexploited'. This is 


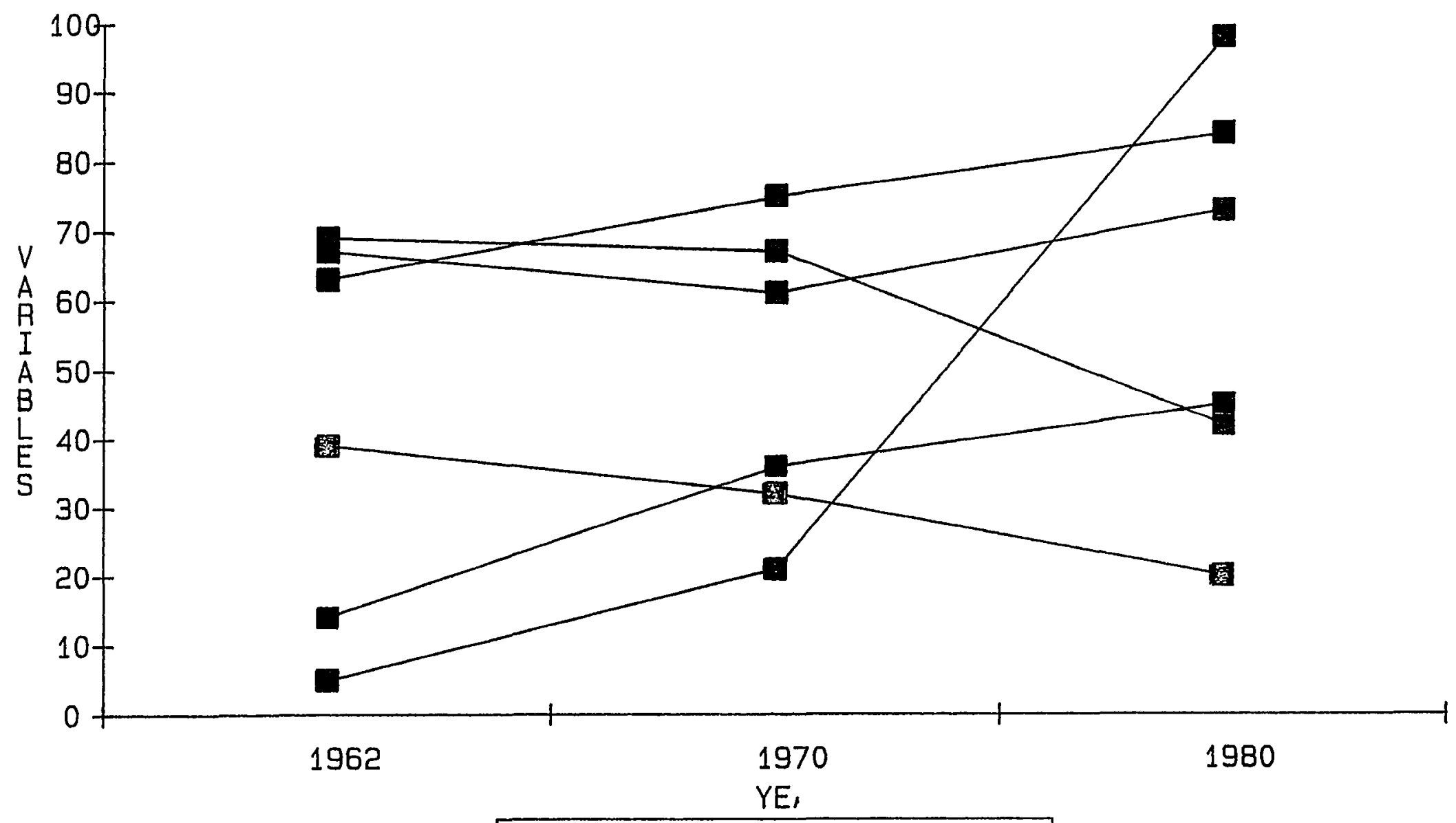

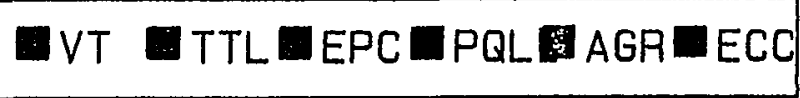

Figure 7. National Variables for South Korea. 
made possible, it is argued, by repression of both trade unions and political opposition to the ruling regimes. Their general conclusion is that access to the markets of the developed countries will not lead to creation of any indigenous, self expanding capitalist development in the third world, but to a dependent industrialization as part of a new form of imperialist domination ${ }^{1}$.

\section{DEVIATIONS FROM CENTER AND PERIPHERY HYPOTHESIS}

In terms of center-periphery distinction, there are countries that do not resemble the characteristics expected in dependency theory. Most notably are Canada, Australia and New Zealand. These countries are both highly developed with comparable national incomes to the European industrialized countries and yet their economies are commonly perceived to be highly dependent on exports of mineral and agricultural products.

A close look at the percent of agriculture in their GDPs reveals that in 1960 the figure for Australia was 12 per cent which dropped to 6.3 per cent by 1980 and for New Zealand the figure was 11.7 per cent in 1980 which in all cases these figures are moderately low. The positions of these two countries are also found to be very high on the vertical division of trade. The Vertical Trade Index for Australia is $\mathbf{- 0 . 6 1}$ for $1962,-0.63$ for 1970 , and -0.50 for 1980 . Same index for New Zealand is -0.72 for $1962,-.65$ for 1970 , and -0.46 for 1980 . Therefore, it seems that the impression that these countries' economies are based on agriculture is not supported by the present findings. Canada also exhibits a low dependence of GDP

1- M. Landesberg, "Export-led Industrialization in the Third World: Manufacturing Imperialism," Review of Radical Political Economics, 11(4), p. 5063. 
on agriculture. The per cent share of agriculture in Canada's GDP was 5.7 per cent in 1962 which by 1980 has dropped further to 4.0 per cent. Canada also ranks high in terms of Vertical Trade index. From 1962 to 1980, Canada's Vertical Trade Index varies from -0.38 to -0.25 .

What seems to be more important is that these countries have never been colonies of the West but they were colonized by white settlers of the European origin in regions without a great native population. This is a crucial factor since they all had the freedom to develop their own agricultural and mineral outputs and build and protect their own industries from the economic effects that the colonies had to endure. Control over their own trade and industry enabled them to import capital equipment from Britain and develop their own economies without running into debt. These countries were able to protect their infant industries from the British competition and invest their earnings for the growth of local economies whereas in colonies the earnings were always allowed to get diverted to the European countries. 


\section{CHAPTER V}

\section{RESULTS OF THE SYSTEM WIDE AND REGIONAL STUDIES}

\section{INTERNATIONAL TRADE HIERARCHIES}

An implicit property of the center-periphery relationship and the existence of economic dependence is the inherent hierarchical nature of the international trade system. The trade hierarchy is based upon the fact that trade relationships among various nations are not reciprocal in the sense that center countries are not as closely tied to the periphery countries they trade with as periphery countries are tied to their associated center countries. For example, while in 1962100 percent of Oman's exports were sent to United Kingdom, the share of the United Kingdom's exports to Oman was negligible. The trade ties for center countries who have numerous trading partners are not as extreme as for the peripheries. For example, 10 percent of West Germany's exports were sent to France in 1962, while the percent of exports of France to West Germany was 17 for the same period.

Based upon the preceding examples of the direct ties that exist among countries, trade hierarchies were constructed. Trade hierarchies were constructed by first identifying the largest export partner of any country and then listing countries under their largest export market countries. For example in Figure 9.1, 94 percent of the Bahamas' exports in 1962 went to the United States. Dependent trade relationship can also exist through a third country. For example, of Iran's exports, 38 percent go to Japan while Japan exports 33 percent to United States. Such hierarchical relationships are thus not limited only to the pairwise dominantsubordinant trade relationships that exist among nations. 
Trade hierarchies developed here are based on export data since dependence on export markets in accordance to the literature on dependency and imperialism, which suggest that export markets are better indicators of dependence than import since in a competitive world, import markets can be substituted far more easier than obtaining export markets ${ }^{1,2,3}$. Therefore, in this term, a dominant country is the one that provides a major market for other countries' products. By definition, given in terms of trade partner concentration, it is expected that the periphery countries' dependence on their largest export partners are much higher than the center countries' dependence on their largest export partners. Figures 8 through 13 present the hierarchies that are identified. These hierarchies as has been stated earlier, are constructed by clustering countries by their largest common export markets. These hierarchies and their temporal behaviors are discussed below:

Five dominant hierarchies have emerged from the data. These countries are the United States, the United Kingdom, France, West Germany, and the Soviet Union. The hierarchies associated with each of these countries are individually examined here.

A high level of export dependence is said to exist if the country's exports to its largest market is over 40 percent; a medium level of dependence exists if the share of the export market is between 20 and 39 percent; finally, a low level of dependence exists if the share of exports to its largest market is below 20 percent. The range selected here is consistent with other studies. It should however be

1- J.D. Coppock, International Economic Instability: The Experience after World War II ( New York: McGraw-Hill, 1962), p.18.

2- Peter J. Lloyd, International Trade Problems of Small Nations (North Carolina: Duke University, 1968), p. 14.

3- O'Conner, James "The Meaning of Economic Imperialism". in Imperialism and Underdevelopment: A Reader, ed. Robert I. Rhodes, pp. 101-50. New York: Monthly Review, 1970, D. 147. 
stated that these cutoff points are arbitrary and hence are useful only as guidelines to carry out the analysis.

\section{THE WORLD HIERARCHIES IN 1938}

For the year 1938, four hierarchies are identified. These countries are: United Kingdom, United States, Germany, and France. While the number of countries sampled for 1938 are limited to 64, the degree of depedency for almost all countries range from midium to high and compared to the hierarchy figures of the later years studied, thay are rather substantial, e.g. high degrees of trade partner concentration. United Kingdom's hierarchy consists of countries from most regions of the world while other heirarchies are primarily regional. United States' hierarchy is concentrated mainly of Latin American countries, the German hierarchy is only European, and France's hierarchy consists of North African and Middle Eastern countries. Therefore, Figure 8 constructs the structure of the world system before the era of decolonization, and thus, the comparison of the 1938 structure to the world structure of the later years would reveal important information on the ascent or decline of countries in the world system.

\section{THE UNITED STATES HIERARCHY}

Figures 9.1, 9.2, and 9.3 present the United States hierarchies and the degree of export dependence of the countries that are directly linked to the United States.

The United States, historically, provided the principal market for the Latin American countries. Of the 30 countries which had direct links to the U.S. in 1962, only 9 were not part of Latin America. In 1962, the U.S. hierarchy included virtually the entire Western Hemisphere with the exception of Cuba which had recently left the U.S. hierarchy to join the Soviet Union hierarchy. Further, the 


\begin{tabular}{|c|c|c|c|c|c|c|}
\hline \multirow{2}{*}{\multicolumn{2}{|c|}{$\frac{\text { U.K. }}{\text { FALKLAND } 100}$}} & \multicolumn{2}{|l|}{ U.S.A. } & \multicolumn{2}{|l|}{ GERMANY } & \multirow{2}{*}{$\frac{\text { FRANCE }}{\text { ALGERIA } 85}$} \\
\hline & & PANAMA 10 & 100 & BULGARIA & 52 & \\
\hline IRELAND & 93 & PHILIPP. 8 & 84 & TURKEY & 45 & TUNISIA 59 \\
\hline NEW 2EA. & 87 & HONDORAS 8 & 83 & VugOSLAV. & 40 & IRAQ \\
\hline BOLIVIA & 81 & CUBA & 77 & GREECE & 40 & MOROCCO 50 \\
\hline TRINIDAD & 67 & GUATEMALA 7 & 75 & SPAIN & 33 & \\
\hline AUSTRALIA & 64 & EL SALV. & 67 & HUNGARY & 29 & (ALGERIA 21) \\
\hline CEYLON & 62 & COLOMBIA & 61 & ITALY & 24 & \\
\hline JAMAICA & 58 & VENEZUELA & 60 & POLAND & 22 & \\
\hline DENMARK & 58 & MEXICO & 52 & ROMAINIA & 21 & \\
\hline NIGERIA & 51 & HAITI & 50 & AUSTRIA & 20 & \\
\hline S. AFR. & 50 & NICARAGUA & 50 & CZECHOS. & 18 & \\
\hline FINLAND & 47 & COSTA RICA & A44 & SWITZER. & 18 & \\
\hline CANADA & 44 & MALAYSIA & 40 & & & \\
\hline DOMINICAN & 43 & BRAZIL & 38 & (NETHERLA & NDS & 0) \\
\hline EGYPT & 41 & ECUADOR & 30 & & & \\
\hline IRAN & 38 & PERU & 29 & & & \\
\hline ARTOENTTHIIE & 36 & CHILE & 27 & & & \\
\hline U.S.S.R. & 36 & & & & & \\
\hline NETHERLAND & D31 & (U.K. 21) & & & & \\
\hline NORWAY & 30 & & & & & \\
\hline PORTUGAL & 29 & & & & & \\
\hline URUGUAY & 28 & & & & & \\
\hline SWEDEN & 26 & & & & & \\
\hline
\end{tabular}

Figure 8. The world trade hierarchies in 1938. 


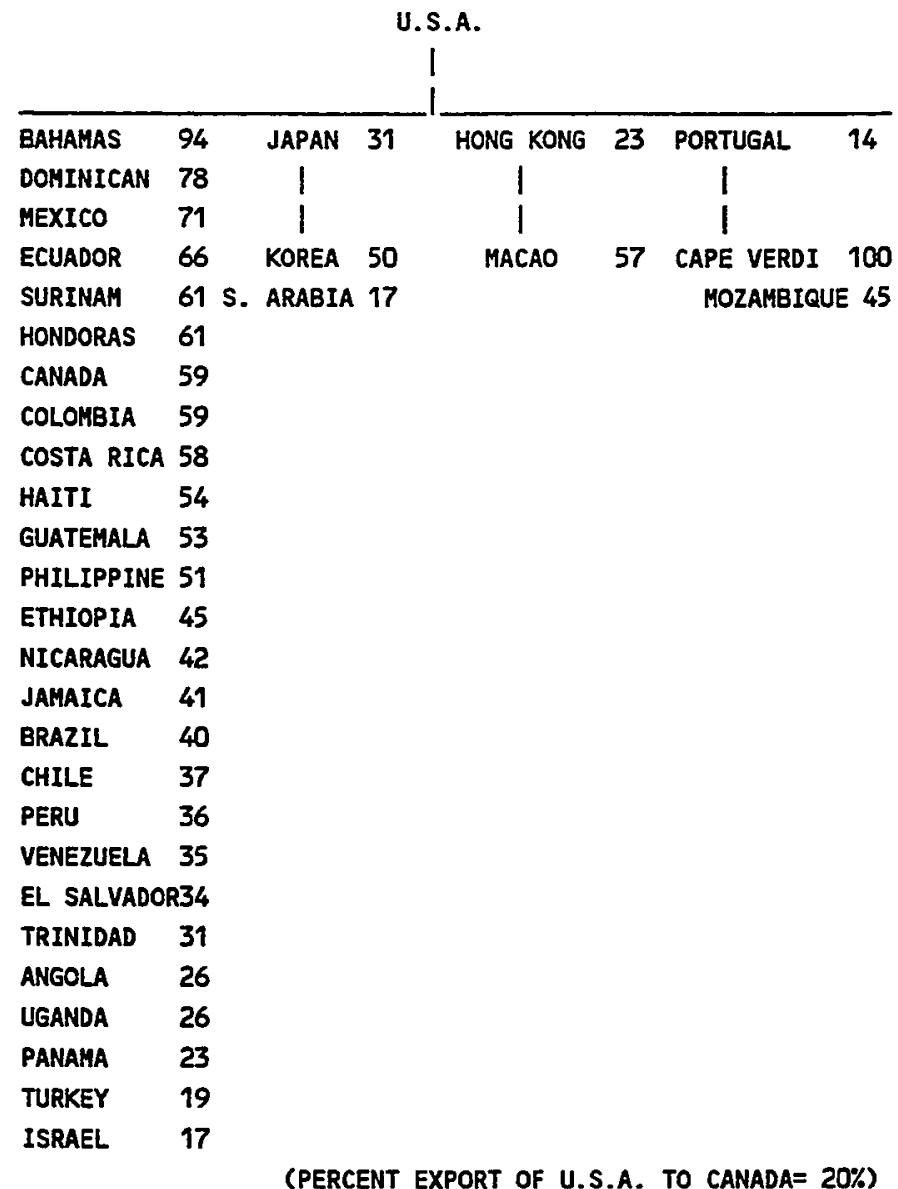

Fiqure 9.1. U.S.A. hierarchy in 1962. 


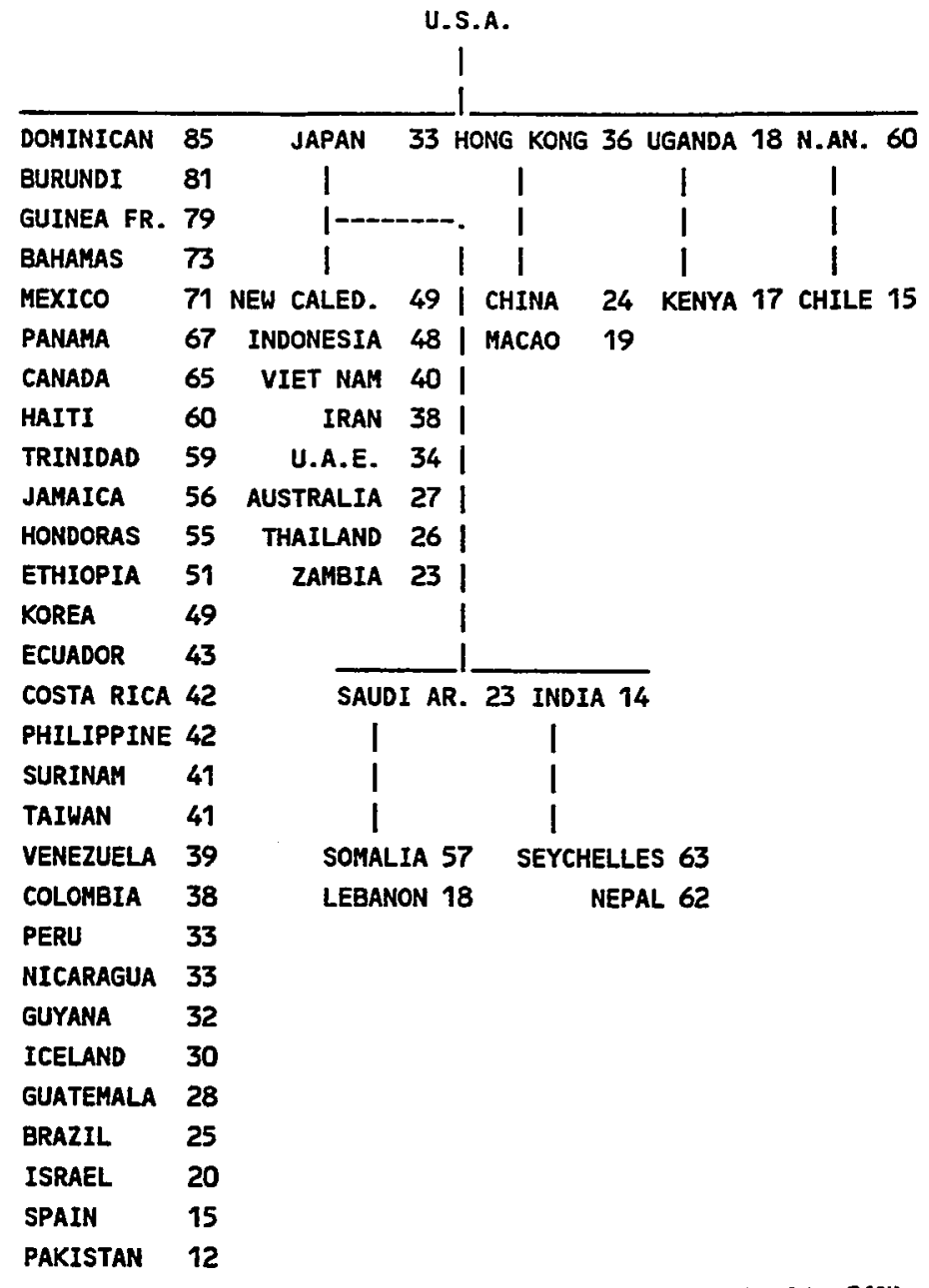

(PERCENT EXPORT OF U.S.A. TO CANADA= 21\%)

Figure 9.2. U.S.A. hierarchy in 1970 
U.S.A.

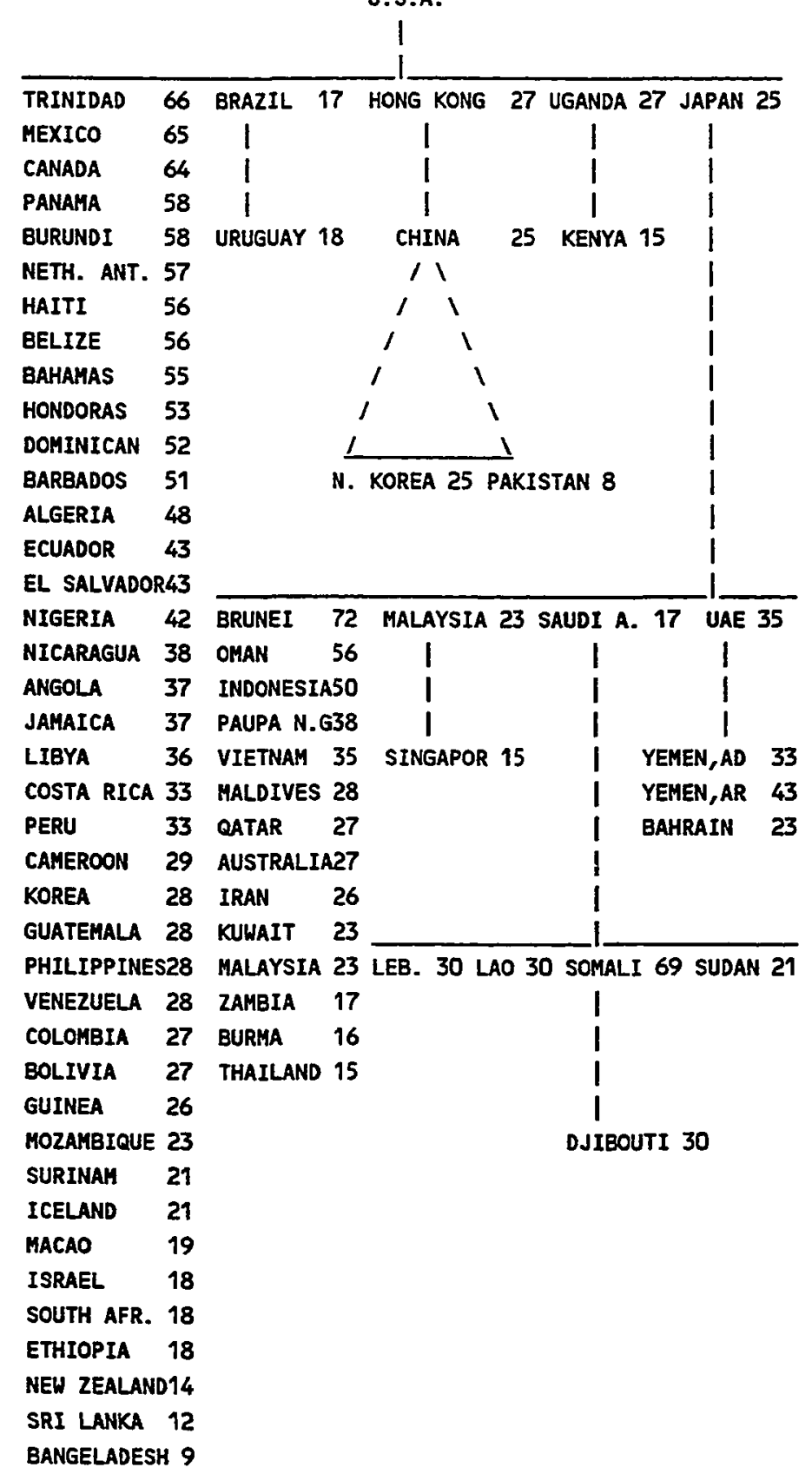

(PERCENT EXPORT OF U.S.A. TO CANADA= 16\%)

Figure 9.3. U.S.A. hierarchy in 1980. 
U.S. was able to provide markets for those countries previously linked to the United Kingdom hierarchy, namely, the Dominican Republic, Canada, Jamaica, Trinidad and Portugal. Turkey also left West German hierarchy to join the U.S. hierarchy in 1962.

At the 40 percent level of dependence, all the countries except Canada, Ethiopia and Philippines, belonged to Latin America. This demonstrates the high degree of dominance which the U.S. maintained over the external economic relationships of Canada and the South American countries.

Some changes did occur during the $1962-1970$ period. Although the Latin American countries remained substantially ependent on the U.S. market, the levels of their dependence were not as great as in the previous decade. The countries in the high dependence category were basically now the smaller countries of the region. The larger countries of Colombia and Brazil, along with Nicaragua and Guatemala, lowered their level of dependence from the high to medium category.

In 1970, Japan emerged as a new sub-hierarchy. Japan, which did not have a major trade hierarchy prior to 1970 , developed its own hierarchy with a small number of countries tied to its market. While 10 countries were linked to Japan in 1970 , by 1980 this figure had reached 18. A significant number of countries that were tied to Japan were the oil producing countries of the Middle East. Except for Zambia in the years 1970 and 1980, the rest of countries linked directly to Japan were from the two regions of the Middle East and South East Asia.

In view of dependency theory, the trade relationship between Japan and United States Suggests that United States has become a periphery to Japan, since Japan's exports to United States is highly concentrated on manufactured products whereas United States' exports to Japan are mainly agricultural. But, since Japan 
heavily depends on the United States market, it is included in the United States hierarchy. Japan's dependence on the U.S. market was 31 percent in 1962 which increased to 33 percent in 1970 and decreased to 25 percent in 1980.

Perhaps the most significant development in the U.S. hierarchy was the addition of the People's Republic of China. This addition, through Hong Kong, reflects the significance of linkage through a third country. The reason behind this addition is found in the political and ideological split that occurred between the Soviet Union and China during the 1960s. China therefore, had to rely for exports on Hong Kong rather than the Soviet Union and Eastern European countries.

Finally, the last significant development in this hierarchy was the addition of the African countries. There were only three African countries in the U.S. hierarchy in 1962, but by 1980 this number had risen to 10 . All these countries were in medium to high dependence categories; all were at one time the colonial possessions of other center countries who became dependent on United States as the influence of other center countries in Africa declined.

\section{THE UNITED KINGDOM HIERARCHY}

The end to formal colonialism had the greatest impact on the hierarchy of the United Kingdom (Figures 10.1, 10.2, and 10.3). While in 1962, over 30 countries were linked directly to the U.K., the number of countries dropped to 21 in 1970 and to 11 in 1980, providing evidence for the decline of the U.K.'s role in the world.

Not only the number of countries in the United Kingdom's hierarchy declined, the degree of dependence of the remaining countries to United Kingdom's market also declined. By 1970, Cyprus, Bolivia, Fiji, New Zealand, Nigeria, Ghana and Tanzania, which were the largest trading countries with the 


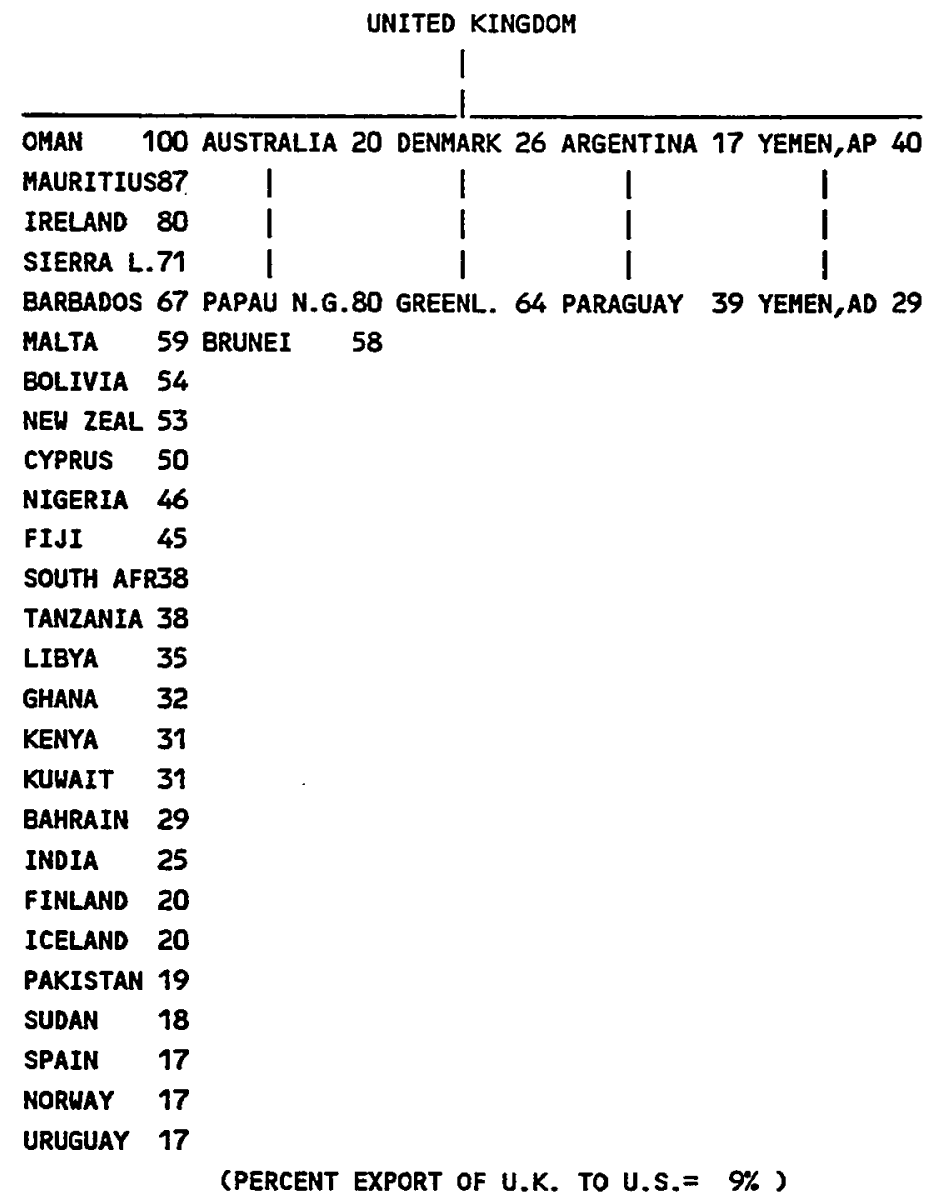

Fiqure 10.1. United Kingdom hierarchy in 1962. 


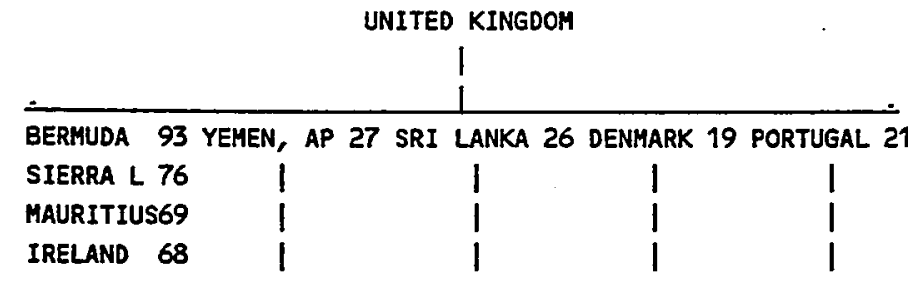

MALAWI 64 YEMEN, AD 52 BURMA 15 GREENLAND 81 MOZAR. 45

BARBADOS 54

ANGOLA 35

GAMBIA 49

MALTA 44

CYPRUS 39

BOLIVIA 39

FIJI 38

NEW ZEAL 38

SOUTH AFR37

QATAR 32

NIGERIA 30

GHANA 23

TANZANIA 20

KUWAIT 19

NORWAY 18

FINLAND 17

SWEDEN 13

(PERCENT EXPORT OF U.K. TO U.S.= 12\% )

Fiqure 10.2. United Kingdom hierarchy in 1970.

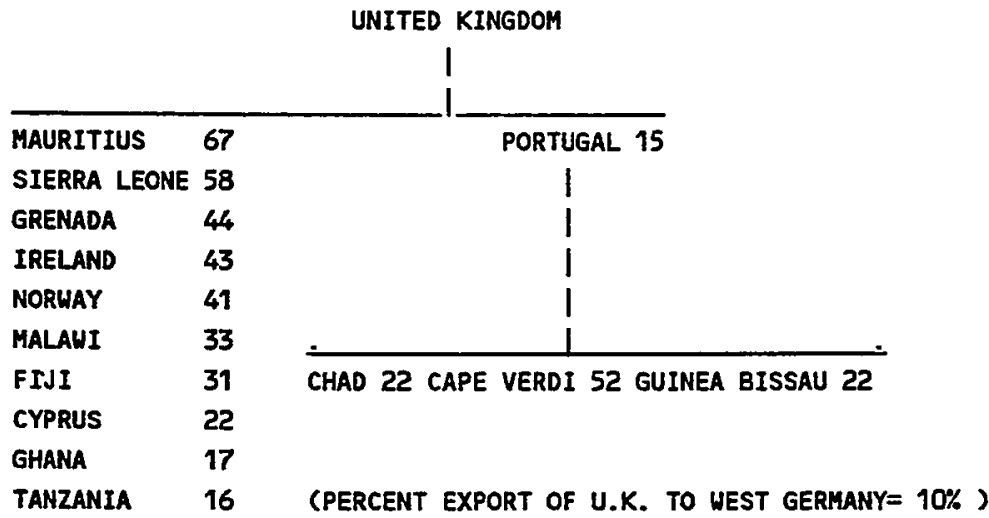

Fiqure 10.3. United Kingdom hierarchy in 1980. 
U.K. in the previous decade, had reduced their dependence on the U.K. market; by 1980, they had actually left the hierarchy. By 1980 only the smallest countries of the 1970 hierarchy remained with United Kingdom. Ireland, by 1980, had become the largest trading country exporting to the U.K. Therefore, a pattern of diminishing dependence upon the U.K. market and the decline of British dominance were very evident from these hierarchies.

\section{THE WEST GERMAN HIERARCHY}

West Germany's economic trade dominance in the European Economic Community could be seen in all years of the study (Figures 11.1, 11.2, and 11.3). Since most of the countries attached to West Germany's hierarchy are from Europe, the level of their dependence on West Germany has been in the medium to low categories. This is of course due to the proximity of other advanced nations in Europe that have served as export outlets to those countries. Even the nonEuropean countries' dependence upon West Germany is not high. Iran, which had the highest dependence in 1962, left that hierarchy to join Japan's. While the number of countries in the West German hierarchy rose from 7 in 1962 to 11 in 1970 and 1980, these additions were basically of smaller countries that were once part of the United Kingdom or French hierarchies.

\section{THE FRENCH HIERARCHY}

Figure 12.1 indicates that up to 1962, France more than any other hierarchy was able to keep its trade domination over its colonies. Of the 20 countries in the 1962 hierarchy, all but two were in the high dependence category with four countries sending 100 percent of their exports to France. However, by 1970 a significant drop in the levels of dependence had occurred among the larger 


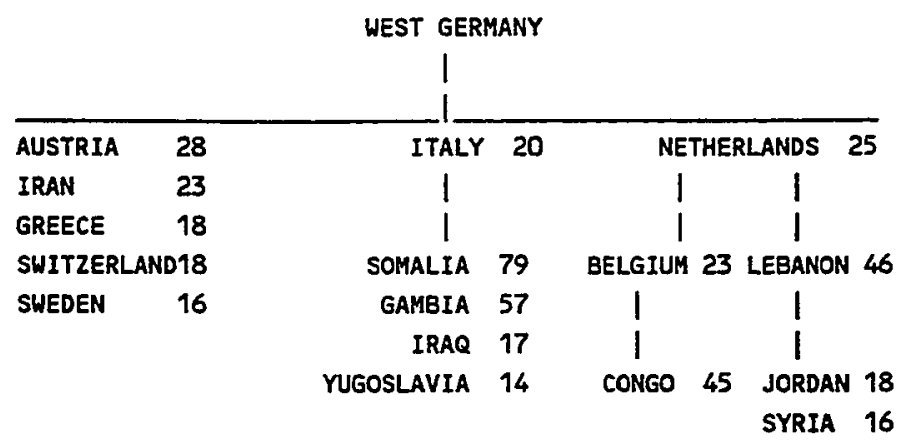

(PERCENT EXPORT OF WEST GERMANY FRANCE $=10 \%$ )

Figure 11.1. West German hierarchy in 1962.

WEST GERMANY

\begin{tabular}{|c|c|c|c|c|}
\hline \multicolumn{2}{|c|}{ NETHERLANDS 23} & \multicolumn{2}{|l|}{ ITALY } & \multirow{4}{*}{$\begin{array}{c}\text { BELGIUM } 24 \\
\text { I } \\
\text { ZAIRE } 43\end{array}$} \\
\hline EL SALVADOR & 25 & I & & \\
\hline CONGO & 24 & i & & \\
\hline OMAN & 22 & 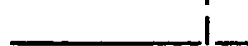 & & \\
\hline AUSTRIA & 22 & SYRIA 22 IRAQ 28 & ARGENTINA 15 & \\
\hline GREECE & 20 & LIBYA 26 & I & \\
\hline TURKEY & 20 & I YUGOSLAVI 15 & i & \\
\hline SWITZERLAND & 15 & 1 & I & \\
\hline URUGUAY & 13 & JORDAN 16 & PARAGUAY 34 & \\
\hline
\end{tabular}

(PERCENT EXPORT OF WEST GERMANY TO FRANCE = 12\% )

Figure 11.2. West German hierarchy in 1970.

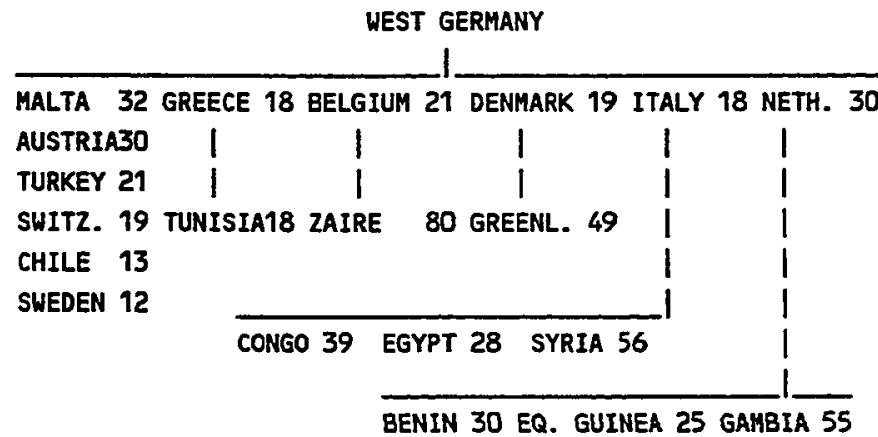

(PERCENT EXPORT OF WEST GERMANY TO FRANCE= 13\% )

Figure 11.3. West German hierarchy in 1980. 


\begin{tabular}{lr}
\multicolumn{2}{c}{ FRANCE } \\
NEW CALEDONIA & 100 \\
MARTINIQUE & 100 \\
MAURITANIA & 100 \\
BENIN & 100 \\
GUADALOUPE & 99 \\
CENTRAL AFR. & 98 \\
REUNION & 94 \\
SENEGAL & 85 \\
ALGERIA & 81 \\
CAMEROON & 68 \\
GABON & 67 \\
NIGER & 67 \\
CHAD & 63 \\
MADAGASCAR & 62 \\
TUNISIA & 55 \\
IVORY COAST & 49 \\
VIETMAM & 41 \\
MOROCCO & 40 \\
QATAR & 28 \\
GUINEA & 27
\end{tabular}

\begin{tabular}{lc}
\multicolumn{1}{c}{ FRANCE } \\
\multicolumn{1}{c}{$\quad$ I } \\
MARTINIQUE & 92 \\
REUNION & 77 \\
CHAD & 74 \\
SENEGAL & 73 \\
GUADALOUPE & 71 \\
CENTRAL AFR. & 57 \\
ALGERIA & 56 \\
VIETNAM & 50 \\
NIGER & 49 \\
GABON & 44 \\
BENIN & 41 \\
MOROCCO & 37 \\
IVORY COAST & 35 \\
MALI & 32 \\
CAMEROON & 31 \\
TUNISIA & 26 \\
MAURITANIA & 22 \\
\hline
\end{tabular}

(PERCENT EXPORT OF FRANCE TO TO WEST GERMANY= 20\%)

(PERCENT EXPORT OF FRANCE

TO WEST GERMANY= $17 \%$ )

Fiqure 12.2. French hierarchy in 1970.

Figure 12.1. French hierarchy in 1962.

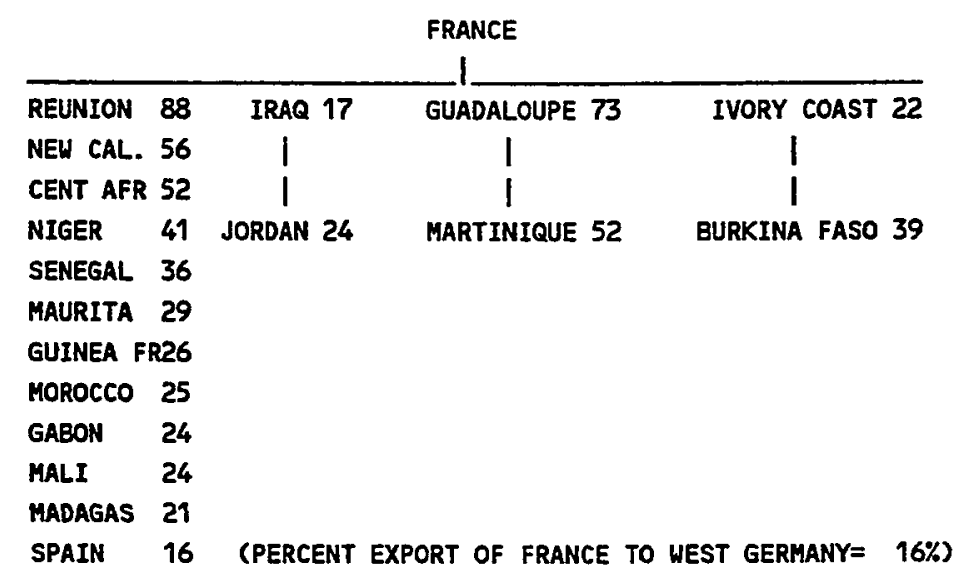

Figure 12.3. French hierarchy in 1980. 
countries of Algeria, the Ivory Coast, Cameroon, Tunisia, and Mauritania. The levels of dependence of countries on France had further decreased by 1980 when only 4 countries were left in the high dependence category.

\section{THE SOVIET UNION HIERARCHY}

In 1962, the Soviet hierarchy consisted of the newly acquired Eastern European countries along with Cuba, Mali, Afghanistan, and Egypt(Figures 13.1 through 13.3). The most significant change in this hierarchy by 1970 , as stated before, was the loss of China to the U.S. hierarchy. In 1980, little change was observed in the Soviet hierarchy except the loss of the Sudan and the gain of Finland and Yugoslavia.

The Soviet Union's own export dependence on United Kingdom for 36 percent in 1938 shifted to East Germany by 1962. Since 1962 the principal trading partner of the Soviet Union has been East Germany. However, East Germany's dependence on Soviet Union has been rather high. This dependence, which was 59 percent in 1962, dropped to 45 percent in 1970 and by 1980 East Germany's Primary market actually shifted to Romania. This suggests a gradual reduction in the degree of East Germany's dependence on the Soviet Union's market.

The Soviet Union and Eastern European countries have also demonstrated increasing participation in the international division of labor. Eastern European trade with Western European countries has grown faster than the trade among the socialist states. By the late 1970s more than half of total Eastern European trade was made with Western Europe 4 .

4- A.G. Frank, The European Challenge: From Atlantic Alliance to PanEuropean Entente for Peace and Jobs (Connecticut: Lawrence Hill \& Company, 1984), p. 69. 
U.S.S.R.

\begin{tabular}{ll}
\multicolumn{1}{c}{ I } \\
BULGARIA & 64 \\
EAST GERMANY & 59 \\
CUBA & 58 \\
MALI & 52 \\
ROMANIA & 51 \\
HUNGARY & 47 \\
CZECHOSLOVAK & 44 \\
CHINA & 44 \\
POLAND & 35 \\
AFGHANISTAN & 33 \\
EGYPT & 17
\end{tabular}

(PERCENT EXPORT OF U.S.S.R. TO EAST GERMANY= 21\% )

Figure 13.1. Soviet Union hierarchy in 1962.

\begin{tabular}{lr}
\multicolumn{1}{c}{ U.S.S.R. } \\
IULGARIA & \\
I & \\
EAST GERMANY & 45 \\
HUNGARY & 39 \\
EGYPT & 39 \\
CUBA & 38 \\
POLAND & 37 \\
CZECHOLSOVAK & 35 \\
ROMANIA & 29 \\
SUDAN & 16
\end{tabular}

(PERCENT EXPORT OF U.S.S.R. TO EAST GERMANY= 17\% )

Figure 13.2. Soviet Union hierarchy in 1970.

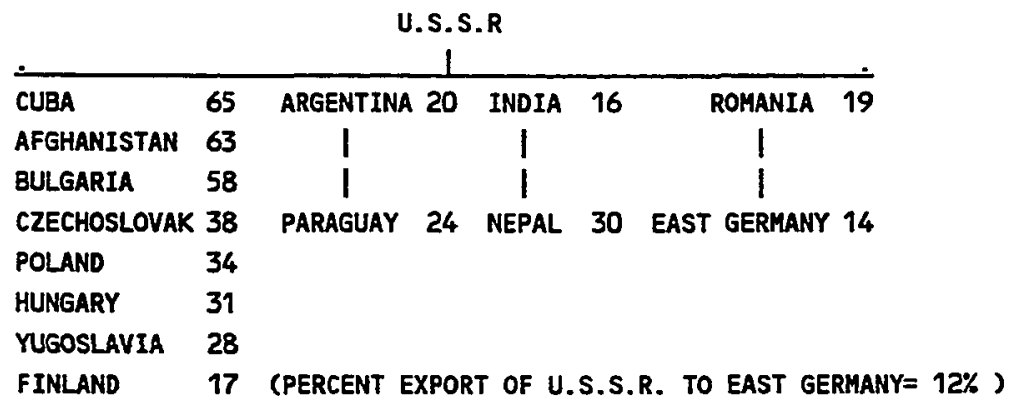

Fiqure 13.3. Soviet Union hierarchy in 1980. 
The international hierarchy system is commonly viewed in terms of EastWest (socialist-capitalist) and North-South( center-periphery) relations( conflicts). While the center-periphery relationship has been growing since the expansion of European overseas trade in the fifteenth century, the capitalist-socialist conflict has been growing since the establishment of a socialist society in the Soviet Union.

If hierarchies were to be drawn based on these four categories( Figure 14), the dominant position of countries could be determined according to their position on the two axes.

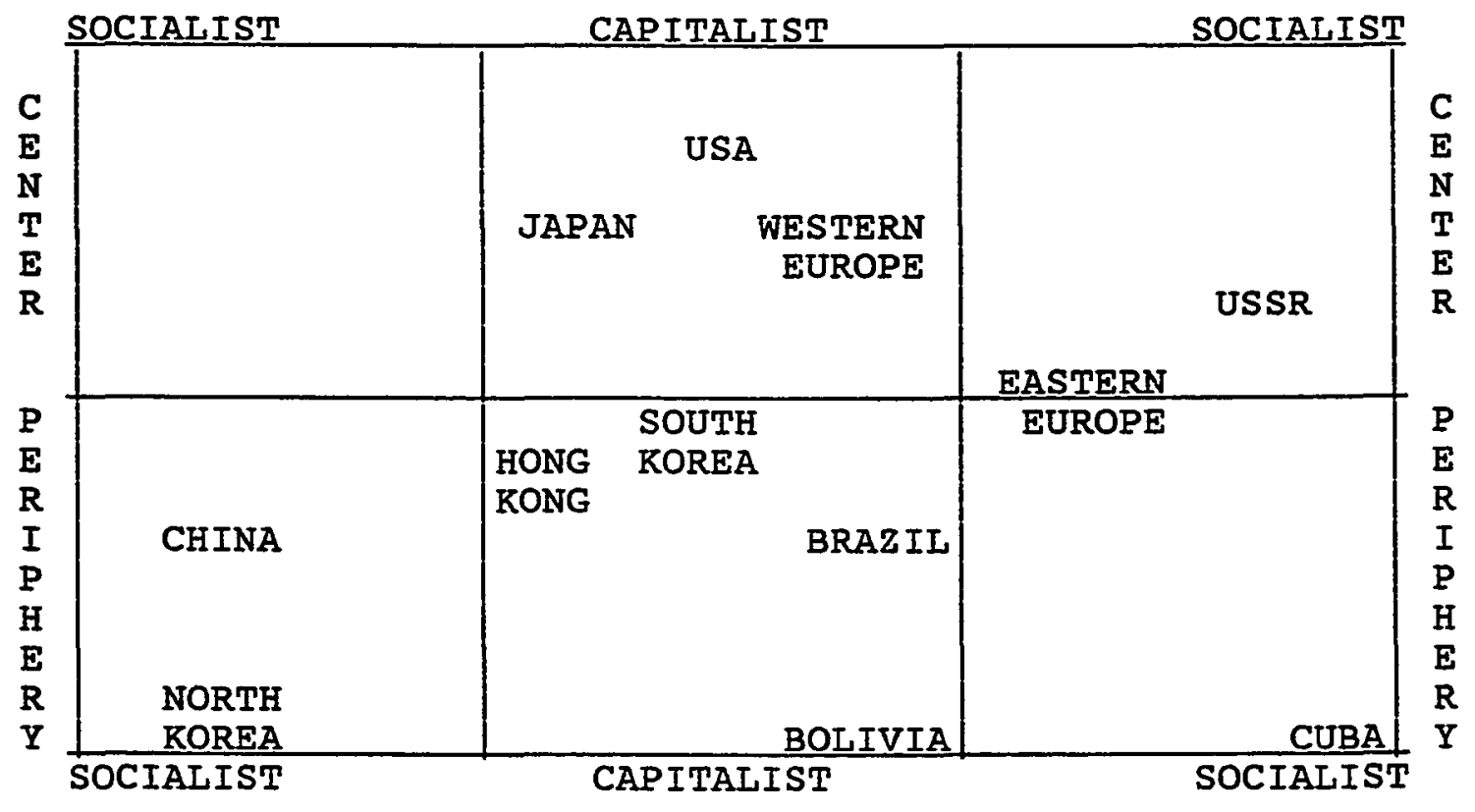

Figure 14. Center/periphery-capitalist/socialist axes.

Since World War II, the world capitalist system has been dominated by the emergence of the United States. Japan which has also emerged as a major trade power seems for now to be limited to domination of the Asian and Middle-Eastern countries. The pattern of this domination to some extent resembles the United States' domination of the Latin American countries earlier in the century. 
countries. The pattern of this domination to some extent resembles the United States' domination of the Latin American countries earlier in the century.

The growth of the United States' hierarchy has been at the expense of the older European hierarchies of the United Kingdom, France, Germany and other smaller European powers. This, by and large, has been due to the fact that after World War II, the United States found itself in an unchallenged position in the world system and therefore sought the independence of the colonies that have traditionally been the exclusive trade zones of other major hierarchies. The decolonization process for the U.S.A. not only meant the opening of those markets to American products and investments, it also provided sources of vital raw materials that were previously denied to United States. With independence, most colonies therefore found themselves uncoupled to some extent from their former European centers only to be coupled with the United States' hierarchy.

If trade hierarchies of Western European centers were in the making for centuries, the Soviet Union's hierarchy has only been a post World War Two phenomenon. While geopolitical considerations and the emerging cold war with the West have been used to explain this hierarchy, the Soviet Union's benefits from this domination deserve some investigation. With regard to COMCON, more favorable conditions of trade that have traditionally been for the DDR and Czechoslovakia( i.e. the export of investment goods in trade for raw materials and agricultural products) has been manipulated by the Soviet Union to favor their own position vis-a-viz trade with the rest of COMCON countries. China's break with the Soviet Union likewise can be viewed as a reaction against the Soviet Unions' dominance over her political and economic life. It is, however, important to state that the concept of 'social imperialism' originated in the Chinese political debate and is best illustrated by the relations between the Soviet Union and China 
during the first ten years after the Chinese Revolution, when China relied heavily on the Soviet Union.

An interesting question often asked about the Soviet Union and the Eastern European countries, is whether they are a part of the capitalist world system or not. Originally, it was believed that there would be an emergence of a socialist division of labor based on the solidarity of the socialist states. In addition, there was hope for emancipation from the world system, but a recent return of the socialist states to commodity production for the world market and their extensive dealings with capitalist multinational companies has made this emancipation an unrealistic promise. Also the rapidly rising importation of Western capital goods has been financed in large by agricultural and mineral exports used by the Western European countries. Since 1967, while the world economy has been contracting, Western capitalists have seemed to be anxious to increase trade with the socialist states. Frank suggests that this expanded 'effective division of labor' has cushioned the crisis of over production in the West ${ }^{5}$. The East-West trade, by the late 1970s, accounted for more than half of the Eastern European countries' total trade with the West, while the West's exports to the East as a whole only accounted for about 6 per cent of the West's total trade ${ }^{6}$. This suggests an increasing economic dependence of the East on the West. In addition to trade, the socialist states have become more open to foreign capital investment. This has also deepened their

5- A.G. Frank, "Long Live Transideological Enterprise! The Socialist Economies in the Capitalist International Division of Labor and West-East-South Political Economic Relations," pp. 178-262 in A.G.Frank, Crisis: In the World Economy, New York: Holmes \& Meier, 1980.

6- Frank, A.G."The European Challenge: From Atlantic Alliance to PanEuropean Entente for Peace and Jobs, " 1984, Lawrence Hill \& Company, Westport, Connecticut, p. 69. 
dependence on the West. While the Eastern European countries had a total debt of 8 billion dollars in 1971 , this debt grew to over 80 billion dollars in $1981^{7}$.

\section{RESULTS OF THE REGIONAL COMPARISONS}

To calculate regional averages for the national variables, the world is divided into seven regions, as defined by the International Monetary Fund. The regions are: 1- Industrial countries, 2- African countries, 3- Asian countries, 4Developing European countries, 5- Middle Eastern countries, 6- Latin American countries, and 7- East European countries. In addition to calculating the regional averages, significance tests for differences of means are also done: (1) between the industrialized region and other regions for 1962, 1970, and 1980; and (2) between each region at two times (1962-1970, 1970-1980, and 1962-1980). The world averages for each variable which are included in the tables of regional results, are discussed in the global studies section of this chapter.

\section{Export Partner Concentration}

Some important observations about the structure of the regional export partner concentrations can be made based on findings of Table XVIII:

1- There is no significant change in the averages for export partner concentration between any two decades for the industrialized region except for 1970-1980.

2- There is no significant change in the averages for export partner concentration for the regions of Middle East (for all three comparisons), Asia (for the 1962-70 comparison), Latin America (for the 1962-70 comparison), and Eastern Europe (for the $1970-80$ and the $1962-80$ comparisons). Other comparisons are statistically significant. However, the F-test value of 1.46 for the $1962-80$ 
TABLE XIIX

SUMMARY OF REGIONAL STATISTICS

Export Partner Concentration

\begin{tabular}{|c|c|c|c|c|c|c|c|c|c|}
\hline & \multicolumn{2}{|c|}{1962} & \multicolumn{2}{|c|}{1970} & \multicolumn{2}{|c|}{1980} & \multicolumn{3}{|c|}{ SIGNIFICANCE } \\
\hline & & & & & & & \multicolumn{2}{|c|}{$62-7070-80$} & \multirow{2}{*}{$\begin{array}{r}62-80 \\
80 \\
\end{array}$} \\
\hline & AVE. & So & AVE. & So & AVE. & SD & 62 & 70 & \\
\hline \multirow[t]{2}{*}{ INDUSTRIAL COUNTRIES } & .42 & .14 & .42 & .12 & .39 & .09 & $0.50 *$ & 2.07 & $1.17 *$ \\
\hline & (20) & & (20) & & $(20)$ & & & & \\
\hline \multirow[t]{2}{*}{ AFRICAN COUNTRIES } & .71 & .17 & .60 & .13 & .56 & .12 & 5.42 & 2.69 & 6.30 \\
\hline & (30) & & (30) & & (30) & & 6.35 & 4.89 & 5.29 \\
\hline \multirow[t]{2}{*}{ ASIAN COUNTRIES } & .60 & .19 & .56 & .17 & .52 & .15 & $1.90 x$ & 2.09 & 3.37 \\
\hline & (19) & & (19) & & (19) & & 3.58 & 2.83 & 3.15 \\
\hline \multirow[t]{2}{*}{ DEVELOPING EUR COUNTRIES } & .51 & .13 & .45 & .06 & .40 & .07 & 2.24 & 3.24 & 3.11 \\
\hline & (8) & & (8) & & (8) & & $1.65 *$ & $.49 *$ & $.29 t$ \\
\hline \multirow{2}{*}{ MIDDLE EASTERN COUNTRIES } & .54 & .16 & .49 & .10 & .51 & .09 & $1.45 *$ & $0.47 *$ & $1.13 \star$ \\
\hline & (15) & & (15) & & (15) & & 2.47 & $1.84 t$ & 3.70 \\
\hline \multirow[t]{2}{*}{ LATIN AMERICAN COUNTRIES } & .67 & .15 & .63 & .16 & .57 & .12 & $1.77 *$ & 3.24 & 5.15 \\
\hline & (29) & & (29) & & (29) & & 5.84 & 4.96 & 5.47 \\
\hline \multirow[t]{2}{*}{ EAST EUROPEAN COUNTRIES } & .57 & .12 & .50 & .07 & .49 & .11 & 3.04 & $0.38 \pi$ & $1.87 t$ \\
\hline & (6) & & (6) & & (6) & & 2.52 & $1.50 *$ & 2.18 \\
\hline \multirow[t]{2}{*}{ WORLD AVERAGE = } & .60 & .19 & .55 & .15 & .51 & .13 & 155.02 & 68.00 & 187.51 \\
\hline & (127) & & (127) & & (127) & -TEST & 1.26 & 1.15 & $1.46 t$ \\
\hline
\end{tabular}

Notes:

- Sample sizes are'given under average figures.

- The first line under significance indicates the difference of means for a given region (or the wor(d) between two different times. An * denotes that the paired t-test value is not significant.

- The second line indicates the difference of means between the industrialized region and other regions for a given time. An * denotes that t-test value (for small samples) is not significant.

- The assumption of constant world variance is tested by the F-test. An * indicatesfailure of this test (rejection of null hypothesis of no change); for this case, comparisons of regions (or the world) between these two times are not valid. 
comparison reveals that the change in the world variance is statistically significant. Since the paired t-test used in these calculations assumes constant population variance, no conclusions can be made for any of the regional averages for the 1962-1980 interval.

3- There are significant differences in the averages between the industrialized region and all other regions except for the Developing European region. This result is noteworthy since it is primarily the Industrialized region that has all the characteristics of the center and it is the other regions which constitute the periphery.

\section{Import Partner Concentration}

It is observed that the import partner concentration averages reflect some of the same characteristics as the export partner concentration averages. Averages for the Industrialized region show no significant change during the study period. Averages for the Industrialized region have significant differences with each other region, except the Developing European region. The F-test fails for both 1962-70 and 1962-80 intervals; therefore, it is only valid to discuss differences over time of particular regions during 1970-80. According to Table XX, the ratios of export and import partner concentration are always close to 1.0 for both industrialized and non-industrialized regions; this expresses the finding, discussed earlier, that EPC and IPC vary directly with one another and not inversely. The ratios show no significant change for most of the regions ( except for Africa in 62-70 and 62-80, for the Middle East in 62-80, and for Latin America for 70-80).

\section{Total Trade Linkages}

As Table XXI demonstrates, while countries in the Industrialized region on average traded with 68 countries in 1962, 84 countries in 1970, and 112 countries in 1980 , the world's averages for all region were only 25,34 , and 49 countries for 
TABLE XIX

SUMMARY OF REGIONAL STATISTICS

Import Partner Concentration

\begin{tabular}{|c|c|c|c|c|c|c|c|c|c|}
\hline & \multicolumn{2}{|c|}{1962} & \multicolumn{2}{|c|}{1970} & \multicolumn{2}{|c|}{1980} & \multicolumn{3}{|c|}{ SIGNIFICANCE } \\
\hline & \multirow[b]{2}{*}{ AVE. } & \multirow[b]{2}{*}{ SD } & \multirow[b]{2}{*}{ AVE. } & \multirow[b]{2}{*}{ SD } & \multirow[b]{2}{*}{ AVE. } & \multirow[b]{2}{*}{ SD } & \multicolumn{2}{|c|}{$62-7070-80$} & \multirow{2}{*}{$\begin{array}{r}62-80 \\
80 \\
\end{array}$} \\
\hline & & & & & & & 62 & 70 & \\
\hline \multirow[t]{2}{*}{ INDUSTRIAL COUNTRIES } & .43 & .11 & .46 & .12 & .43 & .09 & $1.19 *$ & $1.74 t$ & $0.90 *$ \\
\hline & (20) & & (20) & & (20) & & & & \\
\hline \multirow[t]{2}{*}{ AFRICAN COUNTRIES } & .71 & .18 & .55 & .10 & .51 & .09 & 6.53 & 3.89 & 7.46 \\
\hline & (30) & & (30) & & (30) & & 5.94 & 3.11 & 3.46 \\
\hline \multirow[t]{2}{*}{ ASIAN COUNTRIES } & .63 & .17 & .56 & .12 & .54 & .13 & 4.44 & $1.61 *$ & 4.85 \\
\hline & (19) & & (19) & & (19) & & 4.30 & 2.76 & 3.03 \\
\hline \multirow[t]{2}{*}{ DEVELOPING EUR COUNTRIES } & .50 & .10 & .44 & .05 & .41 & .05 & 2.29 & 4.16 & 3.27 \\
\hline & (8) & & (8) & & (8) & & $1.39 t$ & $.29 *$ & $.54 t$ \\
\hline \multirow{2}{*}{ MIDDLE EASTERN COUNTRIES } & .53 & .14 & .46 & .11 & .43 & .08 & 2.62 & $1.26 t$ & 3.13 \\
\hline & (15) & & (15) & & (15) & & 2.33 & $.12 \star$ & $.19 *$ \\
\hline \multirow[t]{2}{*}{ LATIN AMERICAN COUNTRIES } & .64 & .14 & .58 & .12 & .56 & .11 & 4.00 & $0.84 *$ & 6.22 \\
\hline & (29) & & (29) & & (29) & & 5.47 & 3.54 & 4.56 \\
\hline \multirow[t]{2}{*}{ EAST EUROPEAN COUNTRIES } & .59 & .15 & .52 & .08 & .54 & .10 & $1.96 *$ & $0.78 *$ & $1.58 t$ \\
\hline & (6) & & (6) & & (6) & & 2.68 & $1.29 *$ & 2.44 \\
\hline \multirow[t]{2}{*}{ HORLD AVERAGE = } & .60 & .17 & .52 & .12 & .50 & \multirow{2}{*}{$\begin{array}{c}.11 \\
\text {-TEST }\end{array}$} & 126.02 & 17.152 & 211.61 \\
\hline & (127) & & (127) & & $(127)$ & & $1.41 *$ & 1.09 & $1.54 \pi$ \\
\hline
\end{tabular}

Notes:

- Sample sizes are'given under average figures.

- The first line under significance indicates the difference of means for a given region (or the world) between two different times. An * denotes that the paired t-test value is not significant.

- The second line indicates the difference of means between the industrialized region and other regions for a given time. An * denotes that t-test value (for small samples) is not significant.

- The assumption of constant world variance is tested by the F-test. An * indicatesfailure of this test (rejection of null hypothesis of no change); for this case, comparisons of regions (or the world) between these two tiwes are not valid. 
TABLE XX

SUMMARY OF REGIONAL STATISTICS

IHP/EXP RATIO

\begin{tabular}{|c|c|c|c|c|c|c|c|c|c|}
\hline & \multicolumn{2}{|c|}{1962} & \multirow{2}{*}{\multicolumn{2}{|c|}{1970}} & \multirow{2}{*}{\multicolumn{2}{|c|}{1980}} & \multicolumn{3}{|c|}{ SIGNIFICANCE } \\
\hline & \multirow[b]{2}{*}{ AVE. } & \multirow[b]{2}{*}{ SD } & & & & & \multirow{2}{*}{$\begin{array}{r}62-70 \\
62 \\
\end{array}$} & \multirow{2}{*}{$\begin{array}{r}70-80 \\
70 \\
\end{array}$} & \multirow{2}{*}{$\begin{array}{r}62-80 \\
80 \\
\end{array}$} \\
\hline & & & AVE. & SD & AVE. & SD & & & \\
\hline \multirow[t]{2}{*}{ INDUSTRIAL COUNTRIES } & .95 & .13 & .93 & .12 & .92 & .12 & $1.50 \mathrm{t}$ & $0.14 x$ & $0.90 t$ \\
\hline & (20) & & (20) & & (20) & & & & \\
\hline \multirow[t]{2}{*}{ AFRICAN COUNTRIES } & 1.03 & .16 & 1.11 & .26 & 1.10 & .22 & 2.16 & $0.31 *$ & 2.35 \\
\hline & (30) & & (30) & & (30) & & $1.65 *$ & 2.89 & 3.26 \\
\hline \multirow[t]{2}{*}{ ASIAN COUNTRIES } & .96 & .14 & .98 & .19 & .97 & .20 & $0.74 t$ & $0.45 t$ & $0.32 *$ \\
\hline & (19) & & (19) & & (19) & & $.05 *$ & $1.10 x$ & $.87 \star$ \\
\hline \multirow[t]{2}{*}{ DEVELOPING EUR COUNTRIES } & 1.02 & .09 & 1.00 & .08 & 99 & .06 & $0.62 *$ & $0.53 t$ & $1.07 t$ \\
\hline & (8) & & (8) & & (8) & & $1.31 *$ & 1.63* & $1.43 t$ \\
\hline \multirow[t]{2}{*}{ MIDOLE EASTERN COUNTRIES } & 1.02 & .19 & 1.11 & .25 & 1.21 & .27 & $1.11 *$ & $1.17 *$ & 2.91 \\
\hline & (15) & & (15) & & (15) & & $1.23 t$ & 2.85 & 4.12 \\
\hline \multirow[t]{2}{*}{ LATIN AMERICAN COUNTRIES } & 1.05 & .18 & 1.09 & .15 & 1.01 & .14 & $0.93 t$ & 3.46 & $1.13 *$ \\
\hline & (29) & & (29) & & (29) & & 2.11 & 4.19 & 2.23 \\
\hline \multirow[t]{2}{*}{ EAST EUROPEAN COUNTRIES } & .99 & .09 & .97 & .06 & .92 & .14 & $0.39 *$ & $0.83 *$ & $1.00 *$ \\
\hline & (6) & & (6) & & (6) & & $.58 *$ & $.89 *$ & $.02 \star$ \\
\hline \multirow[t]{2}{*}{ WORLD AVERAGE = } & 1.01 & .16 & 1.05 & .20 & 1.03 & .20 & 99.86 & \multicolumn{2}{|c|}{32.22131 .58} \\
\hline & (127) & & (127) & & (127) & -TEST & 1.25 & 1.00 & 1.25 \\
\hline
\end{tabular}

Notes:

- Sanple sizes are given under average figures.

- The first line under significance indicates the difference of means for a given region (or the world) between two different times. An * denotes that the paired t-test value is not significant.

- The second line indicates the difference of means between the industrialized region and other regions for a given tise. An * denotes that t-test value (for small sanples) is not significant.

- The assumption of constant world variance is tested by the F-test. An * indicatesfailure of this test (rejection of null hypothesis of no change); for this case, comparisons of regions (or the world) between these two times are not valid. 
TABLE XXI

SUMMARY OF REGIONAL STATISTICS

Total Trade Linkages

\begin{tabular}{llrrrrrr}
1962 & 1970 & 1980 & \multicolumn{3}{c}{ SIGNIFICANCE } \\
& & & & & $62-70$ & $70-80$ & $62-80$ \\
AVE. SD & AVE. & SD & AVE. & SD & 62 & 70 & 80 \\
\hline
\end{tabular}

\begin{tabular}{|c|c|c|c|c|c|c|}
\hline \multirow[t]{2}{*}{ INDUSTRIAL COUNTRIES } & 68.2030 .42 & 83.6535 .311 & 111.9528 .76 & 3.96 & 5.73 & 14.13 \\
\hline & $(20)$ & $(20)$ & (20) & & & \\
\hline \multirow{2}{*}{ AFRICAN COUNTRIES } & 10.579 .59 & 15.3010 .25 & 23.6714 .94 & 4.69 & 5.51 & 6.98 \\
\hline & $(30)$ & $(30)$ & (30) & 9.72 & 10.03 & 14.23 \\
\hline \multirow[t]{2}{*}{ ASIAN COUNTRIES } & 22.1118 .78 & 28.1126 .34 & 48.6837 .00 & 2.72 & 4.57 & 4.79 \\
\hline & (19) & (19) & (19) & 5.66 & 5.55 & 5.98 \\
\hline \multirow[t]{2}{*}{ DEVELOPING EUR COUNTRIES } & 25.5013 .50 & 35.2516 .09 & 58.1321 .18 & 5.30 & 5.10 & 8.16 \\
\hline & (8) & (8) & (8) & 3.79 & 3.69 & 4.78 \\
\hline \multirow[t]{2}{*}{ HIDDLE EASTERN COUNTRIES } & 19.4310 .95 & 25.5315 .37 & 36.2717 .17 & 3.82 & 4.19 & 5.71 \\
\hline & (14) & (15) & (15) & 5.91 & 5.95 & 9.04 \\
\hline \multirow[t]{2}{*}{ LATIN AMERICAN COUNTRIES } & 15.0314 .54 & 21.7924 .13 & 32.1424 .03 & 3.07 & 3.15 & 6.03 \\
\hline & (29) & (29) & (29) & 8.18 & 7.30 & 10.54 \\
\hline \multirow[t]{2}{*}{ EAST EUROPEAN COUNTRIES } & 32.3311 .34 & 53.3317 .60 & 68.1716 .22 & 3.38 & 9.94 & 6.97 \\
\hline & (6) & (6) & (6) & 2.80 & $2.01 *$ & 3.53 \\
\hline \multirow[t]{2}{*}{ WORLD AVERAGE = } & 25.4825 .76 & 33.7232 .03 & 349.0138 .02 & 95.12 & 1506.33 & 306.38 \\
\hline & (125) & (127) & (125)F-TEST & 1.24 & 1.18 & $1.47 *$ \\
\hline
\end{tabular}

Notes:

- Sample sizes are-given under average figures.

- The first line under significance indicates the difference of means for a given region (or the world) between two different times. An * denotes that the paired t-test value is not significant.

- The second line indicates the difference of means between the industrialized region and other regions for a given time. An * denotes that $t$-test value (for small samples) is not significant.

- The assumption of constant world variance is tested by the F-test. An $\star$ indicatesfailure of this test (rejection of null hypothesis of no change); for this case, comparisons of regions (or the world) between these two times are not valid. 
the same years. In the same time period, the African and Latin American regions that historically were expected to have suffered most under colonialism had the lowest TTLs. The African region's TTLs were 11 in 1962, 15 in 1970, and 24 in 1980. TTL figures for the Latin American region were 15 in 1962, 22 in 1970, and 32 in 1980.

The improvements made in regional trade linkages suggest that, although each region has had significant improvements in the number of countries to which they export, still no region has averages comparable to those of the Industrialized region.

Comparing the results for TTL with those for EPC, it can be observed that there are many more significant changes for the TTL averages. (Although the Ftest, which shows changes in the world variances, renders inapplicable the $62-80$ comparisons for each region, still the fact that there are significant improvements in TTL from 1962 to 1970 , and from 1970 to 1980 , indicate that regional changes from 1962 to 1980 are also likely to be meaningful.) This implies that when (as measured by EPC) volume is also considered, in addition to the number of links, the level of concentration has not significantly changed, even when the number of trading partners has significantly increased (as measured by TTL). Very likely, volumes of trade with the new trading partners are low and thus do not yet effect EPC. TTL might therefore be considered to reveal new tendencies in trading relations which have not yet had time to become substantially developed.

\section{Commodity Concentration}

Several regions (especially Africa,the Middle East, and Latin America) show significant differences with the Industrialized region. No region except Latin America, exhibits any significant decline over time in its export commodity concentration. There are also significant differences between averages for all 
TABLE XXII

SUMMARY OF REGIONAL STATISTICS

Export Commodity concentration

\begin{tabular}{|c|c|c|c|c|c|c|c|c|c|}
\hline & \multicolumn{2}{|c|}{1962} & \multicolumn{2}{|c|}{1970} & \multicolumn{2}{|c|}{1980} & \multicolumn{3}{|c|}{ SIGNIFICANCE } \\
\hline & & & & & & & \multicolumn{2}{|c|}{$62-7070-80$} & \multirow{2}{*}{$\begin{array}{r}62-80 \\
80 \\
\end{array}$} \\
\hline & AVE. & So & AVE. & SD & AVE. & So & 62 & 70 & \\
\hline \multirow[t]{2}{*}{ INDUSTRIAL COUNTRIES } & .26 & .18 & .28 & .18 & .24 & .12 & $0.30 *$ & $0.96 t$ & $0.91 *$ \\
\hline & (20) & & (20) & & (20) & & & & \\
\hline \multirow[t]{2}{*}{ AFRICAN COUNTRIES } & .48 & .22 & .43 & .19 & .47 & .25 & $1.60 t$ & $1.53 t$ & $0.50 x$ \\
\hline & (24) & & (26) & & (16) & & 3.66 & 2.87 & 3.99 \\
\hline \multirow[t]{2}{*}{ ASIAN COUNTRIES } & .40 & .22 & .36 & .22 & .35 & .25 & $0.95 *$ & $0.81 *$ & $0.41 *$ \\
\hline & (13) & & (14) & & (11) & & 2.13 & $1.26 *$ & $1.75 *$ \\
\hline \multirow[t]{2}{*}{ OEVELOPING EUR COUNTRIES } & .31 & .16 & .23 & .10 & .26 & .16 & $1.85 *$ & $0.95 \star$ & $1.32 t$ \\
\hline & (5) & & (7) & & (7) & & $.65 t$ & $.61 *$ & $.50 *$ \\
\hline \multirow[t]{2}{*}{ MIDDLE EASTERN COUNTRIES } & .50 & .31 & .57 & .31 & .56 & .27 & $0.72 t$ & $0.10 *$ & $0.99 *$ \\
\hline & (10) & & (12) & & (10) & & 2.81 & 3.48 & 4.83 \\
\hline \multirow[t]{2}{*}{ LATIN AMERICAN COUNTRIES } & .57 & .22 & .48 & .22 & .42 & .19 & 2.25 & 2.48 & 3.25 \\
\hline & (24) & & (26) & & (22) & & 5.23 & 3.35 & 3.81 \\
\hline
\end{tabular}

EAST EUROPEAN COUNTRIES

$\begin{array}{llllllllll}\text { HORLD AVERAGE }= & .44 & .24 & .41 & .23 & .38 & .2394 .50 & 83.50 & 75.50 * \\ & \text { (95) } & & (84) & & (76) \text { F-TEST } & 1.04 & 1.00 & 1.04\end{array}$

Notes:

- Sample sizes are given under average figures.

- The first line under significance indicates the difference of weans for a given region Cor the wor(d) between two different times. An * denotes that the paired t-test value is not significant.

- The second line indicates the difference of means between the industrialized region and other regions for a given time. An * denotes that $t$-test value (for small samples) is not significant.

- The assumption of constant world variance is tested by the F-test. An $\star$ indicatesfailure of this test (rejection of null hypothesis of no change); for this case, comparisons of regions (or the world) between these two times are not valid. 
TABLE XXIII

SUMMARY OF REGIONAL STATISTICS

Import Commodity Concentration

\begin{tabular}{|c|c|c|c|c|c|c|c|c|c|}
\hline & \multicolumn{2}{|c|}{1962} & \multirow{2}{*}{\multicolumn{2}{|c|}{1970}} & \multirow{2}{*}{\multicolumn{2}{|c|}{1980}} & \multicolumn{3}{|c|}{ SIGNIFICANCE } \\
\hline & \multirow[b]{2}{*}{ AVE. } & \multirow[b]{2}{*}{ SD } & & & & & \multicolumn{2}{|c|}{$62-7070-80$} & \multirow{2}{*}{$\begin{array}{r}62-80 \\
80 \\
\end{array}$} \\
\hline & & & AYE. & SD & AVE. & SD & 62 & 70 & \\
\hline \multirow[t]{2}{*}{ INDUSTRIAL COUNTRIES } & .11 & .06 & .17 & .11 & .16 & .04 & 2.87 & $0.25 \star$ & 5.60 \\
\hline & (20) & & (20) & & (20) & & & & \\
\hline \multirow[t]{2}{*}{ AFRICAN COUNTRIES } & .29 & .07 & .29 & .06 & .24 & .07 & $0.68 t$ & 2.94 & 2.13 \\
\hline & (21) & & (26) & & (16) & & 9.00 & 4.67 & 4.21 \\
\hline \multirow[t]{2}{*}{ ASIAN COUNTRIES } & .24 & .13 & .21 & .10 & .17 & .05 & $0.92 *$ & $0.28 *$ & $0.00 *$ \\
\hline & (12) & & (13) & & (10) & & 3.71 & $1.25 *$ & $.50 *$ \\
\hline \multirow[t]{2}{*}{ DEVELOPING EUR COUNTRIES } & .16 & .06 & .19 & .06 & .17 & .07 & 2.43 & $1.52 *$ & $0.36 *$ \\
\hline & (5) & & (7) & & (7) & & $1.68 t$ & $.51 *$ & $.20 *$ \\
\hline \multirow[t]{2}{*}{ MIDDLE EASTERA COUNTRIES } & .25 & .08 & .29 & .11 & .27 & .08 & $0.67 t$ & $0.51 *$ & $0.31 t$ \\
\hline & (9) & & (14) & & (11) & & 5.68 & 3.29 & 5.35 \\
\hline \multirow[t]{2}{*}{ LATIN AMERICAN COUNTRIES } & .28 & .10 & .25 & .08 & .24 & .06 & $1.10 *$ & $0.89 *$ & $1.39 \%$ \\
\hline & (23) & & (25) & & (22) & & 6.26 & 2.87 & 4.88 \\
\hline
\end{tabular}

EAST EUROPEAN COUNTRIES

WORLD AVERAGE $=$

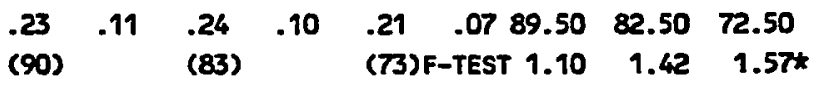

Notes:

- Sample sizes are given under average figures.

- The first line under significance indicates the difference of means for a given region (or the wor(d) between two different times. An * denotes that the paired t-test value is not significant.

- The second line indicates the difference of means between the industrialized region and other regions for a given time. An * denotes that $t$-test value (for snall samples) is not significant.

- The assumption of constant vorld variance is tested by the F-test. An * indicatesfailure of this test (rejection of null hypothesis of no change); for this case, comparisons of regions (or the wor(d) between these two times are not valid. 
regions (except for the Developing European and Asian regions) and that of the Industrialized region. In general, looking at the regional import commodity concentration averages, the same conclusions can be made as those made for export commodity concentration, suggesting similar patterns of concentration in both exports and imports. ( For ICC, the F-test invalidates the paired t-test results for 1962-1980).

\section{Vertical Trade}

Table XXIV reveals that regional averages for non-industrialized regions compared to those of the Industrialized region show significant differences. The industrialized countries have the highest VTs, and African countries, followed by Latin American and Middle Eastern countries, have the lowest VTs during the study period. The Industrialized region does not show any significant improvement except during 70-80; most other regions except for Developing European countries (1970-1980) and Latin America (all comparisons), exhibit significant improvements.

\section{Physical Onality of Life Index}

The Industrialized region exhibits average PQLIs of 91.06 for 1962, 93.67 for 1970 , and 97.24 for 1980 (Table XXV). The worst region, in terms of PQLI, is that of African countries, with average values of 16.76 in 1962, 25.19 in 1970, and 35.97 in 1980 .

The PQLI indicies show consistent improvements over time for all of the regions. With respect to PQLI the gap between Industrialized region and the developing regions has narrowed since 1962. However, significant differences between the Industrialized region and the other regions is still observed. 
TABLE XXIV

SUMMARY OF REGIONAL STATISTICS

Vertical Trade

\begin{tabular}{|c|c|c|c|c|c|c|c|c|c|}
\hline & \multirow{2}{*}{\multicolumn{2}{|c|}{1962}} & \multirow{2}{*}{\multicolumn{2}{|c|}{1970}} & \multirow{2}{*}{\multicolumn{2}{|c|}{1980}} & \multicolumn{3}{|c|}{ SIGNIFICANCE } \\
\hline & & & & & & & \multicolumn{2}{|c|}{$62-7070-80$} & \multirow{2}{*}{$\begin{array}{r}62-80 \\
80 \\
\end{array}$} \\
\hline & AVE. & SD & AVE. & SD & AVE. & SD & 62 & 70 & \\
\hline \multirow[t]{2}{*}{ INOUSTRIAL COUNTRIES } & -.02 & .40 & -.03 & .36 & .04 & .34 & $0.50 *$ & 2.41 & $1.34 t$ \\
\hline & (20) & & (20) & & (20) & & & & \\
\hline \multirow[t]{2}{*}{ AFRICAN COUNTRIES } & -.64 & .12 & -.60 & .18 & -.54 & .18 & $0.93 x$ & $1.82 x$ & $1.02 *$ \\
\hline & (21) & & (26) & & (16) & & 8.01 & 7.42 & 7.87 \\
\hline \multirow[t]{2}{*}{ ASIAN COUNTRIES } & -.41 & .33 & -.39 & .37 & -.15 & .36 & $0.77 t$ & $1.72 *$ & $1.69 *$ \\
\hline & (12) & & (12) & & (10) & & 3.31 & 3.05 & $1.66 t$ \\
\hline \multirow{2}{*}{ DEVELOPING EUR COUNTRIES } & -.40 & .34 & -.26 & .34 & .04 & .17 & $1.00 t$ & 2.49 & 2.29 \\
\hline & (5) & & (6) & & (7) & & 2.32 & $1.50 *$ & $.05 *$ \\
\hline \multirow[t]{2}{*}{ MIDDLE EASTERN COUNTRIES } & -.49 & .26 & -.53 & .29 & -.40 & .36 & $0.45 *$ & $1.07 *$ & $0.15 *$ \\
\hline & (9) & & (14) & & (11) & & 3.91 & 4.35 & 3.75 \\
\hline \multirow[t]{2}{*}{ LATIK AMERICAN COUNTRIES } & -.63 & .16 & -.53 & .20 & -.40 & .27 & 2.93 & 3.16 & 3.86 \\
\hline & (23) & & (25) & & (22) & & 7.38 & 6.27 & 5.14 \\
\hline
\end{tabular}

EAST EUROPEAN COUNTRIES

WORLD AVERAGE =

$$
\begin{array}{rrrrrrrrr}
-.44 & .35 & -.42 & .34 & -.25 & .36 & 87.50 & 80.50 & 72.50 \\
(88) & & \text { (81) } & & (73) \text { F-TEST } & 1.03 & 1.05 & 1.02
\end{array}
$$

Notes:

- Saple sizes are given under average figures.

- The first line under significance indicates the difference of means for a given region cor the wor(d) between two different times. An * denotes that the paired t-test value is not significant.

- The second line indicates the difference of weans between the industrialized region and other regions for a given time. An * denotes that t-test value (for sall samples) is not significant.

- The assumption of constant world variance is tested by the F-test. An * indicatesfailure of this test (rejection of null hypothesis of no change); for this case, comparisons of regions (or the world) between these two tiwes are not valid. 
TABLE XXV

SUMMARY OF REGIONAL STATISTICS

Quality of Life

\begin{tabular}{|c|c|c|c|c|c|c|c|c|c|}
\hline & \multirow{2}{*}{\multicolumn{2}{|c|}{1962}} & \multirow{2}{*}{\multicolumn{2}{|c|}{1970}} & \multirow{2}{*}{\multicolumn{2}{|c|}{1980}} & \multicolumn{3}{|c|}{ SIGNIF ICANCE } \\
\hline & & & & & & & \multicolumn{2}{|c|}{$62-7070-80$} & \multirow{2}{*}{$\begin{array}{r}62-80 \\
80 \\
\end{array}$} \\
\hline & AVE. & SD & AVE. & So & AVE. & so & 62 & 70 & \\
\hline \multirow[t]{2}{*}{ INDUSTRIAL COUNTRIES } & 91.06 & 3.53 & 93.67 & 2.07 & 97.24 & 1.59 & 5.83 & 11.92 & 9.07 \\
\hline & (19) & & (19) & & (20) & & & & \\
\hline \multirow[t]{2}{*}{ AFRICAN COUNTRIES } & 16.76 & 14.12 & 25.19 & 16.02 & 35.97 & 16.78 & 3.81 & 4.00 & 7.73 \\
\hline & (24) & & (17) & & (27) & & 22.99 & 18.95 & 16.22 \\
\hline \multirow[t]{2}{*}{ ASIAN COUNTRIES } & 40.36 & 23.15 & 57.12 & 26.17 & 60.48 & 24.85 & 3.04 & 3.58 & 4.38 \\
\hline & (13) & & (11) & & (15) & & 9.68 & 6.23 & 6.61 \\
\hline \multirow[t]{2}{*}{ DEVELOPING EUR COUNTRIES } & 70.49 & 18.05 & 79.15 & 14.59 & 85.44 & 11.96 & 5.85 & 5.11 & 6.15 \\
\hline & (8) & & (8) & & (8) & & 5.00 & 4.46 & 4.44 \\
\hline \multirow[t]{2}{*}{ MIDDLE EASTERN COUNTRIES } & 36.93 & 20.00 & 47.30 & 21.15 & 54.48 & 20.00 & 2.69 & 4.43 & 3.86 \\
\hline & (10) & & (12) & & (15) & & 11.92 & 9.79 & 9.57 \\
\hline \multirow[t]{2}{*}{ LATIN AMERICAN COUNTRIES } & 53.26 & 18.43 & 66.70 & 17.77 & 73.81 & 14.53 & 6.59 & 6.62 & 7.25 \\
\hline & (23) & & (24) & & (23) & & 9.03 & 6.73 & 7.16 \\
\hline \multirow[t]{2}{*}{ EAST EUROPEAN COUNTRIES } & 86.72 & 3.18 & 91.20 & 1.38 & 93.74 & 1.17 & 3.25 & 3.41 & 3.35 \\
\hline & (5) & & (5) & & (5) & & 2.70 & 2.72 & 4.97 \\
\hline \multirow[t]{2}{*}{ MORLD AVERAGE = } & 51.46 & 30.72 & 63.48 & 28.25 & 66.29 & 26.91 & 127.0 & 117.30 & 134.7 \\
\hline & (88) & & (95) & & $(102) 8$ & F-TEST & 1.08 & 1.04 & 1.14 \\
\hline
\end{tabular}

Notes:

- Sample sizes are given under average figures.

- The first line under significance indicates the difference of means for a given region (or the wor(d) between two different tiaes. An * denotes that the paired t-test value is not significant.

- The second line indicates the difference of means between the industrialized region and other regions for a given time. An * denotes that t-test value (for small samples) is not significant.

- The assumption of constant world variance is tested by the F-test. An * indicatesfailure of this test (rejection of null hypothesis of no change); for this case, comparisons of regions (or the world) between these two times are not valid. 


\section{Economic Dependence on Agriculture}

Regional studies also reveal that the smallest average AGR/GDPs belong to the industrialized countries (Table XXVI), while the African and Asian countries are the regions most dependent on agriculture. Except for Asia (1962-70, 1962-80) and Middle East (for all pairs of years), significant reductions in dependence on agriculture is observed for all other regions.

\section{RESULTS OF REGIONAL DIVERSITY CALCULATIONS}

The regional decomposition analysis is intended to calculate the diversity within and among regions and in the global system as a whole of exporting and importing activity. That is, these calculations reveal to what degree regions are similar to one another with respect to export/import activity and to what degree the countries within each region are similar to one another.

The results of regional entropy calculations for exports and imports for all three years of the study are presented in Tables XXVII through XXX. In each table column (1) represents the per cent export share of each region in the world trade; column (2) represents the within-set entropy calculations for each region; column (3) represents the weighted within-set entropy calculations; and column (4) represent the normalized within-set entropies.

The total entropy diversities (column 1, Table XXVII-A) for the exports over the 127 countries were 5.14 in $1962,5.00$ in 1970 , and 5.16 in 1980 . The maximum entropy value was $\log _{2} 127=6.97$, hence given the number of countries, the observed values were of the order of 72 to 74 per cent of the maximum. These figures are approximately constant in all three years. World export diversity has remained fairly constant over the time period of study. The figures for export diversities at most vary by 1 to 2 percent, suggesting no observable changes in the 
TABLE XXVI

SUHMARY OF REGIONAL STATISTICS

Economic Dependence on Agriculture

\begin{tabular}{|c|c|c|c|c|c|c|c|c|c|}
\hline & \multirow{2}{*}{\multicolumn{2}{|c|}{1962}} & \multirow{2}{*}{\multicolumn{2}{|c|}{1970}} & \multirow{2}{*}{\multicolumn{2}{|c|}{1980}} & \multicolumn{3}{|c|}{ SIGNIFICANCE } \\
\hline & & & & & & & \multicolumn{2}{|c|}{$62-7070-80$} & \multirow{2}{*}{$\begin{array}{r}62-80 \\
80 \\
\end{array}$} \\
\hline & AVE. & SD & AVE. & SD & AVE. & SD & 62 & 70 & \\
\hline \multirow[t]{2}{*}{ INDUSTRIAL COUNTRIES } & 9.28 & 3.94 & 7.86 & 3.62 & 5.82 & 3.32 & 3.95 & 4.64 & 3.91 \\
\hline & (13) & & (16) & & (18) & & & & \\
\hline \multirow[t]{2}{*}{ AFRICAN COUNTRIES } & 43.41 & 17.48 & 38.35 & 15.43 & 34.47 & 16.45 & 4.68 & 2.31 & 3.24 \\
\hline & (23) & & (28) & & (29) & & 8.56 & 8.66 & 7.66 \\
\hline \multirow[t]{2}{*}{ ASIAN COUNTRIES } & 32.77 & 17.04 & 31.58 & 15.12 & 26.12 & 12.77 & $1.87 *$ & 2.43 & $2.01 *$ \\
\hline & (10) & & (12) & & (13) & & 6.00 & 6.82 & 6.87 \\
\hline \multirow[t]{2}{*}{ DEVELOPING EUR COUNTRIES } & 24.33 & 10.53 & 20.67 & 7.78 & 15.00 & 5.76 & 2.60 & 3.88 & 3.40 \\
\hline & (7) & & (7) & & (7) & & 5.60 & 6.03 & 5.33 \\
\hline \multirow[t]{2}{*}{ MIDDLE EASTERN COUNTRIES } & 20.18 & 8.77 & 19.83 & 15.75 & 10.52 & 11.30 & $1.15 t$ & $1.84 t$ & $0.69 t$ \\
\hline & (5) & & (10) & & (11) & & 4.95 & 3.30 & $1.77 *$ \\
\hline \multirow[t]{2}{*}{ LATIN AMERICAN COUNTRIES } & 22.55 & 9.79 & 18.21 & 9.45 & 15.44 & 8.60 & 5.34 & 4.38 & 5.45 \\
\hline & (21) & & (22) & & (21) & & 5.73 & 4.66 & 4.75 \\
\hline
\end{tabular}

EAST EUROPEAN COUNTRIES

WORLD AVERAGE =

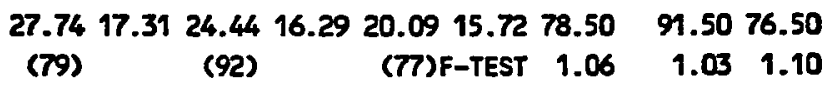

Notes:

- Sapple sizes are given under average figures.

- The first line under significance indicates the difference of weans for a given region (or the world) between two different times. An * denotes that the paired t-test value is not significant.

- The second line indicates the difference of means between the industrialized region and other regions for a given time. An * denotes that t-test value (for small samples) is not significant.

- The assurption of constant world variance is tested by the f-test. An $\star$ indicatesfailure of this test (rejection of null hypothesis of no change); for this case, comparisons of regions (or the world) between these two times are not valid. 
TABLE XXVII-A

SUMHARY OF REGIONAL EXPORT DIVERSITY CALCULATIONS

\begin{tabular}{ccccccccccc} 
& MORLD & \multicolumn{1}{c}{ WEIGHTED UITHIN } \\
YEAR & TOTAL & BETWEEN & UITHIN & 1 & 2 & 3 & 4 & 5 & 6 & 7 \\
\hline 1962 & 5.14 & 1.72 & 3.42 & .15 & .20 & .27 & .03 & .29 & .13 & 2.36 \\
1970 & 5.00 & 1.66 & 3.34 & .11 & .16 & .26 & .03 & .29 & .13 & 2.37 \\
1980 & 5.16 & 1.82 & 3.33 & .12 & .25 & .17 & .03 & .20 & .27 & 2.29
\end{tabular}

TABLE XXVII-B

SUMMARY OF REGIONAL IMPORT DIVERSITY CALCULATIONS

\begin{tabular}{ccccccccccc} 
& MORLD & & \multicolumn{1}{c}{ WEIGHTED WITHIN } \\
YEAR & TOTAL & BETUEEN & WITHIM & 1 & 2 & 3 & 4 & 5 & 6 & 7 \\
\hline 1962 & 5.30 & 1.77 & 3.53 & .15 & .25 & .29 & .06 & .29 & .102 .40 \\
1970 & 5.21 & 1.80 & 3.41 & .13 & .23 & .28 & .06 & .33 & .092 .29 \\
1980 & 5.26 & 1.78 & 3.48 & .13 & .27 & .20 & .07 & .25 & .192 .38
\end{tabular}

Labels 1 through 7 are regions defined as below:

$1=$ African countries,

$2=$ Asian countries,

3 = Eastern European countries,

4 = Developing European countries,

$5=$ Lat in American countries,

6 = Middle Eastern countries,

and $7=$ Industrialized countries. 
TABLE XXVIII-A

SUMMARY OF REGIONAL EXPORT DIVERSITIES

YEAR= 1962

\begin{tabular}{lccccc}
\multicolumn{1}{c}{$\begin{array}{c}\text { (1) } \\
\text { REGIONS }\end{array}$} & $\begin{array}{c}\text { (2) } \\
\text { GLOFAL TREION TO }\end{array}$ & $\begin{array}{c}\text { (3) } \\
\text { UEIGHTED } \\
\text { UITHIN }\end{array}$ & $\begin{array}{c}\text { (4) } \\
\text { NORMAL } \\
\text { UITHIN }\end{array}$ \\
\hline AFRICAN COUNTRIES & 3.81 & 3.88 & .15 & .79 \\
ASIAN COUNTRIES & 5.93 & 3.36 & .20 & .79 \\
EAST EUROPEAN COUNTRIES & 10.90 & 2.47 & .27 & .83 \\
DEVELOPING EUR COUNTRIES & 1.32 & 2.05 & .03 & .80 \\
LATIN AMERICAN COUNTRIES & 7.68 & 3.75 & .29 & .76 \\
MIDDLE EASTERN COUNTRIES & 4.24 & 3.01 & .13 & .77 \\
INDUSTRIAL COUNTRIES & 66.12 & 3.57 & 2.36 & .84
\end{tabular}

BETNEEN REGION DIVERSITY= 1.72

TOTAL MEIGHTED UITHIK= 3.42

GLOBAL DIVERSITY MEASURE $=\mathbf{5 . 1 4}$

MORH GLOBAL DIY. MEASURE= $\quad .74$

NORMLLIZED BETHEEN DIV. $=\quad .61$

TABLE XXVIII-B

SUMMARY OF REGIONAL IMPORT DIVERSITIES

YEAR= 1962

(1) (2) (3)

* OF REgION TO WEIGHTED NORHAL

\begin{tabular}{lccccc}
\multicolumn{1}{c}{ REGIONS } & GLOBAL TRADE & UITHIN & HITHIN & UITHIN \\
\hline AFRICAN COUNTRIES & 3.87 & 3.81 & .15 & .78 \\
ASINH COUNTRIES & 7.21 & 3.45 & .25 & .81 \\
EAST EUROPEAN COUNTRIES & 11.39 & 2.55 & .29 & .85 \\
DEVELOPING EUR COUNTRIES & 2.72 & 2.09 & .06 & .81 \\
LATIN AMERICAN COUNTRIES & 7.16 & 4.02 & .29 & .82 \\
MIDDLE EASTERH COUNTRIES & 2.82 & 3.37 & .10 & .87 \\
IHOUSTRIAL COUNTRIES & 64.83 & 3.71 & 2.40 & .87
\end{tabular}

BETUEEN REGION DIVERSITY= 1.77

TOTAL WEIGHTED WITHIN= 3.53

GLOBAL DIVERSITY MEASURE $=\mathbf{5 . 3 0}$

NORM GLOBAL OIY. MEASURE $=\quad .76$

HORMLIZED BETUEEN DIV. $=\quad .63$ 
TABLE XXIX-A

SUMHARY OF REGIONAL EXPORT DIVERSITIES

$Y E A R=1970$

\begin{tabular}{|c|c|c|c|c|}
\hline REGIONS & $\begin{array}{c}\text { (1) } \\
\% \text { OF REGION TO } \\
\text { GLOBAL TRADE }\end{array}$ & WITHIN & $\begin{array}{l}\text { (3) } \\
\text { WEIGHTED } \\
\text { UITHIN } \\
\end{array}$ & $\begin{array}{l}\text { (4) } \\
\text { NORMAL } \\
\text { UITHIN }\end{array}$ \\
\hline AFRICAN COUNTRIES & 2.73 & 3.94 & .11 & .80 \\
\hline ASIAN COUNTRIES & 4.76 & 3.44 & .16 & .81 \\
\hline EAST EUROPEAN COUNTRIES & 9.82 & 2.64 & .26 & .88 \\
\hline DEVELOPIHG EUR COUNTRIES & 1.53 & 1.92 & .03 & .74 \\
\hline LATIN AMERICAN COUNTRIES & 9.08 & 3.15 & .29 & .64 \\
\hline MIDDLE EASTERN COUNTRIES & 4.27 & 3.13 & .13 & .80 \\
\hline INDUSTRIAL COUNTRIES & 67.82 & 3.49 & 2.37 & .82 \\
\hline
\end{tabular}

BETUEEN REGION DIVERSITY $=1.66$

TOTAL UEIGHTED UITHIN= 3.34

GLOBAL DIVERSITY MEASURE $=5.00$

MORM GLOBAL DIV. MEASURE $=\quad .72$

MORHALIZED BETWEEN DIV.= .59

TABLE XXIX-B

SUHMARY OF REGIONAL IMPORT OIVERSITIES

YEAR $=1970$

\begin{tabular}{lccccc}
\multicolumn{1}{c}{$\begin{array}{c}\text { (1) } \\
\text { K OF REGION TO } \\
\text { GLOBAL TRADE }\end{array}$} & UITHIN & $\begin{array}{c}\text { (2) } \\
\text { WEIGHTED } \\
\text { UITHIN }\end{array}$ & $\begin{array}{c}\text { (4) } \\
\text { MORHAL } \\
\text { UITHIN }\end{array}$ \\
\hline AFRICAN COUNTRIES & 3.45 & 3.68 & .13 & .75 \\
ASIAN COUNTRIES & 6.25 & 3.61 & .23 & .85 \\
EAST EUROPENN COUNTRIES & 10.21 & 2.71 & .28 & .90 \\
DEVELOPING EUR COUNTRIES & 3.12 & 2.01 & .06 & .78 \\
LATIN AMERICAN COUNTRIES & 10.55 & 3.14 & .33 & .64 \\
MIDDLE EASTERN COUNTRIES & 2.68 & 3.41 & .09 & .87 \\
INDUSTRIAL COUNTRIES & 63.74 & 3.59 & 2.29 & .85
\end{tabular}

BETHEEN REGION OIVERSITY $=1.80$

TOTAL MEIGHTED UITHIN= 3.41

GLOBAL DIVERSITY MEASURE $=\mathbf{5 . 2 1}$

MORH GLOBAL DIV. MEASURE= .75

NORHALIZED BETUEEA DIV. $=\quad .64$ 
TABLE XXX-A

SUMMARY OF REGIONAL EXPORT DIVERSITIES

YEAR $=1980$

\begin{tabular}{|c|c|c|c|c|}
\hline REGIONS & $\begin{array}{c}\text { (1) } \\
\text { \% OF REGION TO } \\
\text { GLOBAL TRADE }\end{array}$ & WITHIN & $\begin{array}{c}\text { (3) } \\
\text { WEIGHTED } \\
\text { UITHIN }\end{array}$ & $\begin{array}{l}\text { (4) } \\
\text { NORHAL } \\
\text { UITHIN }\end{array}$ \\
\hline AFRICAN COUNTRIES & 3.90 & 3.13 & .12 & .64 \\
\hline ASINN COUNTRIES & 7.28 & 3.39 & .25 & .80 \\
\hline EAST EUROPEAN COUNTRIES & 7.08 & 2.45 & .17 & .82 \\
\hline DEVELOPING EUR COUNTRIES & 1.81 & 1.71 & .03 & .66 \\
\hline LATIN AHERICAN COUNTRIES & 5.42 & 3.69 & .20 & .75 \\
\hline MIDDLE EASTERN COUNTRIES & 10.92 & 2.47 & .27 & .63 \\
\hline INDUSTRIAL COUNTRIES & 63.59 & 3.60 & 2.29 & .85 \\
\hline BETUEEN REGION DIVERSITY= & 1.82 & & & \\
\hline TOTAL WEIGHTED WITHIN & 3.33 & & & \\
\hline GLOBAL DIVERSITY MEASURE= & 5.16 & & & \\
\hline NORH GLOBAL DIV. MEASURE= & .74 & & & \\
\hline NORMALIZED BETWEEN DIV.= & .65 & & & \\
\hline
\end{tabular}

TABLE XOCX-B

SUMHARY OF REGIONAL IMPORT DIVERSITIES

YEAR $=1980$

(1) (2) (3) (4)

\% OF REGION TO WEIGHTED NORHAL

GLOBAL TRADE WITHIN WITHIN WITHIN

AFRICAN COUNTRIES

3.43

3.64

.74

ASIAN COUNTRIES

7.87

3.38

.13

.80

EAST EUROPEN COUNTRIES

7.59

2.67

.27

.89

DEVELOPING EUR COUNTRIES

3.39

1.96

.20

.76

LATIN AMERICAN COUNTRIES

6.43

3.88

.79

MIODLE EASTERN COUATRIES

5.53

3.36

.25

.86

IMDUSTRIAL COUNTRIES

65.76

3.63

.19

.86

BETUEEN REGION OIVERSITY $=1.78$

TOTAL WEIGHTED WITHIN $=3.48$

GLOBAL DIVERSITY MEASURE $=\mathbf{5} .26$

MORH GLOBAL DIV. MEASURE= .75

MORMALIZED BETUEEN DIV. $=\quad .64$ 
world system by this measure ( however, it is not possible to conduct any error estimates, or significance test for this measure.) World import diversities also do not exhibit any changes during the study period. Normalized global diversity figures are constant, fairly high, and similar between exports and imports.

\begin{tabular}{cccc}
\multicolumn{2}{c}{ NORMALIZED REGIONAL } & \multicolumn{2}{c}{ DIVERSITIES } \\
& & & \\
& 1962 & 1970 & 1980 \\
\hline EXPORT & 0.74 & 0.72 & 0.74 \\
IMPORT & 0.76 & 0.75 & 0.75 \\
(taken from Tables 29, & 30, and 31 )
\end{tabular}

The second column of Table XXIIX-A contains the between-set entropies and the third column contains the total within set entropies. The latter consistently exceeds the former and the differences are substantial. The between set entropies varied between 1.66 and 1.82 bits; the maximum was $\log _{2} 7=2.80$; hence representing the diversity among different regions of the world- the observed values are about 59 to 65 per cent of the maximum.

Most of the world export or import diversity (homogeneity) was due to the within-region components. For example, in 1962, 67 per cent of world export diversity of 5.14 was due to the within-region diversity of 3.42 . Even though this figure is largely due to the industrialized region, since it is heavily weighted in the sum (since it has a large proportion of the total trade activity), still the diversities of other regions were high (column 2, tables XXIX-A through XXXI-A), so if weighting were different, one would still get dominance of the within-region contribution. This is clearly shown by the normalized diversity figures (below), which reveal that the within-region diversities are closer to their maximum possible values:

$\begin{array}{cccc} & & \begin{array}{c}\text { NORMALIZED } \\ \text { BETWEEN }\end{array} & \begin{array}{c}\text { NORMALIZED } \\ \text { WITHIN }\end{array} \\ \text { EXPORTS } & 1962 & 0.61 & 0.76 \text { TO } 0.84 \\ 1970 & 0.59 & 0.64 \text { TO } 0.88 \\ & 1980 & 0.65 & 0.66 \text { TO } 0.85\end{array}$


NORMALIZED

BETWEEN
NORMALIZED

WITHIN

$\begin{array}{lllll} & 1962 & 0.63 & 0.78 & \text { TO } 0.87 \\ \text { IMPORTS } & 1970 & 0.64 & 0.64 & \text { TO } 0.90 \\ 1980 & 0.64 & 0.76 \text { TO } 0.89\end{array}$

It can, be concluded that regions are somewhat more homogeneous internally, i.e., with respect to the countries in region, than the world is homogeneous with respect to regions. This is an argument for the existence of differentiation within the world system. However, no evidence48flefiy substantial change in the world system, as measured by diversity indices, are observable over the time period of this study.

It is interesting to note that all regions are fairly diverse (homogeneous). Looking at the column (4) of Tables XXVIII-A through XXX-A, all the normalized within figures are fairly high. Other regions have (internally) diversities comparable to that of the industrialized region.

Particular regions also show greater variability over time in (internal) diversity than the world did as a whole. This is shown by the following figures:

NORMALIZED GLOBAL EXPORT DIVERSITIES

\begin{tabular}{|c|c|c|c|c|}
\hline WORID & $\begin{array}{l}\text { EXPORT } \\
\text { IMPORT }\end{array}$ & $\begin{array}{l}\frac{1962}{0.74} \\
0.76\end{array}$ & $\begin{array}{l}1970 \\
0.72 \\
0.75\end{array}$ & $\begin{array}{l}\frac{1980}{0.74} \\
0.75\end{array}$ \\
\hline $\begin{array}{l}\text { EASTERN } \\
\text { DEV } \\
\text { IATIN } \\
\text { MIDDI }\end{array}$ & $\begin{array}{l}\text { AFRICA } \\
\text { EUROPE } \\
\text { EUROPE } \\
\text { AMERICA } \\
\text { LE EAST }\end{array}$ & $\begin{array}{l}0.79 \\
0.83 \\
0.80 \\
0.76 \\
0.77\end{array}$ & $\begin{array}{l}0.80 \\
0.88 \\
0.74 \\
0.64 \star \\
0.80\end{array}$ & $\begin{array}{l}0.64 * \\
0.82 \\
0.66 * \\
0.75 \\
0.63 *\end{array}$ \\
\hline
\end{tabular}

*- relatively big change. 
These changes would require, for their interpretation some consideration of world and regional historical events over this period, this, however, is not in the domain of the current study.

\section{RESULTS OF SYSTEMWIDE STUDIES}

\section{GLOBAL POLARIZATION}

QUESTION: What level of polarization exists in the world system and for the period of 1962 to 1980 has polarization been increasing or decreasing?

In order to answer the above question, a measure of centrality from graph theory is used as described in the chapter on methodology. The Global Polarization Index measures the degree of world system's centralness in the sense of the tendency of a single country to be more central than all other countries in the world trade network.

This measure has a range of 0 to 1 . An index of 0 would indicate that the trade network is homogeneous with each country being linked to all other countries. An index of 1.0 would indicate that there exists only one central country and all other countries are linked only to that country.

The Global Polarization indices for all three years of the study as indicated by Figure 15 exhibit a high degree of systemic polarization: $0.70,0.69$, and 0.67 . The relative constancy of these values suggest that from 1962 to 1980 , a strong degree of polarization has been a stable property of the world system.

\section{GLOBAL CONCENTRATION}

QUESTION: What level of global concentration exists in the world system and has global concentration in the world system been increasing or decreasing between 1962 and 1980? 
While Global Polarization index considers only the trade linkages, the Global Concentration measures the volume of trade for each of the trading linkages.

This measure has a range of 0 to 1.0 with 1.0 indicating perfect concentration. The calculated results of $0.52,0.53$, and 0.52 shown in Figure 15 indicate moderately high degrees of concentration during the sample years. The pattern of concentration is basically constant as was the index of Global Polarization. Factors contributing to the global concentration are: 1 - the very high total volume of trade for industrialized countries ( 64 per cent of world trade in 1962), and 2- the very high Import and Export Partner Concentrations for all of the other six regions.

\section{GLOBAL A VERAGES}

Global averages and standard deviation values for variables used in the regional study are listed in table XXXI. The following observations can be made about the variation in these indicators:

1- Due to the large sample size for these global average calculations, all changes over time (not including those rejected by the F-test) are substantially significant and are in the direction of a reduction of average world peripheralness. For example, EPC declines, EEC declines, VT increases, and PQLI increases. While such average improvements could be consistent with increasing, diminishing, or constant differences between developed and developing countries, the regional results discussed earlier point to either constancy or reduction of such differences, i.e. to a lesser degree of world system differentiation.

2- The global average for Export Partner Concentration (EPC) exhibited a relative improvement of about 15 per cent from 1962 to 1980 . Global averages for 


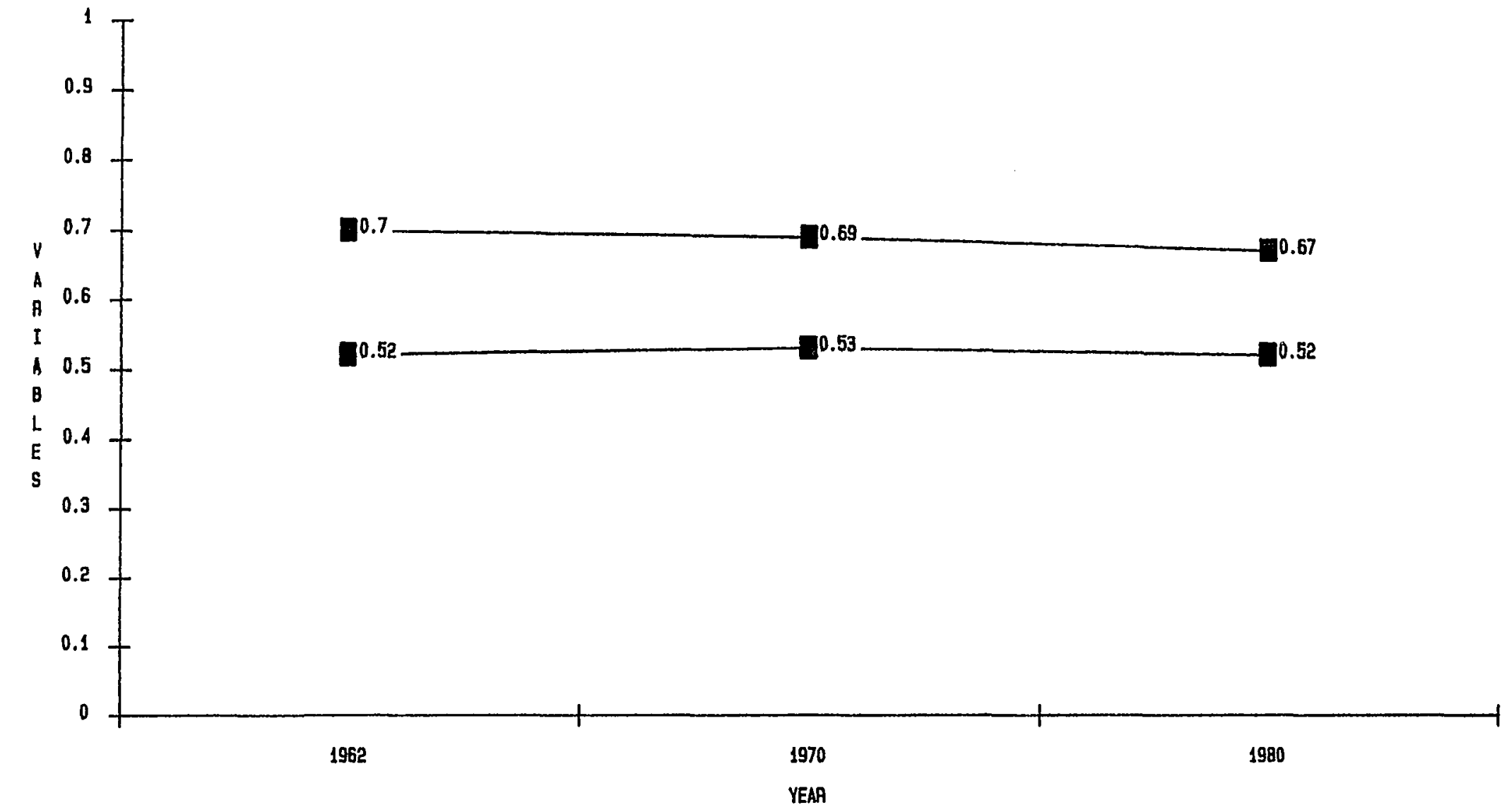

9 polaAization CONCENIRation

Figure 15. Global Measures of Polarization and Concentration. 


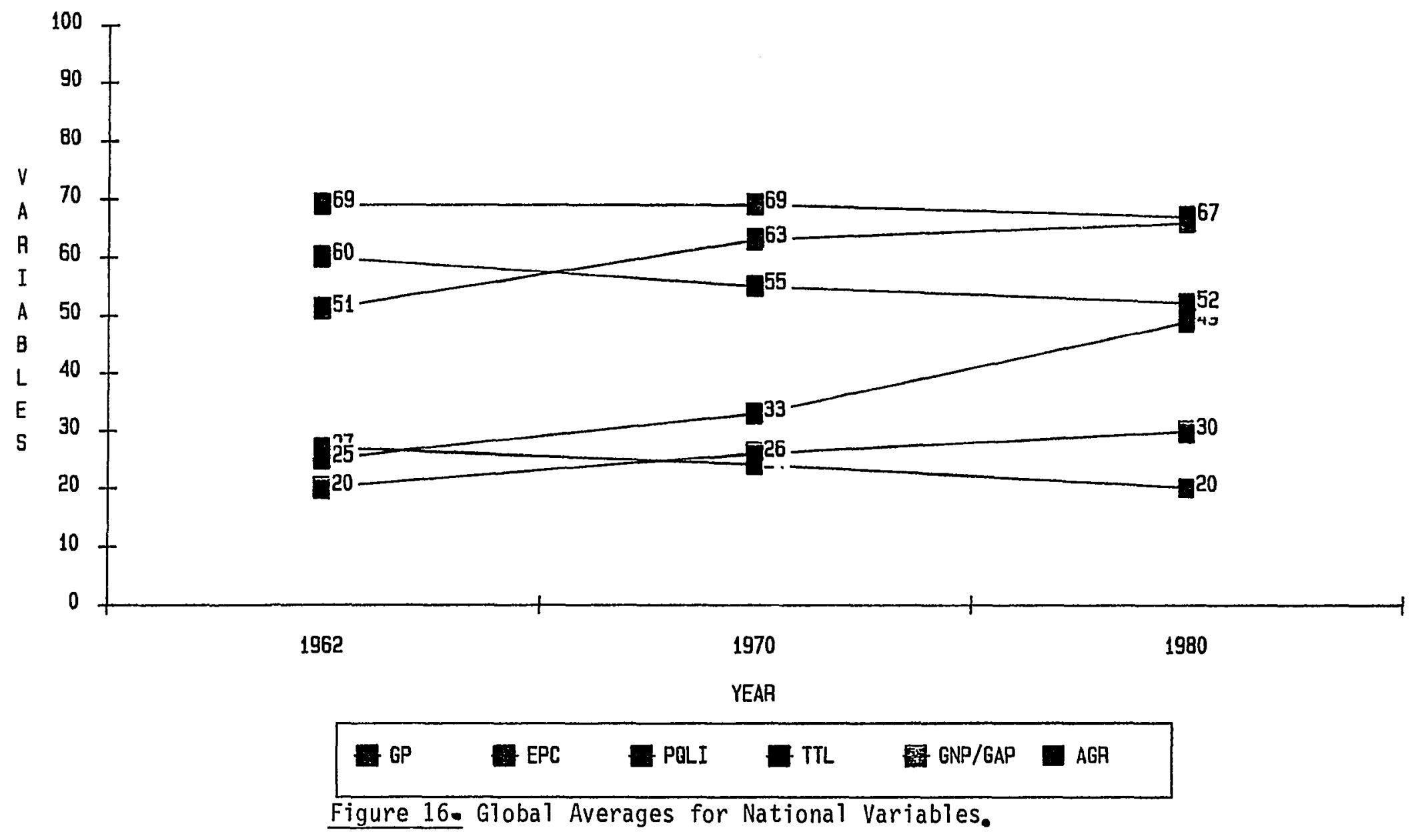


TABLE XXXI

SUMHARY OF GLOBAL STATISTICS

VARIABLES

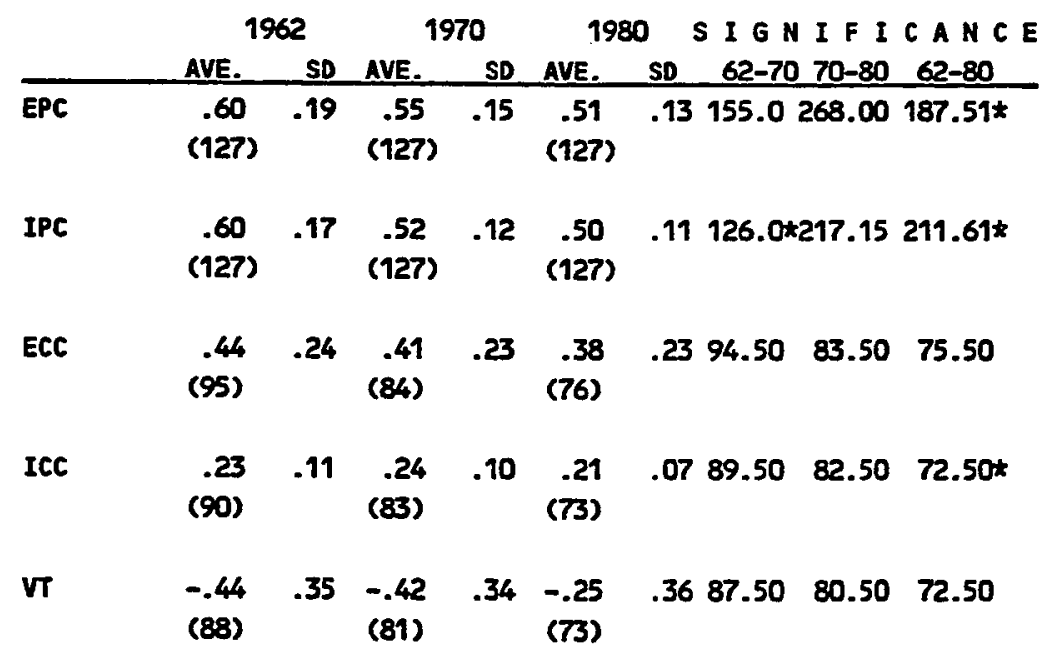

PQLI $\quad 51.4630 .7263 .4828 .2566 .2926 .91 \quad 127.5117 .30134 .70$ (88) (95) (102)

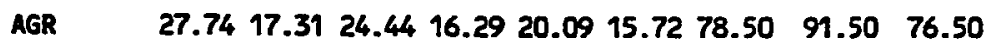
(79) (92) (77)

TTL $\quad 25.4825 .7633 .7232 .0349 .0138 .0295 .15$ 1506.3 306.38t (125) (127) (125)

- The sample sizes are given under averoge figures.

- The difference of means for a given variable between two different time intervals are calculated by the paired t-test. An * denotes that the F-test has failed and significance values for paired $t-$ test not valid. 
Import Partner Concentration (IPC) also exhibited similar change to those of EPC, underlining similarity of import and export activity at the country, regional, and global levels of analysis.

3- While the global averages for Export Commodity Concentration (ECC) and for Import Commodity Concentration (ICC) have significant changes between any two years of the study, commodity concentrations remain basically constant during this period. These variables show the smallest percent change from 1962 to 1970 (ECC: 14\%, ICC: 9\%).

4- The Global averages for the Vertical Trade index show large improvements during 1962 to 1980 (43\%).

5- The averages for the Global Physical Quality of Life exhibited a $29 \%$ improvement from 1962 to 1980 . The average PQLI of 51.46 in 1962 rose to 66.29 by the year 1980 .

6- The averages for the percent of Agriculture in GDP for the world also exhibited a $28 \%$ improvement in this period. The share of agriculture in GDP dropped from 27.74 in 1962 to 20.09 in 1980.

7- The averages for the Total Trade Linkages (TTL) had the largest improvement in the study period. The average global TTL which was 25.48 in 1962 rose about $92 \%$ to 49.01 in 1980.

One can look, not only at the global averages of the national variables, but also at the standard deviations of these average values, particularly where changes in the magnitudes of the averages and of the standard deviation are in opposite directions. The most striking illustration of this is given by PQLI, where the standard deviation declines (from 30.7 to 26.9) even though the average PQLI increases (from 51.5 to 66.3 ). The magnitude of the reduction in variability in PQLI is not very great, but, superimposed on a rise in its average level, this 
observation is an aspect of reduced world system differentiation during this time interval. The boundedness of PQLI coupled with the fact that the industrialized countries have nearly achieved their maximum possible values suggests, however, that this variable may not be a sensitive indicator of degree of differentiation in the world system. 


\section{CHAPTER VI}

\section{CONCLUSIONS}

\section{Theoretical Context}

Two leading perspectives on international trade and economic relations have been identified. The classical view regards the international division of labor through free trade as promoting economic development. The dependency theory, contrary to classical theory, views the international division of labor as an obstacle to the economic development of the now-underdeveloped countries.

The essence of dependency theory and Galtung's structural theory of imperialism is the division of the world into center and periphery countries, themselves divided into center and periphery sectors. The formation of centerperiphery relationships among countries is said by dependency theorists to have started with the expansion of the European capitalist system, which over the past centuries integrated the other regions of the world into its sphere of influence by political domination and colonization.

Dependency theory, in contrast with the orthodox view, asserts that the trade expansion of the European countries with their colonies did not originate from a basis of free trade and comparative advantage, but from the imposition of mercantile regulations, and the restricting the trade of most colonies (if not all) to a single European country. The monopolization of colonial trade by different European countries showed great similarity in their trade practices. All excluded foreign ships from colonial ports, insisted that colonial trade pass through metropolitan ports in both directions, and forbade the competition of colonial 
products or manufactures with those of the metropolis. These restrictions practically shut out the colonial countries' commercial opportunities with other countries.

While the European countries were free to extend their trade with other countries, autonomously develop their own agricultural and mineral outputs, and build their own industries, growth in the colonies was only an extension of developments in the metropolis. According to dependency theory, these mercantile and other colonial regulations established an international division of labor between periphery countries that produced agricultural and mineral products consumed by the center and the center countries that produced manufactured products consumed by both center and periphery. This general division of labor, according to dependency theory, persists to this day, although the location of individual nations within this world system has not necessarily remained constant over time. This "feudal" interaction structure has led to differential spin-off ef fects primarily going to the center countries, and, more generally, has produced a gap in income and in the quality of living conditions between nations. There is, according to Galtung, a dependency of periphery on the center. This dependence, which arises from an artificial division of labor, is said to be the essence of the unequal relationship between the center and periphery countries.

The decolonization of Asian, African, and Latin American countries after World War II opened the possibility that with the gain of independence and the end of direct colonial rule, these countries would follow paths that would allow them to industrialize their economies and improve their living conditions and income levels. Instead, according to dependency theorists, the continuing ability of the center countries and multinational companies to interfere politically and 
economically in the economic activities of the periphery countries allowed a new era of neocolonialism to replace the old colonial period.

The dependency school and other structural theorists who have maintained the existence of this neocolonialism, have described means to identify levels of dependence within the world system and have of fered hypotheses about a number of characteristics of the center-periphery dichotomy in international trade relations.

Dependency theory divides countries into distinct categories of center and periphery with a third category of semi-periphery occupying the middle ground. According to Galtung and others, there is in the periphery, a concentration of trade partners, with each periphery country having most of its trade with one center country, while that center country is free to extend its trade relations in any direction. Second, there is commodity concentration, with periphery countries having only few primary commodities to export. And third, center-periphery coupling has produced a gap in the quality of living conditions between these countries.

These hypotheses have been the subject of a number of studies by Galtung and other researchers. While the findings of most of those studies have been in support of center-periphery hypothesis, the studies themselves have been limited in the time period studied, in the variables used, or in the number of countries studied.

In the present study, the three decade period of 1960 to 1980, during which more than 90 countries (appendix B) gained their independence, is selected to test the center-periphery hypotheses. This period is examined at three particular (and arbitrarily selected) years: 1962, 1970, and 1980. Data from World Bank is more complete for 1962 than for 1960, and thus chosen instead of 1960 . The study 
considers 127 countries, uses variables considered in prior studies, and adds new variables by which both synchronic and diachronic features of the world system at national, regional and global levels could be quantitatively investigated. At the national level, the interrelationship of variables is studied; at the global level, the focus is on temporal changes, and interrelationships are not examined.

The national properties of the center-periphery relationship are operationalized using ten variables: 1- Trade Partner Concentration, 2- Total Trade Linkages, 3- Import Partner Concentration, 4- Export Commodity Concentration, 5Import Commodity Concentration, 6- Vertical Trade, 7- Physical Quality of Life Index, 8- Percent share of GDP on Agriculture, 9- GNP per capita, and 10- Export Dependency. Bivariate correlation calculations are done between all pairs of variables for the 127 countries for the years 1962, 1970, and 1980. Since the concentration of trade Partner had historically been the fundamental tool of colonial rule, and has had the greatest impact on the shaping of the centerperiphery structure,the variable TPC is selected for use as the independent variable in this study. ( This is primarily for clarity of presentation; all of the variables studied should be seen as being interrelated.)

The system-wide behavior of the world system was investigated by three different analyses of import-export data: 1- developing export trade hierarchies, to identify the center and periphery countries and to see how these hierarchies change with time, 2- conducting regional decomposition calculations on world export and imports to find the degree of diversity of import and export shares within and among various regions of the world, and 3- measuring Systemic Polarization and Global Concentration, by which temporal changes in the concentration of import-export linkages in the world system as a whole might be examined; and by 4- calculating regional and global averages for the individual 
variables used in the national study (e.g. TPC, VT, etc.) to see if there were significant changes over the time period of this study.

In addition, using the League of Nations' data for the year 1938, this study also conducts a limited examination for some trade variables for 64 countries.

\section{SUMMARY}

\section{Discussion of the National Hypotheses}

At the national level, for the years sampled, the correlation coefficients among most of the variables range from medium to strong in the expected directions. This confirms the hypotheses suggested by the center-periphery dichotomy. All correlations are statistically significant at 0.05 level.

The correlation results reveal that: 1 - the degree of association between Export Partner Concentration and Import Partner Concentration indices are high for all three years $(0.85,0.73$, and 0.68 for 1962,1970 and 1980 respectively); 2countries with high degrees of Export Partner Concentration also have high degrees of commodity concentrations in their exports as well as their imports. Consistent with this, Import and Export Commodity Concentrations are found to be positively correlated $(0.48,0.47$, and 0.51 for 1962,1970 , and 1980$)$; this result is contrary to the generally held view that periphery countries that export few commodities import a diverse range of commodities that their economies require $^{1}$; 3 - countries that had high Trade Partner Concentrations were also found to be low in Vertical Trade, suggesting that partner concentration is not consistent with a favorable position in the international division of labor; 4- countries whose Export Partner Concentration figures are high are found to be those countries that

1- While the small number of sectors used in the concentration calculations might cast doubt on this result, as discussed in chapter 4, similar results were obtained by Michaely who used more sectors. 
have allowed agriculture to have a major part in their economy $(0.42,0.32$, and 0.25); 5- lower levels of Physical Quality of Life Index is also found to be associated with high Trade Export Partner Concentration ( $-0.48,-0.36$, and 0.45); 6- countries whose exports to their largest trade partner are a large percentage of their national income are also those countries that have higher Export Partner Concentration ( $0.57,0.40$, and 0.55 ) and Export Commodity Concentration ( $0.28,0.40$, and 0.44.) In fact, aside from Import Partner Concentration, Export Partner Concentration has the strongest correlations with Export Dependency; and, 7- low levels of Trade Partner Concentration is found to be associated $(-0.34)$ with high levels of GNP per capita in the single year (1980) for which GNP data is used.

In terms of national income, it is found that low Export Partner Concentration, low share of agriculture in their GDP, and high positions in Vertical Trade, are associated with high capita GNP ( correlations of $\mathbf{- 0 . 6 6 , - 0 . 3 4}$, and $\mathbf{0 . 4 5}$, respectively, for 1980 and for oil exporting countries excluded from the data). High per capita GNP is, as expected, associated with high degrees of processing and industrialization. This is consistent with the argument that over the past two centuries, the widening of the gap in per capita GNP ( as exhibited by Table II) can be explained by the industrialization of the center countries and the export specialization of periphery countries' in primary or extractive commodities. ${ }^{2}$ In 1980 , the GNP gap in terms of ratios between the industrialized region and Africa is 10:1. If the top ten richest countries are compared to the ten

2- The center-periphery structure was established before the industrial revolution and was thus associated initially with moderate GNP per capita gaps; after the industrial revolution the extent of specialization in processed goods versus primary products increased and with it also the GNP gap. The present study does not examine this two century time period; temporal changes in the twenty year period examined in this study are discussed in the next section. 
poorest countries, the gap increases to $49: 1$. When the gap between the richest country and the poorest country is compared, the ratio is $94: 1$. These facts illuminate the sharp differences in the GNP's per capita at the extremes of the center and periphery.

The high correlations between Trade Partner Concentration and other national variables are consistent with the central notions of the center and periphery hypothesis. Trade partner concentration can clearly be used as an indicator of centralness or peripheralness of a given country. It is interesting to observe that a "mere" diversity measure (Trade Partner Concentration) is so clearly linked to many other, more familiar, variables and thus reveals much about center-periphery structure. Total Trade Linkages, conceptually, is a closely related variable; its correlations with other variables are also very high, and it can also be used as a simple indicator of centralness and peripheralness.

Also observed are high correlations between Physical Quality of Life Index with GNP/capita and with the lack of dependence of GDP on agriculture.

One variable that consistently gives results in the opposite direction of what is expected is Import Commodity Concentration. These results agree with a study conducted by Michaely ${ }^{3}$ on 44 countries for the year of 1954, but oppose the assertions made by Leontief ${ }^{4}$. Leontief had expressed the idea that developed countries like the U.S. export a great diversity of manufactured goods and import a few agricultural and mineral commodities and that underdeveloped countries were mirror images of developed countries. The present results suggest that as a country's trade partner concentration increases, so does both its export and import

3- M.Michaely, Concentration in International Trade Amsterdam: North Holland Publishing Company, 1967. 1966), p. 64

4- W. Leontief, Input-Ontput Economics (N.Y:: Oxford University Press, 
concentrations. Therefore, the generally held view that, as Export Commodity Concentration increases, Import Commodity Concentration decreases is rejected by the present findings.

At the national level, the present findings do in fact support the results obtained by the previous tests of the center-periphery hypothesis. Galtung's study which was the only major test that used correlation calculations obtained higher correlation coefficients among variables than the correlation coefficients obtained in the present study. That is due to the fact that more countries have been considered here, which therefore would increase the degree of variability and thus lower correlation coefficients are expected.

\section{Discussion of Global Investigations}

The study found, as expected, that for all the sampled years the industrialized region had the highest volume of trade and trade linkages. The highest share of trade for any other region was made with the industrialized countries while the per cent share of trade among other regions was very small.

The system-wide studies in all the sampled years revealed the existence of five major hierarchies of the United States, Great Britain, West Germany, France, and the U.S.S.R. Based on trade dependence, the existence of these hierarchies gave further evidence of the center-periphery relationship in the world system. A further look at these hierarchies also revealed that while the United States' hierarchy grew in number of countries, other hierarchies declined. The most notable decline could be seen in the United Kingdom's hierarchy. The most important new development in trade hierarchies in this period was the growth of a hierarchy by Japan. Japan's hierarchy, which was nonexistent in 1962, became one of the major world hierarchies encompassing most of Asia and the Middle East. 
One may observe that since Japan's exports to United States is concentrated on Manufactured products and United States' exports to Japan are mostly agricultural, therefore, United States can be identified as Japan's periphery. However, since Japan's dependence on United States' market is great, Japan is considered under the United States hierarchy .

Considering the results of regional averages calculated from the national variables, the following observations can be made. For all variables, statistically significant differences are found in each year of the study between averages obtained for the Industrialized region and those obtained for (at least some of) the other regions. Trade Partner Concentrations for exports improves over the 1962 1970 interval or $1970-1980$ interval or both intervals for all regions except the Middle East. TTl improves for all regions over both these time periods. Commodity Concentrations (for imports as well as exports) and Vertical Trade show a few significant changes in these time periods. Quality of Life for all regions exhibit statistically significant improvements. Agricultural dependency for all regions except for Asia and Middle East show some significant reductions. All temporal changes which are statistically significant, except for a few involving ECC, are in the directionof reduced peripheralization of the regions involved. These changes for the non-industrialized regions are invariabley larger than those for the Industrialized region, and also occur more frequently (for more variables and for more time intervals). This pattern of changes points in the direction of reduced differentiation of the world system over the time period of this study, although it is hard to assess how much importance to give to the specific magnitudes of these changes.

The regional diversity calculations measured the diversity in the distribution of total world imports or exports between regions and nations. It is 
found that regions are more homogeneous internally, i.e. with respect to countries within a region, than the world is homogeneous with respect to regions. That is, regions differ more substantially in shares of import and export activity than countries differ within regions. This is an argument for the existence of global differentiation. As the global diversity figures has been constant during the study period, differentiation as measured by import and export activity have remained constant since the era of decolonization.

Global averages exhibit significant differences for nearly all variables from 1962 to 1970, and from 1970 to 1980. For example, EPC, ECC, VT, PQLI, AGR, and TTL, show significant improvements in both of these time intervals. The differences are all in the direction of lesser peripheralization of the world.

While global and regional averages of some national variables change during the study period, the systemic measures of Global Concentration and Global Polarization have virtually remained unchanged at medium to high levels of concentration during this time ( about 0.53 for Global concentration and 0.69 for polarization for the three sampled years.) Both of these measures reflect the degree of concentration of trade linkages in the world as a whole, i.e., are roughly analogous to the Trade Partner Concentration measure for individual nations 5 .

We thus have two sets of results in apparent conflict with one another. One, the global and many regional averages suggests reduced world systemic differentiation over the twenty year period of the study. The other, the two systemic variables and the decomposition analysis ( and a few regional and global averages), suggest a constant degree of world system differentiation in the study

5- The Global Concentration index measures the degree of concentration of entries in the world import-export table; Global Polarization measures the degree of the world centralness with respect to the tendency of a single country dominating the world trade. 
period. The picture becomes more complex if one considers the assertions made by Bairoch that, over the time period of this study, the GNP gap between developed and underdeveloped countries has widened ( Table II); this might be taken as an indication of increased world differentiation in this period.

The widening of the gap in GNP per capita has been attributed primarily to increasing differences in the levels of processing between center and periphery countries and the international commodity prices, that have led to unfavorable terms of trade for the periphery countries 6,7 . For example, while the GNP increased dramatically for oil producing countries, copper and other minerals have suffered great price reductions in recent years, and this has contributed to lesser GNP's for countries exporting those materials. International price fluctuations has played a major role in influencing income for those export economies that are heavily dependent on foreign trade. That is, even if Vertical Trade and TPC differences between developed and developing countries are diminishing, as global and regional averages suggest, it is still possible that changes in prices have multiplied the effect of the structural differences which still exist and have thus produced an increase in GNP gap.( The increase in income of the oil producing countries due to the increase in international prices of nil has allowed those countries to close the GNP gap very rapidly without any structural transformations of their economic structures.) A secondary reason to the causes of widening GNP

6- Primary commodity prices have registered their fastest drops ever. According to recent study by Peter F. Drucker, by the year 1986, raw material prices were at their lowest levels in relations to the prices of manufactured goods in recorded history. Even in some cases commodity prices were lower than the prices during the Great Depression ( e.g., lead and copper). Even the $\$ 15$ price of pertoleum in 1986 was actually lower than its 1933 prices (when adjusted for the purchasing power of the dollar.) 1986, p. 769.

7- Peter F. Drucker, "The Changed World Economy," Foreign Affairs, 
per capita gap may be attributed to the rapidly growing populations in the developing countries that could diminish any gains in GNP.

While the GNP gap between center and periphery nations has been widening, there is a high correlation between GNP per capita and PQLI, suggesting that improvements in individual country's GNP per capita may have been sufficient to yield better quality of life, even when the GNP gap widens. That is, an increase in GNP gap does not necessarily mean an increase in gap in quality of life.

Other aspects of the world system may indeed be relatively constant. The variables subjected to decomposition analysis, and the two systemic variables, Global Polarization and Global Concentration, all deal with the volume of trade among countries and this characteristic of the world system may not have significantly changed during the study period (i.e. the highest share of trade was still were concentrated within the industrialized region). The absence of any significant changes in Export Commodity Concentration at both regional and global levels may likewise reflect a real constancy.

In summary, it is too simple to speak of the world system in toto as either increasing, decreasing, or preserving its degree of differentiation. Some aspects may be moving in one direction; others in another. This study has been limited in the number and kind of variables studied and in the time duration considered 8 , and differences in the findings of different parts of this study is not surprising. Still the clear implication of this research is that differentiation exists in the international economic system, and has persisted since decolonization. In terms of

8- For some purposes this time period is adequate, e.g. for observing shifts of particular nations such as South Korea in the center-periphery structure, but twenty years may be too short a period of time to see significant structural changes in the overall global system. 
the assertions made by dependency theory, structural dependence has remained a permanent feature of the world economic system. Although there have been changes among the center countries and there has been ascent or decline for some countries, the overwhelming majority of countries have remained as periphery nations. The basic pattern of division of labor has hardly changed. For the majority of those countries which have gained their independence in the post World War Two era, economic independence has not emerged as a consequence of political independence.

\section{DIRECTIONS FOR FUTURE RESEARCH}

While the current research is a test of center-periphery structure in international economic relations, center-periphery structure, as Galtung has repeatedly stated, involves more than just economic factors. This structure is a multidimensional relationship in which not all dependency or imperialism can be understood by economics alone. A more complex study that incorporates noneconomic dimensions such as political, military, cultural, and communication interactions (especially if these can be quantitatively operationalized) would be a desirable next step.

Even considering only economic variables, there are important variables absent from this study. The relationship of multinational corporations to center and periphery countries, issues of foreign investment, international debt, the consequences of international aid on local economies are only a few aspects of the world system that deserve further investigation.

Although the results of the current study on commodity concentrations supports Michaely's test, it would be desirable to calculate commodities in terms of more sectors, i.e., lower levels of SITC. 
The discussion in the previous section about whether the differentiation of the global system has been increasing, decreasing, or remaining constant is very tentative; the issue clearly needs further study. Quite possibly, other systemic measures might be found which are sensitive to those changes which do seem to be occurring. Although some tests were conducted for 1938 , more complete data for that year (or earlier years), if they could be located, would allow a better longitudinal study, which might help explain the origin and the causes of the increase in national income disparities.

The current research has yielded many national and regional statistics that could be further used for comparative synchronic and diachronic investigations at both national and regional levels and even at subregional levels. At the national level, these data could be used to examine the ascent and decline in the world system of any of the nations considered in this study; in this dissertation, only South Korea was looked at specifically in this way. Regional data could be disaggregated into subregional grouping. For example, Samir Amin tracing the effects of colonialism on African economy, identified three particular subregions in Africa. These subregions were: 1- Africa of the colonial economy, 2- Africa of the concession owning companies, and 3- Africa of the labor reserves. Using the available data characteristics and structures of subregions could be evaluated and compared to one another both synchronically and diachronically.

In regards to the center-periphery hypothesis, the existing data could be further examined by using cluster analysis or factor analysis, to see if other relevant observations can be made.

Dependency theory, with its key notion of the center-periphery dichotomy, has contributed significantly to our understanding of global economic relations which goes beyond an analysis of individual nations or multinational entities. It is 
hoped that further research in this area can strengthen our understanding of the global system and of its impact upon individual nations and regions. 


\section{BIBLIOGRAPHY}

Addo, H."Foreign Policy Strategies for Achieving the NIEO: A Third World Perspective," in C.W. Kegley, Jr. and P. McGowan(eds.) The Political Economy of Foreign Policy Behavior. Beverly Hills: Sage, 1980.

Adleman, I., Morris, C.T."Society, Politics and Economic Development." John Hopkins Press: Baltimore, 1971.

Amin, S., et al"Dynamics of Global Crisis,", New York: Monthly Review Press, 1982.

Bairoch, P."Disparities in Economic Development Since the Industrial Revolution", St. Martin Press, 1978.

Bairoch, P., Levy-Leboyer, M."Disparities in Economic Development Since the Industrial Revolution", St. Martin Press, 1978.

Blomstrom, M., Hettne, B."Development Theory in Transition," London: Zed Books, 1984.

Bohannon, P., and Curtin, P."Africa and Africans," New York: Natural History Press, 1971.

Chenery, H., Syrquin, M."Patterns of Development 1950-70." Oxford Univ. Press: London, 1975.

Deutsch, K.W."Theories of Imperialism and Neocolonialism," in "Testing Theories of Economic Imperialism", edited by: Rosen, S.J. and Kurth J.R., Toronto: Lexington Bcoks, 1974.

Donaldson, P."Worlds Apart: The Economic Gulf Between Nations", New York: Viking Press, 1973.

Dos Santos, T."The Structure of Dependence", in "Readings in U.S. Imperialism," edited by Fann, K.T. and Hodges, D.C., Boston: Porter Sargent Publisher, 1971.

. "The Crisis of Development Theory and the Problems of Depenedence in Latin America," in H. Bernstein (ed.), "Underdevelopment and Development", Harmondsworth: Penguin, 1973.

Drucker, P. F.,"The Changed World Economy," Foreign Affairs, 1986, p. 768-791.

Fage, J.D."An Introduction to the History of West Africa," Cambridge:

Cambridge University Press, 1962, 
Fieldhouse, D.K."Economic and Empire: 1830-1914," Ithaca: Cornell University Press, 1973.

—. "The colonial Empires," New york: Delacorte Press, 1967. . "Colonialism: 1870-1945," New York: St. Martin Press, 1981.

Frank, A.G."The Development of Underdevelopment" in "Imperialism and Underdevelopment: A Reader", ed. R.I. Rhodes.

- "The development of Underdevelopment" in "Imperilaism and Underdevelopment: A reader," ed. R.I. Rhodes .

Freeman, L.C."Centrality in Social Networks: Conceptual Clarifications." Social Networks, 1 (1978-79): p. 215-39.

Galtung, J."A Structural Theory of Imperialism", Journal of Peace Research, Vol VIII, No 2.

- "Conflict on a Global Scale: Social Imperialism and the Sub-imperialismContinuities in the Structural Theory of Imperialism," World Development, Vol4, No. 3.

Gidengil, E."Centers and Peripheries: An Empirical Test of Galtung's Theory of Imperialism," Jounal of Peace Research, Vol. 15, No. 1, 1978.

Hancock, W.K."Survey of British Commonwealth Affairs," II, ii, Problems of Economic Policy, 1918-39, London: 1942.

Hegel, G.W.F."Philosophy of Right," Oxford: 1942.

Hobson, J.A."Imperialism," Ann Arbor: The University of Michigan Press, 1965.

Kuznets, S."Economic Growth of Small Nations," The Challenge of Development(Jerusalem: The Eliezer Kaplan School of Economics and Social Sciences, The Hebrew University, 1958).

Lenin, V.I."Imperialism, the Highest Stage of Capitalism," Moscow: 1916.

Leontief, W."The Structure of Development," Scientific America. 1963:September.

Leontief, W."Input-Output Economics", Oxford University Press, N.Y., 1966.

List, F." National System of Political Economy," Philadelphia: J.B. Lippincot, 1856.

McGowan, P.J. and Kordan, B."Imperialism in World-System Perspective: Britain 1870-1914," in World System Structure: Continuity and Change," Edited by: Hollist, W.L. and Rosenau, J.N., Beverly Hills:Sage, 1981.

McGranahan, et al."Contents and Measurement of Socioeconomic Development." Praeger Publishers, N.Y., 1972. 
Marshall, A."Principles of Economics", 8th ed., London, 1920.

Marx, K."Capital," Moscow: Foreign Languages Publishing House, 1962.

Meier, G.M."Leading Issues in Economic Development", Oxford University Press, N.Y., 1984.

Michaely, M."Concentration in International Trade," Amsterdam:North-Holland Publishing Company, 1967.

Mill, J.S."Principles of Political Economics," London, 1848.

Morawetz, D."Twenty-five Years of Economic Development: 1950 to 1975," The World Bank, 1977.

Morris, D.M."Measuring the Condition of the World's Poor." Pergamon Press, N.Y., 1979.

Myrdal, G."Economic Theory and Underdeveloped Regions", London: Gerald Duckworth, 1957.

—. "Asian Drama," New York: Pantheon Books,1968.

"Poverty- Imperialism- Poverty", in "The Economic Causes of Imperialism," edited by Wolfe, M., New York; John Wiley and Sons, 1972.

Nieminen, J."On Centrality in a Graph." Scandinavian Journal of Psychology, 15:322-336.

Patel, S."The Economic Distance Between Nations: Its Origin, Measurement, and Outlook", Economic Journal, 1974(March):119-131.

Prebisch, R."The Economic Development of Latin America and Its Principal Problems," New York: United Nation, 1950.

Richardson, N.R."Political Complience and U.S. Trade Development." American Political Science Review, vol. 70, No. 4, 1976, pp. 1098-1109.

Rostow, W.W."The Stages of Economic Growth", Cambridge: Cambridge University Press, 1960.

Roubiquet P."Discours et Opinions de Jules Ferry," trans. D.K. Fieldhouse.

Singer, H.W."The Distribution of Gains Between Investing and Borrowing Countries", American Economic Review, Vol II, no 2.

—. "Weak States in a World of Powers: The Dynamics of International Relationships." Free Press, New York, 1972.

Smith, A."An Inquiry into the Nature and Cuases of The Wealth of Nations," Edwin Canan(ed.), New York: The Modern Library, 1937. 
Snyder, D., Kick, E.L."Structural Position in the World System and Economic Growth 1955-70: A Multiple Network Analysis of Transnational Interaction." American Journal of Sociology 84(1979):1096-126.

Wakefield, E.G. "A View of the Art of Colonization," London: 1849, p. 83.

Wallerstein, I."The Modern World System," New York: Academic Press, 1974.

Williams, E."Capitalism and Slavery," New York: Perigee Books, 1944.

Theil, H."Economics and In formation Theory." North Holland Publishing Company, Chicago, 1967. 


\section{APPENDIX A}

\section{COUNTRIES USED IN THIS STUDY}


TABLE A.1

LIST OF COUNTRIES USED IN 1938 STUDY

ALGERIA
ARGENTINA
AUSTRALIA
AUSTRIA
BOLIVIA
BRAZIL
BULGARIA
CAMADA
CEYLON
CHILE
CHIMA
COLOKBIA
COSTA RICA
CUBA
CZECHOSLOVAKIA
DENMARK
DOHIMICAN REP.
ECUADOR
EGYPT
EL SALVADOR
FALKLAND
FIMLAND
FRANCE
GERHANY
GREECE
GUATEMALA
HAITI
HONDORAS
HOHG KONG
HUNGARY
IRAN
IRAQ

IRELAND
ITALY
JAMAICA
MALAYSIA
MEXICO
MOROCCO
NETHERLANDS
NEW ZEALAND
NICARAGUA
NIGERIA
NORWAY
PANAM
PARAGUAY
PERU
PHILIPPINES
POLAND
PORTUGAL
ROMANIA
SOUTH AFRICA
SPAIN
SWEDEN
SWITZERLAND
THAILAND
TRINIDAD
TUNISIA
TURKEY
U.K.
URUGUAY
U.S.A.
U.S.S.R.
VENEZUELA
YUGOSLAVIA


TABLE A.2

LIST OF COUNTRIES USED IN 1962 STUDY

\begin{tabular}{|c|c|c|}
\hline AFGHANISTAN & GREECE & NIGER \\
\hline ALGERIA & GREENLAND & NIGERIA \\
\hline ANGOLA & GUADALOUPE & NORWAY \\
\hline ARGENTINA & GUATEMALA & OMAN \\
\hline AUSTRALIA & GUINEA & PAKISTAN \\
\hline AUSTRIA & HAITI & Panama \\
\hline BAHAMAS & HONDORAS & PARAgUaY \\
\hline BAHREIN & HONG KONG & PERU \\
\hline BARBADOS & HUNGARY & PHILIPPINES \\
\hline BELGIUH & ICELAND & POLAND \\
\hline BENIN & INDIA & PORTUGAL \\
\hline BERHUDA & INDONESIA & CATAR \\
\hline BOLIVIA & IRAN & REUNION \\
\hline BRAZIL & IRAQ & ROHAINA \\
\hline BRUNEI & IRELAND & SAUDI ARABIA \\
\hline BULGARIA & ISRAEL & SENEGAL \\
\hline BURHA & ITALY & SIERRA LEON \\
\hline CAMEROON & IVORY COAST & SINGAPORE \\
\hline CANADA & JAYAAICA & SOMALI \\
\hline CAPE VERDI & JAPAN & SOUTH AFRICA \\
\hline CENTRAL AFRICAN REP. & JORDAN & SPAIN \\
\hline CHAD & KENYA & SUDAN \\
\hline CHILE & SOUTH KOREA & SURINAM \\
\hline CHIMA & KUUAIT & SHEDEN \\
\hline COLOHBIA & LAOS & SWITZERLAND \\
\hline CONGO & LEBANON & SYRIA \\
\hline COSTA RICA & LIBYA & TANZANIA \\
\hline CUBA & HACAO & THAILAND \\
\hline CYPRUS & MADAGASCAR & TRINIDAD \\
\hline CZECHOSLOVAKIA & MALAYSIA & TUMISIA \\
\hline DENMARK & MALI & TURKEY \\
\hline DONIMICAN REPUBLIC & MALTA & U.K. \\
\hline EAST GERMANY & MARTINIQUE & U.S.A. \\
\hline ECUADOR & MAURITANIA & U.S.S.R. \\
\hline EGYPT & MAURITIUS & UGANDA \\
\hline EL SALVADOR & MEXICO & URUGUAY \\
\hline ETHIOPIA & MOROCCO & VENEZUELA \\
\hline FIJI & MOZAMBIQUE & VIET MAH \\
\hline FINLAND & NETHERLAND ANT. & YEMEN, ARAB REP. \\
\hline FRANCE & METHERLANDS & YEHEN, PEOPLES \\
\hline GABON & NEU CALEDONIA & YUGOSLAVIA \\
\hline garibia & NEW GUINEA & \\
\hline GERMANY, FEDERAL REP. & MEH ZEALAND & \\
\hline GHakA & NICARAGUA & \\
\hline
\end{tabular}


TABLE A.3

LIST OF COUNTRIES USED IN 1970 STUDY

\begin{tabular}{|c|c|c|c|}
\hline AFGHANISTAN & GREECE & NICARAGUA & VENEZUELA \\
\hline ALGERIA & GREENLAND & NIGER & VIET NAM \\
\hline ANGOLA & GUADALOUPE & MIGERIA & YEMEN, A. \\
\hline ARGENTINA & GUATEMALA & MORTH KOREA & YEHEN, P. \\
\hline AUSTRALIA & GUINEA REPUBLIC & NORTH VIET NAY & YUGOSLAVIA \\
\hline AUSTRIA & GUYANA & NORWAY & ZAIRE \\
\hline BAHAMAS & HAITI & OMAN & \\
\hline BAHREIN & HONDORAS & PAKISTAN & \\
\hline BARBADOS & HONG KONG & PANAMA & \\
\hline BELGIUH & HUNGARY & PAPAU NEW GUINEA & \\
\hline BENIN & ICELAND & PARAGUAY & \\
\hline BERHUDA & INDIA & PERU & \\
\hline BOLIVIA & INDONESIA & PHILIPPINES & \\
\hline BRAzIL & IRAN & POLANo & \\
\hline BRUNEI & IRAQ & PORTUGAL & \\
\hline BULGARIA & IRELAND & QATAR & \\
\hline BURMA & ISRAEL & REUNION & \\
\hline BURUNDI & ITALY & ROMANIA & \\
\hline CAMEROON & IVORY COAST & SAUDI ARABIA & \\
\hline CANADA & JAMAICA & SENEGAL & \\
\hline CENTRAL AFRICAN REP. & JAPAN & SEYCHELLES & \\
\hline CHAD & JORDAN & SIERRA LEON & \\
\hline CHILE & KENYA & SINGAPORE & \\
\hline CHINA & SOUTH KOREA & SOMALIA & \\
\hline COLOHBIA & KUWAIT & SOUTH AFRICA & \\
\hline CONGO & LAOS & SPAIN & \\
\hline COSTA RICA & LEBANON & SRI LANKA & \\
\hline CUBA & LIBYA & SUDAN & \\
\hline CYPRUS & macıo & SURINAN & \\
\hline CZECHOSLOVAKIA & MALAWI & SWEDEN & \\
\hline DENMARK & MALAYSIA & SWITZERLAND & \\
\hline DOHINICAN REPUBLIC & MALI & SYRIA & \\
\hline EAST GERHANY & MALTA & TAIHAN & \\
\hline ECUADOR & MARTINIQUE & TANZANIA & \\
\hline EGYPT & MARITANIA & THAILAND & \\
\hline EL SALVADOR & MAURITIUS & TRINIDAD & \\
\hline ETHIOPIA & MEXICO & TUNISIA & \\
\hline FIJI & MOROCCO & TURKEY & \\
\hline FINLAND & mozambiQue & U.A.E. & \\
\hline FRANCE & NEPAL & U.K. & \\
\hline GABOA & NETHERLAND ANT. & U.S.A. & \\
\hline GAKBIA & NETHERLANDS & U.S.S.R. & \\
\hline GERHANY, FEDERAL REP. & - NEH CALEDONIA & UGANDA & \\
\hline GHANA & MEH ZEALAND & URUGUAY & \\
\hline
\end{tabular}


TABLE A.4

LIST OF COUNTRIES USED IN 1980 STUDY

\begin{tabular}{|c|c|c|}
\hline AFGHANISTAN & GREECE & NICARAGUA \\
\hline ALGERIA & GREENLAND & NIGER \\
\hline ANGOLA & GRENADA & NIGERIA \\
\hline ARGENTINA & GUADALOUPE & NORTH KOREA \\
\hline AUSTRALIA & GUATEMALA & NORHAY \\
\hline AUSTRIA & GUIANA, FRENCH & OMAN \\
\hline BAHAMAS & GUINEA & PAKISTAN \\
\hline BAHREIN & GUINEA-BISSAU & PAKAMA \\
\hline BANGLADESH & HAITI & PARAGUAY \\
\hline BARBADOS & HONDORAS & PAPAU NEW GUINEA \\
\hline BELGIUH & HONG KONG & PERU \\
\hline BENIN & HUNGARY & PHILIPPINES \\
\hline BERHUDA & ICELAND & POLAND \\
\hline BOLIVIA & INDIA & PORTUGAL \\
\hline BRAZIL & INDONESIA & eatar \\
\hline BRUNEI & IRAN & REUNIOH \\
\hline EULGARIA & IRAQ & ROHANIA \\
\hline BURKINA FASO & IRELAND & SAUDI ARABIA \\
\hline BURHA & ISRAEL & SENEGAL \\
\hline BURUNDI & ITALY & SEYCHELLES \\
\hline CAHEROON & IVORY COAST & SIERRA LEONE \\
\hline CANADA & JAMAICA & SINGAPORE \\
\hline CAPE VERDI & JAPAN & SOMALIA \\
\hline CENTRAL AFRICAN REP. & JORDAN & SOUTH AFRICA \\
\hline CHAD & KENYA & SPAIN \\
\hline CHILE & SOUTH KOREA & SRI LANKA \\
\hline CHIMA & KUWAIT & SUDAN \\
\hline COLOHBIA & LAOS & SURIMAY \\
\hline COHGO & LEBANON & SWEDEN \\
\hline COSTA RICA & LIBYA & SWITZERLAND \\
\hline CUBA & MACAO & SYRIA \\
\hline CYPRUS & MADAGASCAR & TANZANIA \\
\hline CZECHOSLOVAKIA & MALAMI & THAILAND \\
\hline DENMIARK & MALAYSIA & TRINIDAD \\
\hline DJIBOUTI & MALDIVES & TUNISIA \\
\hline DOHIHICAN REPUBLIC & MALI & TURKEY \\
\hline EAST GERHANY & HALTA & U.A.E. \\
\hline ECUADOR & MARTINIQUE & U.S.A. \\
\hline EGYPT & MARITANIA & U.S.S.R. \\
\hline EL SALVADOR & MAURITIUS & UGANDA \\
\hline ETHIOPIA & HEXICO & U.K. \\
\hline FIJI & MOROCCO & URUGUAY \\
\hline FINLAKD & MOZAMBIQUE & VENEZUELA \\
\hline FRANCE & NEPAL & VIET NAM \\
\hline GABOH & NETHERLAND ANT. & YENEN, A.D. \\
\hline GAMBIA & NETHERLANDS & YEHEN, A.R. \\
\hline $\begin{array}{l}\text { GERHANY, FED REP. } \\
\text { GHANA }\end{array}$ & $\begin{array}{l}\text { HEU CALEDONIA } \\
\text { NEU ZEALAND }\end{array}$ & $\begin{array}{l}\text { YUGOSLAVIA } \\
\text { ZAIRE }\end{array}$ \\
\hline
\end{tabular}


APPENDIX B

COMPARISON OF ENTROPY AND HIRSCHMAN INDICES OF CONCENTRATION 
TRADE PARTNER CONCENTRATION

\begin{tabular}{|c|c|c|}
\hline COUNTRY & ENTROPY & HIRSCHMANN \\
\hline U.S.A. & 0.29 & 0.24 \\
\hline CANADA & 0.62 & 0.65 \\
\hline AUSTRALIA & 0.39 & 0.32 \\
\hline JAPAN & 0.31 & 0.29 \\
\hline NEH ZEALAN & 0.39 & 0.29 \\
\hline AUSTRIA & 0.40 & 0.35 \\
\hline BELGIUH & 0.45 & 0.35 \\
\hline DENHARK & 0.39 & 0.30 \\
\hline FINLAND & 0.41 & 0.31 \\
\hline FRANCE & 0.31 & 0.26 \\
\hline GERMANY & 0.33 & 0.24 \\
\hline ICELAND & 0.46 & 0.32 \\
\hline IRELAND & 0.51 & 0.46 \\
\hline ITALY & 0.33 & 0.27 \\
\hline NETHERLAND & 0.45 & 0.38 \\
\hline NORUAY & 0.53 & 0.47 \\
\hline SPAIN & 0.31 & 0.25 \\
\hline SUEDEN & 0.33 & 0.25 \\
\hline SUITZERLAN & 0.33 & 0.27 \\
\hline UK & 0.28 & 0.22 \\
\hline ALGERIA & 0.63 & 0.52 \\
\hline ANGOLA & 0.61 & 0.48 \\
\hline BENIN & 0.55 & 0.39 \\
\hline BURKIMA FA & 0.61 & 0.47 \\
\hline BURUNDI & 0.67 & 0.60 \\
\hline CAMEROON & 0.56 & 0.41 \\
\hline CAPE VERDI & 0.72 & 0.58 \\
\hline CEH. AFR. & 0.65 & 0.56 \\
\hline CHAD & 0.57 & 0.38 \\
\hline CONGO & 0.61 & 0.46 \\
\hline DJIBOUTI & 0.63 & 0.44 \\
\hline EQU. GUINE & 0.74 & 0.61 \\
\hline ETHIOPIA & 0.46 & 0.31 \\
\hline GABON & 0.56 & 0.38 \\
\hline GAMBIA & 0.53 & 0.36 \\
\hline Gнами & 0.52 & 0.35 \\
\hline GUINEA & 0.56 & 0.38 \\
\hline GUIHEA-BIS & 0.60 & 0.40 \\
\hline IVORY COAS & 0.47 & 0.33 \\
\hline KENYA & 0.35 & 0.27 \\
\hline MADAGASCAR & 0.53 & 0.36 \\
\hline MLLAUI & 0.54 & 0.41 \\
\hline MALI & 0.53 & 0.36 \\
\hline MAURITANIA & 0.59 & 0.41 \\
\hline MAURITIUS & 0.74 & 0.70 \\
\hline MOROCCO & 0.39 & 0.31 \\
\hline MOZAMBIQUE & 0.44 & 0.31 \\
\hline
\end{tabular}


APPENDIX B- (CONT'D)

\begin{tabular}{|c|c|c|}
\hline COUNTRY & ENTROPY & HIRSCHMANN \\
\hline NIGER & 0.65 & 0.48 \\
\hline MIGERIA & 0.58 & 0.48 \\
\hline REUNION & 0.89 & 0.89 \\
\hline SENEGAL & 0.48 & 0.40 \\
\hline SEYCHELLES & 0.68 & 0.54 \\
\hline SIERRA LEO & 0.76 & 0.63 \\
\hline SOHALIA & 0.77 & 0.72 \\
\hline SOUTH AFRI & 0.49 & 0.33 \\
\hline SUDAN & 0.42 & 0.30 \\
\hline TANzanIa & 0.38 & 0.27 \\
\hline TUMISIA & 0.51 & 0.36 \\
\hline UGANDA & 0.53 & 0.38 \\
\hline ZAIRE & 0.83 & 0.81 \\
\hline ZAMBIA & 0.49 & 0.32 \\
\hline AFGHANISTA & 0.70 & 0.65 \\
\hline BANGLADESH & 0.29 & 0.20 \\
\hline BRUNEI & 0.78 & 0.73 \\
\hline BURMA & 0.41 & 0.28 \\
\hline снIма & 0.45 & 0.37 \\
\hline FIJI & 0.59 & 0.41 \\
\hline HONG KONG & 0.38 & 0.32 \\
\hline INDIA & 0.32 & 0.25 \\
\hline INDONESIA & 0.65 & 0.55 \\
\hline KOREA & 0.42 & 0.36 \\
\hline LAO & 0.61 & 0.43 \\
\hline MACAO & 0.51 & 0.36 \\
\hline maLAYSIA & 0.47 & 0.36 \\
\hline MALDIVES & 0.63 & 0.43 \\
\hline NEPAL & 0.53 & 0.38 \\
\hline NEU CALEDO & 0.80 & 0.66 \\
\hline PAKISTAN & 0.29 & 0.21 \\
\hline PAUPA N GU & 0.64 & 0.49 \\
\hline PHILIPPINE & 0.50 & 0.41 \\
\hline SINGAPORE & 0.34 & 0.26 \\
\hline SRI LANKA & 0.31 & 0.22 \\
\hline THAILAND & 0.39 & 0.28 \\
\hline VIETMAn & 0.56 & 0.43 \\
\hline CYPRUS & 0.41 & 0.30 \\
\hline GREECE & 0.34 & 0.26 \\
\hline HUNGARY & 0.44 & 0.36 \\
\hline MALTA & 0.54 & 0.41 \\
\hline PORTUGAL & 0.37 & 0.27 \\
\hline ROHANIA & 0.34 & 0.25 \\
\hline TURKEY & 0.37 & 0.27 \\
\hline yugosLavia & 0.40 & 0.33 \\
\hline
\end{tabular}


APPENDIX B- (CONT'D)

\begin{tabular}{|c|c|c|}
\hline COUNTRY & ENTROPY & HIRSCHMANN \\
\hline BAHRAIN & 0.47 & 0.33 \\
\hline EGYPT & 0.43 & 0.34 \\
\hline IRAN & 0.45 & 0.33 \\
\hline IRAQ & 0.42 & 0.30 \\
\hline ISRAEL & 0.40 & 0.28 \\
\hline JORDAN & 0.48 & 0.34 \\
\hline KUWAIT & 0.44 & 0.32 \\
\hline LEBANON & 0.50 & 0.42 \\
\hline LIBYA & 0.57 & 0.44 \\
\hline OHAN & 0.70 & 0.59 \\
\hline QATAR & 0.49 & 0.36 \\
\hline SAUDI ARAB & 0.41 & 0.29 \\
\hline SYRIA & 0.64 & 0.59 \\
\hline U.A.E. & 0.48 & 0.40 \\
\hline YEHEN, A.R & 0.60 & 0.48 \\
\hline YENEN, A.D & 0.60 & 0.45 \\
\hline ARGENTINA & 0.37 & 0.28 \\
\hline BAHArAS & 0.67 & 0.59 \\
\hline BARBADOS & 0.66 & 0.56 \\
\hline BELIZE & 0.74 & 0.63 \\
\hline BERHUDA & 0.46 & 0.31 \\
\hline BOLIVIA & 0.56 & 0.40 \\
\hline BRAZIL & 0.30 & 0.24 \\
\hline CHILE & 0.41 & 0.27 \\
\hline COLOHBIA & 0.45 & 0.36 \\
\hline COSTA RICA & 0.51 & 0.40 \\
\hline DOHINICAN & 0.67 & 0.58 \\
\hline ECUADOR & 0.58 & 0.48 \\
\hline EL SALVADO & 0.63 & 0.50 \\
\hline GREENLAND & 0.65 & 0.54 \\
\hline GRENADA & 0.63 & 0.50 \\
\hline GUADALOUPE & 0.82 & 0.76 \\
\hline guatemala & 0.49 & 0.36 \\
\hline GUIANA, FR & 0.61 & 0.43 \\
\hline HAITI & 0.69 & 0.60 \\
\hline HONDORAS & 0.62 & 0.56 \\
\hline JAMAICA & 0.58 & 0.45 \\
\hline MARTINIQUE & 0.79 & 0.66 \\
\hline MEXICO & 0.68 & 0.67 \\
\hline NETHR. ANT & 0.59 & 0.58 \\
\hline NICARAGUA & 0.56 & 0.44 \\
\hline PANArA & 0.64 & 0.60 \\
\hline PARUGUAY & 0.51 & 0.35 \\
\hline PERU & 0.44 & 0.37 \\
\hline SURIMAKE & 0.53 & 0.36 \\
\hline TRINIDAD & 0.68 & 0.67 \\
\hline URUGUAY & 0.41 & 0.29 \\
\hline
\end{tabular}


APPENDIX B- (CONT'D)

$\begin{array}{lll}\text { COUNTRY } & \text { ENTROPY } & \text { HIRSCHMANN } \\ \text { VENEZUELA } & 0.50 & 0.39 \\ \text { BULGARIA } & 0.60 & 0.60 \\ \text { CUBA } & 0.65 & 0.66 \\ \text { CZECHOSLOV } & 0.48 & 0.42 \\ \text { EAST GERHA } & 0.37 & 0.26 \\ \text { MORTH KORE } & 0.54 & 0.38 \\ \text { POLAND } & 0.44 & 0.38 \\ \text { U.S.S.R. } & 0.39 & 0.25\end{array}$

CORRELATION COEFFICIENT BETUEEN ENTROPY AND HIRSCHHAN= 0.97 


\begin{abstract}
APPENDIX C
CORRELATION RESULTS WHEN OIL EXPORTING COUNTRIES ARE EXCLUDED
\end{abstract}


SUMMARY OF PEARSON CORRELATION RESULTS AMONG VARIABLES

\begin{tabular}{|c|c|c|c|}
\hline \% AGRICULTURE-GDP & 1962 & 1970 & 1980 \\
\hline \% AGRICULTURE-GDP & 1.0000 & 1.0000 & 1.0000 \\
\hline VERTICAL TRADE & -.5842 & -.5863 & -.4776 \\
\hline EXP COHM CONCEN & .3345 & .3458 & .2055 \\
\hline IMP COMH CONCEN & .5382 & .3891 & .1833 \\
\hline G.N.P. PER CAPITA & .0000 & .0000 & -.6655 \\
\hline QUALITY OF LIFE & -.8233 & -.8336 & -.7411 \\
\hline \% EXP MANUFAC. & -.5263 & -.5348 & -.5094 \\
\hline \% EXP AGR-MINE & .5447 & .5676 & .5460 \\
\hline \% IMP MANUFAC. & .5017 & .2444 & .0918 \\
\hline * IHP AGR-HINE & -.4982 & -.2333 & -.0905 \\
\hline EXP. PARTNER CON. & .4505 & .3163 & .3023 \\
\hline IMP. PARTNER CON. & .5130 & .2773 & .2132 \\
\hline EPC/IPC RATIO & .0490 & .1909 & .1943 \\
\hline TOT TRADE LINKAGES & -.5533 & -.5840 & -.5722 \\
\hline TOTAL IMPORTS & -.4637 & -.4751 & -.4141 \\
\hline TOTAL EXPORTS & -.4386 & -.4457 & -.4058 \\
\hline ECC/ICC RATIO & -.2163 & -.1166 & .0859 \\
\hline COHM COHC GAP & .1062 & .2071 & .1512 \\
\hline
\end{tabular}


SUMMARY OF PEARSON CORRELATION RESULTS AMONG VARIABLES

\begin{tabular}{|c|c|c|c|}
\hline VERTICAL TRADE & 1962 & 1970 & 1980 \\
\hline X AGRICULTURE-GDP & -.5842 & -.5863 & -.4776 \\
\hline VERTICAL TRADE & 1.0000 & 1.0000 & 1.0000 \\
\hline EXP COHH CONCEN & -.4345 & -.3643 & -.3495 \\
\hline IMP COHM CONCEN & -.6267 & -.5979 & -.6691 \\
\hline G.M.P. PER CAPITA & .0000 & .0000 & .4565 \\
\hline QUALITY OF LIFE & .5831 & .5157 & .5458 \\
\hline \% EXP MANUFAC. & .9406 & .9365 & .9508 \\
\hline \% EXP AGR-MINE & -.9392 & -.9292 & -.9515 \\
\hline \% ImP haNUfaC. & -.7452 & -.5818 & -.6304 \\
\hline \% IMP AGR-HINE & .7393 & .5538 & .6290 \\
\hline EXP. PARTNER CON. & -.5924 & -.3719 & -.5346 \\
\hline IMP. PARTNER CON. & -.4905 & -.3550 & -.3894 \\
\hline EPC/IPC RATIO & -.3277 & -.1800 & -.3586 \\
\hline TOT TRADE LINKAGES & .8301 & .6842 & .6626 \\
\hline TOTAL IMPORTS & .6965 & .5958 & .5112 \\
\hline TOTAL EXPORTS & .6352 & .5567 & .4878 \\
\hline ECC/ICC RATIO & .4491 & .3802 & .1684 \\
\hline COHH CONC GAP & -.1394 & -.1150 & -.1240 \\
\hline
\end{tabular}


SUMMARY OF PEARSON CORRELATION RESULTS AMONG VARIABLES

\begin{tabular}{|c|c|c|c|}
\hline EXP COMH CONCEN & 1962 & 1970 & 1980 \\
\hline X AGRICULTURE-GDP & .3345 & .3458 & .2055 \\
\hline VERTICAL TRADE & -.4345 & -.3643 & -.3495 \\
\hline EXP COMH CONCEN & 1.0000 & 1.0000 & 1.0000 \\
\hline IMP COMH CONCEN & .5094 & .3871 & .3737 \\
\hline G.N.P. PER CAPITA & .0000 & .0000 & -.2654 \\
\hline QUALITY OF LIFE & -.2847 & -.3004 & -.2540 \\
\hline \% EXP MANUFAC. & -.4816 & -.4352 & -.3332 \\
\hline * EXP AGR-MINE & .5081 & .4737 & .3592 \\
\hline \# IMP MANUFAC. & .1297 & -.0530 & .1570 \\
\hline \% IMP AGR-MINE & -.1312 & .0639 & -.1590 \\
\hline EXP. PARTNER CON. & .4784 & .4507 & .5192 \\
\hline IMP. PARTNER CON. & .4402 & .3281 & .4381 \\
\hline EPC/IPC RATIO & .0957 & .3238 & .2690 \\
\hline TOT TRADE LINKAGES & -.4773 & -.4394 & -.5117 \\
\hline TOTAL IHPORTS & -.3889 & -.3046 & -.2827 \\
\hline TOTAL EXPORTS & -.3455 & -.2651 & -.2597 \\
\hline ECC/ICC RATIO & .1277 & .1441 & .6768 \\
\hline CONM CONC GAP & .8644 & .8900 & .9298 \\
\hline
\end{tabular}


SUMMARY OF PEARSON CORRELATION RESULTS AMONG VARIABLES

\begin{tabular}{|c|c|c|c|}
\hline IHP COHM CONCEN & 1962 & 1970 & 1980 \\
\hline \% AGRICULTURE-GDP & .5382 & .3891 & .1833 \\
\hline VERTICAL TRADE & -.6267 & -.5979 & -.6691 \\
\hline EXP COHH CONCEN & .5094 & .3871 & .3737 \\
\hline IMP COHM CONCEN & 1.0000 & 1.0000 & 1.0000 \\
\hline G.N.P. PER CAPITA & .0000 & .0000 & -.3099 \\
\hline QUALITY OF LIFE & -.6344 & -.4446 & -.4525 \\
\hline X EXP MANUFAC. & -.5928 & -.4926 & -.5519 \\
\hline X EXP AGR-MINE & .5983 & .5157 & .5455 \\
\hline \% IHP RANUFAC. & .4355 & .4284 & .6568 \\
\hline \% IMP AGR-MINE & -.4456 & -.4674 & -.6567 \\
\hline EXP. PARTNER CON. & .4448 & .4145 & .4506 \\
\hline IHP. PARTNER CON. & .5787 & .5185 & .4596 \\
\hline EPC/IPC RATIO & -.0540 & .1103 & .1721 \\
\hline TOT TRADE LINKAGES & -.5703 & -.5637 & -.5640 \\
\hline TOTAL IMPORTS & -.5020 & -.4624 & -.3684 \\
\hline TOTAL EXPORTS & -.4434 & -.4267 & -.3445 \\
\hline ECC/ICC RATIO & -.4315 & -.3636 & -.3006 \\
\hline COHH CONC GAP & .0022 & -.0661 & .0132 \\
\hline
\end{tabular}


SUMHARY OF PEARSON CORRELATION RESULTS AMONG VARIABLES

\begin{tabular}{|c|c|c|c|}
\hline G.N.P. PER CAPITA & 1962 & 1970 & 1980 \\
\hline X AGRICULTURE-GDP & .0000 & .0000 & -.6655 \\
\hline VERTICAL TRADE & .0000 & .0000 & .4565 \\
\hline EXP COHM CONCEN & .0000 & .0000 & -.2654 \\
\hline IHP COHA CONCEN & .0000 & .0000 & -.3099 \\
\hline G.N.P. PER CAPITA & .0000 & .0000 & 1.0000 \\
\hline QUALITY OF LIFE & .0000 & .0000 & .6698 \\
\hline Z EXP MANUFAC. & .0000 & .0000 & .5078 \\
\hline * EXP AGR-MINE & .0000 & .0000 & -.5240 \\
\hline ₹ IHP MANUFAC. & .0000 & .0000 & -.0669 \\
\hline \% IMP AGR-MINE & .0000 & .0000 & .0662 \\
\hline EXP. PARTNER CON. & .0000 & .0000 & -.3444 \\
\hline IHP. PARTNER CON. & .0000 & .0000 & -.2026 \\
\hline EPC/IPC RATIO & .0000 & .0000 & -.2686 \\
\hline TOT TRADE LINKAGES & .0000 & .0000 & .6843 \\
\hline TOTAL IMPORTS & .0000 & .0000 & .6139 \\
\hline TOTAL EXPORTS & .0000 & .0000 & .6058 \\
\hline ECC/ICC RATIO & .0000 & .0000 & -.1029 \\
\hline COHA CONC GAP & .0000 & .0000 & -.1647 \\
\hline
\end{tabular}


SUMMARY OF PEARSON CORRELATION RESULTS AMONG VARIABLES

\begin{tabular}{|c|c|c|c|}
\hline QUALITY OF LIFE & 1962 & 1970 & 1980 \\
\hline \% AGRICULTURE-GDP & -.8233 & -.8336 & -.7411 \\
\hline VERTICAL TRADE & .5831 & .5157 & .5458 \\
\hline EXP COMH CONCEN & -.2847 & -.3004 & -.2540 \\
\hline IHP COHH CONCEN & -.6344 & -.4446 & -.4525 \\
\hline G.N.P. PER CAPITA & .0000 & .0000 & .6698 \\
\hline QUALITY OF LIFE & 1.0000 & 1.0000 & 1.0000 \\
\hline * EXP MANUFAC. & .5436 & .5002 & .5498 \\
\hline \% EXP AGR-MINE & -.5633 & -.5209 & -.5813 \\
\hline * IMP MANUFAC. & -.4394 & -.1839 & -.1931 \\
\hline$X$ IMP AGR-MINE & .4366 & .1520 & .1914 \\
\hline EXP. PARTNER CON. & -.5206 & -.3851 & -.4527 \\
\hline IMP. PARTNER CON. & -.5801 & -.3203 & -.3415 \\
\hline EPC/IPC RATIO & -.0824 & -.2646 & -.2544 \\
\hline TOT TRADE LINKAGES & .5843 & .5747 & .6597 \\
\hline TOTAL IHPORTS & .4677 & .4191 & .4117 \\
\hline TOTAL EXPORTS & .4162 & .3829 & .3969 \\
\hline ECC/ICC RATIO & .2770 & .1350 & .0348 \\
\hline COHM CONC GAP & .0096 & -.1303 & -.0858 \\
\hline
\end{tabular}


SUMMARY OF PEARSON CORRELATION RESULTS AMONG VARIABLES

\begin{tabular}{|c|c|c|c|}
\hline X EXP MANUFAC. & 1962 & 1970 & 1980 \\
\hline X AGRICULTURE-GDP & -.5263 & -.5348 & -.5094 \\
\hline VERTICAL TRADE & .9406 & .9365 & .9508 \\
\hline EXP COHH CONCEN & -.4816 & -.4352 & -.3332 \\
\hline IMP COMH CONCEN & -.5928 & -.4926 & -.5519 \\
\hline G.N.P. PER CAPITA & .0000 &.$\infty 000$ & .5078 \\
\hline QUALITY OF LIFE & .5436 & .5002 & .5498 \\
\hline \% EXP MANUFAC. & 1.0000 & 1.0000 & 1.0000 \\
\hline Z EXP AGR-MINE & -.9988 & -.9999 & -.9998 \\
\hline \% IMP MANUFAC. & -.4552 & -.3113 & -.3719 \\
\hline \% IMP AGR-MINE & .4450 & .2589 & .3699 \\
\hline EXP. PARTNER CON. & -.6226 & -.4711 & -.5274 \\
\hline IMP. PARTNER CON. & -.5189 & -.3755 & -.3462 \\
\hline EPC/IPC RATIO & -.3718 & -.3101 & -.3862 \\
\hline TOT TRADE LINKAGES & .8025 & .6986 & .6590 \\
\hline TOTAL IHPORTS & .6609 & .5761 & .4856 \\
\hline TOTAL EXPORTS & .5970 & .5347 & .4683 \\
\hline ECC/ICC RATIO & .3629 & .2541 & .1196 \\
\hline COHH CONC GAP & -.2219 & -.2300 & -.1512 \\
\hline
\end{tabular}


SUMMARY OF PEARSON CORRELATION RESULTS AMONG VARIABLES

\begin{tabular}{|c|c|c|c|}
\hline \% EXP AGR-HINE & 1962 & 1970 & 1980 \\
\hline X AGRICULTURE-GDP & .5447 & .5676 & .5460 \\
\hline VERTICAL TRADE & -.9392 & -.9292 & -.9515 \\
\hline EXP COHH CONCEN & .5081 & .4737 & .3592 \\
\hline IMP COIM CONCEN & .5983 & .5157 & .5455 \\
\hline G.N.P. PER CAPITA & .0000 & .0000 & -.5240 \\
\hline QUALITY OF LIFE & -.5633 & -.5209 & -.5813 \\
\hline X EXP MANUFAC. & -.9988 & -.9999 & -.9998 \\
\hline X EXP AGR-HINE & 1.0000 & 1.0000 & 1.0000 \\
\hline X Imp manufac. & .4728 & .2606 & .3617 \\
\hline X IMP AGR-HINE & -.4640 & -.2172 & -.3599 \\
\hline EXP. PARTNER CON. & .6002 & .4587 & .5075 \\
\hline IMP. PARTNER CON. & .5102 & .3394 & .3115 \\
\hline EPC/IPC RATIO & .3284 & .3243 & .3935 \\
\hline TOT TRADE LINKAGES & -.8072 & -.7031 & -.6679 \\
\hline TOTAL IMPORTS & -.6660 & -.5809 & -.4920 \\
\hline TOTAL EXPORTS & -.6017 & -.5382 & -.4751 \\
\hline ECC/ICC RATIO & -.3509 & -.2469 & -.0907 \\
\hline COHH CONC GAP & .2464 & .2717 & .1843 \\
\hline
\end{tabular}


SUMMARY OF PEARSON CORRELATION RESULTS AMONG VARIABLES

* inp manufac.

X agricUlture-gDP

VERTICAL TRADE

EXP COHM CONCEN

IHP COHH CONCEN

G.N.P. PER CAPITA

QUALITY OF LIFE

X eXp hanufac.

\% EXP AGR-MINE

z Imp manufac.

\% IMP AGR-MINE

EXP. PARTNER CON.

IMP. PARTNER CON.

EPC/IPC RATIO

TOT TRADE LIMKAGES

TOTAL IAPORTS

TOTAL EXPORTS

ECC/ICC RATIO

CONH CONC GAP
$1962 \quad 1970 \quad 1980$

$$
\begin{aligned}
& \begin{array}{lll}
.5017 \quad .2444 & .0918
\end{array} \\
& \begin{array}{lll}
-.7452 & -.5818 & -.6304
\end{array} \\
& \begin{array}{lll}
.1297 & -.0530 \quad .1570
\end{array} \\
& \begin{array}{lll}
.4355 & .4284 & .6568
\end{array} \\
& \begin{array}{llll}
.0000 & .0000 & -.0669
\end{array} \\
& \begin{array}{lll}
-.4394 & -.1839 & -.1931
\end{array} \\
& \begin{array}{lll}
-.4552 & -.3113 & -.3719
\end{array} \\
& \begin{array}{lll}
.4728 & .2606 & .3617
\end{array} \\
& 1.0000 \quad 1.0000 \quad 1.0000 \\
& \begin{array}{llll}
-.9968 & -.9580 & -.9998
\end{array} \\
& \begin{array}{lll}
.3570 & .0114 & .3504
\end{array} \\
& \begin{array}{lll}
.2626 & .2271 \quad .4052
\end{array} \\
& \begin{array}{lll}
.2157 & -.2130 \quad .0974
\end{array} \\
& \begin{array}{lll}
-.5732 & -.2526 & -.3300
\end{array} \\
& \begin{array}{lll}
-.4935 & -.2704 & -.3164
\end{array} \\
& \begin{array}{lll}
-.4669 & -.2605 & -.2888
\end{array} \\
& \begin{array}{lll}
-.4599 & -.4349 & -.2777
\end{array} \\
& \begin{array}{lll}
-.1134 & -.2696 & -.0877
\end{array}
\end{aligned}
$$


SUMMARY OF PEARSON CORRELATION RESULTS AMONG VARIABLES

\begin{tabular}{|c|c|c|c|}
\hline$X$ IMP AGR-MINE & 1962 & 1970 & 1980 \\
\hline$X$ AGRICULTURE-GDP & -.4982 & -.2333 & -.0905 \\
\hline VERTICAL TRADE & .7393 & .5538 & .6290 \\
\hline EXP CONH CONCEN & -.1312 & .0639 & -.1590 \\
\hline IMP COHH CONCEN & -.4456 & -.4674 & -.6567 \\
\hline G.N.P. PER CAPITA & .0000 & .0000 & .0662 \\
\hline QUALITY OF LIFE & .4366 & .1520 & .1914 \\
\hline \% eXP hanufac. & .4450 & .2589 & .3699 \\
\hline$X$ EXP AGR-MINE & -.4640 & -.2172 & -.3599 \\
\hline \% IHP maNufaC. & -.9968 & -.9580 & -.9998 \\
\hline \% IHP AGR-MINE & 1.0000 & 1.0000 & 1.0000 \\
\hline EXP. PARTNER CON. & -.3475 & .0053 & -.3517 \\
\hline IMP. PARTMER CON. & -.2574 & -.2175 & -.4082 \\
\hline EPC/IPC RATIO & -.2057 & .2279 & -.0964 \\
\hline TOT TRADE LINKAGES & .5659 & .2335 & .3305 \\
\hline TOTAL IMPORTS & .4879 & .2482 & .3150 \\
\hline TOTAL EXPORTS & .4613 & .2415 & .2873 \\
\hline ECC/ICC RATIO & .4669 & .4389 & .2750 \\
\hline COHA CONC GAP & .1173 & .2999 & .0854 \\
\hline
\end{tabular}


SUHMARY OF PEARSON CORRELATION RESULTS AMONG VARIABLES

\begin{tabular}{|c|c|c|c|}
\hline EXP. PARTNER CON. & 1962 & 1970 & 1980 \\
\hline X AGRICULTURE-GDP & .4505 & .3163 & .3023 \\
\hline VERTICAL TRADE & -.5924 & -.3719 & -.5346 \\
\hline EXP COHH CONCEN & .4184 & .4507 & .5192 \\
\hline IMP COMH CONCEN & .4448 & .4145 & .4506 \\
\hline G.N.P. PER CAPITA & .0000 & .0000 & -.3444 \\
\hline QUALITY OF LIFE & -.5206 & -.3851 & -.4527 \\
\hline \% EXP MANUFAC. & -.6226 & -.4711 & -.5274 \\
\hline X EXP AGR-MINE & .6002 & .4587 & .5075 \\
\hline \% IMP MANUFAC. & .3570 & .0114 & .3504 \\
\hline X IMP AGR-MINE & -.3475 & .0053 & -.3517 \\
\hline EXP. PARTNER CON. & 1.0000 & 1.0000 & 1.0000 \\
\hline IMP. PARTMER CON. & .8456 & .7337 & .7013 \\
\hline EPC/IPC RATIO & .4371 & .6319 & .5811 \\
\hline TOT TRADE LINKAGES & -.7674 & -.7123 & -.7265 \\
\hline TOTAL IMPORTS & -.5151 & -.4320 & -.4482 \\
\hline TOTAL EXPORTS & -.4591 & -.3980 & -.4171 \\
\hline ECC/ICC RATIO & -.2108 & -.1347 & 1978 \\
\hline ONH CONC & .3133 & .2427 & .4027 \\
\hline
\end{tabular}


SUMMARY OF PEARSON CORRELATION RESULTS AMONG VARIABLES

\begin{tabular}{|c|c|c|c|}
\hline IMP. PARTNER CON. & 1962 & 1970 & 1980 \\
\hline X AGRICULTURE-GDP & .5130 & .2773 & .2132 \\
\hline VERTICAL TRADE & -.4905 & -.3550 & -.3894 \\
\hline EXP COMH CONCEN & .4402 & .3281 & .4381 \\
\hline IMP COHN CONCEN & .5787 & .5185 & .4596 \\
\hline G.N.P. PER CAPITA & .0000 & .0000 & -.2026 \\
\hline QUALITY OF LIFE & -.5801 & -.3203 & -.3415 \\
\hline * exp nanufac. & -.5189 & -.3755 & -.3462 \\
\hline X EXP AGR-MINE & .5102 & .3394 & .3115 \\
\hline X IHP MANUFAC. & .2626 & .2271 & .4052 \\
\hline X IMP AGR-MINE & -.2574 & -.2175 & -.4082 \\
\hline EXP. PARTNER COH. & .8456 & .7337 & .7013 \\
\hline IMP. PARTNER CON. & 1.0000 & 1.0000 & 1.0000 \\
\hline EPC/IPC RATIO & -.0948 & -.0460 & -.1477 \\
\hline TOT TRADE LINKAGES & -.6604 & -.5677 & -.5332 \\
\hline TOTAL IMPORTS & -.4902 & -.3796 & -.4007 \\
\hline TOTAL EXPORTS & -.4408 & -.3458 & -.3740 \\
\hline ECC/ICC RATIO & -.2319 & -.1832 & .1670 \\
\hline COHH CONC GAP & .2168 & .0379 & .3119 \\
\hline
\end{tabular}


SUMMARY OF PEARSON CORRELATION RESULTS AMONG VARIABLES

\begin{tabular}{|c|c|c|c|}
\hline EPC/IPC RATIO & 1962 & 1970 & 1980 \\
\hline X AGRICULTURE-GDP & .0490 & .1909 & .1943 \\
\hline VERTICAL TRADE & -.3277 & -.1800 & -.3586 \\
\hline EXP COHH CONCEN & .0957 & .3238 & .2690 \\
\hline IMP COHY CONCEN & -.0540 & .1103 & .1721 \\
\hline G.N.P. PER CAPITA & .0000 & .0000 & -.2686 \\
\hline QUALITY OF LIFE & -.0824 & -.2646 & -.2544 \\
\hline X EXP manUfAC. & -.3718 & -.3101 & -.3862 \\
\hline \% EXP NGR-MINE & .3284 & .3243 & .3935 \\
\hline \% IMP MANUFAC. & .2157 & -.2130 & .0974 \\
\hline X IMP AGR-HINE & -.2057 & .2279 & -.0964 \\
\hline EXP. PARTNER CON. & .4371 & .6319 & .5811 \\
\hline IMP. PARTNER CON. & -.0948 & -.0460 & -.1477 \\
\hline EPC/IPC RATIO & 1.0000 & 1.0000 & 1.0000 \\
\hline TOT TRADE LIHKAGES & -.3539 & -.4352 & -.4266 \\
\hline TOTAL IMPORTS & -.1739 & -.2382 & -.1920 \\
\hline TOTAL EXPORTS & -.1479 & -.2253 & -.1804 \\
\hline ECC/ICC RATIO & -.0349 & -.02222 & .1066 \\
\hline CONH CONC GAP & .1994 & .3038 & .2285 \\
\hline
\end{tabular}


SUMMARY OF PEARSON CORRELATION RESULTS AMONG VARIABLES

\begin{tabular}{|c|c|c|c|}
\hline TOT TRADE LINKAGES & 1962 & 1970 & 1980 \\
\hline X AGRICULTURE-GDP & -.5533 & -.5840 & -.5722 \\
\hline VERTICAL TRADE & .8301 & .6842 & .6626 \\
\hline EXP COHH COHCEN & -.4773 & -.4394 & -.5117 \\
\hline IMP CONH CONCEN & -.5703 & -.5637 & -.5640 \\
\hline G.N.P. PER CAPITA & .0000 & .0000 & .6843 \\
\hline QUALITY OF LIFE & .5843 & .5747 & .6597 \\
\hline X EXP maNUFAC. & .8025 & .6986 & .6590 \\
\hline X EXP AGR-HINE & -.8072 & -.7031 & -.6679 \\
\hline X IMP MANUFAC. & -.5732 & -.2526 & -.3300 \\
\hline X IMP AGR-MINE & .5659 & .2335 & .3305 \\
\hline EXP. PARTNER CON. & -.7674 & -.7123 & -.7265 \\
\hline IMP. PARTNER CON. & -.6604 & -.5677 & -.5332 \\
\hline EPC/IPC RATIO & -.3539 & -.4352 & -.4266 \\
\hline TOT TRADE LINKAGES & 1.0000 & 1.0000 & 1.0000 \\
\hline TOTAL IMPORTS & .8246 & .7916 & .7180 \\
\hline TOTAL EXPORTS & .7570 & .7519 & .6920 \\
\hline ECC/ICC RATIO & .3736 & .2978 & -.0950 \\
\hline COMH CONC GAP & -.2772 & -.2112 & -.3425 \\
\hline
\end{tabular}


SUMMARY OF PEARSON CORRELATION RESULTS AMONG VARIABLES

\begin{tabular}{|c|c|c|c|}
\hline TOTAL IMPORTS & 1962 & 1970 & 1980 \\
\hline X AGRICULTURE-GDP & -.4637 & -.4751 & -.4141 \\
\hline VERTICAL TRADE & .6965 & .5958 & .5112 \\
\hline EXP COHM CONCEN & -.3889 & -.3046 & -.2827 \\
\hline IHP COHA CONCEN & -.5020 & -.4624 & -.3684 \\
\hline G.N.P. PER CAPITA & .0000 & .0000 & .6139 \\
\hline QUALITY OF LIFE & .4677 & .4191 & .4117 \\
\hline X EXP MANUFAC. & .6609 & .5761 & .4856 \\
\hline X EXP NGR-MINE & -.6660 & -.5809 & -.4920 \\
\hline X IMP MANUFAC. & -.4935 & -.2704 & -.3164 \\
\hline \% IMP AGR-HINE & .4879 & .2482 & .3150 \\
\hline EXP. PARTNER CON. & -.5151 & -.4320 & -.4482 \\
\hline IMP. PARTHER COH. & -.4902 & -.3796 & -.4007 \\
\hline EPC/IPC RATIO & -.1739 & -.2382 & -.1920 \\
\hline TOT TRADE LINKAGES & .8246 & .7916 & .7180 \\
\hline TOTAL IMPORTS & 1.0000 & 1.0000 & 1.0000 \\
\hline TOTAL EXPORTS & .9864 & .9900 & .9890 \\
\hline ECC/ICC RATIO & .2547 & .2664 & -.0291 \\
\hline COHH CONC GAP & -.1910 & -.1159 & -.1694 \\
\hline
\end{tabular}


SUMHARY OF PEARSON CORRELATION RESULTS AMONG VARIABLES

TOTAL EXPORTS

* agriculture-gop

VERTICAL TRADE

EXP CONH CONCEN

IMP COHH CONCEN

G.N.P. PER CAPITA

QUALITY OF LIFE

X EXP manufac.

* EXP AGR-MINE

\% IMP manUfac.

X IMP AGR-MINE

EXP. PARTNER CON.

IHP. PARTNER CON.

EPC/IPC RATIO

TOT TRADE LINKAGES

TOTAL IHPORTS

TOTAL EXPORTS

ECC/ICC RATIO

CONH CONC GAP
$1962 \quad 1970 \quad 1980$

$$
\begin{aligned}
& \begin{array}{lll}
-.4386 & -.4457 & -.4058
\end{array} \\
& \begin{array}{lll}
.6352 & .5567 & .4878
\end{array} \\
& \begin{array}{lll}
-.3455 & -.2651 & -.2597
\end{array} \\
& \begin{array}{lll}
-.4434 & -.4267 & -.3445
\end{array} \\
& \begin{array}{lll}
.0000 \quad .0000 \quad .6058
\end{array} \\
& \begin{array}{lll}
.4162 \quad .3829 & .3969
\end{array} \\
& \begin{array}{lll}
.5970 \quad .5347 & .4683
\end{array} \\
& \begin{array}{lll}
-.6017 & -.5382 & -.4751
\end{array} \\
& \begin{array}{lll}
-.4669 & -.2605 & -.2888
\end{array} \\
& \begin{array}{lll}
.4613 & .2415 \quad .2873
\end{array} \\
& \begin{array}{lll}
-.4591 & -.3980 & -.4171
\end{array} \\
& \begin{array}{lll}
-.4408 & -.3458 & -.3740
\end{array} \\
& -.1479 \quad-.2253 \quad-.1804 \\
& \begin{array}{lll}
.7570 & .7519 \quad .6920
\end{array} \\
& \begin{array}{lll}
.9864 \quad .9900 \quad .9890
\end{array} \\
& 1.0000 \quad 1.0000 \quad 1.0000 \\
& \begin{array}{lll}
.2170 & .2839 & -.0202
\end{array} \\
& \begin{array}{lll}
-.1751 & -.0892 & -.1528
\end{array}
\end{aligned}
$$


SUMMARY OF PEARSON CORRELATION RESULTS AMONG VARIABLES

\begin{tabular}{|c|c|c|c|}
\hline ECC/ICC RATIO & 1962 & 1970 & 1980 \\
\hline × AGRICULTURE-GDP & -.2163 & -.1166 & .0859 \\
\hline VERTICAL TRADE & .4491 & .3802 & .1684 \\
\hline EXP COHH CONCEN & .1277 & .1441 & .6768 \\
\hline IMP COHH CONCEN & -.4315 & -.3636 & -.3006 \\
\hline G.N.P. PER CAPITA & .0000 & .0000 & -.1029 \\
\hline QUALITY OF LIFE & .2770 & .1350 & .0348 \\
\hline X EXP MANUFAC. & .3629 & .2541 & .1196 \\
\hline * EXP AGR-HINE & -.3509 & -.2469 & -.0907 \\
\hline X IHP MANUFAC. & -.4599 & -.4349 & -.2777 \\
\hline X IHP AGR-HINE & .4669 & .4389 & .2750 \\
\hline EXP. PARTNER CON. & -.2108 & -.1347 & .1978 \\
\hline IHP. PARTNER CON. & -.2319 & -.1832 & .1670 \\
\hline EPC/IPC RATIO & -.0349 & -.0222 & .1066 \\
\hline TOT TRADE LINKAGES & .3736 & .2978 & -.0950 \\
\hline TOTAL IAPORTS & .2547 & .2664 & -.0291 \\
\hline TOTAL EXPORTS & .2170 & .2839 & -.0202 \\
\hline ECC/ICC RATIO & 1.0000 & 1.0000 & 1.0000 \\
\hline CONH CONC GAP & .3942 & .3305 & .8457 \\
\hline
\end{tabular}


SUMMARY OF PEARSON CORRELATION RESULTS AMONG VARIABLES

\begin{tabular}{|c|c|c|c|}
\hline COHH CONC GAP & 1962 & 1970 & 1980 \\
\hline X AGRICULTURE-GDP & .1062 & .2071 & .1512 \\
\hline VERTICAL TRADE & -.1394 & -.1150 & -.1240 \\
\hline EXP COHM CONCEN & .8644 & .8900 & .9298 \\
\hline IMP COHM CONCEN & .0022 & -.0661 & .0132 \\
\hline G.N.P. PER CAPITA & .0000 & .0000 & -.1647 \\
\hline QUALITY OF LIFE & .0096 & -.1303 & -.0858 \\
\hline X EXP MANUFAC. & -.2219 & -.2300 & -.1512 \\
\hline X EXP AGR-HINE & .2464 & .2717 & .1843 \\
\hline X IHP MANUFAC. & -.1134 & -.2696 & -.0877 \\
\hline X IMP AGR-MINE & .1173 & .2999 & .0854 \\
\hline EXP. PARTNER CON. & .3133 & .2427 & .4027 \\
\hline IMP. PARTNER CON. & .2168 & .0379 & .3119 \\
\hline EPC/IPC RATIO & .1994 & .3038 & .2285 \\
\hline TOT TRADE LINKAGES & -.2772 & -.2112 & -.3425 \\
\hline TOTAL INPORTS & -.1910 & -.1159 & -.1694 \\
\hline TOTAL EXPORTS & -.1751 & -.0892 & -.1528 \\
\hline ECC/ICC RATIO & .3942 & .3305 & .8457 \\
\hline COHM CONC GAP & 1.0000 & 1.0000 & 1.0000 \\
\hline
\end{tabular}




\section{APPENDIX D}

SUMMARY OF NATIONAL STATISTICS 
This appendix provides national statistics for over twenty variables of which the relationships among ten of those variables are discussed in the text. The statistics are given for all 127 countries, therefore, diachronic comparisons among these countries are made possible. The first three colomns are the values of each variable for the years 1962,1970 , and 1980 . The last two colomns indicate the percent change of each variable from 1962 to 1970 or from 1970 to 1980. 
AFGHANISTA

SUMMARY OF NATIONAL STATISTICS

\begin{tabular}{|c|c|c|c|c|c|}
\hline MATIONAL VARIABLES & 1962 & 1970 & 1980 & $62-70$ & $70-80$ \\
\hline EXP. PARTNER CON. & 0.64 & 0.61 & 0.70 & -4.69 & 14.75 \\
\hline IMP. PARTNER CON. & 0.70 & 0.57 & 0.66 & -18.57 & 15.79 \\
\hline EPC/IPC RATIO & 0.90 & 1.07 & 1.06 & 18.89 & -0.93 \\
\hline EXP COMH CONCEN & 0.39 & 0.20 & NA & -48.72 & - \\
\hline IMP CONM CONCEN & 0.56 & 0.38 & NA & -32.14 & - \\
\hline ECC/ICC RATIO & 0.70 & 0.53 & M & -24.43 & - \\
\hline VERTICAL TRADE & -0.71 & -0.61 & M & -14.08 & - \\
\hline TOT TRADE LINKAgES & 9.00 & 8.00 & 21.00 & -11.11 & 162.50 \\
\hline * AGRICULTURE-GDP & MA & NA & MA & - & - \\
\hline QUALITY OF LIFE & -1.90 & 4.50 & 9.70 & -336.84 & 115.56 \\
\hline \% LRGST EXP TO GDP & NA & NA & MA & - & - \\
\hline * EXP LGST PARTNER & 32.00 & 29.00 & 63.00 & -9.38 & 117.24 \\
\hline \% IHP LGST PARTNER & 53.00 & 27.00 & 58.00 & -49.06 & 114.81 \\
\hline \% EXP MAKUFAC. & 14.00 & 11.00 & Na & -21.43 & - \\
\hline \% EXP AGR-MINE & 85.00 & 88.00 & MA & 3.53 & - \\
\hline \% IMP MANUFAC. & 85.00 & 71.00 & NA & -16.47 & - \\
\hline ₹ IMP AGR-MINE & 14.00 & 28.00 & NA & 100.00 & - \\
\hline TOTAL EXPORTS & 76.00 & 71.00 & 623.00 & -6.58 & 777.46 \\
\hline TOTAL IMPORTS & 73.00 & 77.00 & 776.00 & 5.48 & 907.79 \\
\hline G.N.P. PER CAPITA & MA & $\mathbf{M}$ & 210.00 & - & - \\
\hline
\end{tabular}


ALGERIA

SUMMARY OF NATIONAL STATISTICS

\begin{tabular}{|c|c|c|c|c|c|}
\hline NATIONAL VARIABLES & 1962 & 1970 & 1980 & $62-70$ & $70-80$ \\
\hline EXP. PARTNER CON. & 0.83 & 0.64 & 0.63 & -22.89 & -1.56 \\
\hline IMP. PARTNER CON. & 0.79 & 0.56 & 0.46 & -29.11 & -17.86 \\
\hline EPC/IPC RATIO & 1.04 & 1.14 & 1.36 & 9.62 & 19.30 \\
\hline EXP COMM CONCEN & NA & 0.50 & 0.96 & - & 92.00 \\
\hline IHP COMH CONCEN & NA & 0.29 & 0.26 & - & -10.34 \\
\hline ECC/ICC RATIO & NA & 1.72 & 3.69 & - & 114.15 \\
\hline VERTICAL TRADE & NA & -0.73 & -0.71 & - & -2.74 \\
\hline TOT TRADE LINKAGES & 15.00 & 21.00 & 40.00 & 40.00 & 90.48 \\
\hline X AGRICULTURE-GDP & 16.00 & 13.00 & 7.00 & -18.75 & -46.15 \\
\hline QUALITY OF LIFE & NA & 34.20 & 44.00 & - & 28.65 \\
\hline X LRGST EXP TO GDP & 11.20 & 4.10 & MA & -63.39 & - \\
\hline * EXP LGST PARTNER & 81.00 & 55.00 & 48.00 & -32.10 & -12.73 \\
\hline X IMP LGST PARTNER & 79.00 & 48.00 & 26.00 & -39.24 & -45.83 \\
\hline X EXP manUfac. & NA & 6.00 & M & - & - \\
\hline$X$ EXP AGR-MINE & M & 93.00 & 99.00 & - & 6.45 \\
\hline X IMP MANUFAC. & NA & 79.00 & 71.00 & - & -10.13 \\
\hline X IMP AGR-MINE & NA & 20.00 & 28.00 & - & 40.00 \\
\hline TOTAL EXPORTS & 807.00 & 968.00 & 15621.00 & 19.95 & 1513.74 \\
\hline TOTAL IMPORTS & 705.00 & 1167.00 & 9973.00 & 65.53 & 754.58 \\
\hline G.H.P. PER CAPITA & MA & MA & 2140.00 & - & - \\
\hline
\end{tabular}


ANGOLA

SUMMARY OF NATIONAL STATISTICS

\begin{tabular}{|c|c|c|c|c|c|}
\hline MATIONAL VARIABLES & 1962 & 1970 & 1980 & $62-70$ & $70-80$ \\
\hline EXP. PARTNER CON. & 0.56 & 0.55 & 0.61 & -1.79 & 10.91 \\
\hline IHP. PARTNER CON. & 0.56 & 0.55 & 0.44 & -1.79 & -20.00 \\
\hline EPC/IPC RATIO & 1.00 & 0.99 & 1.40 & -1.00 & 41.41 \\
\hline EXP COMH CONCEN & 0.35 & 0.21 & M & -40.00 & - \\
\hline IMP COMM CONCEN & 0.34 & 0.32 & Ma & -5.88 & - \\
\hline ECC/ICC RATIO & 1.03 & 0.66 & M & -36.25 & - \\
\hline VERTICAL TRADE & -0.63 & -0.57 & Ma & -9.52 & - \\
\hline TOT TRADE LINKAGES & 12.00 & 20.00 & 22.00 & 66.67 & 10.00 \\
\hline \% AGRICULTURE-GDP & 53.20 & 48.40 & 43.30 & -9.02 & -10.54 \\
\hline QUALITY OF LIFE & MA & NA & M & - & - \\
\hline * LRGST EXP TO GDP & NA & NA & MA & - & - \\
\hline * EXP LGST PARTNER & 26.00 & 35.00 & 37.00 & 34.62 & 5.71 \\
\hline \% IMP LGST PARTNER & 40.00 & 29.00 & 14.00 & -27.50 & -51.72 \\
\hline X EXP hanUfaC. & 14.00 & 21.00 & NA & 50.00 & - \\
\hline X EXP AGR-HINE & 85.00 & 78.00 & NA & -8.24 & - \\
\hline * iap tanufac. & 77.00 & 79.00 & MA & 2.60 & - \\
\hline$X$ IMP AGR-MINE & 22.00 & 21.00 & MA & -4.55 & - \\
\hline TOTAL EXPORTS & 139.00 & 411.00 & 1868.00 & 195.68 & 354.50 \\
\hline TOTAL IKPORTS & 109.00 & 486.00 & 1213.00 & 345.87 & 149.59 \\
\hline G.N.P. PER CAPITA & NA & MA & 840.00 & - & - \\
\hline
\end{tabular}


ARGENTINA

SUMMARY OF NATIONAL STATISTICS

\begin{tabular}{|c|c|c|c|c|c|}
\hline NATIONAL VARIABLES & 1962 & 1970 & 1980 & $62-70$ & $70-80$ \\
\hline EXP. PARTNER CON. & 0.42 & 0.38 & 0.37 & -9.52 & -2.63 \\
\hline IHP. PARTNER CON. & 0.46 & 0.44 & 0.42 & -4.35 & -4.55 \\
\hline EPC/IPC RATIO & 0.91 & 0.87 & 0.88 & -4.40 & 1.15 \\
\hline EXP COHA CONCEN & 0.52 & 0.46 & 0.32 & -11.54 & -30.43 \\
\hline IMP COMM COHCEN & 0.30 & 0.19 & 0.24 & -36.67 & 26.32 \\
\hline ECC/ICC RATIO & 1.73 & 2.42 & 1.33 & 39.68 & -44.93 \\
\hline VERTICAL TRADE & -0.77 & -0.59 & -0.54 & -23.38 & -8.47 \\
\hline TOT TRADE LINKAGES & 40.00 & 54.00 & 88.00 & 35.00 & 62.96 \\
\hline X AGRICULTURE-GDP & 17.60 & 13.60 & 9.70 & -22.73 & -28.68 \\
\hline QUALITY OF LIFE & 79.40 & 83.00 & 86.50 & 4.53 & 4.22 \\
\hline * LRGST EXP TO GDP & 1.20 & $\mathbf{M A}$ & Ma & -8350.00 & 0.00 \\
\hline * EXP LGST PARTNER & 16.00 & 15.00 & 20.00 & -6.25 & 33.33 \\
\hline \% IMP LGST PARTNER & 28.00 & 24.00 & 24.00 & -14.29 & 0.00 \\
\hline X EXP MaNUFAC. & 3.00 & 14.00 & 23.00 & 366.67 & 64.29 \\
\hline \% EXP AGR-MINE & 96.00 & 86.00 & 76.00 & -10.42 & -11.63 \\
\hline X IHP MANUFAC. & 80.00 & 73.00 & $\pi . \infty 0$ & -8.75 & 5.48 \\
\hline \% IMP AGR-MINE & 19.00 & 26.00 & 22.00 & 36.84 & -15.38 \\
\hline TOTAL EXPORTS & 1208.00 & 1771.00 & 7982.00 & 46.61 & 350.71 \\
\hline TOTAL IMPORTS & 1299.00 & 1772.00 & 10934.00 & 36.41 & 517.04 \\
\hline G.N.P. PER CAPITA & ma & NA & 2560.00 & - & - \\
\hline
\end{tabular}


AUSTRALIA

SUMHARY OF NATIONAL STATISTICS

\begin{tabular}{|c|c|c|c|c|c|}
\hline NATIONAL VARIABLES & 1962 & 1970 & 1980 & $62-70$ & $70-80$ \\
\hline EXP. PARTNER CON. & 0.42 & 0.41 & 0.39 & -2.38 & -4.88 \\
\hline IMP. PARTNER CON. & 0.46 & 0.45 & 0.42 & -2.17 & -6.67 \\
\hline EPC/IPC RATIO & 0.90 & 0.91 & 0.93 & 1.11 & 2.20 \\
\hline EXP COHH CONCEN & 0.21 & 0.09 & 0.10 & -57.14 & 11.11 \\
\hline IHP COHM CONCEN & 0.19 & 0.29 & 0.25 & 52.63 & -13.79 \\
\hline ECC/ICC RATIO & 1.11 & 0.31 & 0.40 & -71.92 & 28.89 \\
\hline VERTICAL TRADE & -0.61 & -0.63 & -0.50 & 3.28 & -20.63 \\
\hline TOT TRADE LINKAGES & 58.00 & 80.00 & 89.00 & 37.93 & 11.25 \\
\hline X AGRICULTURE-GDP & $12 . \infty 0$ & 9.40 & 6.30 & -21.67 & -32.98 \\
\hline QUALITY OF LIFE & 92.80 & 94.10 & 97.10 & 1.40 & 3.19 \\
\hline X LRGST EXP TO GDP & 2.90 & 3.90 & 5.40 & 34.48 & 38.46 \\
\hline \% EXP LGST PARTNER & 20.00 & 26.00 & 27.00 & 30.00 & 3.85 \\
\hline X IMP LGST PARTNER & 31.00 & 23.00 & 21.00 & -25.81 & -8.70 \\
\hline X EXP manufac. & 11.00 & 18.00 & 26.00 & 63.64 & 44.64 \\
\hline X EXP AGR-MINE & 88.00 & 81.00 & 73.00 & -7.95 & -9.88 \\
\hline \% IMP MANUFAC. & 72.00 & 82.00 & 76.00 & 13.89 & -7.32 \\
\hline \% IMP AGR-MINE & 27.00 & 17.00 & 23.00 & -37.04 & 35.29 \\
\hline TOTAL EXPORTS & 2251.00 & 4707.00 & 21339.00 & 109.11 & 353.35 \\
\hline TOTAL IISPORTS & 2054.00 & 4175.00 & 19305.00 & 103.26 & 362.40 \\
\hline G.N.P. PER CAPITA & NA & $\mathbf{N A}$ & 11080.00 & - & - \\
\hline
\end{tabular}


AUSTRIA

SUMMARY OF NATIONAL STATISTICS

\begin{tabular}{|c|c|c|c|c|c|}
\hline MATIONAL VARIABLES & 1962 & 1970 & 1980 & $62-70$ & $70-80$ \\
\hline EXP. PARTNER CON. & 0.41 & 0.38 & 0.40 & -7.32 & 5.26 \\
\hline IMP. PARTNER CON. & 0.53 & 0.54 & 0.53 & 1.89 & -1.85 \\
\hline EPC/IPC RATIO & 0.76 & 0.70 & 0.76 & -7.89 & 8.57 \\
\hline EXP COHM CONCEN & 0.21 & 0.26 & 0.28 & 23.81 & 7.69 \\
\hline IHP COHH CONCEN & 0.12 & 0.29 & 0.18 & 141.67 & -37.93 \\
\hline ECC/ICC RATIO & 1.75 & 0.90 & 1.56 & -48.77 & 73.50 \\
\hline VERTICAL TRADE & 0.11 & -0.02 & 0.14 & -118.18 & -800.00 \\
\hline TOT TRADE LINKAGES & 53.00 & 81.00 & 101.00 & 52.83 & 24.69 \\
\hline X AGRICULTURE-GDP & 11.00 & 8.40 & 4.90 & -23.64 & -41.67 \\
\hline QUALITY OF LIFE & 88.20 & 91.60 & 95.50 & 3.85 & 4.26 \\
\hline X LRGST EXP TO GDP & 4.70 & 4.40 & 4.90 & -6.38 & 11.36 \\
\hline X EXP LGST PARTNER & 28.00 & 21.00 & 30.00 & -25.00 & 42.86 \\
\hline X IHP LGST PARTNER & 46.00 & 46.00 & 45.00 & 0.00 & -2.17 \\
\hline X EXP MANUFAC. & 73.00 & 80.00 & 82.00 & 9.59 & 2.50 \\
\hline X EXP AGR-MINE & 26.00 & 19.00 & 17.00 & -26.92 & -10.53 \\
\hline X IHP MANUFAC. & 62.00 & 82.00 & 69.00 & 32.26 & -15.85 \\
\hline * IHP AGR-HINE & 37.00 & 17.00 & 30.00 & -54.05 & 76.47 \\
\hline TOTAL EXPORTS & 1249.00 & 3085.00 & 17463.00 & 147.00 & 466.06 \\
\hline TOTAL IMPORTS & 1483.00 & 3356.00 & 23107.00 & 126.30 & 588.53 \\
\hline G.H.P. PER CA & $\mathrm{ma}$ & M & 10210.00 & - & - \\
\hline
\end{tabular}


BAHAMAS

SUMMARY OF NATIONAL STATISTICS

\begin{tabular}{|c|c|c|c|c|c|}
\hline MATIONAL VARIABLES & 1962 & 1970 & 1980 & $62-70$ & $70-80$ \\
\hline EXP. PARTNER CON. & 0.95 & 0.75 & 0.67 & -21.05 & -10.67 \\
\hline IHP. PARTNER CON. & 0.70 & 0.69 & 0.57 & -1.43 & -17.39 \\
\hline EPC/IPC RATIO & 1.36 & 1.09 & 1.17 & -19.85 & 7.34 \\
\hline EXP CONH CONCEN & 0.16 & 0.16 & $\mathbf{M}$ & 0.00 & - \\
\hline IHP COHH CONCEN & NA & 0.20 & $\mathbf{M}$ & - & - \\
\hline ECC/ICC RATIO & NA & 0.80 & MA & - & - \\
\hline VERTICAL TRADE & NA & -0.16 & $\mathbf{M}$ & - & - \\
\hline TOT TRADE LINKAGES & 1.00 & 5.00 & 25.00 & 400.00 & 400.00 \\
\hline \% AGRICULTURE-GDP & NA & MA & MA & - & - \\
\hline QUALITY OF LIFE & NA & Ma & NA & - & - \\
\hline \% LRGST EXP TO GDP & NA & NA & NA & - & - \\
\hline X EXP LGST PARTNER & 94.00 & 73.00 & 55.00 & -22.34 & -24.66 \\
\hline X IMP LGST PARTNER & 49.00 & 59.00 & 35.00 & 20.41 & -40.68 \\
\hline X EXP MANUFAC. & NA & 45.00 & MA & - & - \\
\hline X EXP AGR-MINE & NA & 54.00 & NA & - & - \\
\hline X ImP hanufaC. & MA & 61.00 & $M$ & - & - \\
\hline X IMP AGR-HINE & NA & 38.00 & MA & - & - \\
\hline TOTAL EXPORTS & 8.00 & 85.00 & 2327.00 & 962.50 & 2637.65 \\
\hline TOTAL IMPORTS & 108.00 & 289.00 & 3122.00 & 167.59 & 980.28 \\
\hline G.N.P. PER CAPITA & NA & MA & U & - & - \\
\hline
\end{tabular}


BAHREIN

SUMMARY OF NATIONAL STATISTICS

\begin{tabular}{|c|c|c|c|c|c|}
\hline NATIONAL VARIABLES & 1962 & 1970 & 1980 & $62-70$ & $70-80$ \\
\hline EXP. PARTNER CON. & 0.51 & 0.48 & 0.47 & -5.88 & -2.08 \\
\hline IMP. PARTNER CON. & 0.60 & 0.62 & 0.67 & 3.33 & 8.06 \\
\hline EPC/IPC RATIO & 0.86 & 0.78 & 0.70 & -9.30 & -10.26 \\
\hline EXP COMH CONCEN & NA & 0.54 & 0.88 & - & 62.96 \\
\hline IHP COHH CONCEN & NA & 0.34 & 0.34 & - & 0.00 \\
\hline ECC/ICC RATIO & NA & 1.59 & 2.59 & - & 62.96 \\
\hline VERTICAL TRADE & MA & -0.60 & -0.30 & - & -50.00 \\
\hline TOT TRADE LINKAGES & 16.00 & 16.00 & 36.00 & 0.00 & 125.00 \\
\hline X AGRICULTURE-GDP & MA & MA & 1.30 & - & - \\
\hline QUALITY OF LIFE & 39.80 & 57.20 & 66.20 & 43.72 & 15.73 \\
\hline * LRGST EXP TO GDP & NA & MA & MA & - & - \\
\hline \% EXP LGST PARTNER & 28.00 & 14.00 & 23.00 & -50.00 & 64.29 \\
\hline X IMP LGST PARTNER & 42.00 & 46.00 & 63.00 & 9.52 & 36.96 \\
\hline Z EXP MANUFAC. & MA & 16.00 & 3.00 & - & -81.25 \\
\hline Z EXP AGR-MINE & MA & 83.00 & 97.00 & - & 16.87 \\
\hline X IHP MANUFAC. & $M$ & 76.00 & 32.00 & - & -57.89 \\
\hline \% IMP AGR-MINE & NA & 23.00 & 67.00 & - & 191.30 \\
\hline TOTAL EXPORTS & 153.00 & 159.00 & 2748.00 & 3.92 & 1628.30 \\
\hline TOTAL IHPORTS & 54.00 & 256.00 & 3193.00 & 374.07 & 1147.27 \\
\hline G.N.P. PER CAPITA & MA & MA & 8960.00 & - & - \\
\hline
\end{tabular}


BARBADOS

SUMAARY OF NATIONAL STATISTICS

\begin{tabular}{|c|c|c|c|c|c|}
\hline MATIONAL VARIABLES & 1962 & 1970 & 1980 & $62-70$ & $70-80$ \\
\hline EXP. PARTMER CON. & 0.83 & 0.73 & 0.66 & -12.05 & -9.59 \\
\hline IHP. PARTNER CON. & 0.75 & 0.60 & 0.54 & -20.00 & -10.00 \\
\hline EPC/IPC RATIO & 1.10 & 1.23 & 1.24 & 11.82 & 0.81 \\
\hline EXP COMH CONCEN & 0.90 & 0.49 & 0.35 & -45.50 & -28.57 \\
\hline IHP CONH CONCEN & 0.23 & 0.25 & 0.19 & 8.70 & -24.00 \\
\hline ECC/ICC RATIO & 3.91 & 1.96 & 1.84 & -49.91 & -6.02 \\
\hline VERTICAL TRADE & -0.51 & -0.41 & -0.11 & -19.61 & -73.17 \\
\hline TOT TRADE LINKAGES & 3.00 & 4.00 & 7.00 & 33.33 & 75.00 \\
\hline \% AGRICULTURE-GDP & 25.70 & 18.30 & 9.70 & -28.79 & -46.99 \\
\hline QUALITY OF LIFE & 78.70 & 87.60 & 97.70 & 11.31 & 4.68 \\
\hline \% LRGST EXP TO GDP & 16.10 & 8.70 & 9.10 & -45.96 & 4.60 \\
\hline X EXP LGST PARTNER & 67.00 & 54.00 & 51.00 & -19.40 & -5.56 \\
\hline X IMP LGST PARTHER & 52.00 & 33.00 & 31.00 & -36.54 & -6.06 \\
\hline Z EXP MANUFAC. & 2.00 & 25.00 & 52.00 & 1150.00 & 108.00 \\
\hline X EXP AGR-MINE & 97.00 & 74.00 & 47.00 & -23.71 & -36.49 \\
\hline$X$ IMP MANUFAC. & 53.00 & 66.00 & 63.00 & 24.53 & -4.55 \\
\hline$X$ IHP AGR-HINE & 46.00 & 33.00 & 36.00 & -28.26 & 9.09 \\
\hline TOTAL EXPORTS & 12.00 & 32.00 & 165.00 & 166.67 & 415.63 \\
\hline TOTAL IMPORTS & 28.00 & 86.00 & 430.00 & 207.14 & 400.00 \\
\hline G.N.P.P & A & A & 3500.00 & - & - \\
\hline
\end{tabular}


BELGIUM

SUMMARY OF NATIONAL STATISTICS

\begin{tabular}{|c|c|c|c|c|c|}
\hline NATIONAL VARIABLES & 1962 & 1970 & 1980 & $62-70$ & $70-80$ \\
\hline EXP. PARTNER CON. & 0.42 & 0.48 & 0.45 & 14.29 & -6.25 \\
\hline IMP. PARTNER CON. & 0.41 & 0.46 & 0.46 & 12.20 & 0.00 \\
\hline EPC/IPC RATIO & 1.02 & 1.03 & 0.97 & 0.98 & -5.83 \\
\hline EXP CONH CONCEN & 0.26 & 0.24 & 0.23 & -7.69 & -4.17 \\
\hline IHP CONH CONCEN & 0.05 & 0.09 & 0.13 & 80.00 & 44.44 \\
\hline ECC/ICC RATIO & 5.20 & 2.67 & 1.77 & -48.72 & -33.65 \\
\hline VERTICAL TRADE & 0.22 & 0.17 & 0.14 & -22.73 & -17.65 \\
\hline TOT TRADE LINKAGES & 90.00 & 111.00 & 135.00 & 23.33 & 21.62 \\
\hline X AGRICULTURE-GDP & 6.50 & 5.00 & 2.80 & -23.08 & -44.00 \\
\hline QUALITY OF LIFE & 90.60 & 93.50 & 95.90 & 3.20 & 2.57 \\
\hline$X$ LRGST EXP TO GDP & NA & 11.20 & 10.60 & - & -5.36 \\
\hline X EXP LGST PARTNER & 23.00 & 24.00 & 21.00 & 4.35 & -12.50 \\
\hline X IMP LGST PARTMER & 20.00 & 24.00 & 21.00 & 20.00 & -12.50 \\
\hline X EXP MANUFAC. & 75.00 & 76.00 & 74.00 & 1.33 & -2.63 \\
\hline Z EXP AGR-MINE & 24.00 & 23.00 & 25.00 & -4.17 & 8.70 \\
\hline X IMP manufac. & 53.00 & 59.00 & 60.00 & 11.32 & 1.69 \\
\hline X IHP AGR-MINE & 47.00 & 40.00 & 39.00 & -14.89 & -2.50 \\
\hline TOTAL EXPORTS & \multicolumn{3}{|c|}{4241.0011493 .0063859 .00} & 171.00 & 455.63 \\
\hline TOTAL IHPORTS & 4378.00 & 11429.00 & 70181.00 & 161.06 & 514.06 \\
\hline G.H.P. PER CAPITA & NA & NA & 11920.00 & - & - \\
\hline
\end{tabular}


BENIN

SUMMARY OF NATIONAL STATISTICS

\begin{tabular}{|c|c|c|c|c|c|}
\hline NATIONAL VARIABLES & 1962 & 1970 & 1980 & $62-70$ & $70-80$ \\
\hline EXP. PARTNER CON. & 1.00 & 0.60 & 0.55 & -40.00 & -8.33 \\
\hline IMP. PARTMER CON. & 1.00 & 0.62 & 0.48 & -38.00 & -22.58 \\
\hline EPC/IPC RATIO & 1.00 & 0.97 & 1.15 & -3.00 & 18.56 \\
\hline EXP COHN CONCEN & 0.80 & 0.46 & Ma & -42.50 & - \\
\hline IMP COHH CONCEN & 0.26 & 0.33 & MA & 26.92 & - \\
\hline ECC/ICC RATIO & 3.08 & 1.39 & MA & -54.70 & - \\
\hline VERTICAL TRADE & -0.60 & -0.65 & MA & 8.33 & - \\
\hline TOT TRADE LINKAGES & 1.00 & 8.00 & 10.00 & 700.00 & 25.00 \\
\hline \% AGRICULTURE-GDP & 54.80 & 51.40 & 44.80 & -6.20 & -12.84 \\
\hline QUALITY OF LIFE & 4.40 & 14.40 & 30.30 & 227.27 & 110.42 \\
\hline X LRGST EXP TO GOP & 6.70 & 9.20 & $\boldsymbol{M A}$ & 37.31 & - \\
\hline X EXP LGST PARTNER & 100.00 & 41.00 & 30.00 & -59.00 & -26.83 \\
\hline \% IHP LGST PARTNER & 100.00 & 52.00 & 24.00 & -48.00 & -53.85 \\
\hline X EXP RANUFAC. & 4.00 & 10.00 & MA & 150.00 & - \\
\hline \% EXP AGR-NINE & 95.00 & 89.00 & Ma & -6.32 & - \\
\hline X IMP MANUFAC. & 63.00 & 75.00 & MA & 19.05 & - \\
\hline \% IMP AGR-HINE & 36.00 & 24.00 & MA & -33.33 & - \\
\hline TOTAL EXPORTS & 7.00 & $31 . \infty$ & $\mathbf{5 9 . 0 0}$ & 342.86 & 90.32 \\
\hline TOTAL IHPORTS & 15.00 & 50.00 & 546.00 & 233.33 & 992.00 \\
\hline G.H.P. PEI & MA & $\mathbf{M A}$ & 320.00 & - & - \\
\hline
\end{tabular}


BERMUDA

SUMMARY OF NATIONAL STATISTICS

\begin{tabular}{|c|c|c|c|c|c|}
\hline MATIONAL VARIABLES & 1962 & 1970 & 1980 & $62-70$ & $70-80$ \\
\hline EXP. PARTNER CON. & 0.59 & 0.93 & 0.46 & 57.63 & -50.54 \\
\hline IMP. PARTNER CON. & 0.76 & 0.73 & 0.54 & -3.95 & -26.03 \\
\hline EPC/IPC RATIO & 0.77 & 1.28 & 0.86 & 66.23 & -32.81 \\
\hline EXP COAH CONCEN & MA & 0.76 & 0.46 & - & -39.47 \\
\hline IMP CONM CONCEN & MA & MA & 0.25 & - & - \\
\hline ECC/ICC RATLO & MA & Ma & 1.84 & - & - \\
\hline VERTICAL TRADE & NA & NA & -0.06 & - & - \\
\hline TOT TRADE LINKAGES & 5.00 & 2.00 & 5.00 & -60.00 & 150.00 \\
\hline X AGRICULTURE-GDP & MA & MA & NA & - & - \\
\hline QUALITY OF LIFE & M & Ma & M & - & - \\
\hline X LRGST EXP TO GDP & NA & MA & Ma & - & - \\
\hline \% EXP LGST PARTNER & 22.00 & 93.00 & 19.00 & 322.73 & -79.57 \\
\hline X IMP LGST PARTNER & 54.00 & 56.00 & 28.00 & 3.70 & -50.00 \\
\hline X EXP manufac. & NA & MA & 54.00 & - & - \\
\hline X EXP AGR-MINE & MA & MA & 45.00 & - & - \\
\hline x IMP haNUFAC. & NA & MA & 60.00 & - & - \\
\hline \% IMP AGR-HIHE & NA & MA & 39.00 & - & - \\
\hline TOTAL EXPORTS & 13.00 & 37.00 & 23.00 & 184.62 & -37.84 \\
\hline TOTAL IMPORTS & 68.00 & 161.00 & 479.00 & 136.76 & 197.52 \\
\hline G.N.P. PER CAPITA & MA & MA & 7600.00 & - & - \\
\hline
\end{tabular}


BOLIVIA

SUMMARY OF NATIONAL STATISTICS

\begin{tabular}{|c|c|c|c|c|c|}
\hline MATIOHAL VARIABLES & 1962 & 1970 & 1980 & $62-70$ & $70-80$ \\
\hline EXP. PARTNER CON. & 0.75 & 0.68 & 0.56 & -9.33 & -17.65 \\
\hline IMP. PARTNER CON. & 0.57 & 0.53 & 0.52 & -7.02 & -1.89 \\
\hline EPC/IPC RATIO & 1.30 & 1.27 & 1.08 & -2.31 & -14.96 \\
\hline EXP COMA CONCEN & 0.75 & 0.79 & 0.65 & 5.33 & -17.72 \\
\hline IMP CONH CONCEN & 0.26 & 0.33 & 0.40 & 26.92 & 21.21 \\
\hline ECC/ICC RATIO & 2.88 & 2.39 & 1.62 & -17.01 & -32.12 \\
\hline VERTICAL TRADE & -0.63 & -0.74 & -0.85 & 17.46 & 14.86 \\
\hline TOT TRADE LINKAGES & 6.00 & 9.00 & 18.00 & $\mathbf{5 0 . 0 0}$ & 100.00 \\
\hline X AGRICULTURE-GDP & 25.70 & 20.50 & 17.60 & -20.23 & -14.15 \\
\hline QUALITY OF LIFE & 26.40 & 31.40 & 46.20 & 18.94 & 47.13 \\
\hline X LRGST EXP TO GDP & 9.10 & 8.80 & 7.60 & -3.30 & -13.64 \\
\hline \% EXP LGST PARTNER & 53.00 & $39 . \infty 0$ & 27.00 & -26.42 & -30.77 \\
\hline * IMP LGST PARTNER & 40.00 & 33.00 & 21.00 & -17.50 & -36.36 \\
\hline \% EXP HANUFAC. & 5.00 & 3.00 & 2.00 & $-\$ 0.00$ & -33.33 \\
\hline \% EXP AGR-MINE & 95.00 & 97.00 & 97.00 & 2.11 & 0.00 \\
\hline * IMP MANUFAC. & 68.00 & 76.00 & 87.00 & 11.76 & 14.47 \\
\hline \% IMP AGR-HINE & 31.00 & 23.00 & 12.00 & -25.81 & -47.83 \\
\hline TOTAL EXPORTS & 76.00 & 221.00 & 967.00 & 190.79 & 337.56 \\
\hline TOTAL IHPORTS & 78.00 & 136.00 & 833.00 & 74.36 & 512.50 \\
\hline G.N.P. PER CAPITA & NA & NA & 600.00 & - & - \\
\hline
\end{tabular}


BRAZIL

SUMMARY OF MATIONAL STATISTICS

\begin{tabular}{|c|c|c|c|c|c|}
\hline MATIONAL VARIABLES & 1962 & 1970 & 1980 & $62-70$ & $70-80$ \\
\hline EXP. PARTNER CON. & 0.48 & 0.39 & 0.30 & -18.75 & -23.08 \\
\hline IHP. PARTNER CON. & 0.43 & 0.44 & 0.39 & 2.33 & -11.36 \\
\hline EPC/IPC RATIO & 1.13 & 0.87 & 0.76 & -23.01 & -12.64 \\
\hline EXP COHH CONCEN & 0.45 & 0.29 & 0.15 & -35.56 & -48.28 \\
\hline IMP COMH CONCEN & 0.12 & 0.18 & 0.19 & 50.00 & 5.56 \\
\hline ECC/ICC RATIO & 3.75 & 1.61 & 0.79 & -57.04 & -51.00 \\
\hline VERTICAL TRADE & -0.54 & -0.55 & -0.02 & 1.85 & -96.36 \\
\hline TOT TRADE LINKAGES & 40.00 & 60.00 & 105.00 & 50.00 & 75.00 \\
\hline \% AGRICULTURE-GDP & 17.70 & 11.90 & 13.10 & -32.77 & 10.08 \\
\hline QUALITY OF LIFE & 51.40 & 59.70 & 69.80 & 16.15 & 16.92 \\
\hline \% LRGST EXP TO GDP & 2.70 & 1.70 & 1.10 & -37.04 & -35.29 \\
\hline * EXP LGST PARTNER & 40.00 & 24.00 & 17.00 & -40.00 & -29.17 \\
\hline \% IMP LGST PARTNER & 31.00 & 32.00 & 18.00 & 3.23 & -43.75 \\
\hline Z EXP RANUFAC. & 3.00 & 14.00 & 38.00 & 366.67 & 171.43 \\
\hline \% EXP AGR-MINE & 96.00 & 85.00 & 61.00 & -11.46 & -28.24 \\
\hline \% IMP MANUFAC. & 57.00 & 68.00 & 40.00 & 19.30 & -41.18 \\
\hline X IHP AGR-MINE & 42.00 & 31.00 & 59.00 & -26.19 & 90.32 \\
\hline TOTAL EXPORTS & 1207.00 & 2731.00 & 19740.00 & 126.26 & 622.81 \\
\hline TOTAL IMPORTS & 1361.00 & 2564.00 & 23750.00 & 88.39 & 826.29 \\
\hline G.N.P. PER CAPITA & NA & MA & 2220.00 & 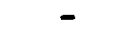 & - \\
\hline
\end{tabular}


BRUNEI

SUMMARY OF NATIONAL STATISTICS

\begin{tabular}{|c|c|c|c|c|c|}
\hline NATIONAL VARIABLES & 1962 & 1970 & 1980 & $62-70$ & $70-80$ \\
\hline EXP. PARTNER CON. & 0.78 & 0.89 & 0.78 & 14.10 & -12.36 \\
\hline IHP. PARTNER CON. & 0.85 & 0.65 & 0.67 & -23.53 & 3.08 \\
\hline EPC/IPC RATIO & 0.91 & 1.36 & 1.18 & 49.45 & -13.24 \\
\hline EXP COHM CONCEN & NA & NA & $\mathbf{M}$ & - & - \\
\hline IMP COHM CONCEN & NA & Ma & MA & - & - \\
\hline ECC/ICC RATIO & NA & MA & $\mathbf{M}$ & - & - \\
\hline VERTICAL TRADE & NA & MA & MA & - & - \\
\hline TOT TRADE LINKAGES & 4.00 & 3.00 & 9.00 & -25.00 & 200.00 \\
\hline \% AGRICULTURE-GDP & NA & NA & MA & - & - \\
\hline QUALITY OF LIFE & NA & NA & NA & - & - \\
\hline * LRGST EXP TO GDP & NA & MA & NA & - & - \\
\hline * EXP LGST PARTNER & 58.00 & 84.00 & 72.00 & 44.83 & -14.29 \\
\hline X IMP LGST PARTNER & 77.00 & 37.00 & 49.00 & -51.95 & 32.43 \\
\hline * eXP manufac. & MA & NA & NA & - & - \\
\hline X EXP AGR-MINE & MA & MA & M & - & - \\
\hline * IHP haNufac. & $\mathbf{M}$ & MA & MA & - & - \\
\hline X IMP AGR-MINE & NA & NA & MA & - & - \\
\hline TOTAL EXPORTS & 29.00 & 97.00 & 4514.00 & 234.48 & 4553.61 \\
\hline TOTAL IHPORTS & 8.00 & 68.00 & 561.00 & 750.00 & 725.00 \\
\hline G.N.P. PER CAPITA & MA & M & Ma & - & - \\
\hline
\end{tabular}


BULGARIA

SUMHARY OF NATIONAL STATISTICS

\begin{tabular}{|c|c|c|c|c|c|}
\hline MATIONAL VARIABLES & 1962 & 1970 & 1980 & $62-70$ & $70-80$ \\
\hline EXP. PARTNER CON. & 0.69 & 0.61 & 0.60 & -11.59 & -1.64 \\
\hline IHP. PARTNER CON. & 0.71 & 0.57 & 0.64 & -19.72 & 12.28 \\
\hline EPC/IPC RATIO & 0.96 & 1.06 & 0.94 & 10.42 & -11.32 \\
\hline EXP COHA CONCEN & $\mathbf{M}$ & $\mathbf{M a}$ & $\mathbf{M}$ & - & - \\
\hline IMP COHA CONCEN & MA & Ma & $\mathbf{M}$ & - & - \\
\hline ECC/ICC RATIO & MA & MA & $\boldsymbol{\mu}$ & - & - \\
\hline VERTICAL TRADE & MA & Ma & $\boldsymbol{M}$ & - & - \\
\hline TOT TRADE LINKAGES & 25.00 & 47.00 & 64.00 & 88.00 & 36.17 \\
\hline ₹ AGRICULTURE-GDP & NA & MA & $\boldsymbol{M}$ & - & - \\
\hline QUALITY OF LIFE & 83.90 & 90.10 & 92.80 & 7.39 & 3.00 \\
\hline \% LRGST EXP TO GDP & MA & MA & M & - & - \\
\hline X EXP LGST PARTMER & 64.00 & 57.00 & 58.00 & -10.94 & 1.75 \\
\hline X IMP LGST PARTNER & 68.00 & 53.00 & 63.00 & -22.06 & 18.87 \\
\hline X EXP MANUFAC. & $\mathrm{MA}$ & NA & $\mathbf{M}$ & - & -2 \\
\hline \% EXP AGR-HINE & NA & NA & MA & - & - \\
\hline X IMP MANUFAC. & NA & NA & NA & - & - \\
\hline X IMP AGR-HINE & NA & NA & NA & - & - \\
\hline TOTAL EXPORTS & 604.00 & 1876.00 & 8770.00 & 210.60 & 367.48 \\
\hline TOTAL IMPORTS & 652.00 & 1767.00 & 8914.00 & 171.01 & 404.47 \\
\hline G.N.P. PER CAPITA & NA & MA & 3400.00 & - & - \\
\hline
\end{tabular}


BURM

SUMMARY OF NATIONAL STATISTICS

\begin{tabular}{|c|c|c|c|c|c|}
\hline MATIONAL VARIABLES & 1962 & 1970 & 1980 & $62-70$ & $70-80$ \\
\hline EXP. PARTMER CON. & 0.45 & 0.41 & 0.41 & -8.89 & 0.00 \\
\hline IMP. PARTNER CON. & 0.48 & 0.47 & 0.52 & -2.08 & 10.64 \\
\hline EPC/IPC RATIO & 0.95 & 0.87 & 0.78 & -8.42 & -10.34 \\
\hline EXP COAN CONCEN & 0.57 & 0.46 & MA & -19.30 & - \\
\hline IMP CONH CONCEN & 0.34 & 0.34 & $\mathbf{M}$ & 0.00 & - \\
\hline ECC/ICC RATIO & 1.68 & 1.35 & MA & -19.30 & - \\
\hline VERTICAL TRADE & -0.77 & -0.82 & MA & 6.49 & - \\
\hline TOT TRADE LINKAGES & 19.00 & 20.00 & 32.00 & 5.26 & 60.00 \\
\hline \% AGRICULTURE-GDP & 35.00 & 36.50 & 44.90 & 4.29 & 23.01 \\
\hline QUALITY OF LIFE & 35.60 & MA & 54.80 & - & - \\
\hline X LRGST EXP TO GDP & NA & NA & Ma & - & - \\
\hline X EXP LGST PARTNER & $15 . \infty 0$ & 14.00 & 16.00 & -6.67 & 14.29 \\
\hline X IHP LGST PARTNER & 25.00 & 23.00 & 36.00 & -8.00 & 56.52 \\
\hline X EXP manUfAC. & MA & 1.00 & MA & - & - \\
\hline X EXP AGR-NINE & 99.00 & 98.00 & $M$ & -1.01 & - \\
\hline X Imp manufac. & 78.00 & 83.00 & MA & 6.41 & - \\
\hline X IHP AGR-MINE & 21.00 & 16.00 & NA & -23.81 & - \\
\hline TOTAL EXPORTS & 201.00 & 101.00 & 424.00 & -49.75 & 319.80 \\
\hline TOTAL IHPORTS & 205.00 & 163.00 & 593.00 & -20.49 & 263.80 \\
\hline G.N.P. PER CAPITA & NA & NA & 190.00 & - & - \\
\hline
\end{tabular}


CAMEROON

SUMMARY OF NATIONAL STATISTICS

\begin{tabular}{|c|c|c|c|c|c|}
\hline MATIONAL VARIABLES & 1962 & 1970 & 1980 & $62-70$ & $70-80$ \\
\hline EXP. PARTNER CON. & 0.78 & 0.56 & 0.56 & -28.21 & 0.00 \\
\hline IHP. PARTNER CON. & 0.69 & 0.60 & 0.55 & -13.04 & -8.33 \\
\hline EPC/IPC RATIO & 1.13 & 0.93 & 1.03 & -17.70 & 10.75 \\
\hline EXP CONH CONCEN & 0.32 & 0.32 & 0.28 & 0.00 & -12.50 \\
\hline IHP CONH CONCEN & 0.28 & 0.34 & 0.29 & 21.43 & -14.71 \\
\hline ECC/ICC RATIO & 1.14 & 0.94 & 0.97 & -17.65 & 2.59 \\
\hline VERTICAL TRADE & -0.68 & -0.73 & -0.74 & 7.35 & 1.37 \\
\hline TOT TRADE LINKAGES & 6.00 & 17.00 & 25.00 & 183.33 & 47.06 \\
\hline \% AGRICULTURE-GDP & MA & 30.90 & 30.50 & - & -1.29 \\
\hline QUALITY OF LIFE & 15.70 & 22.00 & 42.10 & 40.13 & 91.36 \\
\hline * LRGST EXP TO GDP & 9.10 & 5.10 & 8.50 & -43.96 & 66.67 \\
\hline \% EXP LGST PARTNER & 67.00 & 31.00 & 29.00 & -53.73 & -6.45 \\
\hline \% IMP LGST PARTNER & 62.00 & 52.00 & 47.00 & -16.13 & -9.62 \\
\hline Z EXP MANUFAC. & 4.00 & 8.00 & 3.00 & 100.00 & -62.50 \\
\hline Z EXP AGR-MINE & 95.00 & 91.00 & 96.00 & -4.21 & 5.49 \\
\hline Z IMP MANUFAC. & 71.00 & 81.00 & 78.00 & 14.08 & -3.70 \\
\hline X IHP AGR-HINE & 28.00 & 18.00 & 21.00 & -35.71 & 16.67 \\
\hline TOTAL EXPORTS & 99.00 & 230.00 & 1373.00 & 152.75 & 496.96 \\
\hline TOTAL IAPORTS & 78.00 & 212.00 & 1505.00 & 171.79 & 609.91 \\
\hline G.N.P. PER CAPITA & MA & MA & 880.00 & - & - \\
\hline
\end{tabular}


CAMADA

SUMMARY OF NATIONAL STATISTICS

\begin{tabular}{|c|c|c|c|c|c|}
\hline MATIONAL VARIABLES & 1962 & 1970 & 1980 & $62-70$ & $70-80$ \\
\hline EXP. PARTNER CON. & 0.63 & 0.65 & 0.62 & 3.17 & -4.62 \\
\hline IMP. PARTMER CON. & 0.68 & 0.70 & 0.68 & 2.94 & -2.86 \\
\hline EPC/IPC RATIO & 0.92 & 0.93 & 0.91 & 1.09 & -2.15 \\
\hline EXP COHM CONCEN & 0.04 & 0.06 & 0.04 & 50.00 & -33.33 \\
\hline IMP COMH CONCEN & 0.18 & 0.28 & 0.22 & 55.56 & -21.43 \\
\hline ECC/ICC RATIO & 0.22 & 0.21 & 0.18 & -3.57 & -15.15 \\
\hline VERTICAL TRADE & -0.38 & -0.28 & -0.25 & -26.32 & -10.71 \\
\hline TOT TRADE LINKAGES & 71.00 & 90.00 & 112.00 & 26.76 & 24.44 \\
\hline * AGRICULTURE-GDP & 5.70 & 4.80 & 4.00 & -15.79 & -16.67 \\
\hline QUALITY OF LIFE & MA & 93.70 & 97.30 & - & 3.84 \\
\hline * LRGST EXP TO GDP & 8.50 & 12.80 & 13.30 & 50.59 & 3.91 \\
\hline X EXP LGST PARTNER & 59.00 & 65.00 & 64.00 & 10.17 & -1.54 \\
\hline * IHP LGST PARTHER & 68.00 & 71.00 & 68.00 & 4.41 & -4.23 \\
\hline X EXP MNUUFAC. & 33.00 & 51.00 & 48.00 & 54.55 & -5.88 \\
\hline X EXP AGR-MINE & 66.00 & 48.00 & 51.00 & -27.27 & 6.25 \\
\hline X IMP MANUFAC. & 71.00 & 79.00 & 73.00 & 11.27 & -7.59 \\
\hline X IHP AGR-MINE & 28.00 & 20.00 & 26.00 & -28.57 & 30.00 \\
\hline TOTAL EXPORTS & \multicolumn{3}{|c|}{5901.0015991 .0064047 .00} & 170.99 & 300.52 \\
\hline TOTAL IMPORTS & 5618.00 & 12698.00 & 51348.00 & 126.02 & 304.38 \\
\hline G.N.P. PER CAPITA & NA & $\mathbf{M}$ & 11400.00 & - & - \\
\hline
\end{tabular}


CENT. AFR.

SUMHARY OF NATIONAL STATISTICS

\begin{tabular}{|c|c|c|c|c|c|}
\hline NATIONAL VARIABLES & 1962 & 1970 & 1980 & $62-70$ & $70-80$ \\
\hline EXP. PARTNER CON. & 0.98 & 0.68 & 0.65 & -30.61 & -4.41 \\
\hline IHP. PARTNER CON. & 1.00 & 0.68 & 0.65 & -32.00 & -4.41 \\
\hline EPC/IPC RATIO & 0.98 & 1.00 & 1.00 & 2.04 & 0.00 \\
\hline EXP CONH CONCEN & 0.28 & 0.31 & 0.32 & 10.71 & 3.23 \\
\hline IHP CONH CONCEN & 0.32 & 0.35 & 0.30 & 9.38 & -14.29 \\
\hline ECC/ICC RATIO & 0.88 & 0.89 & 1.07 & 1.22 & 20.43 \\
\hline VERTICAL TRADE & -0.58 & -0.36 & -0.48 & -37.93 & 33.33 \\
\hline TOT TRADE LINKAGES & 1.00 & 6.00 & 12.00 & 500.00 & 100.00 \\
\hline X AGRICULTURE-GDP & 50.50 & 40.70 & 36.20 & -19.41 & -11.06 \\
\hline QUALITY OF LIFE & 4.80 & MA & 26.80 & - & - \\
\hline X LRGST EXP TO GDP & 6.70 & 8.10 & M & 20.90 & - \\
\hline \% exp lgst partner & 97.00 & 57.00 & 52.00 & -41.24 & -8.77 \\
\hline X IMP LGST PARTHER & 100.00 & 60.00 & 56.00 & -40.00 & -6.67 \\
\hline X EXP MANUFAC. & 21.00 & 44.00 & 26.00 & 109.52 & -40.91 \\
\hline ※ EXP AGR-HINE & 78.00 & 55.00 & 73.00 & -29.49 & 32.73 \\
\hline X IHP MUNUFAC. & 78.00 & 79.00 & 74.00 & 1.28 & -6.33 \\
\hline \% IMP AGR-MINE & 21.00 & 20.00 & 25.00 & -4.76 & 25.00 \\
\hline TOTAL EXPORTS & 9.00 & $28 . \infty 0$ & 114.00 & 211.11 & 307.14 \\
\hline TOTAL IMPORTS & 11.00 & 34.00 & 101.00 & 209.09 & 197.06 \\
\hline G.N.P. PER CAPITA & NA & M & 320.00 & - & - \\
\hline
\end{tabular}


CHAD

SUMMARY OF MATIONAL STATISTICS

\begin{tabular}{|c|c|c|c|c|c|}
\hline NATIONAL VARIABLES & 1962 & 1970 & 1980 & $62-70$ & $70-80$ \\
\hline EXP. PARTNER CON. & 0.77 & 0.79 & 0.57 & 2.60 & -27.85 \\
\hline IHP. PARTNER CON. & 1.00 & 0.63 & 0.63 & -37.00 & 0.00 \\
\hline EPC/IPC RATIO & 0.77 & 1.26 & 0.91 & 63.64 & -27.78 \\
\hline EXP COHH CONCEN & 0.51 & 0.52 & MA & 1.96 & - \\
\hline IMP COHN CONCEN & 0.21 & 0.20 & M & -4.76 & - \\
\hline ECC/ICC RATIO & 2.43 & 2.60 & M & 7.06 & - \\
\hline VERTICAL TRADE & -0.63 & -0.56 & $\mathbf{M A}$ & -11.11 & - \\
\hline TOT TRADE LINKAGES & 3.00 & 3.00 & 8.00 & 0.00 & 166.67 \\
\hline \% AGRICULTURE-GDP & 52.00 & 49.20 & 51.20 & -5.38 & 4.07 \\
\hline QUALITY OF LIFE & 4.50 & 11.90 & 21.40 & 164.44 & 79.83 \\
\hline \% LRGST EXP TO GDP & NA & 1.90 & Ma & - & - \\
\hline \% EXP LGST PARTNER & 62.00 & 73.00 & 22.00 & 17.74 & -69.86 \\
\hline \% IMP LGST PARTHER & 100.00 & 51.00 & 32.00 & -49.00 & -37.25 \\
\hline X EXP MANUFAC. & 3.00 & 4.00 & M & 33.33 & - \\
\hline X EXP AGR-MINE & 96.00 & 95.00 & M & -1.04 & - \\
\hline$X$ IMP MANUFAC. & 66.00 & 61.00 & Ma & -7.58 & - \\
\hline \& IMP AGR-MINE & 34.00 & 38.00 & MA & 11.76 & - \\
\hline TOTAL EXPORTS & 13.00 & 29.00 & 66.00 & 123.08 & 127.59 \\
\hline TOTAL IMPORTS & 10.00 & 35.00 & 36.00 & 250.00 & 2.86 \\
\hline G.N.P. PER C & MA & MA & 110.00 & - & - \\
\hline
\end{tabular}


CHILE

SUMMARY OF MATIONAL STATISTICS

\begin{tabular}{|c|c|c|c|c|c|}
\hline MATIONAL VARIABLES & 1962 & 1970 & 1980 & $62-70$ & $70-80$ \\
\hline EXP. PARTNER CON. & 0.56 & 0.49 & 0.41 & -12.50 & -16.33 \\
\hline IHP. PARTNER COH. & 0.49 & 0.49 & 0.44 & 0.00 & -10.20 \\
\hline EPC/IPC RATIO & 1.13 & 1.01 & 0.94 & -10.62 & -6.93 \\
\hline EXP CONH CONCEN & 0.67 & 0.70 & 0.30 & 4.48 & -57.14 \\
\hline IHP COHH CONCEN & 0.19 & 0.22 & 0.17 & 15.79 & -22.73 \\
\hline ECC/ICC RATIO & 3.53 & 3.18 & 1.76 & -9.77 & -44.54 \\
\hline VERTICAL TRADE & -0.63 & -0.68 & -0.41 & 7.94 & -39.71 \\
\hline TOT TRADE LINKAGES & 19.00 & 23.00 & 40.00 & 21.05 & 73.91 \\
\hline X AGRICULTURE-GDP & 9.30 & 7.60 & 7.60 & -18.28 & 0.00 \\
\hline QUALITY OF LIFE & 61.50 & 72.70 & $83 . \infty 0$ & 18.21 & 14.17 \\
\hline X LRGST EXP TO GDP & 4.10 & 1.90 & 4.80 & -53.66 & 152.63 \\
\hline X EXP LGST PARTNER & 36.00 & 15.00 & 13.00 & -58.33 & -13.33 \\
\hline \% IHP LGST PARTNER & 34.00 & 35.00 & 27.00 & 2.94 & -22.86 \\
\hline X EXP MANUFAC. & 3.00 & 4.00 & 20.00 & 33.33 & 400.00 \\
\hline X EXP AGR-MINE & 96.00 & 94.00 & 79.00 & -2.08 & -15.96 \\
\hline X IMP MANUFAC. & 66.00 & 72.00 & 60.00 & 9.09 & -16.67 \\
\hline X IHP AGR-MINE & 33.00 & 27.00 & 39.00 & -18.18 & 44.44 \\
\hline TOTAL EXPORTS & 528.00 & 1241.00 & 4515.00 & 135.04 & 263.82 \\
\hline TOTAL IMPORTS & 495.00 & 856.00 & 4921.00 & 72.93 & 474.88 \\
\hline .H.P. PER & NA & MA & 2560.00 & - & - \\
\hline
\end{tabular}


CHIMa

SUMHARY OF MATIONAL STATISTICS

\begin{tabular}{|c|c|c|c|c|c|}
\hline NATIONAL VARIABLES & 1962 & 1970 & 1980 & $62-70$ & $70-80$ \\
\hline EXP. PARTNER CON. & 0.56 & 0.37 & 0.45 & -33.93 & 21.62 \\
\hline IMP. PARTHER CON. & 0.51 & 0.44 & 0.43 & -13.73 & -2.27 \\
\hline EPC/IPC RATIO & 1.11 & 0.84 & 1.05 & -24.32 & 25.00 \\
\hline EXP CONH CONCEN & NA & NA & Ma & - & - \\
\hline IHP CONH CONCEN & NA & NA & Ma & - & - \\
\hline ECC/ICC RATIO & MA & MA & $M$ & - & - \\
\hline VERTICAL TRADE & NA & MA & $M$ & - & - \\
\hline TOT TRADE LINKAGES & 33.00 & 58.00 & 56.00 & 75.76 & -3.45 \\
\hline X agriculture-Gop & MA & MA & 32.50 & - & - \\
\hline QUALITY OF LIFE & 26.20 & MA & 70.10 & - & - \\
\hline * LRGST EXP TO GDP & NA & Ma & MA & - & - \\
\hline X EXP LGST PARTNER & 44.00 & 24.00 & 25.00 & -45.45 & 4.17 \\
\hline X Imp lgst PARTMER & 30.00 & 31.00 & 26.00 & 3.33 & -16.13 \\
\hline Z EXP MANUFAC. & NA & Ma & NA & - & - \\
\hline \% EXP AGR-HINE & NA & MA & MA & - & - \\
\hline \% IHP manufac. & NA & $\mathbf{M A}$ & M & - & - \\
\hline X IHP AGR-HINE & MA & MA & M & - & - \\
\hline TOTAL EXPORTS & 1168.00 & 1748.00 & 16922.00 & 49.66 & 868.08 \\
\hline TOTAL IMPORTS & 776.00 & 1821.00 & 19748.00 & 134.66 & 951.51 \\
\hline G.N.P. PER CAPITA & $\mathrm{MA}$ & $\mathbf{N A}$ & 300.00 & - & - \\
\hline
\end{tabular}


COLOMBIA

SUMMARY OF NATIONAL STATISTICS

\begin{tabular}{|c|c|c|c|c|c|}
\hline MATIONAL VARIABLES & 1962 & 1970 & 1980 & $62-70$ & $70-80$ \\
\hline EXP. PARTNER CON. & 0.65 & 0.50 & 0.45 & -23.08 & -10.00 \\
\hline IMP. PARTNER CON. & 0.57 & 0.55 & 0.50 & -3.51 & -9.09 \\
\hline EPC/IPC RATIO & 1.15 & 0.92 & 0.91 & -20.00 & -1.09 \\
\hline EXP COHH CONCEN & 0.54 & 0.47 & 0.45 & -12.96 & -4.26 \\
\hline INP COHH CONCEN & 0.28 & 0.32 & 0.19 & 14.29 & -40.63 \\
\hline ECC/ICC RATIO & 1.93 & 1.47 & 2.37 & -23.84 & 61.25 \\
\hline VERTICAL TRADE & -0.77 & -0.75 & -0.50 & -2.60 & -33.33 \\
\hline TOT TRADE LINKAGES & 20.00 & 37.00 & 56.00 & 85.00 & 51.35 \\
\hline X AGRICULTURE-GDP & 35.60 & 30.50 & 28.60 & -14.33 & -6.23 \\
\hline QUALITY OF LIFE & 53.00 & 67.20 & 74.40 & 26.79 & 10.71 \\
\hline X LRGST EXP TO GDP & 5.60 & 3.40 & 3.50 & -39.29 & 2.94 \\
\hline X EXP LGST PARTNER & 58.00 & 38.00 & 27.00 & -34.48 & -28.95 \\
\hline * IMP LGST PARTNER & 50.00 & 48.00 & 37.00 & -4.00 & -22.92 \\
\hline \& EXP MANUFAC. & 3.00 & 8.00 & 20.00 & 166.67 & 150.00 \\
\hline \& EXP MGR-MINE & 96.00 & 91.00 & 79.00 & -5.21 & -13.19 \\
\hline \% IMP MANUFAC. & 81.00 & 83.00 & 70.00 & 2.47 & -15.66 \\
\hline \% IMP AGR-MINE & 19.00 & 16.00 & 29.00 & -15.79 & 81.25 \\
\hline TOTAL EXPORTS & 452.00 & 691.00 & 3932.00 & 52.88 & 469.03 \\
\hline TOTAL IMPORTS & 452.00 & 818.00 & 4568.00 & 80.97 & 458.44 \\
\hline .N.P. PE & MA & $\mathbf{M}$ & $1380 . \infty 0$ & - & - \\
\hline
\end{tabular}


conso

SUMMARY OF NATIONAL STATISTICS

\begin{tabular}{|c|c|c|c|c|c|}
\hline NATIOMAL VARIABLES & 1962 & 1970 & 1980 & $62-70$ & $70-80$ \\
\hline EXP. PARTNER CON. & 0.62 & 0.49 & 0.61 & -20.97 & 24.49 \\
\hline IMP. PARTNER CON. & 0.53 & 0.66 & 0.58 & 24.53 & -12.12 \\
\hline EPC/IPC RATIO & 1.17 & 0.75 & 1.06 & -35.90 & 41.33 \\
\hline EXP CONM CONCEN & 0.21 & 0.31 & 0.65 & 47.62 & 109.68 \\
\hline IHP COHH CONCEN & 0.30 & 0.34 & 0.26 & 13.33 & -23.53 \\
\hline ECC/ICC RATIO & 0.70 & 0.91 & 2.50 & 30.25 & 174.19 \\
\hline VERTICAL TRADE & -0.33 & -0.48 & -0.58 & 45.45 & 20.83 \\
\hline TOT TRADE LINKAGES & $\mathbf{1 3 . 0 0}$ & 14.00 & 18.00 & 7.69 & 28.57 \\
\hline * AGRICULTURE-GDP & 23.20 & 19.50 & 13.40 & -15.95 & -31.28 \\
\hline QUALITY OF LIFE & 22.20 & 40.90 & 51.60 & 84.23 & 26.16 \\
\hline * LRGST EXP TO GDP & 4.70 & MA & MA & -2206.38 & 0.00 \\
\hline * EXP LGST PARTNER & 45.00 & 23.00 & 39.00 & -48.89 & 69.57 \\
\hline \% IMP LGST PARTNER & 31.00 & 56.00 & 50.00 & 80.65 & -10.71 \\
\hline \% EXP MANUFAC. & 40.00 & 29.00 & 7.00 & -27.50 & -75.86 \\
\hline * EXP AGR-HINE & 60.00 & 71.00 & 92.00 & 18.33 & 29.58 \\
\hline X InP MANUFAC. & 73.00 & 76.00 & 65.00 & 4.11 & -14.47 \\
\hline X IMP AGR-MINE & 26.00 & 23.00 & 34.00 & -11.54 & 47.83 \\
\hline TOTAL EXPORTS & 406.00 & 67.00 & 954.00 & -83.50 & 1323.88 \\
\hline TOTAL IMPORTS & 221.00 & 75.00 & 517.00 & -66.06 & 589.33 \\
\hline G.N.P. PER CAPITA & NA & MA & 1110.00 & - & - \\
\hline
\end{tabular}


COSTA RICA

SUMMARY OF NATIONAL STATISTICS

\begin{tabular}{|c|c|c|c|c|c|}
\hline MATIONAL VARIABLES & 1962 & 1970 & 1980 & $62-70$ & $70-80$ \\
\hline EXP. PARTNER CON. & 0.71 & 0.54 & 0.51 & -23.94 & -5.56 \\
\hline IHP. PARTHER CON. & 0.58 & 0.50 & 0.50 & -13.79 & 0.00 \\
\hline EPC/IPC RATIO & 1.23 & 1.08 & 1.01 & -12.20 & -6.48 \\
\hline EXP COHI CONCEN & 0.76 & 0.60 & 0.46 & -21.05 & -23.33 \\
\hline IMP COMH CONCEN & 0.35 & 0.33 & 0.25 & -5.71 & -24.24 \\
\hline ECC/ICC RATIO & 2.17 & 1.82 & 1.84 & -16.27 & 1.20 \\
\hline VERTICAL TRADE & -0.72 & -0.62 & -0.38 & -13.89 & -38.71 \\
\hline TOT TRADE LINKAGES & 8.00 & 18.00 & 35.00 & 125.00 & 94.44 \\
\hline X AGRICULTURE-GDP & 26.00 & 23.80 & 20.30 & -8.46 & -14.71 \\
\hline QUALITY OF LIFE & 56.30 & 78.20 & 89.80 & 38.50 & 14.83 \\
\hline * LRGST EXP TO GDP & 9.40 & 9.00 & 9.90 & -4.26 & 10.00 \\
\hline \% EXP LGST PARTNER & 57.00 & 42.00 & 33.00 & -26.32 & -21.43 \\
\hline X IHP LGST PARTMER & 48.00 & 34.00 & 36.00 & -29.17 & 5.88 \\
\hline X EXP maNufaC. & 9.00 & 19.00 & 34.00 & 111.11 & 78.95 \\
\hline X EXP AGR-hINE & 90.00 & 80.00 & 65.00 & -11.11 & -18.75 \\
\hline X IMP MANUFAC. & 81.00 & 82.00 & 72.00 & 1.23 & -12.20 \\
\hline X IMP AGR-HINE & 18.00 & 18.00 & 27.00 & 0.00 & 50.00 \\
\hline TOTAL EXPORTS & 83.00 & 226.00 & 979.00 & 172.29 & 333.19 \\
\hline TOTAL IMPORTS & 102.00 & 274.00 & 1349.00 & 168.63 & 392.34 \\
\hline G.N.P. PER CAPITA & Ma & MA & 1430.00 & - & - \\
\hline
\end{tabular}


CUBA

SUMMARY OF NATIONAL STATISTICS

\begin{tabular}{|c|c|c|c|c|c|}
\hline MATIONAL VARIABLES & 1962 & 1970 & 1980 & $62-70$ & $70-80$ \\
\hline EXP. PARTNER CON. & 0.64 & 0.53 & 0.65 & -17.19 & 22.64 \\
\hline IMP. PARTNER CON. & 0.73 & 0.61 & 0.63 & -16.44 & 3.28 \\
\hline EPC/IPC RATIO & 0.87 & 0.88 & 1.04 & 1.15 & 18.18 \\
\hline EXP COHI CONCEN & NA & MA & MA & - & - \\
\hline IHP COHM CONCEN & MA & MA & MA & - & - \\
\hline ECC/ICC RATIO & MA & NA & MA & - & - \\
\hline VERTICAL TRADE & NA & NA & MA & - & - \\
\hline TOT TRADE LIMKAGES & 21.00 & 25.00 & 40.00 & 19.05 & 60.00 \\
\hline X AGRICULTURE-GDP & Ma & NA & $\mathbf{M a}$ & - & - \\
\hline QUALITY OF LIFE & NA & MA & NA & - & - \\
\hline X LRGST EXP TO GDP & $\mathbf{M A}$ & MA & MA & - & - \\
\hline X EXP LEST PARTNER & 58.00 & 37.00 & 65.00 & -36.21 & 75.68 \\
\hline Z IMP LGST PARTNER & 74.00 & 54.00 & 59.00 & -27.03 & 9.26 \\
\hline Z EXP HANUFAC. & NA & MA & MA & - & - \\
\hline X EXP AGR-MINE & NA & NA & Ma & - & - \\
\hline \% IHP MANUFAC. & MA & MA & MA & - & - \\
\hline$X$ IMP AGR-HINE & Ma & MA & MA & - & - \\
\hline TOTAL EXPORTS & 400.00 & 620.00 & 4614.00 & 55.00 & 644.19 \\
\hline TOTAL IMPORTS & 497.00 & 1183.00 & 5889.00 & 138.03 & 397.80 \\
\hline G.N.P. PER CAPITA & NA & MA & 1200.00 & - & - \\
\hline
\end{tabular}


CYPRUS

SUMHARY OF NATIONAL STATISTICS

\begin{tabular}{|c|c|c|c|c|c|}
\hline MATIOHAL VARIABLES & 1962 & 1970 & 1980 & $62-70$ & $70-80$ \\
\hline EXP. PARTNER CON. & 0.61 & 0.51 & 0.41 & -16.39 & -19.61 \\
\hline IHP. PARTNER CON. & 0.53 & 0.44 & 0.41 & -16.98 & -6.82 \\
\hline EPC/IPC RATIO & 1.15 & 1.15 & 0.98 & 0.00 & -14.78 \\
\hline EXP COMH CONCEN & 0.45 & 0.28 & 0.27 & -37.78 & -3.57 \\
\hline IHP COAH CONCEN & 0.24 & 0.26 & 0.20 & 8.33 & -23.08 \\
\hline ECC/ICC RATIO & 1.88 & 1.08 & 1.35 & -42.56 & 25.36 \\
\hline VERTICAL TRADE & -0.67 & -0.57 & -0.08 & -14.93 & -85.96 \\
\hline TOT TRADE LINKAGES & 8.00 & 15.00 & 39.00 & 87.50 & 160.00 \\
\hline X AGRICULTURE-GDP & 20.90 & 19.60 & 13.60 & -6.22 & -30.61 \\
\hline QUALITY OF LIFE & 81.70 & 86.70 & 91.10 & 6.12 & 5.07 \\
\hline X LRGST EXP TO GDP & 5.90 & 7.60 & 9.20 & 28.81 & 21.05 \\
\hline \% EXP LGST PARTNER & 49.00 & 39.00 & 22.00 & -20.41 & -43.59 \\
\hline X IHP LGST PARTNER & 40.00 & 30.00 & 24.00 & -25.00 & -20.00 \\
\hline X EXP MARUfAC. & 2.00 & 15.00 & 55.00 & 650.00 & 266.67 \\
\hline X EXP AGR-HINE & 97.00 & 84.00 & 44.00 & -13.40 & -47.62 \\
\hline X IMP MANUFAC. & 69.00 & 72.00 & 63.00 & 4.35 & -12.50 \\
\hline X IMP AGR-HIME & 31.00 & 27.00 & 36.00 & -12.90 & 33.33 \\
\hline TOTAL EXPORTS & 53.00 & 106.00 & 491.00 & 100.00 & 363.21 \\
\hline TOTAL IMPORTS & 105.00 & 206.00 & 1480.00 & 96.19 & 618.45 \\
\hline G.N.P. PER CAPITA & NA & NA & 3740.00 & - & - \\
\hline
\end{tabular}


CZECHOSLOV

SUMMARY OF NATIONAL STATISTICS

\begin{tabular}{|c|c|c|c|c|c|}
\hline MATIONAL VARIABLES & 1962 & 1970 & 1980 & $62-70$ & $70-80$ \\
\hline EXP. PARTNER CON. & 0.58 & 0.46 & 0.48 & -20.69 & 4.35 \\
\hline IMP. PARTNER CON. & 0.51 & 0.49 & 0.54 & -3.92 & 10.20 \\
\hline EPC/IPC RATIO & 1.13 & 0.95 & 0.89 & -15.93 & -6.32 \\
\hline EXP COHH CONCEN & MA & NA & 0.27 & - & - \\
\hline IHP CONH CONCEN & MA & M & 0.09 & - & - \\
\hline ECC/ICC RATIO & $\mathbf{M}$ & $\mathbf{M A}$ & 3.00 & - & - \\
\hline VERTICAL TRADE & NA & NA & 0.29 & - & - \\
\hline TOT TRADE LINKAGES & 23.00 & 72.00 & 80.00 & 213.04 & 11.11 \\
\hline X AGRICULTURE-GDP & NA & NA & Ma & - & - \\
\hline QUALITY OF LIFE & 90.10 & 92.00 & 93.80 & 2.11 & 1.96 \\
\hline$X$ LRGST EXP TO GDP & NA & MA & MA & - & - \\
\hline \% EXP LGST PARTNER & 每 00 & 34,00 & 38.00 & -23.73 & 11.76 \\
\hline X IMP LGST PARTMER & 41.00 & 35.00 & 46.00 & -14.63 & 31.43 \\
\hline X EXP manufac. & MA & NA & 84.00 & - & - \\
\hline \% EXP AGR-MINE & мA & NA & $15 . \infty 0$ & - & - \\
\hline * IMP MANUFAC. & MA & MA & 55.00 & - & - \\
\hline X IHP AGR-MINE & NA & MA & 44.00 & - & - \\
\hline TOTAL EXPORTS & 1862.00 & 3519.00 & 13661.00 & 88.99 & 288.21 \\
\hline TOTAL IAPORTS & 1876.00 & 3419.00 & 12196.00 & 82.25 & 256.71 \\
\hline G.H.P. PER CAPITA & NA & $\mathbf{M A}$ & 4400.00 & - & - \\
\hline
\end{tabular}


DENHARK

SUMMARY OF NATIONAL STATISTICS

\begin{tabular}{|c|c|c|c|c|c|}
\hline MATIOANL VARIABLES & 1962 & 1970 & 1980 & $62-70$ & $70-80$ \\
\hline EXP. PARTNER CON. & 0.44 & 0.40 & 0.39 & -9.09 & -2.50 \\
\hline IMP. PARTNER CON. & 0.44 & 0.43 & 0.43 & -2.27 & 0.00 \\
\hline EPC/IPC RATIO & 1.00 & 0.93 & 0.90 & -7.00 & -3.23 \\
\hline EXP COHH CONCEN & 0.25 & 0.16 & 0.14 & -36.00 & -12.50 \\
\hline IHP CONH CONCEN & 0.14 & 0.18 & 0.15 & 28.57 & -16.67 \\
\hline ECC/ICC RATIO & 1.79 & 0.89 & 0.93 & -50.22 & 5.00 \\
\hline VERTICAL TRADE & -0.24 & -0.16 & -0.02 & -33.33 & -87.50 \\
\hline TOT TRADE LINKAGES & 63.00 & 90.00 & 110.00 & 42.86 & 22.22 \\
\hline$X$ AGRICULTURE-GDP & 11.20 & 7.90 & 5.10 & -29.46 & -35.44 \\
\hline QUALITY OF LIFE & 93.70 & 95.70 & 97.90 & 2.13 & 2.30 \\
\hline \% LRGST EXP TO GDP & 5.80 & 4.00 & 4.00 & -31.03 & 0.00 \\
\hline X EXP LGST PARTNER & 25.00 & 19.00 & 19.00 & -24.00 & 0.00 \\
\hline * IMP LGST PARTHER & 24.00 & 20.00 & 20.00 & -16.67 & 0.00 \\
\hline X EXP MANUFAC. & 38.00 & 53.00 & 56.00 & 39.47 & 5.66 \\
\hline * EXP AGR-HINE & 61.00 & 46.00 & 43.00 & -24.59 & -6.52 \\
\hline * IHP MANUFAC. & 62.00 & 69.00 & 58.00 & 11.29 & -15.94 \\
\hline X IMP AGR-MINE & 37.00 & 30.00 & 41.00 & -18.92 & 36.67 \\
\hline TOTAL EXPORTS & 1617.00 & 3253.00 & 16674.00 & 101.18 & 412.57 \\
\hline TOTAL IMPORTS & 1904.00 & 3858.00 & 18028.00 & 102.63 & 367.29 \\
\hline G.N.P. PER CAPITA & MA & MA & 13120.00 & - & - \\
\hline
\end{tabular}


dominican

SUMHARY OF NATIONAL STATISTICS

\begin{tabular}{|c|c|c|c|c|c|}
\hline MATIOHAL VARIABLES & 1962 & 1970 & 1980 & $62-70$ & $70-80$ \\
\hline EXP. PARTNER CON. & 0.78 & 0.85 & 0.67 & 8.97 & -21.18 \\
\hline IMP. PARTNER CON. & 0.63 & 0.61 & 0.61 & -3.17 & 0.00 \\
\hline EPC/IPC RATIO & 1.25 & 1.41 & 1.10 & 12.80 & -21.99 \\
\hline EXP COMH CONCEN & 0.78 & 0.71 & 0.55 & -8.97 & -22.54 \\
\hline IHP COHA CONCEN & 0.25 & 0.22 & 0.16 & -12.00 & -27.27 \\
\hline ECC/ICC RATIO & 3.12 & 3.23 & 3.44 & 3.44 & 6.51 \\
\hline VERTICAL TRADE & -0.70 & -0.69 & -0.30 & -1.43 & -56.52 \\
\hline TOT TRADE LINKAGES & 14.00 & 8.00 & 25.00 & -42.86 & 212.50 \\
\hline * AGRICULTURE-GDP & 26.70 & 24.80 & 20.50 & -7.12 & -17.34 \\
\hline QUALITY OF LIFE & 48.60 & 59.40 & 66.70 & 22.22 & 12.29 \\
\hline \% LRGST EXP TO GDP & 12.80 & 10.00 & 12.70 & -21.88 & 27.00 \\
\hline X EXP LGST PARTNER & 77.00 & 85.00 & 52.00 & 10.39 & -38.82 \\
\hline X IMP LGST PARTNER & 53.00 & 51.00 & 50.00 & -3.77 & -1.96 \\
\hline X EXP manUfaC. & 2.00 & 4.00 & 23.00 & 100.00 & 475.00 \\
\hline X EXP AGR-MIHE & 97.00 & 95.00 & 76.00 & -2.06 & -20.00 \\
\hline X IHP maNUfaC. & 72.00 & 73.00 & 54.00 & 1.39 & -26.03 \\
\hline$X$ IMP AGR-MINE & 27.00 & 26.00 & 45.00 & -3.70 & 73.08 \\
\hline TOTAL EXPORTS & 197.00 & 210.00 & 954.00 & 6.60 & 354.29 \\
\hline TOTAL IMPORTS & 132.00 & 278.00 & 1567.00 & 110.61 & 463.67 \\
\hline G.N.P. PER CAPITA & NA & NA & 1260.00 & - & - \\
\hline
\end{tabular}


EAST GERHA

SUMMARY OF NATIONAL STATISTICS

\begin{tabular}{|c|c|c|c|c|c|}
\hline NATIONAL VARIABLES & 1962 & 1970 & 1980 & $62-70$ & $70-80$ \\
\hline EXP. PARTNER CON. & 0.67 & 0.55 & 0.37 & -17.91 & -32.73 \\
\hline IMP. PARTNER CON. & 0.73 & 0.58 & 0.56 & -20.55 & -3.45 \\
\hline EPC/IPC RATIO & 0.92 & 0.96 & 0.67 & 4.35 & -30.21 \\
\hline EXP CONH CONCEN & MA & MA & $\mathbf{M}$ & - & - \\
\hline IHP COHH CONCEN & MA & $\mathbf{M}$ & MA & - & - \\
\hline ECC/ICC RATIO & MA & $\boldsymbol{M}$ & $\mathbf{M}$ & - & - \\
\hline VERTICAL TRADE & NA & MA & $\mathbf{M A}$ & - & - \\
\hline TOT TRADE LINKAGES & 34.00 & 46.00 & 63.00 & 35.29 & 36.96 \\
\hline \% AGRICULTURE-GDP & MA & $\mathbf{M A}$ & MA & - & - \\
\hline QUALITY OF LIFE & 88.30 & 93.00 & 95.60 & 5.32 & 2.80 \\
\hline X LRGST EXP TO GDP & NA & MA & $M$ & - & - \\
\hline X EXP LGST PARTNER & 59.00 & 45.00 & 14.00 & -23.73 & -68.89 \\
\hline X IHP LGST PARTMER & 69.00 & 49.00 & 48.00 & -28.99 & -2.04 \\
\hline \% EXP HANUFAC. & MA & MA & MA & - & - \\
\hline$X$ EXP AGR-MINE & NA & MA & MA & - & - \\
\hline X IRP MANUFAC. & $\mathbf{M}$ & MA & $\boldsymbol{M}$ & - & - \\
\hline$X$ IHP AGR-HINE & MA & $\mathbf{M}$ & MA & - & - \\
\hline TOTAL EXPORTS & 1812.00 & 3853.00 & 4191.00 & 112.64 & 8.77 \\
\hline TOTAL IMPORTS & 1983.00 & 3933.00 & 15488.00 & 98.34 & 293.80 \\
\hline G.H.P. & MA & $M$ & 5100.00 & - & - \\
\hline
\end{tabular}


ECUADOR

SUMMARY OF NATIONAL STATISTICS

\begin{tabular}{|c|c|c|c|c|c|}
\hline MATIONAL VARIABLES & 1962 & 1970 & 1980 & $62-70$ & $70-80$ \\
\hline EXP. PARTNER CON. & 0.72 & 0.56 & 0.58 & -22.22 & 3.57 \\
\hline IHP. PARTNER CON. & 0.57 & 0.54 & 0.51 & -5.26 & -5.56 \\
\hline EPC/IPC RATIO & 1.25 & 1.05 & 1.15 & -16.00 & 9.52 \\
\hline EXP CONH CONCEN & 0.88 & 0.83 & 0.46 & -5.68 & -44.58 \\
\hline IMP COHN CONCEN & 0.31 & 0.34 & 0.37 & 9.68 & 8.82 \\
\hline ECC/ICC RATIO & 2.84 & 2.44 & 1.24 & -14.00 & -49.07 \\
\hline VERTICAL TRADE & -0.78 & -0.82 & -0.84 & 5.13 & 2.44 \\
\hline TOT TRADE LINKAGES & 11.00 & 18.00 & 33.00 & 63.64 & 83.33 \\
\hline X AGRICULTURE-GDP & 27.50 & 25.20 & 15.10 & -8.36 & -40.08 \\
\hline QUALITY OF LIFE & 46.80 & 56.40 & 69.20 & 20.51 & 22.70 \\
\hline \& LRGST EXP TO GDP & 6.50 & 4.80 & 10.90 & -26.15 & 127.08 \\
\hline \% EXP LGST PARTNER & 65.00 & 42.00 & 43.00 & -35.38 & 2.38 \\
\hline X IMP LGST PARTNER & 46.00 & 43.00 & 39.00 & -6.52 & -9.30 \\
\hline X EXP MANUFAC. & 1.00 & 1.00 & 2.00 & 0.00 & 100.00 \\
\hline X EXP AGR-HINE & 98.00 & 98.00 & 97.00 & 0.00 & -1.02 \\
\hline \% IMP MANUFAC. & 79.00 & 83.00 & 86.00 & 5.06 & 3.61 \\
\hline * IMP AGR-HINE & 20.00 & 16.00 & 13.00 & -20.00 & -18.75 \\
\hline TOTAL EXPORTS & 115.00 & 189.00 & 1861.00 & 64.35 & 884.66 \\
\hline TOTAL IMPORTS & 96.00 & 288.00 & 2210.00 & 200.00 & 667.36 \\
\hline G.N.P. PER CAPITA & NA & MA & 1180.00 & - & - \\
\hline
\end{tabular}


EGYPT

SUMMARY OF NATIONAL STATISTICS

\begin{tabular}{|c|c|c|c|c|c|}
\hline MATIOHAL VARIABLES & 1962 & 1970 & 1980 & $62-70$ & $70-80$ \\
\hline EXP. PARTMER CON. & 0.37 & 0.44 & 0.43 & 18.92 & -2.27 \\
\hline IMP. PARTNER CON. & 0.43 & 0.35 & 0.40 & -18.60 & 14.29 \\
\hline EPC/IPC RATIO & 0.85 & 1.25 & 1.10 & 47.06 & -12.00 \\
\hline EXP COHH CONCEN & 0.27 & 0.23 & 0.38 & -14.81 & 65.22 \\
\hline IMP COHH CONCEN & 0.12 & 0.11 & 0.24 & -8.33 & 118.18 \\
\hline ECC/ICC RATIO & 2.25 & 2.09 & 1.58 & -7.07 & -24.28 \\
\hline VERTICAL TRADE & -0.35 & -0.29 & -0.48 & -17.14 & 65.52 \\
\hline TOT TRADE LINKAGES & 33.00 & 45.00 & 48.00 & 36.36 & 6.67 \\
\hline$X$ AGRICULTURE-GOP & 29.90 & 28.50 & 24.50 & -4.68 & -16.04 \\
\hline QUALITY OF LIFE & 30.80 & Mn & 48.60 & - & - \\
\hline$X$ LREST EXP TO GDP & NA & Ma & $\boldsymbol{M}$ & - & - \\
\hline X EXP LGST PARTHER & 16.00 & 38.00 & 28.00 & 137.50 & -26.32 \\
\hline X IHP LGST PARTHER & 28.00 & 14.00 & 18.00 & -50.00 & 28.57 \\
\hline * EXP manUfac. & 17.00 & 27.00 & 11.00 & 58.82 & -59.26 \\
\hline X EXP AGR-HINE & 82.00 & 72.00 & 89.00 & -12.20 & 23.61 \\
\hline * IHP MANUfAC. & 52.00 & 55.00 & 59.00 & 5.77 & 7.27 \\
\hline * IHP AGR-MINE & 47.00 & 44.00 & 41.00 & -6.38 & -6.82 \\
\hline TOTAL EXPORTS & 387.00 & 758.00 & 3017.00 & 95.87 & 298.02 \\
\hline TOTAL IMPORTS & 833.00 & 866.00 & 10157.00 & 3.96 & 1072.86 \\
\hline G.N.P. PER CAPIT & MA & $M$ & 650.00 & - & - \\
\hline
\end{tabular}


EL SALYADO

SUMMARY OF NATIONAL STATISTICS

\begin{tabular}{|c|c|c|c|c|c|}
\hline MATIONAL VARIABLES & 1962 & 1970 & 1980 & $62-70$ & $70-80$ \\
\hline EXP. PARTNER CON. & 0.63 & 0.58 & 0.63 & -7.94 & 8.62 \\
\hline IMP. PARTNER CON. & 0.55 & 0.53 & 0.56 & -3.64 & 5.66 \\
\hline EPC/IPC RATIO & 1.15 & 1.08 & 1.14 & -6.09 & 5.56 \\
\hline EXP COHH CONCEN & 0.48 & 0.34 & 0.23 & -29.17 & -32.35 \\
\hline IHP CONH CONCEN & 0.28 & 0.33 & 0.23 & 17.86 & -30.30 \\
\hline ECC/ICC RATIO & 1.71 & 1.03 & 1.00 & -39.90 & -2.94 \\
\hline VERTICAL TRADE & -0.63 & -0.51 & -0.25 & -19.05 & -50.98 \\
\hline TOT TRADE LIHKAGES & 10.00 & 13.00 & 16.00 & 30.00 & 23.08 \\
\hline \% AGRICULTURE-GDP & 36.10 & 28.90 & 27.20 & -19.94 & -5.88 \\
\hline QUALITY OF LIFE & 41.80 & 55.20 & 65.60 & 32.06 & 18.84 \\
\hline * LRGST EXP TO GDP & 7.00 & 4.80 & 12.40 & -31.43 & 158.33 \\
\hline X EXP LGST PARTNER & 34.00 & 24.00 & 43.00 & -29.41 & 79.17 \\
\hline X IMP LGST PARTNER & 39.00 & 31.00 & 32.00 & -20.51 & 3.23 \\
\hline X EXP maNUFAC. & 8.00 & 28.00 & 35.00 & 250.00 & 25.00 \\
\hline * EXP AGR-MINE & 91.00 & 71.00 & 64.00 & -21.98 & -9.86 \\
\hline X InP manufac. & 71.00 & 79.00 & 60.00 & 11.27 & -24.05 \\
\hline * IMP AGR-HINE & 28.00 & 20.00 & 39.00 & -28.57 & 95.00 \\
\hline TOTAL EXPORTS & 135.00 & 228.00 & 1017.00 & 68.89 & 346.05 \\
\hline TOTAL IMPORTS & 103.00 & 206.00 & 831.00 & 100.00 & 303.40 \\
\hline G.N.P. PER CAPITA & M & $\mathbf{M A}$ & 650.00 & - & - \\
\hline
\end{tabular}


ETHIOPIA

SUMMARY OF NATIONAL STATISTICS

\begin{tabular}{|c|c|c|c|c|c|}
\hline MATIONAL VARIABLES & 1962 & 1970 & 1980 & $62-70$ & $70-80$ \\
\hline EXP. PARTNER CON. & 0.58 & 0.58 & 0.46 & 0.00 & -20.69 \\
\hline IHP. PARTNER CON. & 0.50 & 0.45 & 0.42 & -10.00 & -6.67 \\
\hline EPC/IPC RATIO & 1.16 & 1.28 & 1.10 & 10.34 & -14.06 \\
\hline EXP CONH CONCEN & 0.66 & 0.68 & 0.54 & 3.03 & -20.59 \\
\hline IHP COHM CONCEN & 0.30 & 0.29 & 0.22 & -3.33 & -24.14 \\
\hline ECC/ICC RATIO & 2.20 & 2.34 & 2.45 & 6.58 & 4.68 \\
\hline VERTICAL TRADE & -0.80 & -0.78 & -0.64 & -2.50 & -17.95 \\
\hline TOT TRADE LINKAGES & 12.00 & 14.00 & 24.00 & 16.67 & 71.43 \\
\hline \% AGRICULTURE-GDP & 64.50 & 58.50 & 51.40 & -9.30 & -12.14 \\
\hline QUALITY OF LIFE & 15.70 & 19.80 & 24.30 & 26.11 & 22.73 \\
\hline * LRGST EXP TO GDP & 3.10 & 2.90 & 2.40 & -6.45 & -17.24 \\
\hline \% EXP LEST PARTNEER & 45.00 & 51.00 & 18.00 & 13.33 & -64.71 \\
\hline X IMP LGST PARTNER & 23.00 & 16.00 & $18 . \infty 0$ & -30.43 & 12.50 \\
\hline X EXP MANUFAC. & NA & 1.00 & MA & - & - \\
\hline X EXP AGR-MINE & 99.00 & 98.00 & 99.00 & -1.01 & 1.02 \\
\hline X ImP manUfac. & 80.00 & 79.00 & 64.00 & -1.25 & -18.99 \\
\hline X IAP AGR-HINE & 19.00 & 20.00 & 35.00 & 5.26 & 75.00 \\
\hline TOTAL EXPORTS & 69.00 & 115.00 & 411.00 & 66.67 & 257.39 \\
\hline TOTAL IMPORTS & 96.00 & 169.00 & 686.00 & 76.04 & 305.92 \\
\hline G.N.P.P & $\boldsymbol{N}$ & M & 140.00 & - & - \\
\hline
\end{tabular}


FIJI

SUMMARY OF NATIONAL STATISTICS

\begin{tabular}{|c|c|c|c|c|c|}
\hline NATIONAL VARIABLES & 1962 & 1970 & 1980 & $62-70$ & $70-80$ \\
\hline EXP. PARTNER CON. & 0.65 & 0.65 & 0.59 & $\mathbf{0 . 0 0}$ & -9.23 \\
\hline IMP. PARTNER CON. & 0.73 & 0.59 & 0.60 & -19.18 & 1.69 \\
\hline EPC/IPC RATIO & 0.90 & 1.09 & 0.99 & 21.11 & -9.17 \\
\hline EXP COMH CONCEN & 0.89 & 0.86 & 0.87 & -3.37 & 1.16 \\
\hline IHP COHM CONCEN & 0.22 & 0.25 & 0.22 & 13.64 & -12.00 \\
\hline ECC/ICC RATIO & 4.05 & 3.44 & 3.95 & -14.97 & 14.96 \\
\hline VERTICAL TRADE & -0.60 & -0.63 & -0.58 & 5.00 & -7.94 \\
\hline TOT TRADE LINKAGES & 8.00 & 7.00 & 10.00 & -12.50 & 42.86 \\
\hline X AGRICULTURE-GDP & $\boldsymbol{\mu}$ & 31.80 & 23.70 & - & -25.47 \\
\hline QUALITY OF LIFE & M & 78.00 & 84.40 & - & 8.21 \\
\hline \% LRGST EXP TO GDP & NA & 8.20 & 8.60 & - & 4.88 \\
\hline X EXP LGST PARTNER & 44.00 & 38.00 & 31.00 & -13.64 & -18.42 \\
\hline X IMP LGST PARTNER & 55.00 & 29.00 & 36.00 & -47.27 & 24.14 \\
\hline X EXP MANUFAC. & NA & 2.00 & 1.00 & - & -50.00 \\
\hline Z EXP AGR-HINE & 99.00 & 97.00 & $98 . \infty$ & -2.02 & 1.03 \\
\hline ※ Imp manufac. & 60.00 & 65.00 & 59.00 & 8.33 & -9.23 \\
\hline X IMP AGR-HINE & 39.00 & 35.00 & 40.00 & -10.26 & 14.29 \\
\hline TOTAL EXPORTS & 32.00 & 58.00 & 288.00 & 81.25 & 396.55 \\
\hline TOTAL IMPORTS & 19.00 & 82.00 & 460.00 & 331.58 & 460.98 \\
\hline G.N.P. PER CAPITA & NA & $\mathbf{M A}$ & 2000.00 & - & - \\
\hline
\end{tabular}


FIMLAND

SUMMARY OF NATIONAL STATISTICS

\begin{tabular}{|c|c|c|c|c|c|}
\hline MATIONAL VARIABLES & 1962 & 1970 & 1980 & $62-70$ & $70-80$ \\
\hline EXP. PARTNER CON. & 0.43 & 0.41 & 0.41 & -4.65 & 0.00 \\
\hline IMP. PARTMER CON. & 0.47 & 0.43 & 0.44 & -8.51 & 2.33 \\
\hline EPC/IPC RATIO & 0.93 & 0.94 & 0.93 & 1.08 & -1.06 \\
\hline EXP CONH CONCEN & 0.25 & 0.22 & 0.21 & -12.00 & -4.55 \\
\hline IHP CONH CONCEN & 0.17 & 0.17 & 0.16 & 0.00 & -5.88 \\
\hline ECC/ICC RATIO & 1.47 & 1.29 & 1.31 & -12.00 & 1.42 \\
\hline VERTICAL TRADE & -0.14 & -0.03 & 0.14 & -78.57 & -566.67 \\
\hline TOT TRADE LINKAGES & 43.00 & 55.00 & 83.00 & 27.91 & 50.91 \\
\hline \% AGRICULTURE-GDP & 16.70 & 13.50 & 8.80 & -19.16 & -36.81 \\
\hline QUALITY OF LIFE & 90.50 & 93.00 & 97.80 & 2.76 & 5.16 \\
\hline X LRGST EXP TO GDP & 4.10 & 4.00 & M & -2.44 & - \\
\hline X EXP LGST PARTNER & 20.00 & 17.00 & 17.00 & -15.00 & 0.00 \\
\hline * IHP LEST PARTNER & 20.00 & 16.00 & 21.00 & -20.00 & 37.25 \\
\hline X EXP MARUFAC. & 55.00 & 66.00 & 69.00 & 20.00 & 4.55 \\
\hline$X$ EXP AGR-HIME & 44.00 & 33.00 & 30.00 & -25.00 & -9.09 \\
\hline X IHP RANBFAC. & 69.00 & 70.00 & 56.00 & 1.45 & -20.00 \\
\hline * IHP AGR-MINE & 30.00 & 29.00 & 43.00 & -3.33 & 48.28 \\
\hline TOTAL EXPORTS & 1093.00 & 2301.00 & 14094.00 & 110.52 & 512.52 \\
\hline TOTAL IMPORTS & 1126.00 & 2635.00 & 13690.00 & 134.01 & 419.54 \\
\hline G.N.P. PER CAPITA & MA & MA & 10680.00 & - & 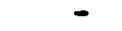 \\
\hline
\end{tabular}


FRANCE

SUMMARY OF NATIONAL STATISTICS

\begin{tabular}{|c|c|c|c|c|c|}
\hline MATIONAL VARIABLES & 1962 & 1970 & 1980 & $62-70$ & $70-80$ \\
\hline EXP. PARTMER CON. & 0.31 & 0.34 & 0.31 & 9.68 & -8.82 \\
\hline IMP. PARTNER CON. & 0.33 & 0.39 & 0.37 & 18.18 & -5.13 \\
\hline EPC/IPC RATIO & 0.94 & 0.86 & 0.84 & -8.51 & -2.33 \\
\hline EXP COHH CONCEN & 0.16 & 0.19 & 0.18 & 18.75 & -5.26 \\
\hline IHP COHM CONCEN & 0.03 & 0.09 & 0.13 & 200.00 & 44.44 \\
\hline ECC/ICC RATIO & 5.33 & 2.11 & 1.38 & -60.42 & -34.41 \\
\hline VERTICAL TRADE & 0.31 & 0.16 & 0.19 & -48.39 & 18.75 \\
\hline TOT TRADE LIMKAGES & 95.00 & 125.00 & 140.00 & 31.58 & 12.00 \\
\hline X AGRICULTURE-GDP & 10.60 & 7.90 & 4.90 & -25.47 & -37.97 \\
\hline QUALITY OF LIFE & 91.20 & 94.10 & 97.90 & 3.18 & 4.04 \\
\hline * LRGST EXP TO GDP & 1.60 & 2.70 & 2.80 & 68.75 & 3.70 \\
\hline X EXP LGST PARTNER & 17.00 & 20.00 & 16.00 & 17.65 & -20.00 \\
\hline X IHP LGST PARTMER & 19.00 & 24.00 & 20.00 & 26.32 & -16.67 \\
\hline X EXP maMUfaC. & 72.00 & 74.00 & 73.00 & 2.78 & -1.35 \\
\hline X EXP $\wedge$ GR-HINE & 27.00 & 25.00 & 26.00 & -7.41 & 4.00 \\
\hline X IMP MANUFAC. & 41.00 & 58.00 & 54.00 & 41.46 & -6.90 \\
\hline X IMP AGR-HINE & 58.00 & 41.00 & 45.00 & -29.31 & 9.76 \\
\hline TOTAL EXPORTS & \multicolumn{3}{|c|}{7172.0017654 .00110827 .90} & 146.15 & 527.78 \\
\hline TOTAL IMPORTS & \multicolumn{3}{|c|}{6934.0017475 .00128211 .90} & 152.02 & 633.69 \\
\hline G.N.P. PER CAPITA & NA & $\mathbf{M}$ & 12190.00 & - & - \\
\hline
\end{tabular}


GABON

SUMMARY OF NATIONAL STATISTICS

\begin{tabular}{|c|c|c|c|c|c|}
\hline MATIONAL VARIABLES & 1962 & 1970 & 1980 & $62-70$ & $70-80$ \\
\hline EXP. PARTNER CON. & 0.76 & 0.59 & 0.56 & -22.37 & -5.08 \\
\hline IMP. PARTNER CON. & 0.90 & 0.67 & 0.66 & -25.56 & -1.49 \\
\hline EPC/IPC RATIO & 0.85 & 0.87 & 0.85 & 2.35 & -2.30 \\
\hline EXP CONH CONCEN & 0.31 & 0.38 & 0.77 & 22.58 & 102.63 \\
\hline IMP CONI CONCEN & 0.29 & 0.37 & 0.33 & 27.59 & -10.81 \\
\hline ECC/ICC RATIO & 1.07 & 1.03 & 2.33 & -3.92 & 127.19 \\
\hline VERTICAL TRADE & -0.57 & -0.74 & -0.75 & 29.82 & 1.35 \\
\hline TOT TRADE LINKAGES & 7.00 & 15.00 & 20.00 & 114.29 & 33.33 \\
\hline X AGRICULTURE-GDP & 32.50 & 25.20 & 7.00 & -22.46 & -72.22 \\
\hline QUALITY OF LIFE & 15.80 & MA & 30.00 & - & - \\
\hline X LRGST EXP TO GDP & 20.30 & 23.20 & 18.90 & 14.29 & -18.53 \\
\hline X EXP LGST PARTNER & 67.00 & 44.00 & 24.00 & -34.33 & -45.45 \\
\hline X IMP LGST PARTMER & 85.00 & 58.00 & 60.00 & -31.76 & 3.45 \\
\hline X EXP RANUFAC. & 14.00 & 9.00 & 2.00 & -35.71 & -77.78 \\
\hline X EXP AGR-MINE & 85.00 & 91.00 & 97.00 & 7.06 & 6.59 \\
\hline$x$ Imp manufaC. & 72.00 & 83.00 & 77.00 & 15.28 & -7.23 \\
\hline X IHP AGR-MINE & 28.00 & 16.00 & 22.00 & -42.86 & 37.50 \\
\hline TOTAL EXPORTS & 52.00 & 114.00 & 2022.00 & 119.23 & 1673.68 \\
\hline TOTAL IMPORTS & 22.00 & 80.00 & 719.00 & 263.64 & 798.75 \\
\hline G.H.P. PER CAPITA & $\mathrm{Nh}$ & HA & 3810.00 & - & - \\
\hline
\end{tabular}


GARBIA

SUMMARY OF NATIONAL STATISTICS

\begin{tabular}{|c|c|c|c|c|c|}
\hline MATIONAL VARIABLES & 1962 & 1970 & 1980 & $62-70$ & $70-80$ \\
\hline EXP. PARTMER CON. & 0.86 & 0.71 & 0.53 & -17.44 & -25.35 \\
\hline IMP. PARTNER CON. & 0.88 & 0.51 & 0.51 & -42.05 & 0.00 \\
\hline EPC/IPC RATIO & 0.98 & 1.40 & 1.04 & 42.86 & -25.71 \\
\hline EXP COHH CONCEN & 1.00 & 0.99 & Ma & -1.00 & - \\
\hline IHP COMM CONCEN & 0.32 & 0.32 & $m a$ & 0.00 & - \\
\hline ECC/ICC RATIO & 3.13 & 3.09 & $\boldsymbol{M}$ & -1.00 & - \\
\hline VERTICAL TRADE & -0.67 & -0.62 & $\boldsymbol{M}$ & -7.46 & - \\
\hline TOT TRADE LINKAGES & 2.00 & 4.00 & 8.00 & 100.00 & 100.00 \\
\hline \% AGRICULTURE-GDP & 39.20 & 39.30 & 33.90 & 0.26 & -13.74 \\
\hline QUALITY OF LIFE & M & $M$ & 9.20 & - & - \\
\hline X LRGST EXP TO GDP & 12.90 & 14.70 & 11.00 & 13.95 & -25.17 \\
\hline X EXP LGST PARTNER & 57.00 & 48.00 & 25.00 & -15.79 & -47.92 \\
\hline X IMP LGST PARTNER & 72.00 & 32.00 & 35.00 & -55.56 & 9.38 \\
\hline \% EXP manufac. & MA & MA & MA & - & - \\
\hline X EXP AGR-HINE & 100.00 & 100.00 & 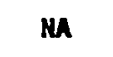 & 0.00 & - \\
\hline Х IMP MANUFAC. & 67.00 & 62.00 & $\mathbf{M A}$ & -7.46 & - \\
\hline X IHP AGR-HIHE & 32.00 & 37.00 & $M$ & 15.63 & - \\
\hline TOTAL EXPORTS & 8.00 & 14.00 & 29.00 & 75.00 & 107.14 \\
\hline TOTAL IMPORTS & 6.00 & 15.00 & 114.00 & 150.00 & 660.00 \\
\hline G.N.P. PER CAPITA & NA & $\mathbf{M}$ & 370.00 & - & - \\
\hline
\end{tabular}


GERHANY

SUMMARY OF NATIONAL STATISTICS

\begin{tabular}{|c|c|c|c|c|c|}
\hline MATIONAL VARLABLES & 1962 & 1970 & 1980 & $62-70$ & $70-80$ \\
\hline EXP. PARTMER CON. & 0.30 & 0.33 & 0.33 & 10.00 & 0.00 \\
\hline IMP. PARTNER CON. & 0.31 & 0.36 & 0.33 & 16.13 & -8.33 \\
\hline EPC/IPC RATIO & 0.97 & 0.91 & 0.98 & -6.19 & 7.69 \\
\hline EXP CONH CONCEN & 0.33 & 0.34 & 0.30 & 3.03 & -11.76 \\
\hline IMP CONH CONCEN & 0.08 & 0.09 & 0.12 & 12.50 & 33.33 \\
\hline ECC/ICC RATIO & 4.13 & 3.78 & 2.50 & -8.42 & -33.82 \\
\hline VERTICAL TRADE & 0.44 & 0.34 & 0.31 & -22.73 & -8.82 \\
\hline TOT TRADE LINKAGES & 101.00 & 125.00 & 141.00 & 23.76 & 12.80 \\
\hline X AGRICULTURE-GDP & 5.70 & 4.40 & 2.80 & -22.81 & -36.36 \\
\hline QUALITY OF LIFE & 89.50 & 91.90 & 95.70 & 2.68 & 4.13 \\
\hline \% LRGST EXP TO GDP & M & MA & $M$ & - & - \\
\hline \% EXP LGST PARTNER & 10.00 & 12.00 & 13.00 & 20.00 & 8.33 \\
\hline X IHP LGST PARTNER & 12.00 & 14.00 & 13.00 & 16.67 & -7.14 \\
\hline X EXP MANUFAC. & 87.00 & 89.00 & 86.00 & 2.30 & -3.37 \\
\hline X EXP AGR-MINE & 12.00 & 10.00 & 13.00 & -16.67 & 30.00 \\
\hline X IMP MANUFAC. & 43.00 & 54.00 & 55.00 & 25.58 & 1.85 \\
\hline * IHP AGR-MINE & 56.00 & 45.00 & 44.00 & -19.64 & -2.22 \\
\hline TOTAL EXPORTS & \multicolumn{3}{|c|}{13111.0034047 .0191223 .90} & 159.68 & 461.65 \\
\hline TOTAL IMPORTS & 10570.00 & 26966.0 & 165170.90 & 155.12 & 512.52 \\
\hline G.N.P. PER CAPITA & MA & MA & 13450.00 & - & - \\
\hline
\end{tabular}


GHANA

SUMMARY OF NATIONAL STATISTICS

\begin{tabular}{|c|c|c|c|c|c|}
\hline MATIONAL VARIABLES & 1962 & 1970 & 1980 & $62-70$ & $70-80$ \\
\hline EXP. PARTNER CON. & 0.54 & 0.50 & 0.52 & -7.41 & 4.00 \\
\hline IMP. PARTNER CON. & 0.51 & 0.46 & 0.48 & -9.80 & 4.35 \\
\hline EPC/IPC RATIO & 1.06 & 1.09 & 1.08 & 2.83 & -0.92 \\
\hline EXP CONH CONCEN & 0.50 & 0.56 & M & 12.00 & - \\
\hline IMP CONM CONCEN & 0.29 & 0.25 & MA & -13.79 & - \\
\hline ECC/ICC RATIO & 1.72 & 2.24 & MA & 29.92 & - \\
\hline VERTICAL TRADE & -0.70 & -0.69 & Ma & -1.43 & - \\
\hline TOT TRADE LINKAGES & 22.00 & 24.00 & 21.00 & 9.09 & -12.50 \\
\hline X AGRICULTURE-GDP & 40.80 & 43.40 & 55.20 & 6.37 & 27.19 \\
\hline QUALITY OF LIFE & 27.90 & 36.50 & 42.90 & 30.82 & 17.53 \\
\hline * LRGST EXP TO GDP & 6.50 & 3.80 & 6.90 & -41.54 & 81.58 \\
\hline \% EXP LGST PARTNNER & 32.00 & 23.00 & 17.00 & -28.13 & -26.09 \\
\hline * IHP LGST PARTNER & 37.00 & 25.00 & 20.00 & -32.43 & -20.00 \\
\hline X EXP MNNUFAC. & 1.00 & MA & MA - & -10000.00 & 0.00 \\
\hline X EXP AGR-MIME & 99.00 & 99.00 & Ma & 0.00 & - \\
\hline \% IIIP MANUFAC. & 70.00 & 69.00 & MA & -1.43 & - \\
\hline X IHP AGR-HINE & 29.00 & 30.00 & Ma & 3.45 & - \\
\hline TOTAL EXPORTS & 312.00 & 457.00 & 1141.00 & 46.47 & 149.67 \\
\hline TOTAL IMPORTS & 276.00 & 356.00 & 1012.00 & 28.99 & 184.27 \\
\hline G.N.P. PER CAPITA & MA & $\boldsymbol{M}$ & 400.00 & - & - \\
\hline
\end{tabular}


GREECE

SUMHARY OF NATIONAL STATISTICS

\begin{tabular}{|c|c|c|c|c|c|}
\hline MATIOHAL VARIABLES & 1962 & 1970 & 1980 & $62-70$ & $70-80$ \\
\hline EXP. PARTNER CON. & 0.39 & 0.39 & 0.34 & $0 . \infty 0$ & -12.82 \\
\hline IHP. PARTNER CON. & 0.39 & 0.40 & 0.39 & 2.56 & -2.50 \\
\hline EPC/IPC RATIO & 1.01 & 0.96 & 0.88 & -4.95 & -8.33 \\
\hline EXP COMN CONCEN & 0.29 & 0.19 & 0.23 & -34.48 & 21.05 \\
\hline IMP COHA CONCEN & 0.17 & 0.21 & 0.16 & 23.53 & -23.81 \\
\hline ECC/ICC RATIO & 1.71 & 0.90 & 1.44 & -46.96 & 58.88 \\
\hline VERTICAL TRADE & -0.59 & -0.38 & -0.13 & -35.59 & -65.79 \\
\hline TOT TRADE LINKAGES & 29.00 & 38.00 & 69.00 & 31.03 & 81.58 \\
\hline X AGRICULTURE-GDP & 26.80 & 21.90 & 17.60 & -18.28 & -19.63 \\
\hline QUALITY OF LIFE & 81.70 & 86.80 & 90.60 & 6.24 & 4.38 \\
\hline ※ LRGST EXP TO GDP & 1.10 & 1.20 & 2.50 & 9.09 & 108.33 \\
\hline X EXP LGST PARTNER & 18.00 & 20.00 & 18.00 & 11.11 & -10.00 \\
\hline ※ IHP LGST PARTNER & 19.00 & 17.00 & 16.00 & -10.53 & -5.88 \\
\hline X EXP MNNUFAC. & 10.00 & 34.00 & 47.00 & 240.00 & 38.24 \\
\hline X EXP AGR-MINE & 89.00 & 65.00 & 52.00 & -26.97 & -20.00 \\
\hline X IMP MANUFAC. & 69.00 & 72.00 & 60.00 & 4.35 & -16.67 \\
\hline X IMP AGR-HINE & 30.00 & $27 . \infty$ & 39.00 & -10.00 & 44.44 \\
\hline TOTAL EXPORTS & 242.00 & 642.00 & 5110.00 & 165.29 & 695.95 \\
\hline TOTAL IMPORTS & 688.00 & 2041.00 & 13770.00 & 196.66 & 574.67 \\
\hline G.M.P. PER & NA & MA & 20.00 & - & - \\
\hline
\end{tabular}


GREENLAND

SUMMARY OF NATIONAL STATISTICS

\begin{tabular}{|c|c|c|c|c|c|}
\hline MATIONAL VARIABLES & 1962 & 1970 & 1980 & $62-70$ & $70-80$ \\
\hline EXP. PARTNER CON. & 0.81 & 0.87 & 0.65 & 7.41 & -25.29 \\
\hline IMP. PARTNER CON. & 0.83 & 0.88 & 0.83 & 6.02 & -5.68 \\
\hline EPC/IPC RATIO & 0.98 & 0.98 & 0.78 & $0 . \infty 0$ & -20.41 \\
\hline EXP CONH CONCEN & NA & NA & $m$ & - & - \\
\hline IHP COHY CONCEN & NA & Ma & $M$ & - & - \\
\hline ECC/ICC RATIO & NA & $\boldsymbol{M}$ & M & - & - \\
\hline VERTICAL TRADE & NA & M & MA & - & - \\
\hline TOT TRADE LINKAGES & 2.00 & 2.00 & 13.00 & 0.00 & 550.00 \\
\hline X AGRICULTURE-GDP & MA & MA & MA & - & - \\
\hline QUALITY OF LIFE & NA & MA & $M$ & - & - \\
\hline * LRGST EXP TO GDP & MA & $\boldsymbol{M}$ & $M$ & - & - \\
\hline ※ EXP LGST PARTNER & 63.00 & 80.00 & 49.00 & 26.98 & -38.75 \\
\hline X IMP LGST PARTNER & 73.00 & 85.00 & 80.00 & 16.44 & -5.88 \\
\hline X EXP makUfac. & MA & MA & MA & - & - \\
\hline \% EXP AGR-MINE & MA & MA & MA & - & - \\
\hline X ImP manUfac. & MA & MA & M & - & - \\
\hline \% IMP AGR-MINE & NA & $M$ & $\boldsymbol{M}$ & - & - \\
\hline TOTAL EXPORTS & 7.00 & 13.00 & 211.00 & 85.71 & 1523.08 \\
\hline TOTAL IHPORTS & 25.00 & 53.00 & 286.00 & 112.00 & 439.62 \\
\hline G.N.P. PER CAPITA & Mn & $\mathbf{M A}$ & 6700.00 & - & - \\
\hline
\end{tabular}


GUADALOUPE

SUMAARY OF NATIONAL STATISTICS

\begin{tabular}{|c|c|c|c|c|c|}
\hline MATIONAL VARIABLES & 1962 & 1970 & 1980 & $62-70$ & $70-80$ \\
\hline EXP. PARTNER CON. & 0.99 & 0.84 & 0.82 & -15.15 & -2.38 \\
\hline IHP. PARTNER CON. & 1.00 & 0.83 & 0.79 & -17.00 & -4.82 \\
\hline EPC/IPC RATIO & 0.99 & 1.02 & 1.04 & 3.03 & 1.96 \\
\hline EXP COHA CONCEN & MA & MA & Ma & - & - \\
\hline IMP CONH CONCEN & Ma & $\boldsymbol{M}$ & MA & - & - \\
\hline ECC/ICC RATIO & MA & $\boldsymbol{M}$ & M & - & - \\
\hline VERTICAL TRADE & MA & MA & MA & - & - \\
\hline TOT TRADE LINKAGES & 1.00 & 2.00 & 4.00 & 100.00 & 100.00 \\
\hline X AGRICULTURE-GDP & NA & M & $\mathbf{M}$ & - & - \\
\hline QUALITY OF LIFE & MA & Ma & $\mathbf{M a}$ & - & - \\
\hline$X$ LRGST EXP TO GDP & 26.70 & 13.20 & $\mathbf{M A}$ & -50.56 & - \\
\hline X EXP LGST PARTHER & 98.00 & 70.00 & 73.00 & -28.57 & 4.29 \\
\hline * IHP LGST PARTMER & 100.00 & 82.00 & 76.00 & -18.00 & -7.32 \\
\hline X EXP MNUFAC. & MM & MA & MA & - & - \\
\hline X EXP AGR-NINE & $\mathbf{M}$ & MA & $\mathbf{M}$ & - & - \\
\hline X IMP MWNUFAC. & NA & MA & MA & - & - \\
\hline X IMP AGR-MINE & MA & MA & MA & - & - \\
\hline TOTAL EXPORTS & 44.00 & 36.00 & 105.00 & -18.18 & 191.67 \\
\hline TOTAL IMPORTS & 38.00 & 92.00 & 610.00 & 142.11 & 563.04 \\
\hline G.M.P. PER CAPITA & $M$ & MA & 3300.00 & - & - \\
\hline
\end{tabular}


GUATEMALA

SUMMARY OF NATIONAL STATISTICS

\begin{tabular}{|c|c|c|c|c|c|}
\hline NATIOHAL VARIABLES & 1962 & 1970 & 1980 & $62-70$ & $70-80$ \\
\hline EXP. PARTNER CON. & 0.68 & 0.51 & 0.49 & -25.00 & -3.92 \\
\hline IMP. PARTNER CON. & 0.59 & 0.53 & 0.50 & -10.17 & -5.66 \\
\hline EPC/IPC RATIO & 1.16 & 0.97 & 0.98 & -16.38 & 1.03 \\
\hline EXP CONH CONCEN & 0.56 & 0.36 & 0.25 & -35.71 & -30.56 \\
\hline IHP COMM CONCEN & 0.27 & 0.36 & 0.23 & 33.33 & -36.11 \\
\hline ECC/ICC RATIO & 2.07 & 1.00 & 1.09 & -51.79 & 8.70 \\
\hline VERTICAL TRADE & -0.68 & -0.55 & -0.40 & -19.12 & -27.27 \\
\hline TOT TRADE LINKAGES & 9.00 & 19.00 & 31.00 & 111.11 & 63.16 \\
\hline X AGRICULTURE-GDP & MA & Ma & Ma & - & - \\
\hline QUALITY OF LIFE & 40.10 & 49.20 & 58.20 & 22.69 & 18.29 \\
\hline * LRGST EXP TO GDP & 5.50 & 4.40 & 6.40 & -20.00 & 45.45 \\
\hline \% EXP LGST PARTHER & 52.00 & 28.00 & 28.00 & -46.15 & 0.00 \\
\hline X IMP LGST PARTNER & 49.00 & $34 . \infty 0$ & 34.00 & -30.61 & 0.00 \\
\hline X EXP maNUFAC. & 7.00 & 28.00 & 24.00 & 300.00 & -14.29 \\
\hline X EXP AGR-HINE & 92.00 & 72.00 & 75.00 & -21.74 & 4.17 \\
\hline X itap manufac. & 74.00 & 83.00 & 64.00 & 12.16 & -22.89 \\
\hline X IHP AGR-HINE & 25.00 & 16.00 & 35.00 & -36.00 & 118.75 \\
\hline TOTAL EXPORTS & 106.00 & 288.00 & 1465.00 & 171.70 & 408.68 \\
\hline TOTAL IHPORTS & 123.00 & 286.00 & 1616.00 & 132.52 & 464.34 \\
\hline G.H.P. PER CAPITA & MA & M & 1140.00 & - & - \\
\hline
\end{tabular}


GUINEA

SUMMARY OF MATIONAL STATISTICS

\begin{tabular}{|c|c|c|c|c|c|}
\hline MATIONAL VARIABLES & 1962 & 1970 & 1980 & $62-70$ & $70-80$ \\
\hline EXP. PARTMER CON. & 0.55 & 0.58 & 0.56 & 5.45 & -3.45 \\
\hline IMP. PARTHER CON. & 0.58 & 0.56 & 0.53 & -3.45 & -5.36 \\
\hline EPC/IPC RATIO & 0.96 & 1.03 & 1.06 & 7.29 & 2.91 \\
\hline EXP COMH CONCEN & MA & MA & Ma & - & - \\
\hline IHP COHH COHCEN & MA & MA & MA & - & - \\
\hline ECC/ICC RATIO & MA & M & $\boldsymbol{M}$ & - & - \\
\hline VERTICAL TRADE & NA & MA & $\mathrm{Ma}$ & - & - \\
\hline TOT TRADE LINKAGES & 8.00 & 9.00 & 15.00 & 12.50 & 66.67 \\
\hline X AGRICULTURE-GDP & MA & $\mathbf{M A}$ & 46.40 & - & - \\
\hline QUALITY OF LIFE & MA & MA & 18.80 & - & - \\
\hline X LRGST EXP TO GDP & 4.90 & MA & Ma & -2120.41 & 0.00 \\
\hline \% EXP LGST PARTMER & 26.00 & 32.00 & 26.00 & 23.08 & -18.75 \\
\hline * IMP LGST PARTNER & 35.00 & 35.00 & 30.00 & 0.00 & -14.29 \\
\hline X EXP MANUFAC. & NA & MA & MA & - & - \\
\hline X EXP AGR-HINE & NA & Ma & MA & - & - \\
\hline Z IHP MANUFAC. & MA & $\mathbf{M a}$ & MA & - & - \\
\hline * IHP AGR-MINE & NA & MA & MA & - & - \\
\hline TOTAL EXPORTS & 43.00 & 47.00 & 407.00 & 9.30 & 765.96 \\
\hline TOTAL IRPORTS & 56.00 & 53.00 & 322.00 & -5.36 & 507.55 \\
\hline G.N.P. PER CAPITA & MA & M & 300.00 & - & - \\
\hline
\end{tabular}


HAITI

SUMMARY OF NATIONAL STATISTICS

\begin{tabular}{|c|c|c|c|c|c|}
\hline NATIONAL VARIABLES & 1962 & 1970 & 1980 & $62-70$ & $70-80$ \\
\hline EXP. PARTNER CON. & 0.68 & 0.69 & 0.69 & 1.47 & 0.00 \\
\hline IMP. PARTNER CON. & 0.72 & 0.59 & 0.63 & -18.06 & 6.78 \\
\hline EPC/IPC RATIO & 0.94 & 1.17 & 1.10 & 24.47 & -5.98 \\
\hline EXP CONM CONCEN & NA & 0.28 & MA & - & - \\
\hline IHP CONM CONCEN & M & 0.30 & $\boldsymbol{m}$ & - & - \\
\hline ECC/ICC RATIO & MA & 0.93 & $\boldsymbol{M}$ & - & - \\
\hline VERTICAL TRADE & MA & -0.46 & $\mathbf{M}$ & - & - \\
\hline TOT TRADE LINKAGES & 7.00 & 6.00 & 9.00 & -14.29 & 50.00 \\
\hline X AGRICULTURE-GDP & NA & M & $\boldsymbol{m}$ & - & - \\
\hline QUALITY OF LIFE & 12.60 & 29.50 & 38.00 & 134.13 & 28.81 \\
\hline X LRGST EXP TO GOP & 6.40 & 6.70 & 6.50 & 4.69 & -2.99 \\
\hline X EXP LGST PARTNER & 54.00 & 60.00 & 56.00 & 11.11 & -6.67 \\
\hline X IMP LGST PARTMER & 66.00 & 50.00 & 58.00 & -24.24 & 16.00 \\
\hline X EXP manufac. & MA & 25.00 & M & - & - \\
\hline X EXP AGR-MINE & Ma & 74.00 & MA & - & - \\
\hline X IHP MANUFAC. & M & 71.00 & MA & - & - \\
\hline \% IHP AGR-NINE & NA & 28.00 & $M$ & - & - \\
\hline TOTAL EXPORTS & 43.00 & 40.00 & 224.00 & -6.98 & 460.00 \\
\hline TOTAL IHPORTS & 36.00 & 66.00 & 528.00 & 83.33 & 700.00 \\
\hline G.N.P. PER CAPITA & MA & $\mathbf{M A}$ & 300.00 & - & - \\
\hline
\end{tabular}


HONDORAS

SUMHARY OF NATIONAL STATISTICS

\begin{tabular}{|c|c|c|c|c|c|}
\hline MATIOHAL VARIABLES & 1962 & 1970 & 1980 & $62-70$ & $70-80$ \\
\hline EXP. PARTNER CON. & 0.69 & 0.63 & 0.62 & -8.70 & -1.59 \\
\hline IMP. PARTNER CON. & 0.70 & 0.58 & 0.54 & -17.14 & -6.90 \\
\hline EPC/IPC RATIO & 1.00 & 1.09 & 1.15 & 9.00 & 5.50 \\
\hline EXP COMH CONCEN & 0.56 & 0.44 & 0.49 & -21.43 & 11.36 \\
\hline IHP COHH CONCEN & 0.34 & 0.15 & 0.26 & -55.88 & 73.33 \\
\hline ECC/ICC RATIO & 1.65 & 2.93 & 1.88 & 78.10 & -35.75 \\
\hline VERTICAL TRADE & -0.77 & -0.54 & -0.60 & -29.87 & 11.11 \\
\hline TOT TRADE LINKAGES & 7.00 & 13.00 & 24.00 & 85.71 & 84.62 \\
\hline X AGRICULTURE-GDP & 41.90 & 37.50 & 30.30 & -10.50 & -19.20 \\
\hline QUALITY OF LIFE & 35.00 & 48.30 & 58.60 & 38.00 & 21.33 \\
\hline X LRGST EXP TO GDP & 10.90 & 16.10 & 18.40 & 47.71 & 14.29 \\
\hline X EXP LGST PARTNER & 61.00 & 55.00 & 53.00 & -9.84 & -3.64 \\
\hline X IhP lgSt PARTNER & 62.00 & 43.00 & 42.00 & -30.65 & -2.33 \\
\hline X EXP maKUFAC. & 2.00 & 8.00 & 12.00 & 300.00 & 50.00 \\
\hline X EXP AGR-MIKE & 98.00 & 92.00 & 87.00 & -6.12 & -5.43 \\
\hline X IHP MANUFAC. & 78.00 & 80.00 & 72.00 & 2.56 & -10.00 \\
\hline * IMP AGR-MINE & 21.00 & 43.00 & 27.00 & $10=.76$ & -37.21 \\
\hline TOTAL EXPORTS & $\pi .00$ & 167.00 & 819.00 & 116.88 & 390.42 \\
\hline TOTAL IMPORTS & 68.00 & 203.00 & 891.00 & 198.53 & 338.92 \\
\hline G.N.P. PER CAPITA & NA & NA & 600.00 & - & - \\
\hline
\end{tabular}


HONG KONG

SUMMARY OF NATIONAL STATISTICS

\begin{tabular}{|c|c|c|c|c|c|}
\hline MATIONAL VARIABLES & 1962 & 1970 & 1980 & $62-70$ & $70-80$ \\
\hline EXP. PARTNER CON. & 0.42 & 0.43 & 0.38 & 2.38 & -11.63 \\
\hline IMP. PARTNER CON. & 0.45 & 0.44 & 0.49 & -2.22 & 11.36 \\
\hline EPC/IPC RATIO & 0.94 & 0.99 & 0.78 & 5.32 & -21.21 \\
\hline EXP COMn COHCEN & 0.67 & 0.65 & 0.60 & -2.99 & -7.69 \\
\hline IMP COHH CONCEN & 0.23 & 0.26 & 0.27 & 13.04 & 3.85 \\
\hline ECC/ICC RATIO & 2.91 & 2.50 & 2.22 & -14.18 & -11.11 \\
\hline VERTICAL TRADE & 0.36 & 0.28 & 0.21 & -22.22 & -25.00 \\
\hline TOT TRADE LINKAGES & 47.00 & 75.00 & 115.00 & 59.57 & 53.33 \\
\hline \% AGRICULTURE-GDP & 3.40 & 2.60 & 1.30 & -23.53 & -50.00 \\
\hline QUALITY OF LIFE & 77.10 & 85.40 & 94.00 & 10.77 & 10.07 \\
\hline * LRGST EXP TO GDP & 11.50 & 30.50 & M & 165.22 & - \\
\hline X EXP LGST PARTNER & 23.00 & 36.00 & 27.00 & 56.52 & -25.00 \\
\hline \% IMP LGST PARTNER & 19.00 & 23.00 & 23.00 & 21.05 & $0 . \infty$ \\
\hline X EXP haNUfac. & 92.00 & 95.00 & 96.00 & 3.26 & 1.05 \\
\hline * EXP AGR-HINE & 7.00 & 4.00 & 3.00 & -42.86 & -25.00 \\
\hline X IMP MANUFAC. & 56.00 & 68.00 & 75.00 & 21.43 & 10.29 \\
\hline$X$ IHP AGR-MINE & 43.00 & 31.00 & 24.00 & -27.91 & -22.58 \\
\hline TOTAL EXPORTS & 684.00 & 2468.00 & 18868.00 & 260.82 & 664.51 \\
\hline TOTAL IHPORTS & 1061.00 & 2920.00 & 20532.00 & 175.21 & 603.15 \\
\hline G.N.P. PER CAPITA & NA & Ma & 5100.00 & - & 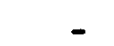 \\
\hline
\end{tabular}


HUNGARY

SUMMARY OF NATIONAL STATISTICS

\begin{tabular}{|c|c|c|c|c|c|}
\hline MATIONAL VARIABLES & 1962 & 1970 & 1980 & $62-70$ & $70-80$ \\
\hline EXP. PARTNER CON. & 0.59 & 0.50 & 0.44 & -15.25 & -12.00 \\
\hline IHP. PARTNER CON. & 0.58 & 0.48 & 0.44 & -17.24 & -8.33 \\
\hline EPC/IPC RATIO & 1.01 & 1.04 & 1.00 & 2.97 & -3.85 \\
\hline EXP CONH CONCEN & M & 0.16 & 0.14 & - & -12.50 \\
\hline IMP COMH CONCEN & MA & 0.10 & 0.12 & - & 20.00 \\
\hline ECC/ICC RATIO & M & 1.60 & 1.17 & - & -27.08 \\
\hline VERTICAL TRADE & $M$ & 0.06 & 0.04 & - & -33.33 \\
\hline TOT TRADE LINKAGES & 29.00 & 48.00 & 50.00 & 65.52 & 4.17 \\
\hline$X$ AGRICULTURE-GDP & 20.40 & 20.00 & 18.10 & -1.96 & -9.50 \\
\hline QUALITY OF LIFE & 85.30 & 88.80 & 91.70 & 4.10 & 3.27 \\
\hline X LRGST EXP TO GDP & MA & $\mathbf{M}$ & $\mathbf{M}$ & - & - \\
\hline X EXP LGST PARTNER & 46.00 & 38.00 & 31.00 & -17.39 & -18.42 \\
\hline X IHP LGST PARTNER & 48.00 & 35.00 & 28.00 & -27.08 & -20.00 \\
\hline X EXP MANUFAC. & MA & 67.00 & 65.00 & - & -2.99 \\
\hline X EXP AGR-HINE & MA & 32.00 & 34.00 & - & 6.25 \\
\hline X IHP MANUFAC. & MA & 62.00 & 62.00 & - & 0.00 \\
\hline * IHP AGR-HINE & MA & 38.00 & 37.00 & - & -2.63 \\
\hline TOTAL EXPORTS & 830.00 & 2077.00 & 8131.00 & 150.24 & 291.48 \\
\hline TOTAL IMPORTS & 856.00 & 2356.00 & 8171.00 & 175.23 & 246.82 \\
\hline G.N.P. PER CAPITA & FA & M & 2100.00 & - & - \\
\hline
\end{tabular}


ICELAND

SUMMARY OF NATIONAL STATISTICS

\begin{tabular}{|c|c|c|c|c|c|}
\hline MATIONAL VARIABLES & 1962 & 1970 & 1980 & $62-70$ & $70-80$ \\
\hline EXP. PARTNER CON. & 0.49 & 0.50 & 0.46 & 2.04 & -8.00 \\
\hline IAP. PARTNER CON. & 0.49 & 0.45 & 0.45 & -8.16 & 0.00 \\
\hline EPC/IPC RATIO & 1.00 & 1.09 & 1.03 & 9.00 & -5.50 \\
\hline EXP CONH CONCEN & 0.83 & 0.59 & 0.53 & -28.92 & -10.17 \\
\hline IHP COMH CONCEN & 0.18 & 0.18 & 0.20 & 0.00 & 11.11 \\
\hline ECC/ICC RATIO & 4.61 & 3.28 & 2.65 & -28.92 & -19.15 \\
\hline VERTICAL TRADE & -0.66 & -0.66 & -0.60 & 0.00 & -9.09 \\
\hline TOT TRADE LIHKAGES & 16.00 & 21.00 & 28.00 & 31.25 & 33.33 \\
\hline X AGRICULTURE-GOP & MA & MA & $\boldsymbol{M}$ & - & - \\
\hline QUALITY OF LIFE & 95.50 & 96.40 & 99.90 & 0.94 & 3.63 \\
\hline$X$ LRGST EXP TO GDP & 7.50 & 9.00 & 7.90 & 20.00 & -12.22 \\
\hline X EXP LGST PARTNER & 19.00 & 30.00 & 21.00 & 57.89 & -30.00 \\
\hline X IHP LGST PARTWER & 17.00 & 15.00 & 11.00 & -11.76 & -26.67 \\
\hline X EXP MANUFAC. & 1.00 & 3.00 & 8.00 & 200.00 & 166.67 \\
\hline$X$ EXP AGR-MINE & 98.00 & 96.00 & 91.00 & -2.04 & -5.21 \\
\hline X IMP RANUFAC. & 67.00 & 69.00 & 68.00 & 2.99 & -1.45 \\
\hline$X$ IHP AGR-MINE & 32.00 & 30.00 & 31.00 & -6.25 & 3.33 \\
\hline TOTAL EXPORTS & 80.00 & 145.00 & 913.00 & 81.25 & 529.66 \\
\hline TOTAL IMPORTS & 78.00 & 149.00 & 917.00 & 91.03 & 515.44 \\
\hline G.N.P. PER CAPITA & NA & NA & 12860.00 & - & - \\
\hline
\end{tabular}


INDIA

SUMHARY OF NATIONAL STATISTICS

\begin{tabular}{|c|c|c|c|c|c|}
\hline MATIOMAL VARIABLES & 1962 & 1970 & 1980 & $62-70$ & $70-80$ \\
\hline EXP. PARTNER CON. & 0.36 & 0.35 & 0.32 & -2.78 & -8.57 \\
\hline IMP. PARTNER CON. & 0.45 & 0.41 & 0.35 & -8.89 & -14.63 \\
\hline EPC/IPC RATIO & 0.80 & 0.84 & 0.91 & $\mathbf{5 . 0 0}$ & 8.33 \\
\hline EXP CONH CONCEN & 0.27 & 0.20 & 0.26 & -25.93 & 30.00 \\
\hline IHP COHH CONCEN & 0.10 & 0.07 & 0.14 & -30.00 & 100.00 \\
\hline ECC/ICC RATIO & 2.70 & 2.86 & 1.86 & 5.82 & -35.00 \\
\hline VERTICAL TRADE & -0.15 & $\mathbf{M}$ & 0.11 & - & - \\
\hline TOT TRADE LINKAGES & 65.00 & 71.00 & 95.00 & 9.23 & 33.80 \\
\hline \& AGRICULTURE-GDP & 48.90 & 48.20 & 40.60 & -1.43 & -15.77 \\
\hline QUALITY OF LIFE & 23.40 & 33.60 & 39.80 & 43.59 & 18.45 \\
\hline x LRGST EXP TO GDP & 1.00 & 0.70 & 0.60 & -30.00 & -14.29 \\
\hline \% EXP LGST PARTNER & 24.00 & 13.00 & 16.00 & -45.83 & 23.08 \\
\hline X IHP LGST PARTNER & 31.00 & 28.00 & 12.00 & -9.68 & -57.14 \\
\hline X EXP manUfac. & 44.00 & 52.00 & 58.00 & 18.18 & 11.54 \\
\hline$X$ EXP AGR-HIHE & 55.00 & 48.00 & 41.00 & -12.73 & -14.58 \\
\hline X IMP manufac. & 59.00 & 52.00 & 47.00 & -11.86 & -9.62 \\
\hline \% IMP AGR-MINE & 40.00 & 47.00 & 52.00 & 17.50 & 10.64 \\
\hline TOTAL EXPORTS & 1358.00 & 2020.00 & 8356.00 & 48.75 & 313.66 \\
\hline TOTAL IMPORTS & 2152.00 & 2040.00 & 13808.00 & -5.20 & 576.86 \\
\hline G.N.P. PER CAPITA & NA & Ma & 260.00 & - & - \\
\hline
\end{tabular}


IMDONESIA

SUMAARY OF MATIONAL STATISTICS

\begin{tabular}{|c|c|c|c|c|c|}
\hline MATIONAL VARIABLES & 1962 & 1970 & 1980 & $62-70$ & $70-80$ \\
\hline EXP. PARTNER CON. & 0.50 & 0.64 & 0.65 & $28 . \infty 0$ & 1.56 \\
\hline IMP. PARTNER CON. & 0.46 & 0.53 & 0.49 & 15.22 & -7.55 \\
\hline EPC/IPC RATIO & 1.09 & 1.21 & 1.32 & 11.01 & 9.09 \\
\hline EXP COHA CONCEN & 0.35 & 0.31 & 0.51 & -11.43 & 64.52 \\
\hline IHP CONH CONCEN & NA & Ma & 0.17 & - & - \\
\hline ECC/ICC RATIO & NA & M & 3.00 & - & - \\
\hline VERTICAL TRADE & NA & $\mathbf{M A}$ & -0.63 & - & - \\
\hline TOT TRADE LINKAGES & 27.00 & 14.00 & 47.00 & -48.15 & 235.71 \\
\hline X AGRICULTURE-GDP & 50.00 & 49.50 & 28.70 & $-1 . \infty 0$ & -42.02 \\
\hline QUALITY OF LIFE & 27.70 & 43.20 & 52.40 & 55.96 & 21.30 \\
\hline \% LRGST EXP TO GDP & 2.80 & 5.40 & 9.60 & 92.86 & 77.78 \\
\hline X EXP LGST PARTNER & 28.00 & 48.00 & 50.00 & 71.43 & 4.17 \\
\hline X IHP LGST PARTNER & 16.00 & 30.00 & 32.00 & 87.50 & 6.67 \\
\hline X EXP manufac. & MA & NA & 2.00 & - & - \\
\hline Z EXP AGR-MINE & Ma & NA & 97.00 & - & - \\
\hline X IMP MANUFAC. & $\mathbf{M}$ & $\mathbf{M A}$ & 65.00 & - & - \\
\hline X IMP AGR-HINE & MA & Ma & 34.00 & - & - \\
\hline TOTAL EXPORTS & 922.00 & \multicolumn{2}{|c|}{929.0021548 .00} & 0.76 & 2219.48 \\
\hline TOTAL IMPORTS & 732.00 & 1049.00 & 10719.00 & 43.31 & 921.83 \\
\hline G.N.P. PER & Na & NA & 530.00 & - & - \\
\hline
\end{tabular}


IRAN

SUMMARY OF NATIONAL STATISTICS

\begin{tabular}{|c|c|c|c|c|c|}
\hline MATIONAL VARIABLES & 1962 & 1970 & 1980 & $62-70$ & $70-80$ \\
\hline EXP. PARTNER CON. & 0.41 & 0.47 & 0.45 & 14.63 & -4.26 \\
\hline IMP. PARTNER CON. & 0.49 & 0.49 & 0.34 & 0.00 & -30.61 \\
\hline EPC/IPC RATIO & 0.83 & 0.95 & 1.30 & 14.46 & 36.84 \\
\hline EXP COHN CONCEN & 0.69 & 0.72 & M & 4.35 & - \\
\hline IMP CONH CONCEN & MA & 0.34 & MA & - & - \\
\hline ECC/ICC RATIO & Ma & 2.12 & MA & - & - \\
\hline VERTICAL TRADE & MA & -0.81 & MA & - & - \\
\hline TOT TRADE LINKAGES & 38.00 & 48.00 & 52.00 & 26.32 & 8.33 \\
\hline X AGRICULTURE-GDP & 29.10 & 23.80 & $\mathbf{M A}$ & -18.21 & - \\
\hline QUALITY OF LIFE & 25.20 & 40.50 & 52.10 & 60.71 & 28.64 \\
\hline * LRGST EXP TO GDP & 9.90 & 8.80 & 6.10 & -11.11 & -30.68 \\
\hline X EXP LGST PARTNER & 23.00 & 38.00 & 26.00 & 65.22 & -31.58 \\
\hline * IAP LGST PARTMER & 21.00 & 21.00 & 13.00 & 0.00 & -38.10 \\
\hline X EXP MANUFAC. & MA & 4.00 & $\boldsymbol{M}$ & - & - \\
\hline X EXP AGR-MINE & MA & 96.00 & $\boldsymbol{M}$ & - & - \\
\hline X IMP MANUFAC. & NA & 85.00 & Ma & - & - \\
\hline X IMP AGR-MINE & MA & 15.00 & $\mathbf{M}$ & - & - \\
\hline TOTAL EXPORTS & 1088.00 & 2361.00 & 14013.00 & 117.00 & 493.52 \\
\hline TOTAL IHPORTS & 454.00 & 1506.00 & 11591.00 & 231.72 & 669.65 \\
\hline G.H.P. PER CAPIT & MA & MA & 1800.00 & - & - \\
\hline
\end{tabular}


IRAQ

SUMHARY OF NATIONAL STATISTICS

\begin{tabular}{|c|c|c|c|c|c|}
\hline MATIONAL VARIABLES & 1962 & 1970 & 1980 & $62-70$ & $70-80$ \\
\hline EXP. PARTNER CON. & 0.46 & 0.45 & 0.42 & -2.17 & -6.67 \\
\hline IMP. PARTNER CON: & 0.40 & 0.33 & 0.39 & -17.50 & 18.18 \\
\hline EPC/IPC RATIO & 1.15 & 1.38 & 1.09 & 20.00 & -21.01 \\
\hline EXP COHA CONCEN & 0.80 & 0.84 & HA & 5.00 & - \\
\hline IHP COHH CONCEN & 0.31 & 0.32 & MA & 3.23 & - \\
\hline ECC/ICC RATIO & 2.58 & 2.63 & Ma & 1.72 & - \\
\hline VERTICAL TRADE & -0.69 & -0.76 & $M$ & 10.14 & - \\
\hline TOT TRADE LINKAGES & 31.00 & 37.00 & 53.00 & 19.35 & 43.24 \\
\hline X AGRICULTURE-GDP & 17.10 & 18.00 & m & 5.26 & - \\
\hline QUALITY OF LIFE & 29.30 & 39.10 & 46.40 & 33.45 & 18.67 \\
\hline$X$ LRGST EXP TO GDP & M & MA & 11.10 & - & - \\
\hline X EXP LGST PARTNER & 17.00 & 28.00 & $17 . \infty$ & 64.71 & -39.29 \\
\hline X IAP LGST PARTNER & 17.00 & 12.00 & 17.00 & -29.41 & 41.67 \\
\hline X EXP MANUFAC. & NA & Ma & MA & - & - \\
\hline \% EXP AGR-MINE & 99.00 & 98.00 & M & -1.01 & - \\
\hline X IMP MANUFAC. & 66.00 & 76.00 & $M$ & 15.15 & - \\
\hline \% IHP AGR-MINE & 30.00 & 23.00 & MA & -23.33 & - \\
\hline TOTAL EXPORTS & 674.00 & 1104.00 & 28289.00 & 63.80 & 2462.41 \\
\hline TOTAL IAPORTS & 324.00 & 473.00 & 12478.00 & 45.99 & 2538.05 \\
\hline G.H.P. PER CAPITA & NA & NA & 2540.00 & - & - \\
\hline
\end{tabular}


IRELAND

SUMMARY OF NATIONAL STATISTICS

\begin{tabular}{|c|c|c|c|c|c|}
\hline MATIOHAL VARIABLES & 1962 & 1970 & 1980 & $62-70$ & $70-80$ \\
\hline EXP. PARTNER CON. & 0.81 & 0.69 & 0.51 & -14.81 & -26.09 \\
\hline IHP. PARTNER CON. & 0.62 & 0.63 & 0.61 & 1.61 & -3.17 \\
\hline EPC/IPC RATIO & 1.31 & 1.11 & 0.84 & -15.27 & -24.32 \\
\hline EXP COHN CONCEN & 0.39 & 0.27 & 0.23 & -30.77 & -14.81 \\
\hline IHP COAH CONCEN & 0.15 & 0.19 & 0.20 & 26.67 & 5.26 \\
\hline ECC/ICC RATIO & 2.60 & 1.42 & 1.15 & -45.34 & -19.07 \\
\hline VERTICAL TRADE & -0.34 & -0.29 & -0.10 & -14.71 & -65.52 \\
\hline TOT TRADE LINKAGES & 11.00 & 29.00 & 90.00 & 163.64 & 210.34 \\
\hline \% AGRICULTURE-GDP & MA & 14.40 & 15.00 & - & 4.17 \\
\hline QUALITY OF LIFE & 89.60 & 92.60 & 95.60 & 3.35 & 3.24 \\
\hline X LRGST EXP TO GDP & 19.40 & 18.90 & 21.90 & -2.58 & 15.87 \\
\hline Z EXP LGST PARTNER & 79.00 & 67.00 & 43.00 & -15.19 & -35.82 \\
\hline \% IMP LGST PARTNER & 57.00 & 60.00 & 57.00 & 5.26 & -5.00 \\
\hline Х EXP ПаNUFAC. & 28.00 & 40.00 & 57.00 & 42.86 & 42.50 \\
\hline Z EXP AGR-HINE & 71.00 & 59.00 & 42.00 & -16.90 & -28.81 \\
\hline X IMP MANUFAC. & 62.00 & 70.00 & 68.00 & 12.90 & -2.86 \\
\hline Z IHP AGR-AINE & 37.00 & 30.00 & 31.00 & -18.92 & 3.35 \\
\hline TOTAL EXPORTS & 446.00 & 1005.00 & 8369.00 & 125.34 & 732.74 \\
\hline TOTAL IMPORTS & 689.00 & 1504.00 & 10799.00 & 118.29 & 618.02 \\
\hline
\end{tabular}

G.N.P. PER CAPITA

MA MA 5230.00 
ISRAEL

SUMHARY OF NATIONAL STATISTICS

\begin{tabular}{|c|c|c|c|c|c|}
\hline MATIOMAL VARIABLES & 1962 & 1970 & 1980 & $62-70$ & $70-80$ \\
\hline EXP. PARTNER CON. & 0.41 & 0.38 & 0.40 & -7.32 & 5.26 \\
\hline IMP. PARTNER CON. & 0.51 & 0.54 & 0.50 & 5.88 & -7.41 \\
\hline EPC/IPC RATIO & 0.80 & 0.70 & 0.81 & -12.50 & 15.71 \\
\hline EXP COMH CONCEH & 0.43 & 0.38 & 0.39 & -11.63 & 2.63 \\
\hline IMP COHM CONCEN & 0.15 & 0.21 & 0.16 & 40.00 & -23.81 \\
\hline ECC/ICC RATIO & 2.87 & 1.81 & 2.44 & -36.88 & 34.70 \\
\hline VERTICAL TRADE & 0.01 & -0.02 & 0.25 & -300.00 & -1350.00 \\
\hline TOT TRADE LINKAGES & 28.00 & 46.00 & 62.00 & 64.29 & 34.78 \\
\hline X AGRICULTURE-GDP & 11.10 & 8.50 & 5.30 & -23.42 & -37.65 \\
\hline QUALITY OF LIFE & 84.20 & 88.90 & 91.70 & 5.58 & 3.15 \\
\hline Z LRGST EXP TO GDP & 1.30 & 2.90 & 3.50 & 123.08 & 20.69 \\
\hline X EXP LGST PARTNER & 16.00 & 19.00 & 18.00 & 18.75 & -5.26 \\
\hline X IMP LGST PARTHER & 36.00 & 39.00 & 31.00 & 8.33 & -20.51 \\
\hline X EXP MANUFAC. & 65.00 & 70.00 & 82.00 & 7.69 & 17.14 \\
\hline Z EXP AGR-MINE & 34.00 & 29.00 & 17.00 & -14.71 & -41.38 \\
\hline X IMP MANUFAC. & 64.00 & 72.00 & 56.00 & 12.50 & -22.22 \\
\hline X IHP AGR-HIME & 35.00 & 27.00 & 43.00 & -22.86 & 59.26 \\
\hline TOTAL EXPORTS & 251.00 & 754.00 & 4771.00 & 200.40 & 532.76 \\
\hline TOTAL IMPORTS & 484.00 & 1511.00 & 6549.00 & 212.19 & 333.42 \\
\hline G.N.P. PER & NA & MA & 5160.00 & - & - \\
\hline
\end{tabular}


ITALY

SUMMARY OF NATIONAL STATISTICS

\begin{tabular}{|c|c|c|c|c|c|}
\hline MATIONAL VARIABLES & 1962 & 1970 & 1980 & $62-70$ & $70-80$ \\
\hline EXP. PARTNER COH. & 0.32 & 0.35 & 0.33 & 9.38 & -5.71 \\
\hline IMP. PARTNER COH. & 0.33 & 0.35 & 0.34 & 6.06 & -2.86 \\
\hline EPC/IPC RATIO & 0.99 & 0.98 & 0.96 & -1.01 & -2.06 \\
\hline EXP COAH CONCEN & 0.20 & 0.27 & 0.30 & 35.00 & 11.11 \\
\hline IHP CONH CONCEN & 0.02 & 0.03 & 0.08 & 50.00 & 166.67 \\
\hline ECC/ICC RATIO & 10.00 & 9.00 & 3.75 & -10.00 & -58.33 \\
\hline VERTICAL TRADE & 0.31 & 0.37 & 0.39 & 19.35 & 5.41 \\
\hline TOT TRADE LINKAGES & 91.00 & 116.00 & 134.00 & 27.47 & 15.52 \\
\hline X AGRICULTURE-GDP & 12.20 & 10.30 & 6.80 & -15.57 & -33.98 \\
\hline QUALITY OF LIFE & 84.90 & 90.10 & 95.50 & 6.12 & 5.99 \\
\hline \% LRGST EXP TO GDP & 2.00 & 3.40 & 4.10 & 70.00 & 20.59 \\
\hline \% EXP LGST PARTNER & 20.00 & 21.00 & 18.00 & 5.00 & -14.29 \\
\hline X IMP LGST PARTMER & 17.00 & 21.00 & 17.00 & 23.53 & -19.05 \\
\hline X EXP manUfac. & 74.00 & 83.00 & 84.00 & 12.16 & 1.20 \\
\hline X EXP AGR-MINE & 25.00 & 16.00 & 15.00 & -36.00 & -6.25 \\
\hline X IHP MANUFAC. & 43.00 & 46.00 & 45.00 & 6.98 & -2.17 \\
\hline \% IHP AGR-MINE & 56.00 & 53.00 & 54.00 & -5.36 & 1.89 \\
\hline TOTAL EXPORTS & \multicolumn{3}{|c|}{4532.0012981 .0076412 .00} & 186.43 & 488.64 \\
\hline TOTAL IMPORTS & 5761.00 & 14373.00 & 93400.00 & 149.49 & 549.83 \\
\hline G.M.P. PER CAPITA & $\boldsymbol{M A}$ & MA & 6960.00 & - & - \\
\hline
\end{tabular}


IVORY COAS

SUMMARY OF NATIONAL STATISTICS

\begin{tabular}{|c|c|c|c|c|c|}
\hline NATIOHAL VARIABLES & 1962 & 1970 & 1980 & $62-70$ & $70-80$ \\
\hline EXP. PARTNER CON. & 0.64 & 0.59 & 0.47 & -7.81 & -20.34 \\
\hline IMP. PARTNER COH. & 0.84 & 0.59 & 0.51 & -29.76 & -13.56 \\
\hline EPC/IPC RATIO & 0.76 & 0.99 & 0.92 & 30.26 & -7.07 \\
\hline EXP COHH CONCEN & 0.58 & 0.46 & 0.39 & -20.69 & -15.22 \\
\hline IMP COHN CONCEN & 0.30 & 0.29 & 0.24 & -3.33 & -17.24 \\
\hline ECC/ICC RATIO & 1.93 & 1.59 & 1.63 & -17.95 & 2.45 \\
\hline VERTICAL TRADE & -0.73 & -0.71 & -0.63 & -2.74 & -11.27 \\
\hline TOT TRADE LINKAGES & 12.00 & 18.00 & 37.00 & $50 . \infty 0$ & 105.56 \\
\hline \% AGRICULTURE-GDP & 43.40 & 33.50 & 26.10 & -22.81 & -22.09 \\
\hline QUALITY OF LIFE & 9.20 & 21.80 & 34.20 & 136.96 & 56.88 \\
\hline X LRGST EXP TO GDP & 14.20 & 9.60 & 8.30 & -32.39 & -13.54 \\
\hline X EXP LGST PARTNER & 48.00 & 34.00 & $22 . \infty 0$ & -29.17 & -35.29 \\
\hline$X$ IMP LGST PARTNER & 82.00 & 49.00 & 43.00 & -40.24 & -12.24 \\
\hline X eXp manufac. & 1.00 & 6.00 & 8.00 & 500.00 & 33.33 \\
\hline X EXP AGR-MINE & 98.00 & 94.00 & 91.00 & -4.08 & -3.19 \\
\hline$X$ ImP manufac. & 73.00 & 77.00 & 71.00 & 5.48 & -7.79 \\
\hline X IMP AGR-MINE & 26.00 & 23.00 & 28.00 & -11.54 & 21.74 \\
\hline TOTAL EXPORTS & 184.00 & 440.00 & 3024.00 & 139.13 & 587.27 \\
\hline TOTAL IRPORTS & 117.00 & 336.00 & 2627.00 & 187.18 & 681.85 \\
\hline G.N.P. PER CAPITA & MA & NA & 1200.00 & - & - \\
\hline
\end{tabular}


JaMuICA

SUHMARY OF NATIONAL STATISTICS

\begin{tabular}{|c|c|c|c|c|c|}
\hline MATIONAL VARIABLES & 1962 & 1970 & 1980 & $62-70$ & $70-80$ \\
\hline EXP. PARTNER CON. & 0.68 & 0.69 & 0.58 & 1.47 & -15.94 \\
\hline IMP. PARTNER CON. & 0.66 & 0.61 & 0.58 & -7.58 & -4.92 \\
\hline EPC/IPC RATIO & 1.04 & 1.14 & 1.01 & 9.62 & -11.40 \\
\hline EXP COMM CONCEN & 0.42 & 0.33 & 0.41 & -21.43 & 24.24 \\
\hline IMP CONH CONCEN & 0.21 & 0.24 & 0.21 & 14.29 & -12.50 \\
\hline ECC/ICC RATIO & 2.00 & 1.38 & 1.95 & -31.25 & 41.99 \\
\hline VERTICAL TRADE & -0.56 & -0.26 & 0.23 & -53.57 & -188.46 \\
\hline TOT TRADE LINKAGES & 9.00 & 14.00 & 25.00 & 55.56 & 78.57 \\
\hline X AGRICULTURE-GDP & 12.50 & 9.20 & 8.20 & -26.40 & -10.87 \\
\hline QUALITY OF LIFE & 76.60 & 88.00 & 90.40 & 14.88 & 2.73 \\
\hline X LRGST EXP TO GDP & 8.80 & 10.90 & 8.60 & 23.86 & -21.10 \\
\hline X EXP LGST PARTNER & 40.00 & 56.00 & 37.00 & 40.00 & -33.93 \\
\hline Х IMP LGST PARTMER & 37.00 & 45.00 & $31 . \infty$ & 21.62 & -31.11 \\
\hline X EXP MAUfAC. & 7.00 & 46.00 & 62.00 & 557.14 & 34.78 \\
\hline$X$ EXP AGR-MINE & 92.00 & 53.00 & 37.00 & -42.39 & -30.19 \\
\hline X IMP MANUFAC. & 63.00 & 72.00 & 39.00 & 14.29 & -45.83 \\
\hline X IHP AGR-MINE & 36.00 & 27.00 & 60.00 & -25.00 & 122.22 \\
\hline TOTAL EXPORTS & 173.00 & 318.00 & 952.00 & 83.82 & 199.37 \\
\hline TOTAL IMPORTS & 172.00 & 478.00 & 981.00 & 177.91 & 105.23 \\
\hline G.N.P. PER CAPITA & NA & MA & 1180.00 & - & - \\
\hline
\end{tabular}


JAPAH

SUMMARY OF NATIONAL STATISTICS

\begin{tabular}{|c|c|c|c|c|c|}
\hline MATIONAL VARIABLES & 1962 & 1970 & 1980 & $62-70$ & $70-80$ \\
\hline EXP. PARTNER CON. & 0.32 & 0.35 & 0.31 & 9.38 & -11.43 \\
\hline IHP. PARTNER CON. & 0.39 & 0.37 & 0.36 & -5.13 & -2.70 \\
\hline EPC/IPC RATIO & 0.84 & 0.94 & 0.86 & 11.90 & -8.51 \\
\hline EXP COHIH CONCEN & 0.38 & 0.41 & 0.46 & 7.89 & 12.20 \\
\hline IHP COHH CONCEN & 0.02 & 0.01 & 0.17 & -50.00 & 1600.00 \\
\hline ECC/ICC RATIO & 19.00 & 41.00 & 2.71 & 115.79 & -93.40 \\
\hline VERTICAL TRADE & 0.64 & 0.68 & 0.76 & 6.25 & 11.76 \\
\hline TOT TRADE LINKAGES & 102.00 & 124.00 & 138.00 & 21.57 & 11.29 \\
\hline X AGRICULTURE-GDP & 12.60 & $\mathbf{8 . 5 0}$ & 5.00 & -32.54 & -41.18 \\
\hline QUALITY OF LIFE & 88.30 & 95.10 & 99.40 & 7.70 & 4.52 \\
\hline \% LRGST EXP TO GDP & 2.10 & 3.40 & 2.30 & 61.90 & -32.35 \\
\hline \% EXP LGST PARTNER & 31.00 & 32.00 & 25.00 & 3.23 & -21.88 \\
\hline * IHP LGST PARTNER & 31.00 & 30.00 & 17.00 & -3.23 & -43.33 \\
\hline \% EXP MANUFAC. & 88.00 & 93.00 & 95.00 & 5.68 & 2.15 \\
\hline X EXP AGR-HINE & 11.00 & 6.00 & 4.00 & -45.45 & -33.33 \\
\hline X IAP MNUFAC. & 24.00 & 25.00 & 19.00 & 4.17 & -24.00 \\
\hline X IMP AGR-MINE & 75.00 & 74.00 & 80.00 & -1.33 & 8.11 \\
\hline TOTAL EXPORTS & \multicolumn{3}{|c|}{4517.0018262 .0123531 .90} & 304.29 & 576.44 \\
\hline TOTAL IMPORTS & 4435.00 & 15464.0 & 121409.90 & 248.68 & 685.11 \\
\hline G.H.P. & NA & MA & 080.00 & - & - \\
\hline
\end{tabular}


JORDAN

SUMMARY OF NATIONAL STATISTICS

\begin{tabular}{|c|c|c|c|c|c|}
\hline MATIONAL VARIABLES & 1962 & 1970 & 1980 & $62-70$ & $70-80$ \\
\hline EXP. PARTNER CON. & 0.57 & 0.54 & 0.48 & -5.26 & -11.11 \\
\hline IMP. PARTNER CON. & 0.46 & 0.46 & 0.40 & $0 . \infty 0$ & -13.04 \\
\hline EPC/IPC RATIO & 1.23 & 1.17 & 1.21 & -4.88 & 3.42 \\
\hline EXP COHI CONCEN & 0.37 & 0.30 & 0.27 & -18.92 & -10.00 \\
\hline IHP COHN CONCEN & 0.25 & 0.25 & 0.19 & 0.00 & $-24 . \infty 0$ \\
\hline ECC/ICC RATIO & 1.48 & 1.20 & 1.42 & -18.92 & 18.42 \\
\hline VERTICAL TRADE & -0.54 & -0.43 & -0.28 & -20.37 & -34.88 \\
\hline TOT TRADE LINKAGES & 7.00 & 7.00 & 23.00 & 0.00 & 228.57 \\
\hline X AGRICULTURE-GDP & NA & $\mathbf{M}$ & 9.20 & - & - \\
\hline QUALITY OF LIFE & 33.00 & $\mathbf{M}$ & 67.40 & - & - \\
\hline X LRGST EXP TO GDP & NA & MA & MA & - & - \\
\hline X EXP LGST PARTNER & 17.00 & 16.00 & 24.00 & -5.88 & 50.00 \\
\hline X IHP LGST PARTNER & 18.00 & 31.00 & 15.00 & 72.22 & -51.61 \\
\hline X EXP MANUFAC. & 6.00 & 16.00 & 33.00 & 166.67 & 106.25 \\
\hline X EXP AGR-HINE & 93.00 & 83.00 & 66.00 & -10.75 & -20.48 \\
\hline X IMP maNuFAC. & 60.00 & 59.00 & 61.00 & -1.67 & 3.39 \\
\hline$X$ IMP AGR-MINE & 39.00 & 40.00 & 38.00 & 2.56 & -5.00 \\
\hline TOTAL EXPORTS & 13.00 & 25.00 & 390.00 & 92.31 & 1460.00 \\
\hline TOTAL IMPORTS & 108.00 & 201.00 & 2695.00 & 86.11 & 1240.80 \\
\hline .N.P. PI & MA & $\mathbf{M}$ & 1620.00 & - & - \\
\hline
\end{tabular}


KENYA

SUHAARY OF NATIONAL STATISTICS

\begin{tabular}{|c|c|c|c|c|c|}
\hline NATIONAL VARIABLES & 1962 & 1970 & 1980 & $62-70$ & $70-80$ \\
\hline EXP. PARTNER CON. & 0.51 & 0.41 & 0.35 & -19.61 & -14.63 \\
\hline IMP. PARTNER CON. & 0.58 & 0.45 & 0.45 & -22.41 & 0.00 \\
\hline EPC/IPC RATIO & 0.88 & 0.91 & 0.79 & 3.41 & -13.19 \\
\hline EXP COHH CONCEN & 0.41 & 0.33 & 0.25 & -19.51 & -24.24 \\
\hline IHP COHH CONCEN & NA & 0.29 & 0.23 & - & -20.69 \\
\hline ECC/ICC RATIO & $\mathbf{M}$ & 1.14 & 1.09 & - & -4.48 \\
\hline VERTICAL TRADE & NA & -0.67 & -0.44 & - & -34.33 \\
\hline TOT TRADE LINKAGES & 17.00 & 32.00 & 60.00 & 88.24 & 87.50 \\
\hline * AGRICULTURE-GDP & 38.80 & 36.70 & 35.10 & -5.41 & -4.36 \\
\hline QUALITY OF LIFE & 23.20 & 36.90 & 52.00 & 59.05 & 40.92 \\
\hline X LRGST EXP TO GDP & 4.10 & $\boldsymbol{\mu}$ & 3.00 & - & - \\
\hline X EXP LGST PARTNER & 30.00 & 16.00 & $15 . \infty 0$ & -46.67 & -6.25 \\
\hline X IMP LGST PARTNER & 41.00 & 29.00 & 24.00 & -29.27 & -17.24 \\
\hline X EXP MANUFAC. & MA & 12.00 & $12 . \infty 0$ & - & 0.00 \\
\hline X EXP AGR-MIHE & MA & 87.00 & 87.00 & - & 0.00 \\
\hline \% IMP huNUFAC. & MA & 80.00 & 55.00 & - & -31.25 \\
\hline \% IMP AGR-HINE & MA & 20.00 & 44.00 & - & 120.00 \\
\hline TOTAL EXPORTS & 106.00 & 275.00 & 1180.00 & 159.43 & 329.09 \\
\hline TOTAL IHPORTS & 203.00 & 431.00 & 2413.00 & 112.32 & 459.86 \\
\hline G.H.P. PER CAPIT & $\mathbf{M}$ & Nh & 420.00 & - & - \\
\hline
\end{tabular}


KOREA

SUMMARY OF MATIONAL STATISTICS

\begin{tabular}{|c|c|c|c|c|c|}
\hline MATIONAL VARIABLES & 1962 & 1970 & 1980 & $62-70$ & $70-80$ \\
\hline EXP. PARTNER CON. & 0.69 & 0.67 & 0.42 & -2.90 & -37.31 \\
\hline IMP. PARTNER CON. & 0.72 & 0.65 & 0.55 & -9.72 & -15.38 \\
\hline EPC/IPC RATIO & 0.96 & 1.03 & 0.77 & 7.29 & -25.24 \\
\hline EXP CONH CONCEN & 0.14 & 0.36 & 0.45 & 157.14 & 25.00 \\
\hline IMP CONH CONCEN & 0.14 & 0.08 & 0.07 & -42.86 & -12.50 \\
\hline ECC/ICC RATIO & 1.00 & 4.50 & 6.43 & 350.00 & 42.86 \\
\hline VERTICAL TRADE & -0.34 & 0.22 & 0.47 & -164.71 & 113.64 \\
\hline TOT TRADE LINKAGES & 5.00 & 21.00 & 98.00 & 320.00 & 366.67 \\
\hline X AGRICULTURE-GDP & 39.20 & 32.00 & 20.30 & -18.37 & -36.56 \\
\hline QUALITY OF LIFE & 60.40 & 75.40 & 83.90 & 24.83 & 11.27 \\
\hline X LRGST EXP TO GDP & 0.80 & 5.80 & 9.80 & 625.00 & 68.97 \\
\hline X EXP LGST PARTNER & 49.00 & 48.00 & 28.00 & -2.04 & -41.67 \\
\hline X IMP LGST PARTNER & 51.00 & $42 . \infty$ & 27.00 & -17.65 & -35.71 \\
\hline X EXP manUfac. & 19.00 & 76.00 & 89.00 & 300.00 & 17.11 \\
\hline X EXP AGR-MINE & 80.00 & 23.00 & $10 . \infty 0$ & -71.25 & -56.52 \\
\hline \% IMP makUfAC. & 53.00 & 54.00 & 43.00 & 1.89 & -20.37 \\
\hline X IHP AGR-HINE & 46.00 & 45.00 & 56.00 & -2.17 & 24.44 \\
\hline TOTAL EXPORTS & 47.00 & 801.00 & 16486.00 & 1604.26 & 1958.18 \\
\hline TOTAL IMPORTS & 415.00 & 1907.00 & 19413.00 & 359.52 & 917.99 \\
\hline G.H.F. PER CAPITA & $\boldsymbol{N}$ & NA & 1700.00 & - & - \\
\hline
\end{tabular}


KUWAIT

SUMHARY OF NATIONAL STATISTICS

\begin{tabular}{|c|c|c|c|c|c|}
\hline MATIONAL VARIABLES & 1962 & 1970 & 1980 & $62-70$ & $70-80$ \\
\hline EXP. PARTNER CON. & 0.57 & 0.46 & 0.44 & -19.30 & -4.35 \\
\hline IHP. PARTNER CON. & 0.53 & 0.40 & 0.41 & -24.53 & 2.50 \\
\hline EPC/IPC RATIO & 1.07 & 1.14 & 1.09 & 6.54 & -4.39 \\
\hline EXP CONH CONCEN & 0.12 & 0.82 & 0.73 & 583.33 & -10.98 \\
\hline IMP COHH CONCEN & 0.32 & 0.34 & 0.36 & 6.25 & 5.88 \\
\hline ECC/ICC RATIO & 0.38 & 2.41 & 2.03 & 543.14 & -15.92 \\
\hline VERTICAL TRADE & -0.24 & -0.73 & -0.72 & 204.17 & -1.37 \\
\hline TOT TRADE LINKAGES & 17.00 & 33.00 & 40.00 & 94.12 & 21.21 \\
\hline \% AGRICULTURE-GDP & MA & 0.50 & 0.30 & - & -40.00 \\
\hline QUALITY OF LIFE & 55.30 & 69.30 & 77.00 & 25.32 & 11.11 \\
\hline X LRGST EXP TO GDP & 25.20 & 11.10 & 21.00 & -55.95 & 89.19 \\
\hline \% EXP LGST PARTNER & 31.00 & 19.00 & 23.00 & -38.71 & 21.05 \\
\hline \% IMP LGST PARTNER & 27.00 & 16.00 & 20.00 & -40.74 & 25.00 \\
\hline \% EXP MANUFAC. & 50.00 & 4.00 & 10.00 & -92.00 & 150.00 \\
\hline I EXP AGR-MINE & 49.00 & 95.00 & 89.00 & 93.88 & -6.32 \\
\hline \& IHP MANUFAC. & 74.00 & 77.00 & 82.00 & 4.05 & 6.49 \\
\hline Z IMP AGR-MINE & 25.00 & 22.00 & 18.00 & -12.00 & -18.18 \\
\hline TOTAL EXPORTS & 1449.00 & 1888.00 & 17623.00 & 30.30 & 833.42 \\
\hline TOTAL IHPORTS & 231.00 & 573.00 & 6163.00 & 148.05 & 975.57 \\
\hline G.N.P. PER CAPITA & MA & NA & 20900.00 & - & - \\
\hline
\end{tabular}


LAOS

SUMMARY OF NATIONAL STATISTICS

\begin{tabular}{|c|c|c|c|c|c|}
\hline NATIONAL VARIABLES & 1962 & 1970 & 1980 & $62-70$ & $70-80$ \\
\hline EXP. PARTNER CON. & 0.80 & 0.70 & 0.61 & -12.50 & -12.86 \\
\hline IMP. PARTNER CON. & 0.66 & 0.63 & 0.65 & -4.55 & 3.17 \\
\hline EPC/IPC RATIO & 1.22 & 1.10 & 0.94 & -9.84 & -14.55 \\
\hline EXP COMH CONCEN & 0.30 & 0.20 & Ma & -33.33 & - \\
\hline IHP COHH CONCEN & 0.26 & 0.21 & MA & -19.23 & - \\
\hline ECC/ICC RATIO & 1.15 & 0.95 & M & -17.46 & - \\
\hline VERTICAL TRADE & -0.37 & -0.21 & $\boldsymbol{M}$ & -43.24 & - \\
\hline TOT TRADE LINKAGES & NA & 3.00 & 5.00 & - & 66.67 \\
\hline * AGRICULTURE-GDP & NA & NA & M & - & - \\
\hline QUALITY OF LIFE & 22.70 & MA & 33.60 & - & - \\
\hline * LRGST EXP TO GDP & NA & NA & $\mathbf{M}$ & - & - \\
\hline \% EXP LGST PARTNER & 57.00 & 40.00 & 30.00 & -29.82 & -25.00 \\
\hline X IHP LGST PARTNER & 34.00 & 32.00 & 43.00 & -5.88 & 34.38 \\
\hline X EXP MANUFAC. & 9.00 & 30.00 & MA & 233.33 & - \\
\hline Z EXP AGR-MINE & 90.00 & 69.00 & MA & -23.33 & - \\
\hline X IHP manufac. & 46.00 & 52.00 & Ma & 13.04 & - \\
\hline X IMP AGR-MINE & 53.00 & 47.00 & MA & -11.32 & - \\
\hline TOTAL EXPORTS & $M$ & 7.00 & 22.00 & - & 214.29 \\
\hline TOTAL IHPORTS & 18.00 & 64.00 & 101.00 & 255.56 & 57.81 \\
\hline G.N.P. PER CAPITA & N & M & 80.00 & - & - \\
\hline
\end{tabular}


LEBANON

SUMHARY OF NATIONAL STATISTICS

\begin{tabular}{|c|c|c|c|c|c|}
\hline NATIONAL VARIABLES & 1962 & 1970 & 1980 & $62-70$ & $70-80$ \\
\hline EXP. PARTNER CON. & 0.56 & 0.39 & 0.50 & -30.36 & 28.21 \\
\hline IMP. PARTNER CON. & 0.41 & 0.35 & 0.37 & -14.63 & 5.71 \\
\hline EPC/IPC RATIO & 1.38 & 1.11 & 1.35 & -19.57 & 21.62 \\
\hline EXP CONH CONCEN & 0.16 & 0.17 & MA & 6.25 & - \\
\hline IMP CONH CONCEN & 0.19 & 0.17 & KA & -10.53 & - \\
\hline ECC/ICC RATLO & 0.84 & 1.00 & $M$ & 18.75 & - \\
\hline VERTICAL TRADE & -0.46 & 0.01 & MA & -102.17 & - \\
\hline TOT TRADE LINKAGES & 16.00 & 22.00 & 37.00 & 37.50 & 68.18 \\
\hline X AGRICULTURE-GDP & MA & Ma & M & - & - \\
\hline QUALITY OF LIFE & MA & 70.50 & 75.80 & - & 7.52 \\
\hline * LRGST EXP TO GDP & NA & NA & MA & - & - \\
\hline \% EXP LGST PARTNER & 45.00 & 17.00 & 38.00 & -62.22 & 123.53 \\
\hline * IMP LGST PARTNER & 13.00 & $13 . \infty 0$ & 12.00 & 0.00 & -7.69 \\
\hline X EXP MANUFAC. & 16.00 & 60.00 & MA & 275.00 & - \\
\hline X EXP AGR-HINE & 83.00 & 39.00 & MA & -53.01 & - \\
\hline X IHP MANUFAC. & 62.00 & 59.00 & $\mathbf{M}$ & -4.84 & - \\
\hline \% IMP AGR-MINE & 37.00 & 40.00 & MA & 8.11 & - \\
\hline TOTAL EXPORTS & 122.00 & 161.00 & 889.00 & 31.97 & 452.17 \\
\hline TOTAL IMPORTS & 315.00 & 643.00 & 3751.00 & 104.13 & 483.36 \\
\hline G.M.P. PER CAPITA & NA & MA & 1200.00 & - & - \\
\hline
\end{tabular}


LIBYA

SUMMARY OF NATIONAL STATISTICS

\begin{tabular}{|c|c|c|c|c|c|}
\hline MATIONAL VARIABLES & 1962 & 1970 & 1980 & $62-70$ & $70-80$ \\
\hline EXP. PARTNER CON. & 0.63 & 0.56 & 0.57 & -11.11 & 1.79 \\
\hline IMP. PARTNER CON. & 0.57 & 0.44 & 0.45 & -22.81 & 2.27 \\
\hline EPC/IPC RATIO & 1.10 & 1.29 & 1.24 & 17.27 & -3.88 \\
\hline EXP COAH CONCEN & 0.88 & $M A$ & M -1 & -11350.00 & $0 . \infty$ \\
\hline IMP COHM CONCEN & 0.33 & 0.29 & 0.34 & -12.12 & 17.24 \\
\hline ECC/ICC RATIO & 2.67 & M & MA & -3812.50 & 0.00 \\
\hline VERTICAL TRADE & -0.83 & -0.72 & -0.78 & -13.25 & 8.33 \\
\hline TOT TRADE LINKAGES & 7.00 & 19.00 & 30.00 & 171.43 & 57.89 \\
\hline X AGRICULTURE-GDP & 13.70 & 3.50 & 1.90 & -74.45 & -45.71 \\
\hline QUALITY OF LIFE & 25.40 & 43.00 & 52.00 & 69.29 & 20.93 \\
\hline X LRGST EXP TO GDP & MA & NA & MA & - & - \\
\hline X EXP LGST PARTNER & 34.00 & 25.00 & 36.00 & -26.47 & 44.00 \\
\hline * IAP LGST PARTNER & 23.00 & 22.00 & 28.00 & -4.35 & 27.27 \\
\hline * eXP manufac. & MA & M & NA & - & - \\
\hline X EXP AGR-HINE & 99.00 & 100.00 & 100.00 & 1.01 & 0.00 \\
\hline X Imp manufac. & 82.00 & 71.00 & 77.00 & -13.41 & 8.45 \\
\hline \% IMP AGR-MINE & 17.00 & 28.00 & 22.00 & 64.71 & -21.43 \\
\hline TOTAL EXPORTS & 138.00 & 2365.00 & 21550.00 & 1613.77 & 811.21 \\
\hline TOTAL IMPORTS & 207.00 & 599.00 & 8946.00 & 189.37 & 1393.49 \\
\hline G.H.P. PER CAPITA & NA & NA & 8450.00 & - & - \\
\hline
\end{tabular}


MACAO

SUMMARY OF NATIONAL STATISTICS

\begin{tabular}{|c|c|c|c|c|c|}
\hline MATIONAL VARIABLES & 1962 & 1970 & 1980 & $62-70$ & $70-80$ \\
\hline EXP. PARTNER CON. & 0.80 & 0.52 & 0.51 & -35.00 & -1.92 \\
\hline IMP. PARTMER CON. & 0.99 & 0.82 & 0.87 & -17.17 & 6.10 \\
\hline EPC/IPC RATIO & 0.81 & 0.63 & 0.59 & -22.22 & -6.35 \\
\hline EXP COHIH CONCEN & NA & NA & Ma & - & - \\
\hline IHP CONH CONCEN & MA & NA & $\mathbf{M A}$ & - & - \\
\hline ECC/ICC RATIO & NA & MA & MA & - & - \\
\hline VERTICAL TRADE & NA & NA & MA & - & - \\
\hline TOT TRADE LINKAGES & 3.00 & 9.00 & 22.00 & 200.00 & 144.44 \\
\hline X AGRICULTURE-GDP & NA & NA & NA & - & - \\
\hline QUALITY OF LIFE & NA & NA & NA & - & - \\
\hline \% LRGST EXP TO GDP & NA & NA & NA & - & - \\
\hline \% EXP LGST PARTMER & 56.00 & 19.00 & 19.00 & -66.07 & 0.00 \\
\hline \% IMP LGST PARTMER & 98.00 & 51.00 & 85.00 & -47.96 & 66.67 \\
\hline \% EXP MANUFAC. & Ma & Ma & NA & - & - \\
\hline * EXP AGR-MINE & NA & NA & MA & - & - \\
\hline \% IHP manUfAC. & NA & NA & $M$ & - & - \\
\hline \% IMP AGR-MINE & NA & NA & NA & - & - \\
\hline TOTAL EXPORTS & 18.00 & 40.00 & 540.00 & 122.22 & 1250.00 \\
\hline TOTAL IMPORTS & 15.00 & 36.00 & 266.00 & 140.00 & 638.89 \\
\hline G.N.P. PER CAPITA & MA & NA & 1500.00 & - & - \\
\hline
\end{tabular}


MALAYSIA

SUMMARY OF NATIONAL STATISTICS

\begin{tabular}{|c|c|c|c|c|c|}
\hline MATIONAL VARIABLES & 1962 & 1970 & 1980 & $62-70$ & $70-80$ \\
\hline EXP. PARTNER CON. & 0.45 & 0.43 & 0.47 & -4.44 & 9.30 \\
\hline IHP. PARTNER CON. & 0.51 & 0.44 & 0.48 & -13.73 & 9.09 \\
\hline EPC/IPC RATIO & 0.89 & 0.98 & 0.97 & 10.11 & -1.02 \\
\hline EXP COHH CONCEN & NA & 0.25 & 0.09 & - & -64.00 \\
\hline IMP COHA CONCEN & NA & 0.13 & 0.18 & - & 38.46 \\
\hline ECC/ICC RATIO & NA & 1.92 & 0.50 & - & -74.00 \\
\hline VERTICAL TRADE & MA & -0.51 & -0.48 & - & -5.88 \\
\hline TOT TRADE LINKAGES & 35.00 & 49.00 & 77.00 & 40.00 & 57.14 \\
\hline \% AGRICULTURE-GDP & 36.00 & 32.10 & 26.40 & -10.83 & -17.76 \\
\hline QUALITY OF LIFE & 54.10 & 65.20 & 72.50 & 20.52 & 11.20 \\
\hline Z LRGST EXP TO GDP & MA & NA & NA & - & - \\
\hline \% EXP LGST PARTNER & 20.00 & 21.00 & 23.00 & $\mathbf{5 . 0 0}$ & 9.52 \\
\hline \& IHP LGST PARTNER & 33.00 & 25.00 & 25.00 & -24.24 & 0.00 \\
\hline X EXP MANUFAC. & NA & 7.00 & 19.00 & - & 171.43 \\
\hline X EXP AGR-MINE & $\mathbf{M A}$ & 92.00 & 80.00 & - & -13.04 \\
\hline X IHP RANUFAC. & NA & 58.00 & 67.00 & - & 15.52 \\
\hline$X$ IHP AGR-MINE & NA & 41.00 & 33.00 & - & -19.51 \\
\hline TOTAL EXPORTS & 846.00 & \multicolumn{2}{|c|}{1678.0012721 .00} & 98.35 & 658.10 \\
\hline TOTAL IMPORTS & 931.00 & \multicolumn{2}{|c|}{1342.0011463 .00} & 44.15 & 754.17 \\
\hline G.N.P. PER CAPITA & NA & NA & 1840.00 & - & - \\
\hline
\end{tabular}


MALI

SUMMARY OF NATIONAL STATISTICS

\begin{tabular}{|c|c|c|c|c|c|}
\hline NATIONAL VARIABLES & 1962 & 1970 & 1980 & $62-70$ & $70-80$ \\
\hline EXP. PARTNER CON. & 0.83 & 0.63 & 0.53 & -24.10 & -15.87 \\
\hline IHP. PARTNER CON. & 0.81 & 0.71 & 0.62 & -12.35 & -12.68 \\
\hline EPC/IPC RATIO & 1.02 & 0.89 & 0.85 & -12.75 & -4.49 \\
\hline EXP CONH CONCEN & 0.64 & 0.44 & MA & -31.25 & - \\
\hline IMP COHN CONCEN & 0.26 & 0.18 & $M$ & -30.77 & - \\
\hline ECC/ICC RATIO & 2.46 & 2.44 & Ma & -0.69 & - \\
\hline VERTICAL TRADE & -0.71 & -0.46 & M & -35.21 & - \\
\hline TOT TRADE LINKAGES & 2.00 & 4.00 & 15.00 & 100.00 & 275.00 \\
\hline \% AGRICULTURE-GDP & 54.80 & 48.60 & 41.60 & -11.31 & -14.40 \\
\hline QUALITY OF LIFE & 5.20 & 12.90 & 18.30 & 148.08 & 41.86 \\
\hline * LRGST EXP TO GDP & 2.10 & 3.00 & Ma & 42.86 & - \\
\hline * EXP LGST PARTNER & 52.00 & 32.00 & 24.00 & -38.46 & -25.00 \\
\hline * IMP LGST PARTNER & 55.00 & 63.00 & 36.00 & 14.55 & -42.86 \\
\hline \% EXP maKUFAC. & 1.00 & 10.00 & MA & 900.00 & - \\
\hline \% EXP AGR-MINE & 98.00 & 89.00 & NA & -9.18 & - \\
\hline X IHP maNUFAC. & 72.00 & 56.00 & MA & -22.22 & - \\
\hline X IMP NGR-MINE & 27.00 & 43.00 & NA & 59.26 & - \\
\hline TOTAL EXPORTS & 8.00 & 16.00 & 145.00 & 100.00 & 806.25 \\
\hline TOTAL IMPORTS & 22.00 & 25.00 & 379.00 & 13.64 & 1416.00 \\
\hline G.N.P. PER CAPITA & MA & MA & 190.00 & - & - \\
\hline
\end{tabular}


MALTA

SUMMARY OF NATIONAL STATISTICS

\begin{tabular}{|c|c|c|c|c|c|}
\hline NATIONAL VARIABLES & 1962 & 1970 & 1980 & $62-70$ & $70-80$ \\
\hline EXP. PARTNER CON. & 0.73 & 0.55 & 0.54 & -24.66 & -1.82 \\
\hline IMP. PARTNER CON. & 0.64 & 0.54 & 0.51 & -15.62 & -5.56 \\
\hline EPC/IPC RATIO & 1.15 & 1.00 & 1.07 & -13.04 & 7.00 \\
\hline EXP COHY CONCEN & MA & 0.41 & 0.61 & - & 48.78 \\
\hline IMP COMH CONCEN & NA & 0.25 & 0.23 & - & -8.00 \\
\hline ECC/ICC RATIO & NA & 1.64 & 2.65 & - & 61.72 \\
\hline VERTICAL TRADE & NA & 0.14 & 0.28 & - & 100.00 \\
\hline TOT TRADE LINKAGES & 2.00 & 7.00 & 23.00 & 250.00 & 228.57 \\
\hline X AGRICULTURE-GDP & 6.40 & 7.20 & 5.10 & 12.50 & -29.17 \\
\hline QUALITY OF LIFE & 77.10 & 88.80 & 91.20 & 15.18 & 2.70 \\
\hline X LRGST EXP TO GDP & 2.50 & 7.80 & NA & 212.00 & - \\
\hline X EXP LGST PARTNER & 59.00 & 44.00 & 32.00 & -25.42 & -27.27 \\
\hline * IHP LGST PARTNER & 47.00 & 40.00 & 23.00 & -14.89 & -42.50 \\
\hline X EXP hanUfaC. & NA & 78.00 & 93.00 & - & 19.23 \\
\hline X EXP ACR-MINE & MA & 21.00 & 6.00 & - & -71.43 \\
\hline * IHP MANUFAC. & NA & 64.00 & 65.00 & - & 1.56 \\
\hline * IMP AGR-HINE & NA & 35.00 & 34.00 & - & -2.86 \\
\hline TOTAL EXPORTS & 7.00 & 33.00 & 456.00 & 371.43 & 1281.82 \\
\hline TOTAL IHPORTS & 65.00 & 152.00 & 854.00 & 133.85 & 461.84 \\
\hline G.N.P. PER CAPITA & MA & NA & 3600.00 & - & - \\
\hline
\end{tabular}


MARTINIQUE

SUMMARY OF NATIONAL STATISTICS

\begin{tabular}{|c|c|c|c|c|c|}
\hline MATIONAL VARIABLES & 1962 & 1970 & 1980 & $62-70$ & $70-80$ \\
\hline EXP. PARTNER CON. & 1.00 & 0.93 & 0.79 & -7.00 & -15.05 \\
\hline IMP. PARTNER CON. & 0.99 & 0.77 & 0.80 & -22.22 & 3.90 \\
\hline EPC/IPC RATIO & 1.01 & 1.20 & 0.99 & 18.81 & -17.50 \\
\hline EXP COHN CONCEN & NA & NA & NA & - & - \\
\hline IMP COHK CONCEN & NA & NA & MA & - & - \\
\hline ECC/ICC RATIO & NA & NA & $\mathbf{M}$ & - & - \\
\hline VERTICAL TRADE & NA & NA & $\mathbf{M}$ & - & - \\
\hline TOT TRADE LIMKAGES & 1.00 & 2.00 & 5.00 & 100.00 & 150.00 \\
\hline \% AGRICULTURE-GDP & NA & MA & NA & - & - \\
\hline QUALITY OF LIFE & Ma & MA & M & - & - \\
\hline * LRGST EXP TO GDP & 36.60 & 10.10 & $M$ & -72.40 & - \\
\hline X EXP LGST PARTINER & 100.00 & 92.00 & 52.00 & -8.00 & -43.48 \\
\hline X IMP LGST PARTNER & 99.00 & 70.00 & 80.00 & -29.29 & 14.29 \\
\hline$X$ EXP manufac. & MA & MA & M & - & - \\
\hline ₹ EXP AGR-HINE & NA & MA & $M$ & - & - \\
\hline X imp manufac. & Ma & $M$ & MN & - & - \\
\hline$X$ IMP AGR-MINE & NA & $\mathbf{M}$ & MA & - & - \\
\hline TOTAL EXPORTS & 43.00 & 29.00 & 116.00 & -32.56 & 300.00 \\
\hline TOTAL IHPORTS & 40.00 & 125.00 & 550.00 & 212.50 & 340.00 \\
\hline G.N.P. PER CAPITA & NA & $\mathbf{M A}$ & 4000.00 & - & - \\
\hline
\end{tabular}


MAURITANIA

SUMMARY OF NATIONAL STATISTICS

\begin{tabular}{|c|c|c|c|c|c|}
\hline NATIOMAL VARIABLES & 1962 & 1970 & 1980 & $62-70$ & $70-80$ \\
\hline EXP. PARTNER CON. & 1.00 & 0.61 & 0.59 & -39.00 & -3.28 \\
\hline IMP. PARTNER CON. & 0.91 & 0.67 & 0.53 & -26.37 & -20.90 \\
\hline EPC/IPC RATIO & 1.10 & 0.91 & 1.11 & -17.27 & 21.98 \\
\hline EXP CONH CONCEN & NA & Ma & Ma & - & - \\
\hline IMP CONA CONCEN & NA & Ma & Ma & 一 & - \\
\hline ECC/ICC RATIO & MA & Ma & M & - & - \\
\hline VERTICAL TRADE & NA & NA & $\mathbf{M}$ & - & - \\
\hline TOT TRADE LINKAGES & 1.00 & 7.00 & 11.00 & 600.00 & 57.14 \\
\hline X AGRICULTURE-GDP & 44.40 & 33.70 & 28.80 & -24.10 & -14.54 \\
\hline QUALITY OF LIFE & 7.70 & 15.50 & 23.80 & 101.30 & 53.55 \\
\hline X LRGST EXP TO GDP & 1.10 & 10.50 & Ma & 854.55 & - \\
\hline X EXP LGST PARTNER & 100.00 & 21.00 & 29.00 & -79.00 & 38.10 \\
\hline X IMP LGST PARTNER & 87.00 & 54.00 & 34.00 & -37.93 & -37.04 \\
\hline X EXP manUfAC. & NA & MA & MA & - & - \\
\hline X EXP AGR-MIHE & M & $\boldsymbol{M}$ & 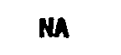 & - & - \\
\hline * IMP haNUFAC. & NA & $\mathrm{m}$ & M & - & - \\
\hline X IMP AGR-MINE & NA & NA & $\mathbf{M A}$ & - & - \\
\hline TOTAL EXPORTS & 1.00 & 79.00 & 234.00 & 7800.00 & 196.20 \\
\hline TOTAL IHPORTS & 30.00 & 31.00 & 289.00 & 3.33 & 832.26 \\
\hline G.N.P. PER CAPITA & NA & $\boldsymbol{N A}$ & 460.00 & - & - \\
\hline
\end{tabular}


MAURITIUS

SUMMARY OF MATIONAL STATISTICS

\begin{tabular}{|c|c|c|c|c|c|}
\hline MATIOHAL VARIABLES & 1962 & 1970 & 1980 & $62-70$ & $70-80$ \\
\hline EXP. PARTNER CON. & 0.87 & 0.80 & 0.74 & -8.05 & -7.50 \\
\hline IHP. PARTNER CON. & 0.64 & 0.44 & 0.43 & -31.25 & -2.27 \\
\hline EPC/IPC RATIO & 1.38 & 1.84 & 1.73 & 33.33 & -5.98 \\
\hline EXP COHH CONCEN & MA & MA & $\boldsymbol{M}$ & - & - \\
\hline IMP COHA CONCEN & MA & NA & M & - & - \\
\hline ECC/ICC RATIO & MA & $\boldsymbol{M}$ & $\mathbf{M}$ & - & - \\
\hline VERTICAL TRADE & NA & MA & MA & - & - \\
\hline TOT TRADE LINKAGES & 4.00 & 4.00 & 12.00 & 0.00 & 200.00 \\
\hline \% AGRICULTURE-GDP & 18.00 & 16.80 & 18.20 & -6.67 & 8.33 \\
\hline QUALITY OF LIFE & 62.30 & 72.90 & 79.70 & 17.01 & 9.33 \\
\hline * LRGST EXP TO GDP & 30.70 & 16.90 & 40.50 & -44.95 & 139.64 \\
\hline X EXP LGST PARTNER & 87.00 & 68.00 & 67.00 & -21.84 & -1.47 \\
\hline * IMP LGST PARTNER & 45.00 & 23.00 & 14.00 & -48.89 & -39.13 \\
\hline Z EXP MANUFAC. & NA & MA & MA & - & - \\
\hline Z EXP AGR-HINE & NA & MA & Ma & - & - \\
\hline X IRP MANUFAC. & MA & MA & MA & - & - \\
\hline X IHP AGR-HINE & Ma & $\mathbf{M}$ & MA & - & - \\
\hline TOTAL EXPORTS & $\mathbf{5 9 . 0 0}$ & 67.00 & 433.00 & 13.56 & 546.27 \\
\hline TOTAL IMPORTS & 37.00 & 59.00 & 384.00 & 59.46 & 550.85 \\
\hline G.N.P. PER CAPITA & MA & MA & 1270.00 & - & - \\
\hline
\end{tabular}


HEXICO

SUMMARY OF NATIONAL STATISTICS

\begin{tabular}{|c|c|c|c|c|c|}
\hline MATIONAL VARIABLES & 1962 & 1970 & 1980 & $62-70$ & $70-80$ \\
\hline EXP. PARTNER CON. & 0.71 & 0.70 & 0.68 & -1.41 & -2.86 \\
\hline IHP. PARTNER CON. & 0.71 & 0.66 & 0.69 & -7.04 & 4.55 \\
\hline EPC/IPC RATIO & 1.00 & 1.06 & 0.99 & 6.00 & -6.60 \\
\hline EXP COMH CONCEN & 0.14 & 0.09 & 0.12 & -35.71 & 33.33 \\
\hline IHP COMM CONCEN & 0.31 & 0.28 & 0.32 & -9.68 & 14.29 \\
\hline ECC/ICC RATIO & 0.45 & 0.32 & 0.38 & -28.83 & 16.67 \\
\hline VERTICAL TRADE & -0.68 & -0.48 & -0.44 & -29.41 & -8.33 \\
\hline TOT TRADE LIMKAGES & 29.00 & 35.00 & 55.00 & 20.69 & 57.14 \\
\hline X AGRICULTURE-GDP & 18.90 & 13.60 & 9.50 & -28.04 & -30.15 \\
\hline QUALITY OF LIFE & 49.00 & 68.20 & 77.00 & 39.18 & 12.90 \\
\hline \% LRGST EXP TO GDP & 3.80 & 2.50 & 2.40 & -34.21 & -4.00 \\
\hline \% EXP LGST PARTNER & 71.00 & 71.00 & 65.00 & 0.00 & -8.45 \\
\hline X IMP LGST PARTNER & 68.00 & 63.00 & 68.00 & -7.35 & 7.94 \\
\hline X EXP MANUFAC. & 15.00 & 32.00 & 39.00 & 113.33 & 21.88 \\
\hline \% EXP AGR-HIME & 84.00 & 67.00 & 60.00 & -20.24 & -10.45 \\
\hline X IMP MANUFAC. & 83.00 & 80.00 & 83.00 & -3.61 & 3.75 \\
\hline * IMP AGR-MINE & 16.00 & 19.00 & 16.00 & 18.75 & -15.79 \\
\hline TOTAL EXPORTS & $77 . \infty$ & 1173.00 & 15267.00 & 50.97 & 1201.53 \\
\hline TOTAL IAPORTS & 1148.00 & 2666.00 & 22156.00 & 132.23 & 731.0 \\
\hline S.N.P. PEI & M & NA & 2250.00 & - & - \\
\hline
\end{tabular}


MOROCCO

SUMHARY OF NATIONAL STATISTICS

\begin{tabular}{|c|c|c|c|c|c|}
\hline MATIONAL VARIABLES & 1962 & 1970 & 1980 & $62-70$ & $70-80$ \\
\hline EXP. PARTHER CON. & 0.48 & 0.46 & 0.39 & -4.17 & -15.22 \\
\hline IMP. PARTNER CON. & 0.49 & 0.46 & 0.45 & -6.12 & -2.17 \\
\hline EPC/IPC RATIO & 0.97 & 0.99 & 0.88 & 2.06 & -11.11 \\
\hline EXP COHM CONCEN & 0.29 & 0.31 & 0.27 & 6.90 & -12.90 \\
\hline IMP COHIH CONCEN & 0.22 & 0.16 & 0.09 & -27.27 & -43.75 \\
\hline ECC/ICC RATIO & 1.32 & 1.94 & 3.00 & 46.98 & 54.84 \\
\hline VERTICAL TRADE & -0.51 & -0.54 & -0.23 & 5.88 & -57.41 \\
\hline TOT TRADE LINKAGES & 34.00 & 36.00 & 56.00 & 5.88 & 55.56 \\
\hline * AGRICULTURE-GDP & 23.30 & 21.80 & 18.20 & -6.44 & -16.51 \\
\hline QUALITY OF LIFE & 22.40 & 32.90 & 44.40 & 46.88 & 34.95 \\
\hline * LRGST EXP TO GDP & 7.00 & 4.90 & $\mathbf{3 . 8 0}$ & -30.00 & -22.45 \\
\hline X EXP LGST PARTHER & 40.00 & 37.00 & 25.00 & -7.50 & -32.43 \\
\hline X IMP LGST PARTHER & 39.00 & 32.00 & 27.00 & -17.95 & -15.63 \\
\hline X EXP matufaC. & 8.00 & 9.00 & 23.00 & 12.50 & 155.56 \\
\hline X EXP MGR-MINE & 91.00 & 90.00 & 76.00 & -1.10 & -15.56 \\
\hline X IMP MANUFAC. & 58.00 & 63.00 & 46.00 & 8.62 & -26.98 \\
\hline * IHP AGR-HINE & 41.00 & 36.00 & 53.00 & -12.20 & 47.22 \\
\hline TOTAL EXPORTS & 338.00 & 477.00 & 2403.00 & 41.12 & 403.77 \\
\hline TOTAL IHPORTS & 387.00 & 665.00 & 4373.00 & 71.83 & 557.59 \\
\hline .N.P. & NA & $\mathbf{M A}$ & 360.00 & - & 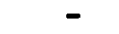 \\
\hline
\end{tabular}


mozambique

SUMMARY OF NATIONAL STATISTICS

\begin{tabular}{|c|c|c|c|c|c|}
\hline NATIOHAL VARIABLES & 1962 & 1970 & 1980 & $62-70$ & $70-80$ \\
\hline EXP. PARTAER CON. & 0.58 & 0.58 & 0.44 & 0.00 & -24.14 \\
\hline IHP. PARTNER CON. & 0.48 & 0.46 & 0.38 & -4.17 & -17.39 \\
\hline EPC/IPC RATIO & 1.22 & 1.26 & 1.13 & 3.28 & -10.32 \\
\hline EXP COMH CONCEN & 0.31 & 0.27 & MA & -12.90 & - \\
\hline IHP COHH CONCEN & 0.24 & 0.27 & MA & 12.50 & - \\
\hline ECC/ICC RATIO & 1.29 & 1.00 & MA & -22.58 & - \\
\hline VERTICAL TRADE & -0.68 & -0.66 & $\mathbf{M}$ & -2.94 & - \\
\hline TOT TRADE LINKAGES & 12.00 & 14.00 & 30.00 & 16.67 & 114.29 \\
\hline \% AGRICULTURE-GDP & 55.70 & 48.50 & 43.10 & -12.93 & -11.13 \\
\hline QUALITY OF LIFE & MA & Ma & MA & - & - \\
\hline \% LRGST EXP TO GDP & Ma & Ma & MA & - & - \\
\hline \% EXP LGST PARTMER & 45.00 & 45.00 & 23.00 & 0.00 & -48.89 \\
\hline X IMP LGST PARTNER & 23.00 & 24.00 & 12.00 & 4.35 & -50.00 \\
\hline \% exp manufac. & 3.00 & 9.00 & Na & 200.00 & - \\
\hline Y EXP AGR-HINE & 96.00 & 90.00 & MA & -6.25 & - \\
\hline X IMP MANUFAC. & 71.00 & 75.00 & $M$ & 5.63 & - \\
\hline X IHP AGR-MINE & 28.00 & 24.00 & M & -14.29 & - \\
\hline TOTAL EXPORTS & 79.00 & 131.00 & 445.00 & 65.82 & 239.69 \\
\hline TOTAL IKPORTS & 120.00 & 349.00 & 590.00 & 190.83 & 69.05 \\
\hline G.N.P. PER CAPITA & MA & NA & 360.00 & - & - \\
\hline
\end{tabular}


METHERLAND

SUMMARY OF NATIONAL STATISTICS

\begin{tabular}{|c|c|c|c|c|c|}
\hline NATIOHAL VARIABLES & 1962 & 1970 & 1980 & $62-70$ & $70-80$ \\
\hline EXP. PARTNER CON. & 0.39 & 0.60 & 0.45 & 53.85 & -25.00 \\
\hline IMP. PARTNER CON. & 0.38 & 0.71 & 0.37 & 86.84 & -47.89 \\
\hline EPC/IPC RATIO & 1.03 & 0.84 & 1.22 & -18.45 & 45.24 \\
\hline EXP COMH CONCEN & 0.07 & 0.84 & 0.10 & 1100.00 & -88.10 \\
\hline IMP COHM CONCEN & 0.11 & 0.48 & 0.14 & 336.36 & -70.83 \\
\hline ECC/ICC RATIO & 0.64 & 1.75 & 0.71 & 175.00 & -59.18 \\
\hline VERTICAL TRADE & -0.07 & -0.18 & -0.04 & 157.14 & -77.78 \\
\hline TOT TRADE LINKAGES & 95.00 & 42.00 & 139.00 & -55.79 & 230.95 \\
\hline X AGRICULTURE-GDP & MA & MA & 4.30 & - & - \\
\hline QUALITY OF LIFE & 95.10 & Ma & 98.60 & - & - \\
\hline \% LRGST EXP TO GDP & 8.10 & 17.50 & 13.90 & 116.05 & -20.57 \\
\hline * EXP LGST PARTNER & 25.00 & 33.00 & 30.00 & 32.00 & -9.09 \\
\hline * Imp lgst partner & 20.00 & 24.00 & 19.00 & 20.00 & -20.83 \\
\hline \% EXP MaNUFAC. & 53.00 & 5.00 & 51.00 & -90.57 & 920.00 \\
\hline \% EXP AGR-HINE & 46.00 & 94.00 & 48.00 & 104.35 & -48.94 \\
\hline X IFP MANUFAC. & 59.00 & 23.00 & 54.00 & -61.02 & 134.78 \\
\hline X IMP AGR-HINE & 40.00 & 76.00 & 45.00 & 90.00 & -40.79 \\
\hline TOTAL EXPORTS & 4438.00 & 653.00 & $720 / 3.00$ & -85.29 & 10932.62 \\
\hline TOTAL IMPORTS & 5860.00 & 988.00 & 92809.00 & -83.14 & 9293.62 \\
\hline G.N.P. PER CAPITA & $\boldsymbol{N}$ & MA & 11790.00 & - & - \\
\hline
\end{tabular}


NETH. ANT.

SUMMARY OF NATIONAL STATISTICS

\begin{tabular}{|c|c|c|c|c|c|}
\hline NATIONAL VARIABLES & 1962 & 1970 & 1980 & $62-70$ & $70-80$ \\
\hline EXP. PARTNER CON. & 0.40 & 0.45 & 0.59 & 12.50 & 31.11 \\
\hline IHP. PARTNER CON. & 0.83 & 0.40 & 0.68 & -51.81 & 70.00 \\
\hline EPC/IPC RATIO & 0.47 & 1.12 & 0.87 & 138.30 & -22.32 \\
\hline EXP COMH CONCEN & 0.96 & 0.10 & Ma & -89.58 & - \\
\hline IHP COHY CONCEN & 0.66 & 0.14 & M & -78.79 & - \\
\hline ECC/ICC RATIO & 1.45 & 0.71 & M & -50.89 & - \\
\hline VERTICAL TRADE & -0.11 & -0.07 & M & -36.36 & - \\
\hline TOT TRADE LINKAGES & 58.00 & 117.00 & 50.00 & 101.72 & -57.26 \\
\hline \% AGRICULTURE-GDP & MA & 5.80 & Ma & - & - \\
\hline QUALITY OF LIFE & NA & 96.30 & M & - & - \\
\hline X LRGST EXP TO GDP & Ma & m & M & - & - \\
\hline X EXP LGST PARTNER & 34.00 & 59.00 & 57.00 & 73.53 & -3.39 \\
\hline X IMP LGST PARTMER & 81.00 & 65.00 & 56.00 & -19.75 & -13.85 \\
\hline \% EXP manufac. & $1 . \infty$ & 56.00 & NA & 5500.00 & - \\
\hline X EXP AGR-HIHE & 98.00 & 43.00 & M & -56.12 & - \\
\hline X IHP MANUFAC. & 11.00 & 64.00 & M & 481.82 & - \\
\hline X IMP AGR-MINE & 88.00 & 35.00 & NA & -60.23 & - \\
\hline TOTAL EXPORTS & \multicolumn{2}{|c|}{654.0011537 .00} & 4231.00 & 1664.07 & -63.33 \\
\hline TOTAL IMPORTS & 761.00 & 14756.00 & 8159.00 & 1839.03 & -44.71 \\
\hline G.H.P. PER CA & Th & MA & 2800.00 & - & - \\
\hline
\end{tabular}


NEW CALEDO

SUMMARY OF NATIONAL STATISTICS

\begin{tabular}{|c|c|c|c|c|c|}
\hline MATIONAL VARIABLES & 1962 & 1970 & 1980 & $62-70$ & $70-80$ \\
\hline EXP. PARTNER CON. & $1 . \infty$ & 0.79 & 0.80 & -21.00 & 1.27 \\
\hline IMP. PARTNER CON. & 0.89 & 0.72 & 0.63 & -19.10 & -12.50 \\
\hline EPC/IPC RATIO & 1.12 & 1.10 & 1.28 & -1.79 & 16.36 \\
\hline EXP CONH CONCEN & NA & NA & $\mu$ & - & - \\
\hline IMP CONH CONCEN & MA & NA & MA & - & - \\
\hline ECC/ICC RATIO & M & NA & $M$ & - & - \\
\hline VERTICAL TRADE & MA & $\mathbf{M}$ & $\boldsymbol{M}$ & - & - \\
\hline TOT TRADE LINKAGES & 1.00 & 6.00 & 4.00 & 500.00 & -33.33 \\
\hline X AGRICULTURE-GDP & NA & NA & $\mathbf{M}$ & - & - \\
\hline QUALITY OF LIFE & MA & MA & $M$ & - & - \\
\hline * LRGST EXP TO GDP & M & M & $M$ & - & - \\
\hline ※ EXP LGST PARTNER & 100.00 & 48.00 & 56.00 & -52.00 & 16.67 \\
\hline X IMP LGST PARTNER & 80.00 & 60.00 & 31.00 & -25.00 & -48.33 \\
\hline X EXP манufac. & MiA & $\boldsymbol{M}$ & $\boldsymbol{M}$ & - & - \\
\hline * EXP AGR-MINE & MA & M & $M$ & - & - \\
\hline X IMP MANUFAC. & Mn & M & $\boldsymbol{\mu} \boldsymbol{\Lambda}$ & - & - \\
\hline X IMP AGR-HINE & $\mathbf{M A}$ & MA & M & - & - \\
\hline TOTAL EXPORTS & 22.00 & 190.00 & 403.00 & 763.64 & 112.11 \\
\hline TOTAL IMPORTS & 27.00 & 173.00 & 466.00 & 540.74 & 169.36 \\
\hline G.M.P. PER CAPITA & M & NA & 6500.00 & - & - \\
\hline
\end{tabular}


NEW ZEALAM

SUHMARY OF NATIONAL STATISTICS

\begin{tabular}{|c|c|c|c|c|c|}
\hline MATIONAL VARIABLES & 1962 & 1970 & 1980 & $62-70$ & $70-80$ \\
\hline EXP. PARTNER CON. & 0.65 & 0.58 & 0.39 & -10.77 & -32.76 \\
\hline IHP. PARTNER COH. & 0.58 & 0.52 & 0.46 & -10.34 & -11.54 \\
\hline EPC/IPC RATIO & 1.12 & 1.10 & 0.85 & -1.79 & -22.73 \\
\hline EXP CONH CONCEN & 0.49 & 0.38 & 0.20 & -22.45 & -47.37 \\
\hline IMP COAIA CONCEN & 0.23 & 0.23 & 0.20 & 0.00 & -13.04 \\
\hline ECC/ICC RATIO & 2.13 & 1.65 & 1.00 & -22.45 & -39.47 \\
\hline VERTICAL TRADE & -0.72 & -0.65 & -0.46 & -9.72 & -29.23 \\
\hline TOT TRADE LINKAGES & 17.00 & 25.00 & 78.00 & 47.06 & 212.00 \\
\hline X AGRICULTURE-GDP & MA & Ma & 11.70 & - & - \\
\hline QUALITY OF LIFE & 93.10 & 94.10 & 96.30 & 1.07 & 2.34 \\
\hline Z LRGST EXP TO GDP & 10.50 & 5.90 & 3.60 & -43.81 & -38.98 \\
\hline * EXP LGST PARTNER & 80.00 & 37.00 & 14.00 & -53.75 & -62.16 \\
\hline * IHP LGST PARTNER & 80.00 & 28.00 & 21.00 & -65.00 & -25.00 \\
\hline \% EXP MANUFAC. & 3.00 & 11.00 & 20.00 & 266.67 & 81.82 \\
\hline \% EXP AGR-MINE & 97.00 & 89.00 & 79.00 & -8.25 & -11.24 \\
\hline \% IHP MANUFAC. & 75.00 & 76.00 & 65.00 & 1.33 & -14.47 \\
\hline X IHP AGR-HINE & 24.00 & 23.00 & 34.00 & -4.17 & 47.83 \\
\hline TOTAL EXPORTS & 738.00 & 1100.00 & 5134.00 & 49.05 & 366.73 \\
\hline TOTAL IMPORTS & 679.00 & 1098.00 & 4755.00 & 61.71 & 333.06 \\
\hline G.A.P. P & Ma & NA & $0 . \infty$ & - & - \\
\hline
\end{tabular}


NICARagUa

SUMMARY OF MATIONAL STATISTICS

\begin{tabular}{|c|c|c|c|c|c|}
\hline MTIONAL VARIABLES & 1962 & 1970 & 1980 & $62-70$ & $70-80$ \\
\hline EXP. PARTNER CON. & 0.62 & 0.54 & 0.56 & -12.90 & 3.70 \\
\hline IMP. PARTNER CON. & 0.63 & 0.54 & 0.52 & -14.29 & -3.70 \\
\hline EPC/IPC RATIO & 0.98 & 0.99 & 1.08 & 1.02 & 9.09 \\
\hline EXP COII CONCEN & 0.40 & 0.33 & 0.48 & -17.50 & 45.45 \\
\hline IMP COHH CONCEN & 0.33 & 0.33 & 0.27 & 0.00 & -18.18 \\
\hline ECC/ICC RATIO & 1.21 & 1.00 & 1.78 & -17.50 & 77.78 \\
\hline VERTICAL TRADE & -0.77 & -0.65 & -0.49 & -15.58 & -24.62 \\
\hline TOT TRADE LINKAGES & 11.00 & 16.00 & 18.00 & 45.45 & 12.50 \\
\hline \% AGRICULTURE-GDP & 23.70 & 24.30 & 23.70 & 2.53 & -2.47 \\
\hline QUALITY OF LIFE & 36.80 & 48.00 & 66.70 & 30.43 & 38.96 \\
\hline X LRGST EXP TO GDP & 8.00 & 7.30 & 9.30 & -8.75 & 27.40 \\
\hline X EXP LGST PARTNER & 52.00 & 33.00 & 38.00 & -36.54 & 15.15 \\
\hline \% IMP LGST PARTNER & 44.00 & 41.00 & 29.00 & -6.82 & -29.27 \\
\hline X EXP MANUFAC. & 2.00 & $16 . \infty 0$ & 13.00 & 700.00 & -18.75 \\
\hline X EXP AGR-NINE & 97.00 & 83.00 & 86.00 & -14.43 & 3.61 \\
\hline X IMP MANUFAC. & 79.00 & 81.00 & 63.00 & 2.53 & -22.22 \\
\hline \% IMP AGR-HINE & 20.00 & 18.00 & 36.00 & -10.00 & 100.00 \\
\hline TOTAL EXPORTS & 88.00 & 180.00 & 413.00 & 104.55 & 129.44 \\
\hline TOTAL IHPORTS & 84.00 & $187 . \infty$ & 849.00 & 122.62 & 354.01 \\
\hline G.N.P. PER CAPITA & M & $\mathbf{M}$ & 860.00 & - & - \\
\hline
\end{tabular}


MIGER

SUMHARY OF MATIONAL STATISTICS

\begin{tabular}{|c|c|c|c|c|c|}
\hline MATIOHAL VARIABLES & 1962 & 1970 & 1980 & $62-70$ & $70-80$ \\
\hline EXP. PARTNER CON. & 0.86 & 0.69 & 0.65 & -19.77 & -5.80 \\
\hline IMP. PARTNER CON. & 1.00 & 0.67 & 0.61 & -33.00 & -8.96 \\
\hline EPC/IPC RATIO & 0.86 & 1.02 & 1.07 & 18.60 & 4.90 \\
\hline EXP COHH CONCEN & 0.76 & 0.76 & $M$ & 0.00 & - \\
\hline IMP COHH CONCEN & 0.23 & 0.30 & Ma & 30.43 & - \\
\hline ECC/ICC RATIO & $\mathbf{3 . 3 0}$ & 2.53 & $\mathbf{M}$ & -23.33 & - \\
\hline VERTICAL TRADE & -0.61 & -0.74 & $\boldsymbol{M}$ & 21.31 & - \\
\hline TOT TRADE LINKAGES & 2.00 & 5.00 & 9.00 & 150.00 & 80.00 \\
\hline X AGRICULTTURE-GDP & 69.00 & 65.10 & 39.30 & -5.65 & -39.63 \\
\hline QUALITY OF LIFE & 5.30 & MA & 21.40 & - & - \\
\hline X LRGST EXP TO GDP & 6.90 & 7.30 & Ma & 5.80 & - \\
\hline X EXP LGST PARTNER & 42.00 & 48.00 & 41.00 & 14.29 & -14.58 \\
\hline X IMP LGST PARTNER & 55.00 & 58.00 & 47.00 & 5.45 & -18.97 \\
\hline X EXP RANUFAC. & 4.00 & 3.00 & $\mathbf{M A}$ & -25.00 & - \\
\hline X EXP AGR-HINE & 96.00 & 96.00 & $\mathbf{M A}$ & $0 . \infty$ & - \\
\hline X IMP MANUFAC. & 65.00 & 77.00 & MA & 18.46 & - \\
\hline \% IHP AGR-HINE & 34.00 & 22.00 & MA & -35.29 & - \\
\hline TOTAL EXPORTS & 12.00 & 30.00 & 555.00 & 150.00 & 1750.00 \\
\hline TOTAL IMPORTS & 11.00 & 40.00 & 451.00 & 263.64 & 1027.50 \\
\hline H.P. PEF & Nh & NA & 30.00 & - & - \\
\hline
\end{tabular}


NIGERIA

SUMMARY OF MATIONAL STATISTICS

\begin{tabular}{|c|c|c|c|c|c|}
\hline MATIONAL VARIABLES & 1962 & 1970 & 1980 & $62-70$ & $70-80$ \\
\hline EXP. PARTNER CON. & 0.64 & 0.53 & 0.58 & -17.19 & 9.43 \\
\hline IMP. PARTNER CON. & 0.53 & 0.47 & 0.44 & -11.32 & -6.38 \\
\hline EPC/IPC RATIO & 1.22 & 1.12 & 1.33 & -8.20 & 18.75 \\
\hline EXP COHH CONCEN & 0.36 & 0.34 & 0.86 & -5.56 & 152.94 \\
\hline IMP COHM CONCEN & 0.30 & 0.35 & 0.31 & 16.67 & -11.43 \\
\hline ECC/ICC RATIO & 1.20 & 0.97 & 2.77 & -19.05 & 185.58 \\
\hline VERTICAL TRADE & -0.69 & -0.87 & -0.77 & 26.09 & -11.49 \\
\hline TOT TRADE LINKAGES & 13.00 & 30.00 & 36.00 & 130.7 & 20.00 \\
\hline \% AGRICULTURE-GDP & 64.30 & 56.70 & 25.40 & -11.82 & -55.20 \\
\hline QUALITY OF LIFE & 12.60 & MA & 34.60 & - & - \\
\hline \% LRGST EXP TO GDP & 6.40 & 7.40 & MA & 15.63 & - \\
\hline X EXP LGST PARTNER & 66.00 & 29.00 & 42.00 & -56.06 & 44.83 \\
\hline X IHP LGST PARTNER & 100.00 & 30.00 & 19.00 & -70.00 & -36.67 \\
\hline X EXP MANUFAC. & 7.00 & 1.00 & $\mathbf{M}$ & -85.71 & - \\
\hline X EXP AGR-HINE & 92.00 & 144.00 & 99.00 & 56.52 & -31.25 \\
\hline X IFP MANUFAC. & 76.00 & 85.00 & 77.00 & 11.84 & -9.41 \\
\hline X IMP AGR-MINE & 23.00 & 14.00 & 22.00 & -39.13 & 57.14 \\
\hline TOTAL EXPORTS & 426.00 & \multicolumn{2}{|c|}{1183.0024362 .00} & 177.70 & 1959.34 \\
\hline TOTAL IMPORTS & 461.00 & \multicolumn{2}{|c|}{897.0014164 .00} & 94.58 & 1479.04 \\
\hline G.N.P. PER & MA & $\mathbf{M}$ & 870.00 & - & - \\
\hline
\end{tabular}


NORWAY

SUMMARY OF NATIONAL STATISTICS

\begin{tabular}{|c|c|c|c|c|c|}
\hline MATIOHAL VARIABLES & 1962 & 1970 & 1980 & $62-70$ & $70-80$ \\
\hline EXP. PARTNER CON. & 0.41 & 0.43 & 0.53 & 4.88 & 23.26 \\
\hline IMP. PARTNER CON. & 0.45 & 0.43 & 0.44 & -4.44 & 2.33 \\
\hline EPC/IPC RATIO & 0.93 & 1.01 & 1.21 & 8.60 & 19.80 \\
\hline EXP CONH CONCEN & 0.05 & G.07 & 0.28 & 40.00 & 300.00 \\
\hline IMP COHA CONCEN & 0.15 & 0.17 & 0.18 & 13.33 & 5.88 \\
\hline ECC/ICC RATIO & 0.33 & 0.41 & 1.56 & 23.53 & 277.78 \\
\hline VERTICAL TRADE & -0.22 & -0.16 & -0.35 & -27.27 & 118.75 \\
\hline TOT TRADE LINKAGES & 51.00 & 66.00 & 98.00 & 29.41 & 48.48 \\
\hline X AGRICULTURE-GDP & 9.00 & 6.90 & 5.30 & -23.33 & -23.19 \\
\hline QUALITY OF LIFE & 95.10 & 96.70 & 98.90 & 1.68 & 2.28 \\
\hline X LRGST EXP TO GDP & 3.90 & 3.80 & 7.40 & -2.56 & 94.74 \\
\hline X EXP LGST PARTNER & 46.00 & 18.00 & 41.00 & -60.87 & 127.78 \\
\hline \% IMP LGST PARTNER & 39.00 & 21.00 & 20.00 & -46.15 & -4.76 \\
\hline X EXP MANUFAC. & 45.00 & 55.00 & 32.00 & 22.22 & -41.82 \\
\hline Z EXP AGR-HIME & 54.00 & 44.00 & 67.00 & -18.52 & 52.27 \\
\hline Z IHP RANUFAC. & 67.00 & 71.00 & 67.00 & 5.97 & -5.63 \\
\hline Z IMP AGR-HINE & 32.00 & 29.00 & 32.00 & -9.38 & 10.34 \\
\hline TOTAL EXPORTS & 950.00 & 2404.00 & 18338.00 & 153.05 & 662.81 \\
\hline TOTAL IMPORTS & 1516.00 & 3418.00 & 14552.00 & 125.46 & 325.75 \\
\hline G.H.P. PER CAPITA & $\mathbf{M A}$ & MA & 14060.00 & - & - \\
\hline
\end{tabular}


SUMAARY OF NATIONAL STATISTICS

\begin{tabular}{|c|c|c|c|c|c|}
\hline MATIONAL VARLABLES & 1962 & 1970 & 1980 & $62-70$ & $70-80$ \\
\hline EXP. PARTNER CON. & 1.00 & 0.56 & 0.70 & -44.00 & 25.00 \\
\hline IMP. PARTNER CON. & 0.76 & 0.68 & 0.45 & -10.53 & -33.82 \\
\hline EPC/IPC RATIO & 1.32 & 0.83 & 1.55 & -37.12 & 86.75 \\
\hline EXP CONH CONCEN & Na & 0.98 & 0.43 & - & -56.12 \\
\hline IHP COHA CONCEN & Ma & 0.56 & 0.25 & - & -55.36 \\
\hline ECC/ICC RATIO & Ma & 1.75 & 1.72 & - & -1.71 \\
\hline VERTICAL TRADE & Ma & -0.92 & 0.05 & - & -105.13 \\
\hline TOT TRADE LINKAGES & MA & 12.00 & 12.00 & - & 0.00 \\
\hline X AGRICULTURE-GDP & $\mathbf{M a}$ & 31.20 & 3.00 & - & -90.38 \\
\hline QUALITY OF LIFE & Ma & 22.20 & 32.60 & - & 46.85 \\
\hline X LRGST EXP TO GDP & Ma & $\mathbf{M}$ & $\mathbf{M}$ & - & - \\
\hline \% EXP LGST PARTHER & 17.00 & 22.00 & 56.00 & 29.41 & 154.55 \\
\hline X IHP LGST PARTNER & 18.00 & 58.00 & 20.00 & 222.22 & -65.52 \\
\hline Z EXP RANUFAC. & MA & MA & 77.00 & - & - \\
\hline \% EXP AGR-MINE & MA & 99.00 & 22.00 & - & -77.78 \\
\hline X IMP MANUFAC. & MA & 92.00 & $72 . \infty 0$ & - & -21.74 \\
\hline X IMP AGR-MINE & NA & 7.00 & 27.00 & - & 285.71 \\
\hline TOTAL EXPORTS & $\mathbf{M A}$ & 419.00 & 2957.00 & - & 605.73 \\
\hline TOTAL IMPORTS & 6.00 & 133.00 & 1468.00 & 2116.67 & 1003.76 \\
\hline G.N.P. PER CAPITA & MA & $\mathbf{M}$ & 5920.00 & - & - \\
\hline
\end{tabular}


PAKISTAN

SUMMARY OF MATIONAL STATISTICS

\begin{tabular}{|c|c|c|c|c|c|}
\hline MATIOHAL VARIABLES & 1962 & 1970 & 1980 & $62-70$ & $70-80$ \\
\hline EXP. PARTNER CON. & 0.37 & 0.28 & 0.29 & -24.32 & 3.57 \\
\hline IMP. PARTNER CON. & 0.53 & 0.46 & 0.37 & -13.21 & -19.57 \\
\hline EPC/IPC RATIO & 0.69 & 0.62 & 0.78 & -10.14 & 25.81 \\
\hline EXP COHH CONCEN & 0.39 & 0.36 & 0.21 & -7.69 & -41.67 \\
\hline IHP COHA CONCEN & 0.20 & 0.19 & 0.14 & -5.00 & -26.32 \\
\hline ECC/ICC RATIO & 1.95 & 1.89 & 1.50 & -2.83 & -20.83 \\
\hline VERTICAL TRADE & -0.46 & -0.09 & -0.05 & -80.43 & -44.44 \\
\hline TOT TRADE LINKAGES & 39.00 & 56.00 & $77 . \infty$ & 43.59 & 37.50 \\
\hline \% AGRICULTURE-GDP & 46.20 & 39.90 & 32.20 & -13.64 & -19.30 \\
\hline QUALITY OF LIFE & 19.90 & $26: 90$ & 33.80 & 35.18 & 25.65 \\
\hline X LRGST EXP TO GDP & 0.70 & 0.60 & 0.70 & -14.29 & 16.67 \\
\hline X EXP LGST PARTNER & 100.00 & 12.00 & 8.00 & -88.00 & -33.33 \\
\hline X IHP LGST PARTNER & $47 . \infty 0$ & 28.00 & 12.00 & -40.43 & -57.14 \\
\hline X EXP MANUFAC. & 24.00 & 57.00 & 48.00 & 137.50 & -15.79 \\
\hline X EXP AGR-MINE & 75.00 & 42.00 & 51.00 & -44.00 & 21.43 \\
\hline$x$ IHP MANUFAC. & 70.00 & 66.00 & 54.00 & -5.71 & -18.18 \\
\hline \% IHP AGR-NINE & 29.00 & 33.00 & 46.00 & 13.79 & 39.39 \\
\hline TOTAL EXPORTS & 367.00 & 693.00 & 2597.00 & 88.83 & 274.75 \\
\hline TOTAL IMPORTS & 729.00 & 1123.00 & 5280.00 & 54.05 & 370.17 \\
\hline G.N.P. PER CAPITA & NA & MA & 350.00 & - & - \\
\hline
\end{tabular}


panara

SUMMARY OF NATIONAL STATISTICS

\begin{tabular}{|c|c|c|c|c|c|}
\hline MATIONAL VARIABLES & 1962 & 1970 & 1980 & $62-70$ & $70-80$ \\
\hline EXP. PARTNER CON. & 0.52 & 0.75 & 0.64 & 44.23 & -14.67 \\
\hline IHP. PARTNER CON. & 0.56 & 0.51 & 0.46 & -8.93 & -9.80 \\
\hline EPC/IPC RATIO & 0.92 & 1.46 & 1.39 & 58.70 & -4.79 \\
\hline EXP COHH CONCEN & 0.48 & 0.55 & 0.48 & 14.58 & -12.73 \\
\hline IMP COHN CONCEN & 0.26 & 0.25 & 0.24 & -3.85 & -4.00 \\
\hline ECC/ICC RATIO & 1.85 & 2.20 & 2.00 & 19.17 & -9.09 \\
\hline VERTICAL TRADE & -0.64 & -0.66 & -0.49 & 3.13 & -25.76 \\
\hline TOT TRADE LINKAGES & 16.00 & 7.00 & 18.00 & -56.25 & 157.14 \\
\hline X AGRICULLTURE-GDP & 19.70 & 16.70 & 11.80 & -15.23 & -29.34 \\
\hline QUALITY OF LIFE & 51.20 & 77.40 & 87.30 & 51.17 & 12.79 \\
\hline X LRGST EXP TO GDP & 4.40 & 4.80 & $\mathbf{5 . 5 0}$ & 9.09 & 14.58 \\
\hline X EXP LGST PARTNER & 18.00 & 67.00 & 58.00 & 272.22 & -13.43 \\
\hline X IHP LGST PARTNER & 38.00 & 31.00 & 31.00 & -18.42 & 0.00 \\
\hline X EXP MANUFAC. & 2.00 & 3.00 & 8.00 & 50.00 & 166.67 \\
\hline X EXP AGR-HIME & 97.00 & 96.00 & 91.00 & -1.03 & -5.21 \\
\hline X IMP MANUFAC. & 66.00 & 69.00 & 58.00 & 4.55 & -15.94 \\
\hline$X$ IMP AGR-HINE & 33.00 & 30.00 & 42.00 & -9.09 & 40.00 \\
\hline TOTAL EXPORTS & 99.00 & 100.00 & 297.00 & 1.01 & 197.00 \\
\hline TOTAL IMPORTS & 224.00 & 653.00 & 4510.00 & 191.52 & 590.66 \\
\hline G.N.P. PE & MA & MA & 1910.00 & - & - \\
\hline
\end{tabular}


PAPAU N.G.

SUMMARY OF NATIONAL STATISTICS

\begin{tabular}{|c|c|c|c|c|c|}
\hline MATIONAL VARIABLES & 1962 & 1970 & 1980 & $62-70$ & $70-80$ \\
\hline EXP. PARTNER CON. & 0.87 & 0.66 & 0.64 & -24.14 & -3.03 \\
\hline IMP. PARTMER CON. & 0.86 & 0.78 & 0.67 & -9.30 & -14.10 \\
\hline EPC/IPC RATIO & 1.01 & 0.85 & 0.95 & -15.84 & 11.76 \\
\hline EXP CONN CONCEN & 0.43 & 0.64 & 0.41 & 48.84 & -35.94 \\
\hline IHP COHH CONCEN & 0.31 & 0.32 & $\mathbf{M n}$ & 3.23 & - \\
\hline ECC/ICC RATIO & 1.39 & 2.00 & $M$ & 44.19 & - \\
\hline VERTICAL TRADE & -0.54 & -0.71 & $\boldsymbol{\mu}$ & 31.48 & - \\
\hline TOT TRADE LINKAGES & 3.00 & 7.00 & 15.00 & 133.33 & 114.29 \\
\hline X AGRICULTURE-GDP & NA & 43.10 & 33.60 & - & -22.04 \\
\hline OUALITY OF LIFE & MA & M & 40.30 & - & - \\
\hline * LRGST EXP TO GDP & MA & MA & $\boldsymbol{M}$ & - & - \\
\hline * EXP LGST PARTNER & 22.00 & 44.00 & 24.00 & 100.00 & -45.45 \\
\hline X IHP LGST PARTNER & 46.00 & 69.00 & 39.00 & 50.00 & -43.48 \\
\hline z EXP nanufac. & 11.00 & 4.00 & M & -63.64 & - \\
\hline X EXP AGR-MINE & 88.00 & 95.00 & M & 7.95 & - \\
\hline y IHP maNUfaC. & 64.00 & 75.00 & MA & 17.19 & - \\
\hline ※ IRP AGR-MINE & 35.00 & 24.00 & $\mathbf{M}$ & -31.43 & - \\
\hline TOTAL EXPORTS & 15.00 & 102.00 & 1107.00 & 580.00 & 985.29 \\
\hline TOTAL IMPORTS & 30.00 & 260.00 & 949.00 & 766.67 & 265.00 \\
\hline G.N.P. PER CAPITA & NA & MA & 840.00 & - & - \\
\hline
\end{tabular}


PARaguaY

SUMMARY OF NATIONAL STATISTICS

\begin{tabular}{|c|c|c|c|c|c|}
\hline MATIONAL VARIABLES & 1962 & 1970 & 1980 & $62-70$ & $70-80$ \\
\hline EXP. PARTNER CON. & 0.69 & 0.60 & 0.51 & -13.04 & -15.00 \\
\hline IMP. PARTNER CON. & 0.53 & 0.55 & 0.58 & 3.77 & 5.45 \\
\hline EPC/IPC RATIO & 1.31 & 1.09 & 0.87 & -16.79 & -20.18 \\
\hline EXP CONH CONCEN & 0.41 & 0.46 & 0.39 & 12.20 & -15.22 \\
\hline IHP COHH CONCEN & 0.21 & 0.22 & 0.23 & 4.76 & 4.55 \\
\hline ECC/ICC RATIO & 1.95 & 2.09 & 1.70 & 7.10 & -18.90 \\
\hline VERTICAL TRADE & -0.50 & -0.56 & -0.50 & 12.00 & -10.71 \\
\hline TOT TRADE LINKAGES & 5.00 & 9.00 & 19.00 & 80.00 & 111.11 \\
\hline X AGRICULTURE-GDP & 38.20 & 34.90 & 31.70 & -8.64 & -9.17 \\
\hline QUALITY OF LIFE & 50.80 & 72.30 & 78.30 & 42.32 & 8.30 \\
\hline$X$ LRGST EXP TO GDP & Ma & Ma & M & - & - \\
\hline X EXP LGST PARTNER & 38.00 & 34.00 & 38.00 & -10.53 & 11.76 \\
\hline X IMP LGST PARTNER & 21.00 & 23.00 & 49.00 & 9.52 & 113.04 \\
\hline X EXP manUfAC. & 11.00 & 9.00 & $11 . \infty$ & -18.18 & 22.22 \\
\hline$X$ EXP AGR-HINE & 88.00 & 91.00 & 88.00 & 3.41 & -3.30 \\
\hline X IHP MANUFAC. & 61.00 & 65.00 & 61.00 & 6.56 & -6.15 \\
\hline X IMP AGR-HINE & 38.00 & 35.00 & 38.00 & -7.89 & 8.57 \\
\hline TOTAL EXPORTS & 24.00 & 51.00 & 300.00 & 112.50 & 488.24 \\
\hline TOTAL IMPORTS & 36.00 & 74.00 & 1042.00 & 105.56 & 1308.11 \\
\hline G.K.P. PER CAPITA & $\mathbf{M}$ & Ma & 1630.00 & - & - \\
\hline
\end{tabular}


PERU

SUHMARY OF MATIONAL STATISTICS

\begin{tabular}{|c|c|c|c|c|c|}
\hline MATIONAL VARIABLES & 1962 & 1970 & 1980 & $62-70$ & $70-80$ \\
\hline EXP. PARTNER CON. & 0.53 & 0.52 & 0.44 & -1.89 & -15.38 \\
\hline IMP. PARTNER COH. & 0.54 & 0.46 & 0.48 & -14.81 & 4.35 \\
\hline EPC/IPC RATIO & 0.99 & 1.14 & 0.91 & 15.15 & -20.18 \\
\hline EXP COHM CONCEN & 0.31 & 0.41 & 0.34 & 32.26 & -17.07 \\
\hline IHP COHH CONCEN & 0.28 & 0.12 & 0.26 & -57.14 & 116.67 \\
\hline ECC/ICC RATIO & 1.11 & 3.42 & 1.31 & 208.60 & -61.73 \\
\hline VERTICAL TRADE & -0.75 & -0.50 & -0.56 & -33.33 & 12.00 \\
\hline TOT TRADE LINKAGES & 25.00 & 38.00 & 53.00 & 52.00 & 39.47 \\
\hline X AGRICULTURE-GDP & 21.70 & 15.60 & 9.90 & -28.11 & -36.54 \\
\hline QUALITY OF LIFE & 36.80 & 53.90 & 64.70 & 46.47 & 20.04 \\
\hline X LRGST EXP TO GDP & 7.50 & 3.80 & 2.80 & -49.33 & -26.32 \\
\hline X EXP LGST PARTNER & 36.00 & 33.00 & 33.00 & -8.33 & 0.00 \\
\hline X IMP LGST PARTNER & 41.00 & 32.00 & 38.00 & -21.95 & 18.75 \\
\hline X Exp maUfac. & 1.00 & 1.00 & 17.00 & 0.00 & 1600.00 \\
\hline X EXP AGR-MIHE & 99.00 & 98.00 & 83.00 & -1.01 & -15.31 \\
\hline z IAP MANUFAC. & 76.00 & 72.00 & 72.00 & -5.26 & 0.00 \\
\hline X IMP AGR-MINE & 23.00 & 54.00 & 27.00 & 134.78 & -50.00 \\
\hline TOTAL EXPORTS & 519.00 & 1047.00 & 3722.00 & 101.73 & 255.49 \\
\hline TOTAL IMPORTS & 446.00 & 659.00 & 3053.00 & 47.76 & 363.28 \\
\hline G.N.P. PER CAPIT & NA & $\mathbf{M A}$ & 1170.00 & - & - \\
\hline
\end{tabular}


PHILIPPINE

SUMMARY OF NATIONAL STATISTICS

\begin{tabular}{|c|c|c|c|c|c|}
\hline MATIONAL VARIABLES & 1962 & 1970 & 1980 & $62-70$ & $70-80$ \\
\hline EXP. PARTNER CON. & 0.67 & 0.69 & 0.50 & 2.99 & -27.54 \\
\hline IHP. PARTMER COH. & 0.58 & 0.56 & 0.45 & -3.45 & -19.64 \\
\hline EPC/IPC RATIO & 1.16 & 1.23 & 1.10 & 6.03 & -10.57 \\
\hline EXP COHH CONCEN & 0.36 & 0.22 & 0.18 & -38.89 & -18.18 \\
\hline IMP COMH CONCEN & 0.08 & 0.16 & 0.19 & 100.00 & 18.75 \\
\hline ECC/ICC RATIO & 4.50 & 1.38 & 0.95 & -69.44 & -31.10 \\
\hline VERTICAL TRADE & -0.53 & -0.61 & -0.22 & 15.09 & -63.93 \\
\hline TOT TRADE LINKAGES & 19.00 & 23.00 & 64.00 & 21.05 & 178.26 \\
\hline X AGRICULTURE-GDP & 25.70 & 26.70 & 25.90 & 3.89 & -3.00 \\
\hline QUALITY OF LIFE & 55.30 & 68.80 & 72.60 & 24.41 & 5.52 \\
\hline X LRGST EXP TO GDP & 4.80 & 5.90 & 5.20 & 22.92 & -11.86 \\
\hline X EXP LGST PARTNER & 51.00 & 41.00 & 28.00 & -19.61 & -31.71 \\
\hline X IHP LGST PARTNER & 44.00 & 34.00 & 24.00 & -22.73 & -29.41 \\
\hline \% exp manufac. & 4.00 & 7.00 & 36.00 & 75.00 & 414.29 \\
\hline X EXP AGR-HINE & 95.00 & 92.00 & 63.00 & -3.16 & -31.52 \\
\hline X IMP MANUFAC. & 65.00 & 68.00 & 58.00 & 4.62 & -14.71 \\
\hline X IMP AGR-MINE & 44.00 & 31.00 & 41.00 & -29.55 & 32.26 \\
\hline TOTAL EXPORTS & 547.00 & 1035.00 & 5664.00 & 89.21 & 447.25 \\
\hline TOTAL IMPORTS & 598.00 & 1307.00 & 8227.00 & 118.56 & 529.46 \\
\hline G.N.P. PER & $\boldsymbol{M A}$ & Ma & 790.00 & - & - \\
\hline
\end{tabular}


POLAND

SUMMARY OF MATIONAL STATISTICS

\begin{tabular}{|c|c|c|c|c|c|}
\hline MATIONAL VARIABLES & 1962 & 1970 & 1980 & $62-70$ & $70-80$ \\
\hline EXP. PARTNER CON. & 0.45 & 0.46 & 0.44 & 2.22 & -4.35 \\
\hline IMP. PARTMER CON. & 0.44 & 0.48 & 0.48 & 9.09 & 0.00 \\
\hline EPC/IPC RATIO & 1.01 & 0.96 & 0.91 & -4.95 & -5.21 \\
\hline EXP CONH CONCEN & MA & $\mathbf{M}$ & $\boldsymbol{M}$ & - & - \\
\hline IHP CONH CONCEN & NA & MA & $\mathbf{M}$ & - & - \\
\hline ECC/ICC RATIO & MA & $\mathbf{M A}$ & $\boldsymbol{M}$ & - & - \\
\hline VERTICAL TRADE & $\mathbf{M A}$ & MA & $\mathbf{M A}$ & - & - \\
\hline TOT TRADE LINKAGES & 42.00 & 61.00 & 79.00 & 45.24 & 29.51 \\
\hline \% AGRICULTURE-GDP & $\mathbf{M A}$ & $\mathbf{M}$ & $\mathbf{M}$ & - & - \\
\hline QUALITY OF LIFE & 82.80 & 89.60 & 93.80 & 8.21 & 4.69 \\
\hline \% LRGST EXP TO GDP & MA & MA & $\mathbf{M A}$ & - & - \\
\hline \% EXP LGST PARTNER & 35.00 & 36.00 & 34.00 & 2.86 & -5.56 \\
\hline \& IMP LGST PARTNER & 31.00 & 38.00 & 39.00 & 22.58 & 2.63 \\
\hline X EXP manUfAC. & Ma & MA & $\mathbf{M}$ & - & - \\
\hline X EXP AGR-HINE & MA & MA & $\mathbf{M}$ & - & - \\
\hline X IMP RANUFAC. & Ma & $\mathbf{M A}$ & 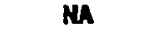 & - & - \\
\hline$X$ IMP AGR-MINE & $\mathbf{N A}$ & $\boldsymbol{M}$ & Ma & - & - \\
\hline TOTAL EXPORTS & 1604.00 & 3406.00 & 15357.00 & 112.34 & 350.88 \\
\hline TOTAL IMPORTS & 1875.00 & 3542.00 & 17053.00 & 88.91 & 381.45 \\
\hline G.H.P. & MA & MA & 3600.00 & - & - \\
\hline
\end{tabular}


portugal

SUMHARY OF NATIONAL STATISTICS

\begin{tabular}{|c|c|c|c|c|c|}
\hline NATIOHLL VARIABLES & 1962 & 1970 & 1980 & $62-70$ & $70-80$ \\
\hline EXP. PARTNER CON. & 0.39 & 0.43 & 0.37 & 10.26 & -13.95 \\
\hline IMP. PARTNER CON. & 0.41 & 0.42 & 0.37 & 2.44 & -11.90 \\
\hline EPC/IPC RATIO & 0.95 & 1.03 & 1.01 & 8.42 & -1.94 \\
\hline EXP CONH CONCEN & 0.28 & 0.22 & 0.23 & -21.13 & 4.55 \\
\hline IMP CONM CONCEN & 0.08 & 0.13 & 0.09 & 62.50 & -30.77 \\
\hline ECC/ICC RATIO & 3.50 & 1.69 & 2.56 & -51.65 & 51.01 \\
\hline VERTICAL TRADE & 0.02 & M & 0.20 & - & - \\
\hline TOT TRADE LINKAGES & 34.00 & 38.00 & 80.00 & 11.76 & 110.53 \\
\hline \% AGRICULTURE-GDP & 28.40 & 20.60 & 13.60 & -27.46 & -33.98 \\
\hline QUALITY OF LIFE & 64.90 & 74.40 & 85.40 & 14.64 & 14.78 \\
\hline X LRGST EXP TO GDP & 1.70 & 3.50 & 2.90 & 105.88 & -17.14 \\
\hline X EXP lGST PARTNER & 13.00 & 20.00 & 15.00 & 53.85 & -25.00 \\
\hline X IMP LGST PARTNER & 16.00 & 17.00 & 12.00 & 6.25 & -29.41 \\
\hline X EXP manufac. & 61.00 & 63.00 & 71.00 & 3.28 & 12.70 \\
\hline X EXP AGR-MINE & 44.00 & 36.00 & 28.00 & -18.18 & -22.22 \\
\hline X IAP MANUFAC. & 55.00 & 64.00 & $51 . \infty 0$ & 16.36 & -20.31 \\
\hline X IMP AGR-MINE & 44.00 & 36.00 & 48.00 & -18.18 & 33.33 \\
\hline TOTAL EXPORTS & 344.00 & 931.00 & 4526.00 & 170.64 & 386.14 \\
\hline TOTAL IMPORTS & 583.00 & 1558.00 & 8976.00 & 167.24 & 476.12 \\
\hline G.N.P. PER CAPIT & MA & MA & 2520.00 & - & - \\
\hline
\end{tabular}


ATAR

SUMMARY OF MATIONAL STATISTICS

\begin{tabular}{|c|c|c|c|c|c|}
\hline MATIONAL VARIABLES & 1962 & 1970 & 1980 & $62-70$ & $70-80$ \\
\hline EXP. PARTNER CON. & 0.62 & 0.59 & 0.49 & -4.84 & -16.95 \\
\hline IHP. PARTNER CON. & 0.68 & 0.63 & 0.44 & -7.35 & -30.16 \\
\hline EPC/IPC RATIO & 0.91 & 0.94 & 1.13 & 3.30 & 20.21 \\
\hline EXP CONH CONCEN & MA & MA & 0.86 & - & - \\
\hline IHP COHM CONCEN & MA & 0.31 & 0.30 & - & -3.23 \\
\hline ECC/ICC RATIO & MA & MA & 2.87 & - & - \\
\hline VERTICAL TRADE & $M$ & -0.72 & -0.73 & - & 1.39 \\
\hline TOT TRADE LINKAGES & 8.00 & 10.00 & 33.00 & 25.00 & 230.00 \\
\hline X AGRICULTURE-GDP & NA & MA & M & - & - \\
\hline QUALITY OF LIFE & NA & 42.10 & 51.80 & - & 23.04 \\
\hline X LRGST EXP TO GDP & NA & MA & $\mathbf{M}$ & - & - \\
\hline X EXP LGST PARTNER & 28.00 & 32.00 & 27.00 & 14.29 & -15.63 \\
\hline X IMP LGST PARTMER & 50.00 & 34.00 & 18.00 & -32.00 & -47.06 \\
\hline X EXP maNUfaC. & NA & M & 5.00 & - & - \\
\hline X EXP AGR-HINE & NA & 100.00 & 92.00 & - & -8.00 \\
\hline \% IMP maNuFaC. & NA & 71.00 & 78.00 & - & 9.86 \\
\hline \% IMP AGR-HINE & NA & 28.00 & 21.00 & - & -25.00 \\
\hline TOTAL EXPORTS & 115.00 & 207.00 & 5300.00 & 80.00 & 2460.39 \\
\hline TOTAL IMPORTS & 27.00 & 51.00 & 1285.00 & 88.89 & 2419.61 \\
\hline G.N.P. PER CAPITA & NA & NA & 10100.00 & - & - \\
\hline
\end{tabular}


REUNION

SUHMARY OF NATIONAL STATISTICS

\begin{tabular}{|c|c|c|c|c|c|}
\hline MATIOALL VARIABLES & 1962 & 1970 & 1980 & $62-70$ & $70-80$ \\
\hline EXP. PARTNER CON. & 0.95 & 0.88 & 0.89 & -7.37 & 1.14 \\
\hline IMP. PARTMER CON. & 0.90 & 0.79 & 0.72 & -12.22 & -8.86 \\
\hline EPC/IPC RATLO & 1.05 & 1.11 & 1.24 & 5.71 & 11.71 \\
\hline EXP COHM CONCEN & NA & M & MA & - & - \\
\hline IHP COHA CONCEN & NA & $\boldsymbol{M}$ & MA & - & - \\
\hline ECC/ICC RATTO & $\mathbf{N A}$ & Ma & $\boldsymbol{M}$ & - & - \\
\hline VERTICAL TRADE & $M A$ & MA & MA & - & - \\
\hline TOT TRADE LINKAGES & 2.00 & 2.00 & 4.00 & 0.00 & 100.00 \\
\hline \& AGRICULTURE-GDP & MA & MA & MA & - & - \\
\hline QUALITY OF LIFE & $\boldsymbol{M}$ & $\mathbf{M}$ & M & - & - \\
\hline * LRGST EXP TO GDP & 20.00 & 10.50 & $\mathbf{M}$ & -47.50 & - \\
\hline X EXP LGST PARTNER & 93.00 & 77.00 & 88.00 & -17.20 & 14.29 \\
\hline X IIIP LGST PARTHER & 85.00 & 79.00 & 69.00 & -7.06 & -12.66 \\
\hline X EXP MANUFAC. & M & $\boldsymbol{M}$ & $\mathbf{M}$ & - & - \\
\hline X EXP AGR-MINE & $\mathbf{M A}$ & $\mathbf{M}$ & Ma & - & - \\
\hline X IRP MUNUFAC. & MA & $\mathbf{M}$ & NA & - & - \\
\hline X IMP AGR-MINE & MA & MA & MA & - & - \\
\hline TOTAL EXPORTS & 43.00 & 48.00 & 125.00 & 11.63 & 160.42 \\
\hline TOTAL IMPORTS & 39.00 & 106.00 & 664.00 & 171.79 & 526.42 \\
\hline G.N.P. PER CAPITA & Ma & Ma & 4200.00 & - & - \\
\hline
\end{tabular}


RUMANIA

SUMHARY OF NATIONAL STATISTICS

\begin{tabular}{|c|c|c|c|c|c|}
\hline MATIONAL VARIABLES & 1962 & 1970 & 1980 & $62-70$ & $70-80$ \\
\hline EXP. PARTNER CON. & 0.59 & 0.42 & 0.34 & -28.81 & -19.05 \\
\hline IMP. PARTNER CON. & 0.58 & 0.42 & 0.35 & -27.59 & -16.67 \\
\hline EPC/IPC RATIO & 1.01 & 1.02 & 0.99 & 0.99 & -2.94 \\
\hline EXP CONH CONCEN & MA & M & $M$ & - & - \\
\hline IMP COMH CONCEN & NA & NA & Nh & - & - \\
\hline ECC/ICC RATIO & NA & NA & M & - & - \\
\hline VERTICAL TRADE & $M$ & MA & M & - & - \\
\hline TOT TRADE LINKAGES & 28.00 & 43.00 & 70.00 & 53.57 & 62.79 \\
\hline \% AGRICULTURE-GDP & MA & MA & NA & - & - \\
\hline QUALITY OF LIFE & 76.10 & 83.70 & 91.20 & 9.99 & 8.96 \\
\hline \% LRGST EXP TO GDP & MA & $\boldsymbol{M}$ & $M$ & - & - \\
\hline \% EXP LGST PARTNER & 51.00 & 29.00 & 19.00 & -43.14 & -34.48 \\
\hline X IMP LGST PARTNEER & 48.00 & 26.00 & 17.00 & -45.83 & -34.62 \\
\hline X EXP maiufac. & NA & M & M & - & - \\
\hline X EXP AGR-MINE & NA & M & Mn & - & - \\
\hline X IHP naNUfaC. & NA & MA & N & - & - \\
\hline X IMP AGR-AIKE & MA & MA & $M$ & - & - \\
\hline TOTAL EXPORTS & 682.00 & 1807.00 & 11806.00 & 164.96 & 553.35 \\
\hline TOTAL IHPORTS & 776.00 & 1880.00 & 12025.00 & 142.27 & 539.63 \\
\hline G.N.P. PER CAPITA & NA & MA & 1890.00 & - & - \\
\hline
\end{tabular}


SAUDI ARAB

SUMMARY OF NATIONAL STATISTICS

\begin{tabular}{|c|c|c|c|c|c|}
\hline MATIONAL VARIABLES & 1962 & 1970 & 1980 & $62-70$ & $70-80$ \\
\hline EXP. PARTNER CON. & 0.42 & 0.43 & 0.41 & 2.38 & -4.65 \\
\hline IMP. PARTMER CON. & 0.47 & 0.41 & 0.42 & -12.77 & 2.44 \\
\hline EPC/IPC RATIO & 0.89 & 1.06 & 0.97 & 19.10 & -8.49 \\
\hline EXP CONH COHCEN & 0.98 & 0.99 & 0.97 & 1.02 & -2.02 \\
\hline IHP CONH CONCEN & 0.32 & 0.30 & 0.36 & -6.25 & 20.00 \\
\hline ECC/ICC RATIO & 3.06 & 3.30 & 2.69 & 7.76 & -18.35 \\
\hline VERTICAL TRADE & -0.62 & -0.63 & -0.82 & 1.61 & 30.16 \\
\hline TOT TRADE LINKAGES & 28.00 & 42.00 & 63.00 & 50.00 & 50.00 \\
\hline X AGRICULTURE-GDP & MA & 7.00 & 1.30 & - & -81.43 \\
\hline QUALITY OF LIFE & 11.80 & 26.60 & 39.40 & 125.42 & 48.12 \\
\hline X LRGST EXP TO GDP & 6.70 & 13.40 & 16.20 & 100.00 & 20.90 \\
\hline X EXP LGST PARTNER & 17.00 & 23.00 & 17.00 & 35.29 & -26.09 \\
\hline X IHP LGST PARTNER & 31.00 & 19.00 & 20.00 & -38.71 & 5.26 \\
\hline X EXP MUNUFAC. & $\mathrm{NA}$ & $\boldsymbol{M}$ & $M$ & - & - \\
\hline X EXP AGR-HINE & 99.00 & $\mathbf{9 9 . 0 0}$ & 99.00 & 0.00 & 0.00 \\
\hline X IAP MANUFAC. & 62.00 & 63.00 & 82.00 & 1.61 & 30.16 \\
\hline Z IMP AGR-MINE & 37.00 & 36.00 & 17.00 & -2.70 & -52.78 \\
\hline TOTAL EXPORTS & 944.00 & \multicolumn{2}{|c|}{2233.0099904 .00} & 136.55 & 4373.98 \\
\hline TOTAL IMPORTS & 246.00 & \multicolumn{2}{|c|}{721.0028045 .00} & 193.09 & 3789.74 \\
\hline G.N.P. PER & NA & MA & 12600.00 & - & - \\
\hline
\end{tabular}


SEMEGAL

SUMMARY OF NATIONAL STATISTICS

\begin{tabular}{|c|c|c|c|c|c|}
\hline MATIONAL VARIABLES & 1962 & 1970 & 1980 & $62-70$ & $70-80$ \\
\hline EXP. PARTNER CON. & 0.88 & 0.75 & 0.48 & -14.77 & -36.00 \\
\hline IIIP. PARTNER CON. & 0.71 & 0.57 & 0.51 & -19.72 & -10.53 \\
\hline EPC/IPC RATIO & 1.23 & 1.32 & 0.94 & 7.32 & -28.79 \\
\hline EXP COHM CONCEN & 0.72 & 0.33 & 0.24 & -54.17 & -27.27 \\
\hline IMP COMH CONCEN & 0.30 & 0.24 & 0.19 & -20.00 & -20.83 \\
\hline ECC/ICC RATIO & 2.40 & 1.38 & 1.26 & -42.71 & -8.13 \\
\hline VERTICAL TRADE & -0.57 & -0.44 & -0.34 & -22.81 & -22.73 \\
\hline TOT TRADE LINKAGES & 5.00 & 10.00 & 34.00 & 100.00 & 240.00 \\
\hline X AGRICULTURE-GDP & 24.30 & 24.70 & 24.60 & 1.65 & -0.60 \\
\hline QUALITY OF LIFE & 8.30 & 15.10 & 19.10 & 81.93 & 26.49 \\
\hline X LRGST EXP TO GDP & 15.00 & 8.20 & 15.70 & -45.33 & 91.46 \\
\hline X EXP LGST PARTNER & 89.00 & 72.00 & 36.00 & -19.10 & -50.00 \\
\hline X IMP LGST PARTNER & 67.00 & 51.00 & 39.00 & -23.88 & -23.53 \\
\hline X EXP muKufaC. & 3.00 & 18.00 & 15.00 & 500.00 & -16.67 \\
\hline Х EXP AGR-HINE & 96.00 & 81.00 & 85.00 & -15.63 & 4.94 \\
\hline X IMP maUfaC. & 60.00 & 62.00 & 48.00 & 3.33 & -22.58 \\
\hline$X$ IMP AGR-HINE & 39.00 & 37.00 & 51.00 & -5.13 & 37.84 \\
\hline TOTAL EXPORTS & 119.00 & 113.00 & 421.00 & -5.04 & 272.57 \\
\hline TOTAL IMPORTS & 161.00 & 176.00 & 1044.00 & 9.32 & 493.18 \\
\hline G.N.P. PER CAP & MA & MA & 430.00 & - & - \\
\hline
\end{tabular}




\begin{tabular}{|c|c|c|c|c|c|}
\hline MATIONAL VARIABLES & 1962 & 1970 & 1980 & $62-70$ & $70-80$ \\
\hline EXP. PARTMER CON. & 0.82 & 0.79 & 0.76 & -3.66 & -3.80 \\
\hline IHP. PARTNER CON. & 0.69 & 0.47 & 0.50 & -31.88 & 6.38 \\
\hline EPC/IPC RATIO & 1.19 & 1.69 & 1.51 & 42.02 & -10.65 \\
\hline EXP COHH CONCEN & 0.30 & 0.39 & $\mathbf{M}$ & 30.00 & - \\
\hline IMP CONH CONCEN & MA & 0.30 & $\mathbf{M}$ & - & - \\
\hline ECC/ICC RATIO & NA & 1.30 & Ma & - & - \\
\hline VERTICAL TRADE & M & -0.07 & $\boldsymbol{m}$ & - & - \\
\hline TOT TRADE LINKAGES & 3.00 & 7.00 & 5.00 & 133.33 & -28.57 \\
\hline X AGRICULTURE-GDP & NA & 34.00 & 33.60 & - & -1.18 \\
\hline QUALITY OF LIFE & 0.80 & 8.40 & 13.30 & 950.00 & 58.33 \\
\hline \% LRGST EXP TO GDP & 9.20 & 13.40 & 11.90 & 45.65 & -11.19 \\
\hline * EXP LGST PARTNER & 70.00 & 75.00 & 58.00 & 7.14 & -22.67 \\
\hline X IAP LGST PARTNER & 58.00 & 32.00 & 26.00 & -44.83 & -18.75 \\
\hline X EXP MANUFAC. & $\boldsymbol{M}$ & 61.00 & $\mathbf{M}$ & - & - \\
\hline Z EXP AGR-HINE & Ma & 38.00 & $\mathbf{M}$ & - & - \\
\hline \% IHP RANUFAC. & $\mathbf{M A}$ & 68.00 & $\mathrm{~m}$ & - & - \\
\hline X IHP AGR-MINE & NA & 31.00 & MA & - & - \\
\hline TOTAL EXPORTS & 45.00 & 96.00 & 153.00 & 113.33 & 59.38 \\
\hline TOTAL IMPORTS & 54.00 & 92.00 & 326.00 & 70.37 & 254.35 \\
\hline G.N.P. PER & NA & NA & 20.00 & - & - \\
\hline
\end{tabular}


SINGAPORE

SUMAARY OF NATIONAL STATISTICS

\begin{tabular}{|c|c|c|c|c|c|}
\hline MATIONAL VARIABLES & 1962 & 1970 & 1980 & $62-70$ & $70-80$ \\
\hline EXP. PARTNER CON. & 0.42 & 0.38 & 0.34 & -9.52 & -10.53 \\
\hline IHP. PARTNER CON. & 0.50 & 0.42 & 0.62 & -16.00 & 0.00 \\
\hline EPC/IPC RATIO & 0.84 & 0.89 & 0.81 & 5.95 & -8.99 \\
\hline EXP CONH CONCEN & 0.05 & 0.03 & 0.07 & -40.00 & 133.33 \\
\hline IMP CONH CONCEN & 0.11 & 0.12 & 0.16 & 9.09 & 33.33 \\
\hline ECC/ICC RATIO & 0.45 & 0.25 & 0.44 & -45.00 & 75.00 \\
\hline VERTICAL TRADE & -0.07 & -0.28 & -0.01 & 300.00 & -96.43 \\
\hline TOT TRADE LINKAGES & 45.00 & 71.00 & 93.00 & 57.78 & 30.99 \\
\hline \% AGRICULTURE-GDP & 3.50 & 2.90 & 1.70 & -17.14 & -41.38 \\
\hline QUALITY OF LIFE & 70.30 & 80.70 & 89.20 & 14.79 & 10.53 \\
\hline * LRGST EXP TO GDP & NA & MA & $\boldsymbol{M}$ & - & - \\
\hline X EXP LGST PARTMER & 30.00 & 22.00 & 15.00 & -26.67 & -31.82 \\
\hline \% IIIP lGST PARTNER & $27 . \infty 0$ & 18.00 & 15.00 & -33.33 & -16.67 \\
\hline X EXP manufac. & 29.00 & 30.00 & 54.00 & 3.45 & 80.00 \\
\hline X EXP AGR-HINE & 70.00 & 69.00 & 46.00 & -1.43 & -33.33 \\
\hline X InP HANUFAC. & 36.00 & 58.00 & 55.00 & 61.11 & -5.17 \\
\hline X IMP AGR-HINE & 63.00 & 41.00 & 44.00 & -34.92 & 7.32 \\
\hline TOTAL EXPORTS & 1009.00 & 1497.00 & 18415.00 & 48.36 & 1130.13 \\
\hline TOTAL IMPORTS & 966.00 & 2292.00 & 25762.00 & 137.27 & 1024.00 \\
\hline G.N.P. PER CAPITA & MA & $\mathbf{M A}$ & 5240.00 & - & - \\
\hline
\end{tabular}


SOMALI

SUMHARY OF NATIONAL STATISTICS

\begin{tabular}{|c|c|c|c|c|c|}
\hline MATIONAL VARIABLES & 1962 & 1970 & 1980 & $62-70$ & $70-80$ \\
\hline EXP. PARTNER CON. & 0.87 & 0.77 & 0.77 & -11.49 & 0.00 \\
\hline IMP. PARTNER COH. & 0.69 & 0.47 & 0.54 & -31.88 & 14.89 \\
\hline EPC/IPC RATIO & 1.26 & 1.65 & 1.43 & 30.95 & -13.33 \\
\hline EXP COHN CONCEN & 0.66 & 0.65 & 0.73 & -1.52 & 12.31 \\
\hline IHP COHA COHCEN & 0.29 & 0.22 & 0.21 & -24.14 & -4.55 \\
\hline ECC/ICC RATIO & 2.28 & 2.95 & 3.48 & 29.82 & 17.66 \\
\hline VERTICAL TRADE & -0.58 & -0.48 & -0.67 & -17.24 & 39.58 \\
\hline TOT TRADE LINKAGES & 2.00 & $\mathbf{3 . 0 0}$ & $\mathbf{8 . 0 0}$ & 50.00 & 166.67 \\
\hline X AGRICULTURE-GDP & 71.10 & 66.80 & 59.20 & -6.05 & -11.38 \\
\hline QUALITY OF LIFE & 6.10 & 11.70 & 33.20 & 91.80 & 183.76 \\
\hline X LRGST EXP TO GDP & MA & $\mathbf{M}$ & $\mathbf{M A}$ & - & - \\
\hline \% EXP LGST PARTMER & 78.00 & 56.00 & 69.00 & -28.21 & 23.21 \\
\hline X IMP LGST PARTHER & 41.00 & 23.00 & 40.00 & -43.90 & 73.91 \\
\hline X EXP MANUFAC. & 2.00 & 6.00 & 1.00 & 200.00 & -83.33 \\
\hline * EXP AGR-MINE & 97.00 & 93.00 & 99.00 & -4.12 & 6.45 \\
\hline X IMP MANUFAC. & 60.00 & 53.00 & 68.00 & -11.67 & 28.30 \\
\hline X IMP AGR-HINE & 40.00 & 46.00 & 31.00 & 15.00 & -32.61 \\
\hline TOTAL EXPORTS & 22.00 & 29.00 & 132.00 & 31.82 & 355.17 \\
\hline TOTAL IMPORTS & 27.00 & 49.00 & 453.00 & 81.48 & 824.49 \\
\hline G.N.P. PER CAPITA & NA & $\mathbf{N A}$ & 280.00 & - & - \\
\hline
\end{tabular}


SOUTH AFRI

SUMMARY OF NATIONAL STATISTICS

\begin{tabular}{|c|c|c|c|c|c|}
\hline NATIONAL VARIABLES & 1962 & 1970 & 1980 & $62-70$ & $70-80$ \\
\hline EXP. PARTNER CON. & 0.51 & 0.55 & 0.49 & 7.84 & -10.91 \\
\hline IMP. PARTNER CON. & 0.47 & 0.49 & 0.52 & 4.26 & 6.12 \\
\hline EPC/IPC RATIO & 1.07 & 1.12 & 0.94 & 4.67 & -16.07 \\
\hline EXP CONH CONCEN & 0.09 & 0.09 & 0.19 & 0.00 & 111.11 \\
\hline IMP COMH CONCEN & MA & 0.30 & 0.38 & - & 26.67 \\
\hline ECC/ICC RATIO & M & 0.30 & 0.50 & - & 66.67 \\
\hline VERTICAL TRADE & Ma & -0.42 & -0.35 & - & -16.67 \\
\hline TOT TRADE LINKAGES & 40.00 & 29.00 & 38.00 & -27.50 & 31.03 \\
\hline X AGRICULTURE-GDP & 12.30 & 10.50 & 7.70 & -14.63 & -26.67 \\
\hline QUALITY OF LIFE & 45.80 & M & 59.60 & - & - \\
\hline X LRGST EXP TO GDP & 5.60 & 3.00 & 3.40 & -46.43 & 13.33 \\
\hline X EXP LGST PARTNNER & 38.00 & 37.00 & 18.00 & -2.63 & -51.35 \\
\hline X IMP LGST PARTNER & 31.00 & 23.00 & 19.00 & -25.81 & -17.39 \\
\hline X EXP maNufAC. & Ma & 40.00 & 53.00 & - & 32.50 \\
\hline \% EXP AGR-MINe & MA & 59.00 & 46.00 & - & -22.03 \\
\hline X IMP MANUFAC. & NA & 83.00 & 88.00 & - & 6.02 \\
\hline X IMP AGR-MINE & MA & 17.00 & 11.00 & - & -35.29 \\
\hline TOTAL EXPORTS & 1056.00 & 1671.00 & 11538.00 & 58.24 & 590.48 \\
\hline TOTAL IMPORTS & 1317.00 & 3365.00 & 13094.00 & 155.50 & 289.12 \\
\hline G.N.P. PER CAPITA & $\boldsymbol{N}$ & $\mathbf{M}$ & 2770.00 & - & - \\
\hline
\end{tabular}


SPAIN

SUMHARY OF NATIONAL STATISTICS

\begin{tabular}{|c|c|c|c|c|c|}
\hline MATIONAL VARIABLES & 1962 & 1970 & 1980 & $62-70$ & $70-80$ \\
\hline EXP. PARTNER CON. & 0.38 & 0.36 & 0.31 & -5.26 & -13.89 \\
\hline IHP. PARTMER COH. & 0.36 & 0.36 & 0.33 & 0.00 & -8.33 \\
\hline EPC/IPC RATIO & 1.05 & 0.99 & 0.94 & -5.71 & -5.05 \\
\hline EXP COMH CONCEN & 0.23 & 0.16 & 0.19 & -30.43 & 18.75 \\
\hline IHP COAH CONCEN & 0.04 & 0.06 & 0.12 & 50.00 & 100.00 \\
\hline ECC/ICC RATIO & 5.75 & 2.67 & 1.58 & -53.62 & -40.63 \\
\hline VERTICAL TRADE & -0.18 & -0.01 & 0.34 & -94.44 & -3500.00 \\
\hline TOT TRADE LINKAGES & 52.00 & 62.00 & 118.00 & 19.23 & 90.32 \\
\hline X AGRICULTURE-GDP & M & 13.10 & 8.20 & - & -37.40 \\
\hline QUALITY OF LIFE & 82.20 & 89.40 & 94.40 & 8.76 & 5.59 \\
\hline X LRGST EXP TO GDP & 1.00 & 1.20 & 1.20 & 20.00 & 0.00 \\
\hline X EXP LGST PARTNER & 16.00 & 14.00 & 16.00 & -12.50 & 14.29 \\
\hline X IHP LGST PARTNER & 15.00 & 17.00 & 10.00 & 13.33 & -49.18 \\
\hline * eXp munufac. & 27.00 & 53.00 & $71 . \infty 0$ & 96.30 & 33.96 \\
\hline \% EXP AGR-HINE & 72.00 & 46.00 & 28.00 & -36.11 & -39.13 \\
\hline \% InP nawUfac. & 46.00 & 54.00 & 38.00 & 17.39 & -29.63 \\
\hline X IMP AGR-MINE & 54.00 & 45.00 & 61.00 & -16.67 & 35.56 \\
\hline TOTAL EXPORTS & 700.00 & 2270.00 & 20348.00 & 224.29 & 796.39 \\
\hline TOTAL IHPORTS & 1533.00 & 4234.00 & 30873.00 & 176.19 & 629.17 \\
\hline G.N.P. PER CAPITA & Mh & M & 5640.00 & - & - \\
\hline
\end{tabular}


SUDAN

SUMAARY OF NATIONAL STATISTICS

\begin{tabular}{|c|c|c|c|c|c|}
\hline NATIONAL VARIABLES & 1962 & 1970 & 1980 & $62-70$ & $70-80$ \\
\hline EXP. PARTNER CON. & 0.43 & 0.43 & 0.42 & 0.00 & -2.33 \\
\hline IMP. PARTNER CON. & 0.44 & 0.40 & 0.40 & -9.09 & 0.00 \\
\hline EPC/IPC RATIO & 0.96 & 1.08 & 1.05 & 12.50 & -2.78 \\
\hline EXP CONH COHCEN & 0.57 & 0.62 & 0.50 & 8.77 & -19.35 \\
\hline IHP CONH CONCEN & 0.29 & 0.23 & 0.21 & -20.69 & -8.70 \\
\hline ECC/ICC RATIO & 1.97 & 2.70 & 2.38 & 37.15 & -11.67 \\
\hline VERTICAL TRADE & -0.77 & -0.67 & -0.59 & -12.99 & -11.94 \\
\hline TOT TRADE LINKAGES & 21.00 & 25.00 & 34.00 & 19.05 & 36.00 \\
\hline X AGRICULTURE-GDP & MA & 41.10 & 38.80 & - & -5.60 \\
\hline QUALITY OF LIFE & 15.00 & 20.50 & 33.50 & 36.67 & 63.41 \\
\hline X LRGST EXP TO GDP & 2.80 & Ma & MA & -3635.71 & 0.00 \\
\hline X EXP LGST PARTNER & 18.00 & 15.00 & 21.00 & -16.67 & 40.00 \\
\hline X IMP LGST PARTNER & 28.00 & 16.00 & 17.00 & -62.86 & 6.25 \\
\hline X EXP MANUFAC. & $\mathbf{M}$ & M & $\mathbf{M A}$ & - & - \\
\hline X EXP AGR-MINE & 99.00 & 99.00 & 99.00 & $0 . \infty$ & 0.00 \\
\hline X IMP MAHUFAC. & $77 . \infty$ & 67.00 & 59.00 & -12.99 & -11.94 \\
\hline$X$ IMP AGR-HINE & 22.00 & 32.00 & 40.00 & 45.45 & 25.00 \\
\hline TOTAL EXPORTS & 220.00 & 294.00 & 533.00 & 33.64 & 81.29 \\
\hline TOTAL IMPORTS & 234.00 & 272.00 & 1730.00 & 16.24 & 536.03 \\
\hline G.M.P. & NA & $\boldsymbol{N}$ & 380.00 & 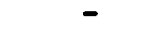 & - \\
\hline
\end{tabular}


SURIMAM

SUAHARY OF MATIONAL STATISTICS

\begin{tabular}{|c|c|c|c|c|c|}
\hline MATIOHAL VARIABLES & 1962 & 1970 & 1980 & $62-70$ & $70-80$ \\
\hline EXP. PARTHER CON. & 0.74 & 0.61 & 0.53 & -17.57 & -13.11 \\
\hline IMP. PARTNER CON. & 0.66 & 0.59 & 0.61 & -10.61 & 3.39 \\
\hline EPC/IPC RATIO & 1.13 & 1.03 & 0.87 & -8.85 & -15.53 \\
\hline EXP COHH CONCEN & 0.62 & 0.77 & $M$ & 24.19 & - \\
\hline IMP CONH CONCEN & 0.23 & 0.15 & 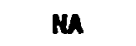 & -34.78 & - \\
\hline ECC/ICC RATIO & 2.70 & 5.13 & M & 90.43 & - \\
\hline VERTICAL TRADE & -0.62 & -0.63 & MA & 1.61 & - \\
\hline TOT TRADE LINKAGES & 5.00 & 12.00 & 20.00 & 140.00 & 66.67 \\
\hline X AGRICULTURE-GDP & 16.10 & 13.00 & 10.60 & -19.25 & -18.46 \\
\hline QUALITY OF LIFE & 70.90 & 76.80 & 76.60 & 8.32 & -0.26 \\
\hline * LRGST EXP TO GDP & 29.30 & 22.10 & 15.20 & -24.57 & -31.22 \\
\hline X EXP LGST PARTMER & 61.00 & 40.00 & 21.00 & -34.43 & -47.50 \\
\hline * IMP lgSt PARTNER & 40.00 & 40.00 & 32.00 & $0 . \infty 0$ & -20.00 \\
\hline Х EXP MANUFAC. & 7.00 & 1.00 & $\mathbf{M}$ & -85.71 & - \\
\hline \% EXP AGR-MINE & 92.00 & 98.00 & MA & 6.52 & - \\
\hline \% IHP MANUFAC. & 69.00 & 65.00 & $\mathbf{M}$ & -5.80 & - \\
\hline Х IHP MGR-MINE & 30.00 & 35.00 & $\mathbf{M}$ & 16.67 & - \\
\hline TOTAL EXPORTS & 52.00 & 127.00 & 519.00 & 144.23 & 308.66 \\
\hline TOTAL IMPORTS & 38.00 & 87.00 & 436.00 & 128.95 & 401.15 \\
\hline G.H.P. PER CAPITA & $\boldsymbol{M}$ & NA & 3030.00 & - & - \\
\hline
\end{tabular}


SUEDEN

SUMMARY OF NATIONAL STATISTICS

\begin{tabular}{|c|c|c|c|c|c|}
\hline MATIONAL VARIABLES & 1962 & 1970 & 1980 & $62-70$ & $70-80$ \\
\hline EXP. PARTNER COH. & 0.37 & 0.36 & 0.33 & -2.70 & -8.33 \\
\hline IMP. PARTNER COH. & 0.41 & 0.40 & 0.39 & -2.44 & -2.50 \\
\hline EPC/IPC RATIO & 0.89 & 0.91 & 0.85 & 2.25 & -6.59 \\
\hline EXP CONH CONCEN & 0.13 & 0.19 & 0.21 & 46.15 & 10.53 \\
\hline IHP COMH CONCEN & 0.12 & 0.17 & 0.18 & 41.67 & 5.88 \\
\hline ECC/ICC RATIO & 1.08 & 1.12 & 1.17 & 3.17 & 4.39 \\
\hline VERTICAL TRADE & 0.04 & 0.06 & 0.17 & 50.00 & 183.33 \\
\hline TOT TRADE LINKAGES & 73.00 & 92.00 & 115.00 & 26.03 & 25.00 \\
\hline X AGRICULTURE-GDP & NA & 5.30 & 3.70 & - & -30.19 \\
\hline QUALITY OF LIFE & 95.20 & 97.10 & 99.60 & 2.00 & 2.57 \\
\hline X LRGST EXP TO GOP & 3.00 & 2.80 & 2.70 & -6.67 & $-3.5 i$ \\
\hline X EXP LEST PARTNER & 15.00 & 12.00 & 12.00 & -20.00 & 0.00 \\
\hline X IMP LGST PARTNER & 23.00 & 19.00 & 17.00 & -17.39 & -10.53 \\
\hline X EXP manufac. & 67.00 & 74.00 & 79.00 & 10.45 & 6.76 \\
\hline X EXP MGR-MINE & 33.00 & 25.00 & 20.00 & -24.24 & -20.00 \\
\hline X IMP MANUFAC. & 62.00 & 68.00 & 62.00 & 9.68 & -8.82 \\
\hline X IMP AGR-MINE & 37.00 & 31.00 & 38.00 & -16.22 & 22.58 \\
\hline TOTAL EXPORTS & 2852.00 & 6694.00 & 30721.00 & 134.71 & 358.93 \\
\hline TOTAL IHPORTS & 2860.00 & 6425.00 & 31316.00 & 124.65 & 387.41 \\
\hline G.H.P. PER CAPITA & MA & MA & 14840.00 & - & - \\
\hline
\end{tabular}


SUITZERLAN

SUMHARY OF MATIONAL STATISTICS

\begin{tabular}{|c|c|c|c|c|c|}
\hline MATIONAL VARIABLES & 1962 & 1970 & 1980 & $62-70$ & $70-80$ \\
\hline EXP. PARTNER CON. & 0.32 & 0.31 & 0.33 & -3.12 & 6.45 \\
\hline IHP. PARTNER CON. & 0.50 & 0.49 & 0.44 & -2.00 & -10.20 \\
\hline EPC/IPC RATIO & 0.64 & 0.64 & 0.75 & 0.00 & 17.19 \\
\hline EXP CONH CONCEN & 0.40 & 0.37 & 0.38 & -7.50 & 2.70 \\
\hline IHP COMH CONCEN & 0.16 & 0.22 & 0.20 & 37.50 & -9.09 \\
\hline ECC/ICC RATIO & 2.50 & 1.68 & 1.90 & -32.73 & 12.97 \\
\hline VERTICAL TRADE & 0.25 & 0.17 & 0.19 & -32.00 & 11.76 \\
\hline TOT TRADE LINKAGES & 74.00 & 98.00 & 118.00 & 32.43 & 20.41 \\
\hline \% AGRICULTURE-GDP & MA & M & $\boldsymbol{m}$ & - & - \\
\hline QUALITY OF LIFE & 92.90 & 94.70 & 98.50 & 1.94 & 4.01 \\
\hline * LRGST EXP TO GBP & 3.80 & 3.60 & 4.00 & -5.26 & 11.11 \\
\hline ₹ EXP LGST PARTNER & 17.00 & 15.00 & 19.00 & -11.76 & 26.67 \\
\hline X IMP LGST PARTNER & 32.00 & 31.00 & 25.00 & -3.13 & -19.35 \\
\hline X EXP manufac. & 91.00 & 89.00 & $90 . \infty 0$ & -2.20 & 1.12 \\
\hline \% EXP AGR-MINE & 8.00 & 10.00 & 9.00 & 25.00 & -10.00 \\
\hline X IHP manufaC. & 66.00 & 72.00 & 71.00 & 9.09 & -1.39 \\
\hline X IHP AGR-HINE & 33.00 & 27.00 & 28.00 & -18.18 & 3.70 \\
\hline TOTAL EXPORTS & 2208.00 & 5081.00 & 29493.00 & 130.12 & 480.46 \\
\hline TOTAL IHPORTS & 3044.00 & 6741.00 & 42436.00 & 121.45 & 529.52 \\
\hline G.N.P. PER CAPIT/ & MA & $\mathbf{M}$ & 17430.00 & - & - \\
\hline
\end{tabular}


SYRIA

SUMMARY OF MATIONAL STATISTICS

\begin{tabular}{|c|c|c|c|c|c|}
\hline MTIONAL VARIABLES & 1962 & 1970 & 1980 & $62-70$ & $70-80$ \\
\hline EXP. PARTNER CON. & 0.40 & 0.43 & 0.64 & 7.50 & 48.84 \\
\hline IHP. PARTNER CON. & 0.39 & 0.35 & 0.37 & -10.26 & 5.71 \\
\hline EPC/IPC RATIO & 1.02 & 1.24 & 1.72 & 21.57 & 38.71 \\
\hline EXP CONH CONCEN & 0.28 & 0.19 & 0.46 & -32.14 & 142.11 \\
\hline IMP CONH CONCEN & 0.27 & 0.19 & 0.17 & -29.63 & -10.53 \\
\hline ECC/ICC RATIO & 1.04 & 1.00 & 2.71 & -3.57 & 170.59 \\
\hline VERTICAL TRADE & -0.65 & -0.45 & -0.50 & -30.77 & 11.11 \\
\hline TOT TRADE LINKAGES & 24.00 & 25.00 & 30.00 & 4.17 & 20.00 \\
\hline X AGRICULTURE-GDP & MA & 25.10 & 19.30 & - & -23.11 \\
\hline QUALITY OF LIFE & 34.50 & $50 . \infty 0$ & 67.70 & 44.93 & 35.40 \\
\hline * LRGST EXP TO GDP & M & M & m & - & - \\
\hline * EXP LGST PARTNER & 15.00 & 21.00 & 56.00 & 40.00 & 166.67 \\
\hline X IHP LGST PARTNER & 14.00 & 8.00 & 15.00 & -42.86 & 87.50 \\
\hline X EXP manUfac. & 5.00 & 11.00 & 7.00 & 120.00 & -36.36 \\
\hline X EXP AGR-MINE & 94.00 & 88.00 & 92.00 & -6.38 & 4.55 \\
\hline X InP manUfaC. & 70.00 & 56.00 & 57.00 & -20.00 & 1.79 \\
\hline$X$ IAP AGR-HINE & 29.00 & 43.00 & 42.00 & 48.28 & -2.33 \\
\hline TOTAL EXPORTS & 166.00 & 201.00 & 2052.00 & 21.08 & 920.90 \\
\hline TOTAL IMPORTS & 203.00 & 294.00 & 4318.00 & 44.83 & 1368.71 \\
\hline G.N.P. PER CAPIT & MA & MA & 1570.00 & - & - \\
\hline
\end{tabular}


TANzanta

SUMMARY OF NATIONAL STATISTICS

\begin{tabular}{|c|c|c|c|c|c|}
\hline MATIONAL VARIABLES & 1962 & 1970 & 1980 & $62-70$ & $70-80$ \\
\hline EXP. PARTNER CON. & 0.54 & 0.39 & 0.38 & -27.78 & -2.56 \\
\hline IMP. PARTHER CON. & 0.64 & 0.43 & 0.39 & -32.81 & -9.30 \\
\hline EPC/IPC RATIO & 0.85 & 0.92 & 0.98 & 8.24 & 6.52 \\
\hline EXP CONH COHCEN & $\mathbf{M}$ & 0.28 & 0.28 & - & 0.00 \\
\hline IMP CONH CONCEN & MA & 0.32 & 0.20 & - & -37.50 \\
\hline ECC/ICC RATIO & MA & 0.88 & 1.40 & - & 60.00 \\
\hline VERTICAL TRADE & NA & -0.70 & -0.49 & - & -30.00 \\
\hline TOT TRADE LINKAGES & 15.00 & 29.00 & 37.00 & 93.33 & 27.59 \\
\hline X AGRICULTURE-GDP & $\mathbf{M}$ & 43.60 & 47.60 & - & 9.17 \\
\hline QUALITY OF LIFE & 18.10 & MA & 56.60 & - & - \\
\hline ※ LRGST EXP TO GDP & M & MA & $\mathbf{M a}$ & - & - \\
\hline X EXP LGST PARTNER & 37.00 & 20.00 & 16.00 & -45.95 & -20.00 \\
\hline X IMP LGST PARTHER & 45.00 & 17.00 & 20.00 & -62.22 & 17.65 \\
\hline \% EXP MANUFAC. & Ma & 12.00 & 14.00 & - & 16.67 \\
\hline X EXP AGR-MIHE & $\mathbf{M A}$ & 87.00 & 85.00 & - & -2.30 \\
\hline$X$ IMP MANUFAC. & M & 82.00 & 62.00 & - & -24.39 \\
\hline X IMP AGR-NIME & MA & $17 . \infty 0$ & 37.00 & - & 117.65 \\
\hline TOTAL EXPORTS & 138.00 & 255.00 & 478.00 & 84.78 & 87.45 \\
\hline TOTAL IMPORTS & 58.00 & 272.00 & 1254.00 & 368.97 & 361.03 \\
\hline G.H.P. PER CAPITA & MA & $\mathbf{M}$ & 280.00 & - & - \\
\hline
\end{tabular}


THAILAND

SUMMARY OF NATIONAL STATISTICS

\begin{tabular}{|c|c|c|c|c|c|}
\hline MATIONAL VARIABLES & 1962 & 1970 & 1980 & $62-70$ & $70-80$ \\
\hline EXP. PARTNER CON. & 0.45 & 0.45 & 0.39 & 0.00 & -13.33 \\
\hline IMP. PARTMER CON. & 0.50 & 0.50 & 0.44 & 0.00 & -12.00 \\
\hline EPC/IPC RATIO & 0.90 & 0.90 & 0.88 & 0.00 & -2.22 \\
\hline EXP COMH CONCEN & 0.37 & 0.27 & 0.15 & -27.03 & -44.44 \\
\hline IHP CONH CONCEN & 0.29 & 0.25 & 0.18 & -13.79 & -28.00 \\
\hline ECC/ICC RATIO & 1.28 & 1.08 & 0.83 & -15.35 & -22.84 \\
\hline VERTICAL TRADE & -0.75 & -0.71 & -0.29 & -5.33 & -59.15 \\
\hline TOT TRADE LIMKAGES & 28.00 & 32.00 & 73.00 & 14.29 & 128.13 \\
\hline X AGRICULTURE-GDP & 39.80 & 33.60 & 27.70 & -15.58 & -17.56 \\
\hline QUALITY OF LIFE & 53.90 & 66.60 & 76.10 & 23.56 & 14.26 \\
\hline * LRGST EXP TO GDP & 2.30 & 2.90 & 4.80 & 26.09 & 65.52 \\
\hline X EXP LGST PARTNER & 15.00 & 25.00 & 15.00 & 66.67 & $-\$ 0.00$ \\
\hline X IMP LGST PARTNER & 29.00 & 38.00 & 22.00 & 31.03 & -42.11 \\
\hline × eXP manufac. & 3.00 & 8.00 & $28 . \infty 0$ & 166.67 & 250.00 \\
\hline X EXP AGR-NINE & 96.00 & 91.00 & 71.00 & -5.21 & -21.98 \\
\hline X IMP nANUFAC. & 78.00 & 78.00 & 57.00 & 0.00 & -26.92 \\
\hline X IHP AGR-HINE & 21.00 & 21.00 & 42.00 & 0.00 & 100.00 \\
\hline TOTAL EXPORTS & 428.00 & 701.00 & 6378.00 & 63.79 & 809.84 \\
\hline TOTAL IMPORTS & 495.00 & 1155.00 & 8575.00 & 133.33 & 642.42 \\
\hline G.K.P. PER CAPITA & M & $\mathbf{M A}$ & 770.00 & - & - \\
\hline
\end{tabular}


TRINIDAD

SUMMARY OF NATIONAL STATISTICS

\begin{tabular}{|c|c|c|c|c|c|}
\hline MATIONAL VARIABLES & 1962 & 1970 & 1980 & $62-70$ & $70-80$ \\
\hline EXP. PARTNER CON. & 0.62 & 0.66 & 0.68 & 6.45 & 3.03 \\
\hline IMP. PARTNER CON. & 0.63 & 0.53 & 0.58 & -15.87 & 9.43 \\
\hline EPC/IPC RATIO & 0.98 & 1.25 & 1.16 & 27.55 & -7.20 \\
\hline EXP CONH CONCEN & 0.69 & 0.55 & 0.80 & -20.29 & 45.45 \\
\hline IHP COHA CONCEN & 0.26 & 0.30 & 0.21 & 15.38 & -30.00 \\
\hline ECC/ICC RATIO & 2.65 & 1.83 & 3.81 & -30.92 & 107.79 \\
\hline VERTICAL TRADE & -0.34 & -0.21 & -0.44 & -38.24 & 109.52 \\
\hline TOT TRADE LINKAGES & 10.00 & 18.00 & 41.00 & 80.00 & 127.78 \\
\hline * AGRICULTTURE-GDP & 8.90 & $\mathbf{5 . 5 0}$ & 3.00 & -38.20 & -45.45 \\
\hline QUALITY OF LIFE & 79.90 & 85.10 & 90.10 & 6.51 & 5.88 \\
\hline X LRGST EXP TO GDP & 14.40 & MA & M & -787.50 & $0 . \infty$ \\
\hline X EXP LGST PARTNER & 31.00 & 58.00 & 66.00 & 87.10 & 13.79 \\
\hline * IMP LGST PARTNER & 27.00 & 24.00 & 31.00 & -11.11 & 29.17 \\
\hline X EXP maNUFAC. & 4.00 & 13.00 & 5.00 & 225.00 & -61.54 \\
\hline X EXP AGR-HINE & 96.00 & 86.00 & 95.00 & -10.42 & 10.47 \\
\hline Z IAP MANUFAC. & 38.00 & 34.00 & 48.00 & -10.53 & 41.18 \\
\hline X IHP AGR-MINE & 62.00 & 65.00 & 51.00 & 4.84 & -21.54 \\
\hline TOTAL EXPORTS & 271.00 & 416.00 & 3488.00 & 53.51 & 738.46 \\
\hline TOTAL IMPORTS & 295.00 & 429.00 & 2330.00 & 45.42 & 443.12 \\
\hline G.N.P. PER & $\mathbf{N} \mathbf{A}$ & $\mathbf{M}$ & 5670.00 & - & - \\
\hline
\end{tabular}


TUMISIA

SUHYARY OF MATIONAL STATISTICS

\begin{tabular}{|c|c|c|c|c|c|}
\hline MATIONAL VARIABLES & 1962 & 1970 & 1980 & $62-70$ & $70-80$ \\
\hline EXP. PARTHER CON. & 0.64 & 0.50 & 0.51 & -21.87 & 2.00 \\
\hline IHP. PARTHER CON. & 0.67 & 0.53 & 0.48 & -20.90 & -9.43 \\
\hline EPC/IPC RATIO & 0.96 & 0.93 & 1.05 & -3.12 & 12.90 \\
\hline EXP CONH CONCEN & 0.38 & 0.26 & 0.37 & -31.58 & 42.31 \\
\hline IHP COMH COACEN & 0.22 & 0.17 & 0.13 & -22.73 & -23.53 \\
\hline ECC/ICC RATIO & 1.73 & 1.53 & 2.85 & -11.46 & 86.09 \\
\hline VERTICAL TRADE & -0.54 & -0.39 & -0.22 & -27.78 & -43.59 \\
\hline TOT TRADE LINKAGES & 15.00 & 21.00 & 42.00 & 40.00 & 100.00 \\
\hline X AGRICULTURE-GDP & MA & 20.50 & 18.30 & - & -10.73 \\
\hline QUALITY OF LIFE & 24.40 & MA & 60.70 & - & - \\
\hline$X$ LRGST EXP TO GDP & 9.80 & $\mathbf{M A}$ & $M$ & -1110.20 & 0.00 \\
\hline X EXP LGST PARTNER & 55.00 & 25.00 & 18.00 & -54.55 & -28.00 \\
\hline X IHP LGST PARTNER & 53.00 & 37.00 & 30.00 & -30.19 & -18.92 \\
\hline X EXP RWNUFAC. & 8.00 & 19.00 & 35.00 & 137.50 & 84.21 \\
\hline Z EXP AGR-HINE & 91.00 & 80.00 & 64.00 & -12.09 & -20.00 \\
\hline Z IMP MARUFAC. & 62.00 & 58.00 & 57.00 & -6.45 & -1.72 \\
\hline * IMP AGR-MINE & 37.00 & 41.00 & 42.00 & 10.81 & 2.44 \\
\hline TOTAL EXPORTS & 110.00 & 174.00 & 2204.00 & 58.18 & 1166.67 \\
\hline TOTAL IHPORTS & 205.00 & 292.00 & 3071.00 & 42.44 & 951.71 \\
\hline G.N.P. & MA & MA & 1420.00 & - & - \\
\hline
\end{tabular}


TURKEY

SUMMARY OF NATIONAL STATISTICS

\begin{tabular}{|c|c|c|c|c|c|}
\hline MATIONAL YARIABLES & 1962 & 1970 & 1980 & $62-70$ & $70-80$ \\
\hline EXP. PARTNER CON. & 0.44 & 0.39 & 0.37 & -11.36 & -5.13 \\
\hline IAP. PARTAER CON. & 0.46 & 0.46 & 0.39 & 0.00 & -15.22 \\
\hline EPC/IPC RATIO & 0.96 & 0.85 & 0.95 & -11.46 & 11.76 \\
\hline EXP COAH CONCEN & 0.46 & 0.28 & 0.21 & -39.13 & -25.00 \\
\hline IMP COAM CONCEN & 0.18 & 0.23 & 0.27 & 27.78 & 17.39 \\
\hline ECC/ICC RATIO & 2.56 & 1.22 & 0.78 & -52.36 & -36.11 \\
\hline VERTICAL TRADE & -0.67 & -0.68 & -0.16 & 1.49 & -76.47 \\
\hline TOT TRADE LINKAGES & 32.00 & 39.00 & 50.00 & 21.88 & 28.21 \\
\hline X AGRICULTURE-GDP & 41.40 & 34.00 & 23.80 & -17.87 & -30.00 \\
\hline QUALITY OF LIFE & 29.30 & 45.30 & 56.50 & 54.61 & 24.72 \\
\hline X LRGST EXP TO GDP & 1.10 & 1.10 & 1.00 & 0.00 & -9.09 \\
\hline X EXP LGST PARTNER & 19.00 & 19.00 & $21 . \infty 0$ & 0.00 & 10.53 \\
\hline X IMP LGST PARTMER & 31.00 & 27.00 & 13.00 & -12.90 & -51.85 \\
\hline X EXP manufac. & 1.00 & 9.00 & 26.00 & 800.00 & 188.89 \\
\hline X EXP AGR-HINE & 98.00 & 91.00 & 73.00 & -7.14 & -19.78 \\
\hline X IMP MANUFAC. & 67.00 & 76.00 & 43.00 & 13.43 & -43.42 \\
\hline X IMP AGR-HINE & 32.00 & 23.00 & 56.00 & -28.13 & 143.48 \\
\hline TOTAL EXPORTS & $\begin{array}{l}390.00 \\
-\end{array}$ & 587.00 & 2874.00 & 50.51 & 389.61 \\
\hline TOTAL IMPORTS & 612.00 & 1144.00 & 7624.00 & 86.93 & 566.43 \\
\hline G.N.P. PER CAPIT & NA & MA & 1540.00 & - & - \\
\hline
\end{tabular}


U.K.

SUMMARY OF MATIONAL STATISTICS

\begin{tabular}{|c|c|c|c|c|c|}
\hline MATIONAL VARIABLES & 1962 & 1970 & 1980 & $62-70$ & $70-80$ \\
\hline EXP. PARTNER COH. & 0.22 & 0.23 & 0.28 & 4.55 & 21.74 \\
\hline IHP. PARTNER CON. & 0.23 & 0.26 & 0.33 & 13.04 & 26.92 \\
\hline EPC/IPC RATIO & 0.94 & 0.92 & 0.85 & -2.13 & -7.61 \\
\hline EXP CONH CONCEN & 0.25 & 0.26 & 0.25 & 4.00 & -3.85 \\
\hline IHP COMH CONCEN & 0.08 & 0.05 & 0.12 & -37.50 & 140.00 \\
\hline ECC/ICC RATIO & 3.13 & 5.20 & 2.08 & 66.40 & -59.94 \\
\hline VERTICAL TRADE & 0.56 & 0.38 & 0.14 & -32.14 & -63.16 \\
\hline TOT TRADE LINKAGES & 112.00 & 126.00 & 141.00 & 12.50 & 11.90 \\
\hline * AGRICULTURE-GDP & 3.40 & 2.80 & 2.20 & -17.65 & -21.43 \\
\hline QUALITY OF LIFE & 90.80 & 93.10 & 96.00 & 2.53 & 3.11 \\
\hline \% LRGST EXP TO GDP & 1.10 & 1.90 & $2 . \infty 0$ & 72.90 & 5.00 \\
\hline X EXP LGST PARTNER & 9.00 & 11.00 & 16.00 & 22.22 & 45.45 \\
\hline X IMP LGST PARTNER & 9.00 & 13.00 & 17.00 & 44.44 & 30.77 \\
\hline X EXP MANUFAC. & 83.00 & 83.00 & 73.00 & 0.00 & -12.05 \\
\hline X EXP AGR-HINE & 17.00 & 16.00 & $22 . \infty 0$ & -5.88 & 37.50 \\
\hline Z IMP MARUFAC. & 27.00 & 45.00 & 61.00 & 66.67 & 35.56 \\
\hline$X$ IMP AGR-MINE & 72.00 & 54.00 & 38.00 & -25.00 & -29.63 \\
\hline TOTAL EXPORTS & \multicolumn{3}{|c|}{10725.0019114 .0108916 .90} & 78.22 & 469.83 \\
\hline TOTAL IMPORTS & \multicolumn{3}{|c|}{10993.0019299 .0102525 .90} & 75.56 & 431.25 \\
\hline G.N.P. PER & M & MA & 9110.00 & - & - \\
\hline
\end{tabular}


U.S.A.

SUMMARY OF NATIONAL STATISTICS

\begin{tabular}{|c|c|c|c|c|c|}
\hline MATIONAL VARIABLES & 1962 & 1970 & 1980 & $62-70$ & $70-80$ \\
\hline EXP. PARTNER CON. & 0.29 & 0.31 & 0.29 & 6.90 & -6.45 \\
\hline IMP. PARTNER CON. & 0.32 & 0.39 & 0.33 & 21.87 & -15.38 \\
\hline EPC/IPC RATIO & 0.90 & 0.81 & 0.88 & -10.00 & 8.64 \\
\hline EXP CONH CONCEN & 0.12 & 0.14 & 0.13 & 16.67 & -7.14 \\
\hline IHP COMH CONCEN & 0.06 & 0.13 & 0.16 & 116.67 & 23.08 \\
\hline ECC/ICC RATIO & 2.00 & 1.08 & 0.81 & -46.15 & -24.55 \\
\hline VERTICAL TRADE & 0.23 & 0.06 & 0.16 & -73.91 & 166.67 \\
\hline TOT TRADE LINKAGES & 96.00 & 115.00 & 131.00 & 19.79 & 13.91 \\
\hline X AGRICULTURE-GDP & 4.00 & 3.10 & 3.00 & -22.50 & -3.23 \\
\hline QUALITY OF LIFE & 91.00 & 92.80 & 97.00 & 1.98 & 4.53 \\
\hline X LRGST EXP TO GDP & M & Ma & м & - & - \\
\hline * EXP LGST PARTNER & $20 . \infty 0$ & $21 . \infty$ & 12.00 & 5.00 & -42.86 \\
\hline \% IMP LGST PARTNER & 22.00 & 26.00 & 9.00 & 18.18 & -65.38 \\
\hline \% EXP muHUfAC. & 65.00 & 70.00 & 67.00 & 7.69 & -4.29 \\
\hline X EXP AGR-HINE & 34.00 & 29.00 & 32.00 & -14.71 & 10.34 \\
\hline * imp hanufac. & 42.00 & 63.00 & $51 . \infty$ & 50.00 & -19.05 \\
\hline X IMP AGR-HINE & 57.00 & 36.00 & $48 . \infty 0$ & -36.84 & 33.33 \\
\hline TOTAL EXPORTS & \multicolumn{3}{|c|}{19086.0042974 .0214549 .90} & 125.16 & 399.26 \\
\hline TOTAL IMPORTS & 15505.00 & 38735.02 & 230553.90 & 149.82 & 495.21 \\
\hline G.N.P. PER CAPITA & NA & MA & 12820.00 & - & - \\
\hline
\end{tabular}


U.S.S.R.

SUMHARY OF MATIONAL STATISTICS

\begin{tabular}{|c|c|c|c|c|c|}
\hline MATIONAL VARIABLES & 1962 & 1970 & 1980 & $62-70$ & $70-80$ \\
\hline EXP. PARTNER CON. & 0.42 & 0.41 & 0.39 & -2.38 & -4.88 \\
\hline IMP. PARTNER CON. & 0.40 & 0.40 & 0.36 & 0.00 & -10.00 \\
\hline EPC/IPC RATIO & 1.04 & 1.03 & 1.08 & -0.96 & 4.85 \\
\hline EXP COHH CONCEN & M & MA & $\mathbf{M}$ & - & - \\
\hline IMP CONM CONCEN & MA & MA & MA & - & - \\
\hline ECC/ICC RATIO & M & MA & M & - & - \\
\hline VERTICAL TRADE & MA & MA & M & - & - \\
\hline TOT TRADE LINKAGES & 49.00 & 69.00 & 83.00 & 40.82 & 20.29 \\
\hline X AGRICULTURE-GDP & MA & Ma & MA & - & - \\
\hline QUALITY OF LIFE & 88.50 & 91.30 & 92.70 & 3.16 & 1.53 \\
\hline X LRGST EXP TO GDP & MA & MA & MA & - & - \\
\hline X EXP LGST PARTNER & 21.00 & 17.00 & 27.00 & -19.05 & 58.82 \\
\hline X IHP LGST PARTNER & 17.00 & 16.00 & 40.00 & -5.88 & 150.00 \\
\hline X EXP manufAc. & MA & MA & M & - & - \\
\hline X EXP AGR-HINE & MA & MA & M & - & - \\
\hline X IMP MANUFAC. & M & M & MA & - & - \\
\hline$X$ IAP AGR-MINE & MA & MA & MA & - & - \\
\hline TOTAL EXPORTS & \multicolumn{3}{|c|}{6305.0011167 .0061490 .00} & 77.11 & 450.64 \\
\hline TOTAL IMPORTS & \multicolumn{3}{|c|}{6138.0010843 .0053996 .00} & 76.65 & 397.98 \\
\hline G.N.P. PER CAPITA & MA & MA & 3200.00 & - & - \\
\hline
\end{tabular}


UGANDA

SUHAARY OF NATIONAL STATISTICS

\begin{tabular}{|c|c|c|c|c|c|}
\hline MATIONAL VARIABLES & 1962 & 1970 & 1980 & $62-70$ & $70-80$ \\
\hline EXP. PARTNER CON. & 0.52 & 0.42 & 0.53 & -19.23 & 26.19 \\
\hline IMP. PARTNER CON. & 0.74 & 0.53 & 0.57 & -28.38 & 7.55 \\
\hline EPC/IPC RATIO & 0.70 & 0.79 & 0.93 & 12.86 & 17.72 \\
\hline EXP COMH CONCEN & 0.45 & 0.48 & M & 6.67 & - \\
\hline IMP COHA CONCEN & 0.56 & 0.41 & $M$ & -26.79 & - \\
\hline ECC/ICC RATIO & 0.80 & 1.17 & M & 45.69 & - \\
\hline VERTICAL TRADE & -0.96 & -0.89 & M & -7.29 & - \\
\hline TOT TRADE LINKAGES & 15.00 & 28.00 & 19.00 & 86.67 & -32.14 \\
\hline X AGRICULTURE-GOP & 52.40 & 51.80 & 73.70 & -1.15 & 42.28 \\
\hline QUALITY OF LIFE & 24.80 & Ma & 45.40 & - & - \\
\hline X LRGST EXP TO GDP & M & 3.90 & NA & - & - \\
\hline X EXP LGST PARTNER & 26.00 & 18.00 & 10.00 & -30.77 & -44.44 \\
\hline \% IHP LGST PARTNER & 58.00 & 35.00 & 12.00 & -39.66 & -65.71 \\
\hline \% exp manufac. & $m$ & NA & $\mathbf{M}$ & - & - \\
\hline$X$ EXP AGR-MINE & 99.00 & 99.00 & MA & 0.00 & - \\
\hline X IHP RAUUFAC. & 96.00 & 89.00 & $\mathbf{M}$ & -7.29 & - \\
\hline \% IHP AGR-MINE & 3.00 & 10.00 & $M$ & 233.33 & - \\
\hline TOTAL EXPORTS & 107.00 & 276.00 & 435.00 & 157.94 & 57.61 \\
\hline TOTAL IMPORTS & 21.00 & 132.00 & 437.00 & 528.57 & 231.06 \\
\hline G.M.P. PER CAPITA & NA & NA & 220.00 & - & - \\
\hline
\end{tabular}


URUGUAY

SUMHARY OF MATIONAL STATISTICS

\begin{tabular}{|c|c|c|c|c|c|}
\hline MATIONAL VARIABLES & 1962 & 1970 & 1980 & $62-70$ & $70-80$ \\
\hline EXP. PARTNER CON. & 0.44 & 0.39 & 0.41 & -11.36 & 5.13 \\
\hline IMP. PARTNER CON. & 0.43 & 0.43 & 0.43 & 0.00 & 0.00 \\
\hline EPC/IPC RATIO & 1.01 & 0.90 & 0.95 & -10.89 & 5.56 \\
\hline EXP COHH CONCEN & 0.48 & 0.31 & 0.23 & -35.42 & -25.81 \\
\hline IMP COHI CONCEN & 0.12 & 0.11 & 0.16 & -8.33 & 45.45 \\
\hline ECC/ICC RATIO & 4.00 & 2.82 & 1.44 & -29.55 & -48.99 \\
\hline VERTICAL TRADE & -0.57 & -0.40 & -0.18 & -29.82 & -55.00 \\
\hline TOT TRADE LINKAGES & 20.00 & 27.00 & 40.00 & 35.00 & 48.15 \\
\hline \% AGRICULTURE-GDP & 18.00 & 13.00 & 10.20 & -27.78 & -21.54 \\
\hline QUALITY OF LIFE & 81.80 & 84.20 & 88.10 & 2.93 & 4.63 \\
\hline X LRGST EXP TO GDP & 2.70 & 0.80 & 2.20 & -70.37 & 175.00 \\
\hline X EXP LGST PARTNER & 16.00 & 13.00 & 18.00 & -18.75 & 38.46 \\
\hline X IHP LGST PARTMER & 21.00 & 16.00 & 18.00 & -23.81 & 12.50 \\
\hline Z EXP MANUFAC. & 4.00 & 20.00 & 38.00 & 400.00 & 90.00 \\
\hline \% EXP AGR-MINE & 95.00 & 79.00 & 61.00 & -16.84 & -22.78 \\
\hline z IhP manufac. & 61.00 & 60.00 & 56.00 & -1.64 & -6.67 \\
\hline * IMP AGR-MINE & 38.00 & 39.00 & 43.00 & 2.63 & 10.26 \\
\hline TOTAL EXPORTS & 150.00 & 232.00 & 1048.00 & 54.67 & 351.72 \\
\hline TOTAL IHPORTS & 207.00 & 241.00 & 1701.00 & 16.43 & 605.81 \\
\hline G.N.P. PER CAPIT & NA & NA & 2820.00 & - & - \\
\hline
\end{tabular}


VENEZUELA

SUMHARY OF NATIONAL STATISTICS

\begin{tabular}{|c|c|c|c|c|c|}
\hline MATIOHAL VARIABLES & 1962 & 1970 & 1980 & $62-70$ & $70-80$ \\
\hline EXP. PARTNER CON. & 0.55 & 0.55 & 0.50 & $0 . \infty 0$ & -9.09 \\
\hline IHP. PARTNER CON. & 0.59 & 0.57 & 0.54 & -3.39 & -5.26 \\
\hline EPC/IPC RATIO & 0.92 & 0.97 & 0.93 & 5.43 & -4.12 \\
\hline EXP COHH CONCEN & 0.80 & 0.89 & 0.92 & 11.25 & 3.37 \\
\hline IHP COHM CONCEN & 0.31 & 0.32 & 0.50 & 5.23 & -6.25 \\
\hline ECC/ICC RATIO & 2.58 & 2.78 & 3.07 & 7.77 & 10.26 \\
\hline VERTICAL TRADE & -0.72 & -0.81 & -0.77 & 12.50 & -4.94 \\
\hline TOT TRADE LINKAGES & 44.00 & 44.00 & 54.00 & $0 . \infty$ & 22.73 \\
\hline X AGRICULTURE-GDP & 6.00 & 6.50 & 5.90 & 8.33 & -9.23 \\
\hline QUALITY OF LIFE & 59.50 & 72.80 & 80.80 & 22.35 & 10.99 \\
\hline$X$ LRGST EXP TO GDP & 11.70 & 9.80 & 12.50 & -16.24 & 27.55 \\
\hline X EXP LGST PARTNER & 34.00 & 38.00 & 28.00 & 11.76 & -26.32 \\
\hline \% ImP lgst partiner & 53.00 & 47.00 & 45.00 & -11.32 & -4.26 \\
\hline X EXP MANUFAC. & 6.00 & 1.00 & 1.00 & -83.33 & 0.00 \\
\hline$X$ EXP AGR-MINE & 93.00 & 98.00 & 98.00 & 5.38 & $0 . \infty$ \\
\hline x IAP MANUFAC. & 78.00 & 82.00 & 79.00 & 5.13 & -3.66 \\
\hline X IMP AGR-MINE & 21.00 & 17.00 & 20.00 & -19.05 & 17.65 \\
\hline TOTAL EXPORTS & 2570.00 & 3148.00 & 19026.00 & 22.49 & 504.38 \\
\hline TOTAL IMPORTS & 880.00 & 1610.00 & 10115.00 & 82.95 & 528.26 \\
\hline G.N.P. PER CAPITA & MA & MA & 4220.00 & - & - \\
\hline
\end{tabular}


VIET MNN

SUMMARY OF NATIONAL STATISTICS

\begin{tabular}{|c|c|c|c|c|c|}
\hline MATIONAL VARIABLES & 1962 & 1970 & 1980 & $62-70$ & $70-80$ \\
\hline EXP. PARTNER CON. & 0.60 & 0.65 & 0.56 & 8.33 & -13.85 \\
\hline IMP. PARTNER CON. & 0.62 & 0.59 & 0.44 & -4.84 & -25.42 \\
\hline EPC/IPC RATIO & 0.97 & 1.09 & 1.27 & 12.37 & 16.51 \\
\hline EXP CONH CONCEN & M & MA & M & - & - \\
\hline IMP COAH CONCEN & $\mathbf{M}$ & MA & Ma & - & - \\
\hline ECC/ICC RATIO & MA & MA & MA & - & - \\
\hline VERTICAL TRADE & $\mathbf{M}$ & MA & M & - & - \\
\hline TOT TRADE LINKAGES & 8.00 & 1.00 & 12.00 & -87.50 & 1100.00 \\
\hline X AGRICULTURE-GDP & Ma & NA & Ma & - & - \\
\hline QUALITY OF LIFE & NA & NA & M & - & - \\
\hline X LRGST EXP TO GDP & 0.60 & 0.05 & Ma & - & - \\
\hline X EXP LGST PARTNER & 41.00 & 49.00 & 35.00 & 19.51 & -28.57 \\
\hline X IMP LGST PARTNER & 40.00 & 42.00 & 15.00 & 5.00 & -64.29 \\
\hline Z EXP MANUFAC. & MA & MA & MA & - & - \\
\hline \% EXP AGR-HINE & NA & Ma & MA & - & - \\
\hline$\chi$ IHP RANUFAC. & NA & Ma & NA & - & - \\
\hline * IHP AGR-MINE & NA & NA & NA & - & - \\
\hline TOTAL EXPORTS & 49.00 & 7.00 & 124.00 & -85.71 & 1671.43 \\
\hline TOTAL IMPORTS & 259.00 & 834.00 & 721.00 & 222.01 & -13.55 \\
\hline G.H.P. PER CAPITA & MA & NA & 150.00 & - & - \\
\hline
\end{tabular}


YEMEN, ARA

SUMMARY OF MATIONAL STATISTICS

\begin{tabular}{|c|c|c|c|c|c|}
\hline MATIONAL VARIABLES & 1962 & 1970 & 1980 & $62-70$ & $70-80$ \\
\hline EXP. PARTNER CON. & 0.70 & 0.77 & 0.60 & 10.00 & -22.08 \\
\hline IHP. PARTNER CON. & 0.88 & 0.45 & 0.47 & -48.86 & 4.44 \\
\hline EPC/IPC RATIO & 0.79 & 1.70 & 1.27 & 115.19 & -25.29 \\
\hline EXP CONH CONCEN & MA & 0.63 & Ma & - & - \\
\hline IMP CONM CONCEN & MA & 0.39 & Ma & - & - \\
\hline ECC/ICC RATIO & MA & 1.62 & M & - & - \\
\hline VERTICAL TRADE & NA & -0.29 & NR & - & - \\
\hline TOT TRADE LINKAGES & 4.00 & 1.00 & 19.00 & -75.00 & 1800.00 \\
\hline X AGRICULTURE-GDP & NA & NA & 15.80 & - & - \\
\hline QUALITY OF LIFE & MA & MA & 32.00 & - & - \\
\hline X LRGST EXP TO GDP & 20.60 & 5.60 & M & -72.82 & - \\
\hline X EXP LGST PARTNER & 28.00 & 52.00 & 33.00 & 85.71 & -36.54 \\
\hline X IHP LGST PARTNER & 78.00 & 16.00 & 28.00 & -79.49 & 75.00 \\
\hline X EXP MANUFAC. & NA & NA & NA & - & - \\
\hline$X$ EXP AGR-MINE & MA & 99.00 & NA & - & - \\
\hline X imp hanufac. & MA & 28.00 & MA & - & - \\
\hline * IHP AGR-MINE & Ma & $71 . \infty 0$ & $\mathbf{M}$ & - & - \\
\hline TOTAL EXPORTS & $6 . \infty 0$ & 2.00 & 511.00 & -66.67 & 25450.00 \\
\hline TOTAL IMPORTS & 11.00 & 42.00 & 1539.00 & 281.82 & 3564.29 \\
\hline G.N.P. PER CAPITA & NA & NA & 500.00 & - & - \\
\hline
\end{tabular}


YEMEN, PEO

SUHMARY OF NATIONAL STATISTICS

\begin{tabular}{|c|c|c|c|c|c|}
\hline MATIOMAL VARIABLES & 1962 & 1970 & 1980 & $62-70$ & $70-80$ \\
\hline EXP. PARTNER CON. & 0.50 & 0.47 & 0.60 & -6.00 & 27.66 \\
\hline IMP. PARTNER CON. & 0.44 & 0.41 & 0.39 & -6.82 & -4.88 \\
\hline EPC/IPC RATIO & 1.13 & 1.14 & 1.55 & 0.88 & 35.96 \\
\hline EXP COHN CONCEN & MA & MA & 0.26 & - & - \\
\hline IHP COHH CONCEN & M & $\mathbf{M A}$ & 0.27 & - & - \\
\hline ECC/ICC RATIO & Ma & NA & 0.96 & - & - \\
\hline VERTICAL TRADE & Ma & MA & -0.13 & - & - \\
\hline TOT TRADE LINKAGES & 15.00 & 20.00 & 6.00 & 33.33 & -70.00 \\
\hline X AGRICULTURE-GDP & MA & 52.20 & 33.80 & - & -35.25 \\
\hline QUALITY OF LIFE & Ma & 18.20 & 16.50 & - & -9.34 \\
\hline \% LRGST EXP TO GDP & MA & $\boldsymbol{M}$ & $\boldsymbol{M}$ & - & - \\
\hline X EXP LGST PARTNER & 40.00 & 27.00 & 43.00 & -32.50 & 59.26 \\
\hline X IMP LGST PARTHER & 21.00 & 19.00 & 17.00 & -9.52 & -10.53 \\
\hline \% EXP manufac. & MA & NA & 50.00 & - & - \\
\hline X EXP AGR-MINE & MM & MA & 49.00 & - & - \\
\hline X IMP MANUFAC. & NA & NA & 63.00 & - & - \\
\hline X IMP AGR-MINE & NA & NA & 36.00 & - & - \\
\hline TOTAL EXPORTS & 86.00 & 131.00 & 22.00 & 52.33 & -83.21 \\
\hline TOTAL IMPORTS & 216.00 & 168.00 & 1639.00 & -22.22 & 875.60 \\
\hline G.N.P. PER CAPITA & NA & NA & 460.00 & - & - \\
\hline
\end{tabular}


YUGOSLAVIA

SUMMARY OF MATIONAL STATISTICS

\begin{tabular}{|c|c|c|c|c|c|}
\hline MATIONAL VARIABLES & 1962 & 1970 & 1980 & $62-70$ & $70-80$ \\
\hline EXP. PARTNER CON. & 0.36 & 0.38 & 0.40 & 5.56 & 5.26 \\
\hline IMP. PARTNER CON. & 0.39 & 0.39 & 0.39 & 0.00 & $\mathbf{0 . 0 0}$ \\
\hline EPC/IPC RATIO & 0.93 & 0.98 & 1.02 & 5.38 & 4.08 \\
\hline EXP CONH CONCEN & 0.07 & 0.09 & 0.16 & 28.57 & 7.78 \\
\hline IMP COHH CONCEN & 0.12 & 0.16 & 0.11 & 33.33 & -31.25 \\
\hline ECC/ICC RATIO & 0.58 & 0.56 & 1.45 & -3.57 & 158.59 \\
\hline VERTICAL TRADE & -0.07 & -0.10 & 0.16 & 42.86 & -260.00 \\
\hline TOT TRADE LINKAGES & 42.00 & 54.00 & 84.00 & 28.57 & 55.56 \\
\hline X AGRICULTURE-GDP & 26.00 & 21.40 & 13.20 & -17.69 & -38.32 \\
\hline QUALITY OF LIFE & 67.80 & 78.70 & 85.80 & 16.08 & 9.02 \\
\hline X LRGST EXP TO GDP & NA & NA & MA & - & - \\
\hline ※ EXP LGST PARTNER & 14.00 & 15.00 & 28.00 & 7.14 & 86.67 \\
\hline X IHP LGST PARTNER & 17.00 & 22.00 & 19.00 & 29.41 & -13.64 \\
\hline$X$ EXP HANUFAC. & 49.00 & 59.00 & 73.00 & 20.41 & 23.73 \\
\hline X EXP AGR-MINE & 40.00 & 40.00 & 26.00 & 0.00 & -35.00 \\
\hline$X$ IAP MANUFAC. & 69.00 & 69.00 & 56.00 & 13.11 & -18.84 \\
\hline * IHP AGR-MINE & 38.00 & 30.00 & 43.00 & -21.05 & 43.33 \\
\hline TOTAL EXPORTS & 677.00 & 1678.00 & 8873.00 & 147.86 & 428.78 \\
\hline TOTAL IMPORTS & 865.00 & 2824.00 & 14693.00 & 226.47 & 420.29 \\
\hline G.N.P. PER CAPIT & NA & NA & 2790.00 & - & - \\
\hline
\end{tabular}

